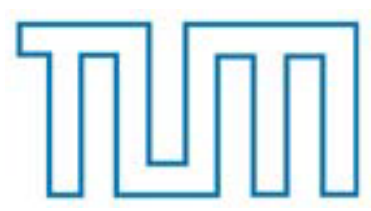

TECHNISCHE UNIVERSITÄT MÜNCHEN

Ingenieurfakultät Bau Geo Umwelt

Lehrstuhl für Ingenieurgeologie

\title{
Abbauwerkzeugverschleiß und empirische Verschleißprognose beim Vortrieb mit Hydroschild TVM in Lockergesteinen
}

\section{Florian Köppl}

Vollständiger Abdruck der von der Ingenieurfakultät Bau Geo Umwelt der Technischen Universität München zur Erlangung des akademischen Grades eines Doktor-Ingenieurs (Dr.-Ing.) genehmigten Dissertation.

Vorsitzende(r):

Prüfer der Dissertation:
Univ.-Prof. Dr.-Ing. habil. Gerhard H. Müller

1. Univ.-Prof. Dr. rer. nat. Kurosch Thuro

2. Univ.-Prof. Dr.-Ing. Markus Thewes,

Ruhr-Universität Bochum

Die Dissertation wurde am 18.03.2014 bei der Technischen Universität München eingereicht und durch die Ingenieurfakultät Bau Geo Umwelt am 27.06.2014 angenommen. 



\section{Zusammenfassung}

Der Verschleiß der Abbauwerkzeuge ist eine zentrale Herausforderung beim Vortrieb mit Hydroschild Tunnelvortriebsmaschinen (TVM) in Lockergesteinen, weil dieser eine häufige Ursache für Vortriebsunterbrechungen darstellt. Dabei sind die Kosten für die Vortriebsunterbrechungen zur Inspektion und für den Wechsel der Abbauwerkzeuge insbesondere bei Druckluftarbeiten in der Abbaukammer häufig um ein vielfaches höher als die Kosten für die zu wechselnden Abbauwerkzeuge. Zusätzlich liegen aufgrund der Unzugänglichkeit der beteiligten Prozesse und der hohen Bandbreite an Einflussfaktoren bisher keine zuverlässigen Prognosemethoden für die Standgrößen der Abbauwerkzeuge, die Anzahl an Werkzeugwechseln und die dafür benötigten Vortriebsunterbrechungen vor.

Die Ziele der vorliegenden Arbeit sind deshalb für Vortriebe mit Hydroschild TVM in Lockergesteinen:

- Identifikation und Quantifizierung der maßgeblichen Einflussfaktoren auf die Standgrößen der Abbauwerkzeuge.

- Aufbau eines Prognosemodells für den Verschleiß der Abbauwerkzeuge.

Als Grundlage für die Datenanalyse konnten in 10 Referenzprojekten mit 13 Hydroschild TVM mit einem Durchmesser von 6,3-15,4 m in einer Vortriebsstrecke von 45,4 km und einem Ausbruchvolumen von ca. 5.6 Mio. $\mathrm{m}^{3}$ in Summe 5.850 Werkzeugwechsel recherchiert und dokumentiert werden. Durch eine empirische, prozessorientierte Analyse dieser Daten in Kombination mit Baustellenversuchen können die Haupteinflussfaktoren auf den Primär- und Sekundärverschleiß der Abbauwerkzeuge aus den Boden- und Gebirgseigenschaften, der Auslegung der Hydroschild TVM und aus der Steuerung des Abbauprozesses qualifiziert und weitgehend quantifiziert werden.

Zentraler Bestandteil der Ergebnisse ist die Entwicklung des neuen Soil Abrasivity Index (SAI) zur Beschreibung der Abrasivität des Bodens im Vortrieb. Der SAl weist sehr gute Korrelationen mit dem Laufweg (Standweg) der Abbauwerkzeuge auf, die als Ausgangspunkt zur Entwicklung eines empirischen Prognosemodells für den Verschleiß der Abbauwerkzeuge genutzt werden können. Nach Korrektur der Einflussfaktoren aus der Auslegung der TVM und der Steuerung des Abbauprozesses ermöglicht das vorgestellte Modell:

- Schätzung des Laufwegs (Standweg) der Abbauwerkzeuge.

- Schätzung der Anzahl der verschleißbedingten Werkzeugwechsel.

- Schätzung der Stationierung und Anzahl der Vortriebsunterbrechungen für Werkzeugwechsel.

- Baubetriebliche Anpassung der Stationierung der Vortriebsunterbrechungen an die Rahmenbedingungen im Projekt für den Zugang zur Abbaukammer.

Die Validierung des Prognosemodells liefert in Bezug auf die Referenzprojekte gute Ergebnisse, zeigt jedoch auch den Bedarf für weitere Untersuchungen auf, insbesondere zur Entstehung und Quantifizierung von Schäden an den Abbauwerkzeugen durch Steine und Blöcke im Boden.

Die Identifikation der Haupteinflussfaktoren auf den Verschleiß der Abbauwerkzeuge in der Datenanalyse bietet für zukünftige Projekte wertvolle Anhaltspunkte für die geotechnische Vorerkundung und Dokumentation im Vortrieb. Das Prognosemodell bietet den beteiligten Bauherren (AG), Bauunternehmungen (AN) und Maschinenlieferanten eine erhebliche Verbesserung der Diskussionsgrundlage für die Planung und Steuerung des Vortriebsablaufs und erlaubt die Berücksichtigung unterschiedlicher Projektziele im Hinblick auf die Verschleißkosten, Vortriebsdauer und Planungssicherheit im Projekt. 


\section{Abstract}

Wear of the cutting tools is a major challenge in Hydroshield tunnelling in soft ground, because it is a common reason for maintenance stops. Thereby the costs for the maintenance stops for inspection and changing of the cutting tools often exceed the costs for the changed cutting tools, especially in case of hyperbaric works in the excavation chamber of the TBM. However, due to the inaccessibility of the processes involved and the variety of influencing factors, there are no prognosis tools available, for reliable estimation of the cutting tool life, the number of tool changes and the required maintenance stops.

Hence, this thesis pursues the following objectives for Hydroshield TBM in soft ground:

- Identification and quantification of the main influencing factors on the life of the cutting tools.

- Development of a prognosis model for the wear of the cutting tools.

As a basis for the intended analysis a total of 5.860 tool changes in 10 reference projects using 13 hydroshield TBM in a diameter range of $6.3-15.4 \mathrm{~m}$ over an advance distance of $45.4 \mathrm{~km}$ and a excavation volume of 5.6 mio. $\mathrm{m}^{3}$ were researched and documented. The empiricla and process-oriented analysis of these data in combination with on-site test results in a qualification and quantification of the main influencing factors on primary and secondary wear of the cutting tools, originating from the properties of the soil, the design of the TBM and the excavation process (TBM operation). A major result of the analysis is the new Soil Abrasivity Index (SAI) for the description of the abrasivity of the soil in actual TBM advance. The SAI shows very good correlations with the life (cutting distance) of the cutting tools, which are used as starting point for the development of a prognosis model. Considering the influencing factors originating from TBM design and operation the developed prognosis model enables:

- Estimation of the cutting distance (tool life) of the cutting tools.

- Estimation of the required number of cutting tool changes.

- Estimation of the number and chainage of maintenance stops for cutting tool inspection and changes.

- Adoption of the chainage of the maintenance stops considering construction operation and the boundary conditions for access to the excavation chamber of the TBM.

Validation results of the prognosis model are in good accordance with the actual data from the reference projects. However, the results also show further demand for research, focusing on the quantification of damages of the cutting tools, cause by cobbles and boulders in the soil.

The identification of the main influencing factors on the wear of the cutting tools in the data analysis gives valuable background for the preliminary geotechnical reconnaissance and documentation during advance in future projects using hydroshield TBMs. The prognosis model provides considerable impovement of the basis for discussion of the planning and actual construstion operation between the involved project owners, construction contractors and TBM suppliers. In addition the model allows for the consideration of different objectives in a project, in order to balance wear related costs, duration of the advance and planning reliability. 


\section{Vorwort \& Dank}

Die Idee zu dieser Arbeit und die Motivation zur Umsetzung entstanden aus der baupraktischen Erfahrung in maschinellen Tunnelvortrieben weltweit während meiner Tätigkeit bei der Herrenknecht AG. Im Gegensatz zu Vortrieben in Festgesteinen ergab sich bei Schildvortrieben in Lockergesteinen eine erhebliche Differenz zwischen der hohen Bedeutung des Verschleißes der Abbauwerkzeuge für die Vortriebsdauer und die Verschleißkosten und dem geringen Kenntnisstand zu den ursächlichen Mechanismen. Daraus entstand die Initiative mit der vorliegenden Arbeit einen Beitrag zur Erhöhung des Kenntnisstandes im Hinblick auf den Verschleiß der Abbauwerkzeuge beim Vortrieb mit Hydroschild TVM in Lockergesteinen zu leisten.

Für die Förderung dieser Idee, die Orientierung und tatkräftige Unterstützung in der Startphase gilt mein Dank in erster Linie Dr.-Ing. C. Frenzel (ehem. Herrenknecht AG).

Die Arbeit wurde an der Technischen Universität München als Dissertation angenommen und von Prof. Dr. rer. nat. K. Thuro am Lehrstuhl für Ingenieur- und Hydrogeologie in hervorragender Weise betreut. Für seine Initiative, langjährige Begleitung und Unterstützung bin ich zu außerordentlichem Dank verpflichtet. Mein Dank gilt darüber hinaus den Mitarbeitern des Lehrstuhls für Ingenieurgeologie, hier insbesondere Herrn Dr. rer. nat. H. Käsling für die Anregungen, Unterstützung und fachliche Diskussion der vorliegenden Arbeit.

Die zweite Prüfung der Arbeit hat Herr Prof. Dr.-Ing. M. Thewes an der Ruhr Universität Bochum mit Freude und hohem Engagement übernommen. Für seine Beratung, die fachliche Diskussion, wertvolle Hinweise und kritische Durchsicht der Arbeit bin ich zu großem Dank verpflichtet.

Die vorliegende Arbeit entstand neben meiner Tätigkeit als Projekt- und Abteilungsleiter im Bereich Field Service der Herrenknecht AG. Mein ausdrücklicher Dank für die Förderung und Unterstützung dieser Arbeit gilt in erster Linie Herrn Dr. E.h. M. Herrenknecht, Herrn Dipl.-Ing. G. Lehmann und Herrn Dipl.-Ing. G. Richter. Besonderer Dank für die langjährige Begleitung und die Schaffung der notwendigen Freiräume zur Erstellung der Arbeit gilt Herrn Dipl.-Ing. O. Boiger. Der Inhalt der Arbeit reflektiert die praktischen Erfahrungen zahlreicher Projektleiter, Maschinenmeister und Mineure in Projekten mit Hydroschild TVM weltweit. Erst der Erfahrungsaustausch mit innen hat die notwendige Grundlage für diese Arbeit geschaffen.

Meiner Frau Susanne und unserer Tochter Johanna Lina gilt mein Dank für den Rückhalt und die moralische Unterstützung, ohne die diese Arbeit nicht möglich gewesen wäre.

„Te saxa loquuntur.“ 


\section{Inhaltsverzeichnis}

Zusammenfassung.................................................................................................

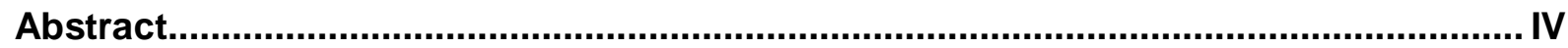

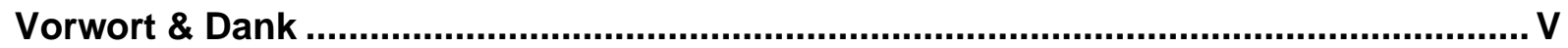

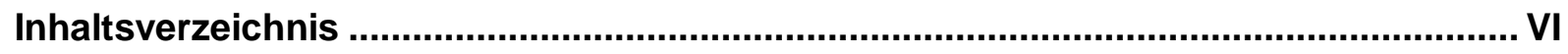

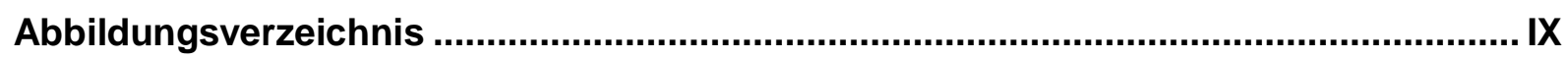

Tabellenverzeichnis ................................................................................................. XIII

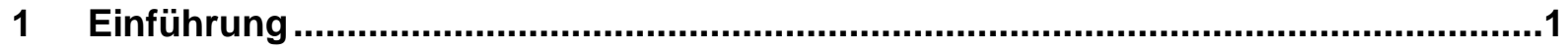

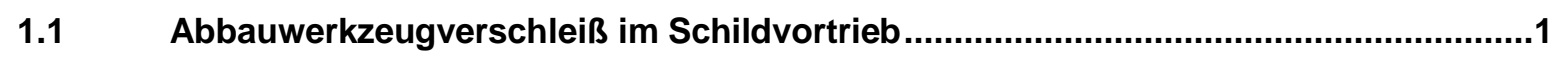

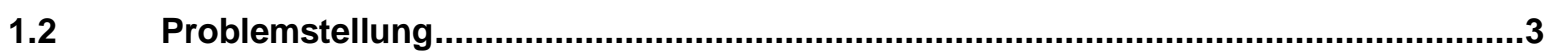

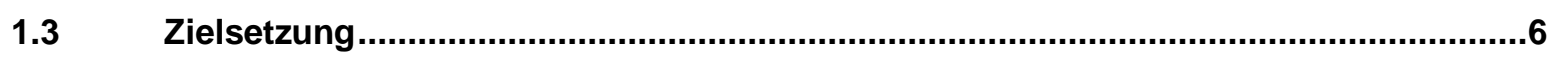

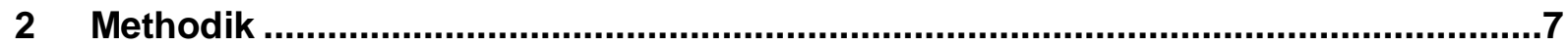

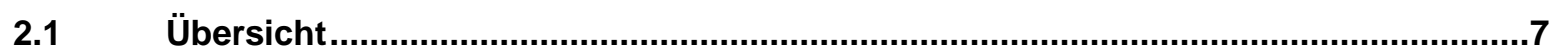

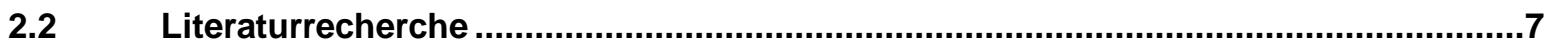

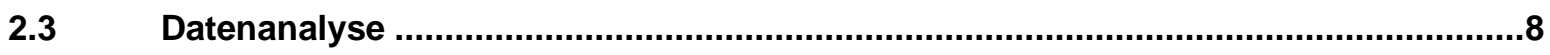

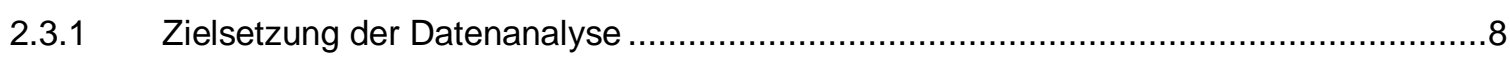

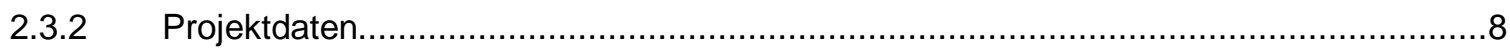

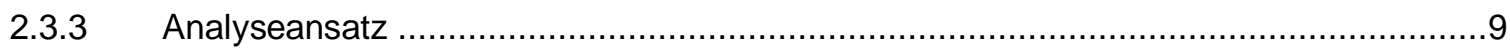

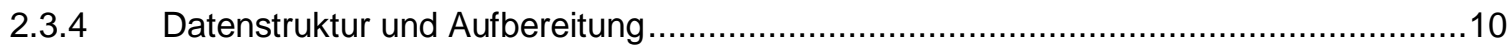

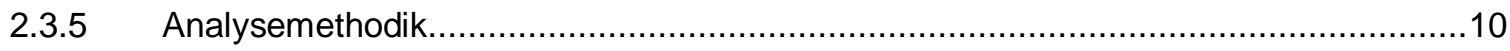

2.4 Aufbau des empirischen Prognosemodells .......................................................12

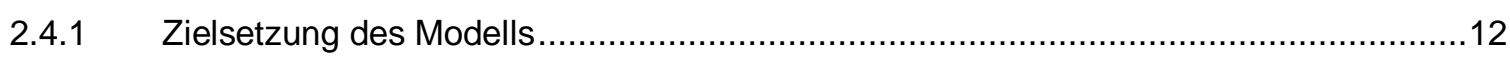

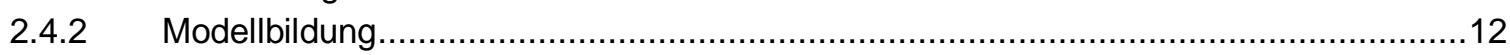

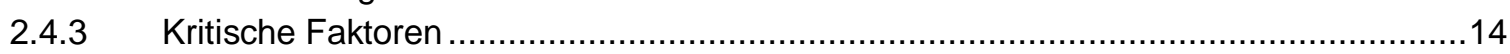

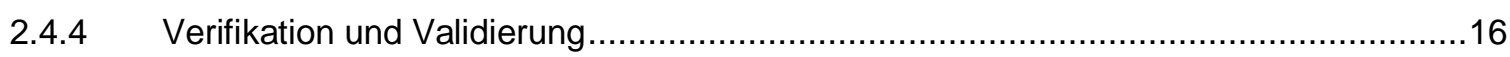

3 Hydroschild TVM Technik \& Vortrieb...................................................................18

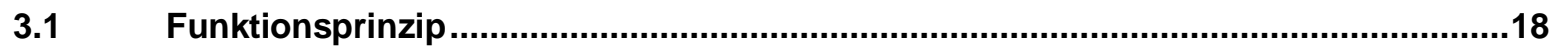

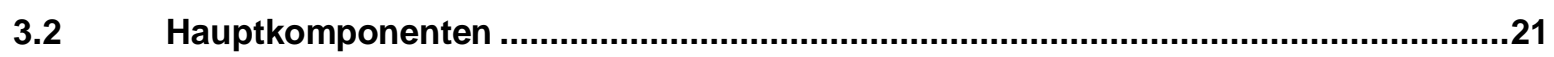

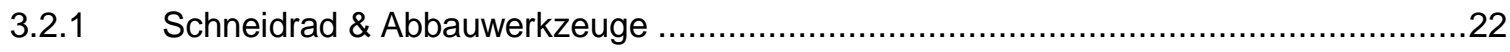

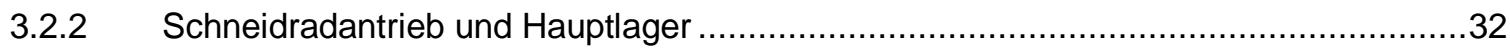

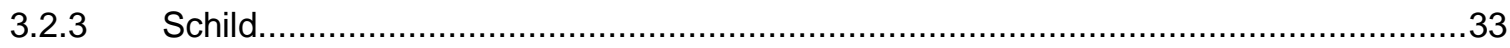

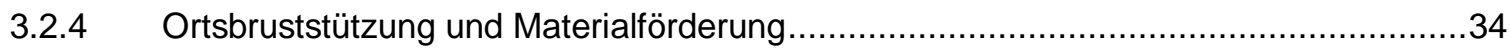

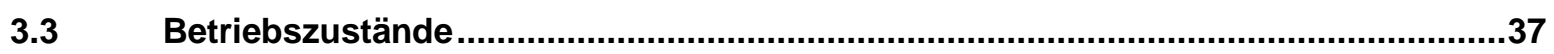

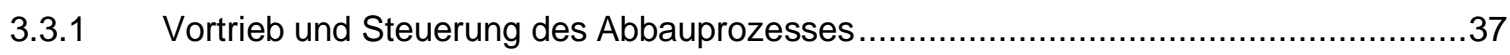

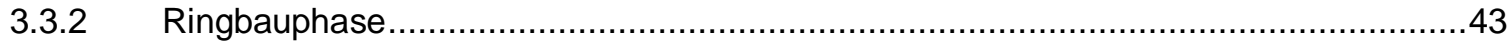

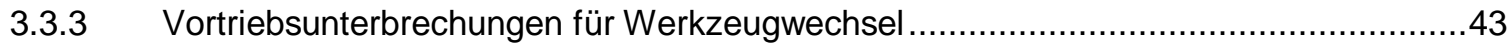

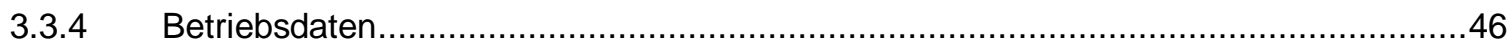

4 Wissensstand zur Abbaumechanik und zum Werkzeugverschleiß .........................48

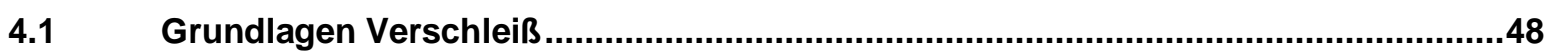

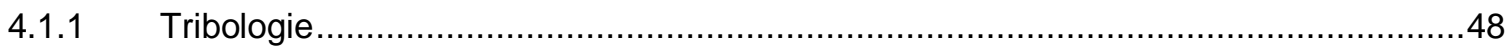

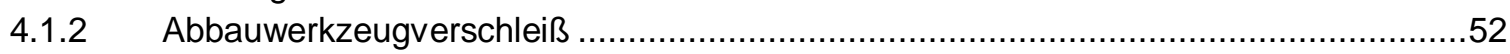




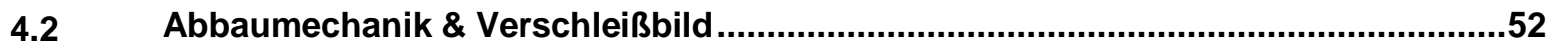

4.2.1 Modelle und Laborversuche zur Abbaumechanik...................................................53

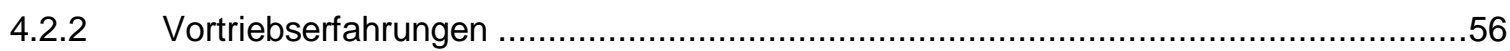

4.3 Maßgebliche Einflussfaktoren \& Verschleißkennwerte........................................59

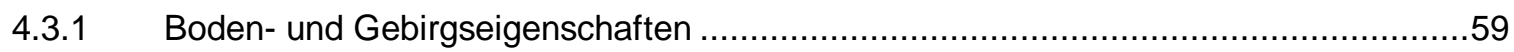

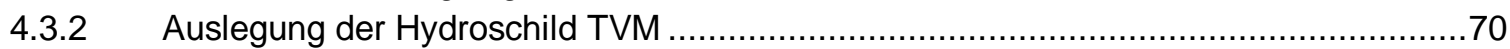

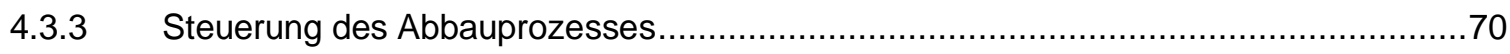

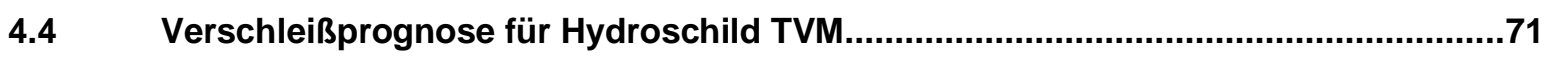

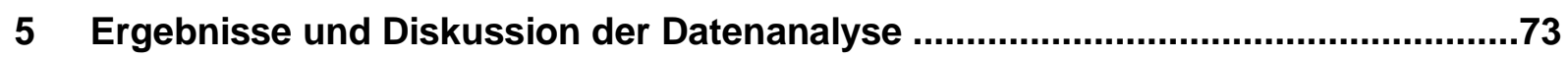

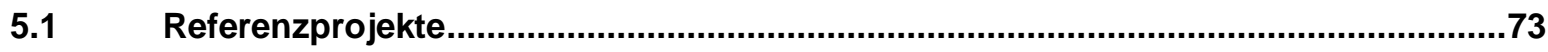

$5.2 \quad$ Dokumentation in den Referenzprojekten .............................................................

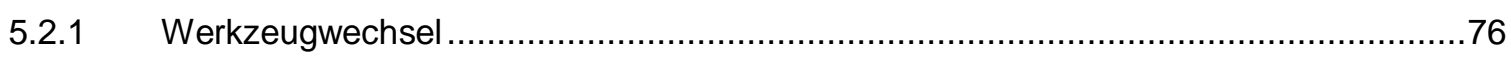

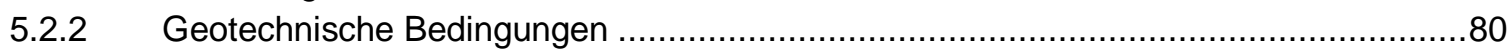

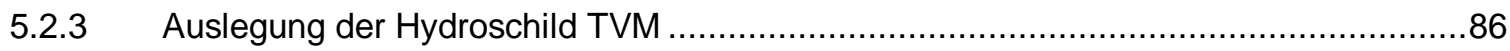

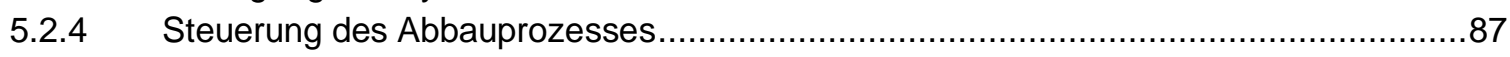

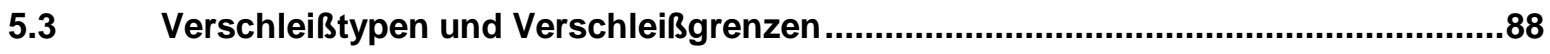

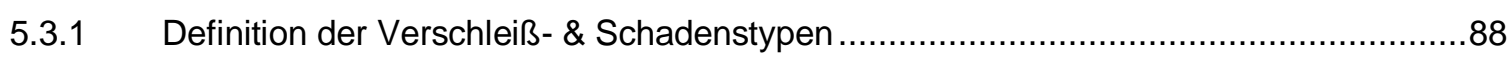

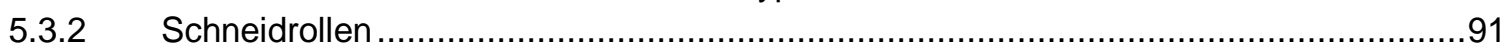

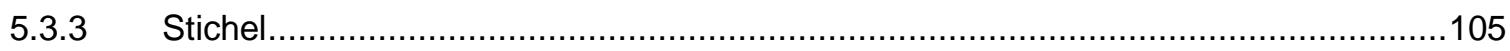

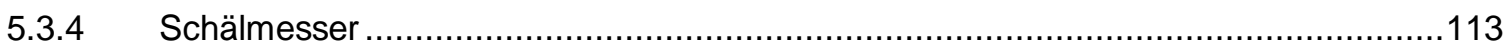

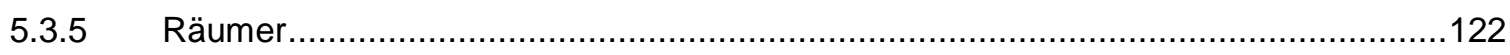

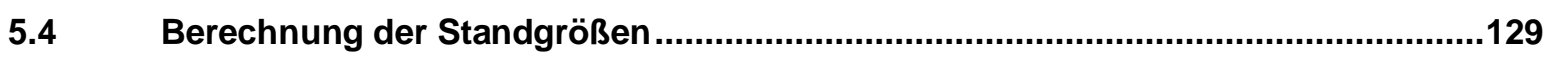

5.4.1 Definition der Standgrößen für Abbauwerkzeuge ...............................................129

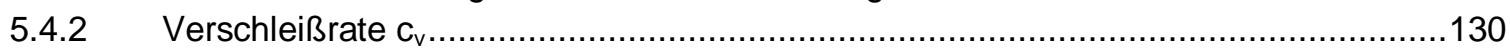

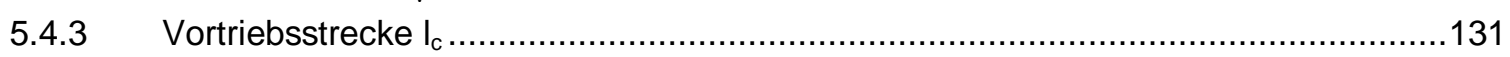

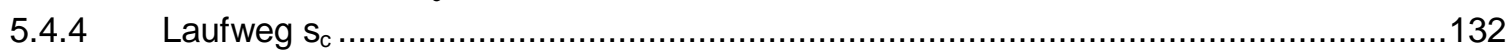

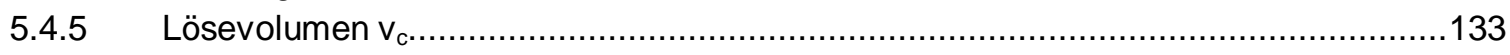

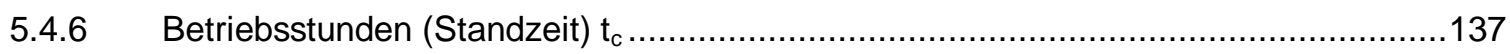

5.4.7 Auswahl der geeigneten Standgröße für die Datenanalyse ...................................138

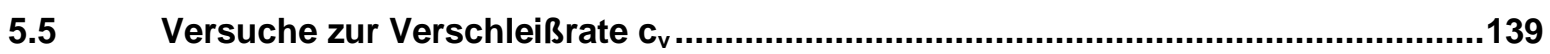

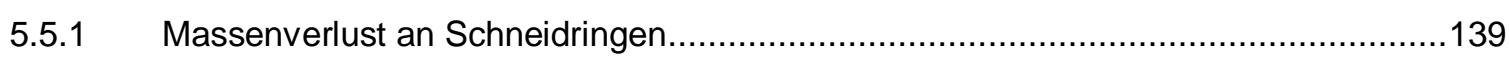

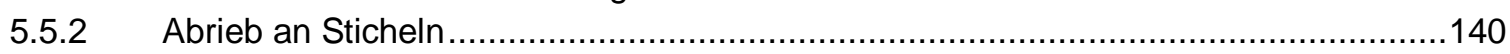

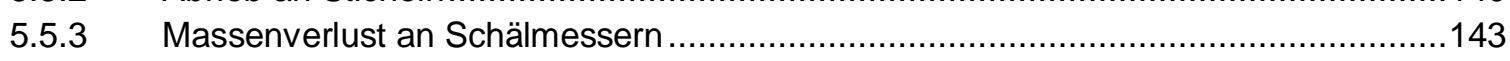

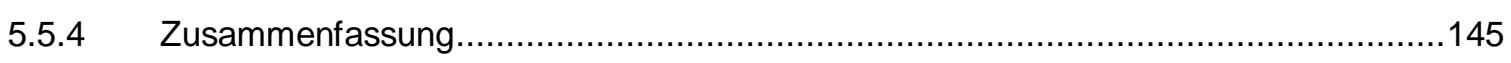

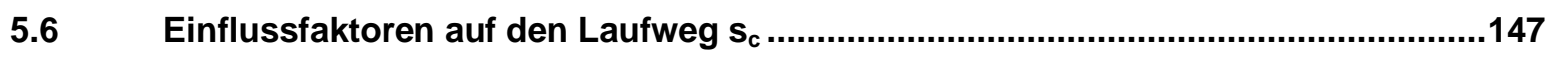

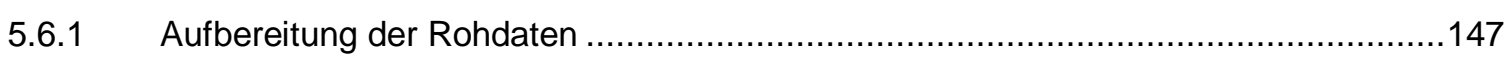

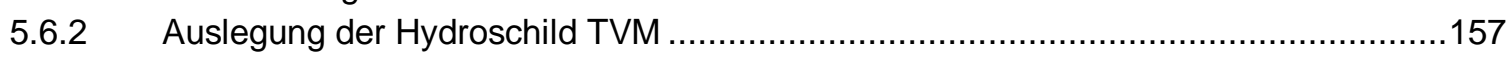

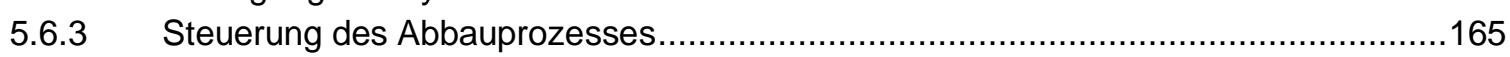

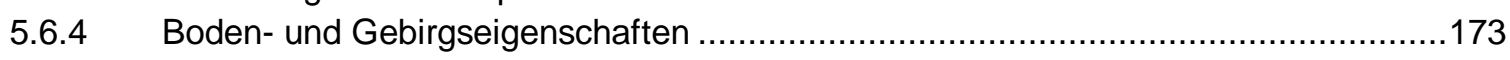

6 Empirische Verschleißprognose ...................................................................195

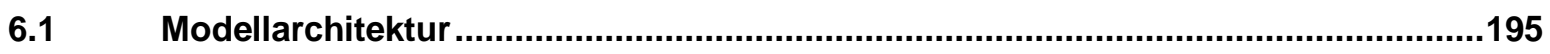

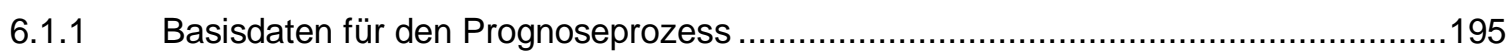

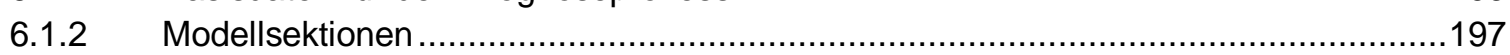

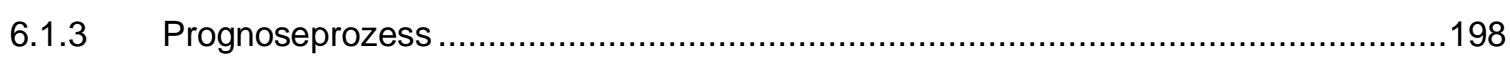




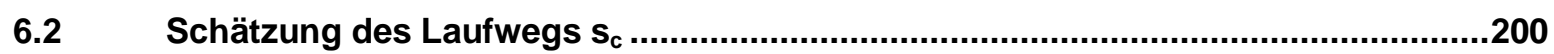

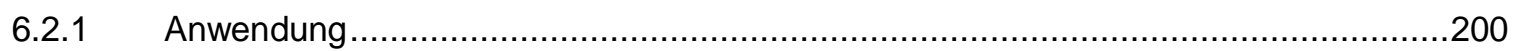

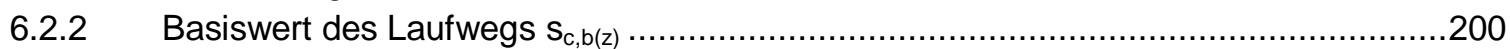

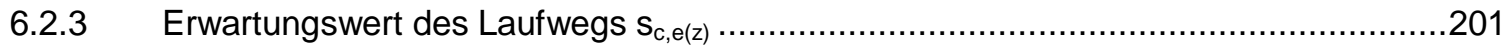

6.3 Prognose der Vortriebsunterbrechungen I für Werkzeugwechsel .........................206

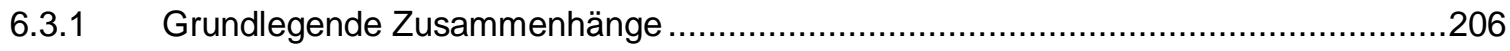

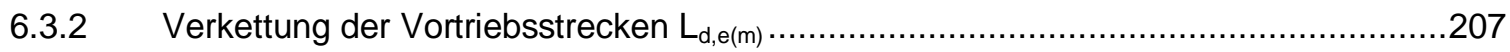

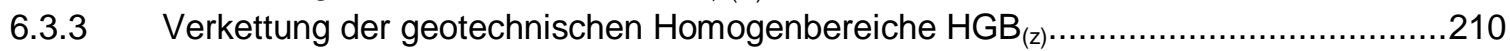

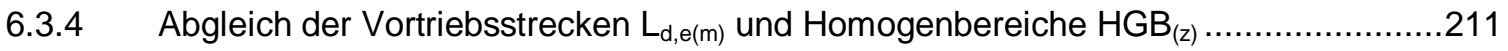

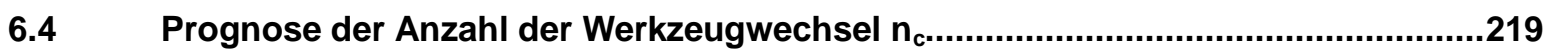

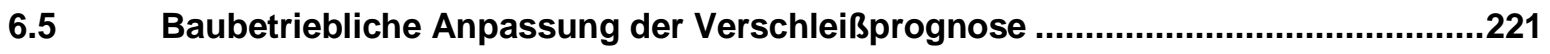

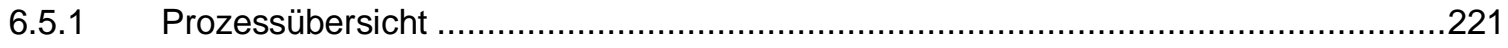

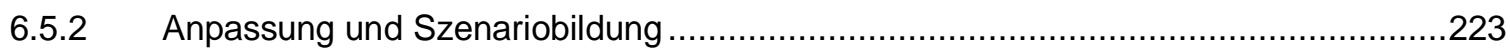

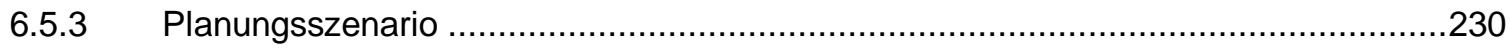

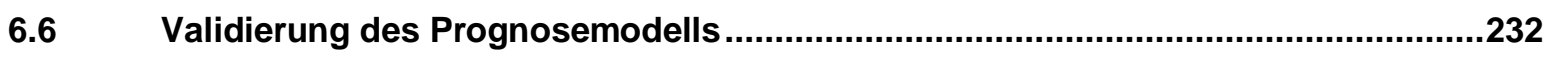

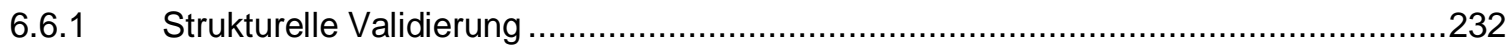

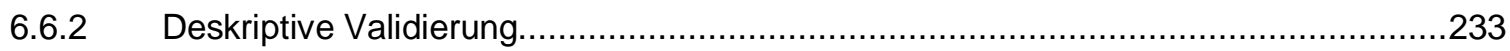

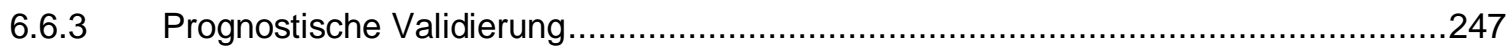

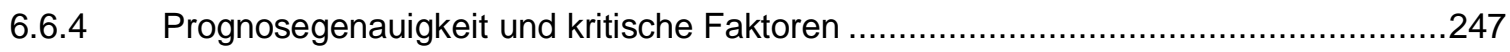

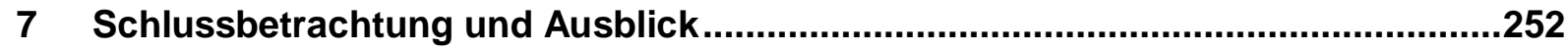

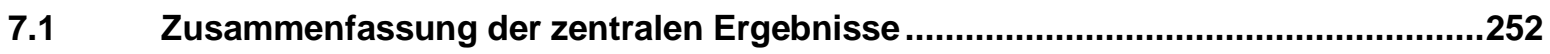

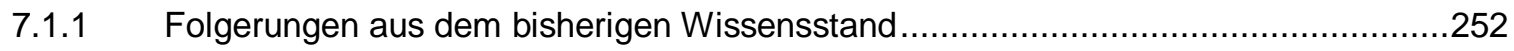

7.1.2 Eregbnisse aus der Analyse der 18 untersuchten Vortriebe ....................................252

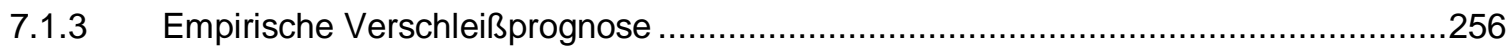

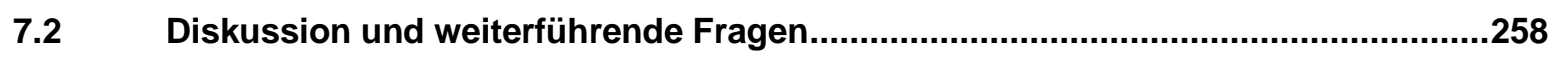

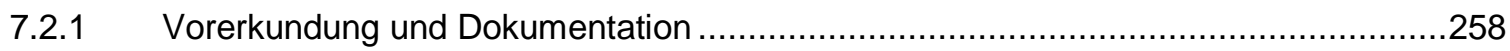

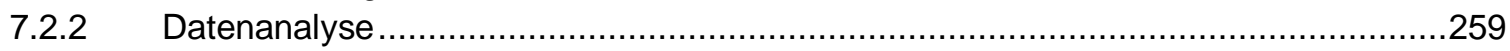

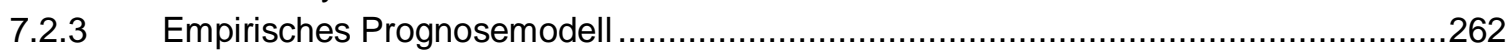

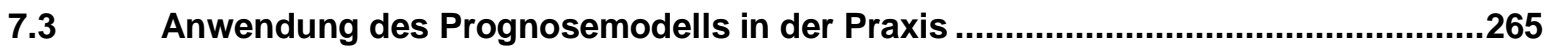

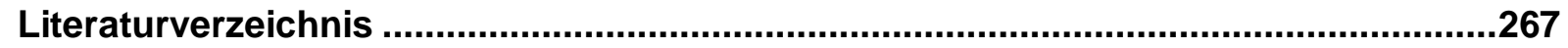

\section{Anhang: Abkürzungen \& Formlzeichen}




\section{Abbildungsverzeichnis}

Abb. 1: 3D-Schemazeichnung einer Hydroschild-TVM

Abb. 2: Blick auf die Ortsbrust durch eine Schneidradöffnung während eines Drucklufteinstiegs. (Herrenknecht AG) ...... 19

Abb. 3: Separationsanlage im Montagezustand. (Herrenknecht AG, verändert) 20

Abb. 4: Typischer Einsatzbereich für Hydroschild TVM anhand der Korngrößenverteilung des Bodens. (Herrenknecht AG, verändert)

Abb. 5: Sternförmiges Speichenschneidrad ohne umlaufende Felge...... 22

Abb. 6: Schneidrad mit umlaufender Felge. (Herrenknecht AG)

Abb. 7: Außenliegendes Felgenschneidrad mit begehbaren Armen für drucklosen Werkzeugwechsel. (Herrenknecht AG) .......23

Abb. 8: 3D-Darstellung einer Schneidrolle mit Befestigungselementen in einem Wedgelock-Gehäuse. (Herrenknecht AG,

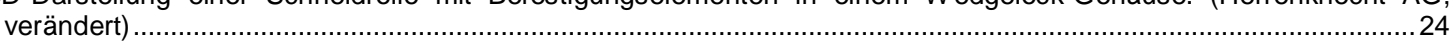

Abb. 9: Querschnitt durch eine 1-Ring Schneidrolle. (Herrenknecht AG, verändert)

Abb. 10: Querschnitt durch den Rollengrundkörper, Schneidring und Sicherungsring einer 1-Ring Schneidrolle.

Abb. 11: 3D-Darstellung dreier gängiger Schälmessertypen mit zugehörigem Halter und Befestigungselemente. (Herrenknecht AG, verändert)

Abb. 12: Querschnitt durch ein Schälmesser mit Halter und Schneidradstahlstruktur.

Abb. 13: 3D-Darstellung eines Stichels mit Befestigungselementen in einem Wedgelock-Gehäuse. (Herrenknecht AG,

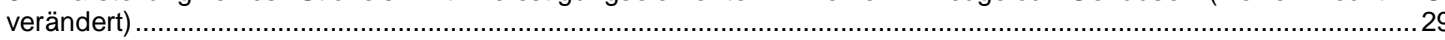

Abb. 14: 3D-Darstellung eines Räumersystems mit 4 Segmenten und zugehörigem Räumerhalter......

Abb. 15: Absolute Höhe bzw. Vorlauf von Schneidrollen $\left(h_{S R}\right)$, Stichel $\left(h_{S T}\right)$ und Schälmesser ( $\left.h_{S M}\right)$ in Bezug auf die

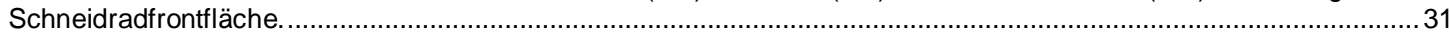

Abb. 16: Beispiel für die Kennkurve aus Schneidraddrehmoment $M_{\text {TVM }}$ [kNm] und Schneidraddrehzahl $U$ [min ${ }^{-1}$ ] eines Schneidradantriebs. (Herrenknecht AG, verändert)

Abb. 17: Schematische Darstellung des Flüssigkeitskreislaufs zwischen der Hydroschild TVM („Schildmaschine“) im Tunnel, der Leitungen und Pumpstationen in der bereits fertiggestellten Tunnelstrecke und der Separationsanlage auf der Baustelleneinrichtungsfläche („BE-Fläche“).

Abb. 18: Schematische Darstellung der Laufbahn der Abbauwerkzeuge im Vortrieb mit dem Spurradius $r_{s}$ [mm], der Penetration $\mathrm{p}[\mathrm{mm} / \mathrm{U}]$ und den anliegenden Schneidkräften $\mathrm{F}_{\mathrm{TC}}[\mathrm{kN}]$ und $\mathrm{F}_{\mathrm{SC}}[\mathrm{kN}]$.

Abb. 19: Schematischer Längsschnitt durch eine Hydroschild TVM mit in bzw. gegen die Vortriebsrichtung anliegenden Widerstands- und Kraftkomponenten im Vortrieb.

Abb. 20: Graphische Darstellung der maximalen Arbeitszeit unter Druckluft (rot) und zugehörige Ausschleusungszeit (blau) in Abhängigkeit des Arbeitsdrucks aus der Verordnung über Arbeiten in Druckluft.

Abb. 21: Allgemeine Beschreibung eines triologischen Systems mit Input, Output, Störungen und durch die Störungen verursachte Verluste. (ZUM GAHR 1981: 81; verändert)

Abb. 22: Schematische Darstellung der vier physikalischen Mechanismen, die bei Abrasion zum Abtrag von Material an der Oberfläche des Grundkörpers führen. (ZUM GAHR 1981: 96, verändert)

Abb. 23: Intensität des abrasiven Verschleißes in Abhängigkeit der Härte der abrasiven Partikel im Verhältnis zur Härte des Grundkörpers.

Abb. 24: Bruchmechanismus und Zerkleinerungsgrad des Bodens in Abhängigkeit der Korngrößenverteilung des Bodens, der Dichte, der Plastizität und des Wassergehalts. (BERETITSCH 1992: 57, verändert)..... 
Abb. 25: Spannungsverteilung an der Frontfläche der Schneide in Abhängigkeit des Schneidwinkels $\alpha_{c}\left[{ }^{\circ}\right.$ und der Schneidgeschwindigkeit $\mathrm{v}_{\mathrm{sc}}[\mathrm{m} / \mathrm{s}]$. (BERETITSCH 1992: 106, verändert)

Abb. 26: Abbauverhalten eines Blocks in einer Matrix durch Schneidrollen in Abhängigkeit des Festigkeitsverhältnisses zwischen Block und Matrix und dem bereits abgebauten Anteil des Blocks. (KIEFFER et al. 2008: 135, verändert).....55

Abb. 27: Qualitative Einschätzung des Verklebungspotentials von bindigen Böden mit der Konsistenzzahl $I_{C}[-]$ und der Plastizitätszahl IP [\%]. (HOLLMANN \& THEWES 2011a: 239, verändert) ......................................................................57

Abb. 28: Grafische Darstellung der drei Kategorien an Einflussfaktoren auf Leistung und Verschleiß im Tunnelvortrieb nach THURO (2002: 1), übertragen auf den Vortrieb mit Hydroschild TVM in Lockergesteinen.

Abb. 29: Korrelation des Werkzeugverbrauchs [Stk./m] mit dem Schneidraddrehmoment $M_{\text {TVM }}$ [MNm]. (WENDL 2012: 85; verändert)......

Abb. 30: Abhängigkeit des LCPC Abrasivitätskoeffizienten LAK [g/t] von der Korngröße $D_{50}[\mathrm{~mm}]$ für verschiedene Lockergesteine. (THURO \& KÄSLING 2009a: 185)

Abb. 31: Korrelation des Cerchar Abrasivitäts Index CAl mit dem Äquivalenten Quarzgehalt ÄQu [\%].

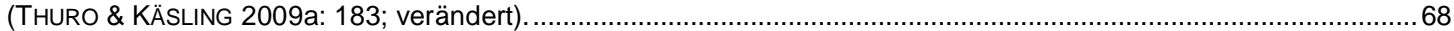

Abb. 32: Korrelation des LCPC Abrasivitätskoeffizienten (LAK) mit dem Cerchar Abrasivitäts Index (CAI). (THURO \& KÄSLING 2009a: 183, verändert).

Abb. 33: Korrelation des LCPC Abrasivitätskoeffizienten (LAK) [g/t] mit dem Äquivalenten Quarzgehalt (ÄQu) [\%].(THURO \& KÄSLING 2009a: 185, verändert).

Abb. 34: Absolute Häufigkeit der Werkzeugkontrollen $n_{1((c)}$ zwischen Ein- und Ausbau der Werkzeuge auf dem Schneidrad über alle Referenzprojekte.

Abb. 35: Histogramm zur Verteilung der Schneidgeschwindigkeit $\mathrm{v}_{\mathrm{sc}}$ der Abbauwerkzeuge in den Referenzprojekten....... 81

Abb. 36: Schwerer Verschleißschaden an der Schneidradstahlstruktur im Bereich des Schneidradzentrums.

Abb. 37: Blick auf die Ortsbrust durch eine Schneidradöffnung in feinkörnigem Geschiebemergel mit eingelagerten Kieskomponenten. (Herrenknecht AG).....

Abb. 38: Blick auf die Ortsbrust durch eine Schneidradöffnung in mitteldicht gelagerten sandigen Kiesen. (Herrenknecht AG) . 92

Abb. 39: Schneidrolle nach dem Einsatz in dicht gelagerten, sandigen Kiesen. .93

Abb. 40: Schematische, unmaßstäbliche Darstellung der Bodenverdrängung durch Schneidringe und Bodenabbau durch die nachlaufenden Schälmesser in der Ortsbrust als Quer- und Längsschnitt einer Schneidspur.

Abb. 41: Geöffnete Schneidrolle mit verschlissener Gleitringdichtung. Die Lagerkammer ist mit Bentonitsuspension gefüllt. (Herrenknecht AG).

Abb. 42: Verklebtes Schneidrollengehäuse während einem Drucklufteinstieg.

Abb. 43: Blick durch eine Schneidradöffnung auf die Frontseite des Schneidradzentrums. Der sichtbare Bereich ist vollständig mit bindigem Material verklebt. (Herrenknecht AG, verändert)

Abb. 44: Seitenansicht einer 1-Ring Schneidrolle mit Verschleißschaden.

Abb. 45: 1-Ring Schneidrolle mit gerissenem Schneidring und weiteren Abplatzungen am Schneidring nach Durchfahren eines Bohrpfahls mit Stahlbewehrung.

Abb. 46: Blick durch eine Schneidradöffnung auf die Ortsbrust mit einem Stahlträger, der in Beton eingebunden ist. Am Stahlträger sind deutlich die Spuren der Schneidrollen sichtbar. (Herrenknecht AG)

Abb. 47: Blick auf die Rückenfläche eines Stichels. Sowohl am Grundkörper als auch an den Hartmetalleinsätzen sind Kratzer parallel zur Schneidrichtung des Stichels zu erkennen.....

Abb. 48: Blick durch eine Schneidradöffnung auf einen Stichel nach dem Bodenabbau in steinigem Kies. 
Abb. 50: Blick auf die Rückenfläche dreier Stichel, die zum Bodenabbau in steinigem Kies eingesetzt wurden.

Abb. 51: Blick auf einen Stichel und die Ortsbrust in Abbau einer Dichtwand aus stahlbewährtem Beton mit ca. 35 MPa Würfeldruckfestigkeit.

Abb. 52: Stichelkopf mit entsprechendem Halter. Die Verschleißgrenze $h_{d, \max (S T)}$ des Stichelkopfs ergibt sich aus der Einbindetiefe der Hartmetalleinsätze in der Rückenfläche.

Abb. 53: Blick durch eine Schneidradöffnung auf zwei Schälmesser und die Ortsbrust in tonigen Schluffen. Die Ortsbrust ist glatt und zeichnet zum Teil detailliert die Form der Schälmesser nach. (Herrenknecht AG) .....

Abb. 54: Blick auf die Ortsbrust in mittel dicht gelagerten, sandigen Kiesen beim Abbau mit Schälmessern ohne vorlaufende Schneidrollen. (Herrenknecht AG)

Abb. 55: Blick auf die Front- \& Rückenfläche eines verschlissenen Schälmessers nach dem Bodenabbau mit vorlaufenden Schneidrollen in sandigen Kiesen. (Herrenknecht AG)

Abb. 56: Frontansicht eines Schneidradarmes nach Vortriebsende. Auf Höhe der Bildmitte sind von links nach rechts fünf Schälmesserpaare bzw. Halterpaare zu sehen. (Herrenknecht AG)

Abb. 57: Blick auf die Rückenfläche eines Schälmessers nach dem Vortrieb in steinigen Kiesen.

Abb. 58: Blick durch eine Schneidradöffnung auf Schälmesserhalter und Ortsbrust in tonigen Schluffen mit eingelagerten Granitblöcken. (Herrenknecht AG)

Abb. 59: Seitenansicht eines Schälmessers im Halter auf dem Schneidrad. Die Einbindetiefe der Hartmetalleinsätze liegt höher als die Obergrenze des Halters.

Abb. 60: Blick auf die Rückenfläche eines Räumersegments nach dem Vortrieb in schluffigen Sanden. (Herrenknecht AG; verändert)

Abb. 61: Blick auf die Rückenfläche eines Räumersegments nach dem Vortrieb in sandigen Schluffen. Der rechte Teil der Rückenfläche ist durch Primärverschleiß gleichmäßig eingeebnet. (Herrenknecht AG)

Abb. 62: Querschnitt durch ein Räumersegment, montiert auf dem Halter. Die Verschleißgrenze $h_{d, \max (\mathrm{RM})}$ ergibt sich aus der Einbindetiefe der Hartmetalleinsätze in der Rückenfläche.

Abb. 63: Bodenabbau durch Schälmesser und Schneidringe bei Furchenbildung durch die Schneidringe in der Ortsbrust...... 134

Abb. 64: Aufteilung der Penetration $p$ auf die verschiedenen aktiven und passiven Schälmesser $\left(S_{a} \& M_{p}\right)$ bei mehrfach besetzten Spuren in Abhängigkeit des Winkelabstandes $\delta$ zwischen den Schälmessern. ......

Abb. 65: Wägung eines Schneidrings mit der Laborwaage auf $+/-1 \mathrm{~g}$ genau.

Abb. 66: Massenverlust $m_{d}[\mathrm{~kg}]$ der Schneidringe angetragen gegen den Laufweg $\mathrm{s}_{\mathrm{c}}[\mathrm{km}]$ der Schneidringe. Die Messpunkte zeigen einen deutlichen Trend zur linearen Abhängigkeit des Massenverlustes $m_{d}$ vom Laufweg $s_{c}$.

Abb. 67: Stichel bei der Messung in der Ortbetonwand. Der Meterstab dient als Maßstab für das Bild. Die Messung der Höhe der Hartmetallzähne wurde mit einer elektronischen Schieblehre auf +/- 0,1 mm genau vorgenommen.

Abb. 68: Höhenverlust $h_{d}[\mathrm{~mm}]$ der Hartmetalleinsätze (Stichel) angetragen gegen den Laufweg $\mathrm{s}_{c}[\mathrm{~km}]$ der Stichel. Die

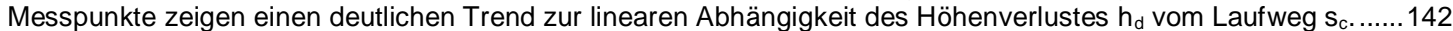

Abb. 69: Wägung eines Schälmessers mit der Laborwaage auf $+/-1 \mathrm{~g}$ genau.

Abb. 70: Massenverlust $m_{d}[g]$ der Schälmesser angetragen gegen den Laufweg $s_{c}$ [km] der Schälmesser. Die Messpunkte zeigen einen deutlichen Trend zur linearen Abhängigkeit des Massenverlustes $\mathrm{m}_{\mathrm{d}}$ vom Laufweg $\mathrm{s}_{\mathrm{c}}$.

Abb. 71: Anteil des verbleibenden Datenvolumens der Abbauwerkzeugwechsel [\%], in Abhängigkeit der akzeptierten Schwankungsbreite des Anteils [\%] unterschiedlicher Bodenarten in der Ortsbrust.

Abb. 72: Verteilung der Länge der geotechnischen Homogenbereiche $L_{d(z)}[\mathrm{m}]$ unterteilt nach Bodengruppen (DIN EN 14688-1).

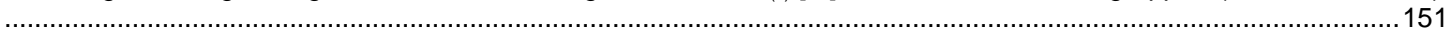

Abb. 73: Beispiel für eine Blockverteilung des Laufwegs $s_{c}$ innerhalb eines geotechnischen Homogenbereiches. Bis auf wenige Abweichungen ergibt sich für jede Kategorie des Laufwegs $\mathrm{s}_{\mathrm{c}}$ die gleiche absolute Häufigkeit. 
Abb. 74: Beispiel für eine links-steile Verteilung des Laufwegs $\mathrm{s}_{\mathrm{c}}$ innerhalb eines geotechnischen Homogenbereiches. 154

Abb. 75: Beispiel für eine rechts-steile Verteilung des Laufwegs $\mathrm{s}_{\mathrm{c}}$ innerhalb eines geotechnischen Homogenbereiches.

Abb. 76: Verteilung der Länge der geotechnischen Homogenbereiche $L_{d(z)}[\mathrm{m}]$ unterteilt nach Bodengruppen (DIN EN 14688 T1) nach Elimination von präventiven Werkzeugwechseln und Gewaltschäden.

Abb. 77: Vergleich des erreichten Laufwegs $\mathrm{s}_{\mathrm{c}}$ von Schälmessern bei primärem Bodenabbau zwischen vorlaufend angeordneten Schneidrollen in Sanden (HGB 2) und Kiesen (HGB 9) und ohne Kontakt zur Ortsbrust bzw. Bodenabbau hinter vorlaufend angeordneten Sticheln in identischen Sanden und Kiesen (HGB 3).......................158

Abb. 78: Frontansicht des Schneidrades einer Hydroschild TVM. Deutlich zu erkennen sind die vier Schneidradarme, auf denen Schälmesser angebracht sind. (Herrenknecht AG).....

Abb. 79: Vergleich des Laufwegs $s_{c}$ zwischen verschiedenen Schneidrollentypen und verschiedenen Schneidenbreiten $b_{S R}$

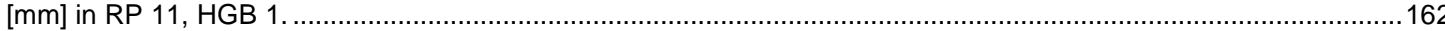

Abb. 80: Vergleich des Laufwegs $\mathrm{s}_{\mathrm{c}}$ verschiedener Schälmesservarianten mit unterschiedlichem Verschleißschutz.

Abb. 81: Im Diagramm ist die Schneidgeschwindigkeit $v_{s c}[\mathrm{~m} / \mathrm{s}]$ der gewechselten Schneidrollen in RP 7 in HGB 9 gegen den Laufweg $\mathrm{s}_{\mathrm{c}}[\mathrm{km}]$ angetragen.

Abb. 82: Im Diagramm ist die Schneidgeschwindigkeit $v_{s c}[\mathrm{~m} / \mathrm{s}]$ der gewechselten Schälmesser in RP 7 in HGB 1 und HGB 11 gegen den Laufweg $\mathrm{s}_{\mathrm{c}}[\mathrm{km}]$ angetragen.

Abb. 83: Laufweg $\mathrm{s}_{\mathrm{c}}[\mathrm{km}]$ baugleicher Schälmesser aus verschiedenen Homogenbereichen bei weitgehend identischen TVMSteuerparametern angetragen gegen den Äquivalenten Quarzgehalt (ÄQu) [\%].....

Abb. 84: Korrelation der normierten Schneidradanpresskraft $F_{n o r m}[k N]$ mit der Scherfestigkeit $T_{c}\left[k N / m^{2}\right]$ des Bodens in der Ortsbrust für unterschiedlichen Schneidradtypen und Bestückungsvarianten mit Schneidrollen und Sticheln. ...........181

Abb. 85: Korrelation des normierten Schneiddrehmomentes $M_{n o r m}[k N m]$ mit der Scherfestigkeit $T_{c}\left[k N / m^{2}\right]$ des Bodens in der Ortsbrust für unterschiedlichen Schneidradtypen und Bestückungsvarianten mit Schneidrollen und Sticheln ....

Abb. 86: Verteilung des Äquivalenten Quarzgehalts (ÄQu) [\%] in den 32 untersuchten geotechnischen Homogenbereichen. .185

Abb. 87: Verteilung der Scherfestigkeit $\mathrm{T}_{\mathrm{c}}\left[\mathrm{kN} / \mathrm{m}^{2}\right]$ in den 32 untersuchten geotechnischen Homogenbereichen. 186

Abb. 88: Verteilung des Korndurchmessers $D_{60}[\mathrm{~mm}]$ in den 32 untersuchten geotechnischen Homogenbereichen. 186

Abb. 89: Theoretische, kumulative Häufigkeitsverteilung des Soil Abrasivity Index (SAI) [-]

Abb. 90: Korrelation des Laufwegs $\mathrm{s}_{\mathrm{c}}[\mathrm{km}]$ von Schneidrollen mit dem Soil Abrasivity Index (SAI) [-]. 190

Abb. 91: Korrelation des Laufwegs $\mathrm{s}_{\mathrm{c}}[\mathrm{km}]$ von Schälmessern mit dem Soil Abrasivity Index (SAI) [-]. 191

Abb. 92: Korrelation des Laufwegs $\mathrm{S}_{\mathrm{c}}[\mathrm{km}]$ von Sticheln mit dem Soil Abrasivity Index (SAI) [-]..... 193

Abb. 93: Sektionen und Prozessschritte des Prognosemodells.

Abb. 94: Vergleich der Vortriebsstrecken $L_{d(m)}[m]$ aus den Referenzprojekten mit den Erwartungswerten für die Vortriebsstrecken $L_{d, e(m)}[m]$ aus der Validierung des Prognosemodells.

Abb. 95: Kumulierte Darstellung der Anzahl der Werkzeugwechsel $\mathrm{n}_{\mathrm{c}(\mathrm{m})}$ je Vortriebsunterbrechung $\mathrm{I}_{(\mathrm{m})}$ über die Vortriebsstationierung $L_{1(m)}$ aus RP 7 (rot) gegenüber der geschätzten Anzahl an Werkzeugwechsel $n_{c, e(m)}$ aus dem

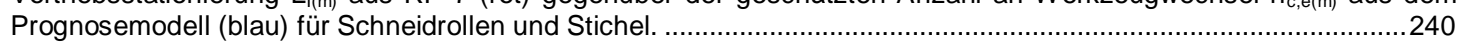

Abb. 96: Kumulierte Darstellung der Anzahl der Werkzeugwechsel $n_{c(m)}$ je Vortriebsunterbrechung $I_{(m)}$ über die Vortriebsstationierung $L_{1(m)}$ aus RP 7 (rot) gegenüber der geschätzten Anzahl an Werkzeugwechsel $n_{c, e(m)}$ aus dem Prognosemodell (blau) für Schälmesser......

Abb. 97: Darstellung der Anzahl der Werkzeugwechsel $n_{c(s)}$ je Schneidspur aus RP 7 (rot) gegenüber der geschätzten Anzahl an Werkzeugwechsel $\mathrm{n}_{\mathrm{c}, \mathrm{e}(\mathrm{m})}$ aus dem Prognosemodell (blau) für Schälmesser.

Abb. 98: Darstellung der Anzahl der Werkzeugwechsel $n_{c(s)}$ je Schneidspur aus RP 7 (rot) gegenüber der geschätzten Anzahl an Werkzeugwechsel $\mathrm{n}_{\mathrm{c}, \mathrm{e}(\mathrm{m})}$ aus dem Prognosemodell (blau) für Schälmesser. 


\section{Tabellenverzeichnis}

Tab. 1: Zusammenstellung an aktuellen Tunnelprojekten mit Einsatz von Hydroschild TVM und EBP TVM bei $>4,0$ bar Stützdruck. Ergänzt nach SMITH (2012: 16). ( $\mathrm{D}_{\text {TVM }}=$ Schneidraddurchmesser) …................................................. 3

Tab. 2: Qualitative Fehlerfortpflanzung im Prognosemodell in Abhängigkeit einer Fehleinschätzung der Standgrößen der

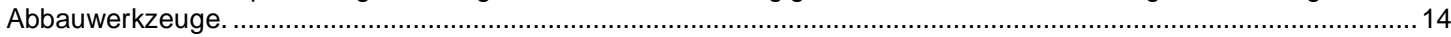

Tab. 3: Gliederung tribologischer Systeme nach beteiligten Systemelementen und Beanspruchungsarten, verändert nach GFT (2002: 14).

Tab. 4: Zusammenfassung der Bodeneigenschaften, Gebirgseigenschaften und Index-Tests, die Einfluss auf den Verschleiß

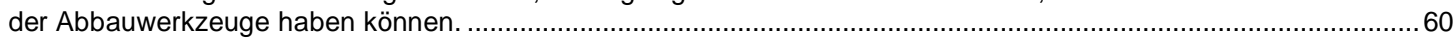

Tab. 5: Zusammenfassung der Mineraldichte, Schleifhärte nach ROSIWAL, Ritzhärte nach MOHS und Härte nach VICKERS für die häufigsten gesteinsbildenden Minerale, verändert nach PLINNINGER (2002: 46), verändert.

Tab. 6: Abrasivitätsklassifikation mit dem LCPC-Test (LAK) für Lockergesteine nach THURO \& KÄSLING (2009a: 184), verändert.

Tab. 7: Abrasivitätsklassifikation mit dem LCPC-Test (LAK) \& Cerchar Abrasivitätsindex (CAI), verändert nach THURO \&

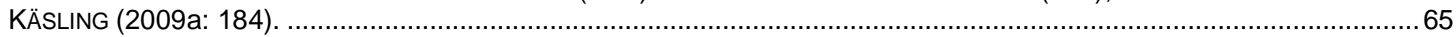

Tab. 8: Übersicht über die Referenzprojekte als Grundlage der empirischen, prozessorientierten Datenanalyse. .74

Tab. 9: Übersicht über die Steuerparameter des Abbauprozesses und die Anzahl an Werkzeugwechseln in den Referenzprojekten als Grundlage für die Datenanalyse. ............................................................................... 75

Tab. 10: Umfang und Detaillierungsgrad der Werkzeugwechseldokumentation in den Referenzprojekten. 78

Tab. 11: Empfehlungen zur geotechnischen Vorerkundung im Hinblick auf den Verschleiß der Abbauwerkzeuge. 82

Tab. 12: Vergleich der Anforderungen an die geotechnischen Dokumentation im Hinblick auf Werkzeugverschleiß mit den tatsächlich bestimmten Kennwerten in den Referenzprojekten.

Tab. 13: Definition und Klassifikation der Verschleiß- und Schadenstypen an Abbauwerkzeugen und Schneidradstruktur. .......90

Tab. 14: Übersicht über die Rohdaten für die Analyse der Werkzeugstandgrößen aus den Referenzprojekten.......

Tab. 15: Übersicht über die verbleibende Anzahl an Werkzeugwechseln für die Datenanalyse nach Bildung der geotechnischen Homogenbereiche.

Tab. 16: Übersicht über die verbleibende Anzahl an Werkzeugwechseln für die Datenanalyse nach Elimination von präventiven Werkzeugwechseln und Gewaltschäden.

Tab. 17: Vergleich des Laufwegs $\mathrm{s}_{c}$ und der prozentualen tatsächlichen Penetration der Schälmesser bei unterschiedlichem

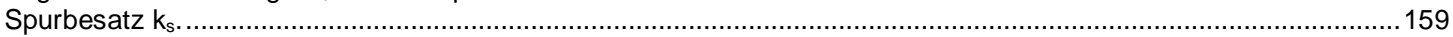

Tab. 18: Verschleißschutz der verschiedenen Schälmesservarianten in Referenzprojekt 1, Homogenbereich 1.

Tab. 19: Eignung der verschiedenen Verfahren zur Bestimmung der Härte der Bodenkomponenten für die vorliegende Datenanalyse und das zu entwickelnde Prognosemodell auf Basis der Bestimmbarkeit (Einschränkungen durch die Testverfahren) und Verfügbarkeit der Kennwerte in den Referenzprojekten.

Tab. 20: Vergleich der theoretisch möglichen Bandbreite von Äquivalentem Quarzgehalt (ÄQu) [\%], Scherfestigkeit $\mathrm{T}_{\mathrm{c}}\left[\mathrm{kN} / \mathrm{m}^{2}\right]$, Korndurchmesser $D_{60}[\mathrm{~mm}]$ und der entsprechenden Bandbreite in den untersuchten Homogenbereichen (HGB). .187

Tab. 21: Erfahrungswerte für die Penetration $p$ aus den Referenzprojekten.

Tab. 22: Zusammenfassung der nicht quantifizierbaren Einflussfaktoren auf den Laufweg $s_{c}$, deren vermutete Auswirkung und qualitative Tendenz in Bezug auf den Laufweg $\mathrm{s}_{\mathrm{c}}$ und der zugehörige Wertebereich in den Basisdaten aus den Referenzprojekten.

Tab. 23: Gliederung der TVM-Verschleißkosten nach Kosten für Abbauwerkzeuge und Wechselkosten (Ausführung der Werkzeugwechsel). 
Tab. 24: Vergleich der tatsächlich erreichten Laufwege $\mathrm{s}_{\mathrm{cv}(\mathrm{m})}$ aus Referenzprojekt 7 mit den geschätzten Laufwegen $\mathrm{S}_{\mathrm{cv}, \mathrm{e}(\mathrm{m})}$ bei Ausbau der Abbauwerkzeuge aus dem Prognosemodell-

Tab. 25.: Abweichungen $d_{s c}$ [\%] (Gl. 139) des geschätzten Laufwegs $S_{\mathrm{cv},(\mathrm{m})}[\mathrm{km}]$ aus dem Prognosemodell vom tatsächlichen Laufweg $\mathrm{s}_{\mathrm{cv}(\mathrm{m})}[\mathrm{km}]$ in den RP 3,6 und 9.

Tab. 26: Abweichungen $d_{n c}[\%]$ der geschätzten Anzahl an Werkzeugwechseln $n_{c, e(m)}[-]$ aus dem Prognosemodell von der tatsächlichen Anzahl an Werkzeugwechseln $\mathrm{n}_{\mathrm{c}(\mathrm{m})}[-]$ in den Referenzprojekten.

Tab. 27: Vergleich der tatsächlichen Anzahl $n_{c(T A)}$ an Werkzeugwechseln aus den Referenzprojekten (RP) und geschätzte Anzahl $n_{c, e(T A)}$ der Werkzeugwechsel über den gesamten Vortrieb bzw. Länge der Tunnelachse (TA).

Tab. 28: Mittlere Abweichung $d_{n c}[\%]$ der geschätzten Anzahl der Werkzeugwechsel $n_{c, e(s)}$ je Schneidspur aus dem Prognosemodell von der tatsächlichen Anzahl an Werkzeugwechseln $n_{c(s)}$ je Schneidspur in den Referenzprojekten $(\mathrm{RP})$.

Tab. 29: Zusammenfassung der wichtigsten Einflussfaktoren auf den Laufweg $\mathrm{s}_{\mathrm{c}}[\mathrm{km}]$ aus der Datenanalyse (Kap. 5.6).......255 


\section{Einführung}

\subsection{Abbauwerkzeugverschleiß im Schildvortrieb}

Im Fokus der vorliegenden Arbeit steht die heute weltweit gängige Vortriebsmethode mit Hydroschild TVM in Lockergesteinen unterhalb des Grundwasserspiegels. Die historische Entwicklung dieser Vortriebsmethode erfolgt ausgehend von konventionellen Vortriebsmethoden. Ursprünglicher Treiber dafür sind zunächst die Anforderungen für Tunnelbauwerke in Lockergesteinen, die aufgrund der fehlenden Standfestigkeit mit konventionellen Methoden nicht durchführbar sind. Ausgehend von der Einführung des weltweit ersten Vortriebsschildes durch M. I. Brunel im Jahr 1806 in London, sind für den bis heute anhaltenden Erfolg des Schildvortriebs vor allem drei zentrale Faktoren maßgeblich:

- Entwicklung der Schildtechnik selbst, insbesondere des statisch günstigen kreisrunden Schildquerschnitts.

- Entwicklung der Hohlraumstützung und Fernhaltung des Grundwassers zunächst durch Druckluft und später durch Stützflüssigkeiten.

- Entwicklung des mechanisierten, vollflächigen Bodenabbaus.

Eine detaillierte Darstellung der entsprechenden historischen und technischen Entwicklung der verschiedenen Schildtypen gibt MAIDL et al. (1995: 9 ff.). Als wesentliche Meilensteine bis zur heutigen Form einer Hydroschild TVM können daraus die folgenden Punkte zusammengefasst werden:

- Einführung des weltweit ersten kastenförmigen Vortriebsschildes mit manuellem Bodenabbau durch M. I. Brunel 1806 und Anwendung in London zur Untertunnelung der Themse 18251843.

- Erfindung der Druckluftschleuse durch Sir T. Cochrane 1830, die Grundvoraussetzung für den Zugang zu unter Über- oder Unterdruck stehenden Hohlräumen ist.

- Entwicklung eines kreisrunden Vortriebsschildes mit manuellem Bodenabbau durch J. H. Greathead und Anwendung zur Unterquerung der Themse in London 1869.

- Entwicklung des ersten Schildes mit flüssigkeitsgestützter Ortsbrust und hydraulischer Abraumförderung durch Greathead 1874.

- Erste Kombination eines Greathead-Schildes mit einer Hohlraumstützung durch Druckluft 1886, abermals in London.

- Entwicklung und Anwendung des ersten Schildes mit vollflächigem, mechanisiertem Bodenabbau durch ein Schneidrad und gleichzeitige mechanische Abraumförderung durch Price 1896.

- Erster erfolgreicher Einsatz eines Schildes mit flüssigkeitsgestützter Ortsbrust durch E. C. Gardner 1959.

- Erstmalige Kombination einer flüssigkeitsgestützten Ortsbrust mit vollflächigem Bodenabbau durch ein Schneidrad und hydraulischer Abraumförderung in Japan 1967.

- Erster Einsatz einer Hydroschild TVM nach heutigen Konstruktionsprinzipien mit bentonitgestützter Ortsbrust, Tauchwand und Steuerung des Stützdrucks über eine Luftblase hinter der Tauchwand 1974 in Deutschland. 
Zusätzlich zur Erschließung bis dahin für den Tunnelbau unzugänglicher Bodenbereiche birgt die Kombination des Schildvortriebs mit Flüssigkeitsstützung, hydraulischer Abraumförderung und vollflächigem Bodenabbau einige weitere wesentliche Vorteile. Der personelle Aufwand für den Vortrieb sinkt, gleichzeitig werden auch die Risiken für Setzungen durch immer bessere Kontrolle des Stützdrucks reduziert. Im Gegenzug nimmt die Vortriebsleistung und Zuverlässigkeit des Vortriebs durch den vollflächigen Abbau und die zunehmende Mechanisierung aller Abläufe, wie z.B. der Abraumförderung, Separation und der Tunnelauskleidung mit Tübbingen, deutlich zu.

Parallel zu dieser positiven Entwicklung erlangen der Abbaumechanismus an der Ortsbrust und Verschleißvorgängen an den Abbauwerkzeugen und der Schneidradstahlstruktur deutlich höhere Bedeutung. Bei den ersten Schildtypen (Brunel \& Greathead) erfolgt der Bodenabbau noch manuell, der Bergmann kann hier im Bedarfsfall ein verschlissenes Werkzeug sofort ungehindert austauschen. Die zusätzliche Stützung des Abbauraums durch Druckluft hat hier keinen Einfluss auf den Werkzugwechsel. Auch bei den ersten Schildtypen mit vollflächigem Bodenabbau durch ein Schneidrad (Price) ist ein Werkzeugwechsel während einer Vortriebsunterbrechung ungehindert möglich. Durch den Einsatz der Stützflüssigkeit bei Hydroschild TVM wird jedoch eine konstruktive Trennung des mit Flüssigkeit gefüllten und unter Druck stehenden Abbauraumes vom übrigen Schildbereich und dem Tunnelhohlraum durch eine durchgehende Druckwand notwendig. Der Zugang zur Abbaukammer zur Inspektion und zum Wechsel der Abbauwerkzeuge während einer Vortriebsunterbrechung wird damit deutlich aufwändiger.

Der Zugang zur Abbaukammer erfolgt bei Hydroschild TVM (Tunnelvortriebsmaschine) durch Schleusen in der Druckwand, um die Druckdifferenz zwischen Abbauraum und Tunnelhohlraum zu überwinden. Sofern keine spezialisierten Tauchtechniken eingesetzt werden, ist dazu eine Absenkung der Stützflüssigkeit in der Abbaukammer notwendig. In Bereichen, in denen die Ortsbrust standsicher ist, oder durch vorlaufende Injektionen bzw. Dichtblöcke stabilisiert wurde, kann dabei auch der Stützdruck abgesenkt werden, um den Werkzeugwechsel unter atmosphärischen Bedingungen auszuführen. Weitaus häufiger werden Werkzeugwechsel jedoch mit Druckluftarbeiten in der Abbaukammer durchgeführt. Dazu wird die Stützflüssigkeit teilweise durch Druckluft ersetzt, welche die Stützung der Ortsbrust aufrechterhält, während die Werkzeuge manuell gewechselt werden. Dabei ist eine genaue Überwachung der Luftverluste aus der Abbaukammer in den Boden hinein notwendig, um Risiken durch eine Instabilitäten der Ortsbrust oder einen Druckverlust in der Abbaukammer durch einen Ausbläser frühzeitig zu erkennen und zu vermeiden (BABENDERERDE 2000: 82).

Die ersten Vortriebe mit Hydroschild TVM, wie z.B. beim Bau der Metro in Lille (Frankreich) 1988 durch Wayss \& Freytag (www-01) weisen noch einen relativ geringen Ausbruchdurchmesser von maximal ca. 8,0 m und kontrollierbare geotechnische Rahmenbedingungen auf. Gleichzeitig liegen die Vortriebe noch relativ nah unter der Geländeoberkante (GOK), sodass der Stützdruck noch unterhalb von ca. 3,0 bar liegt. In diesem Bereich können Druckluftarbeiten unter normaler Atemluft ausgeführt werden. Mit zunehmendem Druck steigt jedoch bereits hier die notwendige Zeit zur Dekompression nach Abschluss der Druckluftarbeiten. Doch bereits mit dem Bau der 4. Röhre des Elbtunnels in Hamburg 1997-2000 mit einem Hydroschild der Firma Herrenknecht (www-02) mit 14,20 m Ausbruchdurchmesser und bis zu 5,5 bar Stützdruck in schwierigen glazial geprägten Bodenbedingungen wird ein Trend hin zu großen Ausbruchdurchmessern, hohen Stützdrücken und anspruchsvollen geotechnischen Bedingungen deutlich.

Dieser Trend hält im Grunde bis heute an, eine eindrucksvolle Zusammenstellung entsprechender Projekte, die unter anderem mit Hydroschild TVM aufgefahren werden gibt SMITH (2012: 16) (Tab. 1). Hier sind aufgrund des hohen Stützdrucks ab ca. 3,0 bar unabhängig von entsprechenden nationalen Normen zunehmend die Grenzen für die Durchführung von Arbeiten in der Abbaukammer unter normaler Atemluft erreicht. Darüber sind spezielle Verfahren aus dem Bereich des Sättigungstauchens notwendig, für die der Aufwand zur Durchführung noch einmal deutlich ansteigt.

Der Verschleiß der Abbauwerkzeuge entwickelt sich deshalb abschließend aufgrund der Notwendigkeit für Vortriebsunterbrechungen und den hohen Aufwand für den Zugang zur Abbaukammer vom wenig bedeutenden Faktor beim manuellen Bodenabbau im Schutz eines Schildes hin zum entscheidenden Faktor für die Vortriebsleistung, Verschleißkosten und die Verfahrenssicherheit bei Vortrieben Hydroschild TVM. Die mit der Durchführung der Werkzeugwechsel verbundenen Risiken, Personaleinsatz und Maschinenstillstand können die eigentlichen Kosten für die zu wechselnden Abbauwerkzeuge bei Weitem übersteigen. 
Tab. 1: Zusammenstellung an aktuellen Tunnelprojekten mit Einsatz von Hydroschild TVM und EBP TVM bei $>4,0$ bar Stützdruck. Ergänzt nach SMITH (2012: 16). ( $D_{\text {TVM }}=$ Schneidraddurchmesser)

\begin{tabular}{|l|c|c|l|l|}
\hline \multicolumn{1}{|c|}{ Projekt } & Ca. Stützdruck [bar] & D D $_{\text {TM }}[\mathrm{m}]$ & Maschinentyp & Hersteller \\
\hline $\begin{array}{l}\text { Hallandsas Rail Tunnels } \\
\text { (Sweden) }\end{array}$ & 13 bar & $10,5 \mathrm{~m}$ & Hydroschild TVM & Herrenknecht \\
\hline $\begin{array}{l}\text { Emisor Oriente } \\
\text { (Mexico City) }\end{array}$ & $6 \mathrm{bar}$ & $8,5-9,0 \mathrm{~m}$ & EPB TVM & $\begin{array}{l}\text { Herrenknecht \& } \\
\text { Robbins }\end{array}$ \\
\hline $\begin{array}{l}\text { Lee Tunnel } \\
\text { (London) }\end{array}$ & $6-7$ bar & $8,5 \mathrm{~m}$ & Hydroschild TVM & Herrenknecht \\
\hline $\begin{array}{l}\text { Lake Meade Intake } \\
\text { (Las Vegas) }\end{array}$ & 17 bar & $7,2 \mathrm{~m}$ & Hydroschild TVM & Herrenknecht \\
\hline $\begin{array}{l}\text { Port Mann Tunnel } \\
\text { (Vancouver) }\end{array}$ & 5 bar & - K.A. - & EPB TVM & Caterpillar \\
\hline $\begin{array}{l}\text { Alaskan Way } \\
\text { (Seattle) }\end{array}$ & 6 bar & $6,2 \mathrm{~m}$ & - Keine Angaben - & Ausschreibung \\
\hline $\begin{array}{l}\text { Singapore Cable Tunnels } \\
\text { (Singapore) }\end{array}$ & 11 bar & $13,7 \mathrm{~m}$ & Hydroschild TVM & Herrenknecht \\
\hline $\begin{array}{l}\text { Istambul Strait Road Crossing } \\
\text { (Istambul) }\end{array}$ & 5 bar & $14,0 \mathrm{~m}$ & - Keine Angaben - & Ausschreibung \\
\hline $\begin{array}{l}\text { Tuen Mun - Chek Lap Kok Link } \\
\text { (Hongkong) }\end{array}$ & EPB TVM & Hitachi Zosen \\
\hline
\end{tabular}

Vor diesem Hintergrund wird einerseits an maschinentechnischen Lösungen gearbeitet, um den Verscheiß der Abbauwerkzeuge an sich zu reduzieren und deren Inspektion und Wechsel zu erleichtern, bis hin zu atmosphärisch wechselbaren Werkzeugen aus begehbaren Schneidrädern heraus. Andererseits sind zunehmend zuverlässige Prognosemöglichkeiten für den Verschleiß der Werkzeuge notwendig, die als Grundlage für die Vortriebsplanung und die Kalkulation der Verschleißkosten dienen können. Diese existieren jedoch bisher nur in wenigen Ansätzen.

\subsection{Problemstellung}

Der Verschleiß der Abbauwerkzeuge, der quantitativ in Form von Standgrößen, wie z.B. der Standzeit $\mathrm{t}_{\mathrm{c}}[\mathrm{h}]$ oder dem Standweg bzw. Laufweg $\mathrm{s}_{\mathrm{c}}[\mathrm{km}]$ (Anhang: Abkürzungen \& Formelzeichen) der Abbauwerkzeuge erfasst werden kann, hat im Vortrieb mit Hydroschild TVM Einfluss auf:

- Vortriebsleistung

- Verschleißkosten

- Verfahrenssicherheit

Der Einfluss des Laufwegs der Abbauwerkzeuge auf die Vortriebsleistung ergibt sich aus der Anzahl und Dauer der für Werkzeugwechsel notwendigen Vortriebsunterbrechungen. Der Laufweg der Abbauwerkzeuge bestimmt die erreichbare Länge der Intervalle zwischen den Vortriebsunterbrechungen für Werkzeugwechsel. Daraus folgt deren Anzahl und Verteilung über die Tunnellänge. Die Dauer der Vortriebsunterbrechungen hängt von der Anzahl der notwendigen Werkzeugwechsel innerhalb einer Vortriebsunterbrechung ab. Diese wird ebenfalls vom Laufweg der Abbauwerkzeuge bestimmt. Der Laufweg der Abbauwerkzeuge wird deshalb über die Anzahl und Dauer der Vortriebsunterbrechungen für Werkzeugwechsel zum Einflussfaktor auf die Vortriebsleistung und die Vortriebsdauer.

Die Verschleißkosten im Vortrieb mit Hydroschild TVM setzen sich allgemein aus zwei Hauptanteilen zusammen, die getrennt betrachtet werden müssen:

- Kosten für die verschlissenen und gewechselten Abbauwerkzeuge

- Kosten für die Durchführung der Werkzeugwechsel 
Die Kosten für die verschlissenen und gewechselten Abbauwerkzeuge hängen über die Anzahl der notwendigen Werkzeugwechsel unmittelbar mit dem Laufweg der Abbauwerkzeuge zusammen. Die Kosten für die Durchführung der Werkzeugwechsel werden direkt von der Anzahl und Dauer der notwendigen Vortriebsunterbrechungen bestimmt, die sich wie beschrieben aus dem Laufweg der Abbauwerkzeuge ergeben. Indirekt hängt der Aufwand für die Durchführung der Werkzeugwechsel jedoch auch von der Zugangsmethode zur Abbaukammer an der gegebenen Stationierung ab. Diese wird primär von den geotechnischen Bedingungen vorgegeben, die maßgeblich sind für die Stabilität der Ortsbrust und die Ausbläsersicherheit. Es lassen sich überblickend folgende Zugangsmethoden unterscheiden:

1. Ist keine Stützung der Ortsbrust notwendig und wird kein Wasserandrang erwartet, können Stützflüssigkeit und Stützdruck abgesenkt werden. Der Werkzeugwechsel in der Abbaukammer kann unter atmosphärischen Bedingungen durchgeführt werden.

2. Ist während des Werkzeugwechsels eine Stützung der Ortsbrust notwendig, kann die Stützflüssigkeit bei ausreichender Stabilität der Ortsbrust und Ausbläsersicherheit teilweise gegen Druckluft ausgetauscht werden. Der Zugang zur Abbaukammer erfolgt über eine Schleuse, der Werkzeugwechsel wird unter Druck durchgeführt. Die Dauer der Vortriebsunterbrechung wird zusätzlich von der zulässigen Arbeitszeit in Abhängigkeit des Stützdrucks und der Entwicklung von Luftverlusten beeinflusst.

3. Bei unzureichender Stabilität der Ortsbrust oder mangelnder Ausbläsersicherheit ist zunächst kein Austausch der Stützflüssigkeit gegen Druckluft möglich. Die Stabilität der Ortsbrust und die Ausbläsersicherheit können einerseits durch zeit- und kostenintensive Zusatzmaßnahmen erhöht werden, um ein Austausch zu ermöglichen. Andererseits können spezialisierte Tauchtechniken für Arbeiten in der Bentonitsuspension eingesetzt werden.

4. Bei Schneidrädern mit begehbaren Schneidradarmen kann ein Teil der Abbauwerkzeuge unter athmosphärischen Bedingungen gewechselt werden. Die einzelnen Abbauwerkzeuge sind hierzu mit Druckluftschleusen ausgestattet. Für die Abbauwerkzeuge ohne Druckluftscheuse kommen die Oben geschilderten Methoden zum Einsatz.

Die Stationierung der Vortriebsunterbrechungen für Werkzeugwechsel ergibt sich im Vortrieb aus der von Vortriebsbeginn aufsteigenden Aneinanderreihung der Intervalle zwischen den Unterbrechungen. Die Vortriebsunterbrechungen sind unumgänglich, um Schäden an den Abbauwerkzeugen und der Schneidradstahlstruktur durch Überschreitung der Verschleißgrenzen zu verhindern. Sie können deshalb im Vortrieb nur eingeschränkt verschoben oder hinausgezögert werden, um z.B. Bereiche zu meiden, in denen wie oben beschrieben der Zugang zur Abbaukammer nicht oder nur mit hohem Aufwand für Zusatzmaßnahmen möglich ist. Der Laufweg der Abbauwerkzeuge hat deshalb in Projekten mit schwierigen geotechnischen Bedingungen auch Einfluss auf die Verfahrenssicherheit im Vortrieb.

Der Verschleiß der Abbauwerkzeuge hat also zusammenfassend in Projekten mit Hydroschild TVM hohen Einfluss auf das Vortriebsgeschehen. Im Gegensatz dazu ist der Kenntnisstand über die Einflussfaktoren auf den Verschleiß und deren Wirkbeziehungen für Hydroschild TVM in Lockergesteinen vergleichsweise gering.

Die Einflussfaktoren auf den Verschleiß der Abbauwerkzeuge, die zu einem bestimmten Laufweg $\mathrm{s}_{\mathrm{c}}$ oder Standzeit $t_{c}$ der Abbauwerkzeuge führen, sind in drei Kategorien unterteilbar (THURO 2002: 1):

- Geotechnik

- Baubetrieb

- Gerätschaft 
In den drei Kategorien sind im Vergleich zu anderen Maschinentypen eine enorm hohe Anzahl an Einflussfaktoren enthalten, welche die Analyse der komplexen Zusammenhänge zwischen der Auslegung der TVM (Gerätschaft), den Bodeneigenschaften (Geotechnik) und der Steuerung des Abbauprozesses (Baubetrieb) erschweren oder gänzlich unmöglich machen (KöHLER et al. 2011: 52). Zudem spielt vermutlich auch die stark eingeschränkte Zugänglichkeit zur Abbaukammer und den im Vortrieb an den Abbauwerkzeugen stattfindenden Verschleißprozessen eine maßgebliche Rolle für den geringen Kenntnisstand.

Als Folge daraus existiert bisher kein anerkanntes Prognosemodell für den Verschleiß von Abbauwerkzeugen in Lockergesteinen. Zwischen einzelnen Laborversuchen und dem tatsächlichen Verschleiß der Abbauwerkzeuge im Vortrieb können kaum aussagekräftige Korrelationen hergestellt werden (JAKOBSEN et al. 2013a: 113). Für die Verschleißprognose wird deshalb empfohlen, eine Einschätzung auf Basis von Erfahrungswerten und einer umfassenden, detaillierten Projektanalyse durchzuführen (KöHLER et al. 2011: 52).

Dieser Prognoseansatz ist aktuell vermutlich der zielführendste Weg, um trotz des geringen Kenntnisstandes überhaupt zu einer nachvollziehbaren Einschätzung zum Verschleiß der Abbauwerkzeuge zu kommen. Obwohl in diesem Ansatz eine hohe Anzahl an möglichen Einflussfaktoren auf den Verschleiß der Abbauwerkzeuge berücksichtigt wird, bleibt das Problem ungelöst, dass deren quantitative Auswirkung und Wechselwirkungen ungeklärt sind. Das Potential für hohe Abweichungen der Einschätzung vom tatsächlich im Vortrieb auftretenden Verschleiß der Abbauwerkzeuge, oder gar vollständige Fehleinschätzungen bleibt auch mit diesem Ansatz erhalten.

Das Verhältnis zwischen dem hohen Einfluss des Verschleiß der Abbauwerkzeuge auf den Vortrieb einerseits und dem geringen Kenntnisstand über die ursächlichen Einflussfaktoren und Zusammenhänge andererseits ergibt in Projekten mit Hydroschild TVM in Lockergesteinen Folgeprobleme bei:

- Ausschreibung von Vortrieben mit Hydroschild TVM

- Vortriebsplanung und Angebotskalkulation

- Auslegung der TVM

- Ausführung des Vortriebs

- Nachverhandlungen im Zusammenhang mit der Standzeit der Abbauwerkzeuge

Für den Bauherren bzw. Auftraggeber besteht in der Ausschreibungsphase für einen Vortrieb mit Hydroschild TVM das Hauptproblem darin, dass nach dem bestehenden Kenntnisstand keine klaren Angaben vorliegen, welche geotechnischen Parameter in der Vorerkundung bestimmt werden müssen, um dem Bieter bzw. Auftragnehmer eine ausreichende Datenbasis zur Vortriebsplanung und Kalkulation der Verschleißkosten zur Verfügung zu stellen.

Die fehlende Möglichkeit zur ausreichend zuverlässigen Prognose der Standzeit der Abbauwerkzeuge erschwert im Anschluss für den Bieter selbst bei umfangreichen Angaben zu den geotechnischen Bedingungen in der Ausschreibung sowohl die Planung der Anzahl und Stationierung der Vortriebsunterbrechungen für Werkzeugwechsel, als auch die Schätzung der Anzahl an notwendigen Werkzeugwechseln. Die Kalkulation der Vortriebsdauer und vor allem der Verschleißkosten unterliegt deshalb einer Unsicherheit.

Für den Hersteller der Hydroschild TVM resultiert aus der geringen Kenntnis der Beziehungen zwischen der Auslegung der TVM, geotechnischen Bedingungen und Betriebsparametern der TVM zunächst das Problem, dass nur eine eingeschränkte Optimierung der Auslegung der TVM im Hinblick auf den Verschleiß der Abbauwerkzeuge möglich ist. Zusätzlich ergeben sich für den Hersteller ähnliche Probleme bei der Einschätzung der Anzahl und Stationierung der Vortriebsunterbrechungen und der Anzahl an notwendigen Werkzeugwechsel, wie für den Bieter. Projektbezogene Leistungszusagen des TVM-Herstellers an den Bieter, z.B. im Hinblick auf die Vortriebsgeschwindigkeit und -dauer, oder Pauschalvereinbarungen zu den Verschleißkosten, unterliegend deshalb ebenfalls einer Unsicherheit.

In der Ausführung des Vortriebs erschwert die fehlende Möglichkeit einer ausreichend zuverlässigen Prognose der Standzeit der Abbauwerkzeuge für den Auftragnehmer die konkrete Entscheidung zur Durchführung einer Vortriebsunterbrechung für Inspektion und Wechsel der Abbauwerkzeuge. 
Die geringe Kenntnis der Beziehungen zwischen der Auslegung der TVM, geotechnischen Bedingungen und Betriebsparametern der TVM schränkt zusätzlich die Möglichkeiten zur Optimierung des Vortriebs im Hinblick auf den Verschleiß der Abbauwerkzeuge durch den Auftragnehmer und den TVMHersteller ein.

Abschließend ergeben sich auch im Fall von Nachverhandlungen in einem Vortrieb mit Hydroschild TVM, z.B. aufgrund einer Überschreitung der geplanten Anzahl an Vortriebsunterbrechungen für Werkzeugwechsel oder einer Überschreitung der Verschleißkosten, Probleme in der Diskussion. Einerseits ist aufgrund der geringen Kenntnis der grundlegenden Wirkbeziehungen zwischen geotechnischen Parametern, Steuerung des Abbauprozesses und Auslegung der TVM nur schwer eine stichhaltige Festlegung möglich, welcher Vertragspartei eine entsprechende Abweichung ursächlich zuzuordnen ist. Andererseits sind die Quantifizierung der Abweichung und deren absolute Auswirkungen aufgrund der fehlenden Möglichkeit zur zuverlässigen Schätzung der Standzeit der Abbauwerkzeuge problematisch.

\section{$1.3 \quad$ Zielsetzung}

Für Hydroschild TVM in Lockergesteinen sollen folgende Ziele erarbeitet werden, die in der Areit in in zwei aufeinander aufbauenden Teilen bearbeitet werden:

- Analyse der Zusammenhänge zwischen den Standgrößen der Abbauwerkzeuge, Auslegung der TVM (Gerätschaft), Boden- \& Gebirgseigenschaften (Geotechnik) und Steuerung des Abbauprozesses (Baubetrieb).

- Aufbau eines Prognosemodells für den Verschleiß der Abbauwerkzeuge

Die Analyse der Zusammenhänge ist im Hinblick auf den Aufbau eines Prognosemodells auf die folgenden Teilziele fokussiert:

- Identifikation der maßgeblichen Einflussfaktoren auf die Standgrößen der Abbauwerkzeuge aus den drei Kategorien.

- Quantifizierung der maßgeblichen Einflussfaktoren.

Der Aufbau des Prognosemodells umfasst unter Berücksichtigung der Problemstellung in Kap. 1.2 folgende Teilziele:

- Prognose der Standgrößen der Abbauwerkzeuge

- Prognose der Stationierung und Anzahl der Vortriebsunterbrechungen für verschleißbedingte Werkzeugwechsel.

- Prognose der Anzahl verschleißbedingter Werkzeugwechsel.

Das Prognosemodell ermöglicht unter anderem die Bestimmung der Stationierung der notwendigen Vortriebsunterbrechungen für Werkzeugwechsel. Aufgrund der Abhängigkeit der Zugangsmöglichkeiten zur Abbaukammer von den geotechnischen Bedingungen an den prognostizierten Stationierungen, soll in das Prognosemodell ein Algorithmus integriert werden, der eine baubetriebliche Anpassung der Prognose an diese Bedingungen ermöglicht. Dieser soll auch projektspezifische Ziele, wie z.B. die Maximierung der Vortriebsgeschwindigkeit, oder Risikominimierung berücksichtigen.

Zusammenfassend soll so mit der vorliegenden Arbeit der Kenntnisstand im Hinblick auf den Verschleiß der Abbauwerkzeuge beim Vortrieb mit Hydroschild TVM in Lockergesteinen signifikant erhöht werden, um eine maßgebliche Verringerung der Folgeprobleme (Kap. 1.2) zu bewirken. Dieses Ziel wird durch die Ergebnisse der Arbeit erreicht. 


\section{Methodik}

\section{1 Übersicht}

Die Vorgehensweise in der Bearbeitung der Zielsetzung und der Aufbau der Arbeit sind in die folgenden Schritte untergliedert:

- Einführung, Problembeschreibung und Zielsetzung (Kap. 1)

- Beschreibung der Arbeitsmethodik (Kap. 2)

- Einbettung des Verschleiß der Abbauwerkzeuge in das Gesamtsystem aus Hydroschild TVM Technik und Vortrieb (Kap. 3).

- Beschreibung des Gesamtsystems Verschleiß der Abbauwerkzeuge beim Vortrieb mit Hydroschild TVM in Lockergesteinen aus bestehendem Wissensstand (Kap. 4) und eigener Datenanalyse (Kap. 5).

- Modellbildung für den Verschleiß der Abbauwerkzeuge beim Vortrieb mit Hydroschild TVM in Lockergesteinen und Validierung (Kap. 6)

- Diskussion und Ausblick (Kap. 7)

Die Informationen zu Hydroschild TVM Technik und Vortrieb wurden in Zusammenarbeit mit der Firma Herrenknecht AG überwiegend aus eigenen Projekterfahrungen zusammengestellt. Die Methodik zur Beschreibung des Gesamtsystems, Erarbeitung des bestehenden Wissensstandes (Kap. 2.2), Datenanalyse (Kap. 2.3), sowie Modellbildung und Validierung (Kap. 2.4) sind im Folgenden im Detail beschrieben.

\section{$2.2 \quad$ Literaturrecherche}

Die Literaturrecherche zur vorliegenden Arbeit wurde mit Fokus zu nachfolgenden Themenkreisen durchgeführt:

- Tribologische Prozesse an Metalloberflächen bei Kontakt mit Locker- und Festgesteinen.

- Vortriebserfahrungen zum Abbauwerkzeugverschleiß in Locker- und Festgesteinen, insbesondere bei Hydroschild TVM.

- Laborversuche und Modelle zur Abbaumechanik und Verschleiß von Abbauwerkzeugen in Lockergesteinen.

- Einflussfaktoren auf den Verschleiß der Abbauwerkzeuge in Lockergesteinen und Beurteilung der Abrasivität von Lockergesteinen.

- Bestehende Modelle zur Verschleißprognose, insbesondere in Lockergesteinen.

Die Ergebnisse der Literaturrecherche sind als bestehender Wissensstand in Kap. 4 zusammengefasst. 


\subsection{Datenanalyse}

\subsubsection{Zielsetzung der Datenanalyse}

Die Datenanalyse zu den Zusammenhängen zwischen den Standgrößen der Abbauwerkzeuge, der Auslegung der TVM, den Boden- und Gebirgseigenschaften und der Steuerung des Abbauprozesses hat die bereits in Kap. 1.3 beschriebenen Ziele:

- Identifikation der maßgeblichen Einflussfaktoren auf die Standgrößen der Abbauwerkzeuge aus den drei genannten Kategorien.

- Quantifizierung der maßgeblichen Einflussfaktoren.

Die Identifikation und Quantifizierung der maßgeblichen Einflussfaktoren bildet die Grundlage für das Prognosemodell (Kap. 2.4). Die Einflussfaktoren bilden hier Systemkomponenten, die durch das Modell in idealisierter Form qualitativ und quantitativ beschrieben werden sollen. Die Ergebnisse der Datenanalyse müssen für den Aufbau des Prognosemodells mindestens folgende Schritte ermöglichen:

- Identifikation der wesentlichen Systemkomponenten für die Modellabstraktion.

- Quantitative Beschreibung der Zusammenhänge zwischen den Systemkomponenten im abstrahierten Modell.

- Erfassung und Beschreibung von nicht quantifizierbaren Systemkomponenten, die Anwendungsgrenzen des Modells bilden und für zukünftige Untersuchungen relevant sind.

Die Datenanalyse liefert folglich auch Anforderungen für die zukünftige Erkundung und Dokumentation der geotechnischen Parameter, der Auslegung der TVM und der Steuerung des Abbauprozesses. Diese sind notwendig sind für:

- Anwendung des Prognosemodells.

- Zukünftige Detaillierung der quantitativen Zusammenhänge zwischen den Komponenten im abstrahierten Modell.

- Zukünftige Erweiterung des Prognosemodells um zusätzliche Systemkomponenten.

Die Ergebnisse der Datenanalyse sind in Kap. 5.6 zusammengefasst.

\subsubsection{Projektdaten}

Die Recherche zu Projektdaten aus Vortrieben mit Hydroschild TVM konzentriert sich auf Basis der Einflussfaktoren auf den Verschleiß der Abbauwerkzeuge (Kap. 1.2) auf die nachfolgenden Datentypen. Diese liegen dabei innerhalb eines Bauprojektes in der Verantwortung verschiedenen Parteien:

- Geotechnische Basisdaten und Baugrundmodell (Erkundung): Auftraggeber (AG)

- Konstruktive Auslegung der TVM: Maschinenhersteller (TL)

- TVM Betriebsdaten aus dem Vortrieb: Auftragnehmer (AN)

- Dokumentation der geotechnischen Verhältnisse im Vortrieb: AG und AN

- Dokumentation von Abbauwerkzeugwechseln im Vortrieb: AN

- Dokumentation der Sanierung von Abbauwerkzeugen: AN, TL oder Zulieferer 
Die angegebenen Daten wurden in Zusammenarbeit mit der Firma Herrenknecht AG als Maschinenhersteller zusammengeführt. Hier liegen die Daten im Rahmen der gegebenen Dokumentationsdichte in größtmöglichem Umfang vor. Die geotechnischen Basisdaten aus der Ausschreibung zu den Projekten werden als Grundlage für die konstruktive Auslegung der Hydroschild TVM durch den Auftragnehmer an den Maschinenhersteller weitergegeben. Die geotechnischen Daten aus der Bauüberwachung, die Betriebsdaten der TVM und die Dokumentation der Abbauwerkzeugwechsel im Vortrieb werden häufig im Hinblick auf Problemlösungen und die Optimierung des Vortriebs an den Maschinenhersteller weitergegeben. Die Lieferung und Sanierung der Abbauwerkzeuge erfolgte in einigen der Referenzprojekte ebenfalls durch die Herrenknecht AG, sodass auch hierzu Daten vorliegen.

Zum Zeitpunkt der Abgabe der vorliegenden Arbeit waren alle untersuchten Projekten die Vortriebsarbeiten abgeschlossen, zum Teil bestanden jedoch noch offene Nachverhandlungen in den Projekten, auch im Hinblick auf den Verschleiß der Abbauwerkzeuge und anderer Maschineneinrichtungen, Verklebungen, sowie im Hinblick auf die Vortriebsleistung. Zudem gewähren vor allem die TVM Betriebsdaten einen sehr detaillierten Einblick in das Vortriebsgeschehen. Alle Projektdaten werden deshalb in anonymisierter Form verwendet, um das für die Analyse benötigte Datenvolumen zu erreichen. Die zugehörigen Projektunterlagen können nicht veröffentlicht werden und werden deshalb weder im Text zitiert, noch im Literaturverzeichnis angegeben. ${ }^{1}$ Die Bearbeitung der in Kap. 1.2 erläuterten Problemstellung liegt letztlich im Interesse aller genannten Parteien, weil daraus eine Erhöhung der Planungssicherheit, Potentiale zur Kostenoptimierung und eine Erhöhung der Verfahrenssicherheit resultiert.

Alle Daten aus den Projekten wurden soweit wie möglich validiert. Dazu wurde einerseits in Zusammenarbeit mit der Herrenknecht AG im überwiegenden Teil der Projekte eine eigene Überprüfung und Dokumentation vor Ort, insbesondere der Werkzeugwechseldaten durchgeführt. Zusätzlich wurden die Daten durch Befragung der Projektleiter, Schichtingenieure und Schildfahrer überprüft und ergänzt. Eine Übersicht zu den zusammengestellten Daten aus den Referenzprojekten gibt Kap. 5.1.

\subsubsection{Analyseansatz}

Für die Analyse der Zusammenhänge zwischen den verschiedenen Einflussfaktoren und dem Verschleiß der Abbauwerkzeuge im Vortrieb mit Hydroschild TVM in Lockergesteinen ist sowohl ein analytischer Ansatz, als auch ein empirischem Ansatz denkbar.

Für einen analytischen Ansatz ist einerseits eine Beschreibung der Verschleißprozesse auf mikroskopischer Ebene im tribologischen System zwischen Werkzeugoberfläche, Boden und der umgebenden Stützflüssigkeit notwendig. Andererseits ist auch eine Beschreibung der Prozesse beim Bodenabbau in der Abbaukammer in einem um ein Vielfaches höheren makroskopischen Maßstab notwendig. Beide Bereiche sind aufgrund der Flüssigkeitsfüllung der Abbaukammer und aufgrund des Stützdrucks während dem Vortrieb unzugänglich. Eine Nachbildung der maßgeblichen Prozesse in theoretischen Betrachtungen oder Laborversuchen über die vorliegende Bandbreite an Größenordnungen und eine sehr hohe Anzahl an Einflussfaktoren sind nur sehr schwer durchführbar. Die Ergebnisse daraus sind deshalb in der Regel auf enge Ausschnitte des komplexen Spektrums an Wechselwirkungen zwischen den Abbauwerkzeugen und dem Boden beschränkt, sodass sie nicht unmittelbar zur Ableitung eines praktisch anwendbaren Prognosemodells für den Verschleiß der Abbauwerkzeuge führen.

Im Gegenzug ergibt die Datenrecherche (Kap. 2.3.2), insbesondere der Projektdaten, einen Datensatz, der eine hohe Bandbreite der makroskopischen Einflussfaktoren auf den Verschleiß der Abbauwerkzeuge abdeckt. Hier bestehen zwar Probleme in der Dichte und Qualität der Dokumentation, diese können im weiteren Verlauf jedoch klar identifiziert und berücksichtigt werden. Auf Basis der Ergebnisse der Literaturrecherche (Kap. 2.2) ist zudem eine erste Beschreibung der makroskopischen Verschleißprozesse in der Abbaukammer und eine Eingrenzung der zugehörigen Haupteinflussfaktoren möglich. Darauf aufbauend können die zu untersuchenden Zusammenhänge in einer empirischen Analyse der Daten, orientiert an den makroskopischen Verschleißprozessen, stark eingegrenzt und in zielführender Weise fokussiert werden. Die Ableitung eines praktisch anwendbaren Prognosemodells für den Verschleiß der Abbauwerkzeuge erscheint auf diesem Weg möglich.

Für die Arbeit wurde deshalb ein empirischer, prozessorientierter Ansatz zur Analyse der zusammengeführten Projektdaten im Hinblick auf den Verschleiß der Abbauwerkzeuge gewählt.

\footnotetext{
${ }^{1}$ Die Basisdaten können der Prüfungskomission zum Zweck der Beurteilung der vorliegenden Arbeit auf Anforderung zugänglich gemacht werden.
} 


\subsubsection{Datenstruktur und Aufbereitung}

Die Analyse der Projektdaten im Hinblick auf den Verschleiß der Abbauwerkzeuge erfordert zunächst die grundlegende Untersuchung der Rahmenbedingungen der Datenentstehung und die Einführung einheitlicher Definitionen und Kategorien im Rahmen der Analyse.

Im ersten Schritt ist eine Analyse, zur Dokumentation der Werkzeugwechsel, der geotechnischen Rahmenbedingungen, der konstruktiven Auslegung der TVM und der Steuerung des Abbauprozesses notwendig (Kap. 5.1). Hieraus ergeben sich theoretische Anforderungen an die Dokumentation, die mit den Möglichkeiten und Grenzen im Vortrieb und mit der Dokumentation in den Referenzprojekten abgeglichen werden können. Hieraus ergibt sich das notwendige Verständnis der Qualität und Aussagekraft der Daten im Hinblick auf den Verschleiß der Abbauwerkzeuge für die weitere Analyse.

Im Anschluss ist eine Zusammenstellung der Erkenntnisse zum Verschleißbild der Abbauwerkzeuge in den Referenzprojekten und den daraus resultierenden Rückschlüssen auf die Abbaumechanik notwendig. So wird eine klare Definition und Unterscheidung der Verschleiß- und Schadensmechanismen nach Ort und Ursache möglich (Kap. 5.3.1). Diese ist Voraussetzung für die weitere einheitliche Beschreibung und Analyse der Projektdaten. Zusätzlich können bisher fehlende Angaben zu den Verschleißgrenzen der unterschiedlichen Abbauwerkzeugtypen in Lockergesteinen entwickelt werden.

Für die Datenanalyse ist weiterhin eine klare Definition der Standbegriff und -größen für Abbauwerkzeuge und eine Methode zur Quantifizierung notwendig, die einen Vergleich der Standgrößen zwischen verschiedenen Referenzprojekten und Hydroschild TVM erlaubt (Kap. 5.4).

Abschließend erfordert sowohl die Datenanalyse, als auch die nachfolgende Erarbeitung des Prognosemodells die Kenntnis des Verlaufs des Abriebs (Verschleißrate) an den Abbauwerkzeugen durch Verschleiß zwischen Ein- und Ausbau der Werkzeuge auf dem Schneidrad. Diese ist allein durch die absolute Angabe zum Ein- und Ausbauzeitpunkt der Abbauwerkzeuge in der Dokumentation der Referenzprojekte nicht gegeben. Der Verlauf des Abriebs an den Abbauwerkzeugen durch Verschleiß wurde deshalb in separaten Baustellenversuchen (Kap. 5.5) untersucht.

\subsubsection{Analysemethodik}

Ziel der Datenanalyse ist es, die Haupteinflussfaktoren auf den Verschleiß der Abbauwerkzeuge anhand der Projektdaten soweit zu Qualifizieren und Quantifizieren, dass abschließend die Ableitung eines Prognosemodells möglich wird.

Dazu ist zunächst die Berechnung der erreichten Standgrößen für alle in den Referenzprojekten gewechselten Abbauwerkzeuge notwendig. Die Berechnung wurde anhand der Formulierungen für die Standgrößen (Kap. 5.4) für jeden einzelnen Werkzeugwechsel vorgenommen.

Anschließend wurden jedem Werkzeugwechsel alle potentiellen Einflussfaktoren aus den gegebenen Rahmenbedingungen zugeordnet. Im Rahmen der vorhandenen Dokumentationsdichte wurden Faktoren aus folgenden Kategorien berücksichtigt:

- Auslegung der TVM und der Abbauwerkzeuge

- Boden- und Gebirgseigenschaften

- Steuerparameter des Abbauprozesses (Betriebsparameter der TVM)

Die Zuordnung eindeutiger geotechnischer Parameter zu einzelnen Werkzeugwechseln erfordert die Bildung geotechnischer Homogenbereiche, innerhalb derer die Parameter einheitlich sind oder gemittelt werden können. Analog zu diesen Homogenbereichen ist auch für die eindeutige Zuordnung von Betriebsparametern der TVM die Bildung von Mittelwerten notwendig. Diese Vorgehensweise bewirkt eine Reduktion des Datenvolumens, weil für die Datenanalyse Abbauwerkzeuge eliminiert werden, die zwischen Ein- und Ausbau auf dem Schneidrad über mehr als einen Homogenbereich im Einsatz waren und deshalb keine Zuordnung eindeutiger Parameter erlauben. Für die verbleibenden Werkzeugwechsel ergibt sich ein Datensatz aus den zugehörigen Standgrößen und Werten für Einflussfaktoren aus den drei genannten Kategorien. 
Im nächsten Schritt ist eine Fokussierung der Datenanalyse auf den stetigen Verschleiß der Abbauwerkzeuge aufgrund der Abrasivität des Bodens notwendig. Dazu ist eine Trennung der Werkzeugwechsel nach dem Wechselgrund in folgende Kategorien erforderlich:

- Werkzeugwechsel aufgrund von stetigem Verschleiß

- Werkzeugwechsel aufgrund von Schäden

Werkzeugwechsel aufgrund von Schäden an den Abbauwerkzeugen sind nicht aussagekräftig für den stetigen Verschleiß der Abbauwerkzeuge. Sie werden deshalb aus dem Datenpool entfernt. Die Werkzeugwechsel aufgrund von stetigem Verschleiß werden weiter in folgende Kategorien unterteilt:

- Präventive Werkzeugwechsel vor Erreichen der Verschleißgrenze

- Werkzeugwechsel an der Verschleißgrenze

- Verspätete Werkzeugwechsel nach Überschreitung der Verschleißgrenze

Die Datenanalyse wird auf die Werkzeugwechsel an der Verschleißgrenze der Abbauwerkzeuge konzentriert. Diese sind für die vorliegende Kombination an Einflussfaktoren maßgeblich. Durch die Elimination der präventiven und verspäteten Werkzeugwechsel ergibt sich eine weitere Reduktion des Datenvolumens. Die Filterung der Werkzeugwechsel nach den Kategorien wird auf Basis einer Analyse der Verteilungsform der Standzeit der Abbauwerkzeuge innerhalb der Homogenbereiche vorgenommen. Diese ist charakteristisch für den dominierenden Wechselgrund und erlaubt zusätzlich die Festlegung auf einen Modalwert für die Standgrößen an der Verschleißgrenze der Abbauwerkzeuge.

Eine übergreifende Analyse der Daten mit naturwissenschaftlichen bzw. statistischen Methoden, wie z.B. einer mehrfaktoriellen Varianzanalyse, ist nicht möglich. Die Anwendung dieser Methoden wird durch die Kombination der folgenden Probleme innerhalb der Projektdaten verhindert:

- Hohe Bandbreite an unterschiedlichen Datentypen.

- Unklare oder fehlende Angaben zu den statistischen Eigenschaften der Basisdaten.

- Stark unterschiedliche Dokumentationsdichte in den Referenzprojekten.

Für die Analyse der Daten im Hinblick auf die Einflussfaktoren auf die Standgrößen der Abbauwerkzeuge wurde deshalb ein vergleichender Ansatz gewählt. Dieser ist an den im Vortrieb ablaufenden Arbeitsprozessen orientiert. Im Vordergrund steht die weitgehende Normierung der Einflussfaktoren, um einzelne Parameter oder Parameterkombinationen gezielt zu untersuchen. Dazu bieten sich folgende Vortriebssituationen an:

- Vergleich der Standgrößen zwischen unterschiedlichen geotechnischen Homogenbereichen, ohne Änderung der Maschinenauslegung und Steuerung des Abbauprozesses.

- Vergleich der Standgrößen zwischen verschiedenen Maschinenauslegungen innerhalb eines geotechnischen Homogenbereichs ohne Änderung der Steuerung des Abbauprozesses.

- Vergleich der Standgrößen zwischen verschiedenen Werten für die Parameter der Steuerung des Abbauprozesses innerhalb eines geotechnischen Homogenbereichs bei parallelen Vortrieben mit identischer Auslegung der TVM.

- Vergleich des Laufwegs $s_{c}$ für verschiedene Werte einzelner Einflussfaktoren, sofern alle anderen Einflussfaktoren normiert werden können.

Innerhalb der Vergleichsansätze können die Zusammenhänge zwischen den Standgrößen der Abbauwerkzeuge und einzelnen Einflussfaktoren oder -kombinationen mit statistischen Methoden untersucht und beschrieben werden. Die Ergebnisse der Datenanalyse sind in Kap. 5 beschrieben. 


\subsection{Aufbau des empirischen Prognosemodells}

\subsubsection{Zielsetzung des Modells}

Das Prognosemodell verfolgt die bereits in Kap. 1.3 beschriebenen Ziele im Hinblick auf den Verschleiß der Abbauwerkzeuge:

- Prognose der Standgrößen der Abbauwerkzeuge

- Prognose der Stationierung und Anzahl der Vortriebsunterbrechungen für verschleißbedingte Werkzeugwechsel.

- Prognose der Anzahl verschleißbedingter Werkzeugwechsel.

- Baubetriebliche Anpassung der Prognose an die geotechnischen Bedingungen für den Zugang zur Abbaukammer und projektspezifische Ziele.

Der Prognoseprozess muss zusätzlich so flexibel gestaltet sein, dass unterschiedliche Ausrichtungen der Prognose verfolgt werden können. In Abhängigkeit des Anwenders und der Motivation für die Prognose sind z.B. folgende Ausrichtungen denkbar:

- Nachbildung des Vortriebsverlaufes in den Referenzprojekten.

- Abbildung eines neuen Vortriebs auf Basis der Gewichtung von Vortriebsgeschwindigkeit, Verschleißkosten und Risikobereitschaft in den Referenzprojekten.

- Abbildung eines neuen oder laufenden Vortriebs, um Potentiale zur Optimierung der Vortriebsgeschwindigkeit, Verschleißkosten oder des Risikomanagements aufzuzeigen.

Das Prognosemodell kann folglich in einem Projekt mit Hydroschild TVM von den Projektbeteiligten zu unterschiedlichen Zwecken eingesetzt werden:

- Ausrichtung der geotechnischen Vorerkundung und Dokumentation im Vortrieb durch den Auftraggeber.

- Vortriebsplanung, Kalkulation der Verschleißkosten, Ausrichtung der geotechnischen Dokumentation im Vortrieb und Optimierung des Vortriebs durch den Auftragnehmer.

- Auslegung der Hydroschild TVM und Optimierung des Vortriebs durch den Maschinenlieferanten.

- Hilfestellung zur Begründung, ursächlichen Zuordnung und Quantifizierung von Abweichungen im Fall von Nachverhandlungen im Hinblick auf den Verschleiß der Abbauwerkzeuge.

Das Prognosemodell (Kap.6) erlaubt zusammenfassend eine maßgebliche Verringerung der in Kap. 1.2 beschriebenen Probleme.

\subsubsection{Modellbildung}

Grundlage für die Modellbildung sind die Ergebnisse der Datenanalyse (Kap. 2.3). Diese liefert nach Abzug von baubetrieblichen Einflüssen und Schadensereignissen (Kap. 2.3.4) eine Identifikation der geotechnischen Parametern, Auslegungsparameter der TVM und Steuerungsparameter des Abbauprozesses, die als Einflussfaktoren in Kombination zu einem bestimmten Wert für die Standgrößen der Abbauwerkzeuge führen. 
Zusätzlich gibt die Datenanalyse Information über den Einfluss und die Quantifizierbarkeit der Parameter, wobei überschlägig die folgenden Kombinationen möglich sind:

- Quantifizierbar, hoher Einfluss.

- Nicht quantifizierbar, vermutlich hoher Einfluss.

- Quantifizierbar, niedriger Einfluss.

- Nicht quantifizierbar, vermutlich niedriger Einfluss.

Ziel der Modellbildung ist, die Zusammenhänge der Einflussfaktoren mit hohem Einfluss abzubilden. Aus der Datenanalyse ergeben sich zwei Einflussfaktoren, für die ein hoher Einfluss vermutet werden kann, die jedoch nicht Quantifiziert werden können. Beide Faktoren unterliegen innerhalb der untersuchten Referenzprojekte keiner oder einer vernachlässigbar geringen Variation. Sie werden deshalb an entsprechender Position im Modell mit einem Korrekturfaktor berücksichtigt, der ohne Einfluss auf das Prognoseergebnis bleibt. Können die beiden Faktoren in zukünftigen Untersuchungen quantifiziert werden, ist so eine Berücksichtigung im Prognosemodell möglich.

Einflussfaktoren mit niedrigem oder vermutlich niedrigem Einfluss werden unabhängig von deren Quantifizierbarkeit im Modell nur in Einzelfällen berücksichtigt. So kann die Komplexität des Prognosemodells im Vergleich zum realen System aus Einflussfaktoren reduziert werden. Ein hoher Anteil der Einflussfaktoren mit niedrigem Einfluss unterliegt innerhalb der Referenzprojekte wiederum keiner oder einer vernachlässigbar geringen Variation. Die Reduktion der Komplexität wirkt sich damit bei der Nachbildung der Referenzprojekte nur in geringem Umfang auf die Prognosequalität des Modells aus (Kap. 2.4.4).

Im Hinblick auf die Anwendung des Prognosemodells auf zukünftige Projekte ist zu beachten, dass die nicht quantifizierbaren Einflussfaktoren mit hohem vermutetem Einfluss Einsatzgrenzen des Modells darstellen. Werden für diese Einflussfaktoren in zukünftigen Projekten Werte erreicht, die außerhalb der geringen Schwankungsbreite in den Referenzprojekten des Modells liegen, verliert das Prognosemodell streng betrachtet seine Gültigkeit. Ein ähnlicher Zusammenhang besteht in abgeschwächter Form auch für die nicht im Modell berücksichtigten Einflussfaktoren mit niedrigem Einfluss.

Die Reduktion der Komplexität hat zusätzliche Vorteile in der Anwendung des Prognosemodells, weil der Umfang an Eingangsparametern und der Aufwand zur Bearbeitung sinken. Die Anwendbarkeit des Prognosemodells in der Projektplanung wird dadurch erhöht.

Zusätzlich zur Reduktion des Prognosemodells auf Einflussfaktoren mit hohem Einfluss kann auf Basis der Datenanalyse bei einzelnen Einflussfaktoren auch eine Reduktion des Detaillierungsgrades vorgenommen werden. Ansatzpunkte sind z.B. Einflussfaktoren, die durch einfacher zu bestimmende Vergleichswerte ersetzt werden können, aber auch der Detaillierungsgrad von Kriterien, die zur Formulierung von Homogenbereichen im Vortrieb eingesetzt werden.

Die Anforderungen an die Struktur des Prognosemodells werden weitgehend durch die Methodik in der Datenanalyse (Kap. 2.3.5) und die Ziele (Kap. 2.4.1) des Modells vorgegeben. Diese legen eine Gliederung des Prognosemodells in Sektionen nahe, die eine Hochrechnung von der Betrachtung einzelner Abbauwerkzeuge ausgehend auf Vortriebsunterbrechungen und Werkzeugwechsel ermöglichen. Die Sektionen müssen also aufeinander aufbauend die folgenden Ergebnisse liefern:

- Sektion 1: Standgrößen der Abbauwerkzeuge

- Sektion 2: Stationierung der Vortriebsunterbrechungen für Werkzeugwechsel

- Sektion 3: Anzahl der verschleißbedingten Werkzeugwechsel.

- Sektion 4: Baubetriebliche Anpassung der Prognose an die geotechnischen Bedingungen für den Zugang zur Abbaukammer und projektspezifischen Ziele.

Die baubetriebliche Anpassung erfordert einen Abgleich des Prognoseergebnisses aus den Sektionen 1 - 3 mit den geotechnischen Bedingungen für den Zugang zur Abbaukammer und projektspezifischen 
Zielen. Aus dem Abgleich ergibt sich der Bedarf für eine Anpassung der Prognose, die durch gezielte Variation einzelner Einflussfaktoren in den Sektionen 1 - 3 erfolgt. Aufgrund der Verzahnung der Sektionen muss jede Variation in einer Sektion auch in den darauf aufbauenden Sektionen berücksichtigt werden, sodass ein verändertes Ergebnis des Prognosemodells entsteht. Dieses muss erneut mit den geotechnischen Bedingungen für den Zugang zur Abbaukammer und projektspezifische Ziele abgeglichen werden, um gegebenenfalls weitere Anpassungen vorzunehmen. Der Anpassungsmechanismus in Sektion 4 muss deshalb als iterativer Algorithmus formuliert werden, der eine schrittweise Annäherung an einen Optimalzustand der Prognose im Hinblick auf die baubetrieblichen Rahmenbedingungen und projektspezifische Ziele ermöglicht. Dieser erlaubt zusätzlich die Bildung verschiedener Prognoseszenarien, die in der Vortriebsplanung und -kalkulation verglichen werden können.

Die Gliederung der Sektionen muss zusätzlich Ansätze für eine zukünftige Erweiterung oder Anpassung des Prognosemodells berücksichtigen. Die Erweiterung betrifft einerseits Schadensmechanismen an den Abbauwerkzeugen, die aus der Datenanalyse (Kap. 2.3.4) und folglich auch aus dem Prognosemodell ausgenommen sind. Andererseits ist auch eine Erweiterung des Prognosemodells auf andere Maschinentypen, z.B. mit erddruckgestützer Ortsbrust denkbar.

\subsubsection{Kritische Faktoren}

Das Prognosemodell gibt als zentrale Ergebnisse die Länge der Intervalle zwischen den Vortriebsunterbrechungen für Werkzeugwechsel, sowie die Anzahl der verschleißbedingten Werkzeugwechsel aus. Beide Parameter hängen direkt von den Standgrößen der Abbauwerkzeuge ab und haben wie beschrieben (Kap. 1.2) hohen Einfluss auf die Vortriebsgeschwindigkeit, die Verschleißkosten und die Verfahrenssicherheit. Sie stellen deshalb die kritischen Faktoren dar, die in der Validierung des Prognosemodells untersucht werden müssen.

Eine Fehleinschätzung der Länge der Intervalle zwischen den Vortriebsunterbrechungen für Werkzeugwechsel und der Anzahl an verschleißbedingten Werkzeugwechseln kann analog zu den Sektionen des Prognosemodells (Kap. 2.4.2) auf drei Ebenen im Prognosemodell erfolgen:

- Fehleinschätzung der Standgrößen der Abbauwerkzeuge (Sektion 1) und Fortpflanzung der Fehler in der Prognose.

- Fehleinschätzung der Intervalle zwischen den Vortriebsunterbrechungen (Sektion 2) und Anzahl der verschleißbedingten Werkzeugwechsel (Sektion 3).

- Fehler in der baubetrieblichen Anpassung der Prognose (Sektion 4).

Zusätzlich haben auch die möglichen Konsequenzen einer Fehleinschätzung der Anzahl und Stationierung der Vortriebsunterbrechungen und der Anzahl an verschleißbedingten Werkzeugwechseln Einfluss auf die Vorgehensweise in der Validierung des Prognosemodells.

Eine Fehleinschätzung der Standgrößen (Sektion 1) pflanzt sich in der Schätzung der Länge der Intervalle zwischen den Vortriebsunterbrechungen für Werkzeugwechsel (Sektion 2) und der Anzahl an verschleißbedingten Werkzeugwechseln (Sektion 3) fort. Der qualitative Zusammenhang zwischen den Parametern ist in Tab. 2 dargestellt.

Tab. 2: Qualitative Fehlerfortpflanzung im Prognosemodell in Abhängigkeit einer Fehleinschätzung der Standgrößen der Abbauwerkzeuge.

\begin{tabular}{|c|c|c|}
\hline Fehleinschätzung & \multicolumn{2}{|c|}{ Fehlerfortpflanzung in der Prognose } \\
\hline Standgrößen & $\begin{array}{c}\text { Länge der Intervalle zw. Vortriebsunter- } \\
\text { brechungen f. Werkzeugwechsel }\end{array}$ & $\begin{array}{c}\text { Anzahl verschleißbedingter Werkzeug- } \\
\text { wechsel }\end{array}$ \\
\hline Überschätzung & Überschätzung & Unterschätzung \\
\hline Unterschätzung & Unterschätzung & Überschätzung \\
\hline
\end{tabular}


Eine Überschätzung der Länge der Intervalle zwischen den Vortriebsunterbrechungen für Werkzeugwechsel hat unabhängig von der Fehlerquelle deutlich höhere Konsequenzen als eine Unterschätzung. Diese kann im Vortrieb nur schwer frühzeitig erkannt werden, ohne dass die Verschleißgrenze einzelner Abbauwerkzeuge überschritten wird. Zusätzlich muss die geplante Länge der Intervalle im Vortrieb reduziert werden. Folglich entstehen Risiken für:

- Verschleißschäden an den Abbauwerkzeugen, Werkzeughaltern und Schneidradstahlstruktur durch verspätete Werkzeugwechsel.

- Erhöhung der Anzahl an Vortriebsunterbrechungen und Positionierung in Bereichen mit schwierigen geotechnischen Bedingungen für den Zugang zur Abbaukammer.

- Erhöhung der Verschleißkosten im Vergleich zur ursprünglichen Vortriebskalkulation.

- Erhöhung der Vortriebsdauer im Vergleich zum ursprünglichen Bauzeitplan.

Eine Unterschätzung der Länge der Intervalle kann im Vortrieb frühzeitig innerhalb der ersten Vortriebsunterbrechungen am Zustand der Abbauwerkzeuge erkannt werden. Die Intervalle können dann auf Basis der gewonnen Erfahrung sinnvoll erhöht werden. Risiken durch eine Unterschätzung der Intervalle liegen eher in der Planungsphase und Angebotskalkulation eines Vortriebes, da tendenziell eine zu hohe Vortriebsdauer und zu hohe Verschleißkosten angesetzt werden, die einen Wettbewerbsnachteil im Bieterverfahren darstellen können.

Für die Anzahl der verschleißbedingten Werkzeugwechsel haben Überschätzung und Unterschätzung der Länge der Intervalle zwischen den Vortriebsunterbrechungen vergleichbare Konsequenzen. Im Fall einer Überschätzung kann diese im Vortrieb frühzeitig innerhalb der ersten Vortriebsunterbrechungen am Zustand der Abbauwerkzeuge erkannt werden. Die Anzahl der verschleißbedingten Werkzeugwechsel kann anschließend auf Basis der gewonnen Erfahrung reduziert werden. In Abhängigkeit vom absoluten Betrag der Überschätzung kann eine Reduktion der geplanten Dauer der Vortriebsunterbrechungen möglich sein, die sich positiv auf die Vortriebsdauer auswirkt. Risiken durch eine Überschätzung der Anzahl verschleißbedingter Werkzeugwechsel liegen wiederum eher in der Planungsphase und Angebotskalkulation eines Vortriebes, da tendenziell zu hohe Verschleißkosten und angesetzt werden.

Im Fall einer Unterschätzung kann diese im Vortrieb ebenfalls frühzeitig innerhalb der ersten Vortriebsunterbrechungen am Zustand der Abbauwerkzeuge erkannt werden. Die Anzahl der verschleißbedingten Werkzeugwechsel kann anschließend auf Basis der gewonnen Erfahrung erhöht werden. In Abhängigkeit vom absoluten Betrag der Unterschätzung kann eine Erhöhung der geplanten Dauer der Vortriebsunterbrechungen notwendig sein, die sich negativ auf die Vortriebsdauer auswirkt. Die Risiken durch eine Unterschätzung der Anzahl verschleißbedingter Werkzeugwechsel liegen folglich hauptsächlich in einer Erhöhung der Verschleißkosten im Vergleich zur ursprünglichen Kalkulation.

Die geschilderten Konsequenzen von Fehleinschätzungen im Prognosemodell zeigen, dass der zutreffenden Schätzung der Länge der Intervalle zwischen den Vortriebsunterbrechungen für Werkzeugwechsel (Sektion 2) eine deutlich höhere Bedeutung zukommt, als der Anzahl an verschleißbedingten Werkzeugwechseln (Sektion 3). Zusätzlich sind die Auswirkungen einer Fehleinschätzung nicht in jeder Richtung gleich. Zusammenfassend müssen also folgende Fehleinschätzungen durch das Prognosemodell vermieden werden:

- Überschätzung der Standgrößen (Sektion 1)

- Überschätzung der Länge der Intervalle zwischen Vortriebsunterbrechungen für Werkzeugwechsel (Sektion 2).

- Unterschätzung der Anzahl verschleißbedingter Werkzeugwechsel.

Die Überprüfung des Prognosemodells im Hinblick auf die kritischen Faktoren erfolgt in der Validierung des Prognosemodells (Kap. 6.6.4). 


\subsubsection{Verifikation und Validierung}

Im Hinblick auf die zukünftige Anwendung des Prognosemodells ist abschließend eine Verifikation und Validierung des Prognosemodells erforderlich (Kap. 6.6). Die Verifikation dient vor allem zur Überprüfung der Anwendbarkeit des Prognosemodells im Hinblick auf folgende Faktoren:

- Durchgängigkeit der Zusammenhänge zwischen den Sektionen und Prozessschritten im Prognosemodell.

- Korrekte Formulierung der Prozessschritte und mathematischen Zusammenhänge.

- Einhaltung korrekter Dimensionen.

Die Überprüfung dieser Faktoren wurde im Zuge der deskriptiven Validierung des Prognosemodells vorgenommen. Hierzu wurde das Prognosemodell anhand der Referenzprojekte ohnehin mehrfach durchgerechnet, sodass Probleme in der Anwendung erfasst und beseitigt werden konnten.

Die Validierung des Prognosemodells dient zur Überprüfung der Eignung des Prognosemodells für den in der Zielsetzung (Kap. 2.4.1) angegebenen Zweck. Als Beurteilungskriterien für die Prognoseergebnisse werden vor allem die in Kap. 2.4.3 erläuterten kritischen Faktoren und Fehlermöglichkeiten herangezogen. Die Validierung wird in drei Teilen durchgeführt:

- Strukturelle Validierung

- Deskriptive Validierung

- Prognostische Validierung

In der strukturelle Validierung (Kap. 6.6.1) wird untersucht, ob das Prognosemodell die Objekte des Realsystems angemessen repräsentiert. Die realen Systemelemente sind hauptsächlich die in der Datenanalyse identifizierten Einflussfaktoren auf den Laufweg $\mathrm{s}_{\mathrm{c}}$ (Kap. 5.6). Diese werden für die Validierung mit den im Prognosemodell berücksichtigten Einflussfaktoren (Kap. 6.1) abgeglichen. Zusätzlich sind die in Kap. 2.4.2 beschriebenen Überlegungen zum Einfluss verschiedener Faktoren und zur Reduktion der Komplexität des Prognosemodells gegenüber dem realen System zu berücksichtigen.

Die deskriptive Validierung (Kap. 6.6.2) beurteilt, ob bzw. mit welcher Genauigkeit das Prognosemodell die Vergangenheitsdaten korrekt wiedergibt. Als Vergangenheitsdaten werden hier die in der Datenanalyse enthaltenen Referenzprojekte (Kap.5.1) eingesetzt. Das Prognosemodell liefert auf Basis der verfügbaren Eingangsdaten aus den Referenzprojekten Ergebnisse, die für die Validierung mit den tatsächlichen Werten aus den Referenzprojekten abgeglichen werden. Der Abgleich erfolgt anhand der kritischen Faktoren (Kap. 2.4.3):

- Prognostizierte und tatsächliche Standgrößen der Abbauwerkzeuge.

- Prognostizierte und tatsächliche Länge der Vortriebsstrecken zwischen den Vortriebsunterbrechungen für Werkzeugwechsel.

- Prognostizierte und tatsächliche Anzahl der Werkzeugwechsel innerhalb der Vortriebsunterbrechungen.

Bei der Beurteilung der Ergebnisse des Abgleichs der genannten Faktoren muss berücksichtigt werden, dass die Datenanalyse auf Werkzeugwechsel aufgrund von stetigem Verschleiß fokussiert ist. Werkzeugwechsel aufgrund von Schäden werden in der Datenaufbereitung (Kap. 2.3.4) eliminiert. Das Prognosemodell kann folglich nur den stetigen Verschleiß der Abbauwerkzeuge abbilden, während im realen System zum Teil eine Überlagerung beider Mechanismen zu beobachten ist. Die Festlegung einer Prognosegenauigkeit in Bezug auf die im realen System erreichten Werte für die Standgrößen der Abbauwerkzeuge, die Länge der Vortriebsstrecken zwischen den Vortriebsunterbrechungen und die Anzahl der Werkzeugwechsel unterliegt deshalb in einigen Fällen zwangsläufig einer hohen Unschärfe. 
Die prognostische Validierung dient dazu, zu untersuchen, ob bzw. mit welcher Genauigkeit mit Hilfe des Modells der Verschleiß der Abbauwerkzeuge in zukünftigen Projkten geschätzt werden kann. Theoretisch kann dazu mit dem Prognosemodell eine Schätzung für die Standgrößen der Abbauwerkzeuge, die Vortriebsstrecken zwischen den Vortriebsunterbrechungen und die Anzahl der Werkzeugwechsel erarbeitet werden. Diese kann mit den später im Vortrieb erzielten Ergebnissen abgeglichen werden. Das Prognosemodell selbst enthält einen rollierenden Anpassungsmechanismus der Verschleißprognose an die tatsächlichen Vortriebsverhältnisse, der den Abgleich der Basisdaten, Schätzungen im Prognoseprozess und der Prognoseergebnisse mit den tatsächlich im Vortrieb vorliegenden Verhältnissen beschreibt (Kap. 6.5.3.2). Für die vorliegende Arbeit konnte jedoch aufgrund des eingeschränkten Zeitrahmens und mangelndem Zugang zu geeigneten Projekten keine Validierung an laufenden Projekten durchgeführt werden. 


\title{
3 Hydroschild TVM Technik \& Vortrieb
}

Das grundsätzliche Funktionsprinzip einer Hydroschild TVM ist in Kap. 3.1 überblickend beschrieben. Im Anschluss sind in Kap. 3.2 detaillierte Informationen zu den einzelnen Maschinenkomponenten angegeben, die für die weiteren Untersuchungen in der vorliegenden Arbeit maßgeblich sind. Die unterschiedlichen Betriebszustände der TVM sind in Kap. 3.3 ausführlich dargestellt.

\subsection{Funktionsprinzip}

In der vorliegenden Arbeit wird mit dem Begriff Hydroschild TVM (Tunnelvortriebsmaschine) eine Vortriebsmaschine mit vollflächigem Bodenabbau durch ein kreisrundes Schneidrad beschrieben, bei der die Ortsbrust durch eine Flüssigkeit mit variabler, den Bodenverhältnissen anpassbarer Viskosität gestützt wird, die gleichzeitig zur Förderung des abgebauten Materials dient. Der Druck der Stützflüssigkeit wird dabei durch ein Druckluftpolster eingestellt. Der Begriff Hydroschild TVM stimmt folglich mit dem Maschinentyp SM-V4 in der von DAUB (2010: 11) vorgestellten Einteilung der verschiedenen Typen an Vortriebsmaschinen überein.

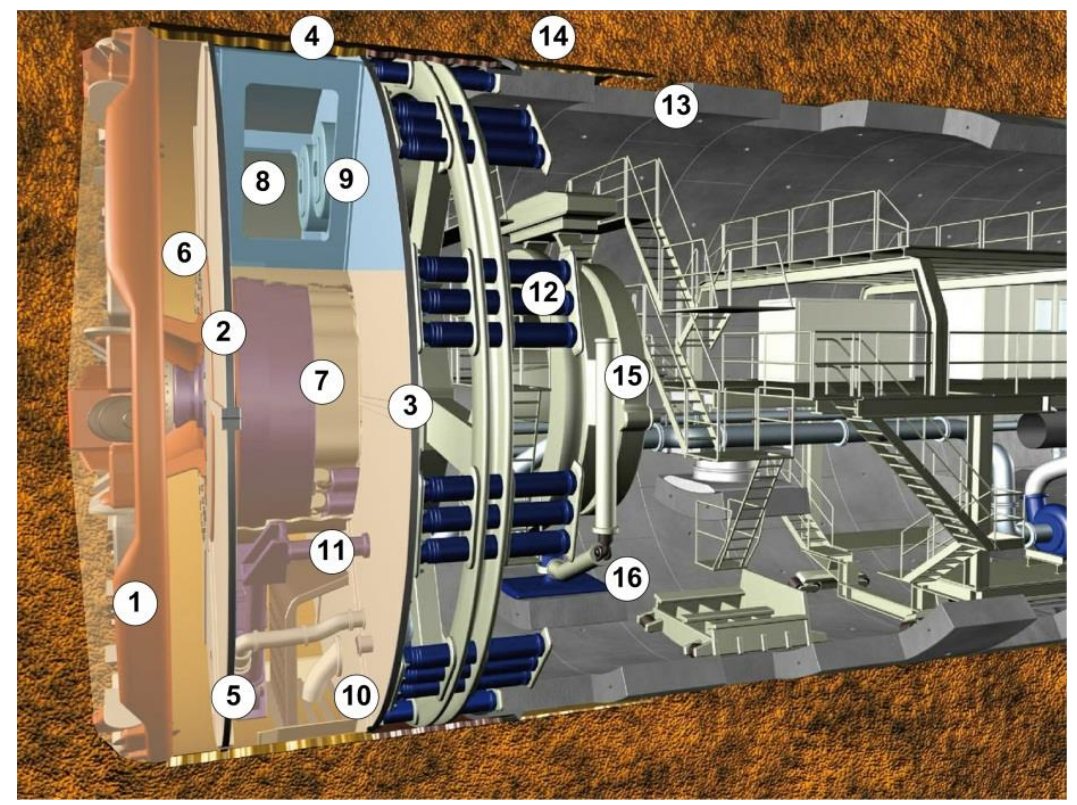

\begin{abstract}
Abb. 1: 3D-Schemazeichnung einer Hydroschild-TVM. Als Baugruppen sind gekennzeichnet: (1) Schneidrad, (2) Tauchwand, (3) Druckwand, (4) Schildmantel, (5) Tauchwandöffnung, (6) Abbaukammer, (7) mit Bentonit gefüllter Teil der Arbeitskammer, (8) mit Druckluft gefüllter Teil der Arbeitskammer, (9) Druckluftschleusen, (10) Förderleitung, (11) Speiseleitung, (12) Vortriebspressen, (13) Tübbingschale, (14) Bürstendichtungen, (15) Erektor, (16) Erektorsaugplatte mit Tübbingsegment. (Herrenknecht AG, verändert.)
\end{abstract}

In der Praxis hat sich für diesen Maschinentyp zeitgleich auch der Begriff Mixschild TVM (www-03) etabliert. Dieser wird in der vorliegenden Arbeit aus Gründen der eindeutigen Abgrenzung nicht verwendet, da mit diesem Begriff in der Vergangenheit auch Maschinen mit umbaubarer Materialförderung beschrieben wurden. Durch die Steuerung des Drucks der Stützflüssigkeit durch ein Druckluftpolster ergibt sich zugleich eine Abgrenzung zum Maschinentyp Slurryschild TVM, bei dem der Druck der Stützflüssigkeit ausschließlich über die Menge an zu- und abgepumpter Flüssigkeit in der Abbaukammer gesteuert wird. Dieser Schildtyp kommt nach MAIDEL et al. (1995: 248) vor allem in Japan zum Einsatz. 
An der Frontseite der Maschine wird an der Ortsbrust der Boden durch Abbauwerkzeuge gelöst, die in Haltern auf der Stahlstruktur des Schneidrads fixiert sind (Kap. 3.2.1). Das Schneidrad ist zusätzlich mit Öffnungen ausgestattet, sodass der gelöste Boden zur Förderung hinter das Schneidrad gelangen kann. Das Schneidrad selbst ist rückseitig durch Verbindungsstreben und einen Flanschring mit dem Schneidradantrieb verbunden (Kap. 3.2.2). Dieser ist über das Hauptlager im Schild fixiert, rückseitig greifen Motoren an, die das Schneidrad antreiben. Zeitgleich werden Schild und Schneidrad in der Vortriebsphase mithilfe der Vortriebspressen in Vortriebsrichtung verschoben. Die dazu notwendigen Kräfte zur Überwindung der Vortriebswiderstände (Kap. 3.3.1) werden durch die Vortriebspressen in den Tübbingausbau eingeleitet.

Der vordere Teil des Schildes ist durch die Druckwand vom hinteren Teil des Schildes getrennt (Kap. 3.2.3). Vor der Druckwand befindet sich der unter Druck stehende Arbeitsbereich, in dem die Stützflüssigkeit und das Druckluftpolster zur Regulierung des Drucks gehalten werden. Hinter der Druckwand befindet sich der unter atmosphärischem Druck stehende Arbeitsbereich der TVM.

Der unter Druck stehende Arbeitsbereich wird durch die Tauchwand in zwei Kammern geteilt, die über eine Öffnung in der Tauchwand in der Schildsohle (Tauchwandöffnung) verbunden sind. Der vor der Tauchwand liegende Bereich wird als Abbaukammer bezeichnet, hier findet der Bodenabbau durch das Schneidrad statt. Die Abbaukammer ist vollständig mit Stützflüssigkeit gefüllt, um während dem Vortrieb die Stützung der Ortsbrust aufrecht zu erhalten und gleichzeitig das abgebaute Material zu fördern. Der hinter der Tauchwand liegende Bereich wird als Arbeitskammer bezeichnet. Hier wird im Schildfirst das Druckluftpolster zur Regulierung des Drucks der Stützflüssigkeit gehalten, der restliche Bereich der Arbeitskammer ist ebenfalls mit der Stützflüssigkeit gefüllt. Der durch das Luftpolster aufgebrachte Druck wird zwischen Arbeitskammer und Abbaukammer über die Tauchwandöffnung nach dem Prinzip der kommunizierenden Röhren ausgeglichen.

Als Stützflüssigkeit wird in der Regel eine Bentonitsuspension eingesetzt. Bentonit ist ein Gemisch aus verschiedenen Tonmineralen. Hauptbestandteil ist quellfähiger Montmorillonit, der mit Wasser zu einer Suspension angerührt wird. Der Druck der Stützflüssigkeit wird über das Luftpolster so gesteuert, dass dieser den in der Ortsbrust vorliegenden Wasser- und Erddruck ausgleicht. An der Ortsbrust wird durch den Stützdruck Wasser aus der Stützflüssigkeit in den Boden gedrückt, dabei bleiben die plättchenförmigen Tonminerale an der Ortsbrust zurück, sodass sich hier eine dünne angelagerte Schicht aus Tonmineralen aufbaut. Diese wird als Filterkuchen bezeichnet (Abb. 2) und hilft, den Druck der Stützflüssigkeit in den Boden zu übertragen.

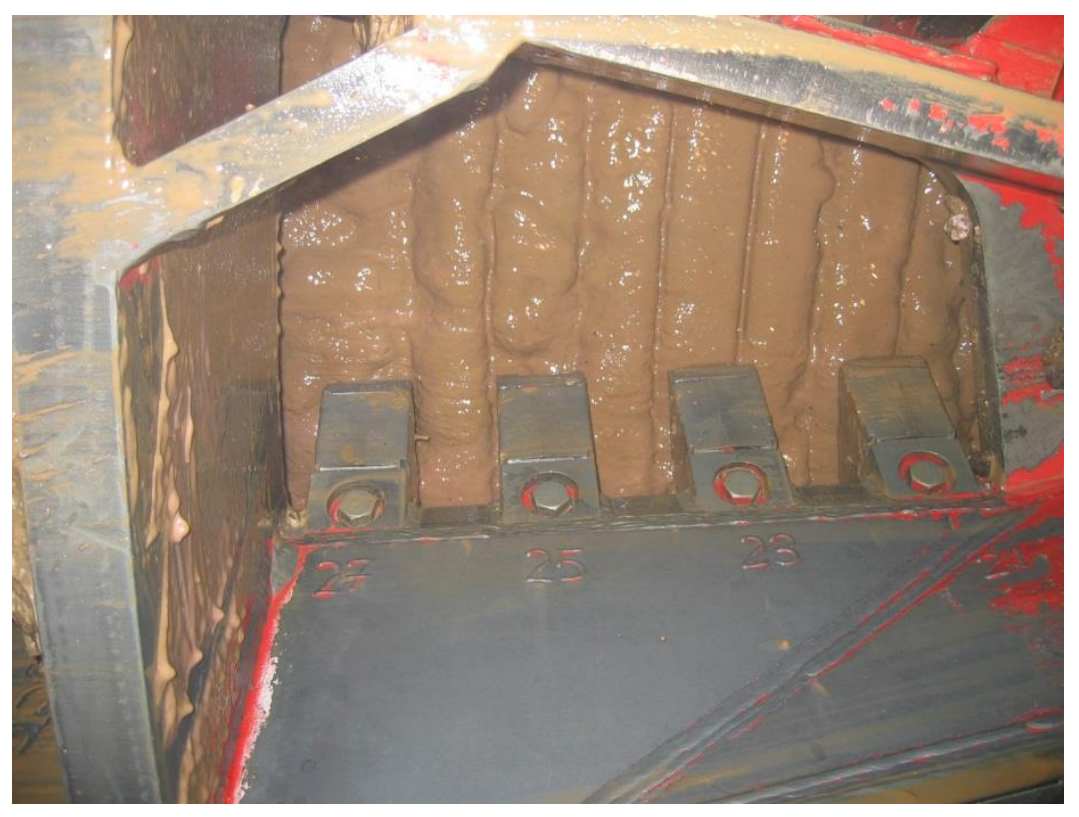

Abb. 2: Blick auf die Ortsbrust durch eine Schneidradöffnung während eines Drucklufteinstiegs. Die Ortsbrust in stark sandigen Kiesen ist mit einem Filterkuchen aus Bentonit überzogen. Im Vordergrund ist die Frontseite und die Verschraubung der Schälmesser auf den Spuren 21, 23, 25 und 27 zu sehen. (Herrenknecht AG) 
Während dem Vortrieb wird die Stützflüssigkeit zur Förderung des abgebauten Bodens aus der Abbaukammer genutzt. Die mit dem abgebauten Boden beladene Stützflüssigkeit wird dazu in der Sohle des Schildes über die Förderleitung hinter der Tauchwandöffnung abgesaugt. Um zu verhindern, dass Steine die Förderleitung blockieren oder zu Schäden in der nachfolgenden Förderpumpe führen, ist zwischen Tauch- und Druckwand ein Rechen vor der Förderleitung angebracht, der die Korngröße der geförderten Komponenten limitiert. Vor dem Rechen ist deshalb zusätzlich ein Brecher angebracht, um gröbere Komponenten zu zerkleinern. Um die Menge an Stützflüssigkeit in der Abbau- und Arbeitskammer konstant zu halten, wird über die Speiseleitung an verschiedenen Stellen der Tauchwand frische Stützflüssigkeit zugegeben. In einer Separationsanlage (Abb. 3) außerhalb des Tunnels wird der abgebaute Boden von der Stützflüssigkeit abgetrennt, sodass die Stützflüssigkeit größtenteils wieder eingesetzt werden kann.

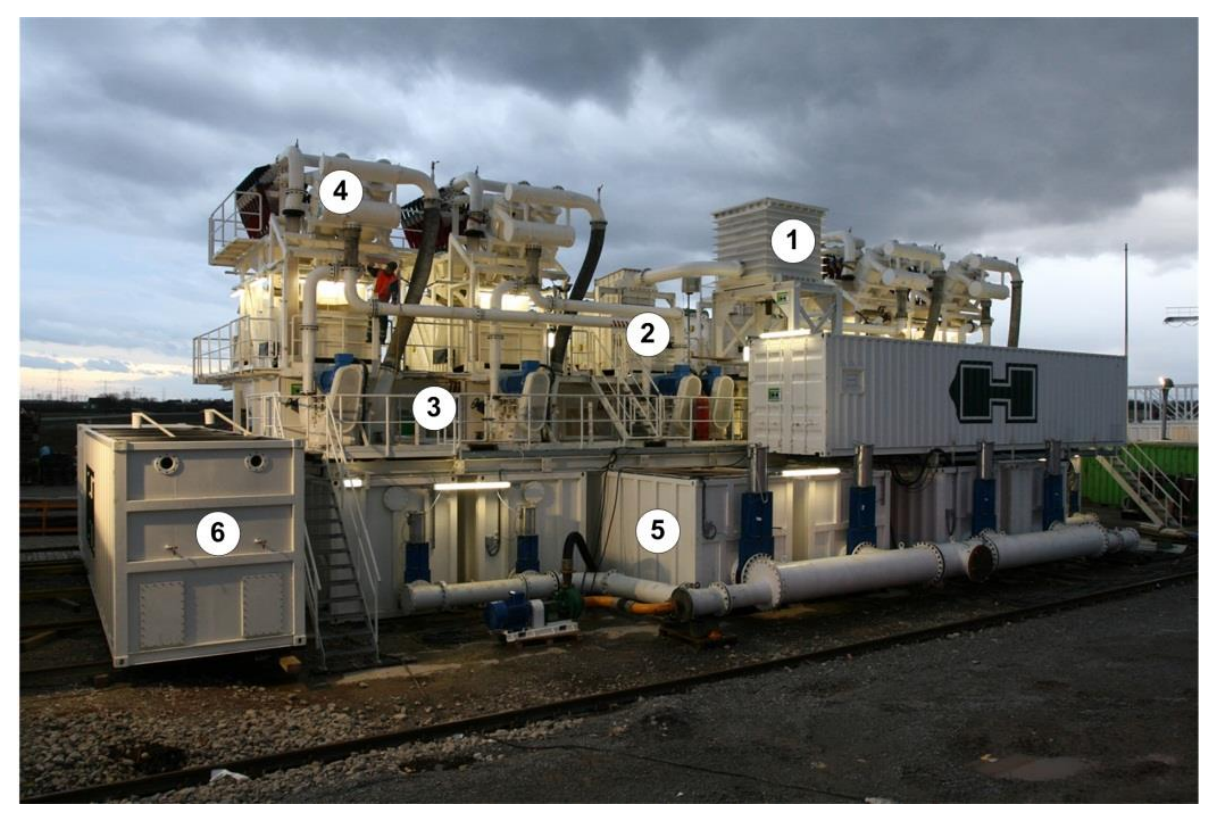

Abb. 3: Separationsanlage im Montagezustand. Die Hauptkomponenten der Anlage sind der Spülungsverteiler (1), Siebstufen (2), Transferpumpen (3), Zyklonstufen (4), Puffertanks (5) und Frischbentonittank (6). (Herrenknecht $A G$, verändert)

Die Vortriebsphase der TVM ist abgeschlossen, sobald die Vortriebspressen weit genug ausgefahren sind, um im Schutz des Schildes den nächsten Tübbingring einbauen zu können. Die einzelnen Segmente der Tübbingringe werden mit dem Erektor in der Schildsohle aufgenommen und an die entsprechende Position in der Tunnellaibung gehoben. Hier werden die entsprechenden Vortriebspressengruppen zurückgezogen, der Stein in Position gebracht und durch Ausfahren der Vortriebspressen gehalten. Sind alle Tübbingsegmente eingesetzt und zu einem vollständigen Tübbingring geschlossen, kann der Vortrieb wieder aufgenommen werden.

Für den Wechsel von Abbauwerkzeugen auf dem Schneidrad ist ein Zugang in die Abbaukammer notwendig, ohne den Stützdruck zu verringern. Dazu sind im Firstbereich des Schildes eine oder mehrere Druckluftschleusen angebracht. Diese sind an der Vorderseite über eine Tür an die Druckwand angeschlossen. Die rückwärtige Tür ist vom atmosphärischen Bereich der TVM zugänglich. Zusätzlich ist im Firstbereich auch in der Tauchwand eine entsprechende Tür vorgesehen, um den Durchgang in die Abbaukammer zum Schneidrad zu ermöglichen. Für den Zugang zum Schneidrad wird die Stützflüssigkeit in der Abbaukammer abgesenkt und durch Druckluft ausgetauscht. Dabei wird der entsprechende Stützdruck durch den Filterkuchen an der Ortsbrust auch mithilfe der Druckluft in den Boden übertragen. Beim Ein- und Ausschleusen von Personen in die Abbaukammer wird der Druck in der Schleuse in Abhängigkeit des Stützdrucks langsam angepasst. Nach Beendigung eines Einstiegs unter Druckluft in die Abbaukammer wird diese wieder vollständig mit Stützflüssigkeit gefüllt, sodass der Vortrieb fortgesetzt werden kann. 


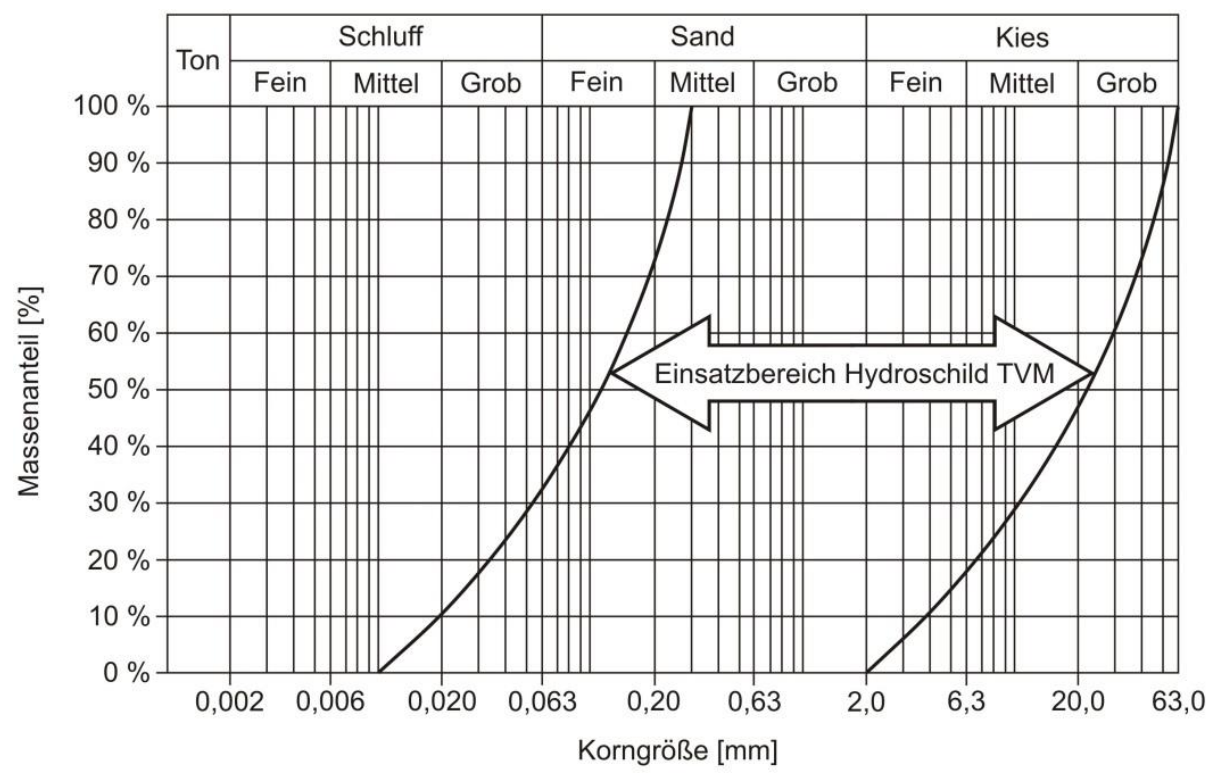

Abb. 4: Typischer Einsatzbereich für Hydroschild TVM anhand der Korngrößenverteilung des Bodens. (Herrenknecht $A G$, verändert)

Der Haupteinsatzbereich von Hydroschild TVM liegt in rolligen Böden mit einer Korngröße oberhalb von Feinsand (Abb. 4) und einem Durchlässigkeitsbeiwert $k_{f}$ größer $10^{-7} \mathrm{~m} / \mathrm{s}$ (www-04). Diese Böden können leicht in der Stützflüssigkeit in Suspension gebracht werden, weil sie beim Abbau häufig dazu neigen, in Einzelkörner zu zerfallen. Aufgrund der Korngröße können sie anschließend in der Separation durch Siebung relativ leicht wieder aus der Stützflüssigkeit entfernt werden. Durch den Einsatz zusätzlicher Additive zur Stützflüssigkeit kann der Einsatzbereich darüber hinaus auch auf bindige Böden erweitert werden (BABENDERERDE 2003: 3). Die Additive dienen hier einerseits dazu, eine Wasseraufnahme des abgebauten Bodens aus der Stützflüssigkeit zu verhindern und Verklebungen zu vermeiden. Andererseits dienen die Additive dazu zu verhindern, dass sich die abgebauten Bodenteile in der Stützflüssigkeit in Einzelkörner kleiner als Feinsand auflösen. Diese erfordern in der Separationsanlage einen wesentlich höheren Aufwand zur Abtrennung aus der Stützflüssigkeit mit Kammerfilterpressen oder Zyklonen.

\subsection{Hauptkomponenten}

Im Anschluss werden die wesentlichen konstruktiven Hauptkomponenten einer Hydroschild TVM und deren Funktion im Einzelnen beschrieben. Die Beschreibung konzentriert sich dabei auf die Komponenten, die für den Bodenabbau, Ortsbruststützung und Abraumförderung verantwortlich sind, weil diese Grundlagen für die weiteren Untersuchungen in der vorliegenden Arbeit sind. Alle weiteren Komponenten, die nicht unmittelbar für die genannten Funktionen entscheidend sind, werden aus Gründen der Übersichtlichkeit nicht im Detail beschrieben. Dazu zählen vor allem:

- Tübbingkran, Segmentfeeder und Erektor für den Ausbau des Tunnels mit Betonsegmenten im Schutz des Schildes.

- Mörtelsystem zur Hinterfüllung der Tübbingsegmente.

- Elektrische und hydraulische Ausrüstung der TVM.

- Nachläufersystem und Logistik.

Detaillierte Informationen zu diesen Punkten sind einerseits in MAIDL et al. (2011:248) und GIRMSCHEID (2004: 119) enthalten, können zusätzlich aber auch über das Internet z.B. über www-05 abgerufen werden. 


\title{
3.2.1 Schneidrad \& Abbauwerkzeuge
}

Das Schneidrad erfüllt im Vortrieb und bei Arbeiten in der Abbaukammer drei Hauptaufgaben:

- Befestigung der Abbauwerkzeuge und Führung über die Ortsbrust.

- Aufnahme der resultierenden Schneidkräfte und Übertragung auf den Antrieb und das Hauptlager.

- Schutzfunktion des Personals bei Arbeiten in der Abbaukammer.

Einen Überblick über die technische Entwicklung verschiedener Schneidradtypen gibt BuRGER (2006: 11). Aus konstruktiver Sicht können Schneidräder demnach einerseits nach ihrem Aufbau und andererseits nach der Lage in Bezug auf den Schildmantel bzw. Schildschneide unterteilt werden. Hinzu kommen begehbare Schneidräder bei großen Maschinendurchmessern.

Vor allem bei älteren Projekten wurden häufig Speichenschneidräder eingesetzt, die aus sternförmig angeordneten Armen bestehen, die im Radius nicht durch eine umlaufende Felge verbunden sind. Deutlich häufiger werden mittlerweile Schneidräder mit breiteren Armen eingesetzt, die im Radius mit einer umlaufenden Felge verbunden sind. Die breiteren Arme erlauben deutlich mehr Werkzeuge auf dem Schneidrad unterzubringen, während die umlaufende Felge die Stabilität des Schneidrades erhöht.

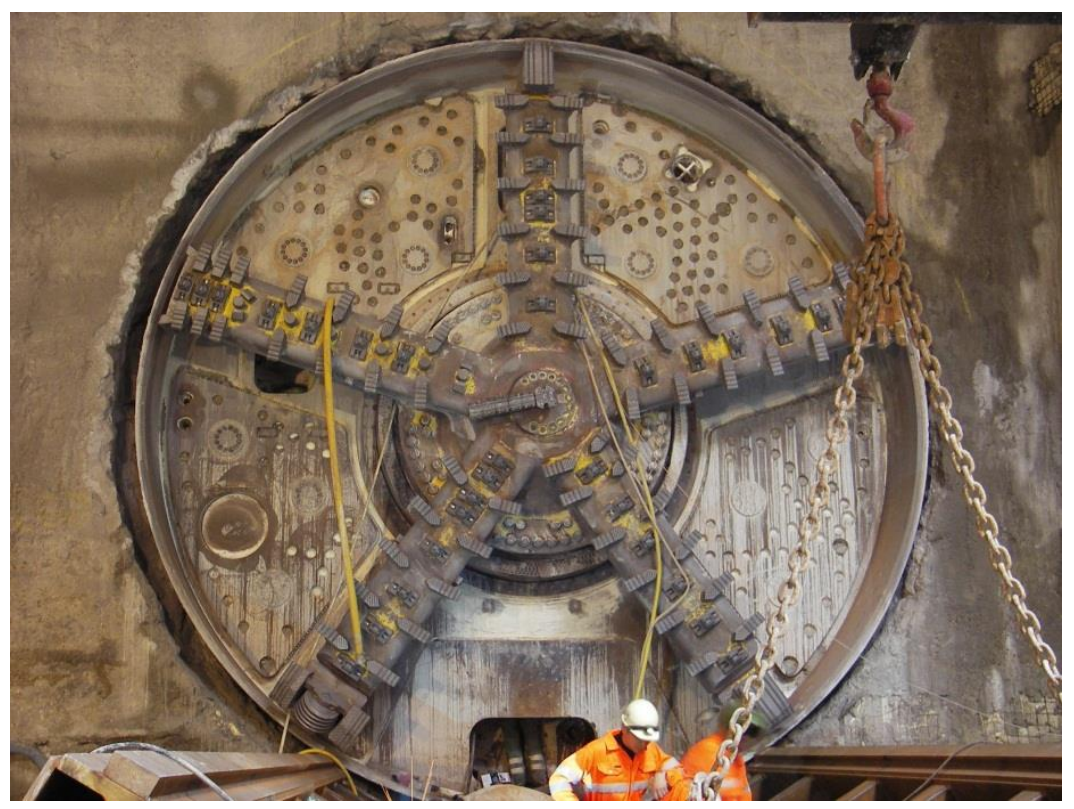

\begin{abstract}
Abb. 5: Sternförmiges Speichenschneidrad ohne umlaufende Felge. Das Schneidrad ist im Schild innenliegend angebracht. Der Antrieb ist verschiebbar gelagert, sodass das Schneidrad auch vor das Schild ausgefahren werden kann. Der Bohrdurchmesser kann dann durch hydraulisch ausfahrbare Überschneider an den Armen erhöht werden.
\end{abstract}

In Bezug auf den Schildmantel kann das Schneidrad vor der Schildschneide, also außenliegend, angeordnet werden. Der Bohrdurchmesser kann damit größer gewählt werden als der Schilddurchmesser, sodass sich ein Überschnitt ergibt. Im Gegensatz dazu kann das Schneidrad auch innerhalb des Schildes, also innenliegend, angeordnet werden. Hier entspricht der Bohrdurchmesser dem Schilddurchmesser, wobei die über dem Schneidrad liegende Schildschneide während dem Vortrieb in den Boden gedrückt wird. 


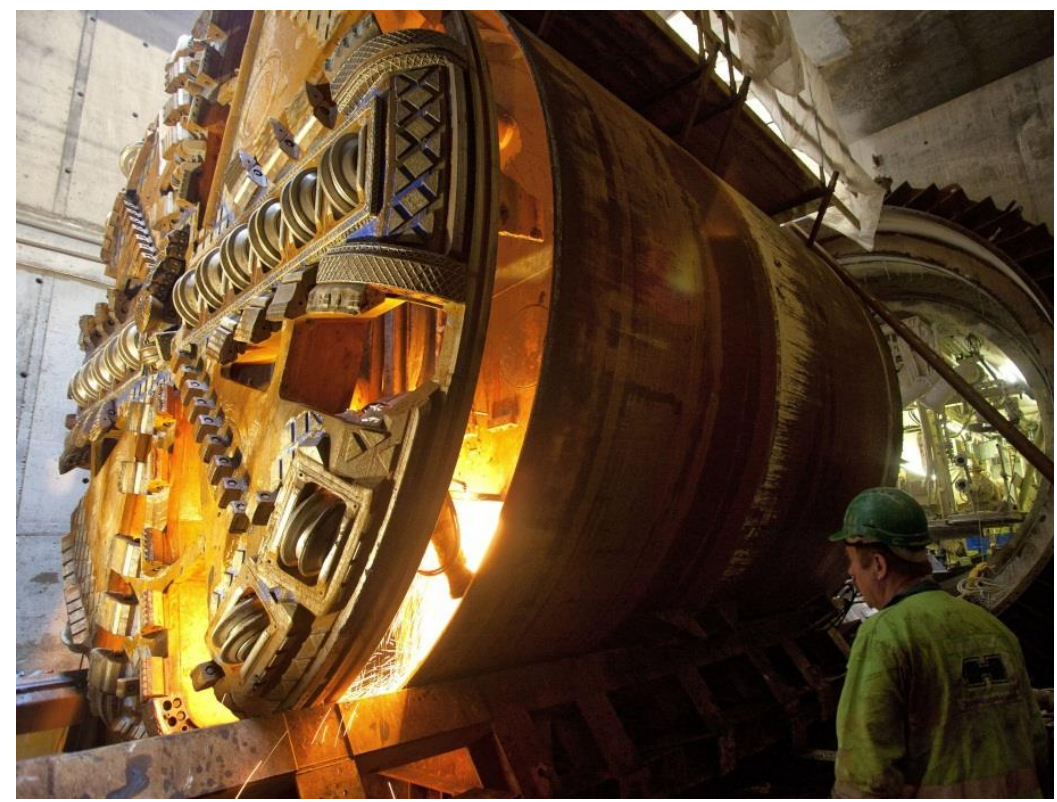

Abb. 6: Schneidrad mit umlaufender Felge. Das Schneidrad ist außenliegend bzw. vor der Schildschneide angebracht. (Herrenknecht AG)

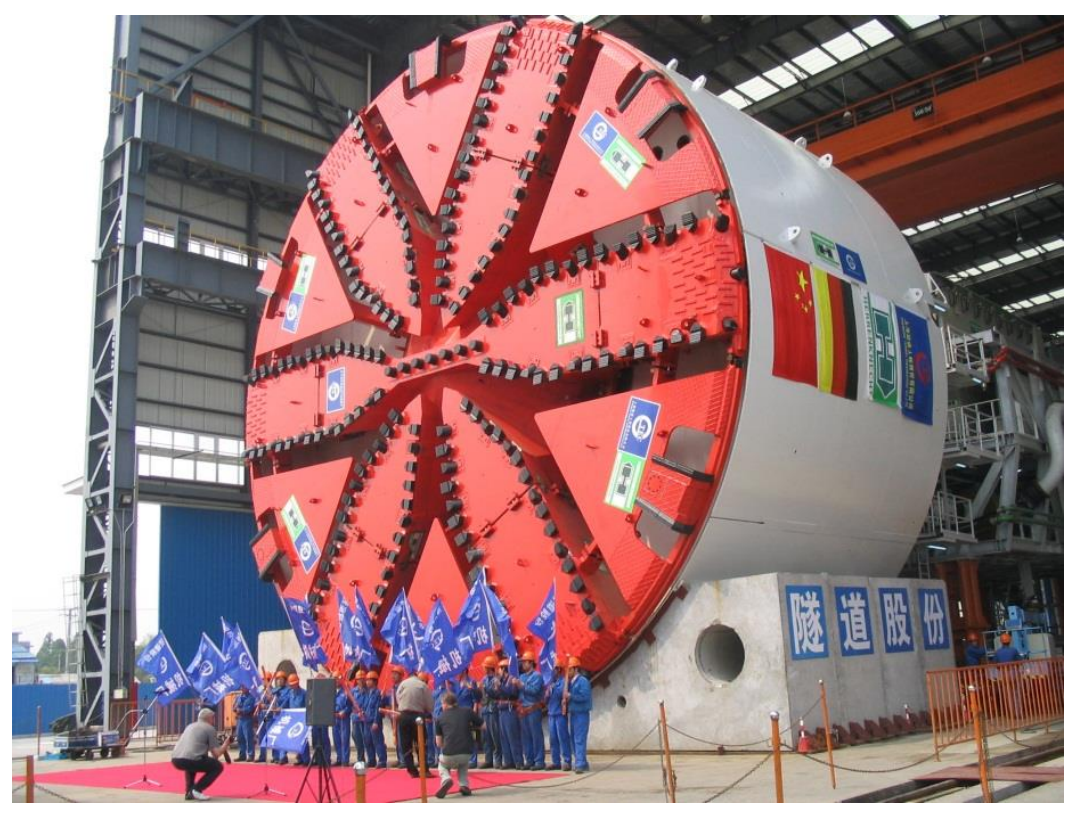

Abb. 7: Außenliegendes Felgenschneidrad mit begehbaren Armen für drucklosen Werkzeugwechsel. (Herrenknecht AG)

Ab einem Maschinendurchmesser $D_{\text {TVM }}$ größer als ca. $12 \mathrm{~m}$ sind aus konstruktiver Sicht begehbare Schneidräder möglich (Abb. 7). Hier ist die Innenseite der Schneidradarme durch entsprechende Öffnungen aus dem atmosphärischen Bereich der TVM hinter der Druckwand begehbar. Daraus ergeben sich Vorteile für den Werkzeugwechsel bei hohem Stützdrück in der Abbaukammer, da die Arbeiten unter atmosphärischen Bedingungen durchgeführt werden können. Dazu wird jedes einzelne Werkzeug mit einer Druckschleuse versehen, über die den Ein- und Ausbau der Werkzeuge aus dem Innenraum der Schneidradarme ermöglicht. 
Die zwischen den Schneidradarmen verbleibenden Öffnungen dienen dem Materialfluss von der Ortsbrust in die Abbaukammer hinter das Schneidrad, wo das Material mit der Stützflüssigkeit gefördert wird. BURGER (2006: 14) gibt für das Schneidradöffnungsverhältnis $O_{T V M}$, also das Verhältnis der Fläche der Öffnungen zur Ortsbrustfläche, in der Regel 25 - 35\% an. Dabei kommt den Öffnungen im Zentrumsbereich des Schneidrades höhere Bedeutung zu als im Außenbereich, da hier häufige Verklebungen entstehen. Die Größe der Schneidradöffnungen ist zusätzlich maßgeblich für die maximal mögliche Größe der Bodenkomponenten die hinter das Schneidrad gelangen können. Diese sollte nicht höher liegen als die mit dem Steinbrecher bewältigbare Korngröße, um zu verhindern, dass sich in der Abbaukammer Blöcke ansammeln, die zu hohem Verschleiß und Schäden führen und zusätzlich den Materialfluss behindern. Bei relativ großen Öffnungen im Außenbereich des Schneidrades werden deshalb zusätzliche Bleche als Korngrößenbegrenzer angebracht.

Der Stahlbau der Schneidradarme ist im Regelfall kastenförmig aus Blechen aufgebaut, wobei die Innenseite mit Ausnahme von begehbaren Schneidrädern im Vortrieb nicht mehr zugänglich ist. An der Schneidradrückseite schließen Verbindungsstreben an, die das Schneidrad mit dem Flanschring verbinden. Dieser ist über Bolzen mit dem Hauptantrieb verbunden. Bei Hydroschild TVM kann das Schneidrad mit dem Hauptantrieb im Regelfall in beide Drehrichtungen gedreht werden, um damit die Verrollung der Schildmaschine zu kontrollieren. Entsprechend werden auch für beide Drehrichtungen Abbauwerkzeuge auf dem Schneidrad vorgesehen.

Die Gehäuse für Schneidrollen werden in den kastenförmigen Stahlbau der Schneidradarme eingesetzt und verschweißt, sodass die Schneidrollen von der Rückseite des Schneidrades aus ein- und ausgebaut werden können. Für die Fixierung der Schneidrollen im Gehäuse existieren hauptsächlich zwei weit verbreitete Systeme. Beim sogenannten Wedgelock-System (Abb. 8) wird die Schneidrolle von der Rückseite in das Gehäuse eingesetzt und anschließend seitlich verschoben, sodass die Schneidrollenachse direkt auf dem Gehäuse bzw. einem entsprechenden Einsatz aufliegt. Die Achse wird dann seitlich durch einen Keil gesichert, der mit einer Schraube und einem Klemmblock gegen das Gehäuse gesichert ist.

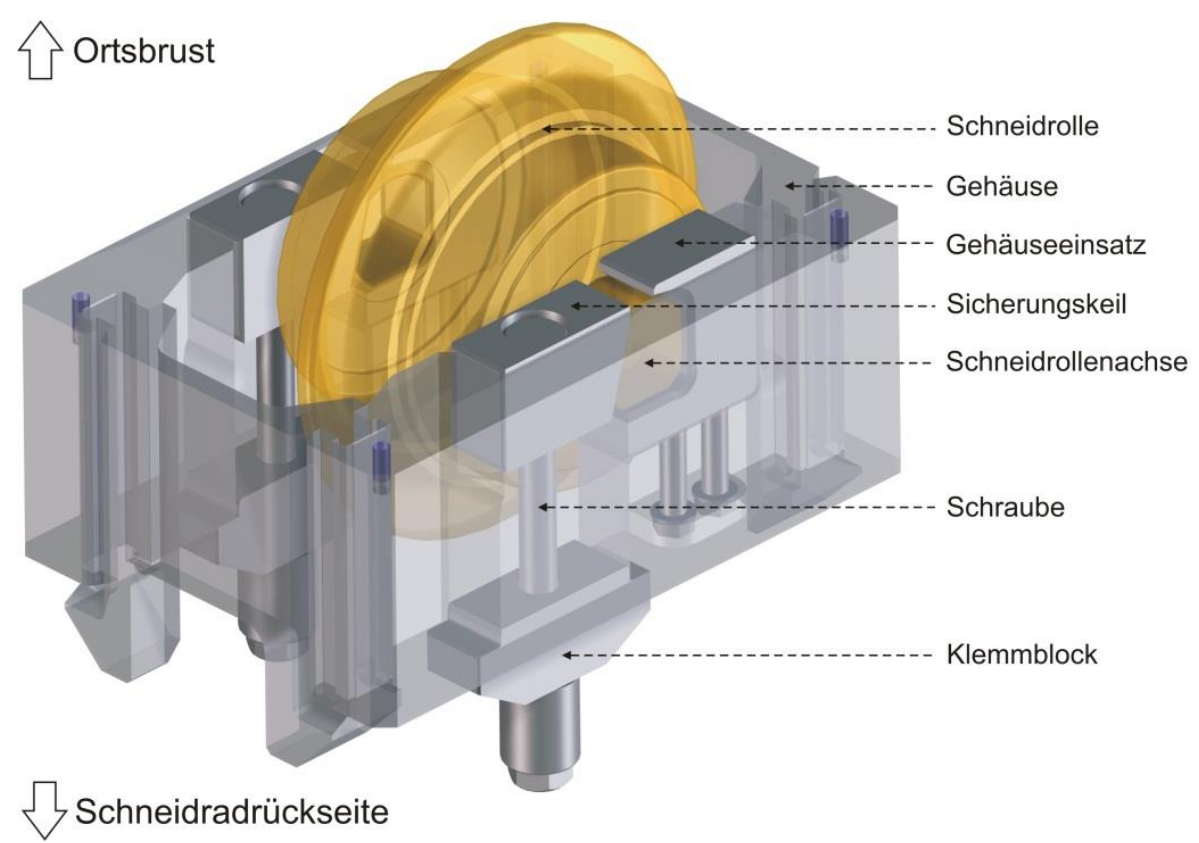

Abb. 8: 3D-Darstellung einer Schneidrolle mit Befestigungselementen in einem Wedgelock-Gehäuse. (Herrenknecht $A G$, verändert)

Beim sogenannten Schrägkeil-System wird die Schneidrollenachse in einen dreieckigen Sitz im Gehäuse eingesetzt und durch einen schräg angeordneten Keil gesichert. Der Hauptunterschied zwischen den beiden Systemen besteht in der Übertragung der Schneidkräfte in das Gehäuse und den Schneidradstahlbau. Beim Wedgelock-System wird durch die seitliche Verschiebung der Schneidrolle 
in den Gehäusesitz ein deutlich höherer Anteil der Schneidkräfte direkt in das Gehäuse übertragen. Die Belastung der Befestigungselemente (Keile, Schraube, Klemmblock) fällt hier deutlich geringer aus. Im Gegenzug ergibt sich durch die seitliche Verschiebung eine leichte Erhöhung des Aufwands im Montage- und Demontageprozess.

Der Aufbau der Schneidrollen selbst folgt trotz zahlreicher Varianten verschiedener Hersteller immer dem nachfolgenden Grundaufbau (Abb. 9). Zentraler Teil einer 1-Ring Schneidrolle ist die Achse, mit der die Schneidrolle auch im Gehäuse fixiert wird. Auf der Achse werden zwei Kegelrollenlager aufgezogen, auf denen der Rollengrundkörper gelagert ist. Auf die Achse werden zusätzlich zwei seitliche Deckel aufgezogen, die zusammen mit dem Rollengrundkörper eine Lagerkammer bilden. Diese wird über verschließbare Öffnungen in den Deckeln entweder mit Öl oder mit Fett gefüllt. Der Spalt zwischen dem im Einsatz drehenden Rollengrundkörper und den feststehenden Deckel (Dichtspalt) wird durch zwei Gleitringdichtungen abgedichtet. Auf den Rollengrundkörper wird abschließend der Schneidring mit einer Presspassung zentrisch aufgezogen und durch einen Sicherungsring fixiert.

Von diesem Grundaufbau gibt es drei wesentliche Varianten, die für die weiteren Untersuchungen in der vorliegenden Arbeit von Bedeutung sind. Bei oben beschriebenem Aufbau können Rollengrundkörper und Schneidring auch aus einem Stück hergestellt werden. Damit entfällt der Sicherungsring für den Schneidring. Diese Variante wird als 1-Fach Monoblock Schneidrolle bezeichnet. Ebenfalls auf Basis dieses Aufbaus können auch zwei Schneidringe mit einem Zwischenring nebeneinander auf den Rollengrundkörper aufgezogen werden. Diese Variante wird als 2-Ring Schneidrolle bezeichnet, wobei die beiden Schneidringe nicht separat gelagert sind. Analog zur 1-Ring Schneidrolle ist auch hier die Auslegung als 2-Fach Monoblock Schneidrolle möglich.

Im Gegensatz zu diesen Varianten werden im Schneidradzentrum aus Platzgründen vier Kegelrollenlager und zwei Rollengrundkörper auf eine verlängerte Achse gezogen. Diese Zentrumsschneidrolle besitzt zwei separat gelagerte Rollengrundkörper, die mit jeweils einem Schneidring bestückt werden.

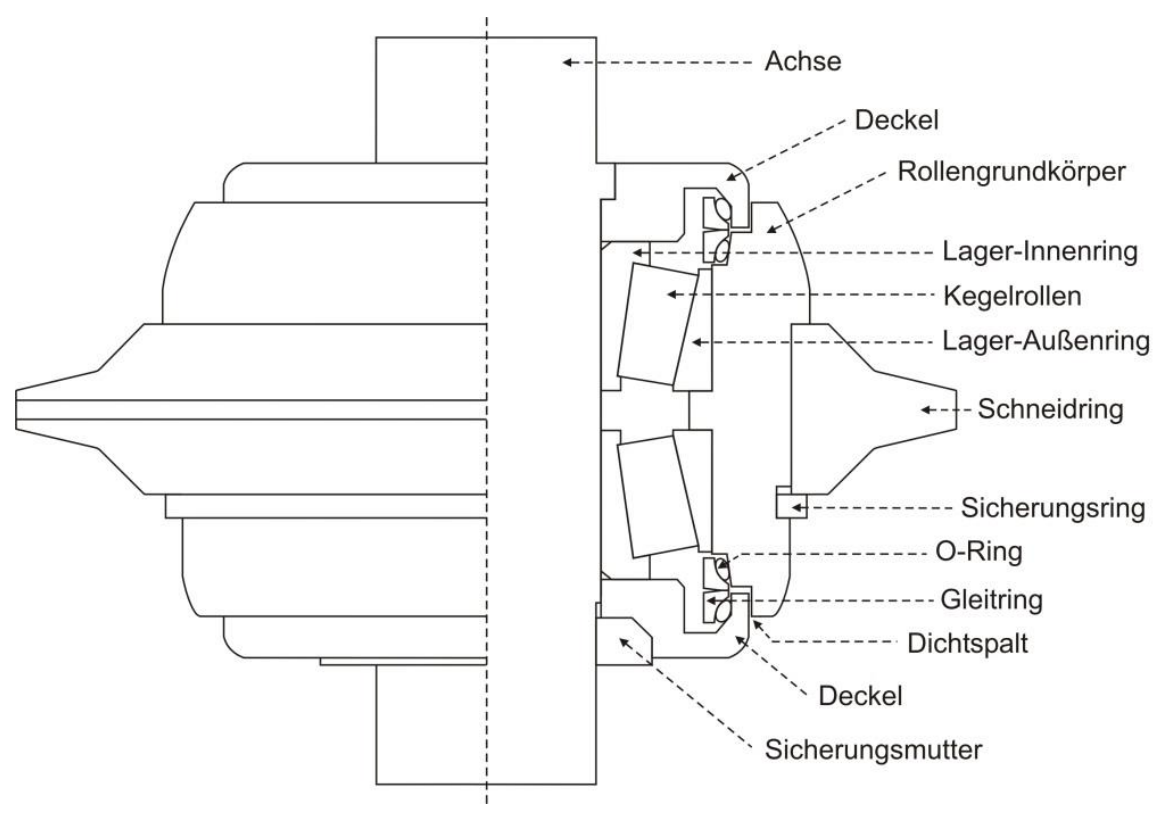

Abb. 9: Querschnitt durch eine 1-Ring Schneidrolle. (Herrenknecht AG, verändert)

Für den Außendurchmesser der Schneidrollen haben sich für Maschinendurchmesser $D_{\text {TVM }}>6,0 \mathrm{~m}$ zwei Varianten etabliert. Häufigste Variante ist ein Außendurchmesser von 17 Zoll (432 mm), wobei die Schneidrollen ein Gewicht von 120 kg (1-Ring Schneidrolle) bis zu 280 kg (Zentrumsschneidrolle) erreichen. Darüber werden auch 19 Zoll $(483 \mathrm{~mm})$ Schneidrollen eingesetzt, die entsprechend ein Gewicht von $190 \mathrm{~kg}$ bis zu $360 \mathrm{~kg}$ erreichen. Die beiden Varianten unterscheiden sich in der Größe der Kegelrollenlager und des Rollengrundkörpers. Als Abstufungen auch 18 Zoll Schneidrollen gängig. Diese basieren auf dem Grundaufbau der 17 Zolll Schneidrolle. Die Änderung des Außendurchmessers wird jeweils durch eine Erhöhung des Schneidringquerschnitts im Bereich der Schneide erreicht. 
Der Schneidring ist das einzige Bauteil der Schneidrolle, das planmäßig Kontakt mit dem Boden in der Ortsbrust hat. Die aktuell in Verwendung befindlichen Schneidringe werden aus verschleißfesten, hochlegierten Werkzeugstählen hergestellt. Diese werden durch Wärmebehandlung auf eine durchgehende Härte nach ROCKWELL von 54 - 58 HRC gebracht. Die Herausforderung besteht dabei darin, eine möglichst hohe Härte des Schneidrings zu erreichen und gleichzeitig die für die Aufnahme der Schneidkräfte notwendige Zähigkeit des Materials zu erhalten. In Einzelfällen werden Schneidringe eingesetzt, die mit Hartmetalleinsätzen und zusätzlichem Hartauftrag ausgestattet sind.

Für die Querschnittsform der Schneidringe haben sich ausgehend von keilförmigen Querschnitten Schneidringe mit einer gleichbleibenden Schneidringbreite $b_{S R}[\mathrm{~mm}]$ etabliert (Abb. 10). Diese bestehen aus einer breiteren Basis, die benötigt wird, um den Schneidring sicher auf dem Rollengrundkörper zu fixieren und die notwendige Widerstandsfähigkeit gegen Verformungen zu erreichen. Auf die Basis ist ein Übergangsbereich aufgesetzt, der in einer schmäleren Schneide mündet, die bis zum Außenradius des Schneidrings eine annähernd gleichbleibende Breite aufweist. Diese liegt üblicherweise in einem Bereich von $12 \mathrm{~mm}$ (1/2 Zoll) bis zu $25 \mathrm{~mm}$ (1 Zoll).

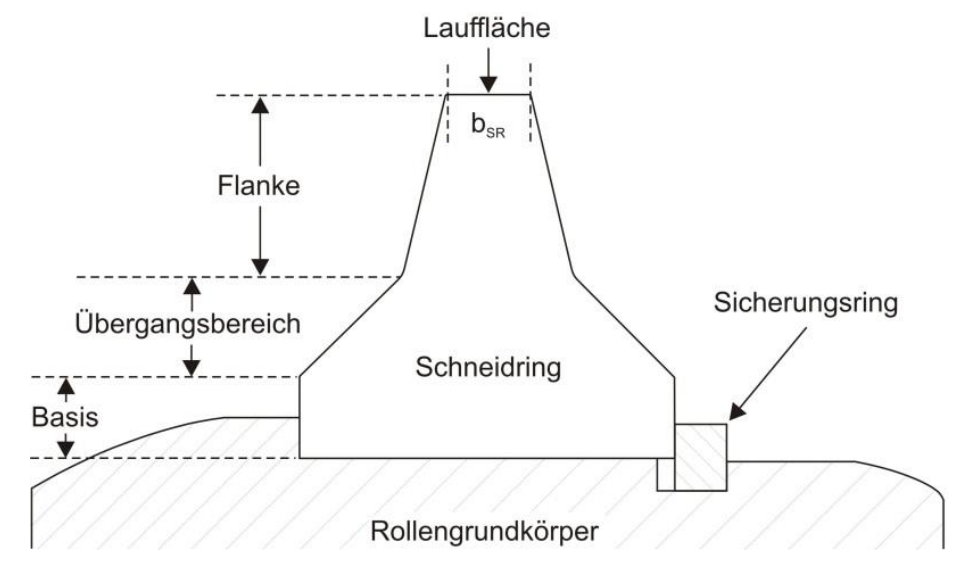

Abb. 10: Querschnitt durch den Rollengrundkörper, Schneidring und Sicherungsring einer 1-Ring Schneidrolle. Die Breite der Lauffläche des Schneidrings wird als Schneidringbreite b $_{\mathrm{SR}}[\mathrm{mm}]$ bezeichnet.

Die Kegelrollenlager in der Schneidrolle erfordern ein bestimmtes Maß an Lagervorspannung. Diese wird während dem Montagevorgang eingestellt. Aus der Lagervorspannung und aus der Reibung zwischen den Gleitringen der Dichtungen entsteht ein Eigendrehmoment der Schneidrolle, das im Vortrieb durch die Schneidwiderstände zu überwinden ist. Als Erfahrungswert hat sich ein Mindestwert von 23 bis $25 \mathrm{Nm}$ für 17" Schneidrollen etabliert, bei dem eine ausreichende Lagervorspannung und zuverlässige Dichtheit der Schneidrolle gewährleistet ist. Zusätzlich liegt die radiale Belastungsgrenze für die Kegelrollenlager in 17" Schneidrollen bei 270 - 295 kN.

Die Halter für Schälmesser werden auf den Stahlbau des Schneidrades aufgesetzt und verschweißt. Die Schälmesser ragen so in die Schneidradöffnungen hinein und können im Vortrieb von der Rückseite des Schneidrades durch die Schneidradöffnungen gewechselt werden. Es können dabei drei verschiedene Halterungssysteme unterschieden werden. Die erste Form besteht aus einer Halterplatte mit zwei bis vier Gewindelöchern. Das Schälmesser wird mit entsprechenden Schrauben auf der Halterplatte verschraubt (Abb. 11). Bei der zweiten Halterform wird der Halter an der Kante zwischen Frontfläche und Seitenfläche des Schneidradstahlbaus angebracht. Der Halter besitzt eine schlitzförmige Öffnung zur Schneidradöffnung hin, in die der Schaft des Schälmessers eingeschoben wird. Der Kopf des Schälmessers liegt auf der Frontfläche des Halters auf. Das Schälmesser wird zusätzlich durch einen Bolzen durch den Halter und den Schaft gesichert. Die Dritte Halterform wird auf der Frontfläche des Schneidradstahlbaus aufgesetzt. Der Halter ist hier mit einer rechteckigen Aufnahme für den Schaft des Schälmessers ausgestattet. Das Schälmesser wird in diese Aufnahme eingesetzt und mit einem Bolzen durch Halter und Schaft gesichert. 
Das Schälmesser selbst besteht aus einem Kopf und einem Schaft zur Befestigung im Halter. Schaft und Kopf werden aus einem Stück aus einem Schmiederohling oder als Guss- oder Brennteil aus Werkzeugstahl gefertigt. Der Werkzeugstahl des Grundkörpers selbst besitzt eine Härte von ca. 4448 HRC. Im Anschluss daran werden die durch mechanische Bearbeitung die abschließenden Konturen des Werkzeuges und entsprechende Aufnahmen für den Verschleißschutz hergestellt. Der Verschleißschutz in Form von Hartmetalleinsätzen und Hartaufträgen wird im letzten Arbeitsschritt in der Schneide, Frontfläche und Rückenfläche (Abb. 12) auf den Schälmesserkopf aufgebracht.
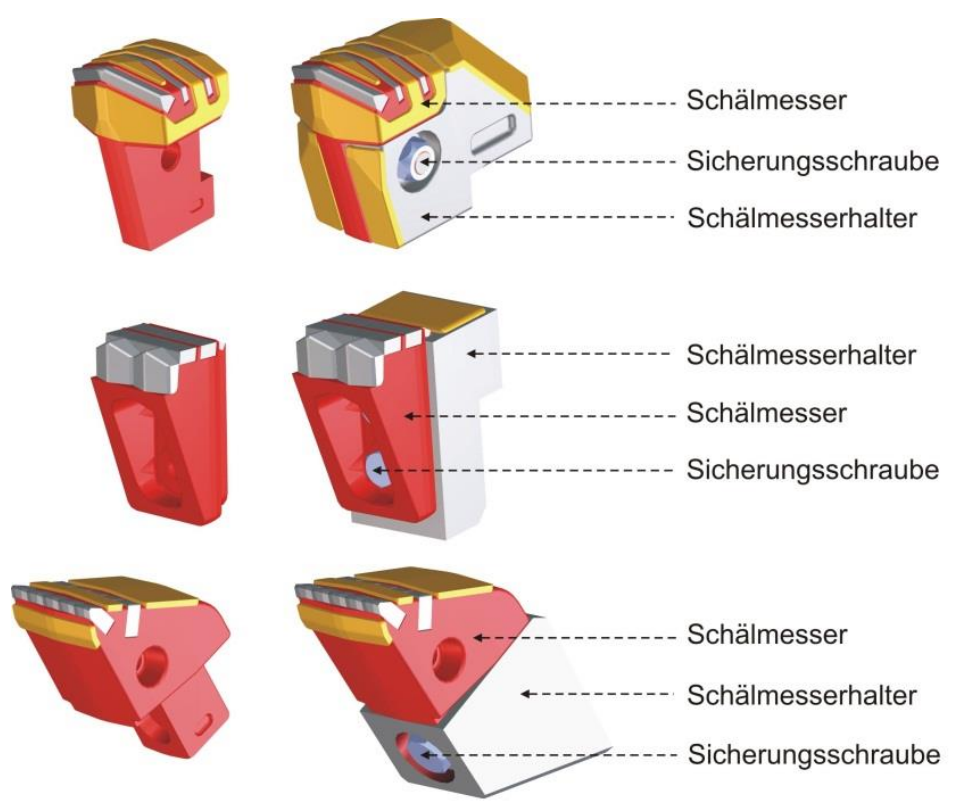

Abb. 11: 3D-Darstellung dreier gängiger Schälmessertypen mit zugehörigem Halter und Befestigungselemente. (Herrenknecht AG, verändert)

Unter dem Begriff Hartmetall werden hier Verbundwerkstoffe zusammengefasst, die aus extrem harten Wolframcarbid-Partikeln (WC) in einer zähen Metallmatrix zusammengesetzt sind. Die Korngröße der Wolframcarbid-Partikel, die chemische Zusammensetzung der Matrix, das prozentuale Verhältnis zwischen Wolframcarbid-Partikel und Metallmatrix und die daraus resultierende Härte sind stark vom Hersteller und dem Anwendungsbereich abhängig. Beispiele geben www-006 und www-07. Die Korngröße der Partikel liegt in der Regel zwischen 0,1 und $50 \mu \mathrm{m}$. Für die umgebende Matrix werden hauptsächlich Nickel $(\mathrm{N})$ und Chrom $(\mathrm{Cr})$ haltige Werkstoffe eingesetzt. Der Anteil der WolframcarbidPartikel am Gesamtwerkstoff beträgt zwischen $70 \%$ und $85 \%$, damit ergibt sich eine mögliche Bandbreite für die Härte dieser Werkstoffe von ca. 900 - 1800 HV (Härte n. VICKERS). Aufgrund der extremen Härte können diese Werkstoffe kaum noch nachbearbeitet werden und sind auch nicht schweißbar. Die Einsätze erhalten ihre endgültige Form bereits während der Fertigung, sie werden je nach Herstellungsverfahren durch einen autogenen oder induktiven Lötprozess mit dem Grundkörper des Schälmessers aus Werkzeugstahl verbunden. Hier werden entsprechende Nuten für die Hartmetalleinsätze in der mechanischen Bearbeitung des Rohlings hergestellt.

Unter dem Begriff Hartauftrag ist ebenfalls eine sehr breite Gruppe an Werkstoffen zusammengefasst, die im Unterschied zu den Hartmetallen jedoch mit unterschiedlichen Methoden schweißbar sind. Zusammensetzung und Eigenschaften sind wiederum stark vom Hersteller und Einsatzbereich abhängig. Eine Übersicht über die Bandbreite an Werkstoffen gibt z.B. www-08. Zu unterscheiden sind hier homogene Werkstoffe, die auf Basis hoher Kobalt (Co), Chrom (Cr), Kohlenstoff (C) und Nickel (Ni) Legierungen nach dem Aufschweißen auf den Grundkörper des Schälmessers eine relativ hohe Härte von ca. 58 - $60 \mathrm{HRC}$ erreichen. Dem gegenüber sind auch Verbundwerkstoffe verfügbar, die nach dem Aufschweißen aus einer zähen Matrix mit eingelagerten Wolframcarbid-Partikeln bestehen. Die Härte der Matrix liegt dabei in einem ähnlichen Bereich wie die Härte des Grundkörpers des Schälmessers. 
Im Hinblick auf die Geometrie der Schälmesser sind für den Bodenabbau vor allem der Schneidwinkel, der Freiwinkel und die Breite entscheidend (BERETITSCH 1995: 98 ff.). Die Breite der eingesetzten Schälmesser beträgt zwischen $100 \mathrm{~mm}$ und $250 \mathrm{~mm}$, der Schneidwinkel $\alpha_{c}\left[^{\circ}\right]$ und der Freiwinkel $\beta_{c}\left[^{\circ}\right]$ wird je nach Bodenart variiert. Schälmesser erreichen so in Abhängigkeit der Breite ein Gewicht von ca. $5,0 \mathrm{~kg}$ bis $35,0 \mathrm{~kg}$. Das Spacing der Schälmesser $S_{S M}[\mathrm{~mm}]$ wird so gewählt, dass die Schälmesser die Ortsbrust vollständig abdecken. Das Spacing $S_{S M}$ entspricht dann der Breite der Schälmesser $b_{S M}$. Zum Teil wird das Spacing $S_{S M}[\mathrm{~mm}]$ jedoch auch kleiner gewählt als die Breite $b_{S M}$, sodass sich eine seitliche Überlappung der Schälmesser zwischen den Schneidspuren ergibt.

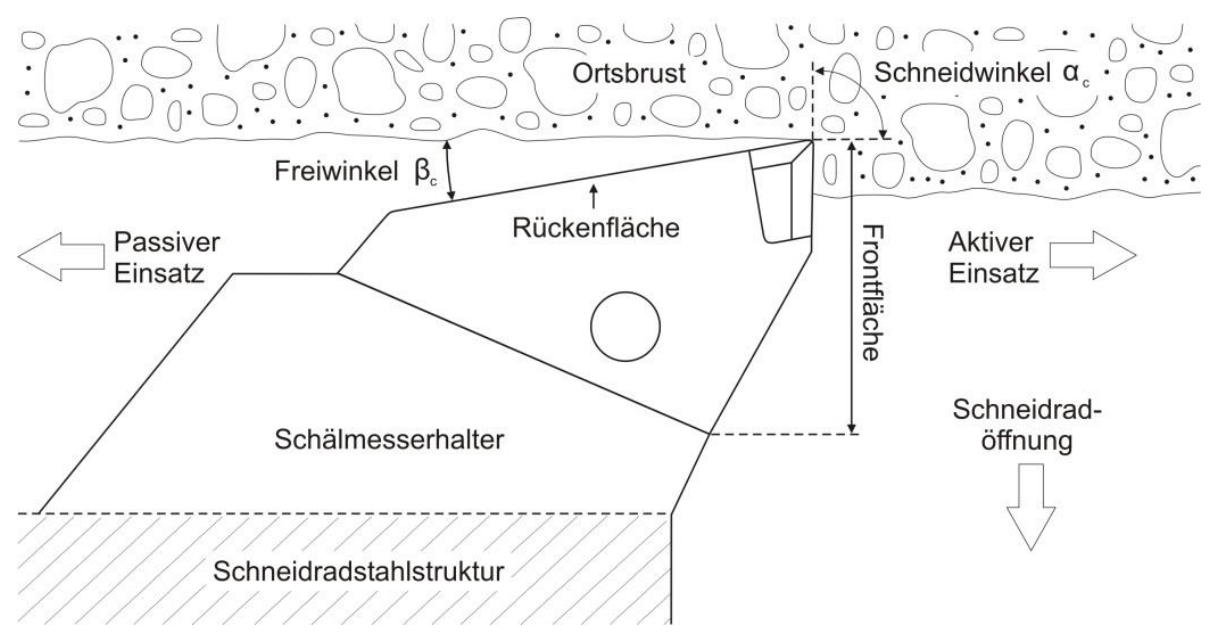

Abb. 12: Querschnitt durch ein Schälmesser mit Halter und Schneidradstahlstruktur.

In Abhängigkeit des Schneidraddurchmessers $D_{T V M}$ und des Spurradius $r_{s}[\mathrm{~mm}]$ werden die Schneidspuren der Schälmesser mit 2 - 8 identischen Schälmessern je Schneidraddrehrichtung bestückt. Der so entstehende Mehrfachbesatz wird bezogen auf eine Schneidraddrehrichtung als Spurbesatz $\mathrm{k}_{\mathrm{s}}$ bezeichnet.

Zusätzlich zu Schneidrollen und Schälmessern werden bei Hydroschild TVM zum Teil auch Stichel zum Bodenabbau eingesetzt. Während diese bei älteren Maschinen häufig in separate Halter auf den Speichen eines Schneidrades angebracht waren (MAIDL et al. 1995: 65 \& BURGER 2006: 11), haben sich bei aktuellen Schneidradauslegungen Stichel etaliert, die alternativ zu Schneidrollen in die Schneidrollengehäuse eingesetzt werden können (Abb. 13).

Dazu ist ein Stichelhalter notwendig, der analog zur Schneidrollenachse mit Keilen und Klemmblock im Schneidrollengehäuse fixiert wird. Auf dem Halter ist an der Vorderseite ein Stichelkopf angebracht, der durch eine Schraube gesichert wird. In einigen konstruktiven Varianten kann der Stichelhalter durch Lösen von zwei Zusatzkeilen im Schneidrollengehäuse gedreht werden, sodass der Stichelkopf auf der Schneidradrückseite liegt. Der Stichelkopf kann damit ohne Ausbau des Halters von der Schneidradrückseite aus gewechselt werden. Stichel, die fest auf der Frontseite der Schneidradstahlstruktur verschweißt sind, kommen kaum noch zum Einsatz, weil zu deren Kontrolle oder Wechsel im Vortrieb Zugang zwischen die Frontseite der Schneidradarme und die Ortsbrust notwendig wäre. Dieser ist aus technischer Sicht kaum möglich und wird auf Grund der Risiken für das Personal vermieden.

Der Stichelkopf ist ähnlich wie ein Schälmesser aufgebaut, mit dem entscheidenden Unterschied, dass beidseitig für beide Schneidraddrehrichtungen eine Schneide vorgesehen ist. Als Verschleißschutz kommen die gleichen Materialien wie für Schälmessern zum Einsatz. Der Stichelkopf hat in Abhängigkeit der Auslegung eine Breite $b_{S T}[\mathrm{~mm}]$ von $50 \mathrm{~mm}$ bis zu $200 \mathrm{~mm}$. 


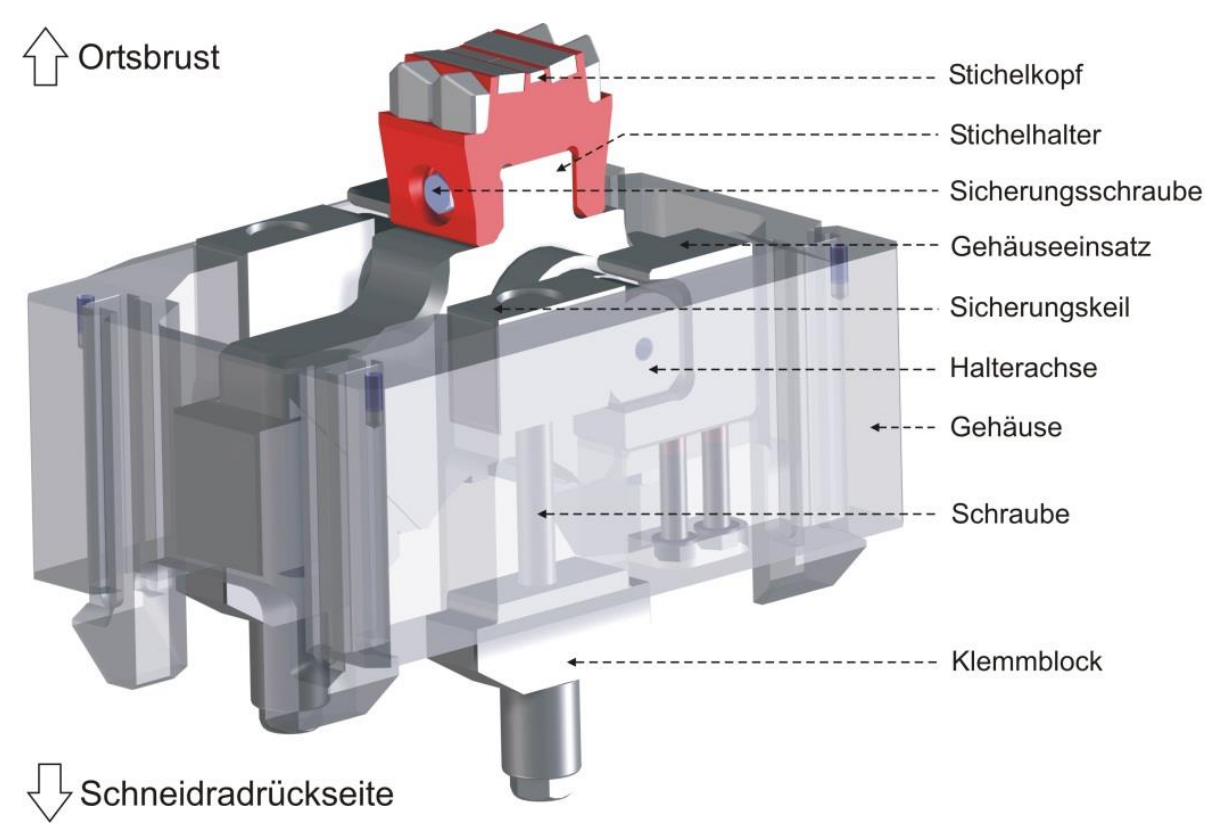

Abb. 13: 3D-Darstellung eines Stichels mit Befestigungselementen in einem Wedgelock-Gehäuse. (Herrenknecht $A G$, verändert)

Werden die Stichel in den Gehäusen der Schneidrollen eingesetzt, entspricht das Spacing der Stichel $\mathrm{S}_{\mathrm{ST}}[\mathrm{mm}]$ dem ursprünglich vorgesehenen Spacing der Schneidrollen $\mathrm{S}_{\mathrm{SR}}[\mathrm{mm}]$. Aufgrund der höheren Breite des Stichelkopfs $b_{S T}[\mathrm{~mm}]$ im Vergleich zur Breite des Schneidrings $b_{S R}$, ergibt sich beim Einsatz der Stichel jedoch ein deutlich höhere Abdeckung der Ortsbrust. Wird die Breite der Stichelköpfe $b_{S T}$ größer oder gleich dem ursprünglichen Spacing der Schneidrollen $S_{S R}$ gewählt, wird die Ortsbrust vollständig durch die Stichel abgedeckt.

Im Kaliberbereich des Schneidrades werden anstatt von Schälmessern Räumer angebracht. Diese decken mit einer durchgehenden Kontur die Rundung des Ausbruchprofils im Übergang zwischen Ortsbrust und Tunnellaibung ab. Im Unterschied zu Vortrieben im Festgestein nehmen die Räumer bei Hydroschild TVM in Lockergesteinen im Kaliberbereich planmäßig aktiv am Bodenabbau teil. Sie werden deshalb meist mit einem ähnlichen Verschleißschutz wie die Schälmesser ausgestattet. Einen Überblick über die konstruktiven Merkmale der Räumer gibt Abb. 14.
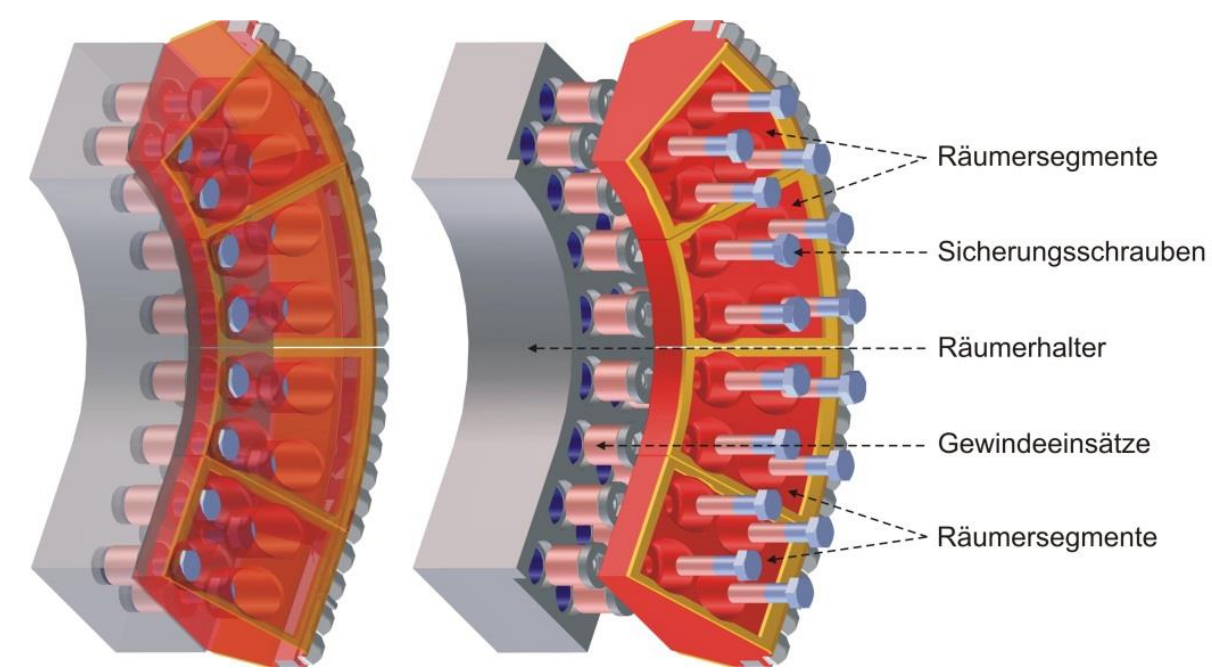

Abb. 14: 3D-Darstellung eines Räumersystems mit 4 Segmenten und zugehörigem Räumerhalter. (Herrenknecht AG, verändert) 
In Abhängigkeit der Auslegung des Schneidrades werden der Radius der Räumerkontur, die Segmentteilung der Räumer und die Verschraubung der Räumersegmente variiert. Eine Teilung in Segmente wird vor allem deshalb vorgenommen, um das Gewicht der Einzelteile im Hinblick auf den Wechsel im Vortrieb gering zu halten. Die Segmente werden jeweils auf einer entsprechenden Halterplatte am Schneidradstahlbau verschraubt. Dabei kann die Verschraubung einreihig oder zweireihig ausgeführt sein, wobei eine zweireihige Verschraubung den Räumersegmenten vor allem in grobkörnigen Böden deutlich mehr Stabilität gibt. Die Auslegung des Schneidwinkels $\alpha_{c}$ und des Freiwinkel $\beta_{c}$ erfolgt analog zu den Schälmessern. Die Räumersegmente erreichen je nach Segmentteilung ein Gewicht von ca. $15,0 \mathrm{~kg}$ bis $50,0 \mathrm{~kg}$.

Das Schneidrad von Hydroschild TVM für den Einsatz in Lockergesteinen ist für zwei Drehrichtungen ausgelegt. Diese werden üblicherweise mit „links" und "rechts“ bezeichnet. Entscheidend ist dabei die Blickrichtung des Betrachters. Im Vortrieb hat sich aus praktischen Gründen die Betrachtung entlang der Tunnelachse (TA) in Vortriebsrichtung (VTR) bewährt, diese wird auch in dieser Arbeit durchgängig angewendet. Daraus ergeben sich folgende Definitionen:

- Schneidraddrehrichtung links: Das Schneidrad wird bei Blick entlang der Tunnelachse in Vortriebsrichtung gegen den Uhrzeigersinn gedreht. Ein beliebiger Punkt auf dem Schneidrad durchläuft die Verbindungslinie zwischen Tunnelachse und Firstlinie („12 Uhr-Position“) stets von rechts nach links.

- Schneidraddrehrichtung rechts: Das Schneidrad wird bei Blick entlang der Tunnelachse in Vortriebsrichtung im Uhrzeigersinn gedreht. Ein beliebiger Punkt auf dem Schneidrad durchläuft die Verbindungslinie zwischen Tunnelachse und Firstlinie („12 Uhr-Position“) stets von links nach rechts.

Die beiden Schneidraddrehrichtungen werden auch bei der Anordnung der verschiedenen Werkzeugtypen auf dem Schneidrad berücksichtigt. Während Schneidrollen und Stiche unabhängig von der Schneidraddrehrichtung funktionieren, sind Schälmesser und Räumer nur für den Bodenabbau in einer Schneidraddrehrichtung ausgelegt. Die Schneidspuren von Schälmessern und Räumern werden deshalb mit zwei separaten Werkzeugen für die beiden Schneidraddrehrichtungen besetzt. Die beiden Werkzeuge werden dazu häufig auf den beiden gegenüberliegenden Seiten eines Schneidradarmes angeordnet. Daraus ergibt sich ein definierter Winkelabstand zwischen den beiden Werkzeugen.

Die Definition der Schneidraddrehrichtungen ist also auch auf einzelne Schälmesser und Räumer übertragbar:

- Abbauwerkzeuge für die Schneidraddrehrichtung links: Das Werkzeug ist durch sein Auslegung (Form \& Verschleißschutz) und Ausrichtung auf dem Schneidrad für den Bodenabbau bei Schneidraddrehrichtung links bestimmt.

- Abbauwerkzeuge für die Schneidraddrehrichtung rechts: Das Werkzeug ist durch sein Auslegung (Form \& Verschleißschutz) und Ausrichtung auf dem Schneidrad für den Bodenabbau bei Schneidraddrehrichtung rechts bestimmt.

Abbauwerkzeuge, die nur für eine der beiden Schneidraddrehrichtung ausgelegt sind, nehmen im Vortrieb hauptsächlich in dieser Drehrichtung aktiv am Bodenabbau teil. In entgegengesetzter Schneidraddrehrichtung werden diese Abbauwerkzeuge passiv über die Ortsbrust geführt. Aus geometrischen Gründen dringen sie dabei geringfügig in die Ortsbrust ein und leisten einen geringen Teil des Bodenabbaus (Kap. 5.4.5). Entsprechend können die folgenden Einsatzarten für diese Abbauwerkzeuge definiert werden:

- Aktiver Einsatz: Das Werkzeug wird planmäßig in der vorgesehenen Richtung über die Ortsbrust geführt.

- Passiver Einsatz: Das Werkzeug wird planmäßig entgegen der vorgesehenen Richtung über die Ortsbrust geführt. 
Mit steigendem Schneidraddurchmesser werden die Spuren von Schälmessern zum Kaliberbereich hin und auch die Räumer zunehmend mehrfach besetzt. Dazu werden jeweils bis zu acht Werkzeuge für eine Drehrichtung auf dem Schneidrad angebracht. Der Besatz erfolgt dabei symmetrisch für beide Schneidraddrehrichtungen. Der Mehrfachbesatz hat verschieden Vorteile, einerseits ergibt sich eine homogenere Belastung des Schneidrades und eine Homogenisierung des Materialflusses, andererseits ergibt die Erhöhung der Anzahl der Werkzeuge eine höhere Sicherheit im Falle des Verlustes einzelner Werkzeuge durch Schäden.

Die Werkzeuge werden in Vortriebsrichtung, bezogen auf die Frontfläche der Schneidradstahlstruktur, in unterschiedlicher Höhe angebracht (Abb. 15). Die Höhe der Abbauwerkzeuge über Schneidradstahlbau ist einerseits maßgeblich für den Abstand der Frontfläche des Schneidrades von der Ortsbrust, hat jedoch auch Einfluss auf die Aufteilung des Bodenabbaus zwischen den verschiedenen Werkzeugtypen. Schälmesser und Räumer werden in der Regel auf gleicher Höhe angeordnet. Sie bilden die niedrigste Werkzeugebene. Die Schneidrollen werden deutlich höher bzw. vorlaufend angeordnet. Im Fall von Blöcken in der Ortsbrust können diese so theoretisch mit den Schneidrollen abgebaut werden, ohne dass an den dahinterliegenden Schälmesser Schäden durch Kontakt mit einem Block entstehen. Die Differenz zwischen Schneidrollen und Schälmessern beträgt dazu häufig ca. $35 \mathrm{~mm}$. Hintergrund ist, dass die Verschleißgrenze für 17-Zoll Schneidringe bei ca. $25 \mathrm{~mm}$ liegt. Mit $35 \mathrm{~mm}$ Abstand zu den dahinterliegenden Schälmessern entsteht so selbst bei vollständig verschlissenen Schneidringen kein Kontakt der Schälmesser zu Blöcken in der Ortsbrust. Bezogen auf die absolute Höhe der Werkzeuge muss der Abstand der Schneidradfrontseite zur Ortsbrust hoch genug sein, um die abgebauten Bodenkomponenten möglichst leicht hinter das Schneidrad transportieren zu können. Als Erfahrungswert wird für Schneidrollen häufig ein Vorlauf $h_{S R}$ von $175 \mathrm{~mm}$ eingesetzt. Für Schälmesser liegt der Vorlauf $h_{S M}$ häufig bei $140 \mathrm{~mm}$.

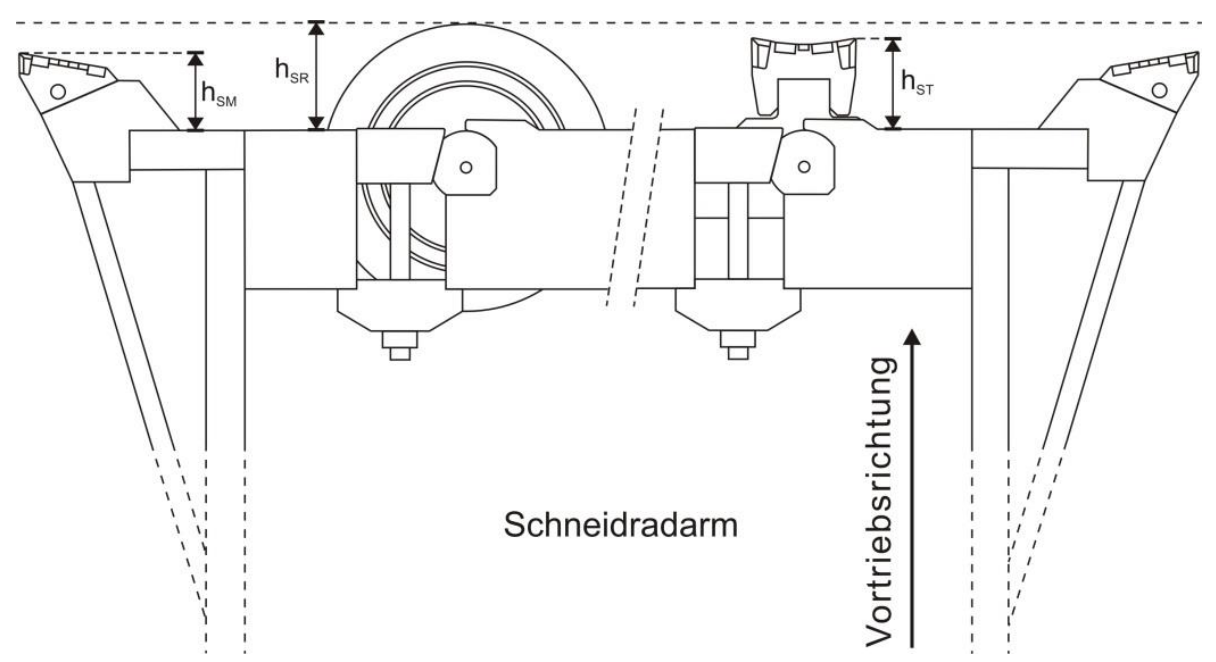

Abb. 15: Absolute Höhe bzw. Vorlauf von Schneidrollen $\left(h_{\mathrm{SR}}\right)$, Stichel $\left(h_{\mathrm{ST}}\right)$ und Schälmesser $\left(h_{\mathrm{SM}}\right)$ in Bezug auf die Schneidradfrontfläche.

Der Abstand der Schneidspuren der einzelnen Werkzeuge in radialer Richtung auf dem Schneidrad wird als Spacing S $[\mathrm{mm}]$ bezeichnet. Das Spacing $S_{S M}$ von Schälmessern wird so gewählt, dass die Schälmesser die Ortsbrust vollflächig abdecken oder sich zum Teil sogar seitlich überlappen. Damit ergibt sich wiederum ein teilweiser Mehrfachbesatz in den Überlappungsbereichen. Das Spacing der Schneidrollen wird so gewählt, dass beim Antreffen von Blöcken diese noch sicher abgebaut werden können. Häufigster Wert sind hier $100 \mathrm{~mm}$ Abstand zwischen den Schneidspuren. Bei einer Schneidringbreite $b_{S R}[\mathrm{~mm}]$ von deutlich kleiner als $100 \mathrm{~mm}$ ergibt sich folglich keine vollflächige Abdeckung der Ortsbrust durch die Schneidrollen. Der Boden zwischen den Schneidrollenspuren wird in Lockergesteinen planmäßig von den Schälmessern abgebaut. Das Spacing der Stichel richtet sich beim Einbau in die Schneidrollengehäuse nach dem Spacing der Schneidrollen. Im Unterschied zu den Schneidrollen weisen die Stichel jedoch häufig eine Breite von mindestens $100 \mathrm{~mm}$ auf, sodass sich eine vollflächige Abdeckung der Ortsbrust ergibt. Räumer besitzen eine durchgehende Schneidkontur, sodass kein Spurabstand im eigentlichen Sinne existiert. 
Alle genannten Informationen über die Anordnung der Werkzeuge, Besatz, Höhe über Schneidradstahlstruktur und Spacing der Schneidspuren werden im sogenannten Spurplan zusammengefasst.

Abschließend wird auch die Stahlstruktur des Schneidrades mit Verschleißschutz ausgestattet. Hierzu zählen die sogenannten Frontbleche, die auf der Frontseite der Schneidradarme flächendeckend angebracht werden. Diese bestehen im Regelfall aus hochlegiertem aber noch gut schweißbarem Stahl, z.B. mit Mangan (Mn), Chrom ( $\mathrm{Cr}$ ) und Nickel (Ni). Eine Übersicht hierzu gibt z.B. www-09. Dieser erreicht mit einer Härte von 370 bis 450 HBW (Härte n. BRINELL) eine deutlich höhere Härte und Verschleißfestigkeit als der Stahl der Schneidradstruktur. Aus vergleichbarem verschleißfestem Stahl wird auch die sogenannte Grillbar hergestellt, die als Verschleißschutz umlaufend um die Schneidradfelge angebracht ist.

Zum Verschleißschutz des Schneidrades zählen im weiteren Sinne auch sogenannte Anlaufkeile, die vor den Schneidrollengehäusen angebracht werden können. Sie schützen einerseits die Befestigungselemente der Schneidrollen vor Verschleiß und andererseits den Grundkörper der Schneidrollen vor Schäden in Böden mit Steinen oder Blöcken.

\subsubsection{Schneidradantrieb und Hauptlager}

Der Schneidradantrieb der TVM dient zum Drehen des Schneidrades. Dazu wird ein entsprechender Zahnkranz im Hauptlager gelagert. Am Zahnkranz greifen rückseitig je nach Maschinenauslegung Elektro- oder Hydraulikmotoren an, die den Zahnkranz antreiben. An der Vorderseite des Zahnkranzes ist der Flanschring des Schneidrades mit Bolzen befestigt.

Die Auswahl eines elektrischen oder hydraulischen Antriebs bestimmt die Kennkurve des Antriebs, wobei bei aktuellen Hydroschild TVM überwiegend elektrische Antriebe eingesetzt werden. Die Kennkurve des Antriebs stellt die von der Auslegung der Motoren und des Antriebs abhängige Beziehung wischen Schneidraddrehzahl U [min $\left.{ }^{-1}\right]$, Schneidraddrehmoment $\mathrm{M}_{\mathrm{TVM}}[\mathrm{kNm}]$ und Antriebsleistung $\mathrm{P}_{\mathrm{TVM}}$ $[\mathrm{kW}]$ als Diagramm dar (Abb. 16).

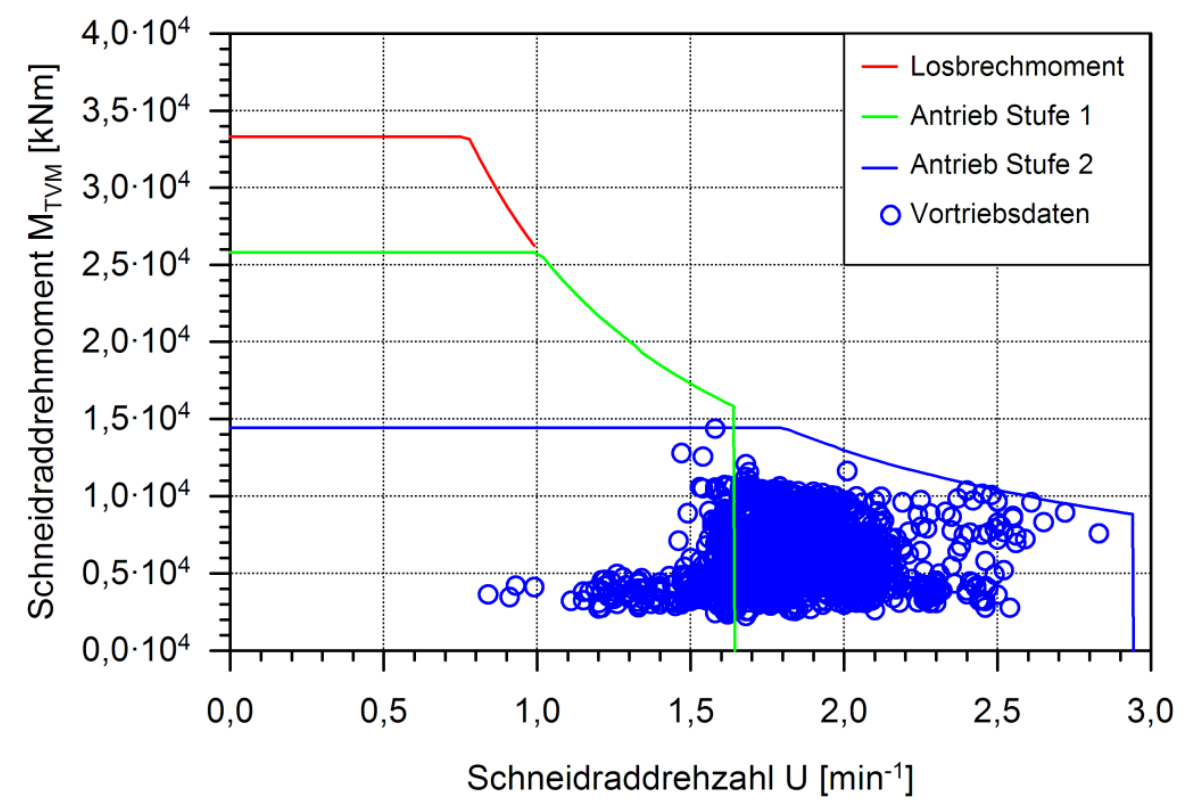

\footnotetext{
Abb. 16: Beispiel für die Kennkurve aus Schneidraddrehmoment $\mathrm{M}_{\mathrm{TVM}}[\mathrm{kNm}]$ und Schneidraddrehzahl $U$ [ $\left.\min ^{-1}\right]$ eines Schneidradantriebs. Darunter sind als Beispiel die tatsächlichen Daten aus dem zugehörigen Referenzprojekt eingetragen. Der Schneidradantrieb wurde hier in Stufe 2 betrieben, die Datenpunkte liegen folglich unterhalb der zugehörigen Leistungskurve (blau). Die Schneidraddrehzahl wurde überwiegend auf 1,6 - 2,2 min $^{-1}$ eingestellt, da sich hier unter den vorliegenden geotechnischen Bedingungen noch keine Einschränkung der Vortriebsgeschwindigkeit $V_{\text {TVM }}[\mathrm{mm} / \mathrm{min}]$ durch das Schneidraddrehmoment ergibt. (Herrenknecht AG, verändert)
} 
Formal wird die Beziehung zwischen Schneidraddrehzahl U, Schneidraddrehmoment $M_{T V M}$ und Antriebsleistung $\mathrm{P}_{\mathrm{TVM}}$ durch den Zusammenhang in Gleichung 1 (GI. 1) ausgedrückt:

$$
\mathrm{P}_{\mathrm{TVM}}=\frac{\mathrm{U} \cdot 360^{\circ}}{60 \mathrm{~s}} \cdot \mathrm{M}_{\mathrm{TVM}}
$$

Die Antriebsleistung $\mathrm{P}_{\mathrm{TVM}}$ ist durch die verbauten Motoren begrenzt. Das Schneidraddrehmoment $\mathrm{M}_{\text {TVM }}$ resultiert hauptsächlich aus den Schneidkräften der Abbauwerkzeuge beim Bodenabbau Die Schneidraddrehzahl ist im Vortrieb ein Einstellwert des Schildfahrers (Kap. 3.3.1). Aus der eingestellte Schneidraddrehzahl $U$ und dem Schneidraddrehmoment $M_{\text {TVM }}$ ergibt sich in der graphischen Darstellung von Schneidraddrehzahl $U$ und Schneidraddrehmoment $M_{T V M}$ (Abb. 16) der sogenannte Betriebspunkt der TVM. Dieser kann nur unterhalb bzw. auf der Kennlinie des Schneidradantriebs liegen.

Durch das Hauptlager erfolgt die Übertragung der Antriebsmomente in die Stahlbaustruktur des Schildes. Dabei existieren drei Varianten für den Einbau des Hauptlagers im Schild:

- Festeinbau

- Einbau mit Axiallager

- Einbau mit Gelenklager

Beim Festeinbau wird das Hauptlager unbeweglich im Schild fixiert. Folglich entfallen die entsprechenden Schneidradverschiebezylinder, über die bei den weiteren Einbauvarianten das Hauptlager zusammen mit dem Antrieb verschoben werden kann.

Bei der zweiten Einbauvariante wird das Hauptlager zusätzlich über ein Axiallager im Schildstahlbau gelagert. Zusätzlich werden Schneidradverschiebezylinder verbaut, über die so das Schneidrad zusammen mit Antrieb und Hauptlager in axialer Richtung, also in bzw. gegen die Vortriebsrichtung, verschoben werden kann. Die Verschiebezylinder sind gegen den Stahlbau des Schildes verspannt.

Bei der dritten Einbauvariante wird das Hauptlager zusätzlich in einem Gelenklager im Schildstahlbau gelagert. Mit den Schneidradverschiebezylindern kann das Schneidrad so sowohl in axialer Richtung verschoben werden, als auch in Bezug auf die Tunnelachse gekippt werden. Durch das Kippen kann der Überschnitt des Schneidrades über den Schildstahlbau verändert werden, sodass sich in Bezug auf die Tunnelachse ein exzentrischer Überschnitt ergibt.

Das Verschieben oder Kippen des Schneidrades erfolgt bei drehendem Schneidrad, um ein unkontrolliertes Eindrücken der Werkzeuge in die Ortsbrust oder die Tunnellaibung zu vermeiden. Zusätzlich wird die Position des Schneidrades in der speicherprogrammierbaren Steuerung (SPS) (Kap. 3.3.4) überwacht, sodass durch die axiale Verschiebung und das Kippen kein Kontakt zwischen Schneidrad und Schildschneide entstehen kann.

\subsubsection{Schild}

Das Schild selbst besteht aus einer umfangreichen Stahlkonstruktion, die im Wesentlichen drei Hauptaufgaben erfüllt:

- Trägerstruktur für alle wichtigen Einbauten (Antrieb, Hauptlager, Erektor, Schleusen, Vortriebspressen)

- Übertragung von Schneidkräften (Schneidrad), Schneidraddrehmoment und Stützkräften (Stützdruck) in den Tübbingausbau.

- Abschottung des Abbauraumes (Stützdruck) vom Ausbauraum und dem bereits ausgebauten Tunnel (atmosphärisch) und Mörtelverpressung des Ringspalts. 
Der Stahlbau des Schildes besteht aus dem kreisrunden Schildmantel, senkrecht dazu verlaufen Tauchwand und Druckwand. Im Zentrum der Stahlstruktur befindet sich die Aufnahme für das Hauptlager. Als Trägerstruktur für die Einbauten im Schild und zur Übertragung der Vortriebskräfte, des Schneidraddrehmoments und der Stützkräften in den Tübbingausbau muss der Stahlbau des Schildes vor allem statischen Kriterien genügen. Die wichtigsten Einbauten im Schild sind Schneidradantrieb und Hauptlager, Verschiebezylinder und Vortriebspressen, Erektor und Schleusen, Steinbrecher, Förderleitung, Förderleitung und Speiseleitung.

Die Abschottung des unter Druck stehenden Abbauraumes vom atmosphärischen Teil der TVM erfolgt durch die Druckwand. Der Druck zwischen Arbeitskammer und Abbaukammer wird in der Sohle durch die Tauchwandöffnung ausgeglichen. Bei starken Verklebungen im Bereich der Tauchwand besteht hier das Risiko, dass kein Druckausgleich zwischen Arbeitskammer und Abbaukammer mehr stattfinden kann. Arbeitskammer und Abbaukammer sind deshalb zusätzlich über eine Rohrleitung verbunden, die in der Arbeitskammer unterhalb der Tunnelachse ansetzt und oberhalb der Tunnelachse in die Abbaukammer mündet. Damit ist jederzeit ein zuverlässiger Druckausgleich gewährleistet. Um den Materialfluss aus der Abbaukammer in die Förderleitung zu optimieren, kann mit dieser Lösung das Sohlsegment der Arbeitskammer auch vollständig vom restlichen Ringraum der Arbeitskammer abgeschottet werden (REHM \& WEHRMEYER 2008: 38), sodass über die Tauchwandöffnung keine direkte Verbindung zwischen den beiden Kammern mehr besteht. Der Druckausgleich erfolgt dann ausschließlich über die Verbindungsrohrleitungen.

Der Stützdruck liegt zusätzlich auch am hinteren Ende des Schildes zwischen Schildmantel und Tübbingausbau an. Hier wird der Ringspalt zwischen Schildmantel und Tübbingausbau umlaufend durch drei bis vier Reihen Bürstendichtungen abgedichtet. Diese werden über Lisenen im Schildmantel, radial umlaufend, kontinuierlich mit hochviskosem Fett gespeist. In der Vortriebsphase gleiten die Bürstendichtungen über den Tübbingausbau, während der hinter dem Schildmantel frei werdende Raum ebenfalls durch Lisenen im Schildmantel mit Mörtel verpresst wird. Dieser dient dazu, den Tübbingausbau zu stabilisieren und weiter abzudichten.

\subsubsection{Ortsbruststützung und Materialförderung}

Sämtliche Einrichtungen auf der TVM für die Flüssigkeitsstützung der Ortsbrust und die Materialförderung bilden einen nahezu geschlossenen Kreislauf zwischen der Abbaukammer der TVM und einer entsprechenden Separationsanlage außerhalb des Tunnels, in dem die Stützflüssigkeit zirkuliert (Abb. 17). Im Optimalfall wird in der Separationsanlage lediglich der abgebaute Boden aus der Stützflüssigkeit entnommen, bevor diese wieder in den Flüssigkeitskreislauf eingespeist wird.

Von der Separationsanlage wird die Stützflüssigkeit über die Speiseleitung zur TVM gepumpt. Die entsprechende Speisepumpe dazu ist ebenfalls in der Separationsanlage enthalten. In der Speiseleitung kommt es aufgrund der Wandreibung in der Leitung mit zunehmender Leitungslänge zu Druckverlust in der Leitung. Dieser wird bei langen Tunnelstrecken ca. $>1.000 \mathrm{~m}$ durch zusätzliche Pumpenstationen zwischen Separationsanlage und TVM wieder erhöht, um den notwendigen Volumenstrom zur TVM aufrecht zu erhalten. Die Stützflüssigkeit wird an verschiedenen Stellen der Tauchwand durch Düsen in die Abbaukammer eingespeist. Die Anordnung und Ausrichtung der Düsen zur Einspeisung der Stützflüssigkeit dient zudem häufig der Spülung kritischer Schildbereiche, vor allem dem Bereich vor der Tauchwandöffnung, um zu verhindern, dass sich hier abgebautes Material ansammelt.

In der Sohle der Arbeitskammer wird hinter der Tauchwandöffnung die Stützflüssigkeit mit dem darin enthaltenen abgebauten Boden mithilfe einer Förderpumpe abgepumpt. Die entsprechende Pumpe ist im Regelfall auf dem ersten Nachläufer installiert. Analog zur Speiseleitung ergibt sich auch in der Förderleitung ein Druckverlust in Abhängigkeit der Leitungslänge, jedoch auch z.B. durch den Aufstieg der Förderleitung in Schächten oder das Tunnelgefälle. Der Druckverlust wird bei langen Strecken entsprechend durch Pumpstationen im Tunnel ausgeglichen, um den notwendigen Volumenstrom in der Förderleitung zur Separationsanlage aufrecht zu halten. Sowohl in der Speiseleitung, als auch in der Förderleitung wird eine Fließgeschwindigkeit $\mathrm{v}_{\mathrm{FL}}$ von maximal ca. $4 \mathrm{~m} / \mathrm{s}$ angestrebt. Bei einer Dichte der Stützflüssigkeit $\rho_{\mathrm{SF}}$ von $1,1 \mathrm{~g} / \mathrm{cm}^{3}$ (Speiseleitung) bis $1,3 \mathrm{~g} / \mathrm{cm}^{3}$ (Förderleitung) und einem Leitungsdurchmesser von $400-500 \mathrm{~mm}$ herrscht hier also weitgehend turbulentes Fließen vor (Kap. 5.2.2.1). 


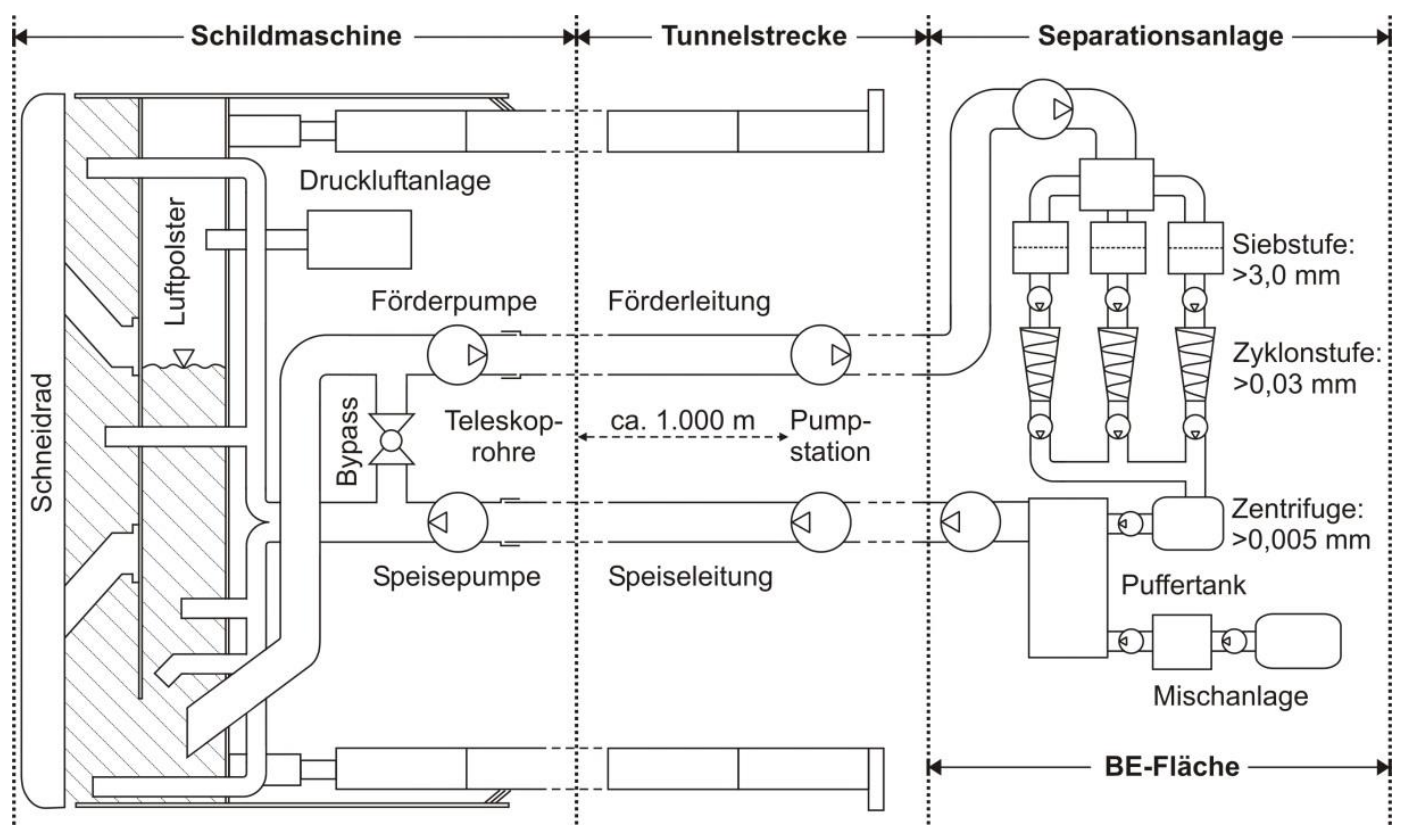

Abb. 17: Schematische Darstellung des Flüssigkeitskreislaufs zwischen der Hydroschild TVM („Schildmaschine") im Tunnel, der Leitungen und Pumpstationen in der bereits fertiggestellten Tunnelstrecke und der Separationsanlage auf der Baustelleneinrichtungsfläche („BE-Fläche“). Die Stützflüssigkeit wird von der Separationsanlage über Speiseleitung und Speisepumpen in die Abbaukammer der TVM gepumpt. Die mit abgebautem Boden aufgeladene Stützflüssigkeit wird über Förderpumpen und Förderleitung zurück zur Separationsanlage transportiert. Hier erfolgt die Abtrennung des abgebauten Bodens aus der Stützflüssigkeit.

Für den Anfahrvorgang der TVM und für die Einstellung des Vortriebs ist ein Bypass zwischen Speiseleitung und Förderleitung notwendig. Über den Bypass kann der Flüssigkeitskreislauf von der Abbaukammer abgetrennt werden. Die entsprechende Verbindungsleitung und zugehörige Schieber befinden sich in Abhängigkeit der Maschinenauslegung auf dem ersten Nachläufer. Im hinteren Bereich der Nachläufer befinden sich zusätzlich Teleskoprohre oder flexible Rohrleitungen auf Gleitschuhen. Diese erlauben während dem Vortrieb die Verlängerung der Förder- und Speiseleitung entsprechend dem Vorschub der TVM im Tunnel. Die ausziehbare Länge der Teleskoprohre bzw. flexiblen Rohrleitungen liegt in Abhängigkeit der Maschinenauslegung zwischen fünf und zehn Meter. Ist diese erreicht, ist eine Verlängerung der Rohrleitungen notwendig. Dazu werden hinter der TVM neue Rohrleitungen eingesetzt, sodass die Teleskoprohre bzw. flexiblen Rohrleitungen wieder vollständig eingefahren werden können. Für die Rohrverlängerung muss der Flüssigkeitskreislauf vollständig eingestellt werden.

Das Luftvolumen und der Stützdruck $P_{\mathrm{SF}}$ im Druckluftkissen hinter der Tauchwand werden durch eine Druckluftanlage gesteuert. Die Genauigkeit der Steuerung liegt bei +/-0,1 bar (BABENDERERDE 2003: 4). Von hier führen Leitungen durch die Druckwand in die Firste der Arbeitskammer. Zusätzlich ist im Schildfirst eine Verbindungsleitung durch die Tauchwand zwischen Arbeitskammer und Abbaukammer installiert. Diese ist für Drucklufteinstiege in die Abbaukammer zum Ein- und Auslassen von Druckluft in die Firste der Abbaukammer notwendig und wird über einen Kugelhahn geöffnet bzw. geschlossen. Die Regelung des Stützdrucks erfolgt im Vortrieb nicht aktiv durch den Schildfahrer, sondern wird an der Druckluftanlage für den jeweiligen Vortriebsabschnitt eingestellt. Der notwendige Stützdruck wird dazu anhand verschiedener Modelle in Abhängigkeit der geotechnischen Rahmenbedingungen errechnet. Einen Überblick über die verschiedenen Modelle geben SCHWARZ et al. (2006: 120) und GRÜBL \& SPEIER (2013: 49). Häufig eingesetzte werden die Modelle nach ANAGNOSTOU \& KoVARI (1996: 379), JANCSECZ \& STEINER (1994: 531) und DIN 4085 (2011: 9).

Der Betrag des Stützdrucks $P_{S F}$ liegt unabhängig vom ausgewählten Modell höher als die Summe aus dem Erddruck und dem vorliegenden Grundwasserdruck an der Ortsbrust. So wird ein unkontrolliertes Eingleiten des Bodens in die Abbaukammer, sowie Wasserzufluss zur Abbaukammer verhindert und das Gebirge an der Ortsbrust stabilisiert (MAILD et al. 1995: 247). Aufgrund der Druckdifferenz 
zwischen dem Stützdruck $P_{S F}$ und dem Wasserdruck dringt die Stützflüssigkeit in Abhängigkeit der Bodeneigenschaften in die Ortsbrust ein. Dabei werden Tonteilchen aus der Stützflüssigkeit an der Ortsbrust angelagert, die eine dünne Membran, den sogenannten Filterkuchen, bilden (MAIDL et al. 1995: 248). Die Übertragung des Stützdrucks auf das Korngerüst des Bodens erfolgt einerseits mechanisch über den Filterkuchen und andererseits durch Strömungskräfte beim Eindringen der Stützflüssigkeit in den Boden (NAGEL et al. 2010: 273, BABENDEREDE \& HolZHÄUSER 1999: 235 \& BABENDERERDE 2000: 81). Beim Eindringen der Stützflüssigkeit in die Ortsbrust ergibt sich ein Anstieg des Porenwasserdrucks im Boden vor der Ortsbrust (NAGEL et al. 2010: 280). BEZUIJEN \& TALMON (2006: 381) und WENDL \& THURO (2011: 224) können einen entsprechenden Anstieg des Grundwasserspiegels im Nahbereich der Ortsbrust zeigen.

Die Separationsanlage dient dazu die abgebauten Bodenteile aus der Stützflüssigkeit abzutrennen, sodass diese mit möglichst geringem Aufwand zurück auf die TVM in den Flüssigkeitskreislauf eingespeist werden kann. Die mit abgebauten Bodenteilen beladene Stützflüssigkeit aus der Förderleitung der TVM wird dazu in mehreren Stufen bearbeitet. Die Auslegung der Anlage berücksichtigt dabei den Ausbruchdurchmesser der TVM, sowie die geplante Vortriebsleistung, sodass sich zum Teil deutliche Unterschiede vor allem in der Größe und Leistung der Anlagen ergeben. Die grundsätzlichen Bearbeitungsstufen der Stützflüssigkeit bleiben jedoch unabhängig von der Größe weitgehend gleich (Abb. 17).

Zunächst werden aus der beladenen Stützflüssigkeit aus der Förderleitung durch ein Grobsieb alle Komponenten mit einem Korndurchmesser ca. $>15 \mathrm{~mm}$ abgetrennt und durch ein weiteres darunter liegendes Feinsieb alle Komponenten mit Korndurchmesser ca. $>3 \mathrm{~mm}$. Die abgetrennten Bodenkomponenten werden über Rutschen und Förderbänder direkt auf einen Abwurf gegeben.

Die verbleibende Stützflüssigkeit wird unter den Siebstufen in Unterlaufwannen aufgefangen und durch Zyklone weiter bearbeitet. In einem Zyklon wird die Stützflüssigkeit durch tangentiale Einleitung in den Zyklonzylinder in starke Rotation versetzt. Die darin enthaltenen Bodenkomponenten werden durch die Fliehkraft an die konische Wand des Zyklons gedrückt und rieseln anschließend entlang der Wand über die unter Öffnung (Unterlaufdüse) des Zyklons ab. Die in Rotation versetzte Stützflüssigkeit staut sich im Gegensatz dazu im konisch verengten Bereich des Zyklons an und bildet im Zentrum des Konus eine gegenläufigen, aufwärts gerichteten Wirble. Hier wird durch die obere Öffnung des Zyklons (Oberlaufdüse) die abgetrennte Stützflüssigkeit entnommen. In der ersten Zyklonstufe können Bodenkomponenten bis ca. $>0,1 \mathrm{~mm}$ abgetrennt werden. Durch eine weitere Zyklonstufe kann der Korndurchmesser der abgetrennten Bodenkomponenten auf ca. 0,03 mm reduziert werden. Die abgetrennten Bodenkomponenten aus beiden Stufen werden wiederum über Rutschen und Förderbänder auf einen Abwurf gegeben.

Vor der Wiederverwendung der Stützflüssigkeit werden durch Zentrifugen oder Kammerfilterpressen alle weiteren Bodenkomponenten bis zu einer Größe von 0,005 mm aus der Stützflüssigkeit entfernt. Die so gereinigte Stützflüssigkeit wird abschließend in einen Puffertank gepumpt, von wo aus sie über die Speisepumpe wieder zur TVM gepumpt wird.

Sämtliches durch die Separationsanlage abgetrennte Material weist einen Restgehalt (Restfeuchte) auf, sodass das Volumen an Stützflüssigkeit durch die Separation leicht sinkt. Zusätzlich steigt durch die Verlängerung der Rohrleitungen zur TVM das notwendige Volumen an Stützflüssigkeit im Flüssigkeitskreislauf mit dem Vortrieb stetig an. Die Separationsanlage beinhaltet deshalb zusätzlich eine Mischanlage zur Herstellung frischer Bentonitsuspension, die in einem Tank aufbewahrt wird und bei Bedarf zur Kompensation des Volumens im Flüssigkeitskreislauf genutzt wird. 


\subsection{Betriebszustände}

Die nachfolgende Beschreibung der Betriebszustände einer Hydroschild TVM unterscheidet folgende Fälle:

- Vortrieb \& Steuerung des Abbauprozesses

- Sicherung und Ausbau des Tunnels

- Wartungsstillstände (Vortriebsunterbrechungen)

Das Hauptaugenmerk liegt dabei in den für die vorliegende Arbeit wichtigen Vortriebsphase und den Vortriebsunterbrechungen für Werkzeugwechsel. Die Ringbauphase ist aus Gründen der Übersichtlichkeit mit deutlich reduziertem Detaillierungsgrad beschrieben, da diese Phase für die vorliegende Arbeit kaum Bedeutung hat. Alle weiteren denkbaren Betriebszustände, insbesondere Stillstände z.B. aus logistischen Gründen sind aus demselben Grund nicht beschrieben. Im Gegensatz dazu ist die Erfassung der Betriebsdaten der TVM von grundlegender Bedeutung für die weitere Arbeit und deshalb abschließend im Detail beschrieben.

\subsubsection{Vortrieb und Steuerung des Abbauprozesses}

Die zentrale Herausforderung in der Vortriebsphase eines Hydroschildes besteht darin, eine hohe Vortriebsleistung zu erzielen und gleichzeitig die Stützung der Ortsbrust zu gewährleisten. Ausgehend von einer vollständig stillstehenden und ausgeschalteten TVM folgt der Beginn des Vortriebs deshalb normalerweise den nachfolgenden Schritten, auch wenn sich je nach Auslegung der TVM im Detail Unterschiede ergeben können. Die Schritte sind aufeinander aufbauend und erzeugen jeweils entsprechende Freigaben, ohne die der nächste Schritt nicht eingeleitet werden kann:

- Einschalten und Freigabe aller notwendigen Maschinenkomponenten über das Bedienpult im Steuerstand (Förder- und Speisepumpen, Steinbrecher, Pumpstationen, Schmiersysteme) und Überprüfung aller wichtigen Tankfüllstände (Getriebe).

- Inbetriebnahme der Förderpumpe, Speisepumpe und der Pumpenstationen und Stabilisierung des Flüssigkeitskreislaufs zwischen TVM und Separation. Die Stützflüssigkeit wird dabei nicht durch die Abbaukammer gepumpt, sondern über den Bypass zwischen Speise- und Förderleitung. Der Volumenstrom in Speise- und Förderleitung $\left[\mathrm{m}^{3} / \mathrm{h}\right]$ wird durch den Schildfahrer eingestellt.

- Inbetriebnahme des Schneidrades, Einschalten der Antriebsmotoren, Auswahl der Drehrichtung, der Antriebsstufe und der Schneidraddrehzahl U.

- Inbetriebnahme des Flüssigkeitskreislaufs. Der Kreislauf wird vom Bypass auf die Arbeits- und Abbaukammer umgestellt.

- Inbetriebnahme der Vortriebspressen. Einstellung der Vorschubgeschwindigkeit über ein zentrales Potentiometer. Steuerung der Vortriebsrichtung über die Einstellung des relativen Vorschubs der einzelnen Pressengruppen zueinander (Vorlauf) über die zugehörigen Potentiometer.

- Der Stützdruck $P_{S F}$ wird bereits vor dem Vortrieb durch den Schichtingenieur in der Regel nach Vorgabe durch die Bauleitung an der Druckluftanlage eingestellt.

Der beschriebene Prozess nimmt in der Praxis vor allem zur Stabilisierung des Flüssigkeitskreislaufs insgesamt ca. 10 bis 15 Minuten in Anspruch.

Ist der Vortrieb über den oben beschriebenen Prozess angelaufen, so wird an der Ortsbrust bei drehendem Schneidrad und gleichzeitigem Vorschub durch die Vortriebspressen Boden durch die Abbauwerkzeuge gelöst. Der gelöste Boden wird durch Räumer und Schälmesser hinter das Schneidrad 
transportiert, fällt hinter dem Schneidrad in die Schildsohle und wird hinter der Tauchwandöffnung mit der Stützflüssigkeit über die Förderleitung in der Sohle abgesaugt. Durch die Speiseleitung wird an verschiedenen Stellen der Arbeitskammer eine äquivalente Menge an frischer Stützflüssigkeit zugegeben. Die Abbaukammer bleibt dabei immer vollständig mit Stützflüssigkeit gefüllt. Bei konstantem Flüssigkeitsspiegel in der Arbeitskammer ergibt sich entsprechend wenig Regelungsaufwand zum Ausgleich des Stützdrucks durch entsprechende Luftvolumina hinter der Tauchwand durch die Druckluftanlage.

Die weitere Steuerung des Abbauprozesses erfolgt im Wesentlichen über sechs manuelle Einstellwerte, die von DüLLMANN et al. (2013: 622) auch als Aktivparameter bezeichnet werden:

- Schneidraddrehzahl $U\left[\min ^{-1}\right]$

- Vortriebsgeschwindigkeit $\mathrm{V}_{\mathrm{TVM}}[\mathrm{mm} / \mathrm{min}]$

- Druckbegrenzung der Vortriebspressengruppen

- Stützdruck $P_{S F}[$ bar $]$

- Fördervolumen in $v_{\mathrm{FL}}\left[\mathrm{m}^{3} / \mathrm{h}\right]$

- Speisevolumen in $\mathrm{V}_{\mathrm{SL}}\left[\mathrm{m}^{3} / \mathrm{h}\right]$

Der Abbauprozess ist also grundsätzlich weggesteuert und druckbegrenzt. Wesentlicher Vorteil dieser Steuerung ist, dass sich dadurch eine Verstetigung des Abbauprozesses ergibt. Das bedeutet der Volumenstrom der Stützflüssigkeit in der Förder- und Speiseleitung ist relativ konstant, genauso wie der Massenstrom des abgebauten Bodens in der Stützflüssigkeit in der Förderleitung. Damit ergibt sich theoretisch auch ein relativ konstanter Volumenstrom in der Separation.

Alle weiteren zentralen Größen des Abbauprozesses sind Reaktionsgrößen bzw. Passivparameter (DÜLLMANN et al. 2013: 622), die sich aus den geotechnischen Verhältnissen in der Ortsbrust, der Maschinenauslegung und den genannten Einstellwerten resultieren. Dazu zählt die Penetration $p$ $[\mathrm{mm} / \mathrm{U}]$, die ein wesentlicher Parameter im Hinblick auf den Verschleiß der Abbauwerkzeuge ist. Die Penetration $p$ entspricht dem Vorschub des Schneidrades je Schneidradumdrehung und ist der Ausgangswert zur Ermittlung der Eindringtiefe einzelner Abbauwerkzeuge in die Ortsbrust. Sie ergibt sich mit Gl. 2 direkt aus der ausgewählten Schneidraddrehzahl U $\left[\mathrm{min}^{-1}\right]$ und der Vortriebsgeschwindigkeit $\mathrm{V}_{\mathrm{TVM}}[\mathrm{mm} / \mathrm{min}]$ :

$$
\mathrm{p}=\frac{\mathrm{V}_{\mathrm{TVM}}}{\mathrm{U}}
$$

Zu den Reaktionsgrößen zählt darüber hinaus vor allem auch die an den Abbauwerkzeugen anliegende Schneidkraft $F_{C}[k N]$. Diese ergibt sich aus dem Schneidwiederstand $W_{C}[k N]$, den der Boden dem Abbau entgegensetzt. Durch das Drehen des Schneidrades und gleichzeitigen Vorschub durch die Vortriebspressen laufen die Abbauwerkzeuge auf einer Bahn, die einer in Vortriebsrichtung ansteigenden Helix entspricht (Abb. 18). Der Radius der Helix entspricht dem Spurradius $r_{s}[\mathrm{~mm}]$ des jeweiligen Werkzeuges, die Ganghöhe der Helix entspricht der Penetration $p$ (Gl. 2). Sowohl der Schneidwiderstand $W_{C}$ des Bodens, als auch die zugehörige Schneidkraft $F_{C}$ können für jedes Werkzeug entsprechend in zwei zueinander senkrecht stehende Komponenten aufgeteilt werden. In Vortriebsrichtung ergibt sich der Eindringwiderstand des Bodens $W_{T C}[k N]$, der an den Abbauwerkzeugen als Anpresskraft $\mathrm{F}_{\mathrm{TC}}[\mathrm{kN}]$ anliegt. In Schneidrichtung des Werkzeuges resultiert aus dem Scherwiderstand des Bodens $W_{\mathrm{SC}}[\mathrm{kN}]$ die Scherkraft $F_{\mathrm{SC}}[\mathrm{kN}]$. 


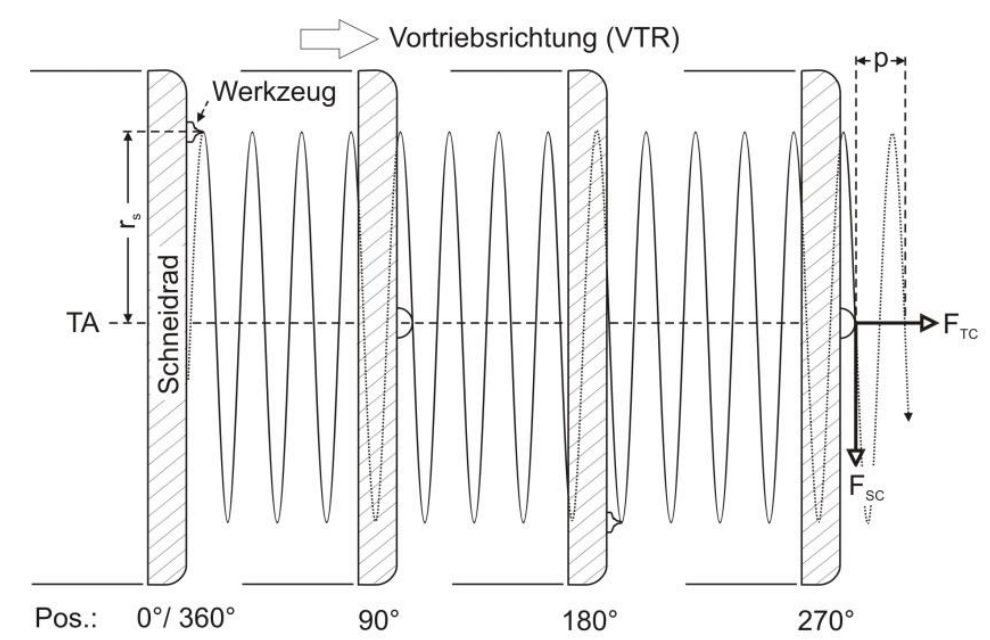

\begin{abstract}
Abb. 18: Schematische Darstellung der Laufbahn der Abbauwerkzeuge im Vortrieb mit dem Spurradius $r_{s}$ [mm], der Penetration $\mathrm{p}[\mathrm{mm} / \mathrm{U}]$ und den anliegenden Schneidkräften $F_{\mathrm{Tc}}[\mathrm{kN}]$ und $\mathrm{F}_{\mathrm{Sc}}[\mathrm{kN}]$. Die Laufbahn der Abbauwerkzeuge gleicht einer liegenden Helix, deren Durchmesser dem Spurradius $r_{s}$ und die Ganghöhe der Penetra-
\end{abstract} tion $\mathrm{p}$ entspricht.

Die Summe der Anpresskräfte $\mathrm{F}_{\mathrm{TC}}$ aller Abbauwerkzeuge auf dem Schneidrad wird als Schneidradanpresskraft $F_{\mathrm{SR}}[\mathrm{kN}]$ bezeichnet. Sie entspricht der Summe aller Eindringwiderstände des Bodens $\mathrm{W}_{\mathrm{SR}}$ [kN] (Gl. 3).

$$
\mathrm{F}_{\mathrm{SR}}=\sum \mathrm{F}_{\mathrm{TC}}
$$

Zur Schneidradanpresskraft kommt ein stark variabler Anteil an mechanischer Stützwirkung zwischen Schneidrad und Ortsbrust hinzu, die aus einer mechanisch wirksamen Verbindung zwischen Schneidradfrontfläche und Ortsbrust durch bereits abgebaute Bodenkomponenten entsteht. Daraus ergibt sich entgegen der Vortriebsrichtung eine zusätzliche Widerstandskomponente $\mathrm{W}_{\mathrm{ME}}[\mathrm{kN}]$ mit der entsprechenden Kraftkomponente in Vortriebsrichtung $F_{M E}[k N]$.

In Abhängigkeit der Schildkonstruktion ergibt sich aus dem Stützdruck $P_{S F}[b a r]$ in der Abbaukammer und der Fläche $A_{S B}\left[m^{2}\right]$ des Flanschrings bzw. der mitdrehenden Zentrumsplatte (Befestigung des Schneidrades am Hauptlager) in der Tauchwand eine weitere Widerstandskomponente $\mathrm{W}_{\mathrm{SB}}[\mathrm{kN}]$ entgegen der Vortriebsrichtung. Die zugehörige Kraftkomponente in Vortriebsrichtung $F_{\mathrm{SB}}[\mathrm{kN}]$ ergibt sich folglich aus Gl. 4:

$$
\mathrm{F}_{\mathrm{SB}}=\mathrm{A}_{\mathrm{SB}} \cdot \mathrm{p}_{\mathrm{SF}} \cdot 100
$$

Zusätzlich liegen innerhalb der Schneidradverschiebezylinder und des Axiallagers des Schneidradantriebes Reibungswiderstände an. Diese sind nach DüLLMANN et al. (2013: 627) nicht getrennt zu erfassen und werden deshalb im Folgenden in Summe als Reibungswiderstand $\mathrm{W}_{\mathrm{RA}}[\mathrm{kN}]$ betrachtet. Die Wirkrichtung des Reibungswiderstandes $W_{R A}$ und der zugehörigen Kraftkomponenten $F_{R A}[k N]$ ist im Vortrieb entgegengesetzt zu den Widerständen und Kräften aus dem Bodenabbau, Stützdruck und mechanischer Stützung (Abb. 19). 
Die bisher beschriebenen Kraft- und Widerstandskomponenten in axialer Richtung sind in der an den Verschiebezylindern anliegende Kraft $F_{S V}[k N]$ aufsummiert (Gl. 6). Sie entspricht der Summe der am Schneidrad anliegenden Widerstandskomponente entgegen der Vortriebsrichtung $W_{\mathrm{SV}}[\mathrm{kN}]$ (Gl. 5).

$$
\begin{aligned}
& \mathrm{W}_{\mathrm{SV}}=\mathrm{W}_{\mathrm{SR}}+\mathrm{W}_{\mathrm{ME}}+\mathrm{W}_{\mathrm{SB}}-\mathrm{W}_{\mathrm{RA}} \\
& \mathrm{F}_{\mathrm{SV}}=\mathrm{F}_{\mathrm{SR}}+\mathrm{F}_{\mathrm{ME}}+\mathrm{F}_{\mathrm{SB}}-\mathrm{F}_{\mathrm{RA}}
\end{aligned}
$$

In umgekehrter Richtung kann die Kraft an den Verschiebezylindern $F_{\text {Sv }}$ eine Analyse der Daten rechnerisch nicht vollständig in die vier Kraftkomponenten aufgeteilt werden. Die Stützkraft $F_{S B}$ kann mit Gl. 4 abgetrennt werden. Eine Trennung der Schneidradanpresskraft $F_{S R}$, der mechanischen Stützkraft $F_{M E}$ und der Reibungskraft $F_{R A}$ ist jedoch nicht möglich, da für beide Kraftkomponenten keine Berechnungsmöglichkeiten existieren. DüLLMANN et al. (2013: 626) zeigen darüber hinaus, dass $\mathrm{F}_{\mathrm{SR}}$ entgegen bisheriger Annahmen in Abhängigkeit der Bodeneigenschaften und des Stützdrucks $P_{S F}$ nicht zwingend die dominierende Kraftkomponente in $\mathrm{F}_{\mathrm{SV}}$ darstellt.

Die an den Verschiebezylindern anliegenden Kraft $F_{S V}$ wird über die Vortriebspressen in den Tübbingausbau eingeleitet und ist damit in der Vortriebspressenkraft $F_{V T P}[k N]$ enthalten. In der Vortriebspressenkraft sind noch fünf weitere Komponenten aufsummiert, die rechnerisch nicht einzeln von $\mathrm{F}_{\mathrm{VTP}}$ abtrennbar sind. Ein entsprechender Längsschnitt zur Darstellung der Widerstands- und Kraftkomponenten ist in Abb. 19 gegeben.

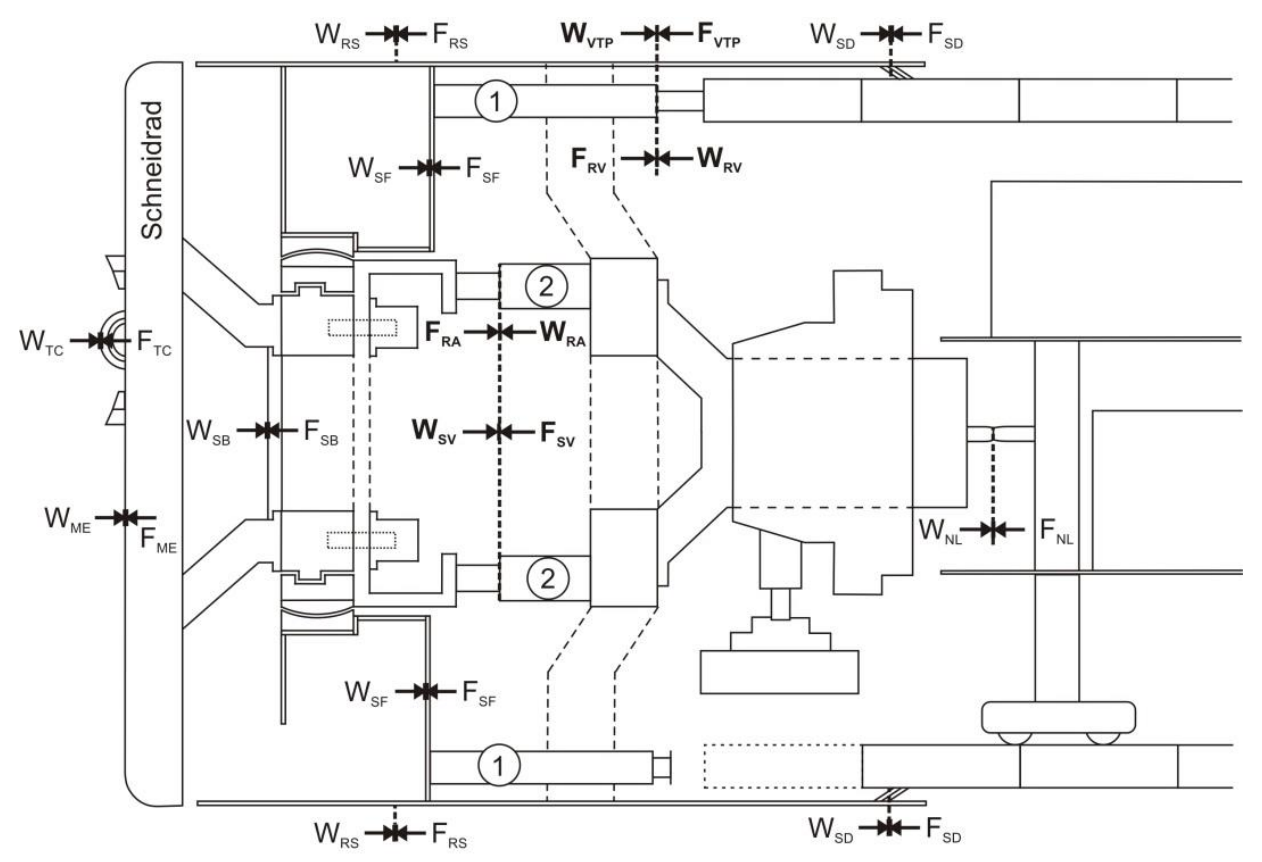

\begin{abstract}
Abb. 19: Schematischer Längsschnitt durch eine Hydroschild TVM mit in bzw. gegen die Vortriebsrichtung anliegenden Widerstands- und Kraftkomponenten im Vortrieb. Die an den Vortriebspressen (1) anliegenden KraftbzW. Widerstandskomponenten $W_{\text {VTP }}$ \& $F_{V T P}$, sowie $F_{R V} \& W_{R V}$ sind aus Gründen der Übersichtlichkeit nur an der Vortriebspresse in der Firste eingetragen, liegen jedoch auch symmetrisch an allen anderen Vortriebspresse an. In gleicher Weise liegen die Kraft- bzw. Widerstandskomponenten $F_{R A}$ \& $W_{R A}$ an allen Schneidradverschiebezylindern (2) an, sind im Längsschnitt jedoch nur am oberen Zylinder eingetragen. Die Einrichtungen des Flüssigkeitskreislaufs (Abb. 17) sind im Längsschnitt nicht dargestellt.
\end{abstract}


Die erste Komponente resultiert aus dem Widerstand $W_{\mathrm{SF}}[\mathrm{kN}]$ entgegen der Vortriebsrichtung, der sich aus der Ortsbrustfläche $A_{\text {TVM }}$ und dem Stützdruck $P_{S F}$ ergibt. Davon ist der Anteil abzuziehen, der bereits für die Fläche der Schneidradbefestigung $A_{S B}$ durch $W_{S B}$ in $G l .7$ berücksichtigt ist:

$$
F_{S F}=\left(A_{T V M}-A_{S B}\right) \cdot P_{S F} \cdot 100
$$

Die dazu notwendige Ortsbrustfläche $A_{T V M}\left[m^{2}\right]$ ergibt sich aus $\mathrm{Gl}$. 8:

$$
\mathrm{A}_{\mathrm{TVM}}=\mathrm{D}_{\mathrm{TVM}}^{2} \cdot \frac{\pi}{4}
$$

Die zweite Komponente ergibt sich aus der Reibungswiderstand $W_{R S}[k N]$ beim Vorschub des Schildes im Tunnel. Ein Modell zur Berechnung der Kraftkomponente $F_{R S}[k N]$ in Vortriebsrichtung stellen MAIDL et al. (1995: 51) vor.

Die dritte Komponente stellt die Reibkraft der Schildschwanzdichtung $\mathrm{W}_{\mathrm{SD}}[\mathrm{kN}]$ auf den bereits eingebauten Tübbingringen dar. Die entsprechende Kraft in Vortriebsrichtung $F_{S D}[k N]$ ist ebenfalls in der Vortriebspressenkraft $F_{\mathrm{VTP}}$ enthalten.

Die vierte in $F_{V T P}$ enthaltene Komponente resultiert aus dem Schleppwiderstand $W_{N L}[k N]$ der Nachläufer, die an die Schildmaschine angehängt sind und somit mit den Vortriebspressen durch den Tunnel gezogen werden. Die entsprechende Schleppkraft $F_{\mathrm{NL}}[\mathrm{kN}]$ in Vortriebsrichtung ergibt sich aus der Masse der Nachläufer $M_{N L}[t]$, dem Reibungskoeffizienten $\mu_{N L}[-]$ zwischen den Laufwerken der Nachläufer und den Tübbingringen bzw. dem Schienensystem. Zusätzlich muss der Gradient der Tunnelachse berücksichtigt werden. Der Wert von $\mu_{N L}$ hängt im Einzelfall von den eingesetzten Fahrwerken und Schienensystemen ab. Die Masse der Nachläufer $M_{N L}$ ist im Vortrieb durch wechselnde Beladung mit Tübbingen, Mörtel und gegebenenfalls Tunnelfahrzeugen oder Bahnen leichten Schwankungen unterworfen.

Ähnlich wie bei den Verschiebezylindern liegen auch in den Vortriebspressen Reibungswiderstände vor, die in im Vortriebswiderstand $W_{\text {VTP }}$ enthalten sind. Die Wirkrichtung des Reibungswiderstandes $\mathrm{W}_{\mathrm{RV}}$ in $\mathrm{kN}$ in den Vortriebspressen und der zugehörigen Kraftkomponenten $\mathrm{F}_{\mathrm{RV}}[\mathrm{kN}]$ ist im Vortrieb wiederum entgegengesetzt zu den bisher beschriebenen Widerstands- und Kraftkomponenten in WVTP bzw. F VTP $_{\text {(Abb. 19). }}$

Die Vortriebspressenkraft $F_{V T P}$ bzW. die entsprechende Widerstandskomponente $W_{\text {VTP }}$ ergibt sich folglich unter Berücksichtigung von aus dem Zusammenhang in Gl. 9 bzw. Gl. 10:

$$
\begin{gathered}
\mathrm{W}_{\mathrm{VTP}}=\mathrm{W}_{\mathrm{SV}}+\mathrm{W}_{\mathrm{SP}}+\mathrm{W}_{\mathrm{RS}}+\mathrm{W}_{\mathrm{SD}}+\mathrm{W}_{\mathrm{NL}}-\mathrm{W}_{\mathrm{RV}} \\
\mathrm{F}_{\mathrm{VTP}}=\mathrm{F}_{\mathrm{SV}}+\mathrm{F}_{\mathrm{SP}}+\mathrm{F}_{\mathrm{RS}}+\mathrm{F}_{\mathrm{SD}}+\mathrm{F}_{\mathrm{NL}}-\mathrm{F}_{\mathrm{RV}}
\end{gathered}
$$

Ist die Schildmaschine nicht mit einer Schneidradverschiebung ausgerüstet, so ergibt sich aufgrund der fehlenden Verschiebezylinder keine Möglichkeit, die am Schneidrad anliegenden Kräfte $F_{S v}$ von den übrigen in der Vortriebspressenkraft $F_{\text {VTP }}$ summierten Kraftkomponenten zu trennen. 
Das Schneidraddrehmoment $\mathrm{M}_{\mathrm{TVM}}[\mathrm{kNm}]$ ist ebenfalls eine Reaktionsgröße die im Wesentlichen aus den Schneidkräften der Abbauwerkzeuge resultiert. Dabei ergibt sich grundsätzlich für jedes einzelne Werkzeug aus der in Schneidrichtung gelegenen Kraftkomponente $F_{S C}$ der Schneidkraft $F_{C}$ und dem Spurradius $r_{s}$ ein entsprechendes Schneidmoment $M_{S C}[k N m]$. Die Summe der Schneidmomente $M_{S C}$ entspricht dem Schneiddrehmoment $\mathrm{M}_{\mathrm{SR}}[\mathrm{kNm}]$ und ergibt sich demnach aus:

$$
\mathrm{M}_{\mathrm{SR}}=\sum \mathrm{M}_{\mathrm{SC}}=\sum \mathrm{F}_{\mathrm{SC}} \cdot \frac{\mathrm{r}_{\mathrm{s}}}{1000}
$$

Im Schneidraddrehmoment $M_{T V M}[k N m]$ sind zusätzlich zumn Schneiddrehmomenten $M_{S R}$ noch drei weitere Komponenten enthalten. Die erste Komponente ist das Eigendrehmoment des Schneidrades $M_{E S}[\mathrm{kNm}]$, das sich im Wesentlichen aus der Überwindung der Reibung in der Lagerung des Schneidrades (Hauptlager) ergibt. Die zweite Komponente $M_{R S}[k N m]$ resultiert aus dem Rührwiderstand des Schneidrades in der Stützflüssigkeit, der im Wesentlichen von der Dichte der Stützflüssigkeit $\rho_{\mathrm{SF}}$ und der Schneidraddrehzahl $U$ abhängt. Die dritte Komponente $\mathrm{M}_{\mathrm{ME}}[\mathrm{kNm}]$ ergibt sich aus dem Reibungswiderstand, der durch Kontakt des Schneidrades über bereits abgebauten Boden zur Ortsbrust und der Tunnellaibung entsteht. Die genannten Komponenten des Schneidraddrehmoments $\mathrm{M}_{\mathrm{TVM}}$ sind in der Praxis nicht voneinander trennbar, $\mathrm{M}_{\mathrm{TVM}}$ ergibt sich jedoch grundsätzlich aus $\mathrm{Gl}$. 12:

$$
\mathrm{M}_{\mathrm{TVM}}=\mathrm{M}_{\mathrm{SR}}+\mathrm{M}_{\mathrm{ES}}+\mathrm{M}_{\mathrm{RS}}+\mathrm{M}_{\mathrm{ME}}
$$

Über die eingestellte Schneidraddrehzahl $U\left[\mathrm{~min}^{-1}\right]$ und das Schneidraddrehmoment $\mathrm{M}_{\mathrm{TVM}}[\mathrm{kNm}]$ ergibt sich abschließend analog zu Gl. 1 auch die Antriebsleistung $P_{\text {TVM }}[\mathrm{kW}]$, die durch die Elektro- oder Hydraulikmotoren am Hauptantrieb aufgebracht wird. Dabei ist zu berücksichtigen, dass die so entstehende Kombination aus Schneidraddrehzahl $U$ und Schneidraddrehmoment $M_{T V M}$ innerhalb der Leistungsgrenze des Hauptantriebes liegen muss (Kap. 3.2.2). Aus dem Schneidraddrehmoment $\mathrm{M}_{\mathrm{TVM}}$ $[\mathrm{kNm}]$, dem Ausbruchdurchmesser der TVM $\mathrm{D}_{\text {TVM }}[\mathrm{m}]$, sowie aus der Vortriebsgeschwindigkeit $\mathrm{V}_{\text {TVM }}$ $[\mathrm{mm} / \mathrm{min}]$ kann abschließend die Spezifische Energie $\mathrm{SE}_{\mathrm{TVM}}\left[\mathrm{MJ} / \mathrm{m}^{3}\right]$ berechnet werden. Diese gibt die Energie an, die für den Abbau je Kubikmeter Boden eingesetzt wird:

$$
\mathrm{SE}_{\mathrm{TVM}}=\frac{\mathrm{M}_{\mathrm{TVM}} \cdot 24.000}{\mathrm{D}_{\mathrm{TVM}}^{2} \cdot \pi \cdot \mathrm{V}_{\mathrm{TVM}}}
$$

Die Vortriebsphase endet, sobald die Vortriebspressen weit genug ausgefahren sind, um den nächsten Tübbingring einzubauen. Der Vortrieb wird durch Abregeln der Vortriebsgeschwindigkeit und der Schneidraddrehzahl eingestellt. Im Regelfall wird zusätzlich für die Dauer der Ringbauphase der Flüssigkeitskreislauf über den Bypass von der Arbeitskammer abgekoppelt, jedoch weiter aufrechterhalten. Nach Abschluss des Ringbaus wird das Schneidrad wieder in Betrieb genommen, der Flüssigkeitskreislauf auf die Arbeitskammer umgestellt und der Vortrieb über die Einstellung der Vorschubgeschwindigkeit wieder aufgenommen. Hierbei entfällt die Phase der Stabilisierung des Flüssigkeitskreislaufs, sodass der Vortrieb im Regelfall innerhalb von 1 bis 2 Minuten wieder aufgenommen werden kann.

In Abhängigkeit des Schneidraddurchmessers $D_{T V M}$ beträgt die mittlere Länge eines Tübbingrings $I_{(b)}$ [m] in Vortriebsrichtung 1,2 bis 2,0 m. Die Zeit, die für den Vortrieb der entsprechenden Strecke benötigt wird, hängt von der Vortriebsgeschwindigkeit ab und beträgt nach Abschluss der Lernkurve zu Vortriebsbeginn ca. 30 bis 60 min. 


\subsubsection{Ringbauphase}

Die Beschreibung der Ringbauphase erfolgt im Anschluss nur im Überblick, da die entsprechenden Prozesse keine wesentliche Bedeutung für die weiteren Untersuchungen in der vorliegenden Arbeit haben. Eine detaillierte Beschreibung ist z.B. in GIRMSCHEID (2008: 511) und GÖRTZ (2002: 51) gegeben.

In Vorbereitung für die Ringbauphase werden im Regelfall bereits während der Vortriebsphase die entsprechenden Tübbingsegmente mit speziellen Tunnelfahrzeugen, Lastwagen oder per Bahn auf der TVM angeliefert. Hier werden die Segmente im hinteren Bereich der Nachläufer mit einem Tübbingkran abgeladen, in den vorderen Teil der Nachläufer transportiert und auf Segmentfeeder in der Maschinensohle abgeladen. Dieser transportiert die Tübbingsegmente in den Schildschwanz unter den Erektor.

Der Erektor wird über eine Fernsteuerung auf der Maschine gesteuert. Das Tübbingsegment wird mit dem Erektor aufgenommen. Die Fixierung des Tübbingsegments am Erektor erfolgt dabei entweder über eine mechanische Kupplung durch einen Bolzen am Erektor oder durch Ansaugen des Tübbingsegments (Vakuumhebeplatte). Um das Tübbingsegment an die vorgesehene Stelle in der Schildlaibung zu setzen, werden die entsprechenden Vortriebspressen zurückgezogen, das Tübbingsegment eingesetzt und durch Ausfahren der Vortriebspressen fixiert.

Die radiale Ausrichtung der Tübbingringe wird durch ein Ringfolgeprogramm im Steuerstand der TVM vorgegeben, welches aus der aktuellen Lage der TVM zur Tunnelachse die notwendige Ausrichtung der Asymmetrie des Tübbingrings berechnet. Da der Einbau der Tübbingsegmente von der Sohle aus erfolgt, müssen die Tübbingsegmente in entsprechender Reihenfolge auf dem Segmentfeeder platziert werden. Diese Reihenfolge wird häufig bereits beim Beladen der Segmente auf die Transportfahrzeuge oder Wagons für die Bahn berücksichtigt, da auf der Maschine in Abhängigkeit der räumlichen Bedingungen nur noch eingeschränkt die Möglichkeit besteht, die Reihenfolge der Tübbingsegmente zu ändern.

Der Ringbau wird durch Setzen des konischen Schlusssteins abgeschlossen. Anschließend werden die Tübbingsegmente im Regelfall noch untereinander verschraubt. Mit dem Ausfahren der letzten Vortriebspressen kann der Vortrieb wieder aufgenommen werden (Kap. 3.3.1).

\subsubsection{Vortriebsunterbrechungen für Werkzeugwechsel}

Die Inspektion und der Wechsel von Abbauwerkzeugen erfordern bei Hydroschild TVM den Zugang zur Abbaukammer. Dieser wird im Vortrieb erschwert durch:

- $\quad$ Füllung der Abbaukammer mit Bentonitsuspension

- Stützdruck in der Abbaukammer

Für den Zugang zur Abbaukammer werden deshalb in Abhängigkeit der geotechnischen Bedingungen die bereits in Kap. 1.2 beschriebenen Methoden eingesetzt:

- Absenkung der Stützflüssigkeit und des Stützdrucks innerhalb von Dichtblöcken und Ausführung des Werkzeugwechsels unter atmosphärischen Bedingungen.

- Austausch der Stützflüssigkeit gegen Druckluft und Ausführung des Werkzeugwechsels unter Druckluft.

- Erhöhung der Standsicherheit der Ortsbrust und der Ausbläsersicherheit durch Zusatzmaßnahmen oder Ausführung des Werkzeugwechsels unter Einsatz spezialisierter Tauchtechniken.

Die am häufigsten angewendete Methode ist der Austausch der Stützflüssigkeit gegen Druckluft und Ausführung des Werkzeugwechsels unter Druckluft. Der teilweise Austausch der Stützflüssigkeit gegen Druckluft stellt einen veränderten Lastfall im Vergleich zu vollflächigen Stützung der Ortsbrst mit 
der Stützflüssigkeit dar. Deshalb wird im Regelfall eine Neuberechnung des erforderlichen Stützdrucks während der Arbeiten unter Druckluft vorgenommen. Für die Ausführung der Arbeiten muss zunächst der Vortrieb eingestellt werden. Für den Austausch der Bentonitsuspension gegen Druckluft muss anschließend als Grundlage ein möglichst dichter und stabiler Filterkuchen an der Ortsbrust aufgebaut werden. Hierfür wird von der Separationsanlage aus frische Bentonitsuspension in den Flüssigkeitskreislauf eingespeist, um zunächst möglichst reine Bentonitsuspension durch die Abbaukammer zu pumpen. Bereits in diesem Stadium wird das Schneidrad leicht von der Ortsbrust zurückgezogen. Die Einwirkzeit zum Aufbau eines ausreichend dichten Filterkuchens hängt stark von den Bodeneigenschaften und den rheologischen Eigenschaften der Bentonitsuspension ab.

Im Anschluss an den Aufbau des Filterkuchens wird in der Firste der Abbaukammer über die Verbindungsleitung zur Arbeitskammer Druckluft aus dem Druckluftpolster in der Arbeitskammer eingespeist. Folglich sinkt der Spiegel der Stützflüssigkeit in der Abbaukammer, im Gegenzug steigt der Pegel der Stützflüssigkeit in der Arbeitskammer. Die Verbindungsleitung wird wieder geschlossen, bevor der Pegel der Stützflüssigkeit den Arbeitsbereich im oberen Teil der Abbaukammer erreicht. Häufig ist dieser erste Niveauausgleich zwischen Arbeits- und Abbaukammer nicht ausreichend, um die notwendige Absenkung des Pegels der Stützflüssigkeit in der Abbaukammer zu erreichen. Deshalb wird im nächsten Schritt über die Förderleitung Stützflüssigkeit aus der Arbeitskammer entnommen. Das entnommene Volumen wird durch ein entsprechendes Volumen an Druckluft in der Abreitskammer ersetzt. Der Pegel der Stützflüssigkeit in der Arbeitskammer darf dabei nicht das Niveau der Tauchwandöffnung in der Schildsohle erreichen. Im Anschluss kann die Verbindungsleitung zwischen Arbeits- und Abbaukammer im Schildfirst erneut geöffnet werden und ein weiterer Niveauausgleich zwischen Arbeits- und Abbaukammer durchgeführt werden. Die Absenkung des Pegels der Stützflüssigkeit in der Abbaukammer wird so schrittweise vorangetrieben, bis das gewünsche Niveau erreicht wird.

Der Mechanismus zur Stützung der Ortsbrust funktioniert mit Druckluft in ähnlicher Weise wie mit der Stützflüssigkeit. In bindigen, undurchlässigen Böden wird der Luftdruck direkt auf das Korngerüst des Bodens übertragen. In durchlässigen Böden bildet einerseits der Filterkuchen eine Membrane, durch die der Stützdruck direkt auf das Korngerüst übertragen wird. Andererseits strömt auch Luft durch den Filterkuchen in den Boden ab, sodass auch durch die Strömungskräfte eine Stützung des Korngerüsts bewirken (BABENDERERDE 2000: 81). Sowohl während dem Absenken der Stützflüssigkeit, als auch während der anschließenden Arbeiten in der Abbaukammer müssen deshalb die Luftverluste aus der Abbaukammer in den Boden genau überwacht werden. Sinkende Luftverluste deuten auf eine zunehmende Erhöhung des Porendrucks im Boden bzw. einen Ausgleich zwischen Porendruck und Stützdruck hin. Dabei geht die Stützung des Korngerüsts verloren, sodass die Gefahr einer instabilen Ortsbrust entsteht. Stark ansteigende Luftverluste deuten dagegen auf die Entwicklung eines Ausbläsers hin (BABENDERERDE 2000: 82).

In beiden Fällen muss die Absenkung der Stützflüssigkeit bzw. die Arbeiten in der Abbaukammer abgebrochen werden, um diese wieder vollständig mit Stützflüssigkeit zu füllen. Dazu wird die Verbindungsleitung zwischen Arbeits- und Abbaukammer im Schildfirst geschlossen und der Spiegel der Stützflüssigkeit über die Speiseleitung wieder angehoben. Gleichzeitig wird die Druckluft im Schildfirst über eine Verbindungsleitung von der Abbaukammer in den atmosphärischen Bereich des Schildes hinter der Druckwand entlassen. Der Druck im Luftpolster zwischen Arbeitsammer und Tauchwand wird hierbei durch die Druckluftanlage konstant gehalten, sodass der Stützdruck erhalten bleibt. Ist die Abbaukammer vollständig mit Stützflüssigkeit gefüllt, wird die Verbindungsleitung zwischen Abbaukammer und dem atmosphärischen Schildbereich über einen Kugelhahn geschlossen.

Der Spiegel der Stützflüssigkeit in der Abbaukammer wird für Werkzeugwechsel folglich nur soweit abgesenkt, wie für die erforderlichen Arbeiten zwingend notwendig ist. Im Fall von Problemen mit einer instabilen Ortsbrust oder ansteigenden Luftverlusten wird so die Zeit zum Aufspiegeln der Stützflüssigkeit minimiert. Bezogen auf den Durchmesser des Schneidrades können mit einer Drittelabsenkung die Werkzeuge im Außenbereich des Schneidrades kontrolliert werden. Für eine Kontrolle der Werkzeuge im Zentrum ist eine Halbabsenkung notwendig. Arbeiten im Bereich der Schildsohle, an der Tauchwandöffnung oder dem Steinbrecher erfordern eine Vollabsenkung. Ist der erforderliche Flüssigkeitsspiegel in der Abbaukammer erreicht, wird der Flüssigkeitskreislauf auf den Bypass umgestellt und aufrecht erhalten, um im Fall von Problemen sofort reagieren zu können. 
Für die Arbeiten am Schneidrad wird das Personal über eine Druckluftschleuse im Firstbereich des Schildes in die Arbeitskammer eingeschleust. Der Druckanstieg erfolgt beim Einschleusen zwar zügig, jedoch langsam genug, um den Personen in der Schleuse einen Druckausgleich zu ermöglichen. Ist in der Schleuse das Druckniveau der Arbeitskammer erreicht, wird die Schleusentür zur Arbeitskammer geöffnet. Der Zugang zur Abbaukammer erfolgt über Türen in der Tauchwand.

Die nach dem Einschleusen zur Verfügung stehende Arbeitszeit in der Arbeits- und Abbaukammer wird neben der Entwicklung der Luftverluste hauptsächlich durch die zulässige Arbeitszeit unter Druckluft mit normaler Atemluft begrenzt. Diese ist in national unterschiedlichen gesetzlichen Vorgaben geregelt. Für Deutschland gilt aktuell die Verordnung über Arbeiten in Druckluft (BGBI. I S. 1909), die zuletzt im Jahr 2008 überarbeitet wurde. Darin sind sowohl die maximale Verweildauer als auch die entsprechenden Ausschleusungszeiten in Abhängigkeit des Arbeitsdrucks bis maximal 3,6 bar geregelt. Während die maximale Verweildauer mit steigendem Arbeitsdruck sinkt, steigt die Ausschleusungszeit mit dem Arbeitsdruck an (Abb. 20), um gesundheitliche Schäden zu vermeiden.

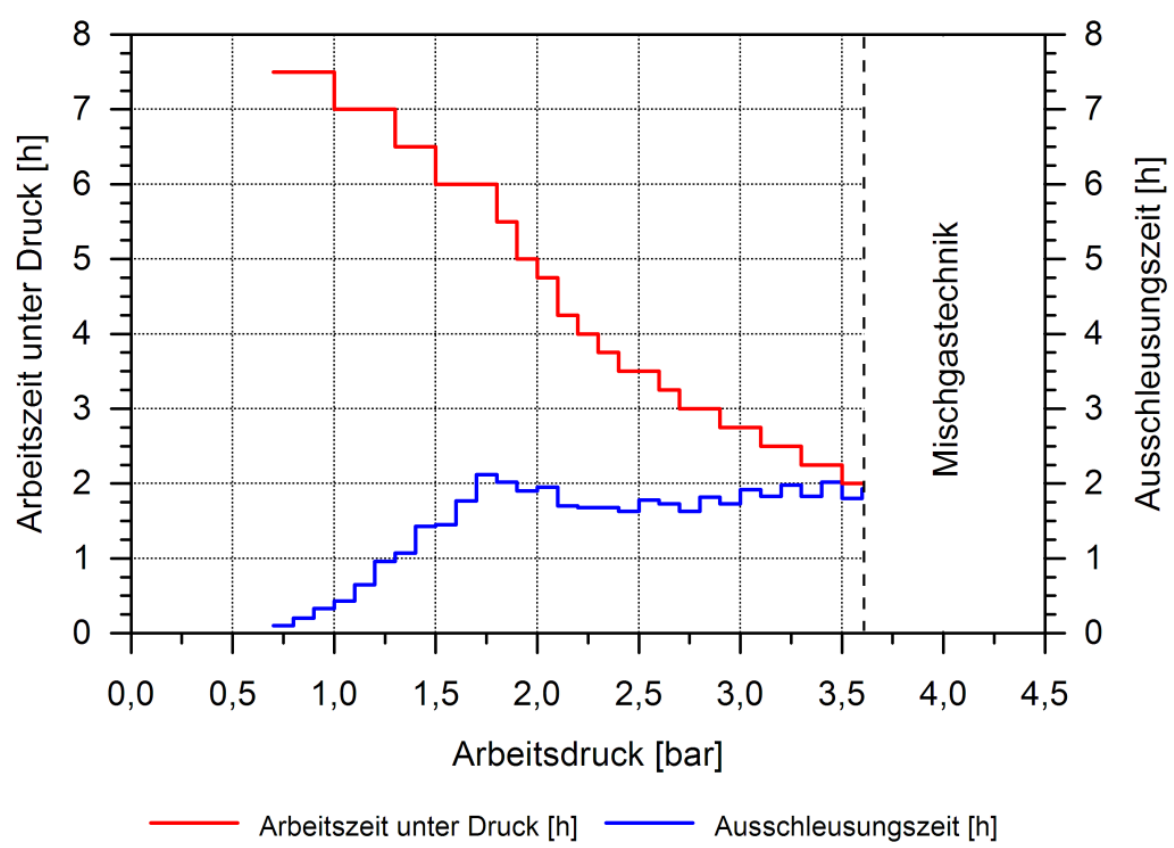

\begin{abstract}
Abb. 20: Graphische Darstellung der maximalen Arbeitszeit unter Druckluft (rot) und zugehörige Ausschleusungszeit (blau) in Abhängigkeit des Arbeitsdrucks aus der Verordnung über Arbeiten in Druckluft. Zusammengestellt aus BGBI. I S. 1909.
\end{abstract}

Oberhalb von 3,6 bar Arbeitsduck wird der Einsatz von normaler Atemluft nach HERRENKNECHT \& BÄPPLER (2007: 1176) aufgrund der sehr hohen Ausschleusungszeiten und sehr geringen Arbeitszeiten unwirtschaftlich. In diesem Bereich werden spezielle Atemgasgemische eingesetzt (SMITH 2012: 11), die längere Arbeitszeiten ermöglichen. Empfohlen werden hier kurze Einsätze im Bereich von 3,6 bis 8,0 bar, bei denen keine Sättigung der im Atemgasgemisch enthaltenen Gase im Blut eintritt. Oberhalb von 8,0 bar werden längere Interventionen empfohlen, bei denen eine Sättigung der im Atemgasgemisch enthaltenen Gase im Blut eintritt (HOLZHÄUSER et al. 2007: 384). In beiden Fällen sind für die Dekompression nach den Arbeiten unter Druckluft spezielle Einrichtungen auf der TVM notwendig, um die erforderliche Dekompressionszeit zu ermöglichen. Einen Überblick über Projekte mit hohem Stützdruck und die eingesetzten Methoden geben sowohl HoLzHÄUSER et al. (2007: 380), als auch HERRENKNECHT \& BÄPPLER (2007: 1176 ff.). Der Zeitaufwand für die Drucklufteinstiege nimmt mit diesen Methoden im Vergleich noch einmal deutlich zu. Zum Teil sind sowohl für die Vorbereitung als auch für das Ausschleusen bis zu einem Tag notwendig. Aus diesem Grund wurden zum Teil bereits Maschinen mit abkoppelbaren und transportablen Schleusen entwickelt, sodass die Dekompression und Umstellung auf Atemluft getrennt vom Vortrieb außerhalb des Tunnels erfolgen kann. 
Für den Transport von Material in und aus der Abbaukammer muss ein von der Personenschleuse unabhängige Materialschleuse eingesetzt werden. Die Personenschleuse wird damit jederzeit frei gehalten, sodass im Falle von Problemen ein sofortiger Rückzug aus der Abbau- und Arbeitskammer möglich ist. Vor allem bei großen Maschinendurchmessern wird häufig eine zweite Personenschleuse verbaut. Diese wird genutzt, um Ausfallzeiten durch die notwendigen Ausschleusungszeiten für das Personal während der Maschinenstillstände für Drucklufteinstiege zu minimieren. So kann über die zweite Personenschleuse eine neue Mannschaft in die Arbeits- und Abbaukammer eingeschleust werden, während die erste Mannschaft die notwendige Ausschleusungszeit in der ersten Personenschleuse durchläuft.

Die Arbeiten in der Arbeitskammer laufen grundsätzlich soweit wie möglich hinter dem Schneidrad ab, sodass das Schneidrad einen mechanischen Schutz gegen eine instabile Ortsbrust bildet. Trotzdem erfordern die Umgebungsbedingungen in der Abbaukammer ein hohes Maß an Sicherheitsvorkehrungen. Die Arbeiten in der Abbaukammer werden deshalb grundsätzlich auf das notwendigste Maß reduziert, um die zur Verfügung stehende Zeit möglichst effektiv zu nutzen. Das Ein- und Aushängen von Podesten an der Tauchwand, die den Zugang zu verschiedenen Bereichen des Schneidrades ermöglichen, sowie das Reinigen des Schneidrades mit Wasser sind meist unumgänglich. Alle Maschinenteile in der Abbaukammer sind im Regelfall mit Bentonitsuspension überzogen. Hinzu kommt zum Teil verklebter Boden am Schneidrad, sodass für die Kontrolle und den Wechsel von Abbauwerkzeuge eine Reinigung erforderlich ist. Dabei darf durch die Reinigungsarbeiten keinesfalls der Filterkuchen an der Ortsbrust beschädigt werden.

Nach dem Ausschleusen des Personals aus der Abbaukammer und dem Aufspiegeln der Stützflüssigkeit in der Abbaukammer wird der Vortrieb erneut aufgenommen. Bei längeren Drucklufteinstiegen können diese auch in Intervallen unterbrochen werden und die Abbaukammer wieder vollständig mit Stützflüssigkeit gefüllt werden, um den Filterkuchen zu erneuern bzw. zu befeuchten.

\subsubsection{Betriebsdaten}

Hydroschild TVM sind grundsätzlich mit einer speicherprogrammierbaren Steuerung (SPS) ausgerüstet. Diese erhält als Eingangswerte alle vortriebsrelevanten Informationen zum aktuellen Betriebszustand der TVM, die durch entsprechende Sensoren auf der TVM aufgenommen werden. Dazu zählen überblickend vor allem folgende Informationen:

- Hydrauliksystem der TVM (Druck, Temperatur, Durchfluss, Tankfüllstände)

- Elektrisches System der TVM (Spannung, Stromstärke)

- Fett-/Ölsystem zur Schmierung des Hauptlagers/-antriebs (Druck, Temperatur \& Füllstände)

- $\quad$ Druckluftsystem der TVM (Druck, Durchfluss)

- Flüssigkeitskreislauf der Stützflüssigkeit (Druck, Durchfluss, Pumpen)

- Mörtel-/Verpresssystem der TVM (Druck, Volumen, Tankfüllstände)

- $\quad$ Einstellwerte und Reaktionsgrößen im Vortrieb (Kap. 3.3.1)

- Vermessungssystem der TVM (Lage, Richtung)

Ein Teil der genannten Eingangswerte wird im Steuerstand der TVM als Information für den Schildfahrer angezeigt. Dazu zählen vor allem die Einstellwerte (Aktivparameter) und Reaktionsgrößen (Passivparameter) (Kap. 3.3.1) aus dem Vortrieb, die Basisdaten aus dem Flüssigkeitskreislauf, dem Hydrauliksystem und die Daten aus dem Vermessungssystem. 
Aus der Kombination der Einstellwerte des Schildfahrers und den Eingangswerten ergeben sich Ausgangswerte aus der SPS. Diese werden über entsprechende Aktoren in eine automatische Reaktion auf der TVM übersetzt. Zum Beispiel werden elektrische Hydraulik- oder Druckluftventile entsprechend geschaltet, oder Änderungen der Schaltung im elektrischen System der TVM vorgenommen.

Aus den Eingangs- und Ausgangswerten werden zusätzlich sogenannte Statusbits erzeugt, die Informationen über den aktuellen Zustand der TVM geben. So wird zum Beispiel ein Statusbit für die Information „Vortrieb“ oder "Ringbau“ erzeugt, der bei gleichzeitigem Drehen des Schneidrades und Vorschub der TVM durch die Vortriebspressen die Information „Vortrieb“ ausgibt. Ein weiteres Beispiel ist die Drehrichtung des Schneidrades, die mit einem Statusbit aufgezeichnet wird, der die Werte "Drehen Links“ und „Drehen Rechts“ annehmen kann.

Zusätzlich dazu sind in der SPS für bestimmte Parameter Grenzwerte gesetzt, bei deren Über- oder Unterschreitung im Steuerstand ein Alarm angezeigt wird. Beispiele dafür sind die Temperatur des Getriebeöls im Hauptantrieb oder die Unterschreitung der Füllstände in Tanks für Hydrauliköl.

Alle genannten Informationen, also Eingangswerte, Ausgangswerte, Statusbits und Alarme werden zur Dokumentation des Vortriebs vollständig aufgezeichnet. Dazu werden im Rhythmus von $10 \mathrm{Se}-$ kunden alle genannten Daten auf einem zentralen Rechner im Steuerstand der TVM gespeichert. Diese Daten werden als Momentanwerte bezeichnet, da sie jeweils den momentanen Zustand der TVM wiedergeben. Aus diesen Momentanwerten werden automatisiert verschiedene weitere Werte gebildet, die in separaten Dateien abgelegt werde. Einerseits werden die Daten anhand der Statusbits vorsortiert, so erfolgt zum Beispiel eine Sortierung der Daten nach Vortriebs-, Ringbau- und Stillstandphase. Andererseits werden außer für Statusbits und Alarmwerte für alle genannten Informationen Mittelwerte über die Vortriebsphase (Ring) gebildet. Diese werden als Mittelwertdaten bezeichnet.

Die Qualität und Aussagekraft der Betriebsdaten der TVM für die Datenanalyse hängt von mehreren Faktoren ab:

- Kalibrierung der Sensoren vor Vortriebsbeginn

- Wartung und Austausch der Sensoren während dem Vortrieb

- Einstellung und Wartung der speicherprogrammierbaren Steuerung

Vor allem in der Startphase des Vortriebs ergeben sich häufig über mehrere Ringe hinweg Probleme, bis eine korrekte Kalibrierung der Sensoren und vollständige Aufzeichnung der Daten erreicht wird. Aber auch in der Vortriebsphase können Probleme durch Verschleiß oder Schäden an den Sensoren und der speicherprogrammierbaren Steuerung entstehen. 


\section{Wissensstand zur Abbaumechanik und zum Werkzeugverschleiß}

Auf Basis der Literaturrecherche (Kap. 2.2) ist nachfolgend der bestehende Wissensstand zum Thema Abbaumechanik und Werkzeugverschleiß für Hydroschild TVM in Lockergesteinen zusammengefasst. Die Ergebnisse sind untergliedern in Grundlagen zum Thema Verschleiß, Abbaumechanik und Verschleißbild, maßgebliche Einflussfaktoren auf den Verschleiß, sowie bestehende Ansätze zur Verschleißprognose für Vortriebe mit Hydroschild TVM in Lockergesteinen.

\subsection{Grundlagen Verschleiß}

\subsubsection{Tribologie}

Grundlage der Betrachtungen zum Werkzeugverschleiß sind die Mechanismen, die bei Kontakt von verschiedenen Stoffen an deren Oberfläche stattfinden. Die Erforschung und Erklärung dieser Mechanismen stellen ein interdisziplinäres Wissenschaftsgebiet dar, das unter dem Begriff Tribologie zusammengefasst ist. Der Begriff Tribologie geht nach ZUM GAHR (1987: 3) auf das griechische Wort tribos zurück, das übersetzt Reiben bedeutet. Der Begriff Verschleiß wird innerhalb der Tribologie von verschiedenen Autoren, z.B. OECD (1969) und in der 1997 zurückgezogenen DIN 50320 (1979: 1) in ähnlicher Weise definiert. Für die vorliegende Arbeit wird daraus die Definition von Verschleiß wie folgt zusammengefasst:

Verschleiß ist ein fortschreitender Massenverlust an der Oberfläche eines Festkörpers, der aus einer Relativbewegung an der Oberfläche gegen einen Festkörper, Flüssigkeit oder Gas und den damit verbundenen mechanischen Einwirkungen resultiert.

\subsubsection{Tribologische Systeme}

Bei der Untersuchung der Mechanismen die an der Oberfläche der Festkörper zu Verschleiß führen, hat sich die Betrachtung in Form von Systemen etabliert. Diese werden als tribologische Systeme bezeichnet. Eine detaillierte Erläuterung dazu gibt ZUM GAHR (1987: $81 \mathrm{ff}$.). Zusammenfassend wird versucht mithilfe eines tribologischen Systems alle Eingangsgrößen, Mechanismen und resultierende Größen in einem möglichst detaillierten Ausschnitt zu beschreiben. Eine schematische Darstellung ist in Abb. 21 gegeben.

Innerhalb der tribologischen Systeme werden vier grundlegende Mechanismen unterschieden, die zum Massenverlust an der Oberfläche führen. Diese sind nach ZUM GAHR (1987: 84 f.):

- Adhäsion

- Abrasion

- Oberflächenermüdung

- Tribochemische Reaktionen

Die vier Mechanismen werden im Folgenden (Kap. 4.1.1.2 - 4.1.1.5) als Zusammnefassung der Erkenntnisse von ZUM GAHR (1987) im Detail beschrieben. Im Hinblick auf den Verschleiß der Abbauwerkzeuge beim Bodenabbau in Lockergesteinen mit Hydroschild TVM werden alle vier Mechanismen in Betracht gezogen. Der Abrasion wird jedoch häufig implizit z.B. von GHARAHBAGH et. al (2011: 605) als der bedeutendste Mechanismus angesehen. DüLLMANN et al. (2014: 96) untermauern diese Annahme, legen jedoch keine detaillierten Untersuchungen dazu vor. Ein entsprechender Nachweis z.B. durch Aufnahmen der verschlissenen Oberflächen mit einem Rasterelektronenmikroskop fehlt jedoch. 


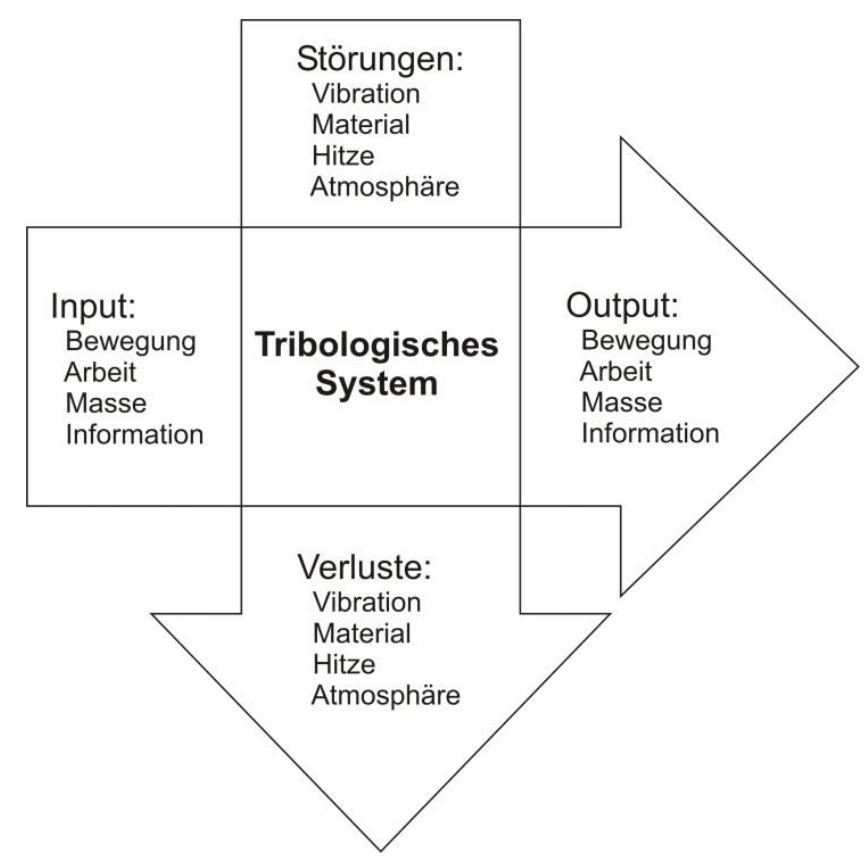

Abb. 21: Allgemeine Beschreibung eines triologischen Systems mit Input, Output, Störungen und durch die Störungen verursachte Verluste. (ZUM GAHR 1981: 81; verändert)

Tribologischen Systeme können anhand der beteiligten Systemelemente und Beanspruchungsarten der Oberflächen gegliedert werden. Eine relativ aktuelle Gliederung gibt die Gesellschaft für Tribologie e.V. (GFT 2002: 14) heraus, die zusammengefasst in Tab. 3 dargestellt ist. Eine ähnliche Gliederung war auch in der 1997 zurückgezogenen DIN 50320 (1979: 5) enthalten.

Tab. 3: Gliederung tribologischer Systeme nach beteiligten Systemelementen und Beanspruchungsarten, verändert nach GFT (2002: 14). Die Tabelle ist auf die im Vortrieb mit Hydroschild TVM vorkommenden Kombinationen an Systemelementen gekürzt. $((\cdot)=$ Mechanismus ist wirksam; $(-)=$ Mechanismus ist nicht wirksam)

\begin{tabular}{|c|c|c|c|c|c|c|}
\hline \multirow[t]{2}{*}{ Systemelemente } & \multirow[t]{2}{*}{$\begin{array}{l}\text { Tribologische } \\
\text { Beanspruchung }\end{array}$} & \multirow[t]{2}{*}{ Verschleißart } & \multicolumn{4}{|c|}{$\begin{array}{c}\text { Wirksame } \\
\text { Mechanismen }\end{array}$} \\
\hline & & & $\begin{array}{l}\frac{.}{9} \\
: \frac{0}{0} \\
\frac{c}{0} \\
\frac{0}{4}\end{array}$ & $\begin{array}{l}\frac{5}{9} \\
\frac{0}{20} \\
\frac{0}{4}\end{array}$ & 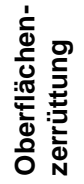 & 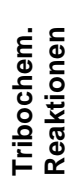 \\
\hline $\begin{array}{l}\text { Festkörper - Zwischenstoff - Festkörper } \\
\text { (Vollständige Filmtrennung) }\end{array}$ & $\begin{array}{l}\text { Gleiten, Rollen, } \\
\text { Wälzen, Prallen, } \\
\text { Stoßen }\end{array}$ & - & - & - & • & • \\
\hline \multirow{3}{*}{$\begin{array}{l}\text { Festkörper - Festkörper } \\
\text { (Festkörperreibung, Grenzreibung, } \\
\text { Mischreibung) }\end{array}$} & Gleiten & Gleitverschleiß & $\bullet$ & $\bullet$ & $\bullet$ & $\bullet$ \\
\hline & $\begin{array}{l}\text { Rollen, } \\
\text { Wälzen } \\
\end{array}$ & $\begin{array}{l}\text { Rollverschleiß, Wälzver- } \\
\text { schleiß }\end{array}$ & $\bullet$ & $\bullet$ & $\bullet$ & $\bullet$ \\
\hline & $\begin{array}{l}\text { Prallen, } \\
\text { Stoßen }\end{array}$ & $\begin{array}{l}\text { Prallverschleiß, Stoßver- } \\
\text { schleiß }\end{array}$ & $\bullet$ & $\bullet$ & $\bullet$ & $\bullet$ \\
\hline \multirow{2}{*}{ Festkörper - Partikel - Festkörper } & Gleiten & $\begin{array}{l}\text { Furchungsverschleiß } \\
\text { Korngleitverschleiß }\end{array}$ & - & $\bullet$ & - & - \\
\hline & Wälzen & Kornwälzverschleiß & - & $\bullet$ & - & - \\
\hline Festkörper - Flüssigkeit mit Partikeln & Strömen & $\begin{array}{l}\text { Spülverschleiß } \\
\text { Erosionsverschleiß }\end{array}$ & - & $\bullet$ & $\bullet$ & $\bullet$ \\
\hline Festkörper - Flüssigkeit & Strömen & $\begin{array}{l}\text { Werkstoffkavitation } \\
\text { Kavitationserosion }\end{array}$ & - & - & $\bullet$ & $\bullet$ \\
\hline
\end{tabular}




\subsubsection{Adhäsion}

Unter dem Begriff Adhäsion werden nach ZUM GAHR (1987: ff.) alle Mechanismen zusammengefasst, bei denen vor allem nach gleitendem Kontakt zwischen zwei Festkörpern unter hoher Normalspannung die Trennung der beiden Festkörper nicht an der ursprünglichen Grenzfläche erfolgt, sondern eine neue Trennfläche durch einen der beiden Festkörper verläuft. Der Festkörper, durch den die neue Trennfläche verläuft, verliert so an der Oberfläche an Masse, diese bleibt an dem zweiten Festkörper haften. Grundvoraussetzung dazu ist, dass sich an der ursprünglichen Trennfläche der beiden Festkörper eine Verbindung einstellt, deren Scherfestigkeit höher liegt als die Scherfestigkeit eines der beiden Festkörper.

In Abhängigkeit der Werkstoffe der beiden Festkörper bestehen unterschiedliche Möglichkeiten für die Art der Verbindung:

- Mechanisches Ineinandergreifen und Verzahnen der beiden Oberflächen

- Diffusion von Atomen oder Molekülen zwischen den beiden Festkörpern

- Elektronentransfer an der Oberfläche zwischen den beiden Festkörpern

- Chemische Bindungen an der Oberfläche (Metallische Bindungen, lonenverbindungen, kovalente Bindungen, Van-der-Waals Kräfte)

\subsubsection{Abrasion}

Abrasion ist nach ZUM GAHR (1987: 93 ff.) die Lösung von Material an der Oberfläche während der Relativbewegung durch harte Partikel oder harte Auswölbungen in einem der beiden Körper. Die harten Partikel können auch in einen der beiden Körper eingebettet sein oder im Zwischenstoff vorliegen. Der Abrasion liegen dabei wiederum vier grundlegende physikalische Mechanismen zu Grunde:

- Mikroermüdung

- Mikropflügen

- Mikroschneiden

- Mikrorisse

Diese vier Mechanismen sind in Abb. 22 schematisch dargestellt.

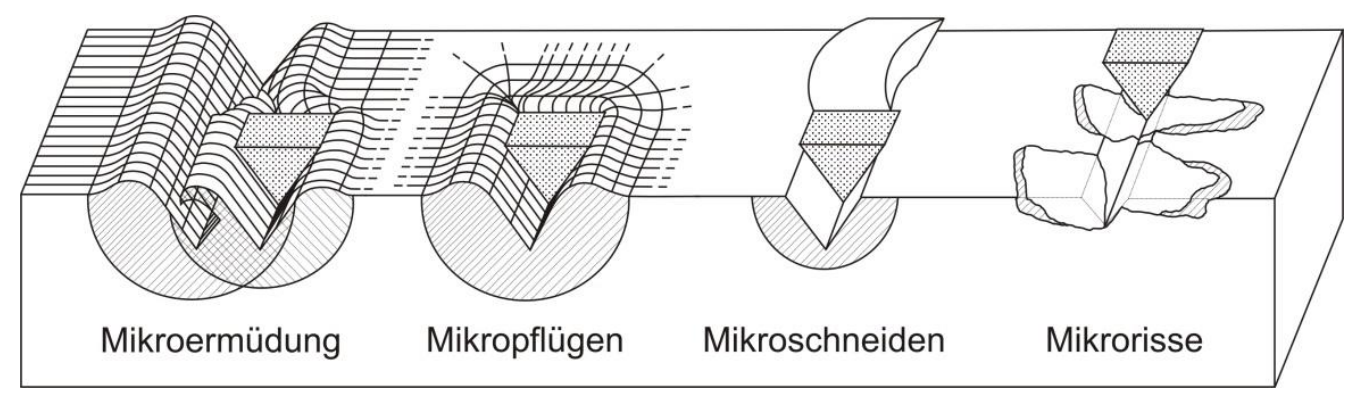

Abb. 22: Schematische Darstellung der vier physikalischen Mechanismen, die bei Abrasion zum Abtrag von Material an der Oberfläche des Grundkörpers führen. (ZUM GAHR 1981: 96, verändert) 
Die vier Mechanismen verursachen unterschiedlich hohen Materialabtrag an der Oberfläche des Grundkörpers. Das abgetragene Volumen nimmt überschlägig vom Mikro-Pflügen hin zu Mikro-Rissen zu. Welcher der vier Mechanismen in einem tribologischen System mit abrasivem Verschleiß dominiert, hängt im Wesentlichen von den Rahmenbedingungen ab. Als bestimmende Faktoren gelten:

- Härte von Grundkörper und Gegenkörper

- Angriffswinkel bzw. Kornform des Gegenkörpers

- Kontaktspannung zwischen Grund- und Gegenkörper

Dem Verhältnis der Härte zwischen Grundkörper und Gegenkörper (Abrasivstoff) kommt ein besonders hohes Maß an Bedeutung zu. Hierzu gibt ZUM GaHR (1987: 94) den in Abb. 23 dargestellten Zusammenhang an. VERHOEF (1997: 43) übernimmt diesen für den Einsatz von Rundschaftmeißeln in Locker- und Festgesteinen. FRENZEL (2010a: 47) ergänzt in seiner Arbeit sowohl eigene, als auch Daten von PLINNINGER (2002: 133), die dem Zusammenhang in Abb. 23 widersprechen. Die Daten zeigen für Abbauwerkzeuge aus inhomogenen Werkstoffen oder einer Kombination verschiedener Werkstoffe eher einen stetigen Anstieg des abrasiven Verschleißes mit ansteigendem Verhältnis zwischen der Härte des Abrasivstoffs und der Härte des Grundkörpers. Ungeachtet dessen bestätigen sie jedoch den hohen Einfluss der Härte der beteiligten Körper auf den abrasiven Verschleiß.

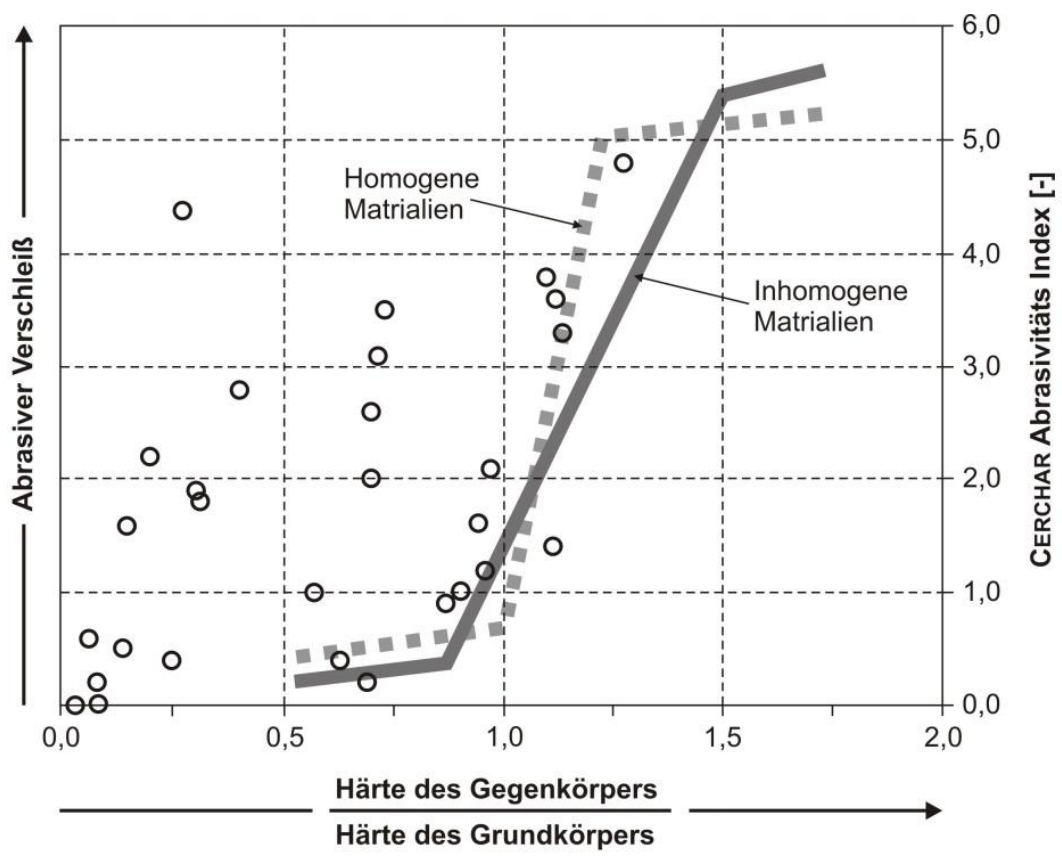

\begin{abstract}
Abb. 23: Intensität des abrasiven Verschleißes in Abhängigkeit der Härte der abrasiven Partikel im Verhältnis zur Härte des Grundkörpers. Die beiden eingetragenen Graphen für homogene bzw. inhomogene Materialien stellen den von ZUM GAHR 1981: 94 angegebenen Zusammenhang dar. Zusätzlich sind Datenpunkte von PLINNINGER (2003: 133) eingetragen. Diese wurden für die Darstellung von FRENZEL (2010a: 47) modifiziert und widersprechen dem von ZUM GAHR beschriebenen Zusammenhang.
\end{abstract}

\title{
4.1.1.4 Oberflächenermüdung
}

Oberflächenermüdung entsteht nach ZUM GAHR (1987: 99 ff.) im Wesentlichen bei wiederholter Beund Entlastung der Oberfläche des Grundkörpers durch den Gegenkörper, die zyklische Spannungen im Werkstoff verursachen. Diese führen zu Mikrorissen an oder unter der Oberfläche des Grundkörpers, die zur Ablösung von flachen Bruchstücken an der Oberfläche des Grundkörpers führen. Die zurückbleibenden Grübchen werden als Pittings bezeichnet. 


\subsubsection{Tribochemische Reaktionen}

Unter tribochemischen Reaktionen werden nach ZUM GAHR (1987: $104 \mathrm{ff}$.) Verschleißmechanismen zusammengefasst, die durch chemische Reaktionen an der Oberfläche eines Festkörpers eine Veränderung des Werkstoffs und Massenverlust entsteht. Unter atmosphärischen Bedingungen zählt dabei zum Beispiel die Oxidation von Metallen.

\subsubsection{Abbauwerkzeugverschleiß}

Eine allgemeine Definition des Begriffs Abbauwerkzeugverschleiß, die auf die tribologischen Grundlagen (Kap. 4.1.1) aufgebaut ist, existiert trotz zahlreicher Veröffentlichungen zu diesem Thema bisher nicht. Im Hinblick auf den Vortrieb mit Hydroschild TVM und Erddruckschilden in Lockergesteinen wird der Verschleiß der Abbauwerkzeuge jedoch häufig in die Begriffe Primärverschleiß und Sekundärverschleiß unterteilt. Die Definition der beiden Begriffe ist dabei nicht einheitlich.

HOLZHÄUSER \& NILSEN (2006: 169) fassen unter Primärverschleiß unabhängig vom Mechanismus oder Ursache alle Verschleißphänomene zusammen, die an Abbauwerkzeugen zu beobachten sind. Unter dem Begriff Sekundärverschleiß werden alle Verschleißphänomene an den Werkzeughaltern und Gehäusen sowie an der restlichen Schneidradstahlstruktur zusammengefasst, die ursächlich auf übermäßigen Verschleiß an den Abbauwerkzeugen zurückzuführen sind. Diese Definition wird von NiLSEN et al. (2006a-c) und NILSEN et al. (2007: 104) weiter verfolgt und auch von GHARAHBAGH et al. (2011: 605) übernommen.

Im Gegensatz dazu definiert FREnZEL (2010b: 302) mit dem Begriff Primärverschleiß alle Verschleißphänomene, die beim planmäßigen Einsatz des Werkzeuges beim Bodenabbau durch den Kontakt mit dem Boden in der Ortsbrust an der Werkzeugschneide entstehen. Unter Sekundärverschleiß werden alle Verschleißphänomene zusammengefasst, die an allen anderen Abbauwerkzeugteilen durch passiven Kontakt mit der Ortsbrust oder bereits abgebautem Boden in der Abbaukammer entstehen. Diese Definition wird von SchOLZ \& WENDL (2010: 3508), WENDL (2012: 126) und FRENZEL\& BABENDERERDE (2011: 37) weiter verfolgt. Der von HolZHÄUSER \& NILSEN (2006: 169) als Sekundärverschleiß bezeichnete Massenverlust an der Schneidradstahlstruktur, den Werkzeughaltern und Gehäusen wird von FRENZEL \& BABENDERERDE (2011: 37) unter dem Begriff Folgeverschleiß (subsequent wear) zusammengefasst.

Die Definition der Verschleißbegriffe ist also in bisherigen Untersuchungen nicht einheitlich. Hinzu kommt, dass durch die vorliegenden Definitionen keine eindeutige Abtrennung zwischen den Begriffen Verschleiß und Schaden erfolgt. Unter dem Begriff Verschleiß werden einerseits Phänomene zusammengefasst, die tatsächlich auf den vier grundlegenden Verschleißmechanismen (Kap. 4.1.1) basieren. Andererseits werden auch Phänomene beschrieben, die aufgrund eines sofortigen makroskopischen Funktionsverlustes der Abbauwerkzeuge oder aufgrund einer Überschreitung der Auslegungsgrenze der Abbauwerkzeuge eher den Charakter eines Schadens tragen. Ein Vergleich der veröffentlichten Ergebnisse wird dadurch deutlich erschwert.

\subsection{Abbaumechanik \& Verschleißbild}

Zur Abbaumechanik verschiedener Werkzeugtypen in Lockergesteinen liegen bisher nur in eingeschränktem Umfang Erkenntnisse vor. Diese stammen nur zum Teil direkt aus dem maschinellen Tunnelbau. Ein wesentlicher Beitrag stammt auch aus dem Umfeld der Bodenbearbeitung in der Agrartechnik, sowie aus dem Bereich des Dredgings von Gräben für die Verlegung von Kabeln oder Rohrleitungen unter Wasser. Eine grundlegende Einteilung verschiedener Schneidtechnologien gibt erstmals STROPPEL (1953).

Die direkten Erkenntnisse zur Abbaumechanik in Lockergesteinen werden ergänzt durch veröffentlichte Vortriebserfahrungen zum Verschleißbild der Abbauwerkzeuge bei Hydroschild TVM. Diese erlauben zum Teil deduktive Schlüsse auf die zu Grunde liegende Mechanik und den Materialfluss beim Bodenabbau. 


\subsubsection{Modelle und Laborversuche zur Abbaumechanik}

Eine erste Betrachtung zur Schneidmechanik einer feststehenden Schneide, die sowohl in der Form als auch in der Anwendung einem Schälmesser auf einem Schneidrad zum Bodenabbau in Lockergesteinen ähnlich ist, geben MCKEYS \& ALI (1977). Bereits hier wird ein dreidimensionaler Bodenkörperansatz gewählt, um anhand verschiedener Parameter die Schneidkräfte zu ermitteln. Ähnliche theoretische Ansätze zur Bruchmechanik und Bruchbild mit zweidimensionalen und dreidimensionalen Ansätzen für den entstehenden Bruchkörper in Tonen und wassergesättigten Sanden verfolgt auch MIEDEMA (1992) und MIEDEMA (1995).

Eine Validierung und Überarbeitung der theoretischen Ansätze zur Bruchmechanik an feststehenden Schneiden in Lockergesteinen auf Basis von großformatigen Laborversuchen erfolgt parallel dazu durch v. Os \& V. LEUSSEN (1987) und BERETITSCH (1992). Eine erste Übertragung der Ansätze für Schneidkräfte einzelner Werkzeuge auf einen vollständigen Schneidkopf für Dredginganwendungen entwickelt MIEDEMA (1989).

Die wesentlichen Erkenntnisse aus dieser Entwicklung der theoretischen Ansätze zur Bruchmechanik und den zugehörigen Laborversuche lassen sich vor allem auf Basis der umfassenden Arbeit von BERETITSCH (1992) wie nachfolgend zusammenfassen. Der Bruchmechanismus des Bodens wird nach den vorliegenden Erkenntnissen vor allem durch die Korngrößenverteilung des Bodens, den Wassergehalt $w[\%]$, die Dichte $\rho\left[\mathrm{g} / \mathrm{cm}^{3}\right]$ und die Schneidgeschwindigkeit $\mathrm{v}_{\mathrm{sc}}[\mathrm{m} / \mathrm{s}]$ bestimmt. Einen Überblick über die beschriebenen Mechanismen und die zughörigen Bodeneigenschaften gibt Abb. 24.

Geotechnischer Einflussfaktoren auf die Schneidkraft $F_{C}[k N]$ sind die dynamischen Schereigenschaften des Bodens. Der Wert der Schergeschwindigkeit $\mathrm{v}_{\mathrm{sc}}[\mathrm{m} / \mathrm{s}]$ liegt sowohl bei den untersuchten Anwendungen (Bodenbearbeitung in der Landwirtschaft \& Dredging), als auch beim Bodenabbau mit Schälmesser um mehrere Größenordnungen höher, als in den in DIN 18137, T1 (2010) und DIN 18137, T2 (2010) normierten Versuchen zur Bestimmung der Scherparameter von Böden. BERETITSCH (1992: 26 f.) führt deshalb hierzu Scherversuche in einem Geschwindigkeitsbereich von 0 bis $50 \mathrm{~m} / \mathrm{min}(0-0,8 \mathrm{~m} / \mathrm{s})$ durch. Diese ergeben, dass sich in bindigen Böden durch die Erhöhung der Geschwindigkeit eine Verschiebung der Schergeraden ergibt. Während der Reibungswinkel $\varphi^{\prime}\left[^{\circ}\right]$ konstant bleibt, steigt die Kohäsion $\mathrm{c}^{\prime}\left[\mathrm{kN} / \mathrm{m}^{2}\right]$ hauptsächlich in einem Übergangsbereich von 0 bis $10 \mathrm{~m} / \mathrm{min}(0-0,2 \mathrm{~m} / \mathrm{s})$ um bis zu $400 \%$ an. Die dynamische Kohäsion $\mathrm{c}_{\mathrm{dyn}}$ unterscheidet sich also wesentlich von der quasistatischen Kohäsion $c^{\prime}$. Die resultierende Schneidkraft $F_{C}$ nimmt unabhängig von der Schergeschwindigkeit mit steigendem Reibungswinkel $\varphi^{\prime}$ und steigender Kohäsion c' zu.

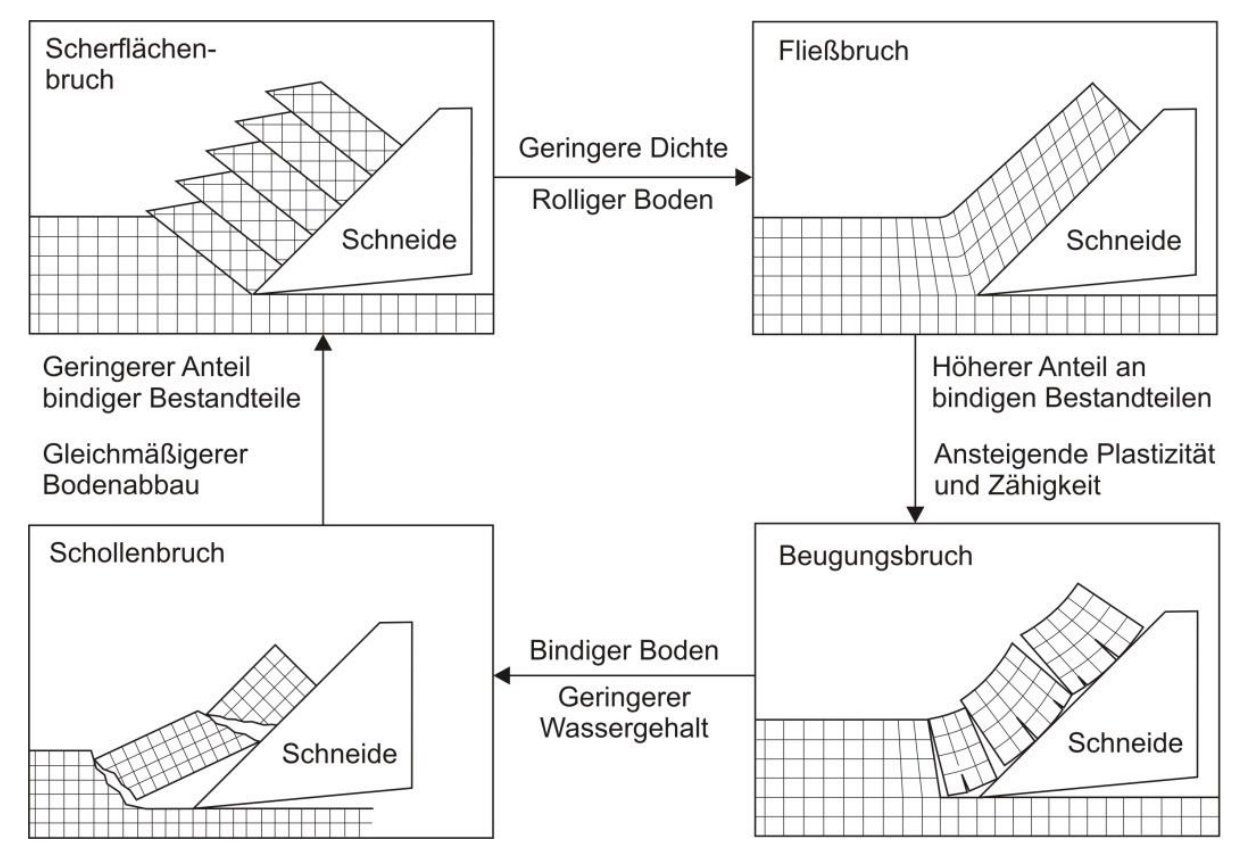

Abb. 24: Bruchmechanismus und Zerkleinerungsgrad des Bodens in Abhängigkeit der Korngrößenverteilung des Bodens, der Dichte, der Plastizität und des Wassergehalts. (BERETITSCH 1992: 57, verändert) 
Zweiter wesentlicher Einflussfaktor auf die resultierende Schneidkraft $F_{C}$ ist die Reibung zwischen Boden und Stahl. Diese beruht nach SCHLICK (1989: 98) im Wesentlichen auf Kapillarkräften an der Grenzfläche zwischen Boden und Stahl. THEWES (1999: 76) fasst deshalb als entscheidende Einflussfaktoren die Korngrößenverteilung, mineralogische Zusammensetzung, den Wassergehalt $\mathrm{w}$ und Porenanteil $\mathrm{n}_{\text {por }}[-]$ des Bodens zusammen. Die Scherspannung zwischen Boden und Stahl [kN/m²] lässt sich folglich nach WILMS (1995: 61) und THEWES (1999: 88) in Abhängigkeit der Konsistenzzahl I I [-] darstellen.

Aus geometrischer Sicht sind die Breite der Schneide $b_{S M}[\mathrm{~mm}]$ und der Schneidwinkel $\alpha_{c}\left[{ }^{\circ}\right]$ des Schälmessers die maßgeblichen Einflussfaktoren auf die Schneidkraft $F_{C}$. Dabei steigt die resultierende Schneidkraft $F_{C}$ sowohl mit steigender Breite der Schneide, als auch mit steigendem Schneidwinkel $\alpha_{c}\left(30-90^{\circ}\right)$ tendenziell an.

Im Hinblick auf die Steuerung des Schneidvorgangs sind einerseits die Schneidgeschwindigkeit $\mathrm{v}_{\mathrm{sc}}$ (Vorschubgeschwindigkeit) und die Penetration $\mathrm{p}$ (Eindringtiefe) für die resultierende Schneidkraft $F_{C}$ von Bedeutung. Die Schneidkraft $F_{C}$ steigt wiederum mit zunehmender Schneidgeschwindigkeit $v_{S C}$ und zunehmender Penetration $p$ tendenziell an. Für große Schneidwinkel $\alpha_{c}>53^{\circ}$, wie sie auch bei Schälmessern häufig eingesetzt werden, verläuft die Zunahme des horizontalen Schneidwiderstands des Bodens bzw. der Schneidkraft FSC nach BERETITSCH (1992: 100) eher exponentiell als linear.

BERETITSCH (1992: 106) kann auf Basis der Laborversuche zudem zeigen, dass die Spannungsverteilung auf der Frontfläche der Schneide ebenfalls stark vom Schneidwinkel $\alpha_{c}$ und der Schneidgeschwindigkeit $\mathrm{v}_{\mathrm{SC}}$ abhängt (Abb. 25).
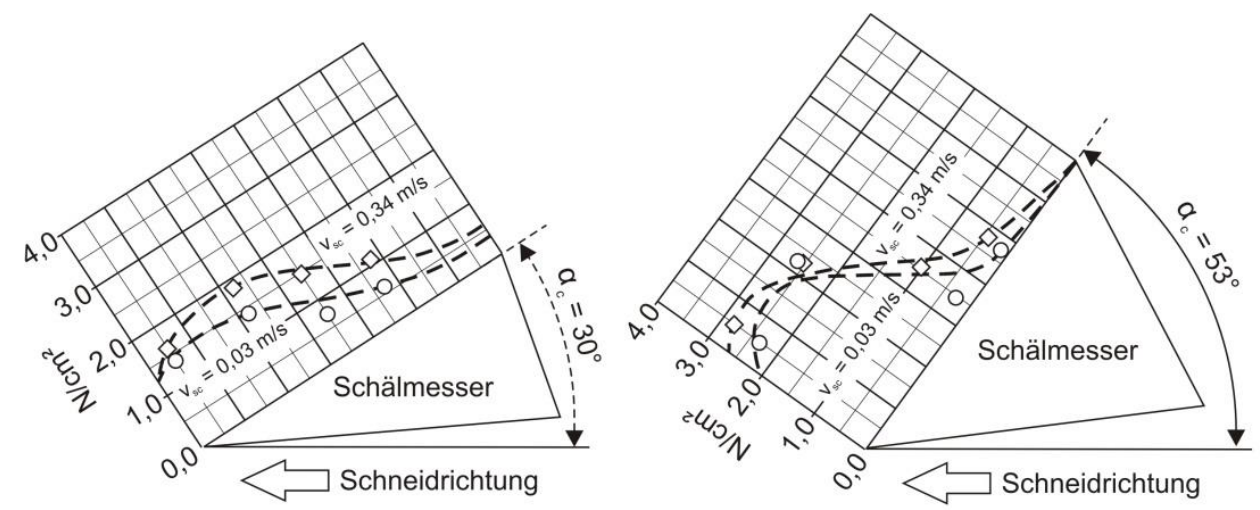

Abb. 25: Spannungsverteilung an der Frontfläche der Schneide in Abhängigkeit des Schneidwinkels $\alpha_{c}\left[^{\circ}\right]$ und der Schneidgeschwindigkeit $\mathrm{v}_{\mathrm{sc}}[\mathrm{m} / \mathrm{s}]$. (BERETITSCH 1992: 106, verändert)

Die genannten Untersuchungen geben zusammenfassend einen guten Überblick über die grundsätzliche Abhängigkeit des Bruchmechanismus und der resultierenden Schneidkraft $F_{C}$ von verschiedenen Einflussfaktoren. Trotz der Ähnlichkeit in der Anwendung der feststehenden Schneide in den genannten Untersuchungen und der Anwendung von Schälmessern auf dem Schneidrad einer Hydroschild TVM in Lockergesteinen bleiben jedoch einige mögliche Einflussfaktoren unberücksichtigt.

Das betrifft zunächst die Umgebungsbedingungen beim Bodenabbau in der Abbaukammer. Der Bodenabbau findet hier im Gegensatz zu den theoretischen Ansätzen und Laborversuchen nicht gegen eine vollständig freie Oberfläche statt. Der Boden in der Ortsbrust wird mit dem Druck der Stützflüssigkeit in der Abbaukammer beaufschlagt, um den in der Ortsbrust anliegenden Erddruck auszugleichen und so die Ortsbrust zu stabilisieren. Der Boden befindet sich folglich auch in einem anderen Spannungszustand als in den genannten Untersuchungen. Zusätzlich findet der Bodenabbau in der Abbaukammer in einer senkrecht stehenden Fläche statt, im Gegensatz zum waagrechten Schneidvorgang in den beschriebenen Ansätzen und Laborversuchen.

Darüber hinaus wurden alle genannten Untersuchungen nur für Böden durchgeführt, in denen die Korngröße sehr viel kleiner ist, als die Dimensionen der Schneide. Die maximal in den Ansätzen und Laborversuchen enthaltene Korngröße liegt bei 2,0 mm (Grobsand). Die minimale Breite der einge- 
setzten Schneiden liegt dagegen bei $100 \mathrm{~m}$. Im Gegensatz dazu sind beim Einsatz von Schälmessern in Lockergesteinen auch deutlich gröbere Korngrößen üblich. Der Haupteinsatzbereich von Hydroschild TVM schließt auch Kiese mit einer Korngröße von 2-63 mm ein. Die Breite der Schneide der Schälmesser liegt dagegen auch in Kiesen häufig bei $100 \mathrm{~mm}$, sodass sich ein von den beschriebenen Untersuchungen deutlich abweichendes Verhältnis zwischen Korngröße und Breite der Schneide ergibt. Dem entsprechend ändert sich vermutlich auch die Abhängigkeit des Bruchmechanismus und der Schneidkraft $F_{\mathrm{C}}$ von den verschiedenen Einflussfaktoren.

Für Schneidrollen existieren mit einer Ausnahme keine entsprechenden Modelle oder Laborversuche zur Abbaumechanik in Lockergesteinen. Die Ergebnisse zum Bruchmechanismus beim Einsatz in Festgesteinen, die z.B. von RostAMI (1997) erarbeitet wurden, sind auf Lockergesteine nicht übertragbar. KIEFER et al. (2008: 129) führen auf Basis der Überlegungen von Goss (2002) Versuche mit einer Linear Cutting Machine (LCM) zum Schneiden von Steinen und Blöcken in Lockergesteinen mit Schneidrollen durch. Dazu werden gut gerundete Granitkomponenten verschiedener Durchmesser $(10-25 \mathrm{~cm})$ und Einaxialer Druckfestigkeit (UCS) [MPa] in ein Zement-Sand-Gemisch eingebettet und mit einer Schneidrolle bearbeitet. Die Ergebnisse der Versuche zeigen, dass der Abbaumechanismus der Granitkomponenten entscheidend von zwei Faktoren abhängt:

- Festigkeitsverhältnis zwischen Matrix und eingebettetem Stein o. Block

- Verhältnis zwischen Ausgangsdurchmesser und bereits abgebautem Anteil des Steins 0. Blocks

Liegen die beiden Parameter jeweils relativ niedrig, bleibt der Block bei der Bearbeitung mit einer Schneidrolle in der Matrix. Es kommt zum Chipping und der Block wird wie in einer Festgesteinsortsbrust stetig abgebaut. Liegen die beiden Parameter jeweils relativ hoch, versagt durch den Lasteintrag der Schneidrolle die Einbindung des verbliebenen Blockteils in der Ortsbrust. Der verbliebene Teil des Blocks wird dann in Abhängigkeit des Lasteintrags entweder als Ganzes aus der Ortsbrust herausgedrückt, oder zerbricht in mehrere Einzelteile, die ebenfalls aus der Ortsbrust herausgedrückt werden.

Der Übergangsbereich zwischen normalem Abbau (Chipping) in der Matrix und Brechen und Herausdrücken der Blöcke aus der Matrix ist in Abb. 26 dargestellt. Damit ist auf Basis des Festigkeitsverhältnisses zwischen Block und Matrix theoretisch eine Prognose der im Vortrieb zu erwartenden Komponentengrößen möglich.

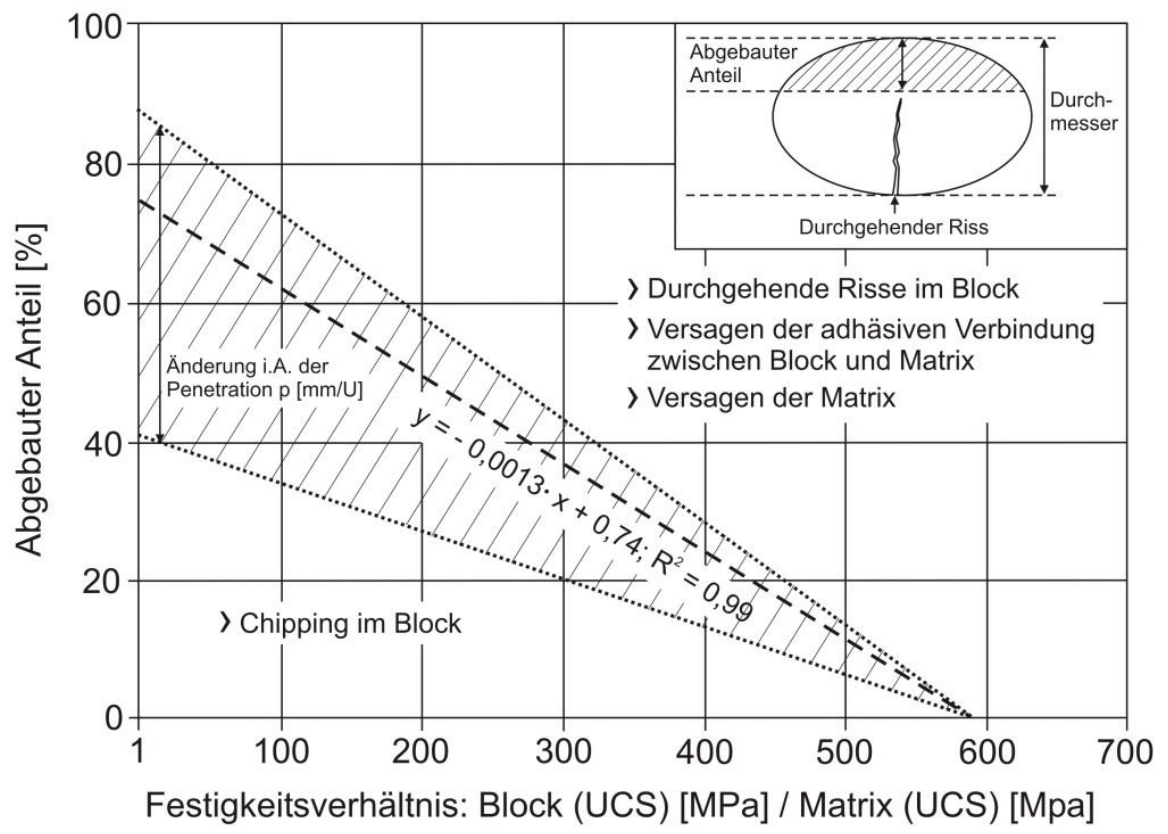

Abb. 26: Abbauverhalten eines Blocks in einer Matrix durch Schneidrollen in Abhängigkeit des Festigkeitsverhältnisses zwischen Block und Matrix und dem bereits abgebauten Anteil des Blocks. (KIEFFER et al. 2008: 135, verändert) 


\subsubsection{Vortriebserfahrungen}

Aus bisherigen Vortrieben mit Hydroschild TVM in Lockergesteinen liegen Erfahrungen zum Verschleißbild und Rückschlüsse auf die Abbaumechanik der verschiedenen Werkzeugtypen in Lockergesteinen vor. Diese sind im Folgenden nach den unterschiedlichen Abbauwerkzeugtypen unterteilt.

\subsubsection{Schneidrollen}

Schneidrollen sind im Regelfall vorlaufend zu Schälmessern und Räumern auf dem Schneidrad angebracht (Kap. 3.2.1). Anders als im Festgestein wird im Lockergestein übereinstimmend nach FRENZEL \& BABENDERERDE (2011: 39) und SCHOLZ \& WENDL (2011: 231) der Boden zwischen den Schneidspuren der Schneidrollen nicht oder nur zum Teil abgebaut. Deshalb entstehen durch den Vorlauf der Schneidrollen Furchen in der Ortsbrust. Beim stetigen Durchlaufen der Furchen haben die Schneidringe sowohl an der Lauffläche, als auch an der Seitenfläche Kontakt zum Boden. Durch den Verschleiß an diesen Flächen ergibt sich eine Selbstschärfung bzw. Zuspitzung des Schneidrings.

Die Schneidrollen tragen damit insgesamt kaum zum Bodenabbau bei. Im Gegenteil wird der Boden in den Furchen nach SCHOLZ \& WENDL (2011: 230) und KöHLER et al. (2010: 49) häufig nur verdrängt, sodass es in Abhängigkeit der Korngrößenverteilung des Bodens und unter Eintrag von Feinmaterial aus der Stützflüssigkeit zu einer Verdichtung des Bodens in der Ortsbrust kommen kann. Innerhalb der Verdichtungszone in der Ortsbrust werden die Bodenkomponenten zum Teil so stark beansprucht, dass es zum Zerbrechen der Komponenten kommen kann. Die Verdichtung der Ortsbrust geht nach SCHOLZ \& WENDL (2011: 233) auch mit einem deutlichen Anstieg der Schneidradanpresskraft $F_{S R}$ und des Schneidradrehmoments $\mathrm{M}_{\mathrm{TVM}}$, sowie des Verschleißes der Abbauwerkzeuge einher.

In Lockergesteinen mit niedriger Lagerungsdichte oder niedriger Konsistenz kann im Gegensatz dazu nach DOWDEN \& ROBINSON (2001: 964) und FRENZEL \& BABENDERERDE (2011: 38) der Schneidwiderstand am Schneidring so niedrig sein, dass dieser nicht ausreicht, das Eigendrehmoment der Schneidrolle zu überwinden. Die Schneidrolle wird deshalb nicht gedreht und der Schneidring einseitig flach abgeschliffen. Dieses Phänomen wird häufig als Blockieren der Schneidrolle bezeichnet.

Nach HolzHÄUSER \& NilSEN (2006: 170) und FRENZEL \& BABENDERERDE (2011: 38) kommen darüber hinaus in bindigen Lockergesteinen auch Verklebungen als Ursache für ein Blockieren der Schneidrolle in Betracht. THEWES (1999: 172) und THEWES (2003: 260) zeigen, dass die Anhaftung von Boden an den Stahlteilen des Schneidrades weitgehend auf Adhäsion zurückgeführt werden kann. Diese entsteht unter atmosphärischen Bedingungen durch Kapillarkräfte. In quellfähigen Tonen wird sie jedoch hauptsächlich von Saugspannungen verursacht, die aus dem Quellvorgang resultieren. Eine qualitative Einschätzung des Verklebungsrisikos kann nach THEWES (1999: 175), THEWES (2003: 254) und THEWES (2004: 92) mithilfe der Plastizitätszahl $I_{P}[\%]$ und der Konsistenzzahl $I_{C}[-]$ vorgenommen werden.

HollmanN \& TheWes (2011: 244) und HollmanN \& THEWES (2012: 578) detaillieren diese Ergebnisse für Hydroschild TVM (Abb. 27). Mit der Stützflüssigkeit steht hier in der Abbaukammer praktisch unbegrenzt Wasser zur Verfügung, sodass auch Böden mit ursprünglich hoher Konsistenzzahl durch die Aufnahme von Wasser in einen für Verklebungen kritischen Zustand verschoben werden können. Bei einer weiteren Erhöhung des Wassergehalts können die Böden darüber hinaus auch in der Stützflüssigkeit in Lösung gehen, sodass ein erhöhter Aufwand für die Separation entsteht.

Wichtig ist zusätzlich, dass bereits geringe Anteile an verklebungsanfälligem Material in der Ortsbrust ausreichend sind, um zu Verklebungen zu führen. Angrenzende gemischtkörnige oder rollige Bodenarten werden in diese Verklebungen eingearbeitet. So kannen aus ursprünglich wenig abrasiven bindigen Bodenarten in der Ortsbrust zum Teil Verklebungsmaterial mit hohem Verschleißpotential entstehen (HolLmANn \& THEWES 2011: 239). 


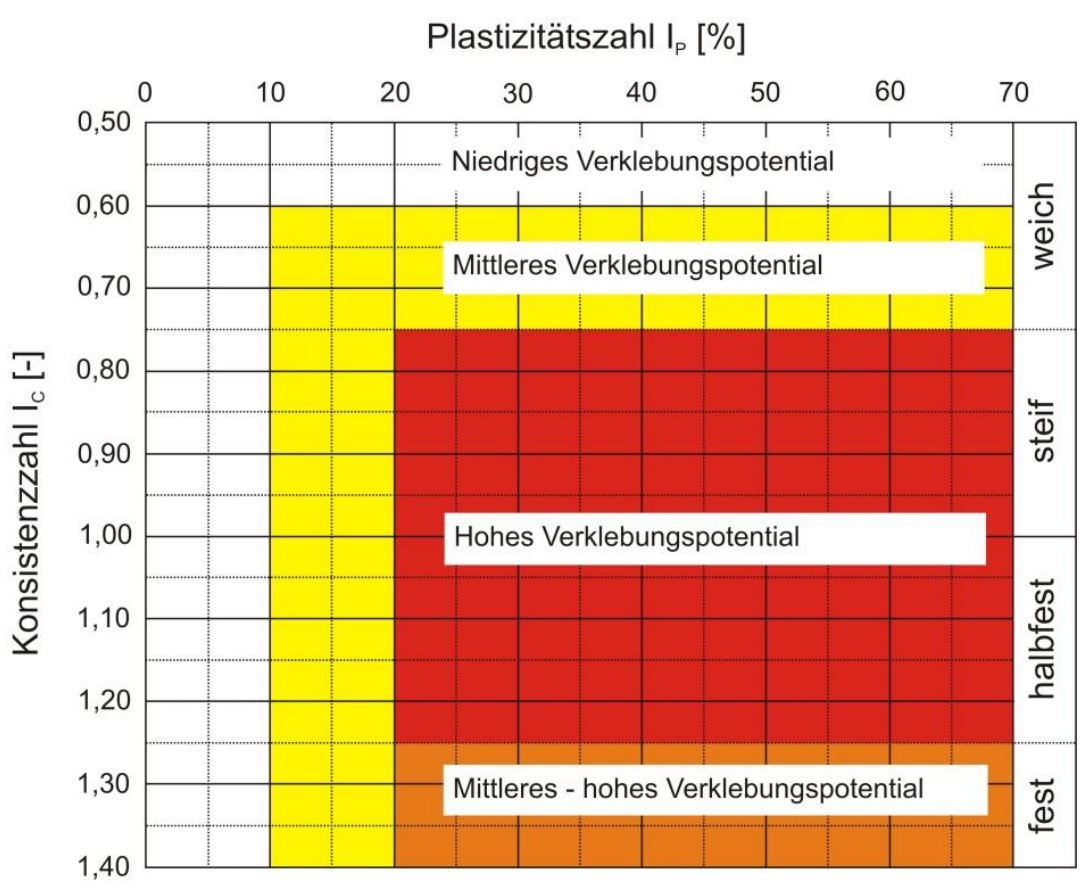

Abb. 27: Qualitative Einschätzung des Verklebungspotentials von bindigen Böden mit der Konsistenzzahl I [-] und der Plastizitätszahl IP [\%]. (HOLLMANN \& THEWES 2011a: 239, verändert)

Praxisbeispiele für Verklebungen in Vortrieben mit Hydroschild TVM geben z.B. THEWES \& BURGER (2003: 186) sowie WeH et al. (2009a: $30 \mathrm{ff}$.) und WeH et al. (2009b: 19). Als Maßnahmen zur Vermeidung von Verklebungen als Ursache für das Blockieren von Schneidrollen nennen THEWES (1999: 177 f.) und THEWES \& BuRger (2003: 185 f.) zusammenfassend folgende Punkte in der Auslegung der TVM und der Steuerung des Abbauprozesses:

- Vermeidung enger Durchlässe im Transportweg des Bodens von der Ortsbrust zur Förderleitung, z.B. in Form eines geschlossenen Schneidradzentrums oder eines Ansaugrechens.

- Vermeidung von Absetzzonen, z.B. in der Schildsohle, durch Agitatoren oder Spüldüsen.

- Maximierung der Austauschrate der Stützflüssigkeit, sowie Spülung der Abbaukammer während der Ringbauzeit.

- Optimierung der Penetration p, um in bindigen Böden möglichst große Späne von der Ortsbrust abzuschälen.

- Einsatz von Additiven zur Verminderung der Verklebungsneigung

KRAUTER (2008: 22) führt Feinmaterial in der Stützflüssigkeit als Ursache für Verschleiß an den Gleitringdichtungen der Schneidrollen an, sodass einerseits ein Verlust des Schmiermittels in der Lagerkammer auftritt, andererseits jedoch auch Feinmaterial in die Lagerkammer eintritt. Dieses führt zum Verschleiß der Kegelrollenlager und damit zum Blockieren der Schneidrolle. Abschließend können die Kegelrollenlager der Schneidrollen nach BURGER (2006: 18) auch durch zu hohe Penetration beim Abbau von Blöcken in der Ortsbrust überlastet werden. Folge daraus sind Lagerschäden, die ebenfalls zum Blockieren der Schneidrolle führen.

Beim Abbau von Blöcken in der Ortsbrust wurde nach KRAUTER (2008: 23) zusätzlich beobachtet, dass auch Schneidringe gewaltsam vom Rollengrundkörper abgedrückt wurden, oder durch wiederholte schlagartige Belastung Risse in den Schneidringen auftraten. Darüber hinaus werden zum Abbau von Blöcken häufig Schneidringe mit Hartmetalleinsätzen eingesetzt, um an diesen Einsätzen eine Spannungskonzentration herbeizuführen und so eher ein Zerbrechen der Blöcke herbeizuführen. 
Durch den Härteunterschied zwischen den Hartmetalleinsätzen und dem umgebenden Stahl des Schneidrings kommt es dabei jedoch häufig zu einem sehr unregelmäßigen Verschleißbild. Die Hartmetalleinsätze werden durch Verschleiß des umliegenden Schneidrings freigelegt und brechen frühzeitig aus.

\subsubsection{Schälmesser}

Die Schälmesser sind in der Regel nachlaufend zu den Schneidrollen auf dem Schneidrad angebracht. Sie bauen den Boden ab, der zwischen den Spuren der Schneidrollen stehen bleibt. Nach SCHOLZ \& WENDL (2011: 232) sind dabei keine spezifischen Schneidspuren der Schälmesser in der Ortsbrust zu erkennen. Das Verschleißbild der Schälmesser bildet jedoch die Form der Furchen der Schneidrollen in der Ortsbrust nach. Im Bereich der Furchen leisten die Schälmesser keinen Bodenabbau, sodass hier der Verschleiß der Schälmesser zum Teil deutlich geringer ist als im angrenzenden Bereich ohne vorlaufende Schneidrolle.

FRENZEL \& BABENDERERDE (2011: 37) beschreiben darüber hinaus, dass an Schälmessern, die am Bodenabbau teilnehmen allgemein sowohl an der Frontseite, als auch an der Rückenfläche Abrieb erkennbar ist. Dieser entsteht an der Frontseite durch Abgleiten des abgebauten Materials, an der Rückenfläche vor allem durch Schleifen an der Ortsbrust (Primärverschleiß) und Sekundärverschleiß. Hinzu kommt, dass an einigen Schälmessern Risse und Sprödbrüche der Hartmetalleinsätze in der Schneide festgestellt wurden. Diese werden auf Hindernisse in der Ortsbrust, z.B. Blöcke, aber auch künstliche Hindernisse wie z.B. Bohrpfähle zurückgeführt, die durch Schälmesser nicht in der Ortsbrust abgebaut werden.

\subsubsection{Stichel}

Für Stichel liegen nur in stark eingeschränktem Umfang Erkenntnisse zum Verschleißbild und zur Abbaumechanik vor. SCHOLZ \& WENDL (2011: 234) ordnen Sticheln eine sehr ähnliche Funktionsweise wie Schälmessern zu, wobei sich auch die Ausprägung des Verschleißbildes an Frontflächen und Rücken mit der allgemeinen Beschreibung von FRENZEL \& BABENDERERDE (2011: 38) deckt.

Im Gegensatz zu den Schneidrollen decken die Stichel in den von SCHOLZ \& WENDL (2011: 229) analysierten Projekten die Ortsbrust vollflächig ab. Es sind wiederum keine Schneidspuren in der Ortsbrust erkennbar. WENDL (2012: 102) beschreibt zusätzlich, dass durch den Einsatz der Stichel im Vergleich zu den Schneidrollen eine deutliche Reduktion der Schneidradanpresskraft $F_{S R}$ und des Schneidraddrehmomentes $M_{\text {TVM }}$ erzielt wurde. Dieser Umstand deutet darauf hin, dass der Abbaumechanismus mit Sticheln trotz vollflächiger Abdeckung der Ortsbrust effektiver abläuft bzw. weniger zur Verdichtung des Bodens in der Ortsbrust (Kap. 4.2.2.1) neigt.

\subsubsection{Räumer}

Für Räumer liegen nahezu keine Erkenntnisse zum Verschleißbild und Abbaumechanismus vor. Nach FRENZEL (2010b: 305) nehmen die Räumer im Kaliberbereich die Funktion von Schälmessern ein. Damit ist die Beschreibung des Verschleißbildes für Schälmesser von FRENZEL \& BABENDERERDE (2011: 37) vermutlich auch auf Räumer übertragbar, direkte Erkenntnisse liegen jedoch nicht vor. Im Unterschied zu Schälmessern hat nach BABENDERERDE (2000: 80) Material das in der Schildsohle bei unzureichendem Materialfluss in der Abbaukammer liegen bleibt, zusätzlichen Einfluss auf den Verschleiß der Räumer. 


\subsection{Maßgebliche Einflussfaktoren \& Verschleißkennwerte}

Sowohl die Löseleistung als auch der Verschleiß hängen bei nahezu allen bohrenden oder fräsenden Abbauverfahren in Locker- und Festgesteinen, von zahlreichen Einflussfaktoren ab, die sich jedoch nach THURO (2002: 1) übergreifend in drei Kategorien unterteilen lassen:

- Geotechnik (Boden- \& Gebirgseigenschaften)

- Gerätschaft (Auslegung der Hydroschild TVM)

- Baubetrieb (Steuerung des Abbauprozesses)

Diese Kategorien können problemlos auf den Vortrieb mit Hydroschild TVM in Lockergesteinen übertragen werden und werden so im Grundsatz auch von DAUB (2010: 251 f.) übernommen. Die bekannten für den Verschleiß maßgeblichen Einflussfaktoren und Kennwerte sind deshalb in den folgenden Kapiteln nach diesen Kategorien gegliedert. Eine schematische Darstellung der drei Kategorien ist in Abb. 28 gegeben.

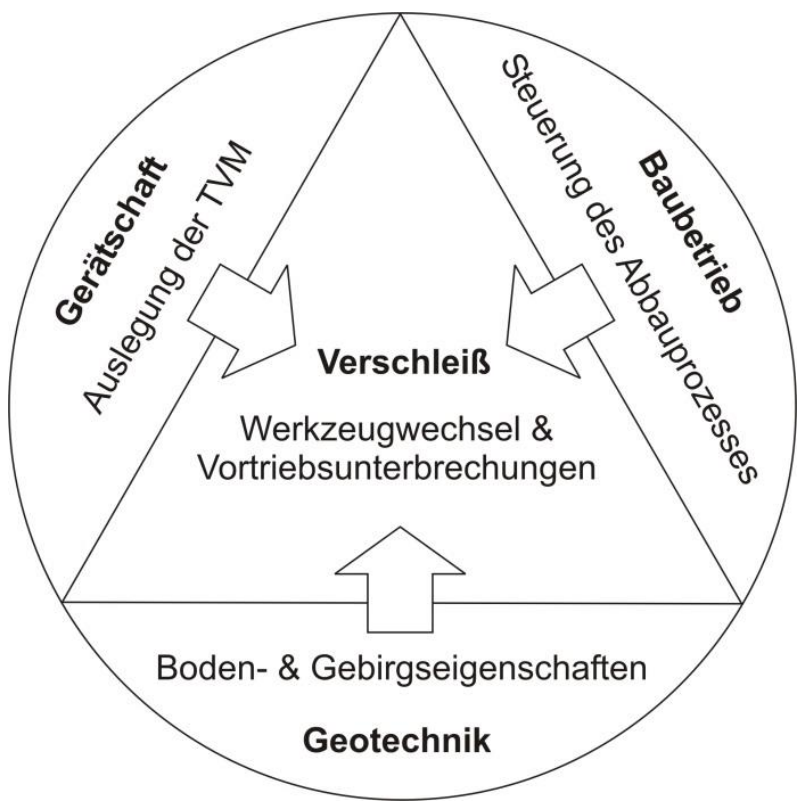

Abb. 28: Grafische Darstellung der drei Kategorien an Einflussfaktoren auf Leistung und Verschleiß im Tunnelvortrieb nach THURO (2002: 1), übertragen auf den Vortrieb mit Hydroschild TVM in Lockergesteinen.

\subsubsection{Boden- und Gebirgseigenschaften}

Einen Überblick über die geotechnischen Kennwerte, die beim Vortrieb mit Hydroschild TVM in Lockergesteinen für den Verschleiß an den Abbauwerkzeugen, der Schneidradstruktur und den Fördereinrichtungen maßgeblich sind, gibt DAUB (2010:267). Darüber hinaus machen auch NILSEN et al. (2006b: 49), WENDL et al. (2010: 3832) und WENDL (2012: 80) Angaben zu weiteren Bodenund Gebirgseigenschaften, die Einfluss auf den Abbauwerkzeugverschleiß haben. Diese Angaben basieren auf einer umfassenden Untersuchung zweier Vortriebe mit Hydroschild TVM von WENDL \& SCHOLZ (2009: 444). Hier wurde eine auf Hydroschild TVM angepassten Dokumentationsmethodik entwickelt, die von WENDL (2011: $128 \mathrm{ff}$.) und WENDL et al. (2012: $169 \mathrm{ff}$.) ausführlich beschrieben wird. Weitere Angaben zu Einflussfaktoren machen GwILDIS et al. (2011: $197 \mathrm{f}$.), WILMS (1995: $117 \mathrm{ff}$.), UetZ (1986) und DrUCKER (2011b: $222 \mathrm{ff}$.$) . Eine Zusammenfassung der ge-$ nannten Parameter ist in Tab. 4 gegeben. 
Tab. 4: Zusammenfassung der Bodeneigenschaften, Gebirgseigenschaften und Index-Tests, die Einfluss auf den Verschleiß der Abbauwerkzeuge haben können. Autoren: 1) NILSEN et al. (2006b); 2) DAUB (2010); 3) SCHOLZ \& WENDL (2010); 4) GWILDIS et al. (2011); 5) WILMS (1995); 6 6) UETZ (1986); 7) DRUCKER (2011b). $((\cdot)=$ maßgeblicher Einflussfaktor)

\begin{tabular}{|c|c|c|c|c|c|c|c|c|c|}
\hline Kennwert & Symbol & Einheit & 1) & 2) & 3) & 4) & 5) & 6) & 7) \\
\hline \multicolumn{10}{|l|}{ Bodeneigenschaften: } \\
\hline Korngrößenverteilung & - & $\%$ & & $\bullet$ & $\bullet$ & & $\bullet$ & & $\bullet$ \\
\hline Wichte unter Auftrieb & $y^{\circ}$ & $\mathrm{kN} / \mathrm{m}^{2}$ & & & $\bullet$ & & & & \\
\hline Lagerungsdichte & $\mathrm{D}$ & $\%$ & & $\bullet$ & $\bullet$ & & & & \\
\hline Kohäsion & $c^{\prime}$ & $\mathrm{kN} / \mathrm{m}^{2}$ & & $\bullet$ & $\bullet$ & & & & \\
\hline Reibungswinkel & $\varphi^{\prime}$ & $\circ$ & & $\bullet$ & $\bullet$ & & & & \\
\hline Wassergehalt & w & $\%$ & & & & & $\bullet$ & $\bullet$ & \\
\hline Plastizitätszahl & $I_{P}$ & $\%$ & & & $\bullet$ & & & & \\
\hline Konsistenzzahl & $\mathrm{I}_{\mathrm{C}}$ & - & & & $\bullet$ & & $\bullet$ & & \\
\hline Durchlässigkeitsbeiwert & $\mathrm{k}_{\mathrm{f}}$ & $\mathrm{m} / \mathrm{s}$ & & & $\bullet$ & & & & \\
\hline Äquivalenter Quarzgehalt & ÄQu & $\%$ & & $\bullet$ & $\bullet$ & $\bullet$ & $\bullet$ & & \\
\hline Mineralogie & - & - & & & $\bullet$ & & & & $\bullet$ \\
\hline Kornform (Sphärizität) & - & - & & & $\bullet$ & & & & $\bullet$ \\
\hline Kornrundungsgrad & - & - & & & $\bullet$ & & & & $\bullet$ \\
\hline Petrographie & - & - & & & $\bullet$ & & & & \\
\hline \multicolumn{10}{|l|}{ Gebirgseigenschaften: } \\
\hline Überlagerung & $\mathrm{H}_{\mathrm{TA}}$ & $\mathrm{m}$ & & & $\bullet$ & & $\bullet$ & & \\
\hline Wasserspiegel & $\mathrm{W}_{\mathrm{TA}}$ & $\mathrm{m}$ & & & $\bullet$ & & $\bullet$ & & \\
\hline Verwitterungszustand & - & - & & & $\bullet$ & & & & \\
\hline \multicolumn{10}{|l|}{ Index-Tests: } \\
\hline LCPC-Abrasivitätsindex & LAK & - & & $\bullet$ & $\bullet$ & & & & \\
\hline LCPC-Brechbarkeitsindex & LBK & - & & $\bullet$ & $\bullet$ & & & & \\
\hline Miller Number & - & - & $\bullet$ & & & $\bullet$ & & & \\
\hline SAT-Index & SAT & - & $\bullet$ & & & $\bullet$ & & & \\
\hline Cerchar Abrasivitätsindex & CAI & - & & & $\bullet$ & & & & \\
\hline TU Wien Abrasimeter & - & - & & & & & & & $\bullet$ \\
\hline
\end{tabular}

Sowohl DAUB (2010) als auch NILSEN et al. (2006a-c) geben keine quantitativen Zusammenhänge zwischen dem Verschleiß der Abbauwerkzeuge und den genannten Bodeneigenschaften, Gebirgseigenschaften und Indextests an.

WENDL (2012: 85) kann dagegen innerhalb einzelner Homogenbereiche in den untersuchten Projekten einen Zusammenhang zwischen Schneidraddrehmoment und Werkzeugverbrauch darstellen (Abb. 29). Der Werkzeugverbrauch wird hier ohne Unterscheidung der verschiedenen Werkzeugtypen auf dem Schneidrad in Stk./m angegeben. Das Schneidraddrehmoment $M_{T V M}$ ist eine Reaktionsgröße des Abbauvorgangs und wird maßgeblich durch den Schneidwiderstand des Bodens in der Ortsbrust bestimmt (Kap. 3.3.1). Der Zusammenhang gibt folglich einen deutlichen Hinweis auf den Einfluss des Schneidwiderstandes bzw. der Scherparameter des Bodens auf den Verschleiß der Abbauwerkzeuge. 


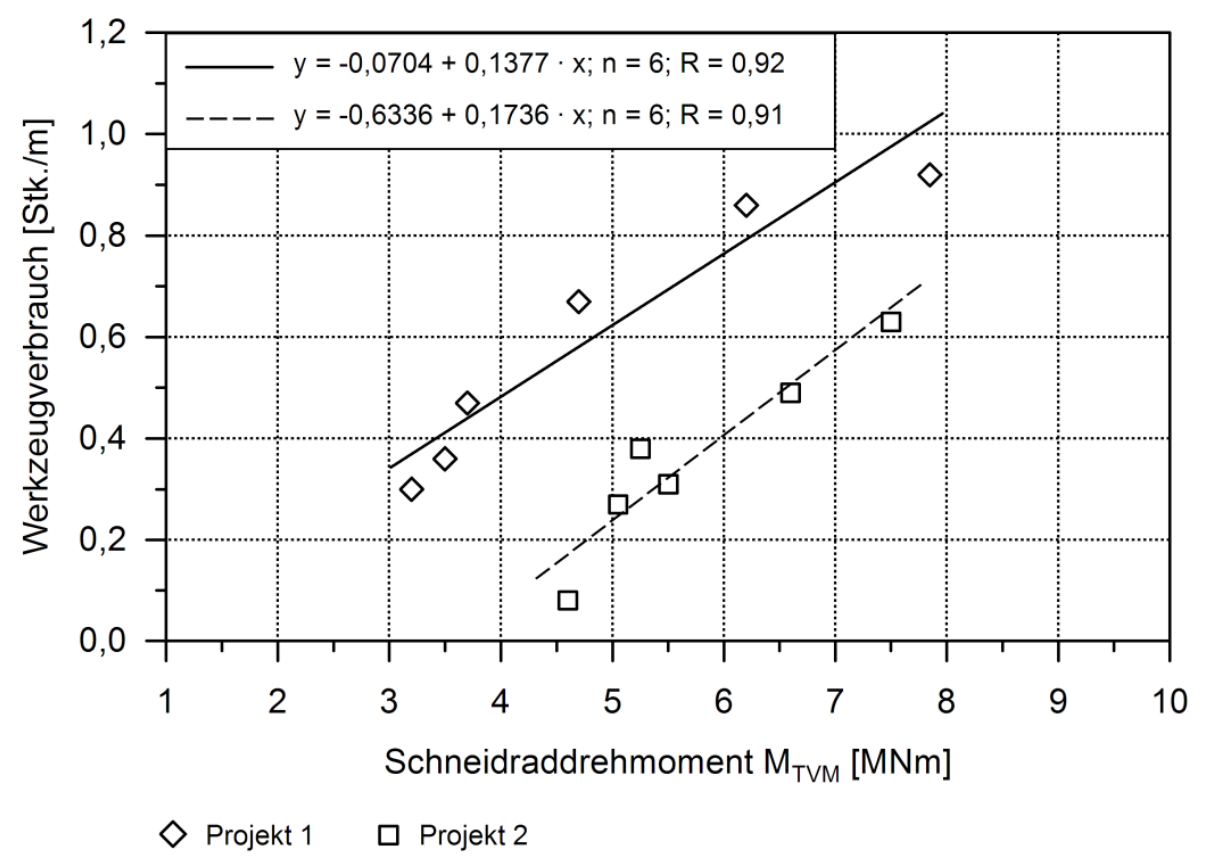

Abb. 29: Korrelation des Werkzeugverbrauchs [Stk./m] mit dem Schneidraddrehmoment $\mathrm{M}_{\mathrm{TVM}}$ [MNm]. (WENDL 2012: 85; verändert)

GwILDIS et al. (2011: 197) wählen einen ähnlichen Ansatz, wobei der Schneidwiderstand über die Spezifischen Energie $\mathrm{SE}_{T V M}\left[\mathrm{~kJ} / \mathrm{m}^{3}\right]$ einfließt und zusätzlich der SAT-Indexwert, die Miller-Number und der Äquivalente Quarzanteil als Parameter für die Bodenabrasivität berücksichtigt wird. Hier kann für den radialen Abrieb an Schneidringen mit steigendem spezifischem Energieverbrauch und mit steigenden Abrasivitätskennwerten eine Zunahme der Abriebrate dargestellt werden.

Beide Untersuchungen von WENDL (2012: 126) und GWILDIS et al. (2011: 197) lassen darüber hinaus jedoch keine Verallgemeinerung in Form eines Prognosemodells für den Verschleiß der Abbauwerkzeuge in Abhängigkeit der Bodeneigenschaften zu.

WILMS (1995: 89 ff.) entwickelt einen Laborversuch zur Verschleißintensität an Stahlprobestiften in konditionierten Bodenproben. Auf Basis dieses Versuchs kann WILMS (1995: $117 \mathrm{ff}$.) innerhalb der Versuche den Druck, die Konsistenz der Bodenprobe, den Äquivalenten Quarzanteil (ÄQu) [\%] und die mittlere Korngröße $D_{50}[\mathrm{~mm}]$ als maßgebliche Einflussfaktoren auf den Verschleiß identifizieren.

UETZ (1982) und UETZ (1986) entwickeln einen dem Verschleißtopf in dem die abrasive Wirkung von Feldspatsand und Quarzsand in Abhängigkeit der Befeuchtung bzw. des Wassergehalts untersucht wird.

Der Äquivalente Quarzgehalt ÄQu [\%] und die wesentlichen Indexversuche zur Charakterisierung der Abrasivität von Lockergesteinen (LAK \& CAI) und deren Korrelationen, sowie der SAT-Test werden nachfolgend im Detail beschrieben. Zusätzlich existiert eine Reihe weitere Indexversuche, die ebenfalls vor dem Hintergrund der Abrasivität von Lockergesteinen entwickelt wurden, wie z.B. die MillerNumber. Diese finden weit weniger Anwendung als LAK, CAI und SAT und sind im Anschluss in (Kap. 4.3.1.5) zusammengefasst.

\subsubsection{1 Äquivalenter Quarzgehalt}

Der Äquivalente Quarzgehalt (ÄQu) [\%] ist eine Methode zur Beschreibung des Gehalts an verschleißscharfen Mineralen in einer Gesteinsprobe. Die Methode beruht auf der absoluten Härtebestimmung von Mineralen nach RosiwaL (1896: 476) und RosIWAL (1916: 118). Dabei wird mit einer definierten Menge Schleifpulver solange Abrieb am Mineralprüfkörper erzeugt, bis das Schleifpulver aufgebraucht ist und kein Gewichtsverlust mehr feststellbar ist. Der Gewichtsverlust des Mineralprüfkörpers ist charakteristisch für die absolute Härte. Die absolute Härteskala wird mit dem Mineral Quarz 
normiert. Quarz wird dabei der Wert 100 auf der Skala zugeordnet. Heute liegen für nahezu alle Minerale entsprechende Werte für die Schleifhärte HRW nach RosIWAL (1916: 118) vor. Einen Überblick über die Schleifhärte HRW der häufigsten gesteinsbildenden Minerale gibt PLINNINGER (2002: 46) (Tab. 5).

Tab. 5: Zusammenfassung der Mineraldichte, Schleifhärte nach RosıWAL, Ritzhärte nach MOHS und Härte nach VICKERS für die häufigsten gesteinsbildenden Minerale, verändert nach PLINNINGER (2002: 46), verändert.

\begin{tabular}{|c|c|c|c|c|}
\hline Mineral & $\begin{array}{c}\text { Mineraldichte } \\
\rho_{m}\left[\mathrm{~g} / \mathrm{cm}^{3}\right]\end{array}$ & $\begin{array}{c}\text { Schleifhärte HRW } \\
\text { n. RoSIWAL }\end{array}$ & $\begin{array}{c}\text { Ritzhärte HMO } \\
\text { n. MOHS }\end{array}$ & $\begin{array}{l}\text { Härte HVI } \\
\text { n. VICKERS }\end{array}$ \\
\hline Anhydrit & $2,90-3,20$ & - & $3,0-3,5$ & 980 \\
\hline Apatit & 3,16 & $3,3-5,5$ & 5,0 & $4450-5950$ \\
\hline Biotit & 3,01 & $1,8-5,6$ & $2,5-3,0$ & $880-1080$ \\
\hline Calcit & 2,72 & $1,9-3,9$ & 3,0 & 1215 \\
\hline Diamant & 3,50 & 117000 & 10,0 & - \\
\hline Dolomit & 2,85 & - & $3,5-4,0$ & $2140-5640$ \\
\hline Epidot & 3,39 & $54-69$ & $6,0-7,0$ & 6670 \\
\hline Fluorit & 3,18 & $2,8-4,4$ & 4,0 & 1295 \\
\hline Gips & 2,32 & $0,3-0,6$ & $1,5-2,0$ & $305-470$ \\
\hline Granat & $3,80-4,20$ & $203-210$ & $6,5-7,5$ & $10400-12410$ \\
\hline Hämatit & 4,90 & 25 & $5,5-6,5$ & 6915 \\
\hline Hornblende & 3,22 & $18-30$ & $5,0-6,0$ & $5990-7160$ \\
\hline Korund & 3,95 & $326-1765$ & 9,0 & $17950-25485$ \\
\hline Magnetit & 5,15 & 34 & 5,5 & 5700 \\
\hline Mikroklin & 2,55 & - & 6,0 & 4340 \\
\hline Muskovit & 2,85 & $1,5-6,1$ & $2,0-3,0$ & 835 \\
\hline Oligoklas & 2,64 & $23-38$ & 6,0 & 7300 \\
\hline Orthoklas & 2,57 & $17-40$ & 6,0 & $6300-9150$ \\
\hline Plagioklas & 2,50 & $3-27$ & 5,0 & - \\
\hline Pyrit & 5,10 & 56 & $6,0-6,5$ & $9515-14060$ \\
\hline Pyroxene & $3,20-3,50$ & $22-65$ & $5,0-6,0$ & $5885-7850$ \\
\hline Quarz & 2,65 & $74-127$ & 7,0 & $8830-13320$ \\
\hline Titanit & 3,48 & 48 & $5,0-5,5$ & $3965-6670$ \\
\hline Topas & 3,56 & $87-148$ & 8,0 & 18150 \\
\hline Turmalin & 3,15 & 102 & $7,0-7,5$ & $11645-13520$ \\
\hline Zirkon & 4,45 & 367 & 7,5 & $10928-14765$ \\
\hline
\end{tabular}


Zur Ermittlung des Äquivalenten Quarzgehalts ÄQu [\%] wird nach THURO (2002: 61) zunächst der quantitative Mineralbestand des Gesteins bestimmt. Dieser kann einerseits an Dünnschliffen im Polarisationsmikroskop mithilfe des Pointcounter-Verfahrens ermittelt werden, jedoch auch an pulverisierten Gesteinsproben durch Röntgendiffraktometrie. Der Äquivalente Quarzgehalt ergibt sich dann aus dem prozentualen Anteil der verschiedenen Minerale $a_{m}[\%]$ und deren Schleifhärte HRW:

$$
\ddot{A} Q u=\sum_{1}^{i} a_{m i} \cdot H R W
$$

Bei der Anwendung des Äquivalenten Quarzgehalts auf Lockergesteine besteht die Herausforderung nach THURO \& KÄSLING (2009a: 182) hauptsächlich darin, unabhängig vom Verfahren zur Bestimmung des quantitativen Mineralbestandes eine repräsentative Bodenproben mit ausreichendem Volumen zu entnehmen, da die eingesetzte Probenmenge jeweils sehr gering ist.

\subsubsection{LCPC-Test}

Der LCPC-Test ist nach dem Laboratoire Central des Ponts et Chaussées (www-10) benannt, wo dieser Test für Festgesteinskörnungen entwickelt wurde. Die Normung der Testeinrichtung erfolgte 1990 durch die Association Francaise de Normalisation (AFNOR) unter der Norm Nr. NF P 18-579. Die erste Einführung des Tests für Anwendungen im Hinblick auf den Verschleiß von Abbauwerkzeugen im Festgestein erfolgte durch BüCHI et al. (1995: 38). THURO et al. (2006: 284 ff.) und THURO et al. (2007: $829 \mathrm{ff}$.) legen eine erste detailliierte Beschreibung der Testprozedur für die Anwendung des Test auf Lockergesteine im Hinblick auf Schildvortriebe und andere Bohrvorgänge in Lockergesteinen vor.

Demnach wird für den LCPC-Test eine Probenmasse $m_{P}$ von $500 \mathrm{~g}+/-2 \mathrm{~g}$ einer Bodenprobe in getrocknetem Zustand in einen Stahlzylinder eingefüllt. Darin wird ein Stahlflügel für die Dauer von 5 Minuten durch einen Elektromotor mit $4.500 \mathrm{~min}^{-1}$ Umdrehungen je Minute in Rotation gesetzt. Beim Rotationsvorgang wird durch die Bodenprobe einerseits Abrieb am Stahlflügel erzeugt, andererseits werden durch den Stahlflügel einzelne Bodenkomponenten gebrochen.

Während das Verhältnis der Masse an Feinmaterial $<1,6 \mathrm{~mm}$ Korndurchmesser vor und nach dem Versuch als $M a ß$ für die Brechbarkeit der eingesetzten Bodenprobe herangezogen werden kann, ist der Massenverlust des Stahlflügels während dem Versuch kennzeichnend für die Abrasivität der Bodenprobe. Der Stahlflügel wird dazu vor und nach dem Versuch gewogen und der daraus resultierende Massenverlust in Bezug zur Probenmenge gesetzt. Daraus ergibt sich der LCPC-Abrasivitätsindex LAK $[\mathrm{g} / \mathrm{t}]$ :

$$
\mathrm{LAK}=\frac{\left(\mathrm{m}_{\mathrm{FA}}-\mathrm{m}_{\mathrm{FV}}\right) \cdot 1.000 \cdot 1.000}{\mathrm{~m}_{\mathrm{P}}}
$$

Dabei ist $m_{F A}[g]$ die Ausgangsmasse des Stahlflügels, $m_{F v}[g]$ die Masse des Stahlflügels nach dem Versuch und $m_{P}[g]$ die Masse der eingesetzten Probe.

Bereits die Untersuchungen von THURO et al. (2006: 287) zeigen, dass der LAK neben dem Gehalt an verschleißscharfen Mineralen in der Bodenprobe auch stark von der Korngröße und der Kornform der eingesetzten Bodenprobe abhängt. Diese Abhängigkeit wird von THURO \& KÄSLING (2009b: 141) noch deutlicher dargestellt (Abb. 30).

Daraus ergibt sich einerseits ein deutlicher Hinweis auf den grundsätzlichen Einfluss der Korngrößenverteilung und Kornform auf Verschleißprozesse bei Bohrvorgängen in Lockergesteinen. Andererseits weisen THURO et al. (2006: 284) jedoch auch darauf hin, dass aufgrund des Abstandes zwischen Stahlflügel und Zylinderwand das Größtkorn in der Bodenprobe 6,3 mm (Feinkies) nicht überschreiten darf. Größere Komponenten müssen entweder aus der Bodenprobe entfernt werden oder durch Bre- 
chen auf eine Korngröße $<6,3 \mathrm{~mm}$ reduziert werden. In beiden Fällen wird die Korngrößenverteilung der Bodenprobe maßgeblich verändert. Beim Brechen der Bodenkomponenten $>6,3 \mathrm{~mm}$ wird zusätzlich auch die Kornform verändert. Damit wird in Summe die Abrasivitätscharakteristik der Bodenprobe für das Testverfahren verändert. Insbesondere in einem Korngrößenbereich, in dem Hydroschild TVM häufig Anwendung finden (Kap. 1.3.1). Der LCPC-Test erlaubt dennoch die Aufstellung einer Abrasivitätsskala, die nach THURO \& KÄSLING (2009a: 184) in Tab. 6 zusammengefasst ist.

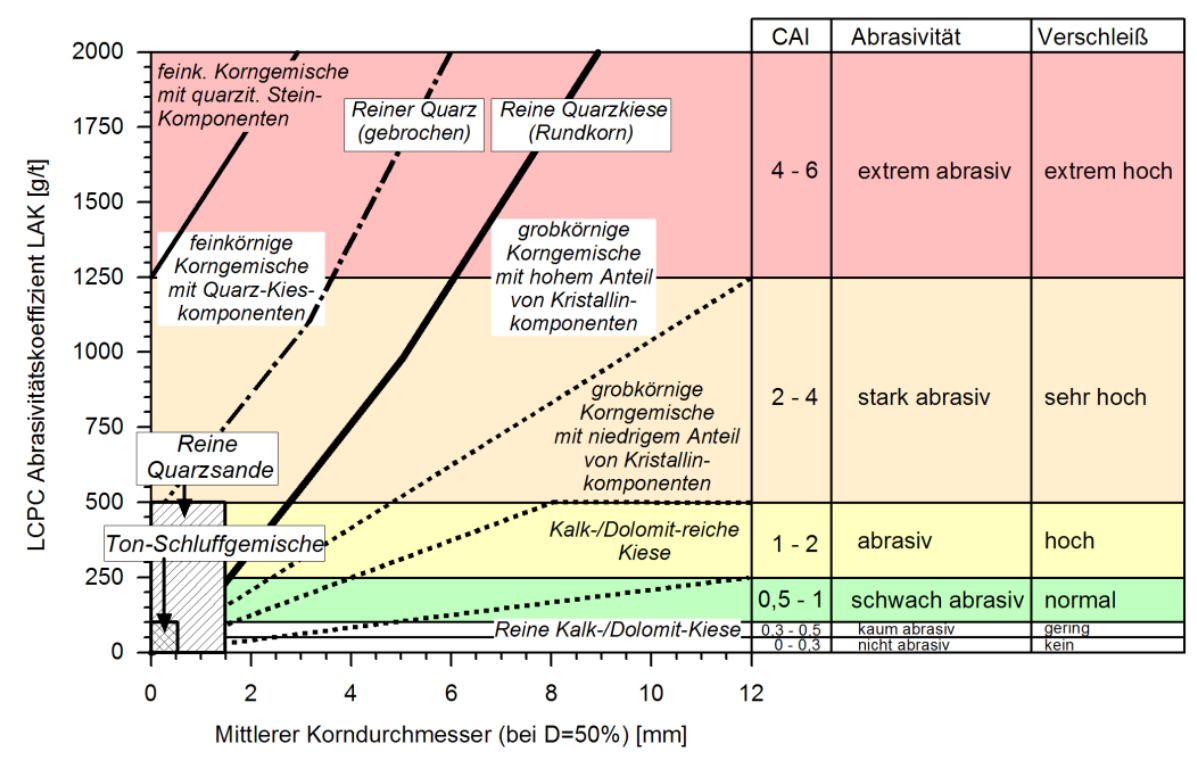

Abb. 30: Abhängigkeit des LCPC Abrasivitätskoeffizienten LAK [g/t] von der Korngröße $D_{50}[\mathrm{~mm}]$ für verschiedene Lockergesteine. Zusätzlich sind die Abrasivitätsklassifikation, das zugehörige Verschleißpotential und der entsprechende CAI-Wert eingetragen. (THURO \& KÄSLING 2009a: 185)

Tab. 6: Abrasivitätsklassifikation mit dem LCPC-Test (LAK) für Lockergesteine nach THURO \& KÄSLING (2009a: 184), verändert.

\begin{tabular}{|c|c|c|c|c|}
\hline $\begin{array}{l}\text { LAK } \\
{[g / t]}\end{array}$ & $\begin{array}{l}\text { Abrasivitäts- } \\
\text { klassifikation }\end{array}$ & $\begin{array}{l}\text { Verschleiß- } \\
\text { potential }\end{array}$ & $\begin{array}{l}\text { Bsp. für die Petrographie der Kom- } \\
\text { ponenten in Lockergesteinen }\end{array}$ & $\begin{array}{l}\text { Beispiele für } \\
\text { Lockergesteine }\end{array}$ \\
\hline $0-50$ & Nicht abrasiv & Sehr niedrig & Holz, Torf, organisches Material & \multirow{2}{*}{$\begin{array}{l}\text { Ton, Schluff und } \\
\text { Kalk- und Dolomit- } \\
\text { sande }\end{array}$} \\
\hline $50-100$ & Kaum abrasiv & Niedrig & Ton- \& Schluffstein, Mergel & \\
\hline $100-250$ & Schwach abrasiv & Moderat & Tonschiefer, Kalk, Dolomit, Marmor & $\begin{array}{l}\text { Kalk- und Dolomit- } \\
\text { reiche Kiese }\end{array}$ \\
\hline $250-500$ & Abrasiv & Hoch & $\begin{array}{l}\text { Verkieselter Kalk, Dolomit, Sandstein, } \\
\text { Phyllit }\end{array}$ & Quarzreiche Sande \\
\hline $500-1250$ & Stark abrasiv & Sehr hoch & $\begin{array}{l}\text { Quarzsandstein, Porphyr, Quarzphyl- } \\
\text { lit, Andesit, Basalt, Glimmerschiefer }\end{array}$ & \multirow{2}{*}{ Quarzreiche Kiese } \\
\hline $1250-2000$ & Extrem abrasiv & Extrem hoch & $\begin{array}{l}\text { (Gang-) Quarz, Quarzit, Granit, Diorit, } \\
\text { Syenit, Gneis, Eklogit, Amphibolit }\end{array}$ & \\
\hline
\end{tabular}

DRUCKER (2011b: 222 ff.) beschreibt einen modifizierten LCPC-Test. Dieser ist in Kap. 4.3.1.5 näher erläutert. 


\subsubsection{CERCHAR Abrasivitätsindex}

Der Cerchar Abrasivitätsindex (CAI) ist nach dem Centre d’Etudes de Charbonnages de France benannt. Der Indextest geht zurück auf die Entwicklung durch VALANTIN (1973), die erste Prüfvorschrift wurde durch CERCHAR (1986) veröffentlicht. Durch WEST (1989) erfolgt eine Überarbeitung des Testgeräts und der Prüfvorschrift. Darüber hinaus ist der Test in der französischen Norm Nr. NF P94-43001 durch AFNOR (2000) beschrieben.

Der Test wurde ursprünglich zur Bestimmung der Abrasivität von Festgesteinen entwickelt. Nach KÄSLING et al. (2007: 229) wird dazu mithilfe eines Testgerätes ein Stahlstift mit definierter Spitze und Härte über eine Prüfstrecke von $10 \mathrm{~mm}$ über die bruchrauhe Oberfläche der Gesteinsprobe geführt. Die Gesteinsprobe wird dazu in einen Schraubstock eingespannt. Der Prüfstift wird zusätzlich mit einem Gewicht von 7,0 kg belastet. Die Prüfstrecke von $10 \mathrm{~mm}$ wird in ca. 10 Sekunden zurückgelegt.

Innerhalb der Prüfstrecke findet Abrieb an der Spitze des Prüfstiftes statt. Zur Bestimmung des CAIWertes wird mithilfe eines Auflichtmikroskops der Durchmesser $d_{v}[\mathrm{~mm}]$ der abgeriebenen Prüfstiftspitze nach dem Versuch bestimmt. Der CAI-Wert ergibt sich dann aus Gl. 16:

$$
\mathrm{CAI}=\mathrm{d}_{\mathrm{V}} \cdot 10
$$

Aufgrund der geringen Länge der Prüfstrecke ergeben sich bei grobkörnigen Festgesteinen, aber auch bei inhomogenen und anisotropen Festgesteinen, zum Teil Probleme bei der Festlegung repräsentativer Prüfstrecken. Diese und andere Probleme im Zusammenhang mit dem Testverfahren, die wesentlichen geologischen Einflussfaktoren auf den CAI-Wert und die daraus resultierende Abrasivitätsklassifikation (Tab. 7) werden hauptsächlich durch AL-AMEEN \& WALLER (1994), PLINNINGER et al. (2004), KÄSLING et al. (2007: $230 \mathrm{ff}$.), THURO \& KÄSLING (2009a: $181 \mathrm{f}$.), THURO \& KÄSLING (2009b: 139), KÄSLING \& THURO (2010a: 1974), KÄSLING \& THURO (2010b: 234) und KÄSLING \& THURO (2010c: 427 f.) bearbeitet. Die Abrasivitätsklassifikation des CAI-Wertes ist aufgrund der guten Korrelation zum LAK (Kap. 4.3.1.5) kompatibel mit der Klassifikation in Tab. 6.

Tab. 7: Abrasivitätsklassifikation mit dem LCPC-Test (LAK) \& Cerchar Abrasivitätsindex (CAI), verändert nach THURO \& KÄSLING (2009a: 184).

\begin{tabular}{|c|c|c|c|}
\hline $\begin{array}{c}\text { LAK } \\
{[\mathbf{g} / \mathbf{t}]}\end{array}$ & $\begin{array}{c}\text { CAI } \\
{[-]}\end{array}$ & $\begin{array}{c}\text { Abrasivitäts- } \\
\text { klassifikation }\end{array}$ & $\begin{array}{c}\text { Verschleiß- } \\
\text { potential }\end{array}$ \\
\hline $0-50$ & $0-0,3$ & Nicht abrasiv & Sehr niedrig \\
\hline $50-100$ & $0,3-0,5$ & Kaum abrasiv & Niedrig \\
\hline $100-250$ & $0,5-1,0$ & Schwach abrasiv & Moderat \\
\hline $250-500$ & $1,0-2,0$ & Abrasiv & Hoch \\
\hline $500-1250$ & $2,0-4,0$ & Stark abrasiv & Sehr hoch \\
\hline $1250-2000$ & $4,0-6,0$ & Extrem abrasiv & Extrem hoch \\
\hline
\end{tabular}

Darüber hinaus ist der CAI in der Verschleißprognose für Tunnelvortriebe in Festgesteinen mit TVM weit verbreitet, weil sich sehr gute empirische Korrelationen zwischen dem CAI und der Abriebrate an Schneidringen bzw. der Standzeit von Schneidrollen herstellen lassen. Die Erarbeitung der Zusammenhänge und Modelle erfolgt überwiegend durch BüCHI et al. (1995: 41), RostAMI (1997: 204 ff.), Rostami (2005: 43 ff.), Rostaml et al. (2005: 739 ff.) und Frenzel (2010a: 46 ff.).

Eine schlüssige Methodik zur Anwendung des Tests auf Lockergesteine fehlt bisher vollständig. Dennoch wurde in einigen Projekten der CAI-Wert an einzelnen Bodenkomponenten bestimmt, die für die Versuchsdurchführung eine Größe $>10 \mathrm{~mm}$ aufweisen müssen. 


\subsubsection{NTNU Soil Abrasion Test}

Der Soil Abrasion Test (SAT) wurde an der NTNU (Norwegian University of Sience and Technology) entwickelt. Grundlage dazu ist eine Testmethode für Festgesteine zur Verschleißprognose für Bohrkronen (Schlagbohren) und TVM-Vortriebe in Festgesteinen. Bei der Anwendung dieser Methode auf Lockergesteine wurden nach NILSEN et al. (2006c: 44) und NILSEN et al. (2007: 110 f.) nur geringe Änderungen an der Versuchseinrichtung und Prüfprozedur für den SAT-Test vorgenommen.

Demnach wird eine getrocknete Bodenprobe mit einer maximalen Korngröße von $4 \mathrm{~mm}$ mit einem konstanten Massenfluss von ca. $80 \mathrm{~g} / \mathrm{min}$ und einer Spurbreite von $30 \mathrm{~mm}$ auf eine rotierende Stahlscheibe gegeben. Über der Stahlscheibe ist mit sehr geringem Abstand ein Prüfkörper aus hochlegiertem Stahl angebracht. Durch die Rotation der Stahlscheibe wird das Probenmaterial unter den Stahlprüfkörper gezogen und zerkleinert. Dabei entsteht Abrieb am Prüfkörper, der nach Abschluss eines Testdurchgangs als Gewichtsverlust [mg] gemessen dem SAT-Wert entspricht.

Eine ähnliche Abrasivitätsklassifikation wie für die zuvor beschriebenen Indexwerte LAK und CAI existiert für den SAT aufgrund einer relativ geringen Anzahl an verfügbaren Versuchen nicht. Anhand erster Versuche stellen NILSEN et al. (2007: 113) mit dem SAT jedoch ein Maximum an Abrasivität bei Sanden fest. Tone, Schluffe und Kiese weisen demnach eine deutlich geringere Abrasivität auf. Dieses Ergebnis widerspricht deutlich allen bisher beschriebenen Ergebnissen. Zu den Versuchen von NILSEN et al. (2006c: 45) und NILSEN et al. (2007: 113) fehlen jedoch jegliche Angaben zum quantitativen Mineralbestand der Proben. Zudem bleibt unklar, wie die Kiesprobe $(2-63 \mathrm{~mm})$ bei einer versuchsbedingt maximalen Korngröße von $4 \mathrm{~mm}$ getestet wurde, wobei die Prüfprozedur kein vorlaufendes Brechen einzelner Komponenten wie beim LCPC-Test erlaubt. Der angegebene Vergleich der verschiedenen Korngrößen ist also kritisch zu betrachten.

JAKOBSEN et al. (2013a: $109 \mathrm{ff}$.) fassen darauf aufbauend neuere Ergebnisse zum SAT-Test zusammen, die einen besseren Überblick über verschiedene Einflussfaktoren ermöglichen. Die vorgestellten Ergebnisse zeigen darüber hinaus die Notwendigkeit für weitere Untersuchungen des Einflusses der Lagerungsdichte, des Wassergehalts und verschiedener Additive auf. Diese werden von JAKOBSEN et al. (2012: 42 f.) und JAKOBSEN et al. (2013b: 399 f.) in einem weiteren neuen Testverfahren zusammengefasst (Kap. 4.3.1.5).

\subsubsection{Weitere Kennwertversuche}

Neben den bisher im Detail beschriebenen Kennwertversuchen zur Klassifizierung der Abrasivität von Lockergesteinen existieren noch eine Anzahl weiterer Versuche mit ähnlichem Charakter, die jedoch bei TVM-Vortrieben nur sehr untergeordnet Anwendung finden. Sie sind im Folgenden aus Gründen der Vollständigkeit zusammengefasst.

SCHIMATZECK \& KNATZ (1976: 116) entwickeln für Festgesteine aufbauend auf ihre Arbeit (SCHIMATZECK \& KNATZ 1970: 274) den sogenannten F-Koeffizienten, der mit dem Verschleiß von Rundschaftmeißeln von Teilschnittmaschinen und Streckenvortriebsmaschinen im Kohlebergbau korreliert. Der FKoeffizient ergibt sich aus dem Volumenanteil $\mathrm{a}_{\mathrm{qz}}$ [\%] von Quarz im Gestein, der mittleren Korngröße der Quarzkörner $d_{\mathrm{qz}}[\mathrm{mm}]$ und der Zugfestigkeit des Gesteins SPZ [kN/m²] (ISRM 1978) mit Gl. 17:

$$
\mathrm{F}=\mathrm{a}_{\mathrm{qz}} \cdot \mathrm{d}_{\mathrm{qz}} \cdot \mathrm{SPZ}
$$

Der F-Koeffizient nach SCHIMATZECK \& KNATZ (1976: 116) ist aufgrund der Einbeziehung der Zugfestigkeit des Gesteins nicht auf Lockergesteine übertragbar.

WILMS (1997: 123 ff.) entwickelt auf Basis eines Laborversuches zur Verschleißintensität an Stahlprobestiften in konditionierten Bodenproben den Verschleißindex $V_{\text {EPB }}$ für die Beschreibung der Abrasivität von Böden für Erddruckschilde. Dieser errechnet sich aus Gl. 18:

$$
\mathrm{V}_{\mathrm{EPB}}=\mathrm{k}_{\mathrm{EPB}} \cdot \ddot{\mathrm{AQ}} \mathrm{u} \cdot \mathrm{D}_{50}^{3}
$$


In Gl. 18 ist $\mathrm{K}_{\mathrm{EPB}}$ ein dimensionsloser Koeffizient mit dem Wert 1.000, ÄQu [\%] der Äquivalente Quarzgehalt und $\mathrm{D}_{50}[\mathrm{~mm}]$ der mittlere Korndurchmesser. Der Verschleißindex $\mathrm{V}_{\mathrm{EPB}}$ korreliert nach den Ergebnissen von WILMS (1997: 126) sehr gut mit der in den Laborversuchen ermittelten Verschleißintensität an den Stahlprobestiften. Dieser Zusammenhang ist zwar aufgrund der systematischen Unterschiede zum Vortrieb mit Hydroschild TVM nicht auf die vorliegende Fragestellung übertragbar. WILMS (1997: 127) kann auch keine direkte Korrelation des Verschleißindexes $V_{\text {EPB }}$ mit dem tatsächlichen Verschleiß der Abbauwerkzeuge in den untersuchten Projekten herstellen. Der Zusammenhang in Gl. 18 zeigt aber dennoch, dass für die aussagekräftige Beschreibung der Bodenabrasivität und deren Korrelation mit dem tatsächlich zu erwartenden Verschleiß der Abbauwerkzeuge auch eine Kombination verschiedener geotechnischer Kennwerte notwendig sein kann.

NILSEN (2006b: 49) fasst als weitere Indextests den Los Angeles Test und Nordic Ball Mill Test zusammen. Das Testkonzept ist bei beiden Indexversuchen sehr ähnlich. Sie wurden entwickelt, um die Abriebfestigkeit von Gesteinskörnungen für Straßenbauanwendungen zu überprüfen. Dazu wird eine Probe der entsprechenden Gesteinskörnung die Korngrößenverteilung bestimmt und anschließend zusammen mit einer definierten Anzahl an Stahlkugeln in eine Trommel gegeben. Die Trommel wird verschlossen und für eine bestimmte Zeit mit festgelegter Drehzahl rotiert. Im Anschluss an den Versuch wird die Korngrößenverteilung erneut bestimmt und der zusätzlich entstandene Feinanteil ins Verhältnis zum Gesamtgewicht der Probe gesetzt. Im Hinblick auf die Beurteilung der Abrasivität von Gesteinskörnungen oder Böden besteht die Möglichkeit den Test in umgekehrter Weise zu nutzen und den Abrieb an den Stahlkugeln durch die Bodenprobe zu bestimmen. Eine entsprechende Testvorschrift und entsprechende Klassifizierung existiert jedoch nicht.

Weiterhin nennt NILSEN (2006b: 49) den Abrasionstest nach DORRY. Dieser hat einen ähnlichen Hintergrund wie die beiden zuvor genannten Versuche. Der Versuch ist in DIN EN 1097-8: 2009 genormt. Die Abriebfestigkeit der Probe wird hier jedoch durch eine rotierende Stahlscheibe getestet, wobei die Abriebfestigkeit anhand des Volumenverlustes der Probe bestimmt wird.

Der vierte von NILSEN (2006b: 49) angeführte Indextest ist die sogenannte Miller-Zahl, die nach der US-Norm ASTM G75-01 bestimmt wird. Der Indexversuch wurde für tiefe Vertikalbohrungen entwickelt, bei denen ähnliche Stützflüssigkeiten zum Einsatz kommen, wie bei Hydroschild TVM. Zur Bestimmung der Miller-Zahl wird ein Stahlblock mit standardisierten Eigenschaften in einem Trog in die zu testende Stützflüssigkeit gegeben, mit 2,3 kg Gewicht beaufschlagt und für 6 Stunden mit einem Hub von $200 \mathrm{~mm}$ linear hin und her bewegt. Die Stützflüssigkeit kann dabei beliebig gewählt werden und enthält im Regelfall den bereits gelösten Boden. Der Gewichtsverlust des Stahlblocks entspricht der Miller-Zahl und gilt als Indexwert für die Abrasivität der getesteten Stützflüssigkeit.

GHARAHBAGH et al. (2011: 607 ff.) beschrieben einen in der Entwicklung befindlichen Indexversuch zur Beschreibung und Klassifizierung der Abrasivität von Lockergesteinen. Hierbei wird ähnlich wie beim LCPC-Test eine Bodenprobe in einen Stahlzylinder gegeben. Anschließend wird ein Stahlflügel in der Bodenprobe in Rotation versetzt. Im Unterschied zum LCPC-Test wird dabei die Drehzahl mit 30 - 60 $\mathrm{min}^{-1}$ deutlich geringer gewählt, um den tatsächlichen Verhältnissen in der Abbaukammer näher zu kommen. Zusätzlich kann die Bodenprobe beliebig konditioniert und bis zu 10 bar mit Druck beaufschlagt werden. Der Stahlflügel selbst besitzt die Form einer Schiffsschraube mit 3 Blättern. Diese sind mit einem Winkel von $10-20^{\circ}$ angestellt. Der Indexwert für die Abrasivität von Lockergesteinen auf Basis des Versuchs wird von GHARAHBAGH et al. (2011) nicht beschrieben. Geplant ist jedoch, dass dieser sowohl den Gewichtsverlust des Stahlflügels, als auch das Drehmoment und die senkrecht dazu am Flügel entstehende Kraftkomponente berücksichtigt.

DRUCKER (2011a: 690 f.) argumentiert auf Basis einer detaillierten Analyse, dass der LCPC-Test für die Anwendung auf Lockergesteine vor allem aufgrund der hohen Drehzahl des Stahlflügels, der geringen Probenmenge und der kurzen Versuchsdauer nicht zur zuverlässigen Beschreibung der Gesamtabrasivität geeignet ist. DRUCKER (2011b: 221) schlägt deshalb eine Modifizierung des LCPCTests vor. Die Versuchseinrichtung weist einen deutlich größeren Stahltopf mit $25 \mathrm{~cm}$ Durchmesser auf, der bis zu $10 \mathrm{~kg}$ Probenmaterial fassen kann und eine Maximalkorngröße in der Bodenprobe von $32 \mathrm{~mm}$ zulässt. Die Drehzahl des Stahlflügels in der Probenmasse wird auf $100 \mathrm{~min}^{-1}$ begrenzt, sodass eine erhöhte Versuchsdauer notwendig wird. Auf die Bodenprobe kann durch Gewichte eine zusätzliche Auflast aufgebracht werden. 
JACOBSEN et al. (2013b: $399 \mathrm{ff}$.) entwickeln nach dem Review der Ergebnisse zum SAT-Test (Kap. 4.3.1.4) durch JAKOBSEN et al. (2013a) ein weiteres Testverfahren in Form eines Verschleißtopfes, um den Einfluss der Lagerungsdichte, des Wassergehalts und verschiedener Additive auf den Abrieb an einem Testflügel zu untersuchen.

BARZEGARI et al. (2013: 64 ff.) entwickeln ebenfalls ein eigenständiges Testgerät, das den Einfluss des Äquivalenten Quarzgehalts auf die Abrasivität des Bodens verdeutlicht. Bisher ist jedoch kein klarer Bezug der Tests zu Hydroschild TVM erkennbar.

\subsubsection{Korrelationen der Kennwerte}

Aufbauend auf die beschriebenen Kennwertversuche stellen THURO \& KäSLING (2009a: 183) und THURO \& KÄSLING (2009b: 140) empirische Korrelationen zwischen Äquivalentem Quarzgehalt (ÄQu), LCPC-Test (LAK) und Cerchar Abrasivitätsindex (CAI) her.

Demnach lassen sich zwischen ÄQu und CAI (Abb. 31), sowie zwischen CAI und LAK (Abb. 32) jeweils lineare Korrelationen mit hoher bis sehr hoher Güte herstellen. Eine entsprechende Korrelation zwischen ÄQu und LAK existiert ebenfalls, weist bei ähnlich hoher Güte jedoch leicht exponentiellen Charakter auf (Abb. 33). Eine Korrelation der Kennwertgruppe von ÄQu, CAI und LAK zum ähnlichen Soil Abrasivity Test (SAT) (Kap. 4.3.1.4) liegt bisher nicht vor.

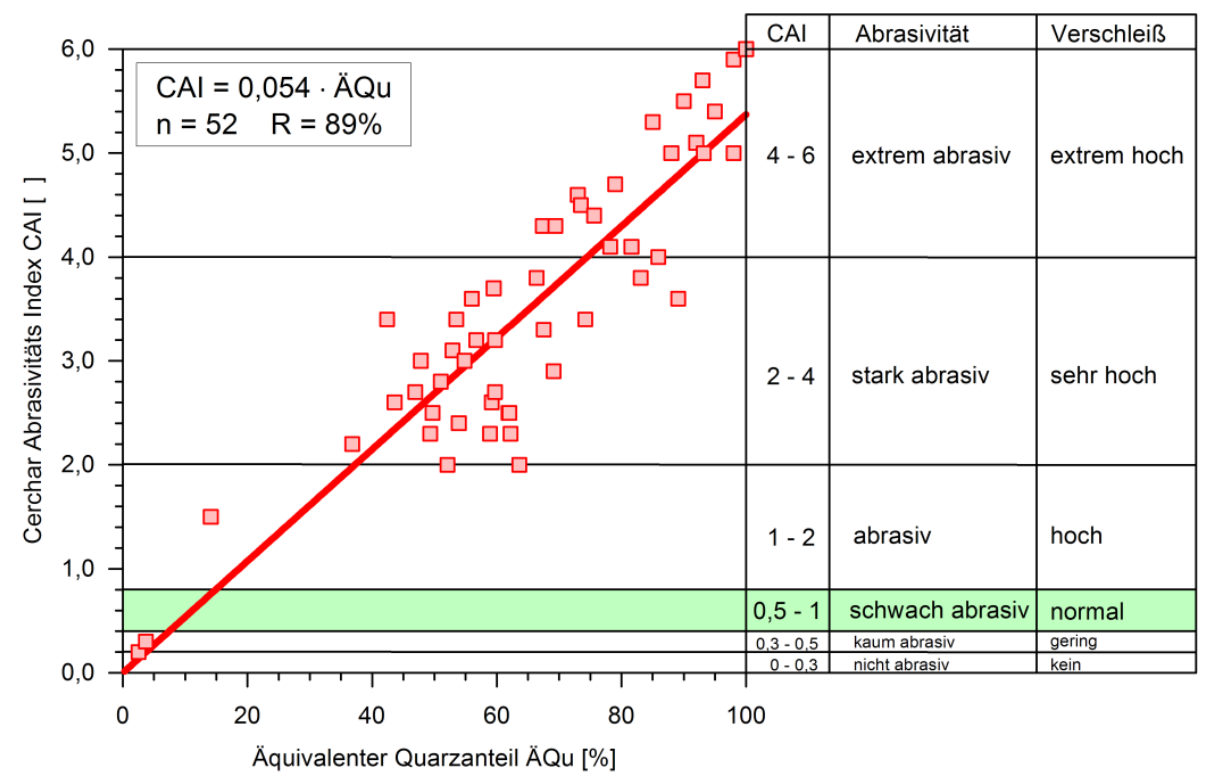

Abb. 31: Korrelation des Cerchar Abrasivitäts Index CAI mit dem Äquivalenten Quarzgehalt ÄQu [\%]. Zusätzlich sind die Abrasivitätsklassifikation, das zugehörige Verschleißpotential und der entsprechende CAI-Wert eingetragen. (THURO \& KÄSLING 2009a: 183; verändert). 


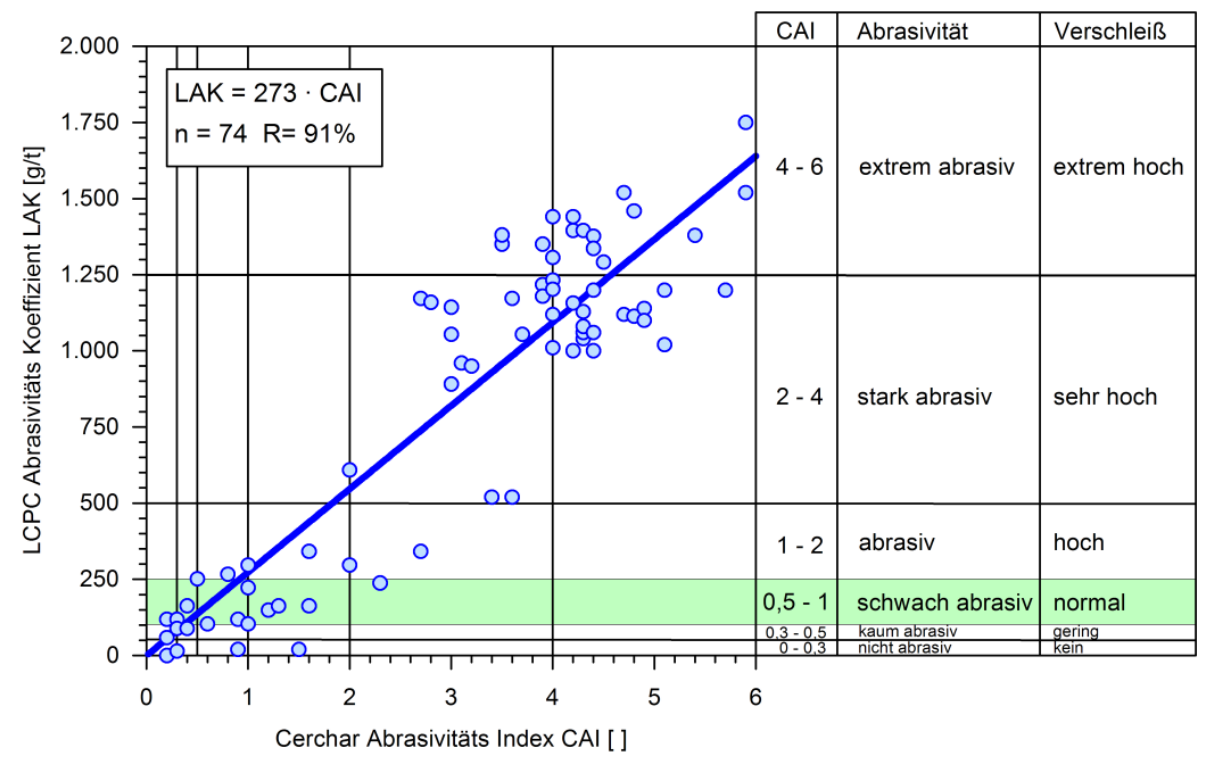

Abb. 32: Korrelation des LCPC Abrasivitätskoeffizienten (LAK) mit dem Cerchar Abrasivitäts Index (CAI). Zusätzlich sind die Abrasivitätsklassifikation, das zugehörige Verschleißpotential und der entsprechende CAI-Wert eingetragen. (THURO \& KÄSLING 2009a: 183, verändert).

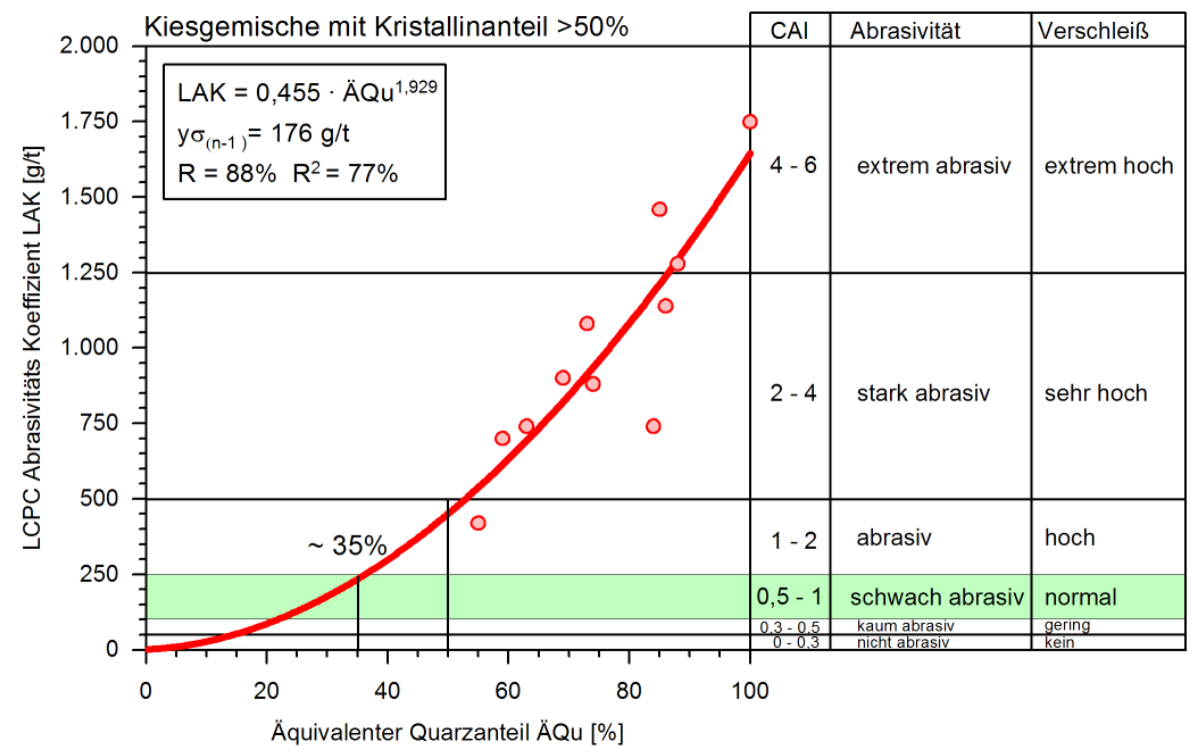

Abb. 33: Korrelation des LCPC Abrasivitätskoeffizienten (LAK) $[\mathrm{g} / \mathrm{t}]$ mit dem Äquivalenten Quarzgehalt (ÄQu) [\%]. Zusätzlich sind die Abrasivitätsklassifikation, das zugehörige Verschleißpotential und der entsprechende CAI-Wert eingetragen. (THURO \& KÄSLING 2009a: 185, verändert). 


\subsubsection{Auslegung der Hydroschild TVM}

Die Auswirkung der Auslegung der TVM auf den Verschleiß der Abbauwerkzeuge wird vor allem von FRENZEL \& BABENDERERDE (2011: $41 \mathrm{ff}$.) bearbeitet und zusammengefasst. Sie nennen dazu drei Hauptfaktoren, die den Verschleiß der Abbauwerkzeuge beeinflussen.

Der erste Einflussaktor ist die Höhe $h_{S R}, h_{S M}, h_{S T} \& h_{R M}[m m]$ der Werkzeuge über dem Schneidradstahlbau. Hier ist einerseits die absolute Höhe der Werkzeuge über dem Schneidradstahlbau maßgeblich. Diese muss einen möglichst günstigen Materialfluss zwischen Ortsbrust und Frontseite des Schneidradstahlbaus hin zu den Schneidradöffnungen gewährleisten, um hohen Sekundärverschleiß an den Abbauwerkzeugen zu vermeiden. Andererseits ist auch die relative Höhe zwischen vor- und nachlaufenden Abbauwerkzeugen ein entscheidender Faktor für den Verschleiß. Bei ausreichendem Abstand zwischen vor- und nachlaufenden Abbauwerkzeugen werden die nachlaufenden Abbauwerkzeuge vor Verschleiß geschützt. Deutlichstes Beispiel ist hier der Schutz von Schälmessern durch vorlaufende Schneidrollen beim Abbau von Böden mit Steinen oder Blöcken.

Der zweite wesentliche Faktor ist das Öffnungsverhältnis des Schneidrades OTVM [\%] bzw. die Anordnung der Öffnungen. Diese müssen einen möglichst optimalen Transport des abgebauten Bodens hinter das Schneidrad zulassen. FRENZEL \& BABENDERERDE (2011: 41) betrachten dazu das Verhältnis zwischen den zur Verfügung stehenden Schneidradöffnungen und dem entsprechenden abgebauten Bodenvolumen je Schneidradumdrehung bezogen auf $20 \mathrm{~cm}$ Abschnitte des Schneidradradius $R_{\text {TVM }}$ [m]. Dieses Verhältnis wird als normalisierter Materialfluss bezeichnet und korreliert zum Teil mit dem Sekundärverschleiß an der Schneidradstahlstruktur. In Bereichen, in denen das Verhältnis von abgebautem Bodenvolumen und zugehörigen Schneidradöffnungen unzureichend ist, wird der Boden zwischen Schneidradfrontseite und Ortsbrust radial nach außen hin zur nächstgelegenen Öffnung hin verdrängt. Dabei steigt auch der Sekundärverschleiß der Abbauwerkzeuge in diesem Bereich an.

Der dritte Einflussfaktor der TVM-Auslegung auf den Verschleiß der Abbauwerkzeuge ist der Spurbesatz $\mathrm{k}_{\mathrm{s}}$. Durch eine mehrfache Belegung der Spuren reduziert sich anteilig die tatsächliche Penetration $\mathrm{p}_{\mathrm{a}}[\mathrm{mm} / \mathrm{U}]$ (Kap. 3.3.1) der einzelnen Abbauwerkzeuge. Als positive Auswirkung auf den Verschleiß der Abbauwerkzeuge nennen FRENZEL \& BABENDERERDE (2011: 39) hier jedoch ausschließlich die Reduktion der Lagerbelastung von Schneidrollen beim Abbau von Steinen oder Blöcken.

\subsubsection{Steuerung des Abbauprozesses}

Der Einfluss der Steuerung des Abbauprozesses auf den Verschleiß der Abbauwerkzeuge im Vortrieb mit Hydroschild TVM wird von verschiedenen Autoren erwähnt. Ähnlich wie für den Einfluss der TVMAuslegung auf den Verschleiß der Abbauwerkzeuge fehlen jedoch bisher detaillierte Untersuchungen zu den einzelnen Einflussfaktoren.

Eine Ausnahme bilden die Untersuchungen von WILMS (1995: $106 \mathrm{ff}$.) zum Einfluss des Umgebungsdrucks auf die Verschleißintensität an Metallstiften in einem Laborversuch in Abhängigkeit des Wassergehalts bzw. der Konsistenz des Bodens. Diese werden zwar für Erddruckschild TVM (EPB TVM) durchgeführt, sodass der überwiegende Teil der Versuche aufgrund des Unterschiedes in der Konsistenz des Materials zur Stützflüssigkeit bei Hydroschild TVM nicht übertragbar ist. Als Grenzzustand wird jedoch ein Fall betrachtet, in dem die Bodenkomponenten vollständig in Wasser dispergiert sind und keine Korn-Korn-Kontakte mehr bestehen.

Für diesen Grenzzustand zeigt WILMS (1995: 152), dass der Umgebungsdruck keinen Einfluss auf den Abrieb an den Metallstiften hat. Dieser bleibt unabhängig vom Umgebungsdruck immer gleich hoch. Dieser Grenzzustand ist mit den Verhältnissen in der Abbaukammer vergleichbar, da auch hier die Bodenkomponenten theoretisch vollständig in der Stützflüssigkeit dispergiert sind und keine KornKorn-Kontakte mehr bestehen. Der Stützdruck in der Abbaukammer entspricht dem Umgebungsdruck. Das lässt den Schluss zu, dass auch hier der Stützdruck $P_{S F}$ [bar] keinen Einfluss auf den Sekundärverschleiß der Abbauwerkzeuge hat. 
NiLSEN et al. (2006a: 37) und HOLZHÄUSER \& NILSEN (2006: 170) beschreiben auf Basis von zwei Referenzprojekten eine deutliche Zunahme des Abbauwerkzeugverschleißes mit zunehmendem Stützdruck $P_{\text {SF }}$ [bar] für EPB TVM. Dieser Zusammenhang stimmt für breiige Konsistenzen des Erdbreis mit den Ergebnissen von WILMS (1995: 106 f.) überein, ist jedoch nicht auf die Verhältnisse in der Abbaukammer einer Hydroschild TVM übertragbar. NILSEN et al. (2006a: 38) weisen darauf deutlich hin und stellen klar, dass grundsätzlich jede Änderung der Eigenschaften des Stützmediums auch Einfluss auf den Verschleiß der Abbauwerkzeuge hat, insbesondere der Unterschied zwischen EPB TVM und Hydroschild TVM.

Der zweite wesentliche Einflussfaktor auf den Verschleiß der Abbauwerkzeuge in der Steuerung des Abbauprozesses ist die Penetration $p$ [mm/U]. Von BURGER (2006: 18) und FRENZEL \& BABENDERERDE (2011: 37) wird darauf hingewiesen, dass die Penetration beim Abbau von Böden mit Steinen oder Blöcken wesentlichen Einfluss auf die Lagerbelastung der Schneidrollen hat. Wird die Penetration hier zu hoch angesetzt, ergibt sich beim Abbau eines Blocks eine Überlastung der Schneidrollenlager, die zu vorzeitigem Verschleiß oder auch Schäden durch sofortiges Blockieren führen kann. Nach den Untersuchungen von KIEFER et al. (2008: $134 \mathrm{ff}$.) werden Blöcken in der Ortsbrust beim Einsatz von Schneidrollen zunächst ähnlich wie im Festgestein durch Chipping abgebaut. Theoretisch sind damit die gängigen Modelle zur Schätzung der Lagerbelastung von Schneidrollen in Abhängigkeit der Penetration p beim Abbau von Festgesteinen, z.B. von RostAMl (1997: 204 ff.), anwendbar.

KÖHLER et al. (2011: 50) geben für den Abbau von Lockergesteinen mit Hydroschild TVM auf Basis von zwei Referenzprojekten verallgemeinert an, dass der Verschleiß der Abbauwerkzeuge mit zunehmender Penetration $\mathrm{p}[\mathrm{mm} / \mathrm{U}]$ und zunehmender Schneidraddrehzahl $U$ [ $\left.\mathrm{min}^{-1}\right]$ ansteigt. Entsprechende Untersuchungen dazu wurden jedoch nicht durchgeführt. SCHOLZ \& WENDL (2011: 233 f.) zeigen anhand von zwei Referenzprojekten, dass die Penetration $\mathrm{p}$ [mm/U] Einfluss auf die Verdichtung des Bodens in der Ortsbrust hat. Diese kann in Abhängigkeit der Bodeneigenschaften wiederum einen maßgeblicher Einflussfaktor auf den Verschleiß der Abbauwerkzeuge sein (Kap.4.2.2.1).

Abschließend hat auch die Wartung maßgeblichen Einfluss auf den Verschleiß der Abbauwerkzeuge. Nach Nilsen et al. (2006a: 37), Scholz \& WendL (2011: 232) und Frenzel \& BABENDER$\operatorname{ERDE}(2011: 41)$ resultiert aus hohem Abrieb bzw. Schäden an vorlaufenden Abbauwerkzeugen (Schneidrollen) häufig auch zunehmender Verschleiß an den nachlaufenden Abbauwerkzeugen (Schälmesser). Der Zeitpunkt des Wechsels und die Werkzeugwechselstrategie insgesamt haben also starken Einfluss auf den Verschleiß der Abbauwerkzeuge.

\subsection{Verschleißprognose für Hydroschild TVM}

Die Notwendigkeit für ein umfassendes Prognosemodell für den Verschleiß der Abbauwerkzeuge in Lockergesteinen wird von verschiedenen Autoren bereits frühzeitig aufgrund unerwarteter Probleme im Vortrieb erkannt. Dennoch stellen NILSEN et al. (2006a: 36) und KöHLER et al. (2010: 52) fest, dass kein entsprechendes Modell zur Verfügung steht. KöHLER et al. (2010: 52) bezweifeln vor dem Hintergrund der hohen Anzahl an Einflussfaktoren sogar, dass eine rein analytische Aufarbeitung der Verschleißprozesse für Hydroschild TVM möglich ist. Die Verschleißprognose für Hydroschild TVM ist deshalb stark auf empirische Werte auf Projektbasis angewiesen.

JAKOBSEN \& DAHL (2011: 62) berichten von ersten Versuchen, die Ergebnisse von SAT-Tests mit dem tatsächlich erreichten Lösevolumen $\mathrm{v}_{\mathrm{c}}\left[\mathrm{m}^{3}\right]$ der Abbauwerkzeuge in Referenzprojekten zu korrelieren. Für vier TVM in drei Referenzprojekten stellen sie einen exponentiellen Zusammenhang zwischen dem Lösevolumen $v_{c}$ und dem SAT-Wert dar, die Güte der Korrelation liegt mit einem Bestimmtheitsmaß von 0,66 jedoch relativ niedrig. JAKOBSEN et al. (2013a: 113) zeigen hierzu neuere Daten, die für verschiedene Abbauwerkzeugtypen untergliedert sind. Das Bestimmtheitsmaß der Korrelation zwischen SAT und dem Standvolumen $v_{c}\left[\mathrm{~m}^{3}\right]$ der Abbauwerkzeuge liegt hier für Schälmesser bei 0,69 und für Schneidrollen bei 0,02. Diese Werte werden einerseits auf das relativ geringe Datenvolumen zurückgeführt und andererseits auf die fehlende Berücksichtigung der Korngrößenverteilung, Kornform und Lagerungsdichte. Im Gegensatz zur vorliegenden Arbeit enthält die Datenbasis hier ausschließlich Projekte mit einem Schneidraddurchmesser $\mathrm{D}_{\mathrm{TVM}}<5,5 \mathrm{~m}$, die überwiegend als Rohrvortriebe ausgeführt wurden. 
Empirische Werte für die Standgrößen der Abbauwerkzeuge beim Vortrieb mit Hydroschild TVM in Lockergesteinen, die zugehörigen geotechnischen Rahmenbedingungen und die resultierenden notwendigen Vortriebsunterbrechungen für Werkzeugwechsel werden selten veröffentlicht. Aus den bisherigen Veröffentlichungen liegen nur drei Angaben zu entsprechenden Werten vor:

- HolZHäUSER \& NiLSEN (2006: 170) nennen als Beispiel für regelmäßige Einstiegsintervalle für Schneidradinspektionen $100-200 \mathrm{~m}$, allerdings ohne eine entsprechende Angabe des Maschinentyps, Ausbruchdurchmessers und geotechnischer Rahmenbedingungen. Für die Reparatur eines verschleißbedingten Schneidradschadens einer EPB TVM untertage geben sie einen Stillstand von 6 Wochen an.

- WENDL et al. (2010: 3827) geben als regelmäßige Einstiegsintervalle für eine Hydroschild TVM mit 13,2 m Ausbruchdurchmesser in fluviatilen Kiesen mit mittlerer bis hoher Lagerungsdichte und wechselndem Anteil an kristallinen Komponenten 50 - 200 m an.

- Frenzel \& BabendeReRde (2011: 39) geben für Stichel im Vergleich zu einer 17-Zoll Schneidrolle einen um ca. Faktor 8 geringeren Zeitbedarf für den Werkzeugwechsel an. 


\section{Ergebnisse und Diskussion der Datenanalyse}

Die Ergebnisse der Datenanalyse (Kap. 2.3) sind in die folgenden in sechs Themenbereiche untergliedert (Kap. 5.1 - 5.6):

- Überblick über die Referenzprojekte (Kap. 5.1)

- Analyse der Dokumentation in den Referenzprojekten (Kap. 5.2)

- Beschreibung der Verschleiß- und Schadenstypen in den Referenzprojekten (Kap. 5.3)

- Berechnung und Auswahl der geeigneten Standgrößen der Abbauwerkzeuge (Kap. 5.4)

- Versuche zur Verschleirate $c_{v}$ (Kap. 5.5)

- Analyse der Einflussfaktoren auf den Laufweg $\mathrm{s}_{\mathrm{c}}$ (Kap. 5.6)

Die Ergebnisse aus den Themenbereichen bauen aufeinander auf. Die Erkenntnisse aus vorlaufenden Themenbereichen bilden jeweils die notwendige Grundlage für die Bearbeitung nachfolgender Themenbereiche. Zusätzlich weisen die Ergebnisse aus den genannten Themenbereichen in Summe einen relativ hohen Umfang auf. Die Diskussion und Interpretation der Ergebnisse erfolgt deshalb direkt in den einzelnene Themenbereichen, um den Zusammenhang innerhalb der Themenbereiche zu erhalten. Eine übergreifende Diskussion der Ergebnisse der Datenanalyse zusammen mit den Ergebnissen zum empirischen Prognosemodell (Kap. 6) erfolgt in Kap. 7.2.

\subsection{Referenzprojekte}

Als Grundlage für die vorliegende Arbeit konnten innerhalb der Datenrecherche 10 Referenzprojekte (RP) mit 13 Hydroschild TVM in 18 Vortrieben (VT) erarbeitet werden. Die enthaltenen Hydroschild TVM umfassen eine hohe Bandbreite an Schneidraddurchmessern $D_{\text {TVM }}$ von 6,32 - 15,43 m. In Summe wurde in den Referenzprojekten dabei auf $45.391 \mathrm{~m}$ Vortriebsstrecke ein Ausbruchvolumen von 5.592.484 $\mathrm{m}^{3}$ erzielt. Eine Übersicht über die Referenzprojekte ist in Tab. 8 gegeben.

Für die empirische, prozessorientierte Datenanalyse konnten in den in Tab. 8 enthaltenen Referenzprojekten in Summe 4.607 Werkzeugwechsel dokumentiert werden. Diese sind zusammen mit Mittelwerten für die wichtigsten Steuerparameter des Abbauprozesses in Tab. 9 zusammengefasst. Mit Ausnahme von Referenzprojekt 8 gibt die angegebene Anzahl an gewechselten Werkzeugen jeweils den vollständigen Vortrieb wieder. In Referenzprojekt 8 konnten nur die Wechsel der Schneidrollen dokumentiert werden. Die genauen Angaben zu den Wechseln der Schälmesser sind für die vorliegende Arbeit nicht verfügbar, sie umfassen jedoch zusätzlich ca. 1.250 Werkzeugwechsel. 


\section{Ergebnisse und Diskussion der Datenanalyse}

Tab. 8: Übersicht über die Referenzprojekte als Grundlage der empirischen, prozessorientierten Datenanalyse

$\left(\mathrm{RP}=\right.$ Referenzprojekt; TVM = Tunnelvortriebsmaschine; VT = Vortrieb; $\mathrm{D}_{\mathrm{TVM}}=$ Schneidraddurchmesser; $\mathrm{O}_{\mathrm{TVM}}=$

Schneidradöffnungsverhältnis; $\mathrm{L}_{\mathrm{TA}}=$ Tunnellänge; $\mathrm{V}_{\mathrm{TA}}=$ Ausbruchvolumen; $\mathrm{SR}=$ Schneidrolle; $\mathrm{ST}=\mathrm{Stichel}$;

$\mathrm{SM}=$ Schälmesser; $\mathrm{HGB}=$ Geotechnischer Homogenbereich; $\mathrm{H}_{\mathrm{TA}}=$ Vertikale Überlagerung der Tunnelachse).

\begin{tabular}{|c|c|c|c|c|c|c|c|c|c|c|c|c|}
\hline \multicolumn{2}{|c|}{ Projekt } & \multicolumn{5}{|c|}{ Vortrieb } & \multicolumn{3}{|c|}{ Werkzeugbesatz } & \multicolumn{3}{|c|}{ Geotechnik } \\
\hline $\mathbf{R P}$ & TVM & VT & $\mathrm{D}_{\mathrm{TVM}}$ & $\mathbf{O}_{\mathrm{TVM}}$ & $\mathrm{L}_{\mathrm{TA}}$ & $V_{T A}$ & SR & ST & SM & \multirow{2}{*}{ HGB } & $\mathrm{H}_{\mathrm{TA}}$ & \multirow{2}{*}{ Bodenarten } \\
\hline Nr. & $\mathrm{Nr}$. & Nr. & [m] & [\%] & [m] & {$\left[\mathrm{m}^{3}\right]$} & Stk. & Stk. & Stk. & & [m] & \\
\hline \multirow{2}{*}{1} & \multirow{2}{*}{1} & 1 & 6,9 & $26 \%$ & 1.203 & 44.984 & 22 & - & 34 & 8 & 23 & $\begin{array}{l}\text { Geschiebemergel, } \\
\text { Schluffe \& Sande }\end{array}$ \\
\hline & & 2 & 6,9 & $26 \%$ & 1.041 & 38.926 & 22 & - & 34 & 8 & 23 & $\begin{array}{l}\text { Geschiebemergel, } \\
\text { Schluffe \& Sande }\end{array}$ \\
\hline 2 & 2 & 3 & 6,8 & $30 \%$ & 268 & 9.733 & 17 & - & 38 & 1 & 15 & Sande \& Kiese \\
\hline \multirow{2}{*}{3} & 3 & 4 & 15,4 & $28 \%$ & 7.170 & 1.335 .220 & - & - & 185 & 1 & 38 & $\begin{array}{l}\text { Schluffige Tone m. } \\
\text { Sandlagen }\end{array}$ \\
\hline & 4 & 5 & 15,4 & $28 \%$ & 7.170 & 1.335 .220 & - & - & 185 & 1 & 38 & $\begin{array}{l}\text { Schluffige Tone m. } \\
\text { Sandlagen }\end{array}$ \\
\hline \multirow{2}{*}{4} & 5 & 6 & 8,4 & $30 \%$ & 2.141 & 118.649 & 19 & - & 170 & 1 & 20 & Sande \& Kiese \\
\hline & 6 & 7 & 8,4 & $30 \%$ & 2.141 & 118.649 & 19 & - & 170 & 1 & 20 & Sande \& Kiese \\
\hline \multirow{4}{*}{5} & \multirow{4}{*}{7} & 8 & 7,4 & $70 \%$ & 490 & 21.074 & - & 36 & 64 & 1 & 13 & Kiese \\
\hline & & 9 & 7,4 & $70 \%$ & 680 & 29.246 & - & 36 & 64 & 3 & 16 & $\begin{array}{l}\text { Tone, Schluffe \& } \\
\text { Kiese }\end{array}$ \\
\hline & & 10 & 7,4 & $70 \%$ & 490 & 21.074 & - & 36 & 64 & 1 & 13 & Kiese \\
\hline & & 11 & 7,4 & $70 \%$ & 680 & 29.246 & - & 36 & 64 & 3 & 16 & $\begin{array}{l}\text { Tone, Schluffe \& } \\
\text { Kiese }\end{array}$ \\
\hline \multirow{2}{*}{6} & 8 & 12 & 14,9 & $28 \%$ & 2.990 & 521.355 & - & - & 185 & 3 & 33 & $\begin{array}{l}\text { Sande, Kiese \& } \\
\text { Schluffstein }\end{array}$ \\
\hline & 9 & 13 & 14,9 & $28 \%$ & 2.990 & 521.355 & - & - & 185 & 5 & 33 & $\begin{array}{l}\text { Sande, Kiese \& } \\
\text { Schluffstein }\end{array}$ \\
\hline 7 & 10 & 14 & 13,0 & $31 \%$ & 5.760 & 764.538 & 69 & (69) & 268 & 11 & 33 & $\begin{array}{l}\text { Schluffe, Sande \& } \\
\text { Kiese }\end{array}$ \\
\hline 8 & 11 & 15 & 13,0 & $31 \%$ & 3.426 & 454.741 & 69 & - & 268 & 4 & 25 & $\begin{array}{l}\text { Schluffe, Sande \& } \\
\text { Kiese }\end{array}$ \\
\hline 9 & 12 & 16 & 6,3 & $28 \%$ & 1.129 & 35.194 & 23 & - & 64 & 4 & 14 & Sande \& Kiese \\
\hline \multirow{2}{*}{10} & \multirow{2}{*}{13} & 17 & 6,6 & $26 \%$ & 2.811 & 96.170 & 22 & (22) & 34 & 4 & 33 & $\begin{array}{l}\text { Geschiebemergel, } \\
\text { Schluffe \& Sande }\end{array}$ \\
\hline & & 18 & 6,6 & $26 \%$ & 2.811 & 96.170 & 22 & (22) & 34 & 4 & 33 & $\begin{array}{l}\text { Geschiebemergel, } \\
\text { Schluffe \& Sande }\end{array}$ \\
\hline \multicolumn{5}{|c|}{ Total: } & 45.391 & 5.592 .484 & & & & & & \\
\hline
\end{tabular}


Tab. 9: Übersicht über die Steuerparameter des Abbauprozesses und die Anzahl an Werkzeugwechseln in den Referenzprojekten als Grundlage für die Datenanalyse. Die Anzahl der Schälmesserwechsel in RP 8 konnte nicht detailliert recherchiert werden, liegt jedoch bei ca. 1.250 Stk. (RP = Referenzprojekt; $\mathrm{D}_{\mathrm{TVM}}=$ Schneidraddurchmesser $; \mathrm{L}_{\mathrm{TA}}=$ Tunnellänge; $\mathrm{V}_{\mathrm{TA}}=$ Ausbruchvolumen; $\mathrm{p}=$ Penetration; $\mathrm{U}=$ Schneidraddrehzahl; $P_{S F}=$ Stützdruck; $M_{T V M}=$ Schneidraddrehmoment; $F_{S V}=$ Vortriebskraft an der Schneidradverschiebung; SR = Schneidrollen; ST = Stichel; SM = Schälmesser)

\begin{tabular}{|c|c|c|c|c|c|c|c|c|c|c|c|}
\hline \multicolumn{4}{|c|}{ Projekt } & \multicolumn{5}{|c|}{ Steuerung Abbauprozess (Vortriebsmittelwerte) } & \multicolumn{3}{|c|}{ Anzahl Werkzeugwechsel } \\
\hline $\mathbf{R P}$ & $\mathbf{D}_{\mathrm{TVM}}$ & $\mathbf{L}_{\mathrm{TA}}$ & $\mathbf{V}_{\mathrm{TA}}$ & p & $\mathbf{U}$ & $\mathbf{P}_{\mathrm{SF}}$ & $\mathbf{M}_{\mathrm{TVM}}$ & $F_{\mathrm{sv}}$ & SR & ST & SM \\
\hline Nr. & {$[\mathrm{m}]$} & [m] & {$\left[\mathrm{m}^{3}\right]$} & {$[\mathrm{mm} / \mathrm{U}]$} & {$\left[\mathrm{min}^{-1}\right]$} & [bar] & [MNm] & [MN] & Stk. & Stk. & Stk. \\
\hline 1 & 6,9 & 2.244 & 83.909 & 12,4 & 1,9 & 3,1 & 1,0 & 2,78 & 115 & 0 & 184 \\
\hline 2 & 6,8 & 268 & 9.733 & 11,0 & 2,0 & 2,3 & 0,45 & 1,60 & 38 & 0 & 2 \\
\hline 3 & 15,4 & 14.340 & 2.671 .041 & 43,9 & 0,9 & 3,9 & 3,0 & 71,79 & 0 & 0 & 64 \\
\hline 4 & 8,4 & 4.282 & 237.299 & 34,3 & 0,9 & 1,9 & 1,4 & 3,52 & 120 & 0 & 191 \\
\hline 5 & 7,4 & 2.340 & 100.640 & 13,3 & 1,9 & 1,8 & 6,6 & 10,19 & 0 & 0 & 0 \\
\hline 6 & 14,9 & 5.980 & 1.042 .710 & 28,3 & 0,8 & 4,0 & 5,2 & 107,60 & 0 & 0 & 408 \\
\hline 7 & 13,0 & 5.760 & 764.538 & 15,7 & 1,9 & 2,7 & 5,0 & 10,08 & 551 & 365 & 1.351 \\
\hline 8 & 13,0 & 3.426 & 456.741 & 12,4 & 1,9 & 1,8 & 5,6 & 9,47 & 560 & 0 & $\begin{array}{c}\text { ca. } \\
1.250 \\
\end{array}$ \\
\hline 9 & 6,3 & 1.129 & 35.194 & 18,5 & 1,8 & 2,4 & 1,0 & 2,56 & 44 & 0 & 142 \\
\hline 10 & 6,6 & 5.622 & 192.340 & 7,7 & 1,9 & 3,2 & 0,7 & 2,97 & 202 & 12 & 258 \\
\hline \multicolumn{2}{|c|}{ Total: } & 45.391 & 5.592 .484 & & & & & & 1.630 & 377 & 3.850 \\
\hline
\end{tabular}

\subsection{Dokumentation in den Referenzprojekten}

Die Dokumentation in den Referenzprojekten hat hohen Einfluss auf die nachfolgenden Beschreibungen zum Verschleißbild, Abbaumechanik und Materialfluss (Kap. 5.3) und die Untersuchung der Einflussfaktoren auf die Werkzeugstandgrößen (Kap. 5.6). Deshalb wurde innerhalb der Referenzprojekte zunächst Art, Umfang und Detaillierungsgrad der Dokumentation für folgende Datenbereiche analysiert:

- Werkzeugwechsel

- Geotechnische Bedingungen im Vortrieb

- Auslegung der TVM und der Abbauwerkzeuge

- Steuerung des Abbauprozesses

Für die vier Bereiche wurden zunächst auf Basis des bestehenden Wissensstandes theoretische Anforderungen an die Dokumentation entwickelt. Diese sollten einerseits in zukünftigen Projekten mit Hydroschild TVM in Lockergesteinen als neuer Standard eingesetzt werden. Andererseits sind bisher nicht für alle erforderlichen Parameter Methoden vorhanden, um diese unter den vorherrschenden Rahmenbedingungen im Vortrieb überhaupt zu ermitteln oder eine ausreichende hohe Dokumentationsdichte zu erreichen. Die theoretischen Anforderungen an die Dokumentation zeigen hier folglich auch über die vorliegende Arbeit hinaus weiteren Untersuchungsbedarf auf. 
Die Dokumentation in den Referenzprojekten kann anschließend mit den theoretischen Anforderungen und den gegebenen Möglichkeiten im Vortrieb abgeglichen werden. Die Analyse der Dokumentation in den Referenzprojekten ist deshalb für die vier oben genannten Bereiche im Folgenden jeweils in drei Teile untergliedert:

- Theoretische Anforderung an die Dokumentation

- Möglichkeiten und Grenzen in der Vortriebspraxis

- Dokumentation in den Referenzprojekten

\subsubsection{Werkzeugwechsel}

\subsubsection{Theoretische Anforderungen an die Dokumentation}

Die Anforderungen an die Dokumentation des Einsatzintervalls eines Werkzeuges umfassen bezogen auf den Vortrieb mit Hydroschild TVM in Lockergesteinen für jeden einzelnen Werkzeugwechsel mindestens die nachfolgend aufgelisteten Informationen:

- Datum

- Vortriebsstationierung (Ring Nr.; Vortriebsstand [m])

- Bearbeiter

- Werkzeugtyp

- Werkzeug ID-Nummer (Schneidrollen)

- Spur Nr.

- Arm Nr. (Schälmesser \& Räumer)

- Drehrichtung (Schälmesser \& Räumer)

- Zustand des Abbauwerkzeuges bei Ausbau

- Wechselgrund

- Fotodokumentation

Die qualitative Zustandsbeschreibung der Abbauwerkzeuge richtet sich nach den Verschleiß- und Schadensarten (Kap. 5.3.1) der unterschiedlichen Werkzeugtypen. Als Referenz für die quantitative Beschreibung des Zustands der Abbauwerkzeuge dient eine festgelegte Verschleißgrenze für die unterschiedlichen Werkzeugtypen. Die Zustandsbeschreibung der Abbauwerkzeuge ist letztlich auch Basis für die Angabe des Wechselgrundes der Abbauwerkzeuge. 
Mit Bezug auf die festgelegten Verschleißgrenzen und Verschleiß- und Schadensarten ist bei der Angabe des Wechselgrundes eine Unterscheidung folgender Kategorien zu empfehlen:

- Präventiver Wechsel (Stetiger Abrieb, Verschleißgrenze nicht erreicht, Wechsel aus baubetrieblichen Gründen.)

- Normaler Wechsel (Stetiger Abrieb, Verschleißgrenze erreicht.)

- Schaden (Verschleißgrenze überschritten oder anderer Schadensmechanismus.)

- Ersatzloser Ausbau (Änderung der Werkzeugbestückung, Ausbau eines Werkzeuges ohne Ersatz durch ein entsprechendes neues Werkzeug.)

- Neubestückung (Änderung der Werkzeugbestückung, Einbau eines neuen Werkzeuges in einen zuvor nicht genutzten bzw. bestückten Halter oder Gehäuse.)

- Werkzeugverlust (Verlust des Werkzeuges während dem Vortrieb, Einbau eines neuen Werkzeuges.)

\subsubsection{Möglichkeiten und Grenzen in der Vortriebspraxis}

Die Dokumentation aller oben aufgelisteten Informationen ist auch in der Praxis durchaus möglich, allerdings treten systembedingt Probleme mit folgenden Faktoren auf:

- Umgebungsbedingungen beim Werkzeugwechsel

- Zugänglichkeit der Abbauwerkzeuge

- Zeitdruck beim Werkzeugwechsel

- Verarbeitung der Informationen

Die Umgebungsbedingungen während der Arbeiten in der Abbaukammer erlauben insbesondere unter Druckluft häufig keine detaillierten schriftlichen Aufzeichnungen und fotographische Dokumentation. Typischerweise herrscht extrem hohe Luftfeuchtigkeit und Abbauwerkzeuge, Schneidradstahlstruktur und Druckwand sind in Abhängigkeit der Reinigungsarbeiten mit Bentonitsuspension und abgebautem Boden überzogen. Um die Informationen aus dem Werkzeugwechsel unter diesen Bedingungen trotzdem zu erfassen, werden diese entweder nach dem Wechsel durch den Bearbeiter schriftlich zusammengefasst oder während dem Wechsel per Funk aus der Abbaukammer weitergegeben.

Der Zugang zu den Abbauwerkzeugen ist während eines Einstieges unter Druckluft deutlich eingeschränkt. Das Schneidrad kann während einer Werkzeugkontrolle nicht oder nur wenige Zentimeter von der Ortsbrust zurückgezogen werden. Damit sind Teile der Werkzeuge, z.B. der Rückenbereich von Schälmessern und Räumern, nicht einsehbar oder können nur durch fühlen per Hand ertastet werden.

Zusätzlich zu den ungünstigen Umgebungsbedingungen und der schweren Zugänglichkeit der Werkzeuge kommt insbesondere bei Arbeiten unter Druckluft ein hoher Zeitdruck während der Werkzeugwechsel hinzu. Dieser ist einerseits bedingt durch die Bemühung die Stillstandzeit der TVM so gering wie möglich zu halten. Andererseits limitieren jedoch auch die zunehmende Austrocknung des Filterkuchens an der Ortsbrust (Rissbildung), die Entwicklung der Luftverluste und länderspezifische gesetzliche Regelungen zur Arbeitszeit unter Druckluft (Kap. 3.3.3) die zur Verfügung stehende Arbeitszeit.

Die genannten Faktoren führen dazu, dass während der Arbeiten in der Abbaukammer nur die aller notwendigsten Informationen dokumentiert werden. Diese unterliegen zusätzlich einer hohen Fehleranfälligkeit. 
Abschließend stellt die Menge und Zusammensetzung der Daten aus den Werkzeugwechseln eine Herausforderung für die Ablage und Verarbeitung der Daten dar. Bei Schneidrädern mit Durchmesser $\mathrm{D}_{\text {TVM }}>10 \mathrm{~m}$ besteht die Werkzeugbestückung häufig aus $>150 \mathrm{Stk}$. Schälmesser, $>60 \mathrm{Stk}$. Schneidrollen und $>48$ Stk. Räumersegmenten (Kap. 5.1), die in der Dokumentation zu erfassen sind. Bei entsprechender Tunnellänge und Bodeneigenschaften kann die Anzahl an Werkzeugwechseln in Summe so deutlich >1.000 Stk. liegen. Entsprechend aufwändig ist die Ablage der Basisdaten, Erstellung von Übersichten und Verbindung verschiedener Datentypen, .z.B. der Fotodokumentation mit schriftlichen Aufzeichnungen. Zum Teil werden hier bereits spezialisierte Datenbanksysteme eingesetzt. Zusätzlich bewirken diese Probleme wiederum eine hohe Fehleranfälligkeit und häufigen Informationsverlust.

\subsubsection{Dokumentation der Werkzeugwechsel in den Referenzprojekten}

Die als Basis für diese Arbeit vorliegenden Daten zu den Werkzeugwechseln in den Referenzprojekten sind in Tab. 10 als Übersicht zusammengefasst. Die Ergebnisse darin verdeutlichen im Vergleich zu den theoretischen Anforderungen an die Dokumentation, dass in den Referenzprojekten zwar alle notwendigen Daten erfasst wurden, um zumindest alle in Kap. 5.4 beschriebenen Standgrößen errechnen zu können. Im Gegensatz dazu sind die Angaben zum Zustand der Abbauwerkzeuge beim Ausbau und der Wechselgrund jedoch in fast allen Projekten unvollständig oder fehlen gänzlich.

Tab. 10: Umfang und Detaillierungsgrad der Werkzeugwechseldokumentation in den Referenzprojekten. Vollständig vorhandene Informationen sind mit (·) gekennzeichnet, unvollständige Informationen mit (०), fehlende Informationen durch (-).

\begin{tabular}{|c|c|c|c|c|c|c|c|c|c|c|c|c|}
\hline 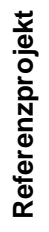 & 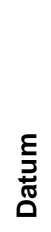 & 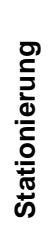 & 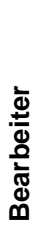 & 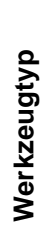 & $\begin{array}{l}\dot{z} \\
\text { ڤ̀ } \\
\text { के }\end{array}$ & $\begin{array}{l}\dot{z} \\
\underline{\underline{z}}\end{array}$ & 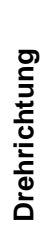 & 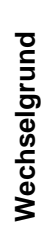 & $\begin{array}{l}\text { D } \\
\frac{\pi}{\pi} \\
\stackrel{N}{n} \\
\end{array}$ & $\begin{array}{l}\text { 루 } \\
\text { 음 } \\
\text { 운 }\end{array}$ & 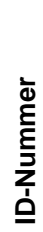 & 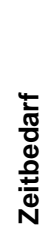 \\
\hline 1 & $\bullet$ & $\bullet$ & - & • & • & ○ & 0 & - & - & - & - & - \\
\hline 2 & $\bullet$ & $\bullet$ & - & $\bullet$ & $\bullet$ & • & $\bullet$ & 0 & - & 0 & - & - \\
\hline 3 & $\bullet$ & $\bullet$ & - & • & $\bullet$ & • & • & - & - & - & - & - \\
\hline 4 & $\bullet$ & $\bullet$ & - & • & $\bullet$ & • & • & O & - & 0 & - & - \\
\hline 5 & $\bullet$ & $\bullet$ & o & $\bullet$ & $\bullet$ & • & $\bullet$ & $\bullet$ & $\bullet$ & $\bullet$ & - & - \\
\hline 6 & $\bullet$ & $\bullet$ & - & $\bullet$ & $\bullet$ & • & • & O & - & - & - & - \\
\hline 7 & $\bullet$ & $\bullet$ & - & $\bullet$ & $\bullet$ & • & $\bullet$ & $\circ$ & - & - & - & - \\
\hline 8 & $\bullet$ & $\bullet$ & • & • & $\bullet$ & • & • & - & - & O & - & 0 \\
\hline 9 & • & $\bullet$ & • & • & $\bullet$ & • & • & O & 0 & O & $\bullet$ & 0 \\
\hline 10 & • & $\bullet$ & • & • & $\bullet$ & • & • & 0 & 0 & 0 & 0 & 0 \\
\hline 11 & $\bullet$ & • & • & $\bullet$ & $\bullet$ & • & $\bullet$ & 0 & - & 0 & 0 & 0 \\
\hline
\end{tabular}

Die Ursachen für die fehlende Dokumentation des Zustandes der Abbauwerkzeuge bei Ausbau und Angabe des Wechselgrundes hat vermutlich mehrere Ursachen. Einerseits wirken sich hier die in Kap. 5.2.1.2 angegebenen Probleme bei der Dokumentation im Vortrieb aus. Andererseits wurde bei der Analyse der Dokumentation der Auslegung der TVM (Kap. 5.2.3) deutlich, dass in der Dokumentation der TVM zum Teil keine Verschleißgrenzen angegeben wurden, oder Verschleißgrenzen, die für die Verschleißbilder der Abbauwerkzeuge im Lockergestein nicht aussagkräftig sind. 
Die fehlende Festlegung von Verschleißgrenzen trifft im Wesentlichen auf Schälmesser, Stichel und Räumer zu. Hier wurde in keinem der Referenzprojekte in der Anwenderdokumentation der TVM des Herstellers eine Festlegung quantitativer Verschleißgrenzen getroffen. Zusätzlich wurden jedoch auch im Anschluss durch den Auftragnehmer keine projektspezifischen Verschleißgrenzen festgelegt.

Für Schneidrollen wurde in fast allen Referenzprojekten in der Anwenderdokumentation die Verschleißgrenze der Schneidrollen für Festgesteinsanwendungen übernommen. Diese entspricht jedoch nicht dem Verschleißbild der Schneidrollen in Lockergesteinen (Kap. 5.3.2). Die Verschleißgrenze für 17"-Schneidrollen im Festgestein wird entsprechend FRENZEL (2010a: 34) als Maximalwert für radialen Abrieb am Schneidring definiert. Der Betrag des radialen Abriebs wird während der Werkzeugkontrolle mit einer Schablone gemessen, die auf den Flanken des Schneidrings als Referenzpunkt aufliegt, da an dieser Stelle im Festgestein üblicherweise kein Abrieb am Schneidring erfolgt.

Im Gegensatz dazu erfolgt der Abrieb am Schneidring im Lockergestein in Abhängigkeit der Bodeneigenschaften (Kap. 5.3.2) überwiegend an den Seitenflächen und Flanken des Schneidrings. Gleichzeitig erfolgt nahezu kein radialer Abrieb an der Lauffläche des Schneidrings. In der Folge wird durch die festgelegte Verschleißgrenze ein Wert bemessen, der für den tatsächlichen Funktionsverlust des Schneidrings im Lockergestein nicht ausschlaggebend ist. Zudem ist die Messmethode für diesen Wert mithilfe der Verschleißschablone irreführend, da sich diese auf eine Auflagefläche an der Schneidringflanke bezieht, die im Lockergestein ebenfalls stetigem Abrieb unterliegt.

Die Konsequenz aus den genannten Problemen ist, dass die Beurteilung des Werkzeugzustandes im Wesentlichen der subjektiven Einschätzung des jeweiligen Bearbeiters und dessen Vorerfahrung unterliegt. Diese wird verstärkt durch eine sehr geringe Anzahl an Kontrollen der einzelnen Abbauwerkzeuge zwischen Ein- und Ausbau.

Um das Verhältnis zwischen der Länge der Intervalle $L_{d}[\mathrm{~m}]$ zwischen zwei Abbauwerkzeuginspektionen und der von den Werkzeugen tatsächlich erreichten Vortriebsstrecke $I_{c}[\mathrm{~m}]$ (Kap. 5.4.3) zu analysieren wurde in den Referenzprojekt der arithmetische Mittelwert über alle Intervalle $L_{d(\mathrm{~m})}[\mathrm{m}]$ gebildet, sowie der arithmetische Mittelwert über die Vortriebsstrecke $I_{c}[\mathrm{~m}]$ aller gewechselten Werkzeuge. Schneidrollen und Schälmesser wurden dabei separat behandelt.

Aus dem Verhältnis der beiden arithmetischen Mittelwerte kann über folgenden Zusammenhang die durchschnittliche Anzahl $n_{1(\mathbb{C})}$ der Kontrollen zwischen Einbau und Ausbau des Werkzeuges auf dem Schneidrad mit Gl. 19 abgeschätzt werden:

$$
\mathrm{n}_{\mathrm{I}(\mathrm{Ic})}=\left(\frac{\sum\left(\frac{\mathrm{l}_{\mathrm{c}}}{\mathrm{n}_{\mathrm{c}(\mathrm{TA})}}\right)}{\sum\left(\frac{\mathrm{L}_{\mathrm{d}(\mathrm{m})}}{\mathrm{n}_{\mathrm{I}(\mathrm{TA})}}\right)}\right)-1
$$

In Gl. 19 steht $\mathrm{n}_{\mathrm{c}(\mathrm{TA})}$ für die Summe aller Werkzeugwechsel über die Vortriebsstrecke bzw. Länge der Tunnelachse $L_{T A}$ und $n_{I(T A)}$ für die Summe aller Vortriebsunterbrechungen über die Länge der Tunnelachse $L_{T A}$. Die Ergebnisse für $n_{\mid l(l)}$ sind in Abb. 34 als Histogramm zusammengefasst. Die Verteilung der Werte zeigt, dass der Modalwert für die Anzahl an Kontrollen der Werkzeuge zwischen Einund Ausbau auf dem Schneidrad bei zwei (Schneidrollen) bzw. drei (Schälmesser) Kontrollen liegt. Eine deutliche Abhängigkeit von $n_{\mathbb{l}(1 \mathrm{c})}$ vom Werkzeugtyp ist nicht erkennbar. Zusätzlich wurde $n_{1(1 \mathrm{c})} \mathrm{im}$ Hinblick auf den zugehörigen TVM-Durchmesser untersucht, eine Abhängigkeit konnte jedoch nicht festgestellt werden. 


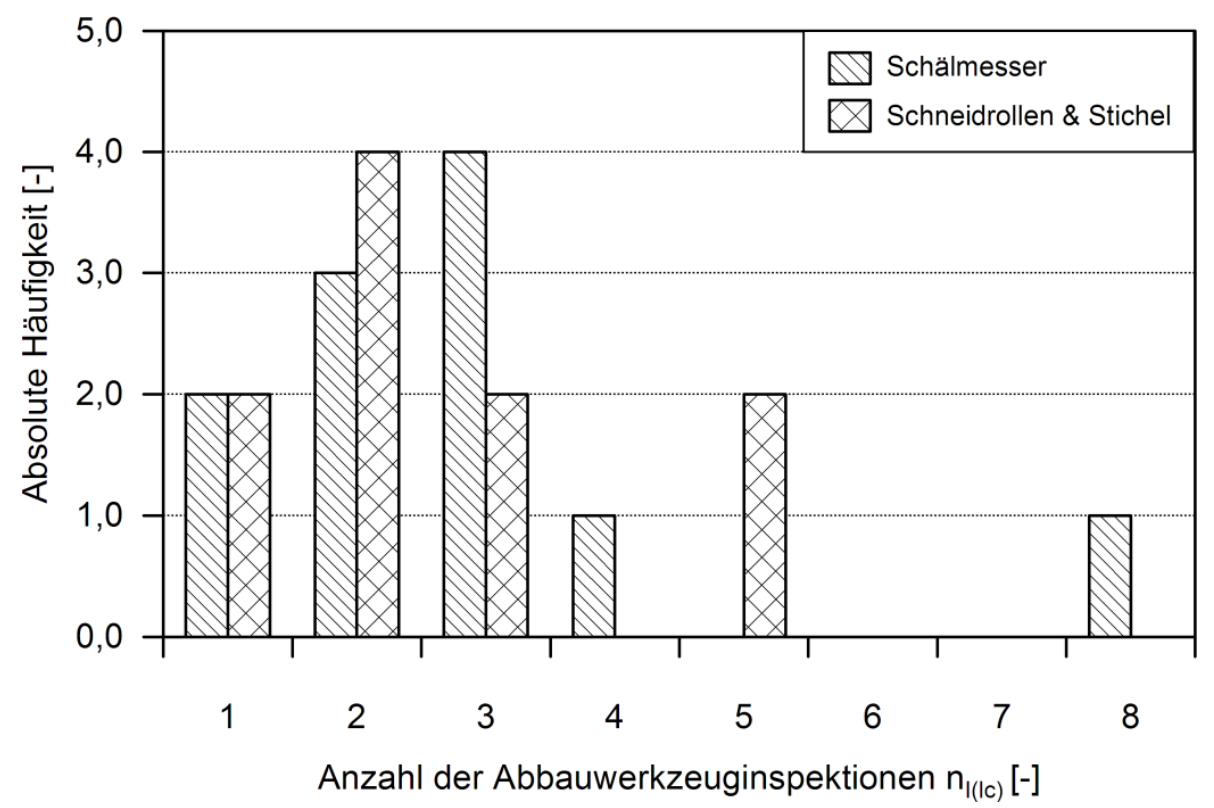

Abb. 34: Absolute Häufigkeit der Werkzeugkontrollen $n_{(\mid l c)} z$ wischen Ein- und Ausbau der Werkzeuge auf dem Schneidrad über alle Referenzprojekte.

Die geringe Anzahl von durchschnittlich zwei bzw. drei Werkzeugkontrollen zwischen Ein- und Ausbau der Werkzeuge wird im Vortrieb im Wesentlichen durch die Minimierung der Einstiege in die Abbaukammer verursacht. Der Effekt der subjektiven Einschätzung des Werkzeugzustandes durch den Bearbeite wird durch die geringe Anzahl an Kontrollen zwischen Ein- und Ausbau des Abbauwerkzeuges vermutlich verstärkt, weil kaum subjektive Erfahrungen zur tatsächlichen Entwicklung der Verschleißrate und zum Betrag der Standgrößen aufgebaut werden.

\subsubsection{Geotechnische Bedingungen}

\subsubsection{Theoretische Anforderungen an die geotechnische Vorerkundung und Dokumentation}

Die wesentlichen geotechnischen Parameter, die nach bestehendem Wissensstand für den Verschleiß der Abbauwerkzeuge maßgeblich sind, sind in Tab. 4 (Kap. 4.3.1) zusammengefasst. Auf Basis der weiteren Ergebnisse in Kap. 4 sind dabei in Ergänzung zu Tab. 4 nachfolgende Überlegungen zum Umfang der Untersuchungen in der Vorerkundung und Dokumentation sinnvoll.

Die Untersuchungen von BERETITSCH (1992: 25 ff.) zeigen, dass die Scherfestigkeit von Lockergesteinen beim Bodenabbau und die daraus resultierenden Schneidkräfte bzw. Kontaktspannungen eine hohe Abhängigkeit von der Schneidgeschwindigkeit $v_{s c}$ aufweist (Kap. 4.2.1). Der dabei untersuchte Bereich der Schneidgeschwindigkeit $v_{s c}$ liegt zwischen $0,05 \mathrm{~m} / \mathrm{s}$ und $0,5 \mathrm{~m} / \mathrm{s}$. Im Vergleich dazu werden in den untersuchten Referenzprojekten durchschnittliche Schneidgeschwindigkeiten $v_{s c}$ der Abbauwerkzeuge von $0,25 \mathrm{~m} / \mathrm{s}$ bis $0,90 \mathrm{~m} / \mathrm{s}$ erreicht. Die Schneidgeschwindigkeit $\mathrm{v}_{\mathrm{sc}}[\mathrm{m} / \mathrm{s}]$ der Abbauwerkzeuge wurde auf Basis der in Kap. 5.6.1.2 dargestellten Unterteilung der untersuchten Vortriebe in Homogenbereiche und Zuordnung von TVM-Betriebsdaten mit Gl. 20 ermittelt:

$$
\mathrm{v}_{\mathrm{sc}}=\frac{2 \cdot \mathrm{r}_{\mathrm{s}} \cdot \pi \cdot \mathrm{U}}{1.000 \cdot 60}
$$


Dabei ist $r_{s}$ der Spurradius der Abbauwerkzeuge $[\mathrm{mm}]$ und $U$ die Schneidraddrehzahl $\left[\mathrm{min}^{-1}\right]$. Einen Überblick über die in den Referenzprojekten erreichten durchschnittlichen Schneidgeschwindigkeiten gibt Abb. 35.

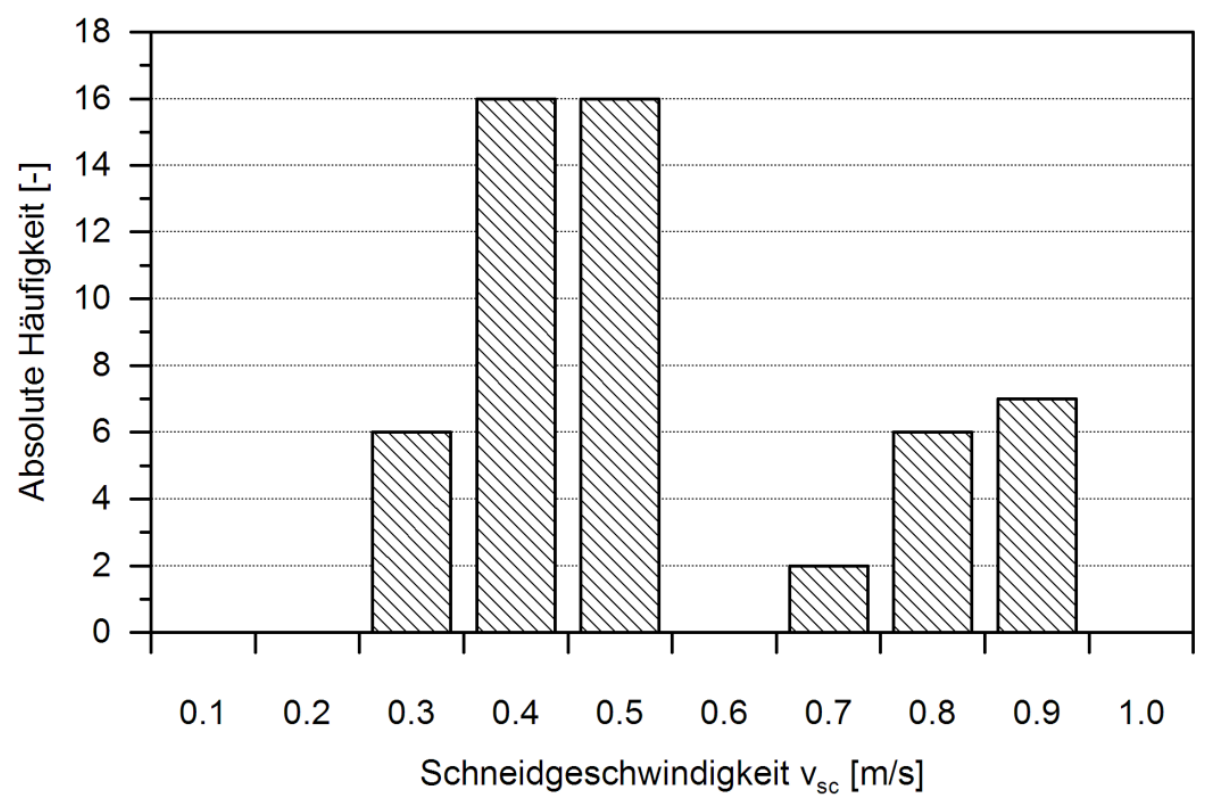

Abb. 35: Histogramm zur Verteilung der Schneidgeschwindigkeit $\mathrm{v}_{\mathrm{sc}}$ der Abbauwerkzeuge in den Referenzprojekten.

In den 53 untersuchten Homogenbereichen liegt der Modalwert der durchschnittliche Schneidgeschwindigkeit $\mathrm{v}_{\mathrm{sc}}$ mit 0,5 m/s bereits am oberen Ende des von BERETITSCH (1992: $26 \mathrm{ff}$.) untersuchten Bereichs. Folglich wären für den Bodenabbau und den damit verbundenen Verschleiß der Abbauwerkzeuge eigentlich die dynamischen Scherparameter des Bodens maßgeblich. Für diese existieren jedoch im Gegenzug keine genormten Versuchsverfahren. Deshalb sind in den Empfehlungen zur geotechnischen Vorerkundung (Tab. 11) weiterhin die quasi statischen Scherparameter berücksichtigt.

Der CeRCHAR Abrasivitätsindex (CAI) ist im eigentlichen Sinne nicht zur Bestimmung der Abrasivität von Lockergesteinen geeignet (Kap. 4.3.1.3). Die Anwendung des CAl auf einzelne Bodenkomponenten erfolgte in drei Referenzprojekten zusätzlich zum LCPC-Test, um die Abrasivität einzelner Bodenkomponenten zu erfassen, die aufgrund einer Korngröße $>4 \mathrm{~mm}$ nicht ohne vorheriges Brechen und Änderung der Kornform eingesetzt werden konnten. Eine statistische Hochrechnung der Abrasivität der Gesamtprobe ist aus diesen Messungen auch in Verbindung mit den LCPC-Tests jedoch nicht möglich. Der CERCHAR Abrasivitätsindex (CAl) ist deshalb in Tab. $11 \mathrm{im}$ Vergleich zum bestehenden Wissensstand nicht enthalten.

Abschließend ergeben sich damit aus dem bestehenden Wissensstand und den oben beschriebenen Ergänzungen die in Tab. 11 zusammengefassten Anforderungen und Empfehlungen für die Vorerkundung der geotechnischen Rahmenbedingungen und Dokumentation im Vortrieb. 


\section{Ergebnisse und Diskussion der Datenanalyse}

Tab. 11: Empfehlungen zur geotechnischen Vorerkundung im Hinblick auf den Verschleiß der Abbauwerkzeuge beim Vortrieb mit Hydroschild TVM in Lockergesteinen.

\begin{tabular}{|c|c|c|c|}
\hline Kennwert & Symbol & Einheit & Prüfvorschrift \\
\hline \multicolumn{4}{|c|}{ Für alle Bodenarten und Homogenbereiche: } \\
\hline Korngrößenverteilung & - & $\%$ & DIN ISO TS 17892-4 (2005) \\
\hline Wichte unter Auftrieb & $\mathrm{Y}^{\prime}$ & $\mathrm{kN} / \mathrm{m}^{2}$ & DIN ISO TS 17892-2 (2005) \\
\hline Lagerungsdichte & $\mathrm{D}$ & $\%$ & DIN 18126 (1996), DIN 18127 (2012) \\
\hline Kohäsion & $c^{\prime}$ & $\mathrm{kN} / \mathrm{m}^{2}$ & DIN 18137, T1 (2010), DIN 18137, T2 (2010) \\
\hline Reibungswinkel & $\varphi^{\prime}$ & $\circ$ & DIN 18137, T1 (2010), DIN 18137, T2 (2010) \\
\hline Porenanteil & $\mathrm{n}_{\text {por }}$ & $\%$ & DIN ISO/TS 17892-2, DIN ISO/TS 17892-3 \\
\hline Porenzahl & $\mathrm{e}_{\mathrm{por}}$ & - & DIN ISO/TS 17892-2, DIN ISO/TS 17892-3 \\
\hline Durchlässigkeit & $k_{f}$ & $\mathrm{~m} / \mathrm{s}$ & DIN 1830, T1 (1998) \\
\hline Wasserspiegel & $\mathrm{W}_{\mathrm{TA}}$ & $\mathrm{m}$ & - \\
\hline Überlagerung & $\mathrm{H}_{\mathrm{TA}}$ & $\mathrm{m}$ & - \\
\hline LCPC-Abrasivitätsindex & LAK & $g / t$ & AFNOR (1990) \\
\hline LCPC-Brechbarkeitsindex & LBK & $\%$ & AFNOR (1990) \\
\hline Äquivalenter Quarzgehalt & $\ddot{A Q u}$ & $\%$ & (Kap. 4.3.1.1) \\
\hline \multicolumn{4}{|l|}{ Zusätzlich für bindige Bodenarten: } \\
\hline Plastizitätszahl & $I_{P}$ & $\%$ & DIN ISO/TS 17892-12 (2005) \\
\hline Konsistenzzahl & $\mathrm{I}_{\mathrm{C}}$ & - & DIN ISO/TS 17892-12 (2005) \\
\hline Fließgrenze & $\mathrm{W}_{\mathrm{L}}$ & $\%$ & DIN ISO/TS 17892-12 (2005) \\
\hline Ausrollgrenze & $\mathrm{w}_{\mathrm{P}}$ & $\%$ & DIN ISO/TS 17892-12 (2005) \\
\hline Schrumpfgrenze & $w_{s}$ & $\%$ & DIN ISO/TS 17892-12 (2005) \\
\hline Wassergehalt & w & $\%$ & DIN ISO/TS 17892-12 (2005) \\
\hline \multicolumn{4}{|c|}{ Zusätzlich für gemischt- und grobkörnige Bodenarten: } \\
\hline Kornform (Sphärizität) & - & - & DIN EN ISO 14688-1:2002 \\
\hline Kornrundungsgrad & - & - & n. TUCKER (1981: 17) \\
\hline Mineralogische Zusammensetzung & - & - & DIN EN ISO 14688-1:2002 \\
\hline
\end{tabular}




\subsubsection{Geotechnische Dokumentation in der Vortriebspraxis}

Im Vergleich zu den oben angegebenen Anforderungen sind die Möglichkeiten zur Ermittlung der in Tab. 4 genannten Index- und Kennwerte in der Praxis zum Teil deutlich eingeschränkt. Die Probleme liegen häufig nicht in der eigentlichen Durchführung der entsprechenden Laborversuche, sondern bei der Gewinnung repräsentativer Proben.

In der Erkundungsphase liegen die Hauptprobleme in der eingeschränkten Zugänglichkeit der Tunneltrasse durch bestehende Bebauung oder natürliche Hindernisse. Zudem werden üblicherweise im späteren Ausbruchquerschnitt und in einem Korridor beiderseits der Tunnelachse so wenig wie möglich Bohrungen oder Sondierung bis auf Niveau der Tunnelachse niedergebracht, da diese anschlieBend wieder aufwändig verfüllt und abgedichtet werden müssen, um die entsprechende Ausbläsersicherheit in der Vortriebsphase wieder herzustellen.

In der Vortriebsphase ist die Gewinnung repräsentativer Bodenproben noch weiter eingeschränkt. Eine Entnahme von Bodenproben ist im Wesentlichen nur an der Ortsbrust oder im Bereich der Separationsanlage möglich.

An der Ortsbrust können im Rahmen eines Einstieges in die Abbaukammer unter Druckluft üblicherweise keine größeren oder ungestörten Proben entnommen werden, da hierzu eine Verletzung des Filterkuchens notwendig ist. Die Folgen dieser Verletzung sind kaum planbar. Im schlechtesten Fall können dadurch die Luftverluste schnell so hoch ansteigen, dass der entsprechende Einstieg in die Abbaukammer unter Druckluft abgebrochen werden muss. Die Beprobung steht deshalb in Konflikt zu den unmittelbar für den Vortrieb notwendigen Arbeiten in der Abbaukammer, z.B. den Wechsel von Abbauwerkzeugen und stellt ein zusätzliches Risiko dar.

Zusätzlich dazu ist bisher nur eingeschränkt untersucht, ob die Eigenschaften des Bodens unmittelbar an der Ortsbrust tatsächlich den ungestörten Bodeneigenschaften im natürlichen Lagerungsverband entsprechen. Durch den aufgebrachten Stützdruck und den zyklischen Vortrieb unterliegt der Porenwasserdruck im Bereich vor der Ortsbrust deutlichen Schwankungen, die vermutlich zu Umlagerungsprozessen im Boden führen. Dabei werden durch die Stützflüssigkeit auch zusätzliche Feinanteile in den Boden unmittelbar vor der Ortsbrust eingetragen. Zusätzlich werden durch die Abbauwerkzeuge beim Bodenabbau und vermutlich auch in eingeschränktem Umfang durch die Schneidradstruktur Spannungen in den Boden eingebracht, die zu einer Änderung der Eigenschaften unmittelbar an der Ortsbrust führen. In bestimmten Fällen konnte eine Verdichtung des Bodens in der Ortsbrust und zerbrochene Bodenkomponenten nachgewiesen werden (Kap. 4.2.2.1).

Im Bereich der Separationsanlage ist eine Beprobung des abgebauten Bodens im Regelfall zwar uneingeschränkt möglich. Allerdings lassen sich auch hier die ursprünglichen Eigenschaften des Bodens nur eingeschränkt rekonstruieren. Zunächst werden, wie oben beschrieben, die Eigenschaften des Bodens bereits beim Abbau verändert.

Anschließend erfolgt der Transport der abgebauten Bodenteile in der Stützflüssigkeit hinter das Schneidrad durch die Abbaukammer zur Förderleitung. Die Strömungsverhältnisse in der Abbaukammer wurden bisher nicht untersucht, die vertikale Sinkgeschwindigkeit der Bodenkomponenten ist jedoch sowohl für laminare als auch für turbulente Strömungsverhältnisse von der Korngröße und Dichte der Bodenkomponenten abhängig. Dieser Zusammenhang lässt die Annahme zu, dass abgebaute Bodenkomponenten in der Abbaukammer unter gleichen Umgebungsbedingungen mit zunehmender Korngröße und Dichte schneller in die Schildsohle absinken und die Förderleitung erreichen. Im Gegensatz dazu werden abgebaute Bodenkomponenten mit sinkender Korngröße und geringerer Dichte tendenziell länger in der Abbaukammer in Suspension gehalten. Entsprechende Untersuchungen zur Verweildauer abgebauter Bodenkomponenten in der Abbaukammer existieren jedoch nicht.

Am Eingang der Förderleitung werden durch den Rechen alle Bodenkomponenten zurückgehalten, die größer sind als die Maschenweite des Rechens. Diese Bodenkomponenten werden entweder durch den Einsatz des Steinbrechers zerkleinert oder so lange in der Abbaukammer gehalten, bis durch Abrieb und Zerbrechen bei Kollision mit anderen Bodenkomponenten oder der Schneidradstruktur die Größe der Bodenkomponenten die Maschenweite des Rechens unterschreitet.

Im Anschluss werden die Bodenkomponenten durch Förderpumpen und Förderleitung zur Separationsanlage transportiert. Untersuchungen zum weiteren Zerbrechen von Bodenkomponenten durch die mechanischen Einwirkungen der Förderpumpen und während dem Transport in der Rohrleitung existieren bisher nicht. 
Eine Aussage über den Fließzustand in der Förderleitung lässt die REYNOLDS-Zahl zu. Diese ergibt sich für Rohrströmungen aus Gl. 21:

$$
\mathrm{R}_{\mathrm{e}}=\frac{\mathrm{v}_{\mathrm{SF}} \cdot \mathrm{d}_{\mathrm{FL}}}{\mathrm{v}_{\mathrm{SF}}}
$$

Dabei ist $\mathrm{v}_{\mathrm{SF}}[\mathrm{m} / \mathrm{s}]$ die Strömungsgeschwindigkeit der Stützflüssigkeit in der Förderleitung, $\mathrm{d}_{\mathrm{FL}}[\mathrm{mm}]$ der Innendurchmesser der Förderleitung und $\mathrm{V}_{\mathrm{SF}}\left[\mathrm{m}^{2} / \mathrm{s}\right]$ die kinematische Viskosität der Stützflüssigkeit. Als Maximalzustand im Regelvortrieb liegt die Fließgeschwindigkeit $\mathrm{v}_{\mathrm{SF}}$ in der Förderleitung üblicherweise bei maximal $4 \mathrm{~m} / \mathrm{s}$, während $d_{\mathrm{FL}}$ häufig mit $300 \mathrm{~mm}$ bis $500 \mathrm{~mm}$ ausgelegt wird. Bei einer kinematischen Viskosität der Bentonitsuspension in der Förderleitung von ca. $1 \cdot 10^{-5}-2 \cdot 10^{-5} \mathrm{~m}^{2} / \mathrm{s}$ ergeben sich für die Reynolds Zahl $R_{e}$ aus Gl. 21 Werte von ca. 100.000 - 200.000. Die Reynolds Zahl liegt damit im Vortrieb deutlich oberhalb dem Grenzwert von 2.320, der als Beginn des Übergangsbereiches zwischen laminarem und turbulentem Fließen in Rohrleitungen angesetzt wird.

Die horizontale Fortbewegungsgeschwindigkeit der Bodenkörner in der Förderleitung und deren vertikale Sinkgeschwindigkeit in der Rohrleitung hängen wiederum von Korngröße und Dichte der Bodenkomponenten ab. Daraus folgt die Annahme, dass sich mit steigender Korngröße und Dichte die horizontale Fortbewegungsgeschwindigkeit der Bodenkomponenten im Vergleich zur Fließgeschwindigkeit der Stützflüssigkeit verringert. Gleichzeitig sinken die Bodenkomponenten zunehmend schneller zum Boden der Rohrleitung, wo sie im Extremfall rollend oder durch Saltation fortbewegt werden. Hier unterliegen die Bodenkomponenten Reibungskräften an der Rohrleitung, sodass die horizontale Fortbewegungsgeschwindigkeit weiter sinkt. Zusammenfassend werden die abgebauten Bodenkomponenten also vermutlich mit zunehmender Größe und Dichte mit sinkender Geschwindigkeit durch die Förderleitung transportiert. Der absolute Unterschied in der Transportdauer zwischen den kleinsten und größten enthaltenen Bodenkomponenten nimmt vermutlich mit steigender Leitungslänge und Steigung der Leitung z.B. am Ausgang eines Schachtes zu.

Die Beschreibung der mechanischen Abläufe beim Transport der abgebauten Bodenkomponenten von der Ortsbrust zur Separationsanlage zeigt, dass das in der Separationsanlage beprobte Kornspektrum eine Verschiebung der verschiedenen Korngrößen zueinander aufweist. Eine detaillierte Untersuchung der Zusammenhänge ist nicht Ziel dieser Arbeit. Wesentlich ist jedoch, dass das in der Separationsanlage beprobte Kornspektrum nicht zwingend der eigentlichen Korngrößenverteilung an der Ortsbrust entsprechen muss. Zudem werden alle in der Ortsbrust enthaltenen Bodenarten durch den Abbau und Transportvorgang durchmischt, sodass keine Aussage über die Verteilung verschiedener Bodenarten in der Ortsbrust möglich ist.

Innerhalb der Separationsanlage selbst sind in Abhängigkeit von deren Aufbau zwei Wege der Probengewinnung möglich. Es können Proben der Stützflüssigkeit aus der Förderleitung entnommen werden, sodass das gesamte aktuell in der Förderleitung befindliche Kornspektrum erfasst wird. Anschließend ist jedoch im Labor eine aufwändige Trennung der Bodenkomponenten von der Stützflüssigkeit notwendig. Die zweite Möglichkeit ist, die bereits aus der Stützflüssigkeit separierten Bodenkomponenten an den verschiedenen Siebstufen und Zyklonen oder Kammerfilterpressen zu beproben. Anschließend wird daraus durch entsprechende anteilige Vermengung der Einzelproben das ungefähre Kornspektrum in der Förderleitung wieder hergestellt.

Zusammenfassend ist bei der alleinigen Probenahme in der Separationsanlage also keine klare Aussage möglich, in wie weit das beprobte Kornspektrum und die Korneigenschaften dem abgebauten Boden an der Ortsbrust entsprechen. Zusätzlich ist keine klare Zuordnung zu einer genauen Vortriebsstationierung möglich. WENDL (2012) und WENDL et al. (2012) zeigen vor dem Hintergrund der beschriebenen Probleme für relativ homogene Baugrundverhältnisse auf, wie mithilfe einer täglichen indirekten Beprobung des Bodens in der Separationsanlage und Referenzierung mit an der Ortsbrust entnommenen Proben dennoch eine relativ umfassende Vortriebsdokumentation möglich ist. 


\subsubsection{Geotechnische Dokumentation in den Referenzprojekten}

Auf Basis der in Kap. 5.2.2.1 beschriebenen Anforderungen an die geotechnische Dokumentation wurden die aus den Referenzprojekten tatsächlich bestimmten geotechnischen Kennwerte ausgewertet. Die Ergebnisse sind als Übersicht in Tab. 12 zusammengefasst. Die Angaben in der Tabelle geben zusätzlich Information darüber, ob die Kennwerte vollständig für alle vorhandenen Bodenarten ermittelt wurden oder ob die Angaben unvollständig sind.

Tab. 12: Vergleich der Anforderungen an die geotechnischen Dokumentation im Hinblick auf den Verschleiß der Abbauwerkzeugverschleiß mit den tatsächlich bestimmten Kennwerten in den Referenzprojekten. Vollständig vorhandene Informationen sind mit $(\cdot)$ gekennzeichnet, unvollständige Informationen mit $(\circ)$, fehlende Informationen durch (-). Kennwerte die aufgrund nicht auftretender Bodenarten nicht zu bestimmen waren sind ausgestrichen.

\begin{tabular}{|c|c|c|c|c|c|c|c|c|c|c|c|}
\hline Referenzprojekt & 1 & 2 & 3 & 4 & 5 & 6 & 7 & 8 & 9 & 10 & 11 \\
\hline \multicolumn{12}{|c|}{ Für alle Bodenarten und Homogenbereiche: } \\
\hline Korngrößenverteilung & $\bullet$ & $\bullet$ & $\circ$ & $\bullet$ & $\bullet$ & $\bullet$ & $\bullet$ & $\bullet$ & $\bullet$ & ० & $\bullet$ \\
\hline Wichte unter Auftrieb $\gamma^{\prime}$ & $\circ$ & $\circ$ & $\circ$ & $\circ$ & $\circ$ & $\circ$ & $\circ$ & $\bullet$ & $\bullet$ & $\circ$ & $\circ$ \\
\hline Lagerungsdichte $\mathrm{I}_{\mathrm{D}}$ & $\circ$ & $\circ$ & $\circ$ & $\circ$ & $\circ$ & $\circ$ & - & - & - & $\circ$ & - \\
\hline Kohäsion c' & $\circ$ & $\circ$ & $\circ$ & $\circ$ & $\circ$ & $\circ$ & $\circ$ & $\bullet$ & $\bullet$ & $\circ$ & $\circ$ \\
\hline Reibungswinkel $\varphi^{\prime}$ & ० & $\circ$ & $\circ$ & $\circ$ & $\circ$ & $\circ$ & $\circ$ & $\bullet$ & $\bullet$ & $\circ$ & $\circ$ \\
\hline Porenanteil $n_{\text {por }}$ & $\bullet$ & - & - & - & - & - & $\circ$ & ० & $\circ$ & - & - \\
\hline Porenzahl $e_{\text {por }}$ & $\bullet$ & - & - & - & - & - & $\circ$ & ० & $\circ$ & - & - \\
\hline Durchlässigkeit $k_{f}$ & $\bullet$ & $\bullet$ & $\circ$ & $\bullet$ & $\bullet$ & $\circ$ & $\circ$ & $\bullet$ & $\bullet$ & $\circ$ & $\bullet$ \\
\hline Wasserspiegel $\mathrm{W}_{\mathrm{TA}}$ & $\bullet$ & $\bullet$ & $\bullet$ & $\bullet$ & $\bullet$ & $\bullet$ & $\bullet$ & $\bullet$ & $\bullet$ & $\bullet$ & $\bullet$ \\
\hline Überlagerung $\mathrm{H}_{\mathrm{TA}}$ & $\bullet$ & $\bullet$ & $\bullet$ & $\bullet$ & $\bullet$ & $\bullet$ & $\bullet$ & $\bullet$ & $\bullet$ & $\bullet$ & $\bullet$ \\
\hline LCPC-Abrasivitätsindex LAK & - & $\circ$ & - & $\circ$ & $\circ$ & - & - & $\bullet$ & $\bullet$ & - & - \\
\hline LCPC-Brechbarkeitsindex LBK & - & $\circ$ & - & ० & $\circ$ & - & - & $\bullet$ & $\bullet$ & - & - \\
\hline Äquivalenter Quarzanteil ÄQu & ० & $\bullet$ & $\circ$ & $\bullet$ & $\bullet$ & $\circ$ & $\circ$ & $\bullet$ & $\bullet$ & $\circ$ & $\circ$ \\
\hline \multicolumn{12}{|l|}{ Zusätzlich für bindige Bodenarten: } \\
\hline Plastizitätszahl I & $\bullet$ & - & - & - & $\circ$ & $\circ$ & $\circ$ & $\circ$ & $\circ$ & & $\bullet$ \\
\hline Konsistenzzahl $\mathrm{I}_{\mathrm{C}}$ & $\bullet$ & - & - & - & $\circ$ & $\circ$ & ० & $\circ$ & $\circ$ & & $\bullet$ \\
\hline Fließgrenze $w_{L}$ & $\bullet$ & $\circ$ & - & $\circ$ & $\circ$ & $\circ$ & $\circ$ & $\circ$ & $\circ$ & & $\bullet$ \\
\hline Ausrollgrenze $w_{p}$ & $\bullet$ & $\circ$ & - & ० & $\circ$ & $\circ$ & $\circ$ & $\circ$ & $\circ$ & & $\bullet$ \\
\hline Schrumpfgrenze $\mathrm{w}_{\mathrm{S}}$ & ० & - & - & - & - & - & - & - & - & & $\circ$ \\
\hline Wassergehalt w & $\bullet$ & - & $\circ$ & - & $\circ$ & $\circ$ & $\circ$ & ० & $\circ$ & & $\bullet$ \\
\hline Mineralogie & $\bullet$ & - & - & $\circ$ & - & - & - & - & - & & $\circ$ \\
\hline \multicolumn{12}{|c|}{ Zusätzlich für gemischt- und grobkörnige Böden: } \\
\hline Cerchar Abrasivitätsindex CAI & - & $\circ$ & - & $\circ$ & $\circ$ & - & - & $\circ$ & $\circ$ & - & - \\
\hline Kornform (Sphärizität) & - & $\circ$ & - & $\circ$ & $\circ$ & - & - & - & - & - & - \\
\hline Kornrundungsgrad & - & $\circ$ & - & $\circ$ & $\circ$ & - & - & - & - & - & - \\
\hline Mineralogische Zusammensetzung & - & $\circ$ & - & $\circ$ & $\circ$ & - & - & ० & $\circ$ & - & - \\
\hline
\end{tabular}


Die Ergebnisse in Tab. 12 zeigen im Vergleich zu den oben beschriebenen Anforderungen ein deutliches Problem auf. Im Hinblick auf den Umfang der ermittelten geotechnischen Kennwerte ist festzustellen, dass mit Ausnahme zweier Projekte die genannten Kennwerte durchgehend unvollständig vorliegen oder nicht ermittelt wurden. Die höchste Vollständigkeit ist bei den Kennwerten gegeben, die als Eingangsparameter für die Dimensionierung des Bauwerks (Statik), der TVM (Schildstatik) und des Stützdrucks $P_{S F}$ (Ortsbruststabilität) notwendig sind. Das sind im Wesentlichen die Korngrößenverteilung, die Scherparameter, Überlagerungshöhe $\mathrm{H}_{T A}$ und der Wasserspiegel $\mathrm{W}_{\text {TA }}$. Die Ursachen für die hohe Unvollständigkeit sind primär in den folgenden Punkten zu suchen:

- Technische Einschränkungen für die Probenahme.

- Wirtschaftliche Einschränkungen bei der Erkundung und Dokumentation.

Zusätzlich wurde untersucht, in welcher Form die in Tab. 12 zusammengefassten Daten vorliegen. Dazu wurde ausgewertet, ob alle Basisdaten aus Labor- und Feldversuchen angegeben sind, welche statistischen Maße der zentralen Tendenz aus den Basisdaten abgeleitet wurden und welche Angaben zur Schwankungsbreite der Kennwerte in der Folge gemacht wurden.

Hier ist in den Referenzprojekten keine durchgehende Methodik erkennbar. Die Basisdaten sind in der Regel nicht für alle Labor- oder Feldversuche vollständig angegeben. Als Maß der zentralen Tendenz wird im Regelfall ein Modalwert angegeben. Weniger verbreitet sind arithmetische Mittelwerte, der Median kommt in keinem Referenzprojekt zur Anwendung. Als Maß für die Schwankungsbreite werden im Wesentlich die Minimal- und Maximalwerte aus den Basisdaten verwendet, wobei in knapp einem Drittel der Projekte überhaupt keine Angaben zu möglichen Schwankungsbreiten gemacht werden. Die Verwendung der Standardabweichung als Maß für die Schwankungsbreite beschränkt sich auf die Projekte in denen auch der Arithmetische Mittelwert zur Anwendung kommt.

\subsubsection{Auslegung der Hydroschild TVM}

Die Berechnung und Analyse der Werkzeugstandgrößen macht, bezogen auf die TVM-Auslegung, die Dokumentation der folgenden Parameter erforderlich:

- Schneidraddurchmesser $D_{T V M}[m]$

- Schneidradöffnungsverhältnis $\mathrm{O}_{\mathrm{TVM}}[\%]$

- $\quad$ Länge der Schildmaschine $\mathrm{L}_{\mathrm{TVM}}[\mathrm{m}]$

- Spurradius der Abbauwerkzeuge $r_{s}[\mathrm{~mm}]$

- Spurbesatz $\mathrm{k}_{\mathrm{s}}[-]$

- Winkelabstand $\delta_{\mathrm{a}}$ und $\delta_{\mathrm{p}}\left[{ }^{\circ}\right] \mathrm{zu}$ angrenzenden Werkzeugen

- Höhe $h_{S R}, h_{S M}, h_{S T}, h_{R M}[m m]$ der Werkzeuge über dem Schneidradstahlbau

- Werkzeugabmessungen [mm]

- Angaben zu den Werkstoffeigenschaften der Abbauwerkzeuge 
Zusätzlich sind für eine erweiterte Analyse der Werkzeugstandgrößen Parameter aus der TVMAuslegung notwendig, die den Materialfluss in der Abbaukammer betreffen:

- Anzahl und Position von Rotoren und Statoren

- Anzahl und Position der Öffnungen für die Bentoniteinspeisung in die Arbeitskammer

- Maschenweite des Rechens vor der Förderleitung

- Konstruktive Ausbildung des Schild-Sohlsegmentes.

Alle oben genannten Parameter sind in der Anwenderdokumentation des Maschinenherstellers enthalten. Diese liegt für alle in Kap. 5.1 beschriebenen Referenzprojekten vor, sodass hieraus die notwendigen Daten für die Analyse entnommen werden konnten.

\subsubsection{Steuerung des Abbauprozesses}

Die Anforderungen an die Dokumentation der Steuerung des Abbauprozesses beinhalten im Vortrieb mit Hydroschild TVM sowohl Informationen zum Bodenabbau, als auch Informationen zum Förderkreislauf. Für die Berechnung der Werkzeugstandgrößen und deren Analyse sind dabei folgende $\mathrm{Pa}$ rameter notwendig, die bezogen auf die Bohrhübe $b$ der TVM vorliegen müssen:

- Schneidraddrehzahl $U\left[\mathrm{~min}^{-1}\right]$

- Schneidraddrehmoment $\mathrm{M}_{\mathrm{TVM}}[\mathrm{kNm}]$

- Penetration $\mathrm{p}[\mathrm{mm} / \mathrm{U}]$

- Vortriebspressenkraft $F_{V T P}[k N]$

- Anpresskraft Schneidradverschiebung $F_{S V}[k N]$

- Stützdruck im Abbauraum $P_{S F}$ [bar]

- Dichte der Bentonitsuspension in der Speiseleitung $\rho_{\mathrm{SL}}$ in $\mathrm{g} / \mathrm{cm}^{3}$

- Dichte der Bentonitsuspension in der Förderleitung $\rho_{\mathrm{SF}}$ in $\mathrm{g} / \mathrm{cm}^{3}$

Für eine erweiterte Analyse der Werkzeugstandgrößen kann die Einbeziehung weitere Parameter aus der Steuerung des Abbauprozesses sinnvoll sein. Dazu gehören die Spezifische Energie SE $\left[\mathrm{MJ} / \mathrm{m}^{3}\right]$, Schneidradkippmomente, Aktivität des Steinbrechers (Wischen/Brechen) und Alarmwerte von Verschleißerkennungssystemen sowie Metallabscheidern.

Alle oben genannten Parameter werden durch das Datenerfassungssystem der TVM in bestimmten Intervallen als Momentanwerte aufgezeichnet (Kap. 3.3.1). Diese Daten stehen aus allen in Kap. 5.1 beschriebenen Referenzprojekte zur Verfügung. 


\subsection{Verschleißtypen und Verschleißgrenzen}

Die Mechanik des Abbauprozesses, das daraus resultierende Ortsbrustbild, das entsprechende Verschleißbild der Abbauwerkzeuge und deren Verschleißgrenzen sind sehr eng miteinander verbunden, sodass eine gemeinsame Beschreibung und Analyse sinnvoll erscheint.

Grundlage dazu ist eine neue Definition der unterschiedlichen Verschleiß- und Schadenstypen an den Abbauwerkzeugen, der Schneidradstruktur und dem Förderkreislauf (Kap. 5.3.1). Diese wurde auf Basis der bestehenden Definitionen (Kap. 4.1.2) und den Verschleiß- und Schadensereignissen in den Referenzprojekten für die vorliegende Arbeit und als Empfehlung für zukünftige Projekte entwickelt.

Die Ergebnisse zu Verschleißbild, Ortsbrustbild, Abbaumechanik der Abbauwerkzeuge sind nachfolgend nach den verschiedenen Werkzeugtypen gegliedert. Zusätzlich sind die Ergebnisse zum Teil nach feinkörnigen, gemischtkörnigen und grobkörnigen Bodenarten unterteilt. Dabei ist zwischen den Bodenarten häufig ein fließender Übergang des Ortsbrust- und Verschleißbildes zu beobachten. Die Rückschlüsse auf die Abbaumechanik erfolgen rein deduktiv aus den in den Referenzprojekten angetroffenen Verschleiß- und Ortsbrustbildern.

Die Referenzprojekte decken nicht alle theoretisch möglichen Kombinationen an unterschiedlichen geotechnischen Bedingungen, Werkzeugbestückungen des Schneidrades und Einstellung der Steuerung des Abbauprozesses ab. Es ist deshalb nicht auszuschließen, dass in zukünftigen Projekten weitere Verschleiß- und Ortsbrustbilder und Zusammenhänge zur Abbaumechanik der Abbauwerkzeuge gefunden werden, die in der Definition der Verschleiß- und Schadenstypen (Kap. 5.3.1) und der Beschreibung für die einzelnen Abbauwerkzeugtypen in Kap. 5.3.2 bis Kap. 5.3.5 ergänzt werden müssen.

Zu den Verschleißgrenzen der Abbauwerkzeuge beim Vortrieb mit Hydroschild TVM in Lockergesteinen fehlen bisher klare bzw. passende Angaben (Kap. 5.2.1.3). Deshalb wurden für jeden Werkzeugtyp anhand der zuvor beschriebenen Verschleißbilder entsprechende Verschleißgrenzen als Grundlage für die vorliegende Arbeit und Empfehlung für zukünftige Projekte entwickelt. Die Verschleißgrenzen der Abbauwerkzeuge werden durch den Zustand definiert, in dem das Werkzeug aufgrund von fortschreitendem Verschleiß einen Funktionsverlust erleidet. Im Hinblick auf die drei Hauptaufgaben der Abbauwerkzeuge bedeutet das, dass eine oder mehrere der folgenden Funktionen aufgrund des Verschleißzustandes nicht mehr bewältigt wird:

- Bodenabbau

- Materialförderung (Schälmesser \& Räumer)

- Verschleißschutz des Werkzeughalters und der Schneidradstruktur

Die angegebenen Verschleißgrenzen spiegeln die in den Referenzprojekten dokumentierten Erfahrungen wieder. Sie wurden mit dem Ziel einer möglichst dauerhaften Erhaltung des Ausgangszustandes der Schneidradstahlstruktur und der Werkzughalter entwickelt, um das Risiko für zeit- und kostenintensive Reparaturen an diesen Bauteilen in der Vortriebsphase möglichst gering zu halten. Es ist deshalb darauf hinzuweisen, dass auf Basis einer höheren oder niedrigeren Risikobereitschaft in zukünftigen Projekten die angegebenen Verschleißgrenzen auch entsprechend angepasst werden können.

\subsubsection{Definition der Verschleiß- \& Schadenstypen}

Die Begriffe Primär- und Sekundärverschleiß, sowie verschiedene Schadenstypen werden in der Praxis sehr flexibel verwendet, um den Verschleiß oder Schäden an verschiedenen Maschinenteilen (Abbauwerkzeuge, Schneidradstruktur, Förderkreislauf) zu beschreiben (Kap. 4.1.2). Die bisherigen Definitionen sind einerseits unvollständig, da Schadensereignisse an den Abbauwerkzeugen und sonstigen Fördereinrichtungen nicht ausreichend berücksichtigt werden. Andererseits sind die Definitionen nicht zufriedenstellend, da keine ausreichende Trennung von Verschleiß- und Schadensereignissen nach deren Ort und Ursache erfolgt. 
Im Sinne einer klaren Abtrennung der genannten Begriffe und einer eineindeutigen Verwendung wurden für die vorliegende Arbeit und als Empfehlung für die Anwendung in zukünftigen Projekten die Definitionen der Verschleiß- und Schadensarten in Tab. 13 entwickelt.

Der Grundgedanke zu diesen Definitionen ist eine saubere Trennung zwischen Verschleiß- und Schadensereignissen nach deren Ort an Abbauwerkzeugen oder der Schneidradstruktur. Zusätzlich werden die Begriffe anhand der gegebenen Kriterien nach der jeweiligen Verschleiß- und Schadensursache getrennt. Diese Gliederung ist sowohl für die nachfolgende Beschreibung der Verschleiß- und Schadensbilder an den Abbauwerkzeugen, als auch für die daraus folgenden Rückschlüsse auf die zu Grunde liegende Abbaumechanik zwingend notwendig.

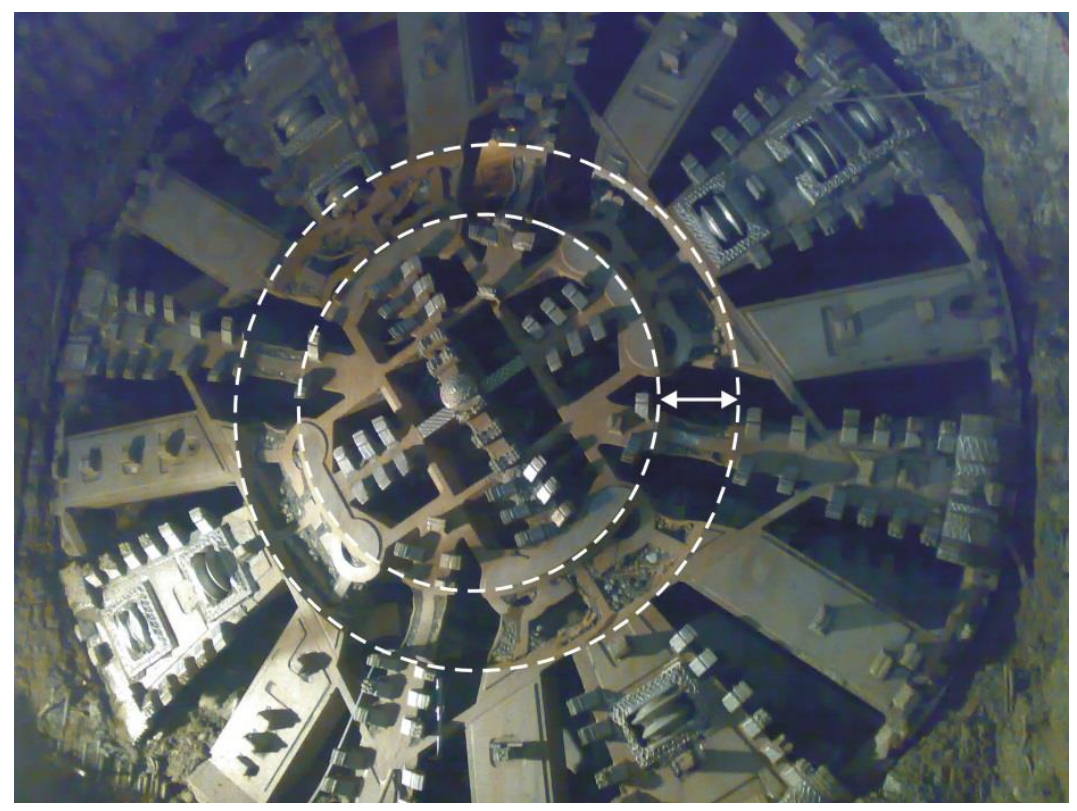

\begin{abstract}
Abb. 36: Schwerer Verschleißschaden an der Schneidradstahlstruktur im Bereich des Schneidradzentrums. Die Stahlstruktur des Schneidrades ist in Form einer umlaufenden Furche (weiße Markierung) bis zur rückwertigen Befestigung der Verbindungsstreben zum Antrieb verschlissen.
\end{abstract}

Die in Tab. 13 angegebenen Definitionen zu Verschleiß und Schäden an der Schneidradstruktur (Abb. 36) und dem Förderkreislauf finden in der vorliegenden Arbeit nur untergeordnet weitere Verwendung. Sie haben dennoch im Verlauf dieser Arbeit bereits eine hohe Praxisrelevanz gewonnen, weil die saubere begriffliche Trennung der Verschleiß- und Schadensarten nach Ort und Ursachen eine sinnvolle Aufarbeitung von Projekterfahrungen und Bearbeitung von Problemen in laufenden Projekten ermöglicht.

Im Hinblick auf Abbauwerkzeuge existiert in der Praxis für Schälmesser, Stichel und Räumer eine Grauzone am Übergang von Primär- und Sekundärverschleiß hin zu einem Verschleißschaden. Sobald die Abbauwerkzeuge ihren Verschleißschutz vollständig verloren haben, besteht nach der Definition in Tab. 13 ein Verschleißschaden. Tatsächlich nehmen die Abbauwerkzeuge aber immer noch mit verminderter Effektivität am Bodenabbau und an der Materialförderung teil, und schützen den Werkzeughalter und die Schneidradstruktur noch bedingt vor Verschleiß. Diese Grauzone wird häufig als Notlaufeigenschaft der Werkzeuge bezeichnet.

Das Überschreiten der Verschleißgrenze zieht also nicht in jedem Fall einen sofortigen Verschleißschaden an Werkzeughaltern oder Schneidradstruktur nach sich. Jedoch nimmt die Sanierbarkeit der Werkzeuge (Schneidrollen) rapide ab und die Verschleißrate $c_{v}$ an den Werkzeugen nimmt aufgrund des fehlenden Verschleißschutzes vermutlich deutlich zu (Kap. 5.5.4). Das Überschreiten der festgelegten Verschleißgrenzen wird deshalb in der Datenanalyse (Kap. 5.6) unmittelbar als Verschleißschaden definiert. 
Tab. 13: Definition und Klassifikation der Verschleiß- und Schadenstypen an Abbauwerkzeugen und Schneidradstruktur.

\begin{tabular}{|c|c|c|}
\hline Тур & $\begin{array}{c}\text { Abbauwerkzeuge } \\
\text { (Schneidrollen, Schälmesser Stichel \& Räumer) }\end{array}$ & $\begin{array}{c}\text { Schneidradstruktur } \\
\text { (Werkzeughalter, Gehäuse, Verschleißschutz, Grill- } \\
\text { bar, Stahlbau) }\end{array}$ \\
\hline 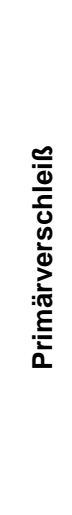 & $\begin{array}{l}\text { Primärverschleiß ist: } \\
\text { - Stetiger Massenverlust bzw. Abrieb } \\
\text { - Bis zum Erreichen der Verschleißgrenze } \\
\text { Primärverschleiß entsteht an: } \\
\text { - Werkzeugteilen die planmäßig beim Bodenabbau } \\
\text { in die Ortsbrust eingreifen. } \\
\text { Primärverschleiß entsteht bei: } \\
\text { - Aktivem Einsatz } \\
\text { - Kontakt mit dem Boden in der Ortsbrust } \\
\text { - Mit dem Ziel bzw. Ergebnis des Bodenabbaus }\end{array}$ & $\begin{array}{l}\text { Primärverschleiß entfällt für die } \\
\text { Schneidradstruktur weil: } \\
\text { - Kein aktiver Einsatz } \\
\text { - Kein Kontakt mit der Ortsbrust } \\
\text { - Mit dem Ziel bzw. Ergebnis des Bodenabbaus }\end{array}$ \\
\hline 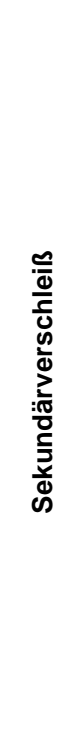 & $\begin{array}{l}\text { Sekundärverschleiß ist: } \\
\text { - Stetiger Massenverlust bzw. Abrieb } \\
\text { - Bis zum Erreichen der Verschleißgrenze } \\
\text { Sekundärverschleiß entsteht an: } \\
\text { - Allen Werkzeugteilen } \\
\text { 1) Sekundärverschleiß entsteht bei: } \\
\text { - Aktivem Einsatz } \\
\text { - Kontakt mit bereits abgebauten Bodenteilen } \\
\text { 2) Sekundärverschleiß entsteht bei: } \\
\text { - Passivem Einsatz } \\
\text { - Kontakt mit der Ortsbrust } \\
\text { 3) Sekundärverschleiß entsteht bei: } \\
\text { - Passivem Einsatz } \\
\text { - Kontakt mit bereits abgebautem Bodenteilen }\end{array}$ & $\begin{array}{l}\text { Sekundärverschleiß ist: } \\
\text { - Stetiger Massenverlust bzw. Abrieb } \\
\text { Sekundärverschleiß entsteht an: } \\
\text { - Allen Teilen der Schneidradstruktur } \\
\text { Sekundärverschleiß entsteht bei: } \\
\text { - Kontakt mit bereits abgebauten Bodenteilen }\end{array}$ \\
\hline 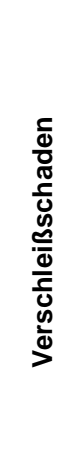 & $\begin{array}{l}\text { Ein Verschleißschaden ist: } \\
\text { - Stetiger Massenverlust bzw. Abrieb } \\
\text { - Überschreiten der Verschleißgrenze } \\
\text { Ein Verschleißschaden entsteht an: } \\
\text { - Allen Werkzeugteilen. } \\
\text { Ein Verschleißschaden entsteht bei: } \\
\text { - Aktivem oder passivem Einsatz } \\
\text { - Kontakt mit der Ortsbrust } \\
\text { - Kontakt mit bereits abgebautem Boden }\end{array}$ & $\begin{array}{l}\text { Ein Verschleißschaden ist: } \\
\text { - Stetiger Massenverlust bzw. Abrieb } \\
\text { - Überschreiten der Verschleißgrenze } \\
\text { Ein Verschleißschaden entsteht an: } \\
\text { - Allen Teilen der Schneidradstruktur. } \\
\text { Ein Verschleißschaden entsteht bei: } \\
\text { - Kontakt mit der Ortsbrust } \\
\text { - Kontakt mit bereits abgebautem Boden }\end{array}$ \\
\hline
\end{tabular}




\begin{tabular}{|c|c|c|}
\hline 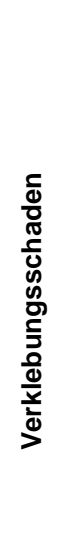 & $\begin{array}{l}\text { Ein Verklebungsschaden ist: } \\
\text { - Stetiger einseitiger Massenverlust bzw. Abrieb } \\
\text { - Unabhängig von der Verschleißgrenze } \\
\text { Ein Verklebungsschaden entsteht an: } \\
\text { - Drehend gelagerten Werkzeugen durch Blockie- } \\
\text { ren } \\
\text { - An allen Werkzeugteilen } \\
\text { Ein Verklebungsschaden entsteht bei: } \\
\text { - Verkleben von bereits abgebauten Bodenteilen } \\
\text { zwischen Werkzeug (drehend) und zugehörigem } \\
\text { Gehäuse (stehend), oder zwischen drehenden } \\
\text { und stehenden Werkzeugteilen. }\end{array}$ & $\begin{array}{l}\text { Verklebungsschäden entfallen für die } \\
\text { Schneidradstruktur. }\end{array}$ \\
\hline 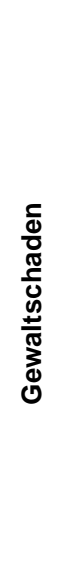 & $\begin{array}{l}\text { Ein Gewaltschaden ist: } \\
\text { - Deformation } \\
\text { - Sprödbruch } \\
\text { - Mit unmittelbarem Funktionsverlust oder vollstän- } \\
\text { digem Verlust des Werkzeuges } \\
\text { Ein Gewaltschaden entsteht an: } \\
\text { - Allen Werkzeugteilen } \\
\text { Ein Gewaltschaden entsteht bei: } \\
\text { - Kontakt mit festen natürlichen oder künstlichen } \\
\text { - Kindernissen in der Ortsbrust } \\
\text { Kontakt mit losen natürlichen oder künstlichen }\end{array}$ & $\begin{array}{l}\text { Ein Gewaltschaden ist: } \\
\text { - Deformation } \\
\text { - Sprödbruch } \\
\text { Ein Gewaltschaden entsteht an: } \\
\text { - Allen Teilen der Schneidradstruktur } \\
\text { Ein Gewaltschaden entsteht bei: } \\
\text { - Kontakt mit festen natürlichen oder künstlichen } \\
\text { Hindernissen in der Ortsbrust } \\
\text { - Kontakt mit losen natürlichen oder Künstlichen } \\
\text { Hindernissen in der Abbaukammer. }\end{array}$ \\
\hline
\end{tabular}

\subsubsection{Schneidrollen}

\subsubsection{Bodenabbau und Primärverschleiß}

\section{Ortsbrustbild}

Schneidrollen werden üblicherweise vorlaufend zu Schälmessern und Räumern eingesetzt. Damit ist das Ortsbrustbild beim Bodenabbau mit Schneidrollen immer auch von den nachlaufenden Werkzeugen beeinflusst. Grundsätzlich kann jedoch in den Referenzprojekten beobachtet werden, dass durch die Schneidringe kein flächiger Bodenabbau in der Ortsbrust erfolgt. Der Boden wird zwischen den Schneidspuren der Schneidringe nicht vollständig gelöst, sodass zwischen den Schneidspuren Grate entstehen, die erst durch die nachlaufenden Schälmesser abgebaut werden. Diese Beobachtungen stimmen mit bestehenden Erfahrungen zum Ortsbrustbild überein (Kap. 4.2.2.1).

Form und Höhe der Grate sind in den Referenzprojekten in Abhängigkeit der Bodeneigenschaften unterschiedlich ausgeprägt. Feinkörnige Böden bilden die Form der Schneidringe und nachlaufenden Schälmesser sehr genau ab, sodass sich eher breite kastenförmige Grate ergeben, die von schmalen Rinnen getrennt werden, die durch die Schneidringe gebildet werden (Abb. 37). Grobkörnige Böden bilden im Gegensatz dazu mit zunehmender Korngröße flachere und unregelmäßig geformte Grate aus, die von breiteren Rinnen getrennt werden (Abb. 38). Die Höhe der Grate bzw. die Tiefe der Rinnen entspricht maximal dem Vorlauf der Schneidrollen vor den Schälmessern. 


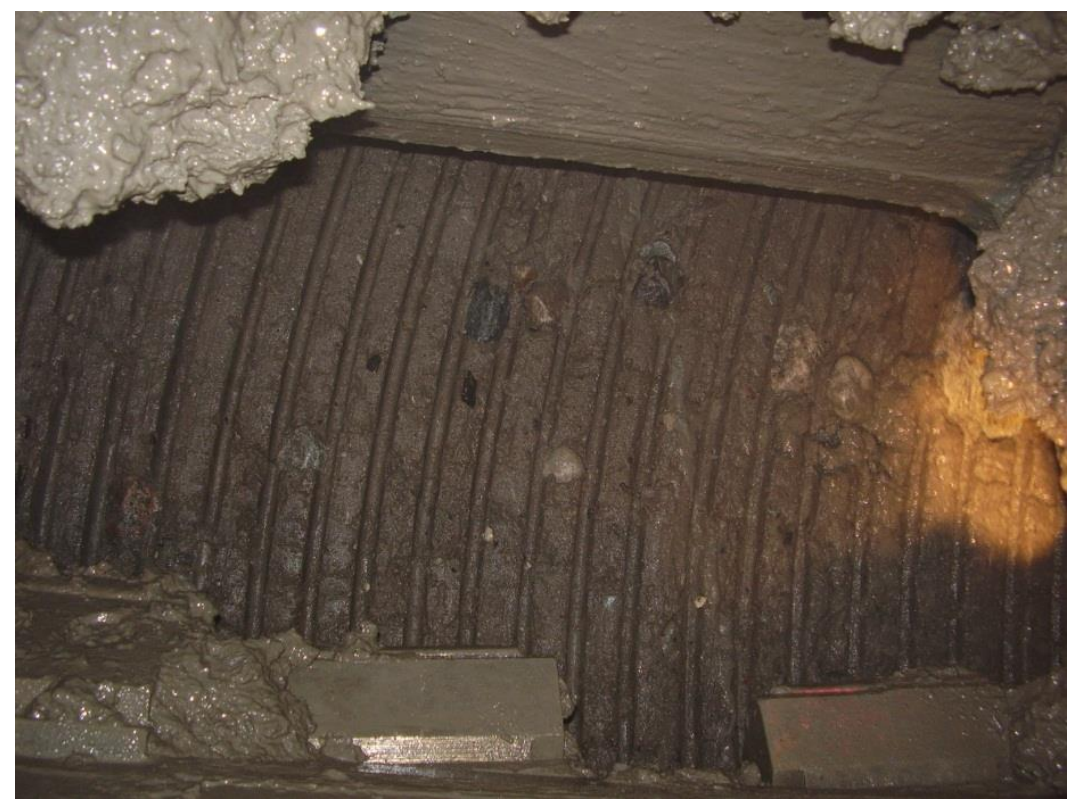

Abb. 37: Blick auf die Ortsbrust durch eine Schneidradöffnung in feinkörnigem Geschiebemergel mit eingelagerten Kieskomponenten. Die Schneidspuren der Schneidrollen werden durch den Boden in der Ortsbrust exakt nachgebildet. (Herrenknecht AG)

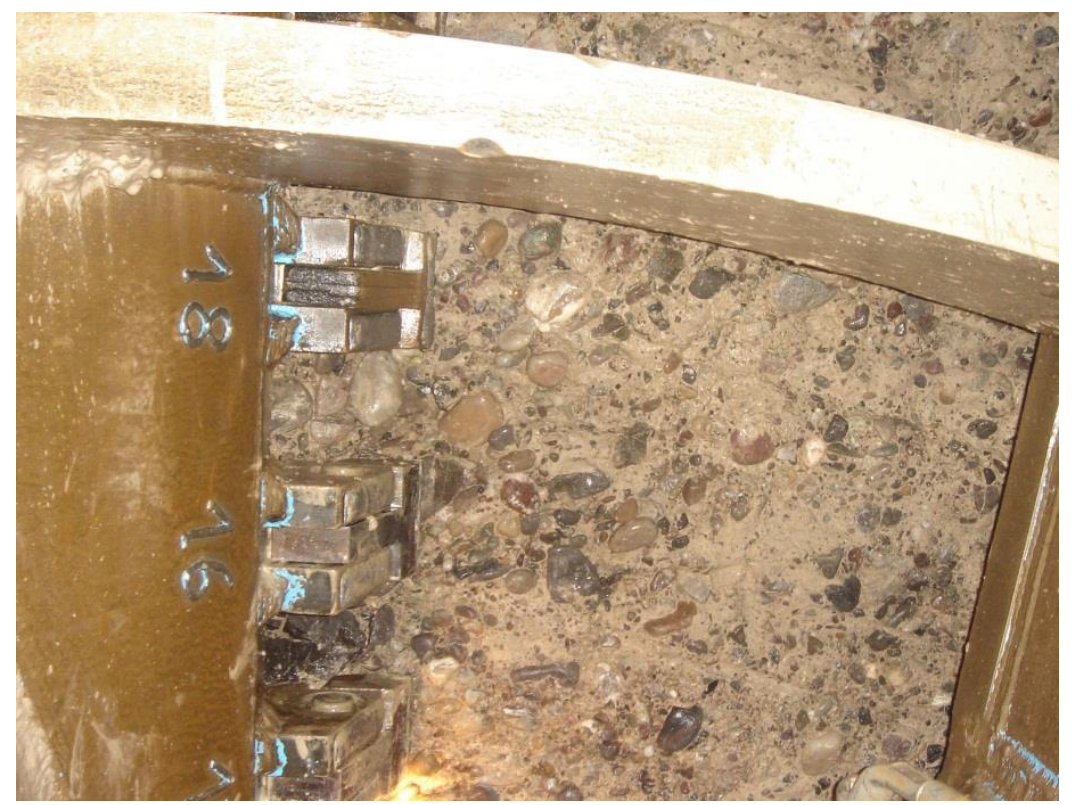

Abb. 38: Blick auf die Ortsbrust durch eine Schneidradöffnung in mitteldicht gelagerten sandigen Kiesen. Der Bodenabbau erfolgt mit vorlaufenden Schneidrollen und nachlaufenden Schälmessern. Die Schneidspuren der Schneidrollen sind nur sehr undeutlich in der Ortsbrust zu erkennen. (Herrenknecht AG)

\section{Verschleißbild}

Die Schneidringe in den Referenzprojekten zeigen grundsätzlich eine Zuspitzung der Schneide. Die Form der Zuspitzung unterscheidet sich ähnlich wie das Ortsbrustbild zwischen feinkörnigen Böden und grobkörnigen Böden.

In feinkörnigen und gemischtkörnigen Böden bis zu einer maximalen Korngröße von ca. Mittelsand $(0,6 \mathrm{~mm})$ findet an den Schneidringen nahezu kein radialer Abrieb statt. Der Abrieb konzentriert sich auf die Seitenflächen des Schneidrings. Die Zuspitzung des Schneidrings kann bei entsprechend lan- 
gem Einsatz dabei nahezu die Form einer Messerschneide erreichen, sobald die beiden Seitenflächen des Schneidrings so stark abgerieben sind, dass der Schneidring keine Lauffläche mehr aufweist. Erst bei Überschreitung dieses Punktes nimmt anschließend auch der radiale Durchmesser des Schneidrings durch den Abrieb deutlich ab. Auf den Seitenflächen der Schneidringe sind deutliche radial verlaufende Kratzer zu sehen (Abb. 39).

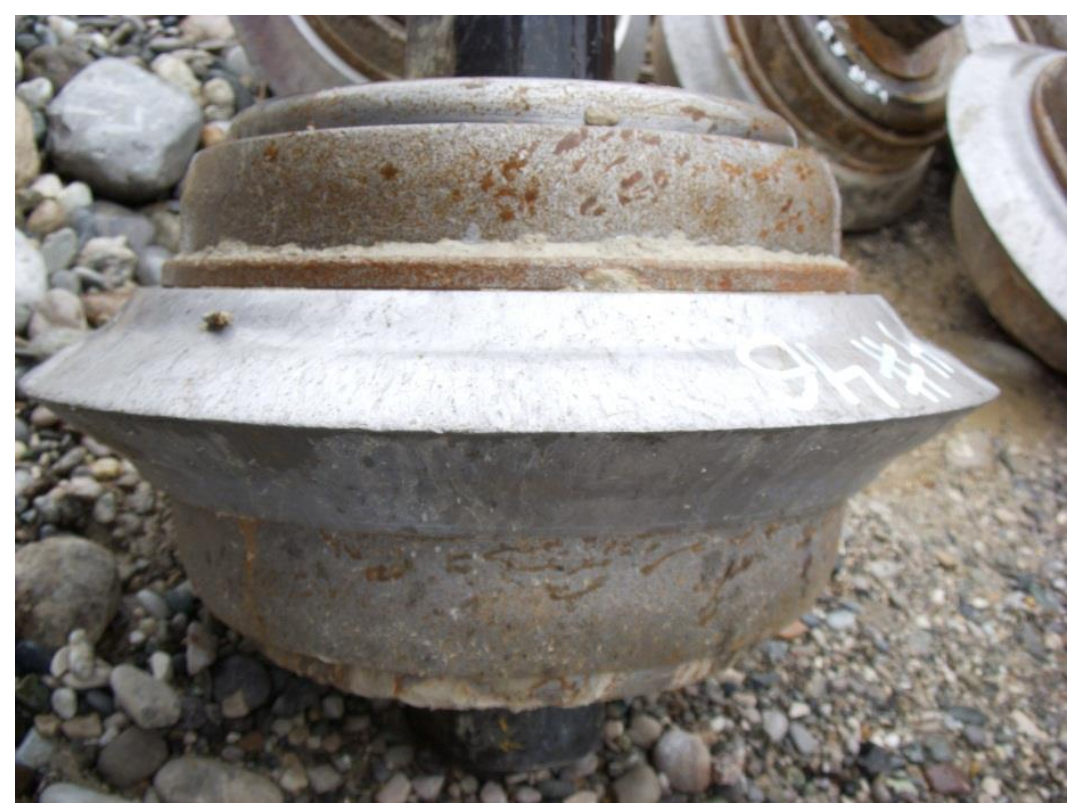

\begin{abstract}
Abb. 39: Schneidrolle nach dem Einsatz in dicht gelagerten, sandigen Kiesen. Die Schneide des Schneidrings ist gegenüber der ursprünglichen Form deutlich zugespitzt. Auf der hell scheinenden Flanke des Schneidrings oberhalb der Bildmitte sind radial verlaufende Kratzer zu erkennen.
\end{abstract}

In gemischtkörnigen Böden mit maximaler Korngröße größer als Mittelsand und grobkörnigen Böden ist im Gegensatz dazu eher eine kegelförmige Zuspitzung der Schneidringe zu beobachten. Der Abrieb konzentriert sich auf die Seitenflächen der Schneidringe, als auch verstärkt an den Kanten zwischen Lauffläche und Seitenflächen. Auf der Lauffläche selbst ist nur in deutlich geringerem Umfang Abrieb zu erkennen. Durch den verstärkten Abrieb an den beiden Kanten wird die Lauffläche des Schneidrings deutlich schneller aufgebraucht als in feinkörnigen Böden, sodass der Durchmesser des Schneidrings früher beginnt abzunehmen. Auf den Seitenflächen des Schneidrings sind ähnlich wie in feinkörnigen Böden radiale Kratzer zu erkennen, die jedoch deutlich unregelmäßiger verlaufen.

\title{
Rückschlüsse auf die Abbaumechanik
}

Das Ortsbrustbild beim Bodenabbau mit Schneidrollen in Lockergesteinen und das zugehörige Verschleißbild der Schneidrollen lassen den Schluss zu, dass durch die Schneidrollen kein durchgehender Bodenabbau zwischen den Schneidspuren erfolgt. Durch die Schneidringe erfolgt vielmehr eine Verdrängung des Bodens in der Ortsbrust.

In feinkörnigen Böden und gemischtkörnigen Böden bis zu einer maximalen Korngröße von ca. Mittelsand wird der Boden unterhalb der Lauffläche des Schneidrings vermutlich in Form eines Spans in die Ortsbrust hinein gedrückt. Dabei findet in der Lauffläche kaum Relativbewegung zwischen Stahl und Boden statt, sodass auch nahezu kein Abrieb entsteht. Das verdrängte Bodenvolumen unterhalb der Lauffläche wird dabei vermutlich ähnlich wie bei einem Grundbruch parallel zur Ortsbrust zur Seite hin abgeschert. Die Scherung erfolgt dabei hin zum Bereich zwischen den Schneidspuren der Schneidrollen. Hier wir der Boden durch die nachlaufenden Schälmesser abgebaut. Eine Überprüfung dieses Mechanismus ist im Vortrieb aufgrund der Unzugänglichkeit nicht möglich. Eine Verdichtung des feinkörnigen Bodens während der Scherung ist aufgrund der Wassersättigung und der geringen Durchlässigkeit nicht gegeben. 
Auch an den Seitenflächen des Schneidrings wird durch dessen Geometrie Boden zur Seite hin verdrängt. Dabei ergibt sich eine deutlich Relativbewegung zwischen Stahl und Boden in der Seitenfläche. Durch den Widerstand des Bodens gegen die Verdrängung zur Seite hin entsteht gleichzeitig eine Spannung normal zu den Seitenflächen des Schneidrings, sodass sich hier Abrieb an der Oberfläche ergibt. Ähnlich wie unterhalb der Lauffläche wird auch der Boden an den Seitenflächen des Schneidrings während der Verdrängung vermutlich zur Seite hin in den Bereich zwischen den Schneidspuren abgeschert und dort durch die nachlaufenden Schälmesser abgebaut. Trotz der seitlichen Verdrängung des Bodens wird aufgrund des bindigen Charakters ursächlich nahezu kein Boden zwischen den Schneidspuren gelöst, sodass die Tröge der Schneidringe in der Ortsbrust deren Form sehr genau nachbilden (Abb. 40).

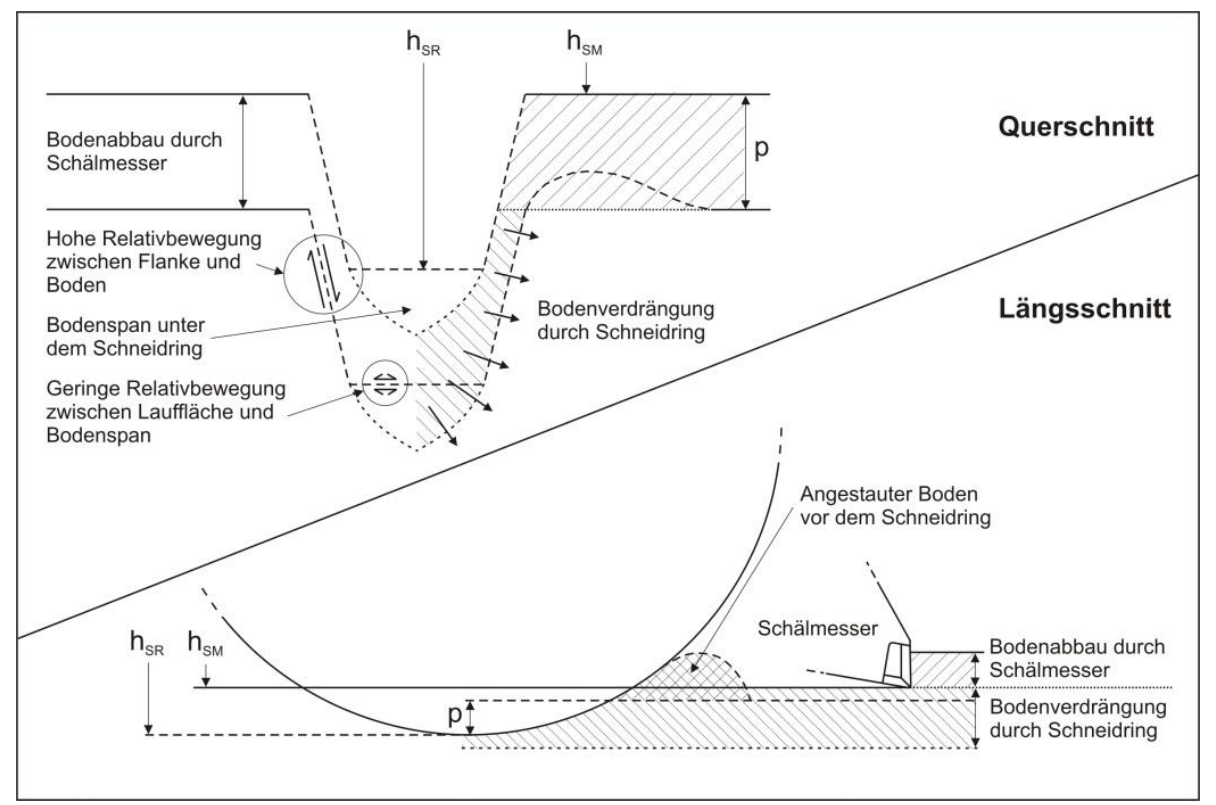

Abb. 40: Schematische, unmaßstäbliche Darstellung der Bodenverdrängung durch Schneidringe und Bodenabbau durch die nachlaufenden Schälmesser in der Ortsbrust als Quer- und Längsschnitt einer Schneidspur.

Der Mechanismus des Bodenabbaus durch Schneidrollen unterscheidet sich in gemischtkörnigen Böden mit einer maximalen Korngröße größer als ca. Mittelsand und in grobkörnigen Böden nicht von feinkörnigen Böden. Unterhalb der Lauffläche des Schneidrings bildet sich aufgrund der Zunahme der Korngröße im Verhältnis zur Breite des Schneidrings jedoch vermutlich kein klar definierbarer Bodenspan mehr aus, der in die Ortsbrust hineingedrückt wird. In der Lauffläche des Schneidrings treten vermutlich zunehmend Kontakte mit Bodenkomponenten auf, die aufgrund ihrer Form und Lage in der Ortsbrust von der Lauffläche zur Seite hin verdrängt werden, bzw. über die Kante zwischen Lauf- und Seitenfläche abgleiten. Dadurch entsteht an dieser Kante deutlicher Abrieb, wie er im Verschleißbild der Schneidringe beobachtet werden kann. Zusätzlich dazu kann es durch die Verdrängung des Bodens unter der Lauffläche bei günstiger Korngrößenverteilung und Wasserdurchlässigkeit, sowie durch Eintrag von Feinmaterial aus der Stützflüssigkeit auch zu einer Verdichtung des Bodens kommen (Kap. 4.2.2.1). Der Verdrängungswiderstand des Bodens kann dann ausreichend hoch sein, dass auch einzelne Bodenkomponenten in der Ortsbrust zerbrochen werden.

An den Seitenflächen des Schneidrings wird der Boden wiederum zur Seite hin in den Bereich zwischen den Schneidspuren verdrängt. Mit zunehmend rolligem Charakter des Bodens werden dabei unmittelbar angrenzend an den Schneidring auch einzelne Bodenkomponenten aus der Ortsbrust gelöst, sodass sich in der Ortsbrust breitere Furchen und flachere Grate zwischen den Schneidspuren ergeben. Analog zu feinkörnigen Böden ergibt sich in der Seitenfläche des Schneidrings eine deutliche Relativbewegung zwischen Stahl und Boden. Durch den Widerstand des Bodens gegen die Verdrängung zur Seite hin entsteht gleichzeitig eine Spannung normal zu den Seitenflächen des Schneidrings, sodass sich wiederum Abrieb an der Oberfläche ergibt. 


\subsubsection{Sekundärverschleiß}

Schneidrollen werden unabhängig von der Schneidraddrehrichtung aktiv eingesetzt. Sekundärverschleiß an Schneidrollen entsteht entsprechend der Definition in Kap. 5.3.1 folglich nur durch bereits abgebauten Boden an Teilen der Schneidrolle, die nicht planmäßig mit der Ortsbrust in Berührung kommen. Der Sekundärverschleiß an Schneidrollen ist damit unabhängig vom Ortsbrustbild, sodass dessen Beschreibung hier entfällt.

\section{Verschleißbild}

Aus den Referenzprojekten lassen sich drei Phänomene zum Sekundärverschleiß an Schneidrollen zusammenfassen.

Zunächst ist häufig unterhalb des Schneidrings sowohl am Sicherungsring, als auch am Grundkörper Abrieb zu erkennen. Dieser kann einerseits relativ gleichmäßig über die Breite des Rollengrundkörpers verteilt sein. Häufig ist jedoch auch eine Konzentration des Abriebs auf den Bereich direkt angrenzend an die Auflagefläche des Schneidrings feststellbar, sodass sich hier zum Teil zwei seichte umlaufende Furchen im Rollengrundkörper ergeben. In beiden Fällen kann in Abhängigkeit des Verschleißzustandes des Schneidrings zum Teil eine erhebliche Ausdünnung des Rollengrundkörpers beobachtet werden.

Der Sekundärverschleiß greift an den Schneidrollen auch im Bereich des Dichtspalts zwischen Rollengrundkörper und Deckel an. Der Spalt kann dabei durch Abrieb an der Innenseite des Rollengrundkörpers und am Außenradius des Deckels zum Teil deutlich erweitert werden.

Abschließend tritt auch an den Gleitringdichtungen der Schneidrollen Verschleiß auf. In ausgebautem Zustand sind auf den Laufflächen der Gleitringdichtungen häufig drei radiale Zonen sichtbar:

- Äußere Zone: Hier sind die Gleitringe bereits verschlissen. Es sind umlaufend feine Kratzer durch feines Bodenmaterial und Korrosionsspuren erkennbar.

- Dicht-Zone: Die eigentliche Abdichtung zwischen den beiden Gleitringen erfolgt in einem schmalen, umlaufend glänzend polierten Band.

- Innere Zone: Hier ist der ursprüngliche matte Bearbeitungszustand der Gleitringe noch vorhanden. Aufgrund der Ölschmierung sind keine Korrosionspuren vorhanden.

Die Dicht-Zone zwischen den beiden Gleitringen wandert durch Verschleiß zunehmend nach innen. Erreicht sie den Innenradius der Gleitringe, so ist die Dichtung vollständig verschlissen. Stützflüssigkeit und Feinmaterial können dann zunehmend in die Lagerkammer der Schneidrollen eindringen (Abb. 41). Im Vortrieb ist der Zustand der Gleitringdichtungen visuell nicht feststellbar. In einigen Fällen erhöht sich jedoch durch eindringendes Feinmaterial und Stützflüssigkeit die Reibung in den Lagern der Schneidrollen, sodass zum Teil eine deutliche Erwärmung der Schneidrollen feststellbar ist.

\section{Rückschlüsse auf den Materialfluss}

Der Verschleiß im Bereich des Dichtspalts der Schneidrollen und der Verschleiß der Gleitringdichtungen haben keine hohe Aussagekraft für den Materialfluss beim Bodenabbau und in der Abbaukammer. Sie zeigen lediglich an, dass in der Stützflüssigkeit feinkörniges, abrasives Material vorhanden ist.

Im Gegensatz dazu lässt der Verschleiß des Rollengrundkörpers und des Sicherungsrings direkt angrenzend an den Schneidring den Schluss zu, dass der während dem Abbau verdrängte Boden zunächst radial am Schneidring Richtung Rollengrundkörper abfließt. Dieser Mechanismus passt zum Bild der seitlichen Bodenverdrängung, das sich aus dem Verschleißbild der Schneidringe (Kap. 5.3.2.1) ergibt. Entscheidenden Einfluss hat dabei vermutlich auch der Abstand zwischen Ortsbrust und Rollengrundkörper. Sinkt der Durchmesser des Schneidrings durch Primärverschleiß, nimmt auch der Abstand des Rollengrundkörpers zur Ortsbrust ab. Die Wahrscheinlichkeit für einen Kontakt zwischen abgebautem Boden und dem Rollengrundkörper steigt damit an. 


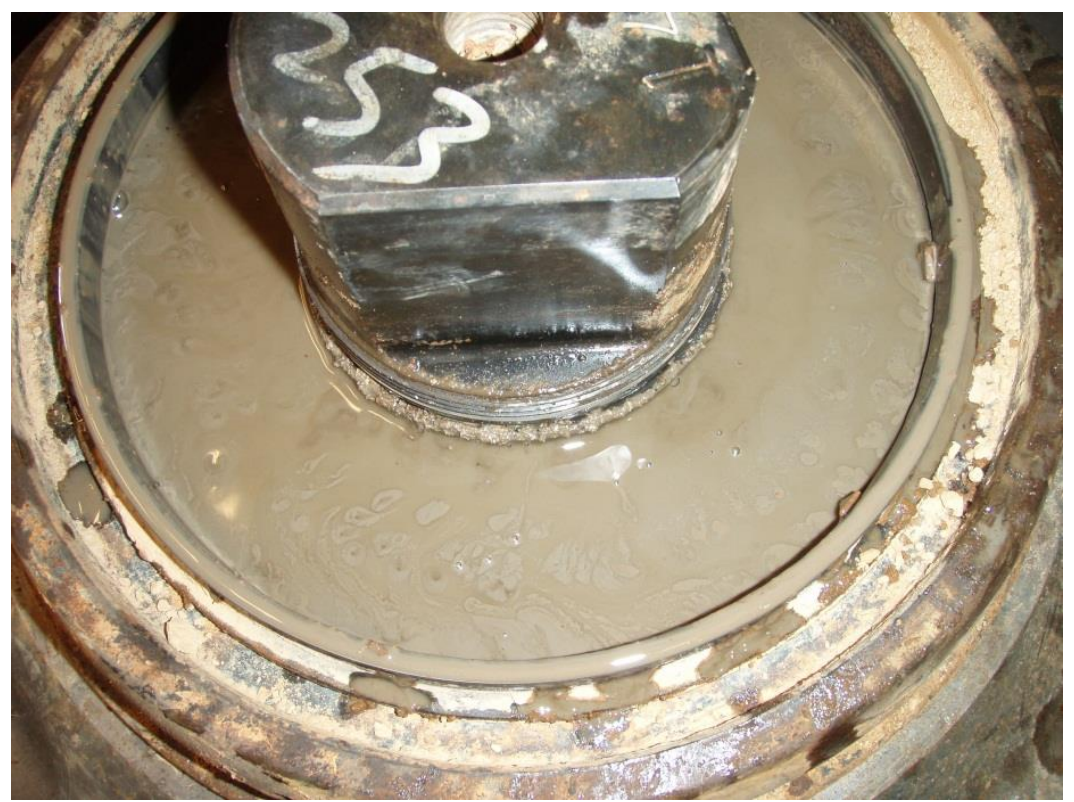

Abb. 41: Geöffnete Schneidrolle mit verschlissener Gleitringdichtung. Die Lagerkammer ist mit Bentonitsuspension gefüllt. (Herrenknecht $A G)$

Zusätzlich hat vermutlich die Menge an bereits abgebauten Bodenkomponenten zwischen Ortsbrust und Schneidradfrontfläche Einfluss auf diesen Prozess. Je mehr Bodenkomponenten je Schneidradumdrehung Raum zwischen Ortsbrust und Schneidradfrontfläche zu den Schneidradöffnungen hin transportiert werden müssen, umso höher ist vermutlich die Wahrscheinlichkeit für einen Kontakt zwischen abgebautem Boden und dem Rollengrundkörper. Die Menge der anfallenden Bodenkomponenten wird hier durch die Penetration $\mathrm{p}$ bestimmt. Die Transportdauer und -wege werden vermutlich maßgeblich vom Schneidradöffnungsverhältnis $\mathrm{O}_{T V M}$ und die Anordnung der Öffnungen beeinflusst.

\subsubsection{Verklebungsschäden}

\section{Schadensbild}

Verklebungsschäden an Schneidrollen treten überwiegend als Folge einer Verklebung des Schneidrollengehäuses (Abb. 42) mit abgebautem Boden oder als Folge einer Verklebung des Dichtspalts zwischen Rollengrundkörper und seitlichen Deckeln mit Feinmaterial auf. Beide Phänomene werden nicht ausschließlich in bindigen Böden beobachtet, die als verklebungsanfällig beschrieben werden (Kap. 4.2.2.1). In einigen Fällen werden die beschriebenen Phänomene bereits bei geringen Anteilen feinkörniger Bodenarten in der Ortsbrust oder in gemischtkörnigen Bodenarten mit hohem Feinkornanteil beobachtet.

Als Schadensbild ist im Vortrieb jeweils das Blockieren der Schneidrolle festzustellen. Der Schneidring wird in der Folge ohne Drehung über die Ortsbrust geschleift. Dabei entsteht eine Flachstelle bzw. einseitiger Abrieb am Schneidring parallel zur Ortsbrust. Wird die entsprechende Schneidrolle nicht ausgewechselt, kann sich der einseitige Abrieb auch auf den Rollengrundkörper und bis auf das Niveau des Schneidradstahlbaus fortpflanzen. Dabei kann direkter Kontakt zwischen Schneidradstrahlbau und der Ortsbrust entstehen, der zu einem Verschleißschaden am Stahlbau führen kann (Abb. 36).

Der einseitige Abrieb am Schneidring ist grundsätzlich nicht auf eine Stelle am Schneidring begrenzt. Innerhalb der Referenzprojekte wurden auch Schneidringe mit mehreren deutlich voneinander abtrennbaren Flachstellen dokumentiert. In seltenen Fällen traten Schneidringe auf, die durch umlaufend direkt aneinander grenzende Flachstellen eine polygonale Form zeigten. 


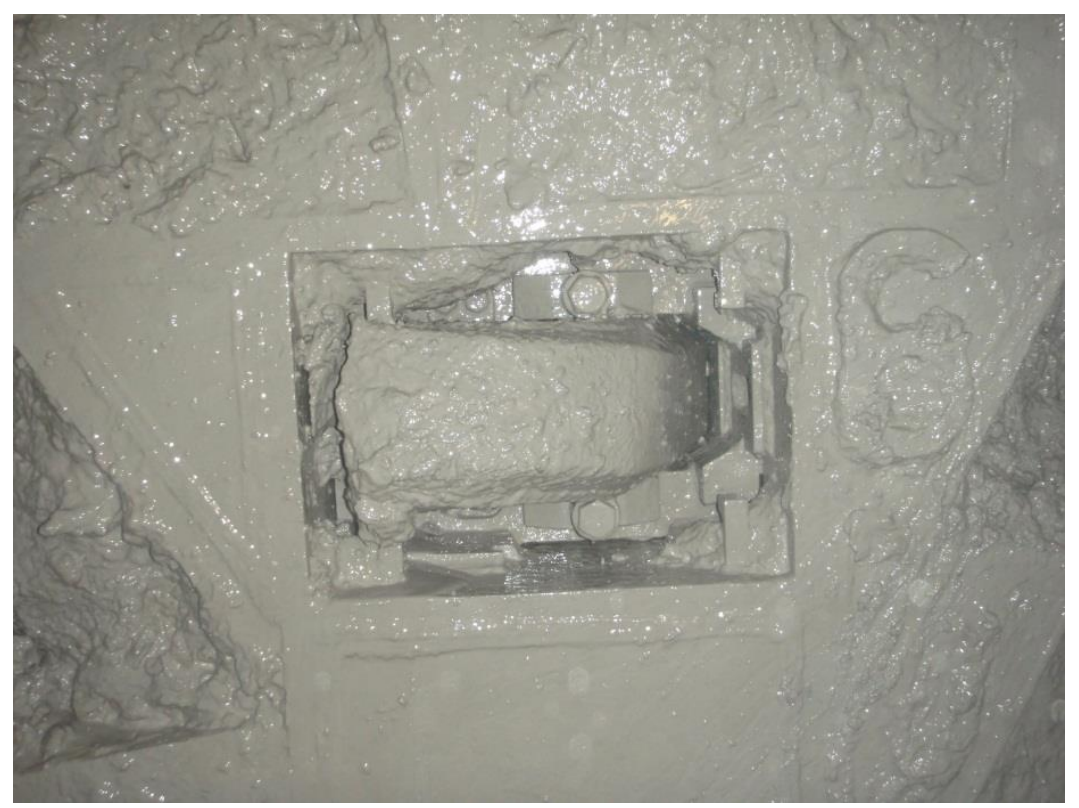

Abb. 42: Verklebtes Schneidrollengehäuse während einem Drucklufteinstieg. Auch die angrenzenden Schneidradöffnungen sind mit abgebautem Boden verklebt. Das Verklebungsmaterial wird zum Teil durch die Öffnungen im Schneidrollengehäuse gepresst und bildet dessen Form nach.

An blockierten Schneidrollen mit einseitigen Flachstellen an den Schneidringen konnten zusätzlich in einigen Fällen auch radiale Risse in den Schneidringen beobachtet werden. In seltenen Fällen haben diese zum Verlust des Schneidrings geführt.

Zusätzlich zu den Flachstellen am Schneidring treten in Zusammenhang mit der Verklebung des Dichtspalts in einzelnen Fällen Schneidrollen auf, deren seitliche Deckel während dem Vortrieb auf der Achse verdreht wurden. In Folge werden zum Teil die O-Ringe zwischen Deckel und Achse oder die mechanische Verdrehsicherung der Deckel beschädigt.

\section{Rückschlüsse auf die Schadensmechanismen}

Die Schadensbilder hängen mit dem Verhältnis zwischen dem Eigendrehmoment der Schneidrollen und den beim Bodenabbau anliegenden Schneidwiderständen des Bodens zusammen.

Im Normalzustand überschreiten die beim Bodenabbau am Schneidring anliegenden Schneidwiderstände das Eigendrehmoment der Schneidrollen deutlich. Die Schneidrolle rollt damit im Vortrieb stetig über die Ortsbrust ab. Innerhalb der Referenzprojekte konnte bis hin zu steifen, tonigen Schluffen mit einem Reibungswinkel $\varphi^{\prime}$ von $33^{\circ}$ und einer Kohäsion c'von $8,6 \mathrm{kN} / \mathrm{m}^{2}$ noch ein stetiges Abrollen der Schneidrollen auf der Ortsbrust beobachtet werden.

Ausgehend vom Normalzustand führen Verklebungen im Schneidrollengehäuse oder im Dichtspalt durch Reibungs- und Adhäsionskräfte an der Oberfläche des Schneidrings, des Rollengrundkörpers und der Deckel zu zusätzlichen Widerständen gegen das Drehen der Schneidrollen. Wird die Summe aus Eigendrehmoment der Schneidrolle und den zusätzlich anliegenden Widerständen größer als der Schneidwiderstand des Bodens, so wird die Schneidrolle in blockiertem Zustand über die Ortsbrust geschleift. Hieraus resultiert die erste Flachstelle am Schneidring (Abb. 43).

Durch die zunehmende Abflachung des Schneidrings nimmt im Anschluss die Kontaktfläche des Schneidrings zur Ortsbrust kontinuierlich zu. Dadurch steigt auch der Schneidwiderstand des Bodens an. Häufig wird der Schneidwiderstand dabei so hoch, dass dieser wieder ausreicht, um das Eigendrehmoment und die Widerstände aus den Verklebungen zu überschreiten. Der Schneidwiderstand kann in ähnlicher Weise auch durch Auftreffen z.B. auf einen Stein in der Ortsbrust oder auf widerstandsfähigere Lagen in der Ortsbrust kurzzeitig deutlich ansteigen. Die Schneidrolle wird dann um einen bestimmten Betrag weiter gedreht, sodass wieder ein im Vergleich wenig verschlissener Teil des Schneidrings auf die Ortsbrust trifft. 
Sinkt der Schneidwiderstand durch die Drehung wieder deutlich unter die Summe aus Eigendrehmoment der Schneidrolle und den zusätzlichen Widerständen durch die Verklebungen, blockiert die Schneidrolle erneut. In der Folge entsteht eine zweite Flachstelle am Schneidring. Dieser Mechanismus kann sich grundsätzlich beliebig oft wiederholen. In seltenen Fällen, vermutlich in sehr homogenen Ortsbrustverhältnissen, entstehen dabei nahezu polygonal geformte Schneidringe mit umlaufend aneinandergrenzenden Flachstellen.

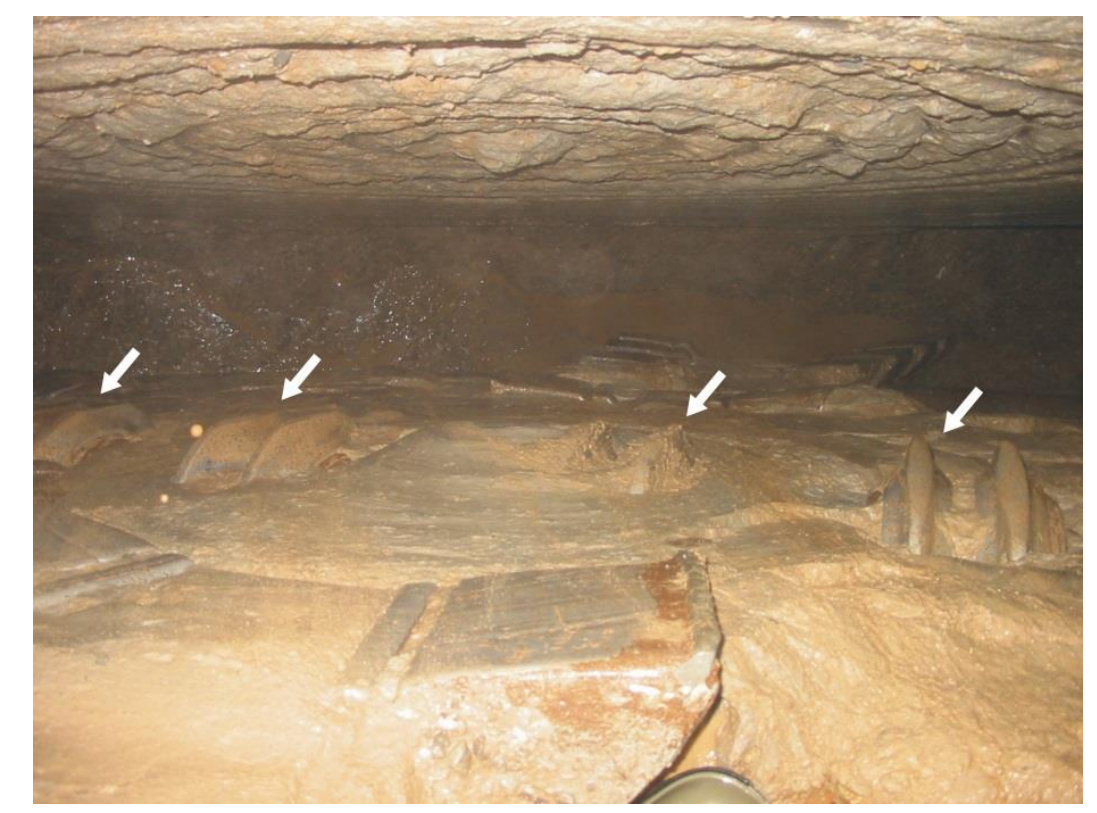

\begin{abstract}
Abb. 43: Blick durch eine Schneidradöffnung auf die Frontseite des Schneidradzentrums. Der sichtbare Bereich ist vollständig mit bindigem Material verklebt. Aus dem anhaftenden Boden stehen die Schneidrollen und Schälmesser hervor. Die Schneidrollen sind durch das anhaftende Material blockiert, die sichtbaren Schneidringe (weiße Markierungen) zeigen deutlich flachgeschliffene Stellen. (Herrenknecht AG, verändert)
\end{abstract}

Die Entstehung von Rissen in den Schneidringen an blockierten Schneidrollen steht in engem Zusammenhang mit der Bildung der Flachstellen. Diese führt einerseits zu einer deutlichen Verringerung des Schneidringquerschnitts. Dabei sinkt die Festigkeit des Schneidrings an der entsprechenden Stelle. Andererseits kommt es an den Flachstellen zum Teil zu einer deutlichen Erhitzung des Schneidring. Hierbei entstehen zusätzliche Spannungen im Schneidring. Beide Faktoren können einzeln oder in Kombination zur Bildung von Rissen führen.

Bei einer Verklebung des Dichtspalts zwischen Rollengrundkörper und den seitlichen Deckeln wird das am Schneidring anliegende Drehmoment auf die eigentlich nicht drehenden Deckel übertragen. Überschreitet das anliegende Moment den Widerstand der Fixierung der Deckel auf der Achse, so werden die Deckel gewaltsam gegen die Achse verdreht. 


\title{
5.3.2.4 Verschleißschäden
}

\section{Schadensbild}

Grundsätzlich sind zwei Typen von Verschleißschäden an den Schneidrollen zu unterscheiden. Die entsprechenden Phänomene können wie folgt zusammengefasst werden:

- Deformation des Rollengrundkörpers und des Sicherungsrings an der Auflagefläche des Schneidrings, Risse im Schneidring, Verlust des Sicherungsrings und des Schneidrings.

- Blockieren der Schneidrolle, Verlust des Schmiermittels, Eindringen von Stützflüssigkeit in die Lagerkammer der Schneidrollen.

Der erste Typ entsteht als Folge von hohem Primärverschleiß an den Schneidringen und gleichzeitig hohem Sekundärverschleiß am Rollengrundkörper und Sicherungsring. Zunächst werden sowohl der Rollengrundkörper als auch der Sicherungsring direkt angrenzend an den Schneidring durch Sekundärverschleiß ausgedünnt. Ab einer Resthöhe der seitlichen Auflagefläche des Schneidrings am Rollengrundkörper von ca. $5 \mathrm{~mm}$ können radial umlaufend Verformungen des Rollengrundkörpers in diesem Bereich beobachtet werden. An der gegenüberliegenden seitlichen Auflagefläche des Schneidrings am Sicherungsring sind häufig Verformungen am Sicherungsring feststellbar. Sobald der Querschnitt des Sicherungsrings durch Sekundärverschleiß auf ca. 50 - 60\% der ursprünglichen Querschnittsfläche reduziert wurde. Mit zunehmender Verformung des Rollengrundkörpers entsteht häufig auch ein bis zu $1 \mathrm{~mm}$ breiter wachsender umlaufender Ringspalt zwischen Sicherungsring und Schneidring (Abb. 44)

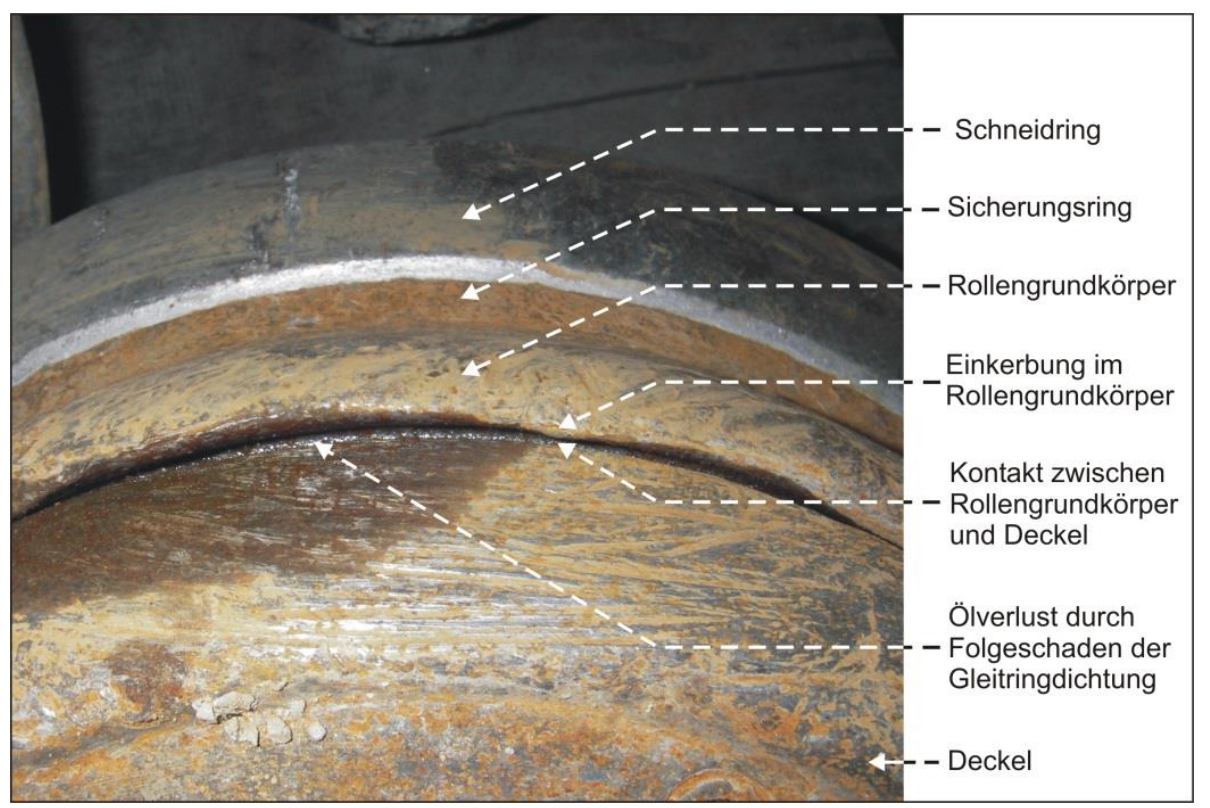

\begin{abstract}
Abb. 44: Seitenansicht einer 1-Ring Schneidrolle mit Verschleißschaden. Der Schneidring ist durch den Bodenabbau zugespitzt. Der Sicherungsring hat durch Sekundärverschleiß bereits deutlich an Material verloren. Der Rollengrundkörper ist seitlich ausgedünnt, zeigt an einer Stelle eine Einkerbung an der der Rollengrundkörper aufgrund der geringen Materialstärker deformiert wurde. Im Bereich der Deformation des Rollengrundkörpers zeigt ein Ölfleck einen Folgeschaden an der Gleitringdichtung an.
\end{abstract}

Bei weiter zunehmendem Abrieb und Verformung des Rollengrundkörpers und des Sicherungsrings können zunehmend Risse in den Schneidringen auftreten, die zum Teil auch zu einem Verlust des Schneidrings führen. Zusätzlich kann in einigen Fällen auch ein vorhergehender Verlust des Sicherungsrings aufgrund von vollständigem Verschleiß der Schweißnaht des Sicherungsrings dokumentiert werden. 
Der zweite Typ an Verschleißschäden entsteht bei Überschreiten der Verschleißgrenze der Gleitringdichtungen. Als Folge treten blockierte Schneidrollen auf, in deren Lagerkammer bei der Demontage kein Öl mehr enthalten ist, jedoch eine teilweise oder vollständige Füllung mit Stützflüssigkeit (Abb. 41). Die im Vortrieb äußerlich an den entsprechenden Schneidrollen feststellbaren Phänomene sind im Wesentlichen identisch mit den Phänomenen an blockierten Schneidrollen aufgrund von Verklebungen (Kap. 5.3.2.3). Im Unterschied ist jedoch keine gewaltsame Verdrehung der seitlichen Deckel auf der Achse festzustellen. Eine klare Unterscheidung der beiden Ursachen ist im Vortrieb folglich nicht möglich, sondern erst bei Zusammenführung der Werkzeugwechseldokumentation und den Ergebnissen aus der Schneidrollensanierung (Demontage).

\section{Rückschlüsse auf die Schadensmechanismen}

Die Deformation des Rollengrundkörpers an der seitlichen Auflagefläche des Schneidrings zeigt, dass während dem Bodenabbau auch erhebliche Seitenkräfte auf den Schneidring wirken, die zur Verformung des Rollengrundkörpers führen. Die zunehmende Bildung eines Ringspalts zwischen Sicherungsring und Schneidring belegt, dass der Schneidring dabei eine seitliche Verschiebung auf dem Rollengrundkörper erfährt. Diese führt vermutlich in einigen Fällen zu einer unsauberen oder verkanteten Auflage des Schneidrings auf dem Rollengrundkörper. Dabei entstehen zusätzliche Spannungen im Schneidring, die die Rissbildung begünstigen. Zusätzlich werden die Querschnittsfläche und die Festigkeit des Schneidrings durch hohen Primärverschleiß vorlaufend reduziert.

Der Verschleiß der Gleitringdichtungen ist im Vortrieb mehr oder weniger stetig und hängt im Wesentlichen vom Anteil an abrasivem Feinmaterial in der Stützflüssigkeit ab. Dieser wird einerseits durch die Bodeneigenschaften (Korngrößenverteilung, Mineralgehalt) gesteuert, andererseits jedoch auch durch die Austauschrate der Stützflüssigkeit und die Qualität der Separation. Sind die Gleitringdichtungen verschlissen, geht das in der Lagerkammer der Schneidrollen befindliche Öl verloren, im Gegenzug kann Stützflüssigkeit eindringen. Dabei werden Reibung und Verschleiß in den Lagern der Schneidrollen deutlich erhöht. Analog zu den Mechanismen bei Verklebungsschäden (Kap. 5.3.2.3) ergibt sich daraus eine Erhöhung des Eigendrehmomentes der Schneidrolle. Liegt dieses höher als die am Schneidring anliegenden Widerstände aus dem Bodenabbau, so wird die Schneidrolle blockiert.

\subsubsection{Gewaltschäden}

\section{Schadensbilder}

Aus den vorliegenden Daten können drei unterschiedliche Typen an Gewaltschäden zusammengefasst werden:

- Blockieren aufgrund von Lagerschäden

- Blockieren aufgrund von Verformungen des Rollengrundkörpers

- Reißen und Brechen der Schneidringe

Die im Vortrieb sichtbaren Phänomene bei blockierten Schneidrollen aufgrund von Lagerschäden sind augenscheinlich identisch mit den Phänomenen bei blockierten Schneidrollen aufgrund von Verklebungen. Eine Unterscheidung der Ursachen ist zum Teil erst mit zusätzlichen Informationen aus der Demontage der Schneidrollen möglich. Bei Schneidrollen mit Lagerschäden sind keine ursächlichen Probleme an den Gleitringdichtungen oder am Rollengrundkörper feststellbar. Die Lagerkammer ist noch ausreichend mit Öl gefült. Dagegen sind zerbrochene Kegelrollen oder deutlich sichtbare oder mit einem Finger tastbare Einkerbungen in der Lauffläche der Lager feststellbar, die zum Blockieren der Rolle führen. Dieses Schadensbild tritt überwiegend beim Auffahren von Blöcken oder künstlichen Hindernissen, wie z.B. Bohrpfählen und Spundwänden in der Ortsbrust auf.

Ein sehr ähnliches Schadensbild liegt bei Schneidrollen vor, die am Rollengrundkörper im Bereich der Lagerkammer deutliche Einschläge oder Verformungen aufweisen. Bei intakten Gleitringdichtungen und vorhandener Ölfüllung sind hier Deformationen an den Käfigen der Kegelrollenlager, gebrochene Kegelrollen oder Einkerbungen in den Laufflächen der Lager als Ursache für das Blockieren der Rolle feststellbar. 
Ein äußerlich ähnliches Schadensbild ergibt sich bei deutlich erkennbaren Einschlägen und Verformungen des Rollengrundkörpers im Bereich der Überlappung des Rollengrundkörpers und der seitlichen Deckel. Das Blockieren der Schneidrolle tritt auf, sobald durch die Verformungen Kontakt zwischen seitlichen Deckeln und dem Rollengrundkörper hergestellt wird. Analog zu Verklebungen des Dichtspalts (Kap. 5.3.2.3) wird der am Schneidring anliegende Widerstand beim Abbau des Bodens auf die seitlichen Deckel übertragen, sodass diese zum Teil gewaltsam auf der Achse der Schneidrolle verdreht werden.

Beide zuvor beschrieben Schadensbilder sind häufig in Zusammenhang mit Metallteilen in der Ortsbrust, losen Metallteilen in der Abbaukammer, aber auch bei Steinen und Blöcken in der Ortsbrust zu beobachten. Metallteile können z.B. bereits beschädigte Teile von Abbauwerkzeugen, verlorene Bohrkronen oder Gestänge, Brunnenrohre, in der Abbaukammer vergessenes Werkzeug oder Podeste sein.

Abschließend sind auch gebrochene und gerissene Schneidringe dokumentiert, die als Gewaltschäden einzustufen sind (Abb. 45). Im Unterschied zu den bisher beschriebenen Rissen in Schneidringen sind hier jeweils deutliche Einkerbungen oder Einschläge auf den Schneidringen sichtbar. Diese treten sehr häufig in Zusammenhang mit Metallteilen in der Ortsbrust und losen Metallteilen in der Abbaukammer auf. Die Risse verlaufen dabei häufig regelloser und weniger radial als z.B. bei entsprechenden Verschleißschäden (Kap. 5.3.3.3).

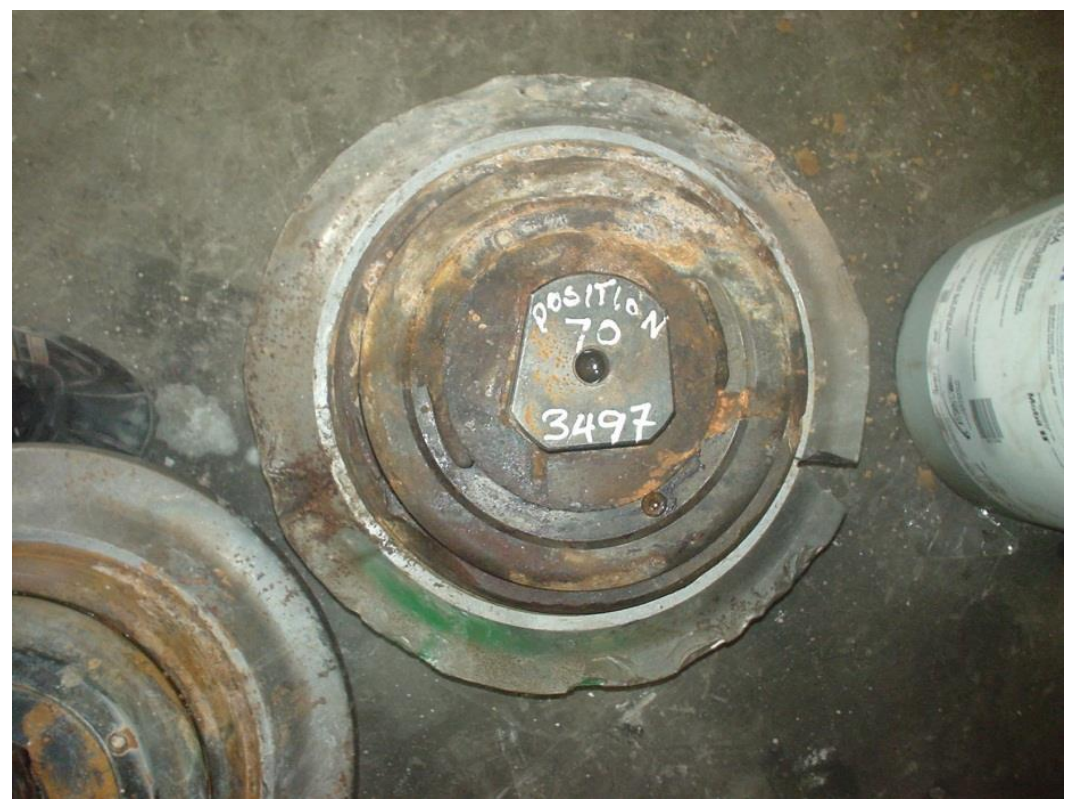

Abb. 45: 1-Ring Schneidrolle mit gerissenem Schneidring und weiteren Abplatzungen am Schneidring nach Durchfahren eines Bohrpfahls mit Stahlbewehrung.

\section{Rückschlüsse auf die Schadensmechanismen}

Schäden an den Lagern der Schneidrollen bei Blöcken oder anderen Hindernissen in der Ortsbrust entstehen durch Überlastung der Lager (Kap. 4.2.2.1). Ursache dafür ist, dass die entsprechenden Hindernisse in der Ortsbrust im Vortrieb häufig nicht rechtzeitig erkannt werden, um die Penetration entsprechend anzupassen. In den umgebenden Lockergesteinen werden mit 17" Schneidrollen häufig Werte für die Penetration $p>15 \mathrm{~mm} / \mathrm{U}$ erreicht (Tab. 9). Beim Einfahren in einen Block oder ein Hindernis wird dieser Wert auch im Festgestein des Blocks bzw. im entsprechenden Hindernis, z.B. Beton, erzwungen. Dabei treten schlagartig sehr hohe Lasten auf die Lager auf, die zum Brechen einzelner Kegelrollen oder zu Einkerbungen in den Laufflächen der Lager führen können. Als überschlägiger Richtwert kann dabei angesetzt werden, dass bei $15,0 \mathrm{~mm}$ Penetration bereits bei einer einaxialen Druckfestigkeit des Blocks oder Hindernisses von ca. 90 - 100 MPa die Belastungsgrenze der Lager in der Schneidrolle von 270 bis 295 kN (Kap. 3.2.1) erreicht wird. 
Die mechanische Beschädigung des Rollengrundkörpers durch Steine oder Blöcke in der Ortsbrust hängt damit zusammen, dass kein vollständiger Abbau in der Ortsbrust möglich ist (Kap. 4.2.1). In Abhängigkeit der Festigkeit der Einbindung des Blocks und der Penetration wird ab einer bestimmten Schnitttiefe der Rest des Blocks vollständig oder in mehreren Einzelteilen aus der Ortsbrust gedrückt (Abb. 26). Diese Restteile ragen dann in den Zwischenraum zwischen Schneidradstahlbau und Ortsbrust hinein. In Abhängigkeit der Lage, Form und Größe der Restteile kann es hier beim Auftreffen auf einen Rollengrundkörper zu mechanischen Schäden kommen.

Ein ähnlicher Mechanismus führt vermutlich auch beim Antreffen von Metallteilen in der Ortsbrust zu mechanischen Schäden an den Rollengrundkörpern. Hindernisse, wie z.B. Spundwandbohlen, Stahlträger, Brunnenrohre, Injektionsrohre oder verlorene Bohrgestänge werden in der Ortsbrust nicht abgebaut, sondern zunehmend freigelegt (Abb. 46) und ragen damit ebenfalls in den Zwischenraum zwischen Schneidradstahlbau und Ortsbrust hinein. In ähnlicher Weise können auch lose Metallteile wie z.B. ein abgerissenes Schälmesser, Räumersegment oder ein Teil eines gerissenen Schneidrings zwischen Ortsbrust und Schneidradstahlbau verklemmt oder mitgewälzt werden, wobei Schäden an den Rollengrundkörpern entstehen können.

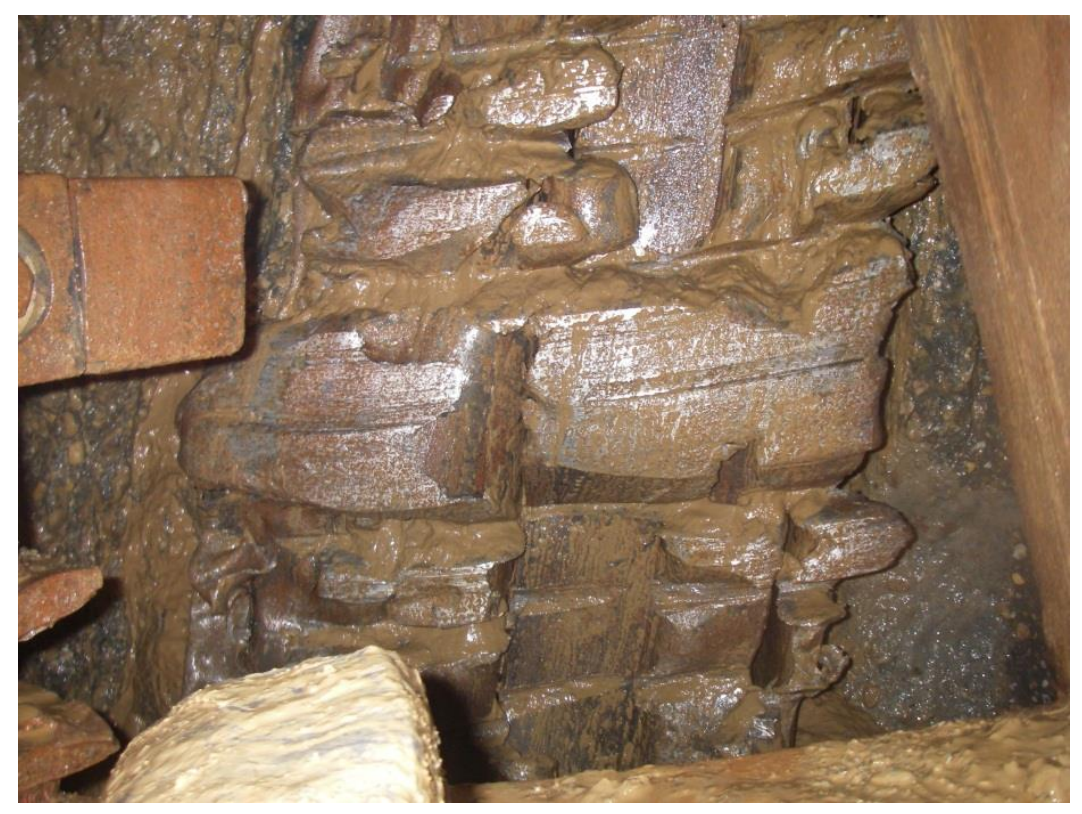

Abb. 46: Blick durch eine Schneidradöffnung auf die Ortsbrust mit einem Stahlträger, der in Beton eingebunden ist. Am Stahlträger sind deutlich die Spuren der Schneidrollen sichtbar. Der Stahlträger wird durch die Abbauwerkzeuge deformiert, aber nicht abgebaut. (Herrenknecht AG)

In ähnlicher Weise entstehen auch Risse in den Schneidringen. Ursache ist hier, dass die Schneidringe eine deutlich höhere Härte (54 - 58 HRC) aufweisen, als der Stahl in Spundwänden, Bohrgestängen oder Bewährungsstahl in Bohrpfählen. Entsprechend ist der Stahl der Schneidringe deutlich spröder als die genannten Bauelemente. Beim Überrollen entstehen an den Schneidringen punktuelle Spannungsspitzen, die aufgrund der relativ niedrigen Zähigkeit zum Sprödbruch des Schneidrings führen. 


\subsubsection{Verschleißgrenzen}

\section{Radialer Abrieb des Schneidrings}

Die Grenze für den radialen Abrieb muss grundsätzlich so gewählt werden, dass selbst bei erreichter Verschleißgrenze und gleichzeitig maximaler Penetration noch ein Überstand von mindestens $10 \mathrm{~mm}$ des Schneidrings über der Schneide der nachlaufenden Schälmesser und Räumer gegeben ist. So wird verhindert, dass es bei Antreffen von Steinen, Blöcken, Festgesteinshorizonten oder anderen festen Hindernissen zu Schäden an den Schälmessern kommt. Diese werden bei ausreichendem Überstand noch von den Schneidrollen abgebaut, ohne dass es zum Kontakt mit Schälmessern kommt. Der Maximalwert für den radialen Abrieb am Schneidring lässt sich also mit Gl. 22 errechnen aus:

$$
\mathrm{h}_{\mathrm{d}, \max (\mathrm{SM})}=\left(\mathrm{h}_{\mathrm{SR}}-\mathrm{h}_{\mathrm{SM}}\right)-10 \mathrm{~mm}
$$

Daraus ergeben sich für 17-Zoll Schneidrollen häufig Werte für den maximalen radialen Abrieb von $25-30 \mathrm{~mm}$. Im Kaliberbereich liegen diese Werte im Regelfall deutlich niedriger, da sich die Schneidkontur der Schneidrollen nach außen hin häufig der Schneidkontur der Räumer annähert. Die äußerste Kaliberschneidrolle steuert zudem den Überschnitt über dem Schildmantel. Hier wird in bestimmten Fällen eine Abweichung von Gl. 22 vorgenommen, um ein bestimmtes Maß an Überschnitt zu erreichen, da dieses Einfluss auf die vortriebsbedingten Setzungen, die Mantelreibung des Schildes und den Kies- bzw. Mörtelverbrauch bei der Hinterfüllung der Tübbinge hat.

Werden noch höhere Werte für den maximalen radialen Abrieb zugelassen, sind zusätzlich die geometrischen Eigenschaften des Schneidrings zu überprüfen. Bei aktuell üblichen 17-Zoll Schneidringen nimmt die Breite des Schneidrings ab einem Abrieb von ca. $25 \mathrm{~mm}$ aus Stabilitätsgründen zum Rollengrundkörper hin deutlich zu (Kap. 5.3.2.1). Wird Abrieb in diesem Bereich zugelassen, sinkt einerseits die Stabilität des Schneidrings, sodass es zu Rissen und Verlust des Schneidrings kommen kann. Andererseits nehmen aufgrund der ansteigenden Kontaktfläche zum Boden die Schneidkräfte vermutlich deutlich zu. Zusätzlich verringert sich der Abstand zum Rollengrundkörper und dem Sicherungsring, sodass hier der Sekundärverschleiß ansteigt. Vor diesem Hintergrund werden zunehmend 18-Zoll Schneidringe eingesetzt, da diese bei entsprechender Anordnung in Bezug auf die Schälmesser radial bis zu $12 \mathrm{~mm}$ mehr Abrieb zulassen, ohne die Stabilität des Schneidrings zu reduzieren.

Problematisch ist nach wie vor die praktische Messung des tatsächlichen Abriebwertes während der Werkzeugkontrollen. Die Auflageflächen, die als Referenz für entsprechende Schablonen zur Verfügung stehen, unterliegen selbst deutlichem Verschleiß, sodass die Messungen des radialen Abriebs tendenziell zu gering ausfallen (Kap. 5.3.2.1). Für genaue und zuverlässige Messungen haben sich bisher entweder das Gehäuse oder der Dichtspalt als Referenz bewährt. Dabei liegt der Aufwand für die Messung jedoch höher als mit den derzeit gängigen Schneidringschablonen.

\section{Seitlicher Abrieb des Schneidrings}

Auch der häufig beobachtete seitliche Abrieb an Schneidringen, der zu einer Zuspitzung der Schneidringe führt (Kap. 5.3.2.1), kann zu einem Verschleißbild an den Schneidringen führen, das einen Wechsel der Schneidrolle erforderlich macht.

Zunächst ist die verbleibende Breite des Schneidrings ein wichtiges Kriterium, da diese die Stabilität des Schneidrings im Bereich der Schneide bestimmt. Besonders in Projekten, in denen Steine, Blöcke oder Festgesteinshorizont erwartet werden, ist eine Mindestbreite des Schneidrings notwendig, um Risse und Sprödbrüche im Schneidring durch hohe Schneidkräfte zu vermeiden. Erfahrungsgemäß liegt der kritische Wert für die Mindestbreite der Schneidringe bei ca. $12 \mathrm{~mm}$. Darunter sollten die entsprechenden Schneidrollen ausgetauscht werden. Vor diesem Hintergrund werden in Lockergesteinen zunehmend Schneidringe mit $24 \mathrm{~mm}$ (1-Zoll) Ausgangsbreite eingesetzt, da diese mehr seitlichen Abrieb zulassen und im Vergleich zu den bisher überwiegend eingesetzten $19 \mathrm{~mm}$ breiten Schneidringen einen wesentlichen Vorteil in der Standzeit erzielen (Kap. 5.6.2.4). 
Die Messung der Schneidringbreite kann im Vortrieb während der Schneidradinspektionen mithilfe einer Schieblehre vorgenommen werden oder die entsprechenden Abmessungen werden zur einfacheren Handhabung in eine Verschleißschablone integriert.

Als zweiter wesentlicher Punkt im Hinblick auf den seitlichen Abrieb an den Schneidringen ist der dadurch zunehmende Sekundärverschleiß am Sicherungsring und Rollengrundkörper zu beobachten. Mit zunehmender Zuspitzung der Schneidringe verdrängen diese immer weniger Boden in der Ortsbrust (Kap. 5.3.2.1). In der Folge nimmt auch der Materialfluss entlang der Schneidringflanken über den Sicherungsring und den Rollengrundkörper zu. Diese unterliegen dabei häufig erheblichem Sekundärverschleiß. Dieser kann ebenfalls ausschlaggebend für einen Wechsel der Schneidrolle sein.

\section{Sekundärverschleiß}

Abschließend kann auch Sekundärverschleiß an verschiedenen Teilen der Schneidrollen zu einem notwendigen Austausch der Schneidrolle führen. Entsprechend müssen hierfür klare Verschleißgrenzen definiert werden. Betroffen sind vor allem der Sicherungsring, der Rollengrundkörper und die Gleitringdichtungen. Diese Verschleißgrenzen werden bei den gängigen, in den Referenzprojekten eingesetzten 17-Zoll Schneidrollen häufig erst nach der ersten oder zweiten Wiederaufbereitung der Rollen erreicht, sodass die nachfolgenden Kriterien auch Anhaltspunkte für den Austausch der entsprechenden Teile bei der Wiederaufbereitung der Schneidrollen darstellen.

Der Sicherungsring an der Basis des Schneidrings verliert durch den Sekundärverschleiß zunehmend an Querschnitt. Daraus entstehen einerseits Probleme mit Verformungen des Sicherungsrings, andererseits besteht die Gefahr, dass die Schweißnaht des Sicherungsrings versagt (Kap. 5.3.2.2). Ein Austausch der Schneidrolle ist folglich notwendig, sobald die ersten Verformungen des Sicherungsringes erkennbar sind oder die Gefahr eines Bruchs der Schweißnaht besteht. Ein kritischer Zustand des Sicherungsrings ist bei den in den Referenzprojekten eingesetzten 17-Zoll Schneidrollen im Regelfall bei 50\% verbleibendem Querschnitt des Sicherungsrings erreicht. Da die Auslegung des Sicherungsrings aufgrund der genannten Probleme in der Praxis einer Überarbeitung durch die Schneidrollenhersteller unterliegt ist diese Grenze für zukünftige Projekte neu zu definieren.

Für den Rollengrundkörper sind im Wesentlichen zwei Stellen maßgeblich, an denen durch Sekundärverschleiß ein kritischer Zustand entstehen kann. Zunächst reduziert sich an der Basis des Schneidrings die seitliche Auflagefläche am Rollengrundkörper. Dadurch kann es zu Verformungen des Rollengrundkörpers kommen, sodass die seitliche Fixierung des Schneidrings verloren geht. Bei den in den Referenzprojekten eingesetzten 17-Zoll Schneidrollen traten die Verformungen jeweils unterhalb einer Resthöhe der Auflagefläche von fünf Millimeter auf (Kap. 5.3.2.2). Ähnlich wie beim radialen Abrieb des Schneidrings fehlt auch hier eine zuverlässiger Ansatzpunkt als Referenz, um im Vortrieb die Resthöhe der Auflagefläche schnell und einfach bestimmen zu können. Da die Verformungen des Rollengrundkörpers an dieser Stelle jedoch selten zu einem sofortigen Versagen der Rolle führen, hat sich in der Praxis ein Wechsel der Rollen bei ersten Anzeichen von Verformungen an der Auflagefläche bewährt.

Der zweite kritische Punkt für Rollengrundkörper ist die Dicke des Rollengrundkörpers an der Außenkante im Bereich der Überlappung mit den seitlichen Deckeln der Schneidrolle. Wird hier ein Mindestmaß von ca. fünf Millimeter unterschritten, kann es bereits allein durch den Materialfluss in der Abbaukammer Verformungen des Rollengrundkörpers kommen, die zu einem Klemmen des Rollengrundkörpers auf den Deckeln führen (Kap. 5.3.2.2). Zudem sinkt die Widerstandsfähigkeit gegen Verformungen beim Auftreffen von Steinen oder Blöcken. Als Verschleißgrenze kann hier also eine verbleibende Reststärke des Rollengrundkörpers an den Außenkanten von $5 \mathrm{~mm}$ angesetzt werden. Dieses Maß kann bei Bedarf im Vortrieb schnell mit einer Schieblehre bestimmt werden.

Abschließend kann auch der Sekundärverschleiß an den Gleitringdichtungen der Rollen zum Verlust des Schmiermittels und nachfolgendem Blockieren der Rolle führen (Kap. 5.3.2.2). Im Vortrieb besteht jedoch keine Möglichkeit, den Zustand der Gleitringdichtungen direkt zu kontrollieren. Da Verschleiß der Gleitringdichtungen nicht ausschließlich von deren Laufleistung, sondern auch vom Gehalt an abrasivem Feinmaterial in der Stützflüssigkeit abhängt, kann keine allgemeingültige Aussage zur erreichbaren Laufleistung gemacht werden. Um trotzdem eine verlässliche Grenzen für den Einsatz der Rollen festzulegen, wurden in der Praxis bisher zwei Vorgehensweisen angewendet. Einerseits kann die Laufleistung der ausgebauten Rollen bestimmt werden und mit dem Zustand der Gleitringdichtungen korreliert werden. Damit kann eine projektspezifische Aussage für die erreichbare Laufleistung als 
Verschleißgrenze erarbeitet werden. Andererseits wurden in einem Referenzprojekt Temperaturmessungen an den Schneidrollen mithilfe eines einfachen Infrarotthermometers vorgenommen. Damit konnten Rollen identifiziert werden, die aufgrund des Schmiermittelverlustes durch erhöhte Reibung in den Lagern eine deutlich höhere Temperatur im Vergleich zu den umgebenden Schneidrollen zeigten. Auch hier ist eine projektspezifische Festlegung der Temperatur als Verschleißgrenze notwendig, da die absolute Temperatur der Schneidrollen stark von der Temperatur der umgebenden Stützflüssigkeit und der Zeitdauer zwischen Einstellung des Vortriebs und der Temperaturmessung abhängt. Für beide Methoden können auf Basis der vorhandenen Daten keine allgemeingültigen Verschleißgrenzen abgeleitet werden.

\subsubsection{Stichel}

\subsubsection{Bodenabbau und Primärverschleiß}

\section{Ortsbrustbild}

Das Ortsbrustbild beim Bodenabbau mit Sticheln hat eine hohe Ähnlichkeit mit dem Ortsbrustbild beim aktiven Bodenabbau mit Schälmessern (Kap. 5.3.4.1). Im Gegensatz dazu ist das Ortsbrustbild jedoch leichter zu beobachten, da die Stichel häufig vorlaufend zu Schälmessern angeordnet sind und keinen mehrfachen Spurbesatz aufweisen. Zusätzlich leisten die Stichel aktiven Bodenabbau in beiden Schneidradrichtungen, sodass keine Trennung von sekundären Phänomenen notwendig ist. Die Stichel decken dabei im Gegensatz zu Schneidrollen die vollständige Ortsbrustfläche ab.

In feinkörnigen Böden entsteht durch den Bodenabbau mit Sticheln wiederum eine nahezu glatte Ortsbrust, die zum Teil detailliert die Schneidkonturen der Stichel nachbildet. Die Stichel hinterlassen dabei zum Teil flache Furchen in der Ortsbrust, die eine maximale Tiefe von ca. $10 \mathrm{~mm}$ erreichen. Der Boden wird beim Abbau vollständig von der Ortsbrust entfernt, jedoch zum Teil noch als Span an der Frontfläche der Stichel mitgeschoben.

In gemischtkörnigen und grobkörnigen Böden werden die Schneidkonturen der Stichel in der Ortsbrust nicht klar abgebildet. Die Ortsbrust weißt mit zunehmender Korngröße eine deutlich unebene Form auf, die Form der Furchen wird undeutlicher. Zum Teil werden auch Bodenkomponenten unterhalb der eigentlichen Schneidkontur der Stichel gelöst, sodass Mulden und Löcher in der Ortsbrust entstehen. Der Boden wird beim Abbau durch die Stichel vollständig von der Ortsbrust entfernt.

\section{Verschleißbild}

Auch das Verschleißbild der Stichel beim aktiven Bodenabbau ähnelt dem Verschleißbild von Schälmessern mit ähnlichem Verschleißschutz beim aktiven Bodenabbau. Die Stichel weisen konstruktiv bedingt jedoch häufig einen deutlich geringeren Freiwinkel auf. Auf der Rückenfläche der Stichel ist unabhängig von der Bodenart, verursacht durch den aktiven Bodenabbau, überwiegend Abrieb parallel zur Ortsbrust zu beobachten. Dieser setzt anfänglich wiederum beidseitig direkt hinter den Schneiden an, greift aufgrund des geringeren Freiwinkels jedoch sehr schnell auf die gesamte Rückenfläche über. Auf der Kontaktfläche sind mit zunehmender Korngröße des Bodens zum Teil auch deutliche Kratzer und Furchen in Schneidrichtung sichtbar (Abb. 47).

Zwischen den Hartmetalleinsätzen der Schneide und der Rückenfläche, sowie zwischen Hartmetalleinsätzen in der Rückenfläche und dem umgebenden Stahl des Grundkörpers ist häufig kein oder nur ein geringer Niveauunterschied im Abrieb erkennbar. Zusätzlich ist an der Schneide selbst ist eine Abstumpfung bzw. Abrundung der Hartmetalleinsätze feststellbar. 


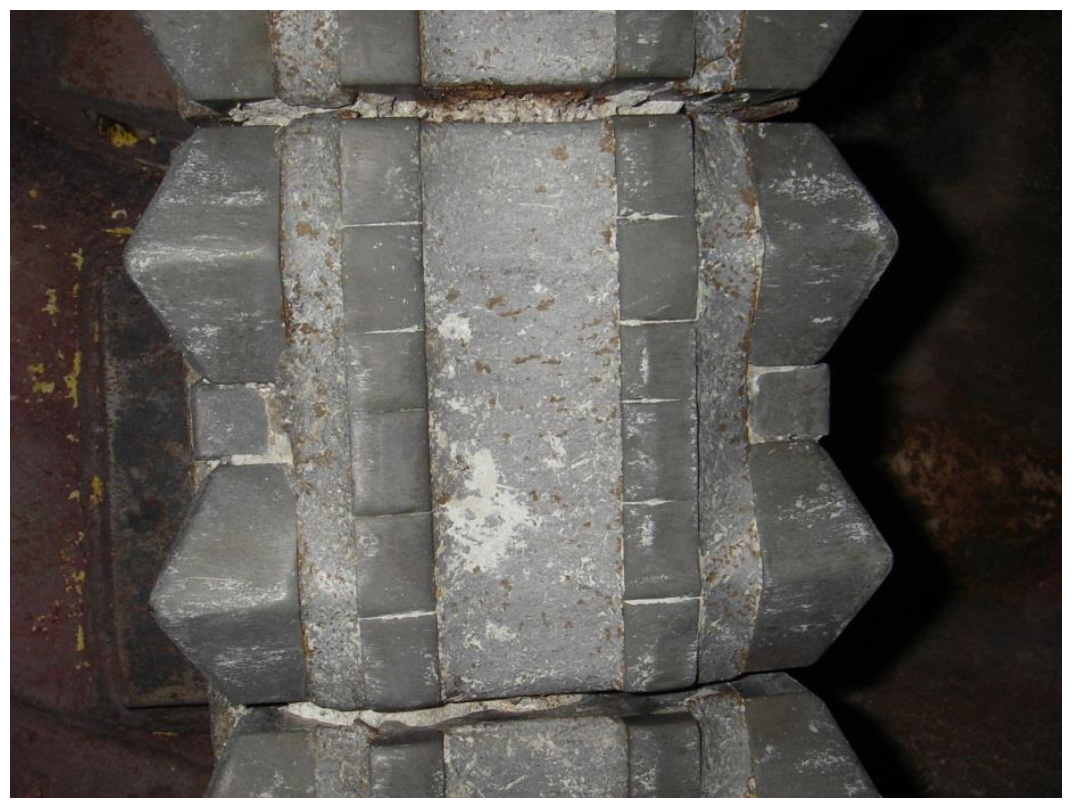

Abb. 47: Blick auf die Rückenfläche eines Stichels. Sowohl am Grundkörper als auch an den Hartmetalleinsätzen sind Kratzer parallel zur Schneidrichtung des Stichels zu erkennen.

An der Frontfläche der Stichel ist analog zu den Schälmessern unterhalb der Schneide häufig ein quer zur Schneidrichtung verlaufender Bereich mit Abrieb erkennbar. Bei zunehmendem Verschleiß entsteht eine Mulde, die bis zur Einbettung der Hartmetalleinsätze an der Schneide der Stichel reichen kann, sodass deren Einbindung in die Stahlmatrix sinkt. Die Intensität des Abriebs in diesem Bereich ist in feinkörnigen und gemischtkörnigen Böden häufig höher als in grobkörnigen Böden.

\section{Rückschlüsse auf die Abbaumechanik}

Das Ortsbrustbild und das zugehörige Verschleißbild der Stichel weisen eine so hohe Ähnlichkeit zum aktiven Bodenabbau mit Schälmessern auf, dass vermutlich auch die Mechanismen beim Bodenabbau weitgehend übertragbar sind.

Beim primären Bodenabbau in feinkörnigen Böden wird durch die Stichel ein Bodenspan aus der Ortsbrust gelöst. Der Abrieb in der Frontfläche der Stichel zeigt, dass dieser über die Frontfläche der Stichel abgleitet. Dennoch werden in der Realität auch beim Bodenabbau mit Sticheln nur sehr selten tatsächlich intakte Bodenspäne in den Separationsanlagen der Hydroschild TVM vorgefunden, da diese beim Transport in der Abbaukammer und in der Förderleitung häufig weiter zerfallen.

Über gemischtkörnige Böden hin zu grobkörnigen Böden wird vermutlich zunehmend kein zusammenhängender Bodenspan mehr ausgebildet, der vollständig über die Frontfläche der Stichel abgleitet. Der Boden zerfällt unmittelbar beim Abbau zunehmend in die einzelnen Bodenkomponenten, die dann mehr oder weniger chaotisch an der Frontfläche der Stichel abgleiten. Die geringere Intensität des Abriebs in der Frontfläche in grobkörnigen Böden deutet darauf hin, dass dabei geringere Kontaktspannungen an der Oberfläche zwischen Stahl und Bodenkomponenten, als beim Abgleiten eines zusammenhängenden Bodenspans entstehen.

Die Bildung der flachen Furchen in der Ortsbrust hängt mit der geometrischen Anordnung der Stichel auf dem Schneidrad zusammen. Die Stichel angrenzender Spuren auf dem Schneidrad sind ähnlich wie Schneidrollen und Schälmesser um bis zu $180^{\circ}$ versetzt angeordnet. Wählt man einen bestimmten Betrachtungspunkt in der Ortsbrust zwischen zwei angrenzenden Schneidspuren von Sticheln, so durchlaufen die beiden Stichel diesen Punkt zu unterschiedlichen Zeitpunkten. Aufgrund des kontinuierlichen Vorschubs des Schneidrades ergibt sich daraus eine Differenz im Bohrfortschritt in Richtung der Tunnelachse zwischen den beiden Durchlaufzeitpunkten. Diese Differenz entspricht der Tiefe der Furchen der Stichel in der Ortsbrust. Der Betrag der Differenz ergibt sich aus geometrischen Gründen (Kap. 5.4.5) aus dem Winkelabstand der beiden Stichel und der Penetration p. 


\subsubsection{Sekundärverschleiß}

Sekundärverschleiß an Sticheln entsteht überwiegend durch bereits abgebaute Bodenkomponenten in der Stützflüssigkeit.

\section{Verschleißbild}

Der Sekundärverschleiß der Stichel konzentriert sich auf die beiden Seitenflächen des Stichelkopfs. Unabhängig von der Bodenart ist häufig eine Ausspülungen am Stahlgrundkörper der Stichel zu beobachten, sodass die Hartmetalleinsätze der Schneide rückseitig freigelegt werden und bei flach liegender Anordnung zum Teil die Hartmetalleinsätze in der Rückenfläche unterspült werden. Die Intensität der Ausspülungen nimmt von feinkörnigen über gemischtkörnige Böden hin zu grobkörnigen Böden zu.

In einigen Fällen ist bei Sticheln auf Schneidspuren mit relativ geringem Spurradius $r_{s} c a .<1,5 \mathrm{~m}$ das Verschleißbild auf der inneren, dem Schneidradzentrum zugewandten Seitenfläche und der äußeren, dem Kaliberbereich zugewandten Seitenfläche leicht unterschiedlich ausgebildet. Auf der inneren Seitenfläche konzentriert sich der Abrieb dann eher im mittleren Bereich des Grundkörpers. Dem gegenüber ist auf der äußeren Seitenfläche die Intensität des Abriebs deutlich auf die Bereiche unmittelbar hinter den Hartmetalleinsätzen fokussiert. Die Intensität des Abriebs ist dabei auf der äußeren Seitenfläche zum Teil deutlich höher als auf der inneren Seitenfläche.

\section{Rückschlüsse auf den Materialfluss}

Die Rückenfläche der Stichel hat bei beiden Schneidraddrehrichtungen kontinuierlich Kontakt zur Ortsbrust. Die beiden Frontflächen dringen wechselseitig beim Bodenabbau in die Ortsbrust ein. Der Sekundärverschleiß durch bereits abgebauten Boden konzentriert sich deshalb zunächst auf die beiden Seitenflächen des Stichelkopfs.

Der Abrieb an den Seitenflächen wird vermutlich durch die beim aktiven Bodenabbau entstehenden Furchen in der Ortsbrust verstärkt. Einzelne Komponenten des bereits abgebauten Bodens werden in der Stützflüssigkeit vermutlich zwischen der Seitenwand der Furche und der Seitenfläche des Stichels kurzzeitig mitgewälzt. Dabei können zwischen Seitenfläche und Wandung der Furche Korn-KornSpannungen aufgebaut werden, die ähnlich dem Sekundärverschleiß von Schälmessern im Freiwinkel (Kap.5.3.4.2) zu einer mahlenden Beanspruchung der Stahloberfläche und damit zu Abrieb führen.

Der Unterschied in der Ausprägung und Intensität des Abriebs an den Seitenflächen der Stichelköpfe auf Spuren mit relativ geringem Spurradius $r_{s}$ ist vermutlich auf zwei geometrische Probleme zurückzuführen.

Zunächst ergibt sich bei einer Breite des Stichelkopfs von $100 \mathrm{~mm}$ unabhängig vom Spurradius ein Unterschied im Spurumfang zwischen äußerer Kante und innerer Kante von 0,63 m je Schneidradumdrehung. Bezogen auf den mittleren Spurumfang des Stichelkopfes entspricht dieser Unterschied bei Schneidspuren mit geringem Spurradius $r_{s}<1,5$ m Differenz von 7 - $10 \%$ zwischen innerer und äußerer Kante. Bei Schneidspuren mit größerem Spurradius $r_{s}$ macht dieser Unterschied nur noch deutlich weniger als $2 \%$ Differenz zwischen innerer und äußerer Kante aus.

Hinzu kommt, dass der langgezogen rechteckige Stichelkopf bei geringem Spurradius $r_{s}$ auf einer relativ engen Kreisbahn über die Ortsbrust geführt wird. Folglich entsteht sowohl an der Außenseite, als auch an der Innenseite Zwickel zwischen der Wandung der Furche und der Seitenfläche des Stichels. Hier werden während des aktiven Einsatzes der Stichel analog zum Freiwinkel von Schälmessern (Kap. 5.3.4.2) Komponenten des bereits abgebauten Bodens aus der Stützflüssigkeit angestaut, sodass Korn-Korn-Kontakte zwischen Stahloberfläche und der Wandung der Furche aufgebaut werden können. Dabei entsteht wiederum mahlender Abrieb an der Oberfläche, wobei die Intensität aufgrund der Zwickel etwas höher liegt als bei Sticheln auf Schneidspuren mit deutlich höherem Spurradius. Aufgrund der Form der Zwickel konzentriert sich der Abrieb an der Außenseite auf die beiden Bereiche nahe der Kante zwischen Seitenfläche und Frontfläche. Auf der Innenseite wird der Abrieb dagegen eher im mittleren Bereich der Seitenfläche verstärkt. 


\subsubsection{Verschleißschäden}

Verschleißschäden an Sticheln treten entsprechend der Definition in Tab. 13 durch Überschreiten der Verschleißgrenze ein. Dabei können die nachfolgenden Schadensbilder beschrieben werden.

\section{Schadensbilder}

Der Abrieb in der Rückenfläche der Stichel beim aktiven Einsatz zum Bodenabbau (Kap. 5.3.3.1) führt bei Überschreiten der Verschleißgrenze zum Verlust des Verschleißschutzes. Die Auswirkung ist abhängig von der jeweiligen Einbindetiefe der Hartmetalleinsätze in der Schneide und der Rückenfläche.

Liegt die Einbindetiefe der Hartmetalleinsätze in der Rückenfläche niedriger als in der Schneide, werden durch den fortschreitenden Abrieb zuerst die Hartmetalleinsätze in der Rückenfläche entfernt. Die Intensität des Abriebs nimmt dann in der Rückenfläche deutlich zu, sodass eine rückwärtige Freilegung der Hartmetalleinsätze in der Schneide bishin zum Verlust der Hartmetalleinsätze in der Schneide zu beobachten ist.

Bei gleicher Einbindetiefe der Hartmetalleinsätze in der Rückenfläche und der Schneide der Stichel geht der Verschleißschutz bei fortschreitendem Abrieb nahezu zeitgleich vollständig verloren. Die Intensität des Abriebs nimmt dann sofort erheblich zu.

In beiden Fällen wird die nach dem vollständigen Verlust des Verschleißschutzes die Rückenfläche der Stichel vollständig eingeebnet und zeigt durchgehende Kratzer in bzw. gegen die Schneidrichtung.

Der Verlust des Verschleißschutzes kann abschließend sowohl in der Rückenfläche als auch an der Schneide durch die Freilegung der Hartmetalleinsätze entstehen. Das dabei entstehende Verschleißbild stimmt mit obiger Beschreibung überein.

\section{Rückschlüsse auf die Abbaumechanik}

Die Mechanismen, die zu den beschriebenen Schadensbildern führen, sind identisch mit den zu Primärverschleiß (Kap. 5.3.3.1) und Sekundärverschleiß (Kap. 5.3.3.2) beschriebenen Mechanismen. Der wesentliche Unterschied besteht im vollständigen Verlust des Verschleißschutzes durch die fortlaufende Wirkung dieser Mechanismen auch nach Überschreiten der Verschleißgrenzel. Die Intensität des Abriebs nimmt aufgrund des fehlenden Verschleißschutzes deutlich zu.

\subsubsection{Gewaltschäden}

Die Schadensbilder an Sticheln durch Gewaltschäden weisen eine hohe Ähnlichkeit zu den entsprechenden Schäden an Schälmessern (Kap. 5.3.4.4) auf. Zusammenfassend können die nachfolgenden Schadensbilder beschrieben werden, die auf Gewaltschäden zurückzuführen sind. Diese treten wiederum häufig in Böden mit Gehalt an Steinen oder Blöcken, sowie bei Antreffen künstlicher Hindernisse wie z.B. verlorene Bohrgestänge, bewehrte Pfähle und Schlitzwände, Spundwände oder -bohlen, auf.

\section{Schadensbilder}

An der Schneide und in der Rückenfläche der Stichel sind häufig Sprödbrüche der Hartmetalleinsätze feststellbar. Diese können als einzelne Risse ausgebildet sein, die jedoch auch zum Abbrechen groBer Teile der Hartmetalleinsätze führen. Dabei bleiben häufig kleine Reste des Hartmetalls am Stahlgrundkörper zurück.

In seltenen Fällen und hauptsächlich im Zusammenhang mit steigender Größe der Blöcke und Hindernisse treten auch Sprödbrüche im Grundkörper der Stichel auf. Diese verlaufen häufig zwischen Schaft und Kopf der Stichel und führen zum vollständigen Verlust des Stichels.

Ein ähnliches Phänomen sind Verformungen und Sprödbrüche der Befestigungselemente der Stichel (Abb. 48). Bei einer konstruktiven Auslegung der Stichel mit wechselbarem Kopf und Halter für Schneidrollengehäuse, können die Verformungen und Sprödbrüche in der Befestigung des Stichelkopfes auf dem Halter oder auch in der Befestigung des Halters im Schneidrollengehäuse auftreten. 
Bei beginnender Verformungen und Brüchen der Befestigungselemente zwischen Stichelkopf und Halter ist zunächst häufig eine seitliche Verkippung (Abb. 49) des Stichelkopfs zu beobachten. Intensivere Schäden haben dagegen den vollständigen Verlust des Stichelkopfs zur Folge.

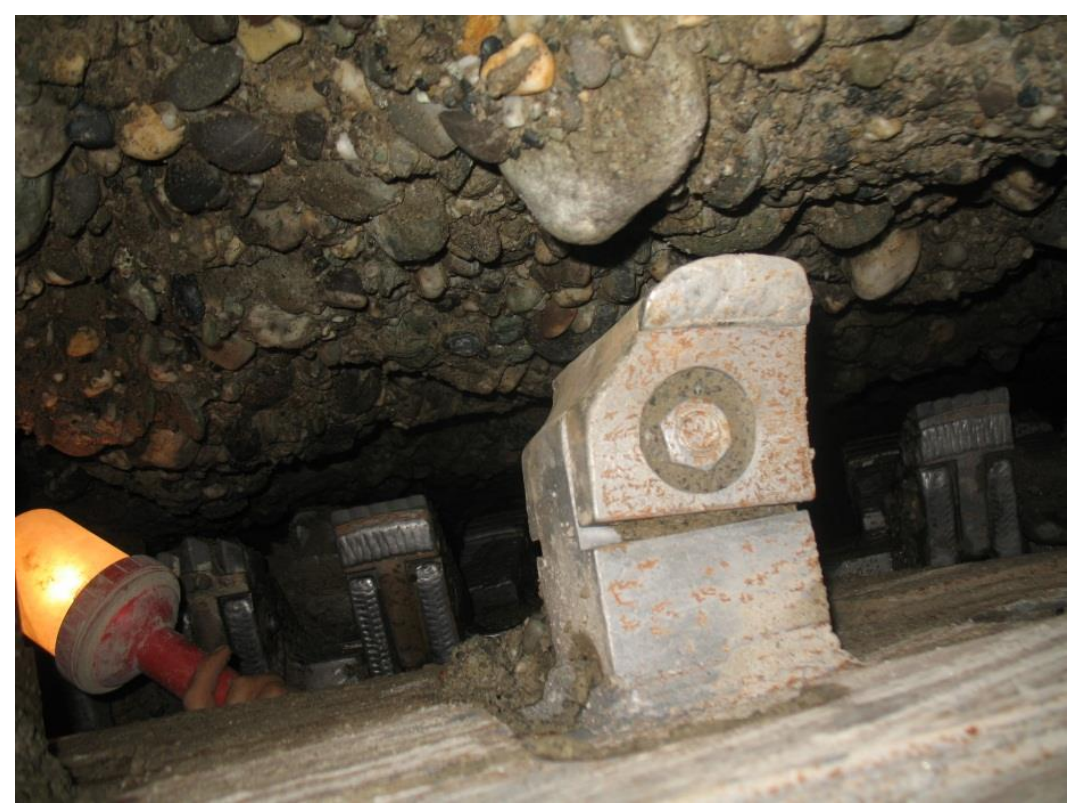

Abb. 48: Blick durch eine Schneidradöffnung auf einen Stichel nach dem Bodenabbau in steinigem Kies. Der Stichelkopf ist durch Verformungen der Befestigungselemente seitlich verkippt. Zusätzlich weist der Stichelkopf einen einseitigen Verschleißschaden auf.

An den Befestigungselementen des Halters im Schneidrollengehäuse konzentrieren sich die Schäden häufig auf die Verdrehsicherung des Halters. Treten hier Verformungen und Sprödbrüche auf, führen diese zur Rotation des Halters im Gehäuse. Der Halter liegt dann direkt auf dem Schneidrollengehäuse auf und wird je nach Schneidraddrehrichtung mehrfach hin und her rotiert. Dabei entstehen sekundäre Folgeschäden am Schneidrollengehäuse.

Sind die Stichel konstruktiv als geschweißte Variante direkt auf dem Schneidradstahlbau fixiert, können in seltenen Fällen auch Sprödbrüche im Halter selbst oder in der Schweißnaht zwischen Halter und Schneidradstahlbau auftreten, die dann zum vollständigen Verlust des Stichels führen.

\section{Rückschlüsse auf den Schadensmechanismus}

Im Unterschied zu Schneidrollen findet durch Stichel vermutlich kein Abbau von Steinen und Blöcken in der Ortsbrust statt. Diese werden eher freigelegt und in einem Stück aus der Ortsbrust gedrückt oder gerissen (Abb. 49).

Die Steine und Blöcke ragen dann in den Zwischenraum zwischen Schneidradstahlbau und Ortsbrust hinein und werden hier mitgewälzt, bis sie in eine Schneidradöffnung gelangen. Dabei entstehen beim Auftreffen auf einen Stichel schlagartige mechanische Belastungen, sodass Sprödbrüchen in den Hartmetalleinsätzen und auch im Grundkörpers entstehen. Analog zu Schälmessern wird dieser Effekt durch die Materialeigenschaften des Hartmetalls verstärkt, das aufgrund der extrem hohen Härte nur eine relativ geringe Zähigkeit aufweist (Abb. 50).

Bei zunehmender Größe der Blöcke nimmt auch der Betrag der mechanischen Belastung zu, sodass auch Schäden an den Befestigungselementen der Stichel bzw. Schäden am Halter oder dessen Verbindung zum Schneidradstahlbau entstehen können. 
Bei künstlichen Hindernissen hängt der Schadensmechanismus stark von der Art des Hindernisses ab. Bei Stahlteilen wie z.B. Spundwänden, Bohrgestängen oder Brunnenrohren sind unmittelbar Sprödbrüche an den Hartmetalleinsätzen feststellbar. Stahlteile werden durch die Stichel nicht abgebaut, sondern verhalten sich ähnlich wie Steine oder Blöcke und werden im Zwischenraum zwischen Schneidradstahlbau und Ortsbrust mitgewälzt, bis sie in eine Schneidradöffnung gelangen. Dabei entstehen, analog zu Steinen und Blöcken, auch schwere Schäden an den Sticheln.

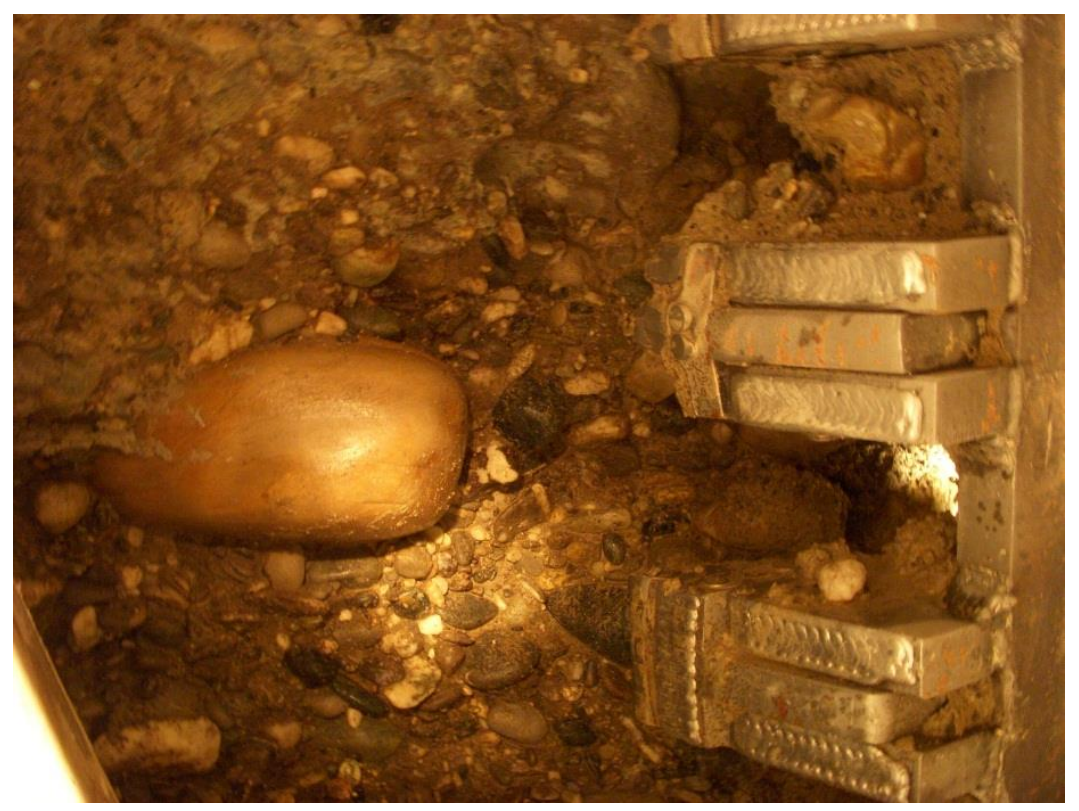

Abb. 49: Blick durch eine Schneidradöffnung auf die Ortsbrust in steinigen, blockigen Kiesen. Das Schneidrad ist mit Schälmessern und vorlaufenden Sticheln bestückt. Der links der Bildmitte sichtbare Stein in der Ortsbrust wurde durch die Stichel freigelegt, aber nicht angebaut

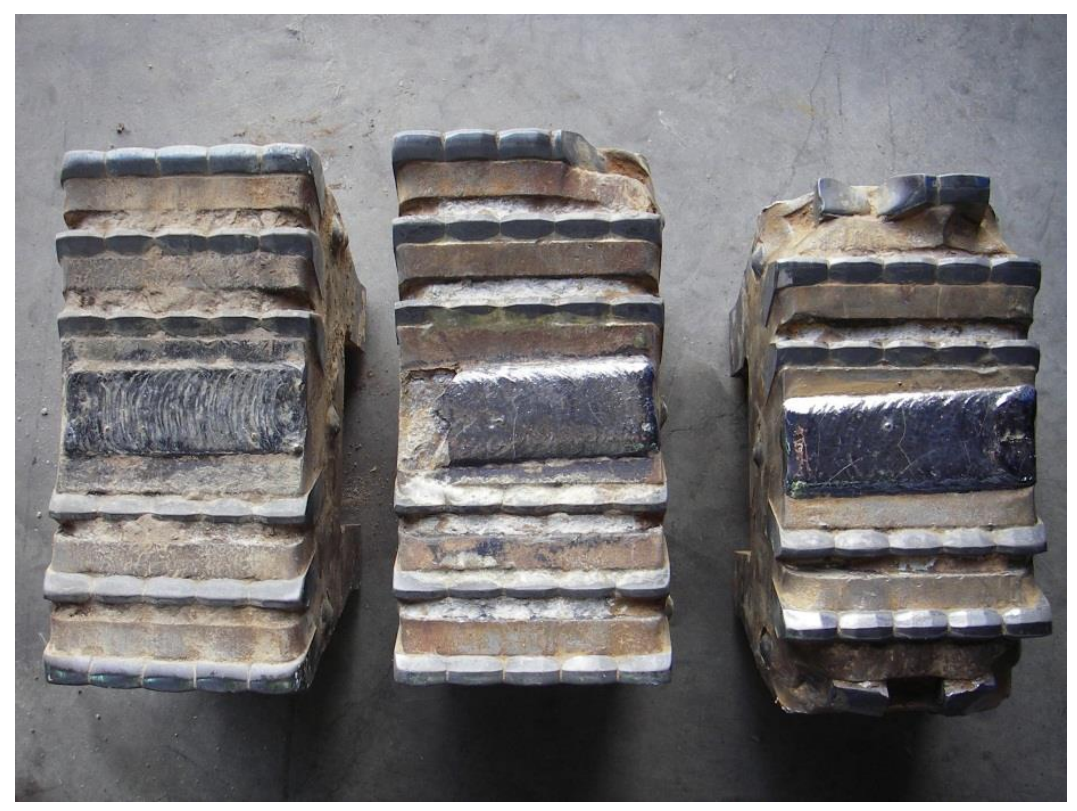

Abb. 50: Blick auf die Rückenfläche dreier Stichel, die zum Bodenabbau in steinigem Kies eingesetzt wurden. Der linke Stichel zeigt leichten Primär- und Sekundärverschleiß am Grundkörper und den Hartmetalleinsätzen. Am mittleren Stichel ist zusätzlich eine Abplatzung am Hartauftrag in der Mitte der Rückenfläche und deutliche Ausbrüche an den oberen Hartmetalleinsätzen zu erkennen. Der rechte Stichel zeigt beidseitig Ausbrüche an den Hartmetalleinsätzen. 
Hindernisse aus bewehrtem Stahlbeton werden dagegen in Abhängigkeit der Festigkeit und der Einbindung noch teilweise in der Ortsbrust abgebaut. Bei Dichtwänden und Bohrpfählen konnte bis zu einer Würfeldruckfestigkeit von $35 \mathrm{MPa}$ und angepasster Penetration $\mathrm{p}$ ca. $<5 \mathrm{~mm}$ noch ein Abbau des Betons mit Sticheln beobachtet werden. Die Stichel hinterlassen entsprechende Schneidspuren im Beton. Im Gegensatz dazu wird die Stahlbewehrung aufgrund der hohen Zähigkeit jedoch nicht abgebaut, sondern freigelegt, sodass immer mehr Stahl in den Zwischenraum zwischen Schneidradstahlbau und Ortsbrust hineinragt. Dieser führt hier wiederum zu den beschriebenen Schäden an den Sticheln (Abb. 51). Teile der Stahlbewährung können sich dabei auch an den Sticheln verfangen, sodass schwere Schäden an Haltern entstehen können.

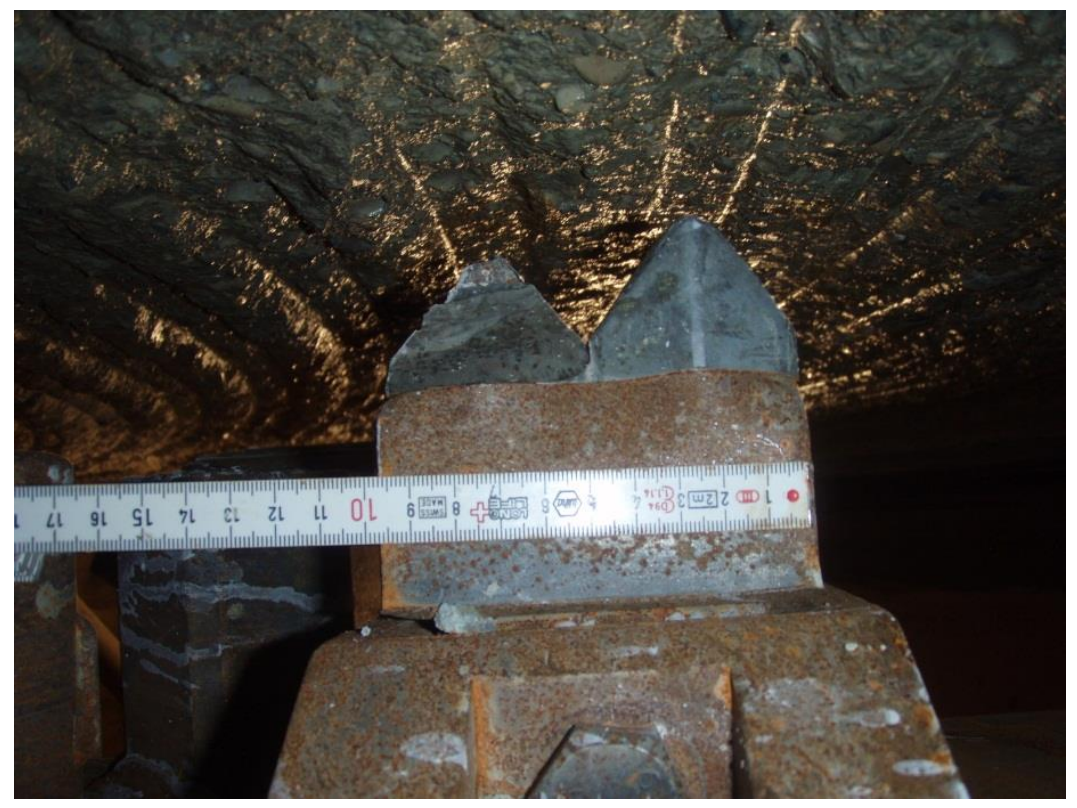

\begin{abstract}
Abb. 51: Blick auf einen Stichel und die Ortsbrust in Abbau einer Dichtwand aus stahlbewährtem Beton mit ca. 35 MPa Würfeldruckfestigkeit. Die Ortsbrust zeigt Furchen, die mit der Form der Hartmetallzähne am Stichel übereinstimmen. Der linke Hartmetallzahn des Stichels weist einen Sprödbruch auf, der durch die Stahlbewährung verursacht wurde.
\end{abstract}

\title{
5.3.3.5 Verschleißgrenzen
}

Die an Sticheln und Schälmessern festgestellten Verschleißbilder sind sich im Grundsatz sehr ähnlich (Kap. 5.3.3). Entsprechend lassen sich auch relativ ähnliche Verschleißgrenzen definieren. Die Hauptunterschiede liegen dabei darin, dass die Stichel üblicherweise vorlaufend zu Schälmessern eingesetzt werden und in beiden Schneidraddrehrichtungen aktiven Bodenabbau leisten. Unabhängig davon ist ein Wechsel der Stichel notwendig, sobald Schäden am Stichel selbst oder dem zugehörigen Halter festgestellt werden.

\section{Primärverschleiß}

Durch den aktiven Bodenabbau der Stichel ist häufig flächiger Abrieb des Stichels in der Kontaktfläche zur Ortsbrust als primäres Verschleißphänomen zu beobachten (Kap. 5.3.3.1).

Entsprechend kann für den Abrieb an der Kontaktfläche zur Ortsbrust eine eindeutige Verschleißgrenze definiert werden. Hier ist ein Wechsel der Stichel notwendig, sobald die Untergrenze der Hartmetalleinsätze erreicht ist. Die Einbindetiefe der Hartmetalleinsätze ist in der Rückenfläche häufig deutlich geringer gewählt als an den Einsätzen an den beiden Schneiden. Damit wird diese Verschleißgrenze auch häufig zuerst an den Hartmetalleinsätzen in der Rückenfläche erreicht (Abb. 52). 


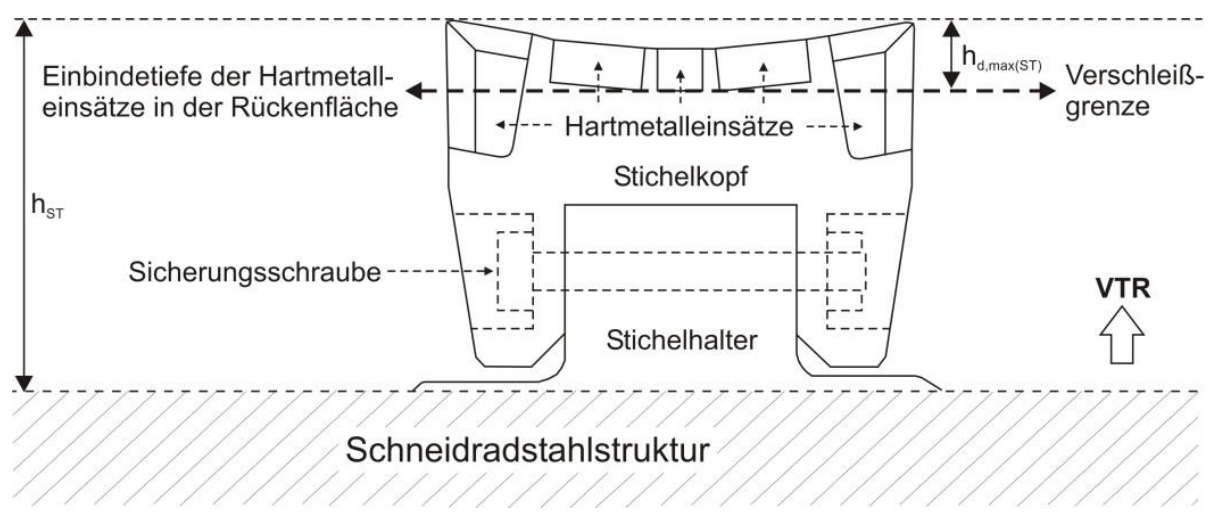

\begin{abstract}
Abb. 52: Stichelkopf mit entsprechendem Halter. Die Verschleißgrenze $h_{d, \max (S T)}$ des Stichelkopfs ergibt sich unabhängig von der Hähe der Stichel $h_{S T}[\mathrm{~mm}]$ über dem Schneidradstahlbau aus der Einbindetiefe der Hartmetalleinsätze in der Rückenfläche. (VTR = Vortriebsrichtung)
\end{abstract}

Im Gegensatz zu den Schneidrollen sind im Hinblick auf den flächigen Abrieb in der Kontaktfläche zur Ortsbrust keine weiteren Grenzen in Bezug auf den Überstand der Stichel zum entsprechenden Halter oder zu den Schälmessern zu berücksichtigen. Der Haupteinsatzbereich von Stichel liegt in Bodentypen, die auch mit Schälmessern problemlos abzubauen sind. Folglich können die Stichel bei entsprechend geringem Überstand über den Schälmessern durchaus bis auf die Höhe der Schälmesser abgeschliffen werden, ohne dass daraus ein Schadenspotential für die Schälmesser entsteht. Gleichzeitig liegt die Oberkante des Halters konstruktionsbedingt deutlich unterhalb der Einbindetiefe der Hartmetalleinsätze im Stichel, sodass in jedem Fall die Unterkante der Hartmetalle erreicht wird, bevor der Halter in direkten Kontakt mit der Ortsbrust kommt.

Der Abrieb an der Kontaktfläche zur Ortsbrust kann im Vortrieb relativ einfach mit einem Meterstab gemessen werden. Allerdings ist der Zugang zu den Sticheln von der Rückseite des Schneidrades bei Sticheln, die in Schneidrollengehäuse eingebaut werden, deutlich eingeschränkt. Stichel, die in der Mitte der Schneidradarme auf dem Frontblech des Schneidrades mit einem Halter angebracht sind oder direkt verschweißt sind, können aufgrund mangelnder Zugänglichkeit im Vortrieb so gut wie nicht mehr kontrolliert oder gewechselt werden.

An den beiden Frontflächen der Stichel wird durch den Verschleiß beim aktiven Bodenabbau zusätzlich häufig die Stahlmatrix unterhalb der Hartmetalleinsätze der Schneide angegriffen. Ein Wechsel des Stichels wird hier notwendig, sobald keine ausreichende Einbindung der Hartmetalleinsätze mehr gegeben ist. Äquivalent zum Primärverschleiß an Schälmessern (Kap. 5.3.4.5) ist eine sichere Beurteilung und Messung der verbleibenden Festigkeit der Einbindung im Vortrieb nicht durchführbar. Entsprechend sollte im Rahmen einer konservativen Werkzeugwechselstrategie der Wechsel des Stichels erfolgen, sobald eine Freilegung der unteren Anlagefläche erkennbar wird.

\title{
Sekundärverschleiß
}

Der Sekundärverschleiß im Rücken der Stichel führt ähnlich wie bei den Schälmessern häufig zu einer Freilegung der Hartmetalleinsätze. Daraus resultiert eine mangelnde Einbindung der Hartmetalleinsätze in die Stahlmatrix, die einerseits das Brechen der Hartmetalleinsätze begünstigt und andererseits bei vollständiger Freilegung zum Verlust der Hartmetalleinsätze führt (Kap. 5.3.3.2).

Entsprechend wird ein Wechsel des Stichels notwendig, sobald durch Freilegung begünstigte Brüche in den Hartmetalleinsätzen im Rücken erkennbar sind. Unabhängig davon stellt auch die Freilegung der Hartmetalle selbst ab einem gewissen Ausmaß einen Wechselgrund dar, wenn ein vollständiges Herausbrechen der Hartmetalleinsätze abzusehen ist.

Kritisch an den genannten beiden Kriterien für den Wechsel eines Stichels ist wiederum, dass einerseits die Rückenfläche der Stichel im Vortrieb nur schwer zugänglich ist. Andererseits kann keine eindeutige und im Vortrieb messbare Verschleißgrenze für diese Kriterien definiert werden. Folglich unterliegt die Beurteilung des Sekundärverschleißes weiterhin der subjektiven, visuellen Einschätzung durch den jeweiligen Bearbeiter. 


\subsubsection{Schälmesser}

\subsubsection{Bodenabbau und Primärverschleiß}

\section{Ortsbrustbild}

Das Ortsbrustbild beim aktiven Bodenabbau durch Schälmesser kann nur in seltenen Fällen direkt beobachtet werden. Einerseits müssen Auswirkungen vorlaufend angeordneter Werkzeuge wie z.B. Schneidrollen abgetrennt werden. Andererseits haben auch die mit geringer Penetration $p_{a}$ (Kap. 5.4.5) passiv mitgeführten Schälmesser Auswirkungen auf das Ortsbrustbild. Die nachfolgende Beschreibung fasst die Phänomene aus dem aktiven Bodenabbau zusammen.

In feinkörnigen Böden entsteht durch den Bodenabbau mit Schälmessern eine nahezu glatte Ortsbrust, die zum Teil detailliert die Schneidkonturen der Schälmesser nachbildet. Der Boden wird beim Abbau vollständig von der Ortsbrust entfernt, jedoch zum Teil noch als Span an der Frontfläche der Schälmesser mitgeschoben (Abb. 53).

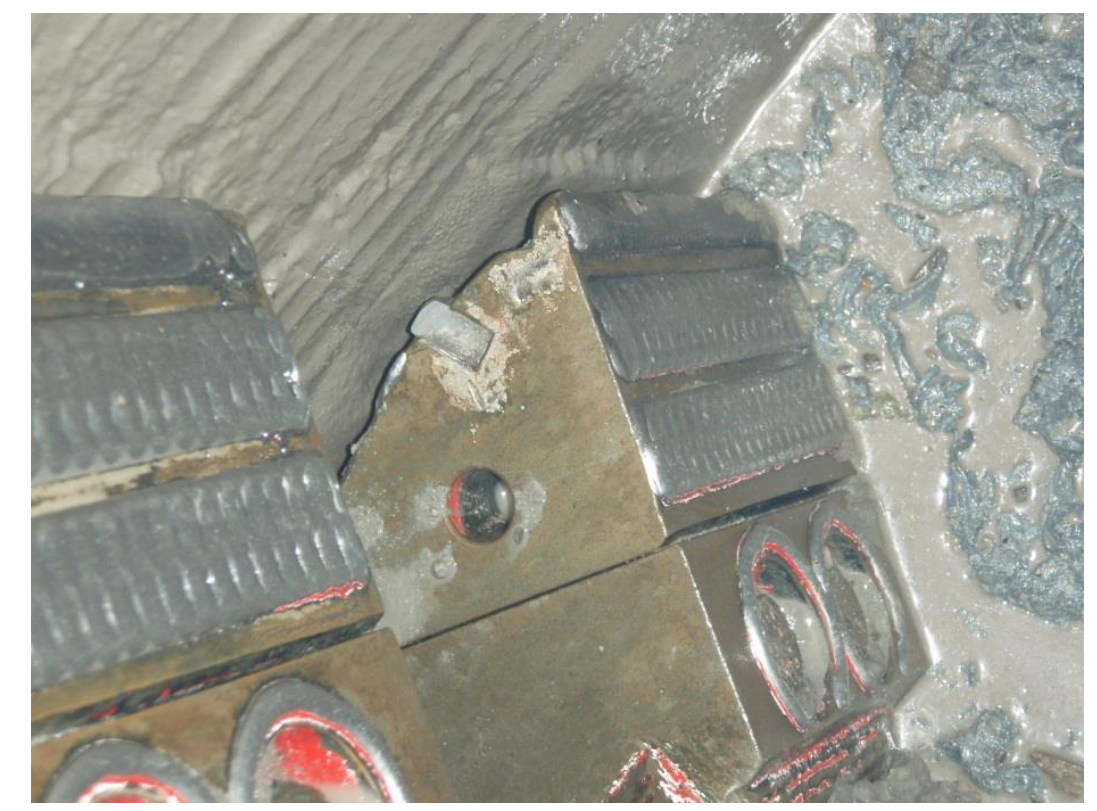

Abb. 53: Blick durch eine Schneidradöffnung auf zwei Schälmesser und die Ortsbrust in tonigen Schluffen. Die Ortsbrust ist glatt und zeichnet zum Teil detailliert die Form der Schälmesser nach. (Herrenknecht AG)

In gemischtkörnigen und grobkörnigen Böden ist keine klare Abbildung der Schneidkonturen der Schälmesser in der Ortsbrust erkennbar. Die Ortsbrust weißt mit zunehmender Korngröße eine deutlich unebenere Form auf, wobei zum Teil auch noch Bodenkomponenten unterhalb der eigentlichen Schneidkontur der Schälmesser entfernt werden, sodass Mulden und Löcher in der Ortsbrust entstehen. Der Boden wird beim Abbau durch die Schälmesser vollständig von der Ortsbrust entfernt (Abb. 54). 


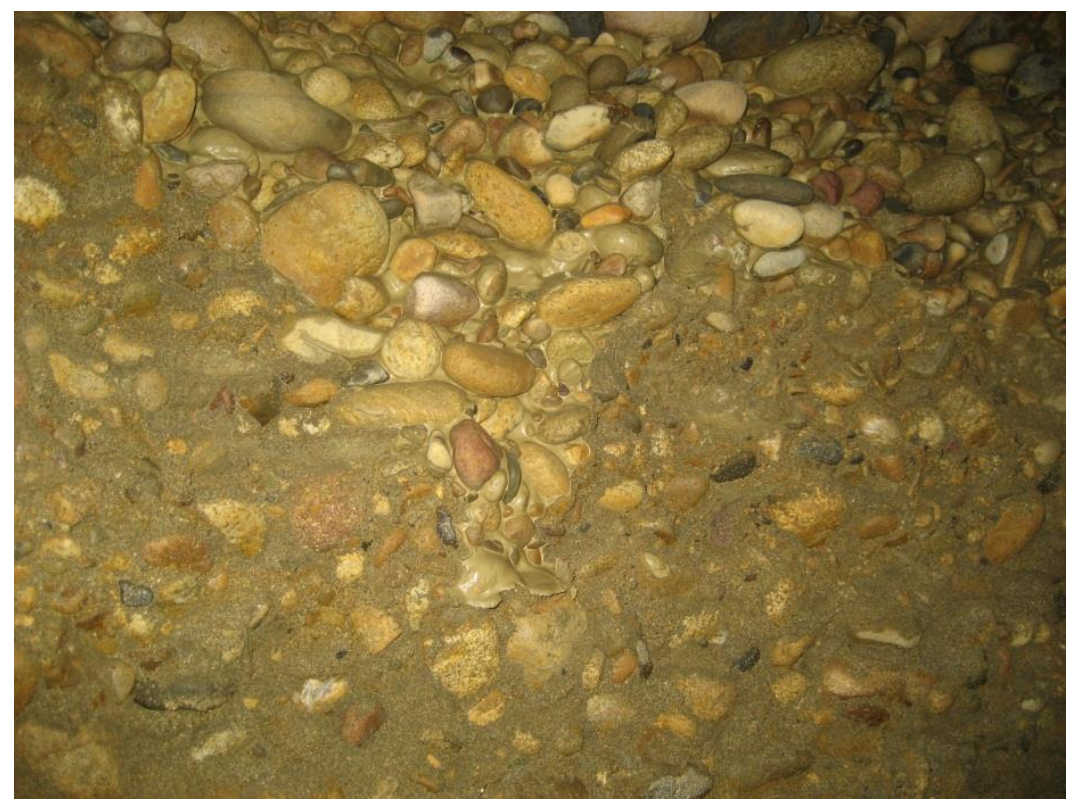

Abb. 54: Blick auf die Ortsbrust in mittel dicht gelagerten, sandigen Kiesen beim Abbau mit Schälmessern ohne vorlaufende Schneidrollen. Der Filterkuchen ist unterhalb der Bildmitte entfernt. Die Ortsbrust ist leicht uneben. Es sind keine Schneidspuren der Schälmesser erkennbar. Bildausschnitt: ca. $40 \mathrm{~cm}$ auf $50 \mathrm{~cm}$. (Herrenknecht AG)

\section{Verschleißbild}

An der Frontfläche der Schälmesser ist häufig unterhalb der Schneide ein Bereich mit Abrieb erkennbar. Bei hohem Abrieb kann dieser aufgrund der Freilegung der Hartmetalleinsätze an der Schneide deren Einbindung in die Stahlmatrix gefährden. Die Intensität des Abriebs in diesem Bereich ist in feinkörnigen und gemischtkörnigen Böden häufig höher als in grobkörnigen Böden (Abb. 55).

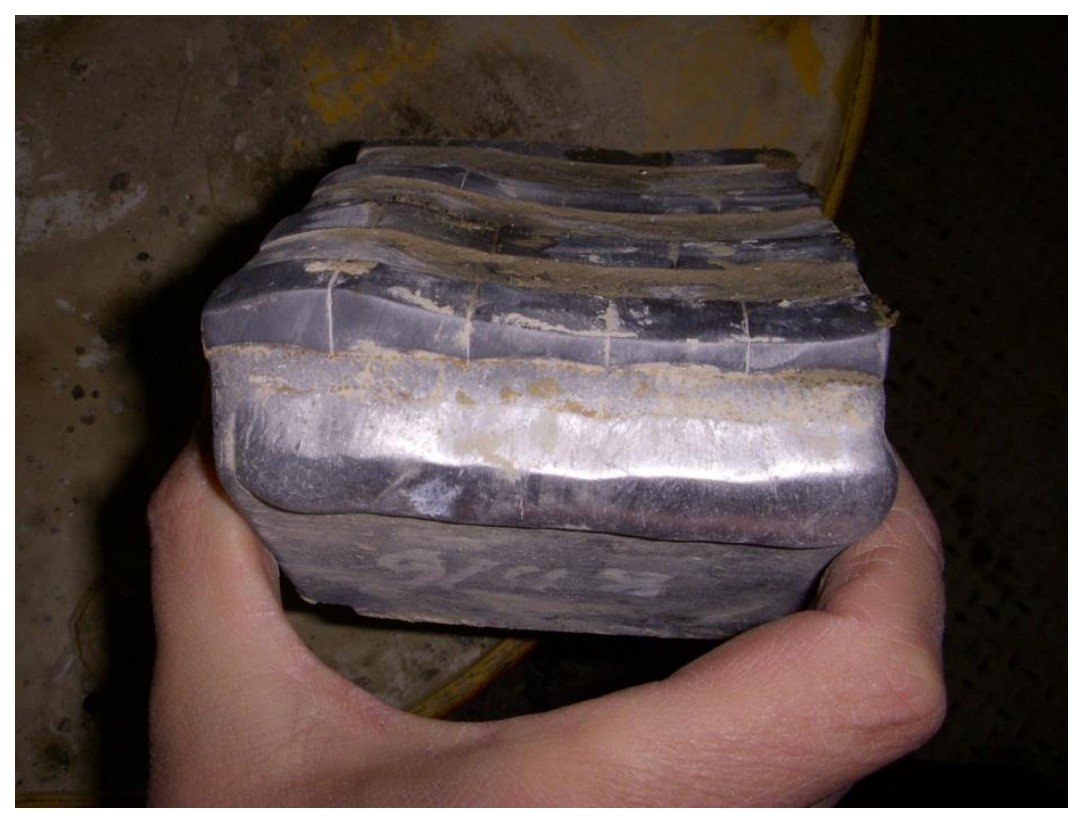

Abb. 55: Blick auf die Front- und Rückenfläche eines verschlissenen Schälmessers nach dem Bodenabbau mit vorlaufenden Schneidrollen in sandigen Kiesen. Die Rückenfläche zeigt auf der linken Seite eine leichte Aufwölbung. Hier wurde das Schälmesser durch die vorlaufende Schneidrolle geschützt. Auf den Hartmetalleinsätzen in der Rückenfläche und der Schneide sind Kratzer in Schneidrichtung des Schälmessers erkennbar. Die Frontfläche des Schälmessers zeigt unterhalb der Schneide deutlichen Abrieb. (Herrenknecht AG) 
Auf der Rückenfläche der Schälmesser ist unabhängig von der Bodenart, verursacht durch den aktiven Bodenabbau, überwiegend Abrieb parallel zur Ortsbrust zu beobachten. Dieser setzt anfänglich direkt hinter der Schneide an, da die übrige Rückenfläche aufgrund des Freiwinkels noch keinen Kontakt zur Ortsbrust hat. Mit zunehmendem Abrieb vergrößert sich die Kontaktfläche zur Ortsbrust, sodass der Freiwinkel zunehmend abgebaut wird. Auf der Kontaktfläche sind mit zunehmender Korngröße des Bodens zum Teil auch deutliche Kratzer und Furchen in Schneidrichtung sichtbar.

Zwischen den Hartmetalleinsätzen der Schneide und der Rückenfläche, sowie zwischen Hartmetalleinsätzen in der Rückenfläche und dem umgebenden Stahl des Grundkörpers ist häufig kein oder nur ein geringer Niveauunterschied im Abrieb erkennbar. Zusätzlich ist an der Schneide selbst eine Abstumpfung bzw. Abrundung der Hartmetalleinsätze erkennbar.

Bei vorlaufenden Schneidrollen ist in gemischtkörnigen und grobkörnigen Böden eine Differenzierung in der Intensität des Abriebs an den Schälmessern zu erkennen. Im Bereich der Schneidspuren der vorlaufenden Schneidrollen entsteht in der Rückenfläche und an der Front der Schälmesser deutlich weniger Abrieb als in den Bereichen zwischen den Schneidspuren der Schneidrollen. Folglich bilden sich in Schneidrichtung verlaufende Grate, die die Form der in der Ortsbrust vorliegenden Furchen entlang der Schneidspuren der Schneidrollen nachbilden.

Beim Einsatz vorlaufender Stichel haben die Schälmesser keinen Kontakt mehr zur Ortsbrust, da die Stichel bei vollständigem Besatz den Boden über die vollständige Ortsbrustfläche abbauen. Dem entsprechend leisten die Schälmesser hier keinen aktiven Bodenabbau.

\section{Rückschlüsse auf die Abbaumechanik}

Das Ortsbrustbild und das zugehörige Verschleißbild der Schälmesser deuten darauf hin, dass beim primären Bodenabbau in feinkörnigen Böden durch die Schälmesser ein Bodenspan aus der Ortsbrust gelöst wird. Der Abrieb in der Frontfläche der Schälmesser zeigt, dass dieser über die Frontfläche der Schälmesser abgleitet wird. Dieser Mechanismus stimmt im Wesentlichen mit den bisherigen Erkenntnissen zum Bodenabbau mit Schälmessern (Kap. 4.2.1) überein. Dennoch werden in der Realität nur sehr selten tatsächlich intakte Bodenspäne in den Separationsanlagen der Hydroschild TVM vorgefunden, da diese beim Transport in der Abbaukammer und in der Förderleitung häufig weiter zerfallen.

Über gemischtkörnige Böden hin zu grobkörnigen Böden wird vermutlich zunehmend kein zusammenhängender Bodenspan mehr ausgebildet, der vollständig über die Frontfläche der Schälmesser abgleitet. Der Boden zerfällt unmittelbar beim Abbau zunehmend in die einzelnen Bodenkomponenten, die dann mehr oder weniger chaotisch an der Frontfläche der Schälmesser abgleiten. Die geringere Intensität des Abriebs in der Frontfläche in grobkörnigen Böden deutet darauf hin, dass hier deutlich geringere Kontaktspannungen an der Oberfläche zwischen Stahl und Bodenkomponenten entstehen, als beim Abgleiten eines zusammenhängenden Spans.

Bei vorlaufend eingesetzten Schneidrollen leisten die Schälmesser im Bereich der Furchen entlang der Schneidspuren der vorlaufenden Schneidrollen nahezu keinen Bodenabbau. Durch die Furchenbildung haben die Schälmesser hier keinen oder nur sehr geringen Kontakt zur Ortsbrust, sodass hier auch deutlich weniger Abrieb an den Schälmessern entsteht.

\subsubsection{Sekundärverschleiß}

Sekundärverschleiß an den Schälmessern entsteht einerseits durch Kontakt mit der Ortsbrust während dem passiven Einsatz, andererseits jedoch auch durch Kontakt mit bereits abgebauten Bodenkomponenten in der Stützflüssigkeit.

\section{Ortsbrustbild}

Das Ortsbrustbild das durch den passiven Einsatz der Schälmesser verursacht wird, entspricht weitgehend dem Ortsbrustbild beim aktiven Bodenabbau. In feinkörnigen Böden werden wiederum die Konturen der Schälmesser zum Teil detailliert nachgebildet. In gemischtkörnigen und grobkörnigen Böden ist kein wesentlicher Unterschied zum Ortsbrustbild beim aktiven Bodenabbau feststellbar. 


\section{Verschleißbild}

Der Verschleiß der Schälmesser beim passiven Einsatz konzentriert sich auf die Rückenfläche der Schälmesser. Die Kontaktfläche zur Ortsbrust, die analog auch beim aktiven Einsatz der Schälmesser entsteht, bleibt auch beim passiven Einsatz erhalten. Entsprechend sind auf der Fläche weiterhin Kratzer in bzw. entgegen der Schneidrichtung sichtbar.

Zusätzlich zum Abrieb an der Kontaktfläche zur Ortsbrust sind verschiedenen Verschleißphänomene an der verbleibenden Rückenfläche feststellbar. Hier ist häufig eine Freilegung bzw. Ausspülung der Hartmetalleinsätze in der Rückenfläche feststellbar. In feinkörnigen Böden sind dabei häufig Spülmarken erkennbar und der Abrieb konzentriert sich auf den Bereich unmittelbar hinter der Kontaktfläche zur Ortsbrust. In gemischtkörnigen und grobkörnigen Böden breitet sich der Abrieb zunehmend auf die vollständige Rückenfläche aus, wobei die Ausbildung von Spülmarken zurückgeht. Zum Teil ist auch eine deutliche Zunahme der Intensität des Abriebs am Grundkörper zu beobachten.

An den Hartmetalleinsätzen in der Rückenfläche selbst ist ebenfalls Abrieb erkennbar, der jedoch deutlich geringer ist als am Stahl des Grundkörpers. Der Abrieb ist zudem zum Teil einseitig und auf der Rückseite bzw. entgegen der geplanten Schneidrichtung höher als an der Frontseite in Schneidrichtung.

\section{Rückschlüsse auf die Abbaumechanik und den Materialfluss}

Der Abrieb an der Kontaktfläche zur Ortsbrust setzt sich analog zum Primärverschleiß fort, da die Schälmesser auch beim passiven Einsatz Kontakt zur Ortsbrust haben, bzw. in Abhängigkeit des Winkelabstandes zum entsprechenden aktiven Schälmesser minimale Penetration $p_{a}[\mathrm{~mm} / \mathrm{U}]$ erfahren (Kap. 5.4.5).

Der Abrieb des Stahlgrundkörpers und die sichtbaren Spülmarken in feinkörnigen Böden zeigen an, dass beim passiven Einsatz der Schälmesser durch die Führung entgegen der geplanten Schneidrichtung Stützflüssigkeit und die darin enthaltenen Bodenkomponenten in den Freiwinkel strömen.

Bei zunehmender Einengung strömt die Stützflüssigkeit seitlich aus dem Freiwinkel heraus. Das dabei entstehende Verschleißbild lässt sich anhand der Ergebnisse von WILMS (1997: 105) (Kap. 4.3.1.5) schlüssig erklären.

In feinkörnigen Böden werden die in der Stützflüssigkeit enthaltenen Bodenkomponenten aufgrund der geringen Korngröße vermutlich vollständig aus dem Freiwinkel entfernt. Somit entstehen keine direkten Kontakte zwischen den Bodenkomponenten in der Stützflüssigkeit, sodass auch keine Korn-KornKontakte zwischen Ortsbrust und Rückenfläche des Schälmessers aufgebaut werden können. In Folge entstehen zwischen den Bodenkomponenten in der Stützflüssigkeit und der Stahloberfläche nur relativ niedrige Spannungen, die etwa in der Größenordnung des umgebenden Stützdrucks liegen. Durch das Überströmen der Stahloberfläche durch die Bodenkomponenten in der Stützflüssigkeit entsteht spülender Abrieb an der Oberfläche. Dieser ist im Strömungsschatten der Hartmetalleinsätze deutlich geringer, sodass Spülmarken entstehen.

In gemischtkörnigen und grobkörnigen Böden bleiben die in der Stützflüssigkeit enthaltenen Bodenkomponenten zunehmend im Freiwinkel hängen. Damit können zwischen Ortsbrust und Rückenfläche des Schälmessers Korn-Korn-Kontakte aufgebaut werden, die eine Übertragung wesentlich höherer Spannungen an der Stahloberfläche ermöglichen. Damit entsteht hier zunehmend mahlender Abrieb an der Oberfläche, wobei die Intensität deutlich höher liegt als bei rein spülendem Abrieb in feinkörnigen Böden. Zeitgleich geht durch die mahlende Beanspruchung der Oberfläche die Ausbildung von Spülmarken zurück.

Grundsätzlich besteht folglich ein Zusammenhang zwischen der Ausbildung des Freiwinkels der Schälmesser, der Korngrößenverteilung des Bodens und der Verschleißart und Intensität in der Rückenfläche. Zusätzlich hat vermutlich die Austauschrate der Stützflüssigkeit in der Abbaukammer einen Einfluss auf die Intensität, da die Wahrscheinlichkeit für Korn-Korn-Kontakte im Freiwinkel sinkt, je geringer die Konzentration der Bodenkomponenten in der Stützflüssigkeit und damit deren Dichte $\rho_{\mathrm{SF}}$ ist. 


\subsubsection{Verschleißschäden}

Verschleißschäden an Schälmessern treten durch Überschreiten der Verschleißgrenze ein. Dabei können die nachfolgenden Schadensbilder beschrieben werden.

\section{Schadensbilder}

Der Abrieb in der Kontaktfläche zwischen Schälmesser und Ortsbrust beim aktiven und passiven Einsatz dehnt sich bei verspätetem Wechsel der Schälmesser auf die vollständige Rückenfläche aus. Dabei erreicht der Abrieb ein Niveau, bei dem sowohl die Hartmetalleinsätze an der Schneide der Schälmesser, als auch der Verschleißschutz in der Rückenfläche vollständig entfernt werden. Die Rückenfläche der Schälmesser ist dann vollständig eingeebnet und zeigt durchgehende Kratzer in bzw. gegen die Schneidrichtung.

Ein ähnliches Schadensbild ergibt sich bei hohem Sekundärverschleiß in der Rückenfläche. Hier können durch den spülenden oder mahlenden Abrieb sowohl der Verschleißschutz in der Rückenfläche, als auch die Hartmetalleinsätze an der Schneide entfernt werden. Die Rückenfläche zeigt dann eine deutlich unebene Oberfläche.

\section{Rückschlüsse auf den Schadensmechanismus}

Die Mechanismen, die zu den beschriebenen Verschleißschäden führen, sind identisch mit den zu Primärverschleiß (Kap. 5.3.4.1) und Sekundärverschleiß (Kap. 5.3.4.2) beschriebenen Verschleißmechanismen in der Rückenfläche der Schälmesser. Der wesentliche Unterschied besteht im vollständigen Verlust des Verschleißschutzes durch die fortlaufende Wirkung dieser Mechanismen auch nach Überschreiten der Verschleißgrenze der Schälmesser (Kap. 5.3.4.5) (Abb. 55). Die Intensität des Abriebs nimmt dabei aufgrund des fehlenden Verschleißschutzes zum Teil deutlich zu.

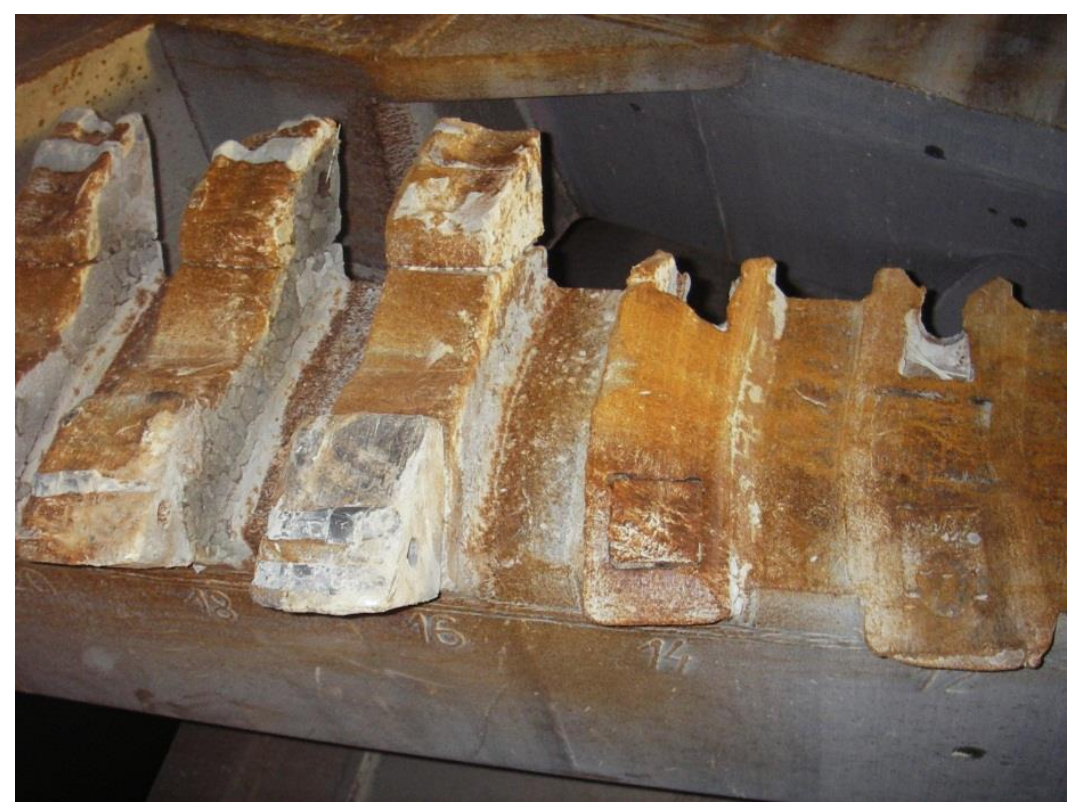

Abb. 56: Frontansicht eines Schneidradarmes nach Vortriebsende. Auf Höhe der Bildmitte sind von links nach rechts fünf Schälmesserpaare bzw. Halterpaare zu sehen. Auf den drei linken Halterpaaren sind noch Schälmesser montiert, die bereits deutlich über die Verschleißgrenze hinaus verschlissen sind (Verschleißschaden). Die Schälmesser der beiden rechten Halterpaare wurden zunächst durch Verschleiß vollständig entfernt, nachfolgend wurden auch die Halter bis auf Höhe der Schneidradstahlstruktur eingeebnet (Verschleißschaden Schneidradstahlstruktur). (Herrenknecht AG) 


\subsubsection{Gewaltschäden}

\section{Schadensbilder}

An Schälmessern können zusammenfassend drei Schadensbilder beschrieben werden, die auf Gewaltschäden zurückzuführen sind. Diese treten häufig in Böden mit Gehalt an Steinen oder Blöcken, sowie bei künstlichen Hindernissen, wie z.B. verlorene Bohrgestänge, bewährte Pfähle und Schlitzwände, Spundwände oder -bohlen, auf.

Hier sind an der Schneide der Schälmesser häufig Sprödbrüche der Hartmetalleinsätze feststellbar (Abb. 57). Diese können als einzelne Risse in den Hartmetalleinsätzen ausgebildet sein, jedoch auch zum Abbrechen großer Teile der Hartmetalleinsätze führen. Dabei bleiben häufig kleine Reste des Hartmetalls am Stahlgrundkörper zurück.

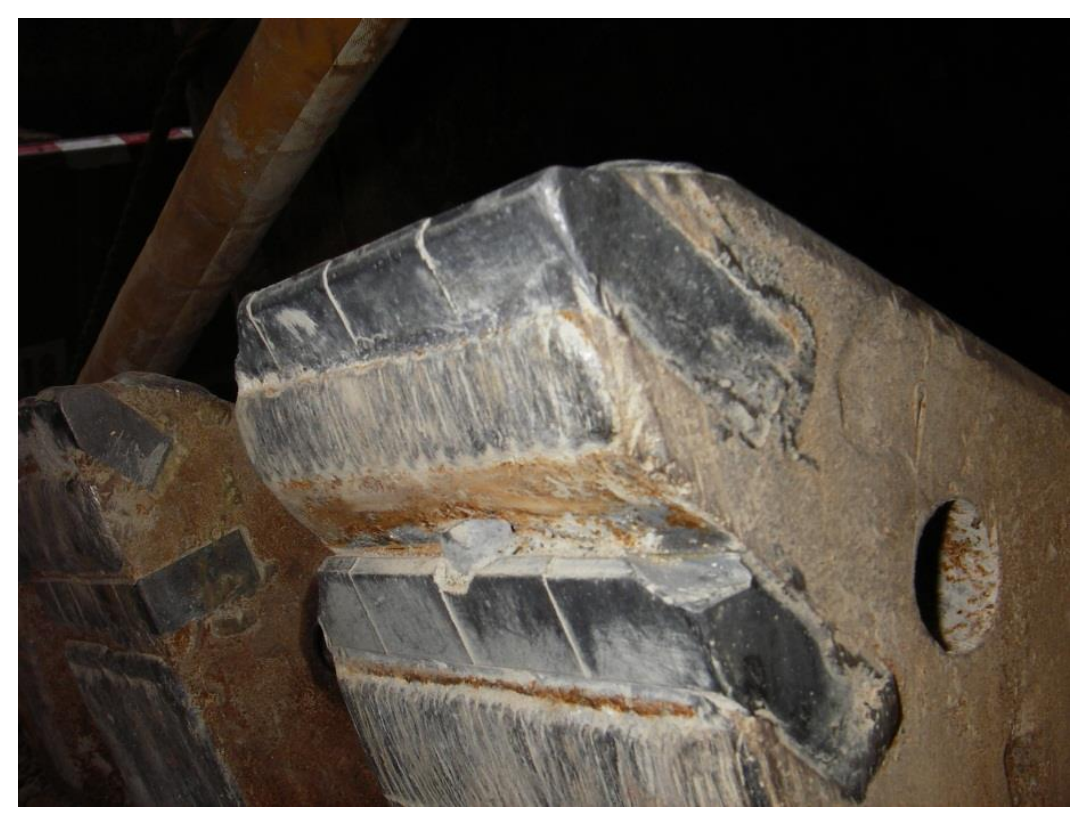

Abb. 57: Blick auf die Rückenfläche eines Schälmessers nach dem Vortrieb in steinigen Kiesen. An den Hartmetalleinsätzen in der Rückenfläche sind zwei Sprödbrüche erkennbar, wobei beim linken der beiden Brüche das Bruchstück noch am Grundkörper anhaftet. Der Hartauftrag in der Rückenfläche zeigt daneben die für den Primärverschleiß typischen, parallel verlaufenden, Kratzer in Schneidrichtung des Schälmessers (Kap. 5.3.4.1).

In seltenen Fällen und hauptsächlich im Zusammenhang mit steigender Größe der Blöcke und Hindernisse treten auch Sprödbrüche im Grundkörper der Schälmesser auf. Diese verlaufen häufig zwischen Schaft und Kopf des Schälmessers und führen zum vollständigen Verlust des Schälmessers.

Ein ähnliches Phänomen stellen Sprödbrüche in den Befestigungselementen der Schälmesser dar, die ebenfalls zum vollständigen Verlust der Schälmesser führen.

\section{Rückschlüsse auf den Schadensmechanismus}

Die bestehenden Erkenntnisse zum Abbau von Blöcken in der Ortsbrust (Kap. 4.2.1) zeigen, dass durch vorlaufende Schneidrollen nur ein teilweiser Abbau in der Ortsbrust stattfindet. In Abhängigkeit der Festigkeit der Einbindung des Blocks und der Penetration wird ab einer bestimmten Schnitttiefe (Abb. 26) der Rest des Blocks vollständig oder in mehreren Einzelteilen aus der Ortsbrust gedrückt. Diese Restteile ragen dann in den Zwischenraum zwischen Schneidradstahlbau und Ortsbrust hinein (Abb. 58). 


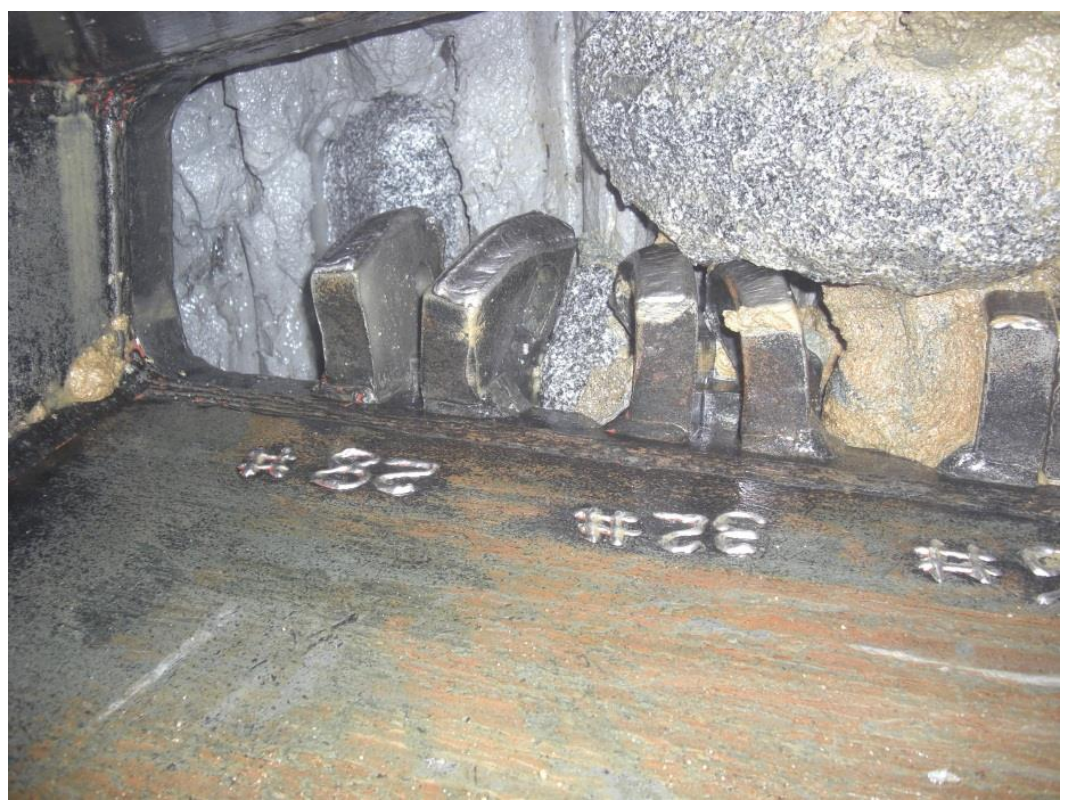

\begin{abstract}
Abb. 58: Blick durch eine Schneidradöffnung auf Schälmesserhalter und Ortsbrust in tonigen Schluffen mit eingelagerten Granitblöcken. Die Schälmesser auf den sichtbaren Spuren Nr. 28 \& 32 fehlen aufgrund von Sprödbrüchen der Schälmesser und Befestigungselemente vollständig. Die zugehörigen Schälmesserhalter wurden durch die eingelagerten Blöcke stark deformiert. (Herrenknecht AG)
\end{abstract}

Die Analyse des Schadensbildes von Sticheln (Kap. 5.3.3.4) zeigt, dass Steine und Blöcke in der Ortsbrust hier nicht abgebaut werden, sondern als Ganzes aus der Ortsbrust entfernt werden. Auch in diesem Fall ragen die Steine und Blöcke in den Zwischenraum zwischen Schneidradstahlbau und Ortsbrust hinein.

In Abhängigkeit der Lage, Form und Größe der Steine, Blöcke oder deren Restteile kommt es hier beim Auftreffen auf ein Schälmesser zu schlagartigen, mechanischen Belastungen der Schälmesser, sodass Sprödbrüchen der Hartmetalleinsätze, aber auch Sprödbrüchen des Grundkörpers entstehen. Dieser Effekt wird verstärkt durch die Materialeigenschaften des Hartmetalls, das aufgrund der extrem hohen Härte nur eine relativ geringe Zähigkeit aufweist. Sprödbrüche sind deshalb in den Hartmetalleinsätzen deutlich häufiger festzustellen, als im Stahlgrundkörper, der eine geringere Härte, jedoch deutlich höhere Zähigkeit besitzt.

Zusätzlich ist das Verhältnis zwischen der Größe der Schneidradöffnungen und dem Durchmesser der Steine, Blöcke und deren Restteile von entscheidender Bedeutung.

Liegt der Durchmesser der Steine, Blöcke und deren Restteile deutlich niedriger als der Durchmesser der Schneidradöffnungen, so werden sie durch die Schälmesser in die Schneidradöffnungen in die Abbaukammer gefördert. Dabei entstehen häufig nur Sprödbrüche in den Hartmetalleinsätzen, da die auftretenden Kräfte für Schäden am Grundkörper der Schälmesser vermutlich zu gering sind.

Der Anstieg der Sprödbrüche im Grundkörper der Schälmesser mit steigender Größe der Blöcke in der Ortsbrust hängt anschließend einerseits mit einer entsprechenden Erhöhung der mechanischen Belastung zusammen. Andererseits wird auch der Transportmechanismus von der Ortsbrust durch die Schneidradöffnungen in die Abbaukammer mit zunehmender Größe erschwert.

Die Schneidradöffnungen weisen häufig nach außen hin einen zunehmenden Durchmesser auf. Liegt der Durchmesser der Steine, Blöcke und deren Restteile noch etwas niedriger als der Durchmesser der Schneidradöffnungen, werden sie zum Teil erst in der Sohle aufgenommen und fallen beim Weiterdrehen des Schneidrads im Bereich der Firste in den Schneidradöffnungen nach unten. Liegt der Durchmesser dagegen nahe an der Größe der Schneidradöffnungen, werden die Steine, Blöcke und deren Restteile zum Teil in den Schneidradöffnungen verklemmt und mitgeschleift, sodass eine Furche in der Ortsbrust entsteht. In beiden Fällen können vermutlich deutlich höhere Kräfte auf die Schälmesser übertragen werden, die zu den beschriebenen Schäden am Grundkörper der Schälmesser führen. 
Künstliche Hindernisse, die kleiner sind als der Durchmesser der Schneidradöffnungen verhalten sich in der Ortsbrust zunächst ähnlich wie Steine oder Blöcke. Im Unterschied dazu werden diese jedoch durch die Schneidrollen bzw. Stichel häufig als Ganzes aus der Ortsbrust entfernt und durch die Schneidradöffnungen in die Abbaukammer gefördert. Dabei kann in Abhängigkeit der Größe der Hindernisse eine schlagartige mechanische Belastung der Schälmesser entstehen, die zu Sprödbrüchen an den Hartmetalleinsätzen der Schälmesser führt.

Größere Hindernisse, wie Spundwände oder Bohrpfähle werden aufgrund der Stahlbewehrung in der Ortsbrust nicht vollständig abgebaut. Der Bewährungsstahl wird durch Schneidrollen bzw. Stichel freigelegt, jedoch nicht geschnitten oder entfernt (Abb. 46). Beim Auftreffen der Schälmesser auf die Stahlbewährung entsteht eine schlagartige mechanische Belastung der Schälmesser, die zu den beschrieben Sprödbrüchen an den Hartmetalleinsätzen führen kann. Durch Verklemmen von Stahlteilen am Schneidrad oder schlicht unbeweglichen Hindernissen in der Ortsbrust können noch deutlich höhere Kräfte übertragen werden, die zu Schäden am Grundkörper der Schälmesser führen können.

\subsubsection{Verschleißgrenzen}

Für Schälmesser können aus den Beobachtungen zum Ortsbrustbild, Verschleißbild und den Rückschlüssen zur Abbaumechanik (Kap. 5.3.3) die nachfolgenden Verschleißgrenzen abgeleitet werden. Schälmesser, an denen während einer Schneidradinspektion ein Schaden festgestellt wird, müssen unabhängig davon sofort gewechselt werden.

\section{Primärverschleiß}

Der Abrieb an den Schälmessern beim aktiven Bodenabbau ist hauptsächlich parallel zur Ortsbrust an der Rückenfläche der Schälmesser, sowie an der Frontfläche in Bewegungsrichtung der Schälmesser festzustellen (Kap. 5.3.4.1).

Für den Abrieb an der Rückenfläche parallel zur Ortsbrust ergeben sich in Abhängigkeit der Auslegung der Schälmesser und des Halters zwei mögliche Verschleißgrenzen, die projektspezifisch zu überprüfen sind. Zunächst ist der Überstand der Schneide der Schälmesser über dem Halter maßgeblich. Ein Wechsel des Schälmessers ist erforderlich, bevor der Halter aufgrund des Abriebs an der Rückenfläche des Schälmessers parallel zur Ortsbrust direkten Kontakt mit dem Boden in der Ortsbrust erhält. Folge wäre in diesem Fall ein Verschleißschaden am Halter, durch den die sichere Fixierung des Schälmessers verloren geht und ein Austausch des Halters notwendig wird. Um den Halter dauerhaft zu schützen, ist deshalb ein Wechsel der Schälmesser bereits $10 \mathrm{~mm}$ vor Erreichen der Höhe des Halters $\left(h_{S M H}\right)$ zu empfehlen. Der maximale Abrieb an den Schälmessern $h_{d, \max (S M)}[\mathrm{mm}]$ ergibt sich mit Gl. 23 aus:

$$
\mathrm{h}_{\mathrm{d}, \max (\mathrm{SM})}=\left(\mathrm{h}_{\mathrm{SM}}-\mathrm{h}_{\mathrm{SMH}}\right)-10 \mathrm{~mm}
$$

Die verbleibende Höhe der Schälmesser kann im Vortrieb dabei relativ einfach entweder mit Bezug auf den Schälmesserhalter oder die Schneidradstahlstruktur mit einem Meterstab in ausreichender Genauigkeit gemessen werden.

Die in Gl. 23 angegebene Methode zur Bestimmung der Verschleißgrenze der Schälmesser im Hinblick auf den Abrieb an der Rückenfläche parallel zur Ortsbrust muss gegen die Einbindetiefe der Hartmetalleinsätze an der Schneide und im Rücken der Schälmesser abgeglichen werden. Bei Auslegungen von Schälmesser und Halter, die eine relativ hohe Differenz zwischen der Höhe der Schälmesser über der Schneidradstahlstruktur $h_{S M}$ und der entsprechenden Höhe des Halters $h_{S M H}$ ergeben, kann die Unterkante der Hartmetalleinsätze in der Schneide oder im Rücken bereits vor der in Gl. 23 angegebenen Verschleißgrenze erreicht werden. Ist dies der Fall, so ist ein Wechsel des Schälmessers bei Erreichen der Unterkante der Hartmetalleinsätze notwendig (Abb. 59). Bei Überschreiten dieser Grenze geht der Verschleißschutz der Schälmesser weitgehend verloren, sodass die Verschleißrate $c_{v}$ rapide ansteigt (Kap. 5.5.4). Ein effektiver Schutz vor einem Verschleißschaden am zugehörigen Halter ist damit durch das Schälmesser nicht mehr gegeben. 


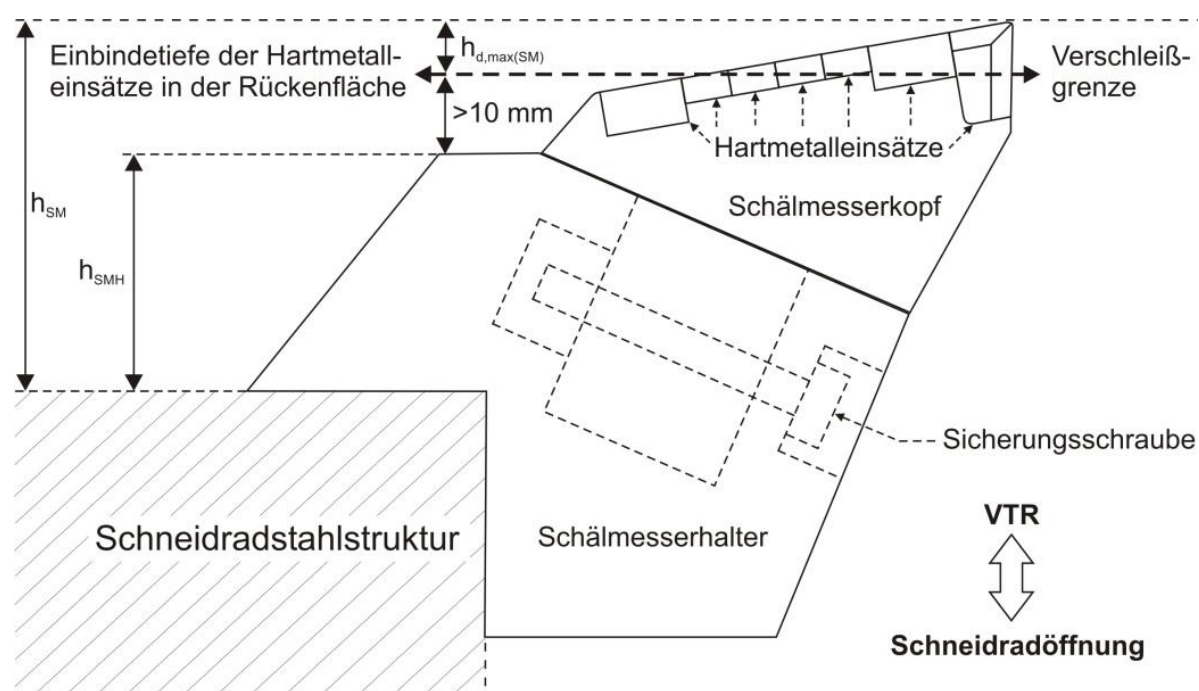

\begin{abstract}
Abb. 59: Seitenansicht eines Schälmessers im Halter auf dem Schneidrad. Die Einbindetiefe der Hartmetalleinsätze liegt höher als die Obergrenze des Halters. Die Unterkante der Hartmetalleinsätze stellt also die Verschleißgrenze $h_{d, \max (S M)}$ dieses Schälmessers, bezogen auf den Abrieb der Rückenfläche, parallel zur Ortsbrust dar.
\end{abstract}

An der Frontfläche der Schälmesser wird durch den Verschleiß beim aktiven Bodenabbau zusätzlich häufig die Stahlmatrix unterhalb der Hartmetalleinsätze der Schneide angegriffen. Ein Wechsel des Schälmessers wird hier notwendig, sobald keine ausreichende Einbindung der Hartmetalleinsätze mehr gegeben ist. Problematisch ist hier die tatsächliche Beurteilung des Zustandes im Vortrieb. Eine sichere Quantifizierung der verbleibenden Festigkeit der Einbindung ist weder visuell noch mit anderen vortriebstauglichen Messmethoden durchführbar. Bei Ansatz einer konservativen Werkzeugwechselstrategie sollte der Wechsel deshalb erfolgen, sobald eine Freilegung der unteren Anlagefläche erkennbar ist.

\title{
Sekundärverschleiß
}

Der Sekundärverschleiß im Rücken der Schälmesser führt ähnlich wie der Verschleiß an der Frontfläche der Schälmesser häufig zum Abrieb an Hartauftrag und Hartmetalleinsätzen, sowie zu einer Freilegung der Hartmetalleinsätze. Daraus resultiert eine mangelnde Einbindung der Hartmetalleinsätze in die Stahlmatrix, die einerseits das Brechen der Hartmetalleinsätze begünstigt und andererseits bei vollständiger Freilegung zum Verlust der Hartmetalleinsätze führt (Kap. 5.3.4.2).

Folglich wird ein Wechsel des Schälmessers notwendig, sobald durch Freilegung begünstigte Brüche in den Hartmetalleinsätzen im Rücken der Schälmesser erkennbar sind. Unabhängig davon stellt auch die Freilegung der Hartmetalle selbst ab einem gewissen Ausmaß einen Wechselgrund dar, wenn ein vollständiges Herausbrechen der Hartmetalleinsätze abzusehen ist.

Zusätzlich kann der Materialverlust durch sekundären Verschleiß im Rücken der Schälmesser so hoch werden, dass auch der Halter auf der Rückseite der Schälmesser angegriffen wird. Folglich ist ein Wechsel des Schälmessers auch notwendig, sobald die Gefahr besteht, dass der Halter angegriffen wird.

Problematisch an allen drei genannten Kriterien ist, dass sich aufgrund der hohen Werkzeug- und Haltervielfalt in den Referenzprojekten keine eindeutige und im Vortrieb messbare Verschleißgrenze für dieses Kriterium definieren lässt. Zusätzlich ist die Rückseite der Schälmesser im Vortrieb nur schwer kontrollierbar. Eine Kontrolle ist nur durch Ertasten der Rückenfläche per Hand möglich. Folglich unterliegt die Beurteilung des Sekundärverschleißes weiterhin der subjektiven, visuellen Einschätzung durch den jeweiligen Bearbeiter. 


\subsubsection{Räumer}

Die Verschleiß- und Schadensmechanismen für Räumer weisen aufgrund der Ähnlichkeit in der Werkzeuggeometrie und der Anwendung auch eine Übereinstimmung mit den entsprechenden Mechanismen bei Schälmessern auf. Allerdings ergeben sich aufgrund der Krümmung, der durchgehenden Schneide der Räumer und der Änderung in der Anordnung der vorlaufenden Werkzeuge auch wesentliche Unterschiede. Die nachfolgende Beschreibung verweist an entsprechender Stelle deshalb auf die analogen Erläuterungen für Schälmesser. Alle wesentlichen Unterschiede werden ausführlich behandelt.

\subsubsection{Bodenabbau und Primärverschleiß}

\section{Ortsbrustbild}

Das Ortsbrustbild beim aktiven Bodenabbau mit Räumern stimmt unabhängig von der Bodenart und trotz der durchgehenden, gekrümmten Schneide mit dem Ortsbrustbild beim aktiven Bodenabbau mit Schälmessern (Kap. 5.3.4.1) überein.

\section{Verschleißbild}

Auch das Verschleißbild der Räumer beim aktiven Bodenabbau weist eine hohe Ähnlichkeit mit dem Verschleißbild von Schälmessern auf (Kap. 5.3.4.1).

An der Frontfläche der Räumer bildet sich analog zu den Schälmesser unterhalb der Schneide häufig eine quer zur Schneidrichtung verlaufender Verschleißmulde, die bis zur Einbettung der Hartmetalleinsätze an der Schneide der Räumer reichen kann, sodass deren Einbindung in die Stahlmatrix sinkt. Die Intensität des Abriebs in diesem Bereich ist in feinkörnigen und gemischtkörnigen Böden häufig höher, als in grobkörnigen Böden.

Auf der Rückenfläche der Räumer ist, unabhängig von der Bodenart, verursacht durch den aktiven Bodenabbau überwiegend Abrieb parallel zur Ortsbrust zu beobachten. Dieser setzt anfänglich direkt hinter der Schneide an. Räumer besitzen häufig einen abgestuften Freiwinkel $\beta_{\mathrm{c}}$. Unmittelbar angrenzend an die Schneide liegt dieser deutlich niedriger als bei Schälmessern. Die Kontaktfläche zur Ortsbrust vergrößert sich in diesem Bereich mit zunehmendem Abrieb deshalb relativ schnell. Etwa ab der Hälfte der Rückenfläche wird der Freiwinkel in einer Stufe häufig stark erhöht, sodass sich ab der Abstufung die Kontaktfläche zur Ortsbrust mit zunehmendem Abrieb nur noch langsam vergrößert.

Im Bereich der Kontaktfläche zur Ortsbrust ist zwischen den Hartmetalleinsätzen der Schneide und der Rückenfläche, sowie zwischen Hartmetalleinsätzen in der Rückenfläche und dem umgebenden Stahl des Grundkörpers häufig kein oder nur ein geringer Niveauunterschied im Abrieb erkennbar. Zusätzlich ist an der Schneide selbst eine Abstumpfung bzw. Abrundung der Hartmetalleinsätze erkennbar.

Bei vorlaufenden Schneidrollen ist in gemischtkörnigen und grobkörnigen Böden eine Differenzierung in der Intensität des Abriebs an den Räumern zu erkennen. Im Bereich der Schneidspuren der vorlaufenden Schneidrollen entsteht in der Rückenfläche und an der Front der Schälmesser deutlich weniger Abrieb als in den Bereichen zwischen den Schneidspuren der Schneidrollen. Folglich bilden sich im Bereich der Räumer mehrere in Schneidrichtung verlaufende Grate, die die Form der in der Ortsbrust vorliegenden Furchen entlang der Schneidspuren der Schneidrollen (Abb. 60) nachbilden.

Beim Einsatz vorlaufender Stichel haben die Räumer keinen Kontakt mehr zur Ortsbrust und leisten keinen aktiven Bodenabbau, sodass kein Primärverschleiß aus dem aktiven Bodenabbau entsteht. 


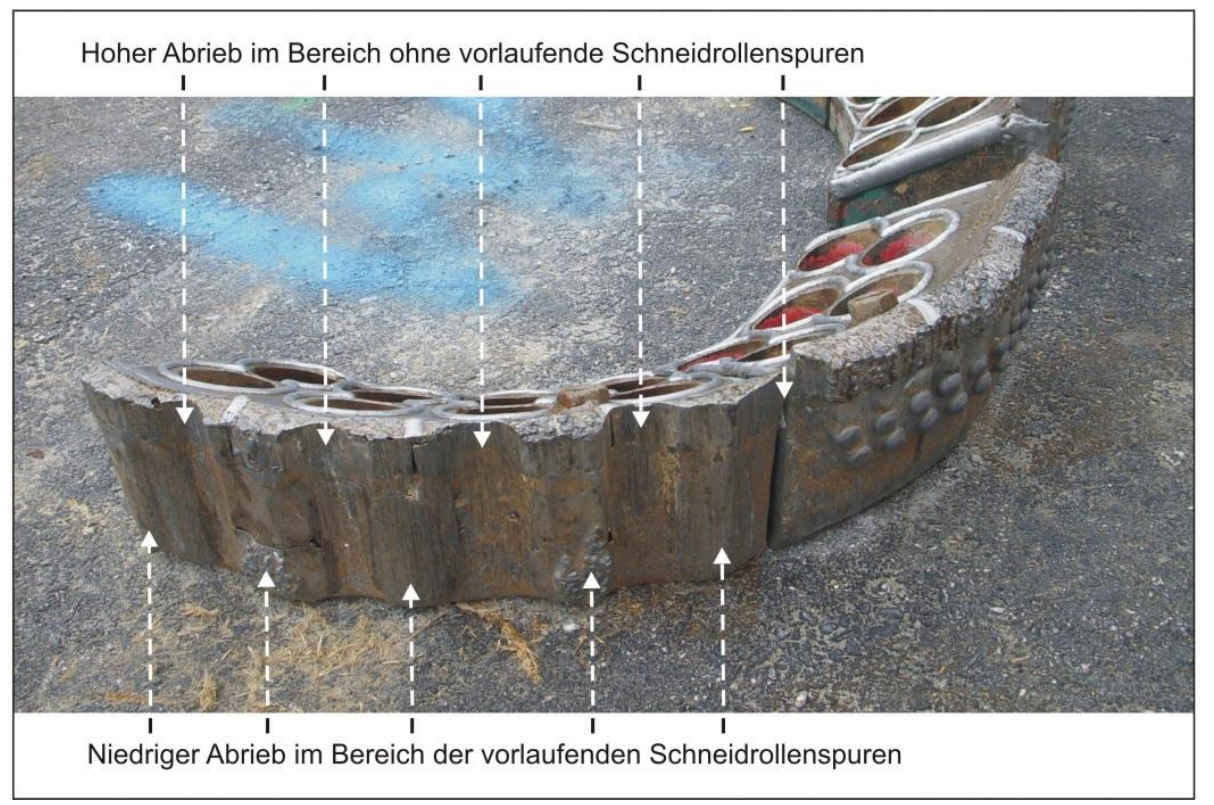

\begin{abstract}
Abb. 60: Blick auf die Rückenfläche eines Räumersegments nach dem Vortrieb in schluffigen Sanden. Der Abrieb in der Rückenfläche ist im Bereich der vorlaufenden Schneidrollenspuren deutlich niedriger als in den dazwischen liegenden Bereichen ohne vorlaufende Schneidrollenspuren. (Herrenknecht AG; verändert)
\end{abstract}

\title{
Rückschlüsse auf die Abbaumechanik
}

Das Ortsbrustbild und das Verschleißbild der Räumer legen aufgrund der hohen Ähnlichkeit nahe, dass die grundsätzlichen Mechanismen beim aktiven Bodenabbau mit Schälmessern (Kap. 5.3.4.1) auch auf die Räumer übertragbar sind.

Die Ergebnisse deuten folglich darauf hin, dass beim primären Bodenabbau in feinkörnigen Böden durch die Räumer ein Bodenspan aus der Ortsbrust gelöst wird, der über die Frontfläche der Räumer abgleitet. Über gemischtkörnige Böden hin zu grobkörnigen Böden wird vermutlich zunehmend kein zusammenhängender Bodenspan mehr ausgebildet, der vollständig über die Frontfläche abgleitet. Der Boden zerfällt unmittelbar beim Abbau zunehmend in die einzelnen Bodenkomponenten, die dann an der Frontfläche der Räumer in Richtung der Schneidradöffnung abgleiten.

Bei vorlaufend eingesetzten Schneidrollen leisten die Räumer im Bereich der Furchen entlang der Schneidspuren der vorlaufenden Schneidrollen nahezu keinen Bodenabbau. Durch die Furchenbildung haben die Räumer hier keinen oder nur sehr geringen Kontakt zur Ortsbrust, sodass hier auch deutlich weniger Abrieb entsteht.

\subsubsection{Sekundärverschleiß}

Sekundärverschleiß an den Räumern entsteht einerseits durch Kontakt mit der Ortsbrust während dem passiven Einsatz, andererseits jedoch auch durch Kontakt mit bereits abgebauten Bodenkomponenten in der Stützflüssigkeit.

\section{Ortsbrustbild}

Das Ortsbrustbild, das durch den passiven Einsatz der Räumer verursacht wird, entspricht weitgehend dem Ortsbrustbild beim aktiven Bodenabbau. In feinkörnigen Böden werden wiederum die Konturen der Räumer zum Teil detailliert nachgebildet. In gemischtkörnigen und grobkörnigen Böden ergibt sich kein wesentlicher Unterschied zum Ortsbrustbild beim aktiven Bodenabbau. 


\section{Verschleißbilder}

Die Kontaktfläche zur Ortsbrust, die analog auch beim aktiven Einsatz der Räumer entsteht, bleibt auch beim passiven Einsatz erhalten. Entsprechend sind auf der Fläche weiterhin Kratzer in bzw. entgegen der Schneidrichtung sichtbar.

Zusätzlich zum Abrieb an der Kontaktfläche zur Ortsbrust sind verschiedenen Verschleißphänomene an der verbleibenden Rückenfläche feststellbar. Hier ist häufig eine Freilegung bzw. Ausspülung der Hartmetalleinsätze feststellbar. In feinkörnigen Böden sind dabei häufig Spülmarken erkennbar und der Abrieb konzentriert sich auf den Bereich unmittelbar hinter der Kontaktfläche zur Ortsbrust. In gemischtkörnigen und grobkörnigen Böden breitet sich der Abrieb zunehmend auf die vollständige Rückenfläche aus, wobei die Ausbildung von Spülmarken zurückgeht. Zum Teil ist auch eine deutliche Zunahme der Intensität des Abriebs am Grundkörper zu beobachten.

An den Hartmetalleinsätzen in der Rückenfläche selbst ist ebenfalls Abrieb erkennbar, der jedoch deutlich geringer ist als am Stahl des Grundkörpers. Der Abrieb ist zudem zum Teil einseitig und auf der Rückseite bzw. entgegen der geplanten Schneidrichtung höher als an der Frontseite in Schneidrichtung.

Zusätzlich zu diesen Phänomenen ist über die vollständige Frontfläche der Räumer zum Teil flächiger Abrieb mit deutlich geringerer Intensität erkennbar.

\section{Rückschlüsse auf die Abbaumechanik}

Der Abrieb an der Kontaktfläche zur Ortsbrust setzt sich analog zum Primärverschleiß fort, weil die Räumer auch beim passiven Einsatz Kontakt zur Ortsbrust haben. In Abhängigkeit des Winkelabstandes zum entsprechenden aktiven Räumer erfahren diese eine minimale Penetration (Kap. 5.4.5) in die Ortsbrust.

Der Abrieb des Stahlgrundkörpers und die sichtbaren Spülmarken (Abb. 61) in feinkörnigen Böden in der verbleibenden Rückenfläche ohne Kontakt zur Ortsbrust zeigen, dass beim passiven Einsatz der Räumer durch die Führung entgegen der geplanten Schneidrichtung Stützflüssigkeit und die darin enthaltenen Bodenkomponenten in den Freiwinkel strömen.

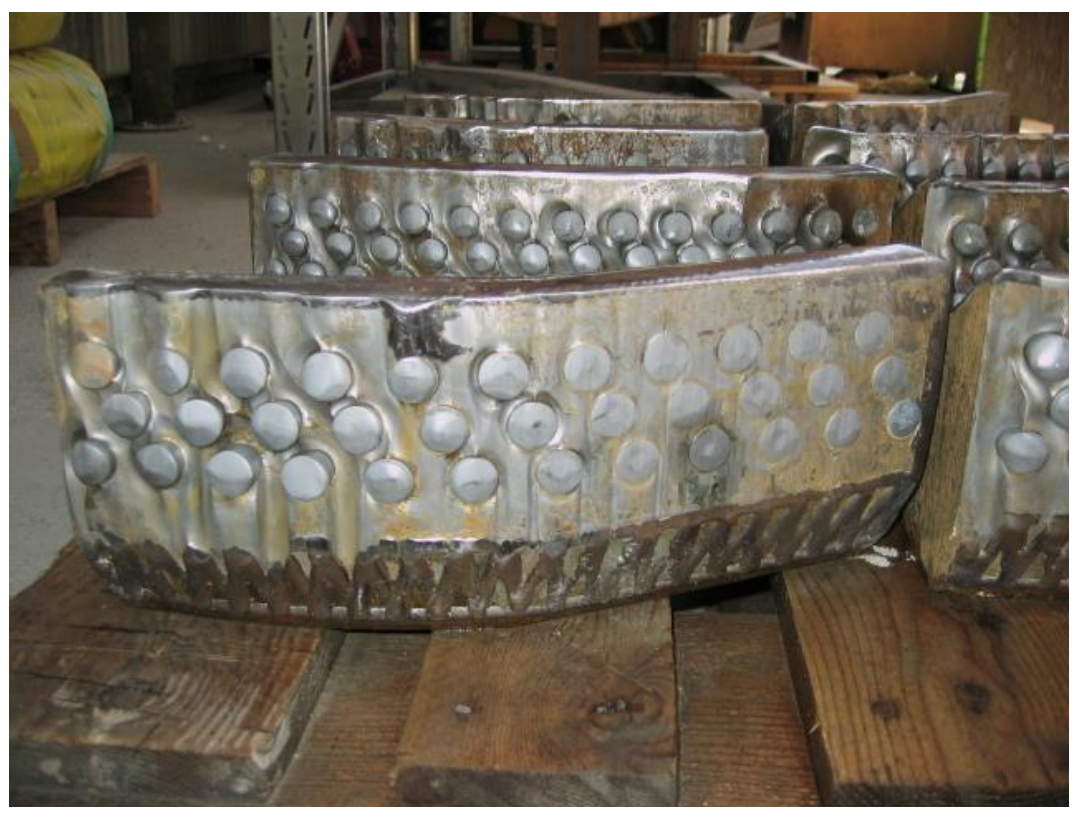

Abb. 61: Blick auf die Rückenfläche eines Räumersegments nach dem Vortrieb in sandigen Schluffen. Der rechte Teil der Rückenfläche ist durch Primärverschleiß gleichmäßig eingeebnet. Zwischen den Hartmetalleinsätzen und dem Grundkörper ist hier kein Unterschied erkennbar. Im linken Teil der Rückenfläche sind die Hartmetalleinsätze in der Rückenfläche durch Sekundärverschleiß freigelegt. Dabei sind deutliche Spülmarken zwischen den Einsätzen erkennbar. (Herrenknecht AG) 
Bei zunehmender Einengung strömt die Stützflüssigkeit seitlich aus dem Freiwinkel heraus. Das dabei entstehende Verschleißbild lässt sich analog zum Sekundärverschleiß der Rückenfläche von Schälmessern schlüssig erklären. Aufgrund der durchgehenden Schneide der Räumer wird der Abstrom der Stützflüssigkeit und der darin enthaltenen Bodenkomponenten vermutlich zusätzlich erschwert (Abb. 61).Ein Unterschied in der Intensität des Abriebs konnte jedoch nicht festgestellt werden.

Der flächige Abrieb der Räumer in der Frontfläche wird vermutlich durch bereits gelöste Bodenkomponenten verursacht, die vor dem Schneidrad in der Schildsohle zum Liegen kommen und durch die Räumer in die Schneidradöffnung transportiert werden. Die Bewegungsrichtung der Räumer ist dabei parallel zur Ortsbrust, sodass während dem Transport der Bodenkomponenten in die Schneidradöffnung keine Korn-Korn-Kontakte gegen die Ortsbrust oder ein anderes Widerlager aufgebaut werden. Die Intensität des flächigen Abriebs in der Frontfläche der Räumer bleibt damit relativ niedrig.

Grundsätzlich besteht folglich auch für Räumer ein Zusammenhang zwischen der Ausbildung des Freiwinkels, der Korngrößenverteilung des Bodens und der Verschleißart und Intensität in der Rückenfläche. Die Austauschrate der Stützflüssigkeit in der Abbaukammer hat einen Einfluss auf die Intensität, da die Wahrscheinlichkeit für Korn-Korn-Kontakte im Freiwinkel sinkt, je geringer die Konzentration der Bodenkomponenten in der Stützflüssigkeit ist. Zusätzlich gewinnt die Austauschrate an Bedeutung, weil sie den Anstau von gelösten Bodenkomponenten in der Schildsohle maßgeblich beeinflusst. Dieser nimmt ebenfalls mit zunehmender Austauschrate ab.

\subsubsection{Verschleißschäden}

Verschleißschäden an Räumern treten durch Überschreiten der Verschleißgrenze ein. Dabei können die nachfolgenden Schadensbilder beschrieben werden. Diese sind nahezu identisch mit den entsprechenden Schadensbildern für Schälmesser (Kap. 5.3.4.3).

\section{Schadensbilder}

Der Abrieb in der Kontaktfläche zwischen Räumer und Ortsbrust beim aktiven und passiven Einsatz dehnt sich bei verspätetem Wechsel der Räumer auf die vollständige Rückenfläche aus. Dabei erreicht der Abrieb ein Niveau, bei dem sowohl die Hartmetalleinsätze an der Schneide, als auch der Verschleißschutz in der Rückenfläche vollständig entfernt werden. Die Rückenfläche der Räumer ist dann vollständig eingeebnet und zeigt durchgehende Kratzer in bzw. gegen die Schneidrichtung.

Ein ähnliches Schadensbild ergibt sich bei hohem Sekundärverschleiß in der Rückenfläche. Hier können durch den spülenden oder mahlenden Abrieb sowohl der Verschleißschutz in der Rückenfläche, als auch die Hartmetalleinsätze an der Schneide entfernt werden. Die Rückenfläche zeigt dann eine deutlich unebene Oberfläche.

\section{Rückschlüsse auf den Schadensmechanismus}

Die Mechanismen, die zu den beschriebenen Verschleißschäden führen, sind identisch mit den zu Primärverschleiß (Kap. 5.3.5.1) und Sekundärverschleiß (Kap. 5.3.5.2) beschriebenen Verschleißmechanismen in der Rückenfläche der Räumer. Der wesentliche Unterschied besteht im vollständigen Verlust des Verschleißschutzes durch die fortlaufende Wirkung dieser Mechanismen auch nach Überschreiten der Verschleißgrenze (Kap. 5.3.5.5). Die Intensität des Abriebs nimmt dabei aufgrund des fehlenden Verschleißschutzes zum Teil deutlich zu. 


\subsubsection{Gewaltschäden}

\section{Schadensbilder}

Die Schadensbilder an Räumern, die auf Gewaltschäden hinweisen, stimmen grundsätzlich mit den entsprechenden Schadensbildern an Schälmessern (Kap. 5.3.4.4) überein. Sie treten ebenfalls häufig in Böden mit Gehalt an Steinen oder Blöcken, sowie bei Antreffen künstlicher Hindernisse wie z.B. verlorene Bohrgestänge, bewährte Pfähle und Schlitzwände, Spundwände oder -bohlen auf und können wie folgt zusammengefasst werden:

- Sprödbrüche der Hartmetalleinsätze in der Schneide der Räumer

- Sprödbrüche und Verformungen im Grundkörper der Räumer

- Sprödbrüche und Verformungen der Befestigungselemente der Räumer

Im Gegensatz zu Schälmessern treten Sprödbrüche und Verformungen im Grundkörper der Räumer oder an den Befestigungselementen deutlich seltener auf.

\section{Rückschlüsse auf die Schadensmechanismen}

Der Abbaumechanismus für Steine und Blöcke in der Ortsbrust ist für Räumer identisch zu Schälmessern (Kap. 5.3.4.4). Lose Steine, Blöcke und deren Restteile führen bei der Förderung in die Schneidradöffnungen aufgrund der schlagartigen mechanischen Belastung auch an den Räumern zu Sprödbrüchen der Hartmetalleinsätze. Sprödbrüche im Grundkörper der Räumer sind aufgrund der Größe der Räumersegmente und der damit verbundenen höheren Widerstandsfähigkeit gegenüber mechanischen Belastungen deutlich seltener als bei Schälmessern. Die Intensität der Schäden nimmt mit steigender Größe der Steine, Blöcke und Restteile in deutlich geringerem Umfang zu.

Das Verhältnis zwischen der Größe der Schneidradöffnungen und dem Durchmesser der Steine, Blöcke und deren Restteile hat für die Gewaltschäden an den Räumern eine ähnlich hohe Bedeutung wie für Schälmesser. Der Größenbereich an Steinen, Blöcken und deren Restteilen, die sich im Bereich der Räumer in den Schneidradöffnungen verklemmen können, ist jedoch deutlich geringer. Die Intensität der Schäden liegt aufgrund der Größe der Räumersegmente und der im Vergleich robusteren Befestigung wiederum niedriger als bei Schälmessern.

Hindernisse, die kleiner sind als der Durchmesser der Schneidradöffnungen verhalten sich in der Ortsbrust zunächst ähnlich wie Steine oder Blöcke. Im Unterschied dazu werden diese jedoch durch die Schneidrollen und Stichel häufig als Ganzes aus der Ortsbrust entfernt. Bei der Förderung durch die Schneidradöffnungen in die Abbaukammer kann, analog zu Schälmessern, in Abhängigkeit der Größe der Hindernisse, eine schlagartige mechanische Belastung der Räumer, die zu Sprödbrüchen an den Hartmetalleinsätzen in der Schneide führt, entstehen.

Größere Hindernisse, wie Spundwände oder Bohrpfähle werden aufgrund der Stahlbewährung in der Ortsbrust nicht vollständig abgebaut. Der Bewährungsstahl wird durch Schneidrollen bzw. Stichel freigelegt, jedoch nicht geschnitten oder entfernt. Beim Auftreffen der Räumer auf die Stahlbewährung entsteht wiederum eine schlagartige mechanische Belastung, die zu den beschrieben Sprödbrüchen an den Hartmetalleinsätzen führen kann. Durch Verklemmen von Stahlteilen am Schneidrad oder schlicht unbeweglichen Hindernissen in der Ortsbrust können noch deutlich höhere Kräfte übertragen werden, die auch zu Sprödbrüchen oder Verformungen am Grundkörper und den Befestigungselementen führen können.

\subsubsection{Verschleißgrenzen}

Die an Räumern festgestellten Verschleißbilder sind im Grundsatz ebenfalls sehr ähnlich zu den Verschleißbildern der Schälmesser (Kap. 5.3.3), sodass auch relativ ähnliche Verschleißgrenzen definiert werden können. Der wesentliche Unterschied liegt in der Krümmung der Räumer. Unabhängig davon ist ein Wechsel der Räumer notwendig, sobald Schäden am Räumer festgestellt werden. 


\section{Primärverschleiß}

Durch Bodenabbau und Materialförderung der Räumer beim aktiven Einsatz entsteht häufig Abrieb entlang der Schneidkontur der Räumer an der Kontaktfläche zur Ortsbrust (Kap. 5.3.5.1).

In Abhängigkeit der Auslegung des Räumers und des Halters kann für den Abrieb entlang der Kontur des Räumers an der Kontaktfläche zur Ortsbrust eine klare Verschleißgrenze definiert werden. In erster Linie ist ein Wechsel des Räumers notwendig, sobald an der Kontur die Einbindetiefe der Hartmetalleinsätze bzw. die Untergrenze des Hartauftrages erreicht wird. Dieses Abriebmaß muss zusätzlich auf den Überstand des Räumers zum Halter hin überprüft werden. Ist die Einbindetiefe der Hartmetalleinsätze größer als der Überstand zum Halter, bzw. liegt die Untergrenze des Hartauftrages in der Frontfläche unterhalb des Halters, so stellt der Halter die maßgebliche Grenze für den Abrieb in der Kontur des Räumers dar. Um einen dauerhaften Schutz des Halters und eine sichere Fixierung der Räumer zu gewährleisten, ist ein Wechsel des Räumers bereits $10 \mathrm{~mm}$ vor Erreichen der Höhe des Halters $\left(h_{\mathrm{SMH}}\right)$ zu empfehlen. Für diesen Fall ergibt sich der maximale Abrieb $h_{\mathrm{d}, \max (\mathrm{RM})}[\mathrm{mm}]$ an den Räumern aus Gl. 24:

$$
\mathrm{h}_{\mathrm{d}, \max (\mathrm{RM})}=\left(\mathrm{h}_{\mathrm{RM}}-\mathrm{h}_{\mathrm{RMH}}\right)-10 \mathrm{~mm}
$$

Der Abrieb in der Kontur der Räumer kann im Vortrieb relativ einfach mithilfe eines Meterstabes bestimmt werden. Die Räumer sind im Regelfall durch die Schneidradöffnungen gut zugänglich. Analog zu Schälmessern sollte die mit Gl. 24 ermittelte Verschleißgrenze nicht unterhalb der Einbindetiefe der Hartmetalle in der Rückenfläche der Räumer liegen. In diesem Fall stellt die Einbindetiefe der Hartmetalleinsätze die Verschleißgrenze der Räumer dar (Abb. 62).

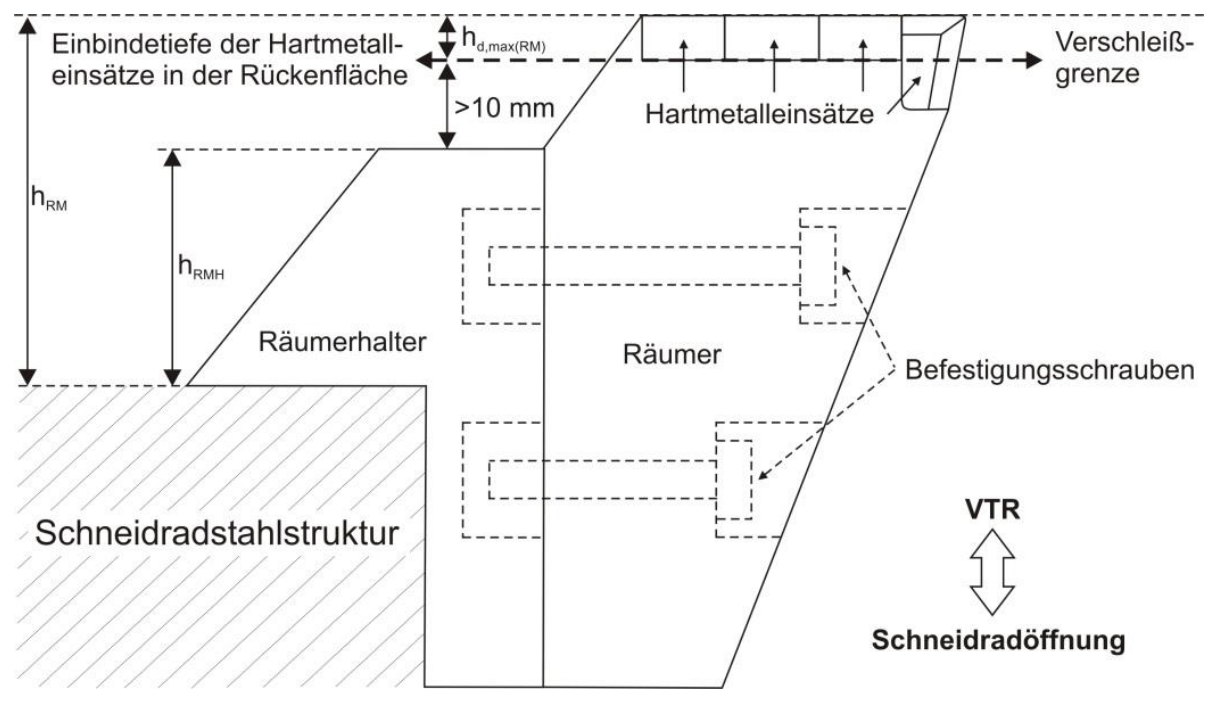

Abb. 62: Querschnitt durch ein Räumersegment, montiert auf dem Halter. Die Verschleißgrenze $h_{d, \max (R M)}$ ergibt sich aus der Einbindetiefe der Hartmetalleinsätze in der Rückenfläche.

Bei Schneidrädern ohne vorlaufende Werkzeuge vor den Räumern spielt zusätzlich der Überschnitt der Räumer über die Schildschneide eine wichtige Rolle für den Vortrieb. Dieser ist dann maßgeblich für den verbleibenden Ringspalt und hat damit Einfluss auf das Setzungsverhalten, die Schildreibung und die Hinterfüllung der Tübbinge.

Unabhängig von den oben genannten Kriterien für den Austausch eines Räumers werden zum Teil projektspezifisch deutlich niedrigere Abriebmaße an der Kontur der Räumer als Grenzwerte festgelegt, um die Materialförderung hinter das Schneidrad möglichst hoch zu halten. Zudem werden im Regelfall alle Räumersegmente eines Armes gleichzeitig ausgetauscht, um einen sauberen Übergang zwischen Ortsbrust und Tunnellaibung zu gewährleisten. 
Zusätzlich zum Abrieb an der Kontur der Räumer entsteht durch den Bodenabbau und die Materialförderung zum Teil auch Abrieb in der Frontfläche der Räumer (Kap. 5.3.4.5). Dadurch wird die Stahlmatrix unterhalb der Hartmetalleinsätze angegriffen bzw. der Hartauftrag in der Frontfläche abgerieben. Ein Wechsel des Räumers wird hier notwendig, sobald keine ausreichende Einbindung der Hartmetalleinsätze mehr gegeben ist, bzw. sobald der Hartauftrag in der Frontfläche vollständig entfernt ist. Äquivalent zum Primärverschleiß an Schälmessern (Kap. 5.3.4.5) ist eine sichere Beurteilung und Messung der verbleibenden Festigkeit der Einbindung der Hartmetalleinsätze im Vortrieb nicht durchführbar. Entsprechend sollte auch der Wechsel eines Räumers im Rahmen einer konservativen Werkzeugwechselstrategie erfolgen, sobald eine Freilegung der unteren Anlagefläche der Hartmetalleinsätze erkennbar ist.

\section{Sekundärverschleiß}

Der Sekundärverschleiß im Rücken der Räumer führt häufig zum Abrieb des Hartauftrages und zu einer Freilegung der Hartmetalleinsätze. Daraus resultiert eine mangelnde Einbindung der Hartmetalleinsätze in die Stahlmatrix, die einerseits das Brechen der Hartmetalleinsätze begünstigt und andererseits bei vollständiger Freilegung zum Verlust der Hartmetalleinsätze führt (Kap. 5.3.5.2).

Entsprechend wird ein Wechsel des Räumers notwendig, sobald durch Freilegung begünstigte Brüche in den Hartmetalleinsätzen im Rücken des Räumers erkennbar sind. Unabhängig davon stellt auch die Freilegung der Hartmetalle selbst ab einem gewissen Ausmaß einen Wechselgrund dar, wenn ein vollständiges Herausbrechen der Hartmetalleinsätze abzusehen ist.

Zusätzlich kann der Materialverlust durch sekundären Verschleiß im Rücken des Räumers so hoch werden, dass auch der Halter angegriffen wird. Folglich ist ein Wechsel des Räumers auch notwendig, sobald die Gefahr besteht, dass der Halter angegriffen wird.

Problematisch an allen drei genannten Kriterien ist, dass sich keine eindeutige und im Vortrieb messbare Verschleißgrenze für dieses Kriterium definieren lässt. Zusätzlich ist die Rückseite der Räumer im Vortrieb nur schwer zugänglich. Eine Überprüfung ist entweder durch Zurückziehen des Schneidrades oder durch Ertasten mit per Hand möglich. Folglich unterliegt die Beurteilung des Sekundärverschleißes weiterhin der subjektiven Einschätzung durch den jeweiligen Bearbeiter. 


\subsection{Berechnung der Standgrößen}

\subsubsection{Definition der Standgrößen für Abbauwerkzeuge}

Der Begriff Standzeit wird im Bereich des maschinellen Tunnelbaus zur Beschreibung des Gebrauchsintervalls eines Abbauwerkzeuges verwendet. Dabei wird üblicherweise nicht unterschieden, welche Messgröße zur Bestimmung des Gebrauchsintervalls verwendet wird. Gebräuchlich sind z.B. die Angabe des Intervalls als Lösevolumen oder Vortriebsstrecke der Tunnelbohrmaschine zwischen dem Zeitpunkt des Ein- und Ausbaus des Werkzeuges auf dem Schneidrad. Unter dem Begriff Standzeit werden also lose verschiedenen Standgrößen für Abbauwerkzeugen zusammengefasst.

Für die vorliegende Arbeit wird eine differenziertere Unterscheidung und Gliederung verschiedener Standgrößen und Einflussfaktoren angewandt. Diese orientiert sich an der Gliederung der Standbegriffe in DIN 6583 (DIN 1981), die für die Zerspanungstechnik von metallischen Werkstoffen einen sehr ähnlichen Themenbereich abdeckt und an bereits bestehenden Untersuchungen zur Gebirgslösbarkeit von THURO (2002: 46 f.) und PLINNINGER et al. (2004: 601).

Die Standgröße eines Abbauwerkzeuges ist das als Zeit-, Weg- oder Volumeneinheit bemessene Intervall, das den Einsatz des Werkzeuges auf dem Schneidrad bis zum Erreichen der Verschleißgrenze angibt. Als Standgrößen können bezogen auf Abbauwerkzeuge also grundsätzlich folgende sinnvolle Größen definiert werden:

- Vortriebsstrecke (Standstrecke) $I_{c}[m]$ : Weg in Metern, den die Tunnelvortriebsmaschine im Einsatzintervall des Werkzeuges auf dem Schneidrad in Vortriebsrichtung zurückgelegt hat.

- Laufweg (Standweg) $\mathbf{s}_{\mathrm{c}}[\mathrm{m}]$ : Weg in Metern, den das Werkzeug auf seiner Spur im Einsatzintervall zurückgelegt hat.

- Lösevolumen (Standvolumen) $\mathbf{v}_{\mathbf{c}}\left[\mathrm{m}^{3}\right]$ : Festvolumen an Boden in Kubikmetern, das durch das Werkzeug im Einsatzintervall abgebaut wurde.

- Betriebsstunden (Standzeit) $\mathbf{t}_{\mathrm{c}}[\mathrm{h}]$ : Zeit in Stunden, die das Werkzeug im Einsatzintervall auf dem Schneidrad verbracht hat.

Das Gebrauchsintervall beginnt frühestens mit Einbau des Abbauwerkzeuges auf dem Schneidrad und endet spätestens mit dem Ausbau des Abbauwerkzeuges auf dem Schneidrad. Die Angabe der Vortriebsstationierung $b_{0}$ und $b_{v}$ bei Ein- und Ausbau der Abbauwerkzeuge wird im Folgenden anhand von Ringnummern vorgenommen. Diese geben die von Vortriebbeginn in Vortriebsrichtung (VTR) ansteigende Anzahl der im Tunnel verbauten Tübbingringe an. Ein Ring entspricht einer Vortriebsstrecke in Höhe der mittleren Breite der Tübbingringe $\mathrm{I}_{(\mathrm{b})}[\mathrm{m}]$. Die einzelnen Ringe werden im Folgenden als Vortriebe $b$ bezeichnet. Die Ringnummern können anhand der mittleren Breite der Tübbingringe $I_{(b)}$ in Vortriebsmeter umgerechnet werden. Hierbei ist zu beachten, dass die Nummerierung der Tübbingringe häufig nicht genau an der Anschlagwand des Tunnels mit Ringnummer 1 beginnt, weil für die Abstützung der TVM bei Vortriebsbeginn Blindringe vor der Anschlagwand gesetzt werden. Die entsprechenden Verschiebungen der Nummerierung wurden in der Berechnung der Standgrößen in den Referenzprojekten berücksichtigt.

Die tatsächliche Stationierung der Ortsbrust zum Ein- und Ausbauzeitpunkt liegt jeweils ca. um die Länge der Schildmaschine $L_{T V M}[\mathrm{~m}]$ in Vortriebsrichtung versetzt, weil die Tübbingringe erst im hinteren Schildbereich verbaut werden. Die genaue Distanz zwischen Ortsbrust und letztem Tübbingring hängt davon ab, wie weit die Vortriebspressen und Schneidradverschiebezylinder aus- bzw. eingefahren wurden. Die aus Ringnummern berechneten Vortriebsstationierungen müssen deshalb theoretisch um diesen Betrag korrigiert werden. Die genaue rückwirkende Ermittlung dieser Distanz anhand der Betriebsdaten der TVM ist dabei nur mit sehr hohem Aufwand möglich.

In der Berechnung der Vortriebsstrecke $I_{c}[\mathrm{~m}]$ und des Lösevolumens $\mathrm{v}_{\mathrm{c}}\left[\mathrm{m}^{3}\right]$ wird jeweils die Differenz zwischen Ein- und Ausbaustationierung ermittelt, sodass der systematische Fehler ohne Korrektur theoretisch einen Maximalbetrag etwa in Höhe der doppelten Breite eines Tübbingrings $I_{(b)}$ erreichen kann. Bezogen auf die erreichten Vortriebsstrecken $I_{c}$ der Abbauwerkzeuge in den Referenzprojekten liegt dieser Betrag bei $95 \%$ der Werkzeugwechsel bei $<1 \%$. In der Berechnung der Standgrößen wur- 
de deshalb keine Korrektur vorgenommen. Bei der anschließenden Zuordnung der Werkzeugwechsel und Standgrößen zu den geotechnischen Bedingungen (Kap. 5.6.1.2) und TVM-Betriebsdaten (Kap. 5.6.1.3) wurde die Differenz zwischen Stationierung der Ortsbrust und Stationierung aus der Umrechnung der Ringnummern entsprechend berücksichtigt.

Der Laufweg $\mathrm{s}_{\mathrm{c}}[\mathrm{km}]$ und die Betriebsstunden $\mathrm{t}_{\mathrm{c}}[\mathrm{h}]$ der Abbauwerkzeuge hängen zusätzlich von der Anzahl der Schneidradumdrehungen $\mathrm{n}_{\cup}$ im Gebrauchsintervall ab. Sowohl der Prozess beim Start des Vortriebs (Kap. 3.3.1), als auch die Vorbereitung einer Vortriebsunterbrechung für Werkzeugwechsel (Kap. 3.3.3) erfordern zum Teil eine hohe Anzahl an Schneidradumdrehungen, ohne dass die TVM gleichzeitig in Vortriebsrichtung (VTR) verschoben wird. Die Abbauwerkzeuge bauen in diesen Phasen also keinen Boden ab und werden nicht passiv über die Ortsbrust geführt. Der genaue Zeitpunkt für Beginn und Ende des Gebrauchsintervalls wird deshalb im Folgenden anhand der Betriebsdaten der TVM an Start und Ende des Vorschubs der TVM bei gleichzeitigem Drehen des Schneidrades gelegt.

Innerhalb des Gebrauchsintervalls unterliegen die Abbauwerkzeuge aufgrund von Primär- und Sekundärverschleiß einem stetigen Verlust an Masse (Kap. 4.1.). Der Massenverlust kann einerseits direkt in Form der Masse des Werkzeuges bestimmt werden. Andererseits ist auch eine Bemessung des Massenverlustes in Form des verlorenen Werkzeugvolumens oder durch den Verlust der Länge bzw. Höhe des Werkzeuges an einer definierten Stelle möglich. Aus dem Quotienten aus Massenverlust und der entsprechenden Standgröße kann die Verschleißrate $c_{v}$ bestimmt werden. Die Verschleißrate gibt also den durch Verschleiß verursachten weg-, volumen- oder zeitabhängigen Massen-, Volumenoder Längenverlust eines Abbauwerkzeuges an. Die verschiedenen Möglichkeiten zur Berechnung der Verschleißrate $c_{v}$ sind im Anschluss in Kap. 5.4.2 beschrieben.

Der Betrag der Verschleißrate $c_{v}$ ist abhängig von der Summe der Einsatzbedingungen während des Bodenabbaus. Der Betrag der Standgrößen ist von der Verschleißrate $c_{v}$ und dem Wechselgrund abhängig. Der Grund für den Ausbau der Abbauwerkzeuge muss nicht zwingend das Erreichen der Verschleißgrenze sein, sondern kann auch aus baubetrieblichen Gründen oder aufgrund von Schäden erfolgen (Kap. 5.2.1.1).

\subsubsection{Verschleißrate $c_{v}$}

Der Massenverlust $m_{d}[g]$ eines Abbauwerkzeugs wird für die Berechnung der Verschleißrate $c_{v}$ aus der Differenz zwischen der Masse bei Einbau des Werkzeuges auf dem Schneidrad $\mathrm{m}_{0}[\mathrm{~g}]$ und der Masse des Werkzeuges bei Ausbau $\mathrm{m}_{\mathrm{v}}$ [g] mit Gl. 25 bestimmt:

$$
\mathrm{m}_{\mathrm{d}}=\mathrm{m}_{0}-\mathrm{m}_{\mathrm{v}}
$$

Der Massenverlust eines Werkzeuges kann theoretisch auch als Volumenverlust $v_{d}\left[\mathrm{~mm}^{3}\right]$ (Gl. 26) oder Längen- bzw. Höhenverlust $h_{d}[\mathrm{~mm}]$ (Gl. 27) an einer definierten Stelle des Abbauwerkzeuges dargestellt werden (Kap. 5.4.1):

$$
\begin{aligned}
& \mathrm{v}_{\mathrm{d}}=\mathrm{v}_{0}-\mathrm{v}_{\mathrm{v}} \\
& \mathrm{h}_{\mathrm{d}}=\mathrm{h}_{0}-\mathrm{h}_{\mathrm{v}}
\end{aligned}
$$


Die Verschleißrate $c_{v}$ ergibt sich aus dem Quotienten des Massen-, Höhen- oder Volumenverlustes und dem Betrag der Standgröße des Abbauwerkzeugs. Für die weiteren Versuche zur Verschleißrate in der vorliegenden Arbeit sind die Varianten der Verschleißrate $c_{v}$ mit Bezug des Massenverlustes $m_{d}$ und des Höhenverlust $h_{d}$ auf den Laufweg $s_{c}[k m]$ notwendig:

$$
\begin{gathered}
\mathrm{c}_{\mathrm{v}}=\frac{\mathrm{m}_{\mathrm{d}}}{\mathrm{s}_{\mathrm{c}}} \\
\mathrm{c}_{\mathrm{v}}=\frac{\mathrm{h}_{\mathrm{d}}}{\mathrm{s}_{\mathrm{c}}}
\end{gathered}
$$

In Gl 28 und Gl. 29 gibt der Laufweg $\mathrm{s}_{\mathrm{c}}[\mathrm{km}]$ als Standgröße jeweils den erreichten Laufweg der Werkzeuge an. Die Berechnung des Laufwegs $\mathrm{S}_{\mathrm{c}}$ ist in Kap. 5.4.4 beschrieben. Theoretisch sind darüber hinaus auch die weiteren Standgrößenvarianten zur Berechnung der Verschleißrate $c_{v}$ in Gl. 28 und Gl 29 einsetzbar. Diese werden jedoch im weiteren Verlauf der Arbeit nicht weiter angewandt.

\subsubsection{Vortriebsstrecke $I_{c}$}

Die Bestimmung der Vortriebsstrecke $I_{\mathrm{c}}[\mathrm{m}](\mathrm{Gl} .30)$ als Standgröße ist für alle Werkzeugtypen gleich. Sie wird durch die zurückgelegte Wegstrecke [m] der TVM zwischen der Stationierung bei Einbau des Werkzeuges und der Stationierung bei Ausbau des Werkzeuges festgelegt:

$$
\mathrm{l}_{\mathrm{c}}=\left(\mathrm{b}_{0}-\mathrm{b}_{\mathrm{v}}\right) \cdot \mathrm{l}_{(\mathrm{b})}
$$

In Gl. 30 ist $b_{v}$ die Ringnummer bei Ausbau des Werkzeuges auf dem Schneidrad, $b_{0}$ die Ringnummer bei Einbau des Werkzeuges auf dem Schneidrad und $I_{(b)}$ die mittlere Breite eines Tübbingrings.

Die Vortriebsstrecke $I_{c}$ kann anhand des Anteils der beiden Schneidraddrehrichtungen weiter in die Aktiv-Vortriebsstrecke $I_{c a}$ und Passiv-Vortriebsstrecke $I_{c p}$ unterteilt werden. Entsprechend der Definition in Kap. 3.2.1 ist die Aktiv-Vortriebsstrecke $I_{c a}$ der Anteil an der Vortriebsstrecke $I_{c}$, in dem das Werkzeug in bestimmungsgemäßer Richtung über die Ortsbrust geführt wurde. Umgekehrt stellt die Passiv-Vortriebsstrecke $I_{c p}$ den Teil der Vortriebsstrecke $I_{c}$ dar, in dem das Werkzeug entgegen der bestimmungsgemäßen Richtung über die Ortsbrust geführt wurde. Die Schneidraddrehrichtung wird innerhalb eines Vortriebes $b$ selten gewechselt, sodass die Länge des Vortriebs $I_{(b)}$ häufig direkt einer der beiden Schneidraddrehrichtungen zugeordnet werden kann. Die Vortriebsstrecken ergeben sich also aus Gl. 31 und Gl. 32:

$$
\mathrm{l}_{\mathrm{ca}}=\sum_{\mathrm{b}_{0}}^{\mathrm{b}_{\mathrm{v}}}\left(\mathrm{b}_{\mathrm{a}} \cdot \mathrm{l}_{(\mathrm{b})}\right)
$$

$$
\mathrm{l}_{\mathrm{cp}}=\sum_{\mathrm{b}_{0}}^{\mathrm{b}_{\mathrm{v}}}\left(\mathrm{b}_{\mathrm{p}} \cdot \mathrm{l}_{(\mathrm{b})}\right)
$$

In Gl. 31 und Gl. 32 sind $b_{a}$ die Vortriebe, in denen das Werkzeug aktiv über die Ortsbrust geführt wurde und $b_{p}$ die Vortriebe, in denen das Werkzeug passiv über die Ortsbrust geführt wurde. 
Wird innerhalb eines Vortriebes die Drehrichtung des Schneidrades gewechselt, so kann der jeweilige Anteil der beiden Drehrichtungen über deren zeitlichen Anteil an der Vorschubzeit $t_{(b)}$ für den Vortrieb $b$ ermittelt werden. Da die aufgenommenen Zeitintervalle in den Momentanwerten der TVM jeweils gleich lang sind (10 s), kann der Längenanteil der Drehrichtungen am jeweiligen Vortrieb mit Gl. 33 bzw. Gl. 34 bestimmt werden:

$$
\begin{aligned}
& \mathrm{l}_{\mathrm{a}(\mathrm{b})}=\mathrm{l}_{(\mathrm{b})} \cdot\left(\frac{\mathrm{t}_{\mathrm{a}(\mathrm{b})}}{\mathrm{t}_{(\mathrm{b})}}\right) \\
& \mathrm{l}_{\mathrm{p}(\mathrm{b})}=\mathrm{l}_{(\mathrm{b})} \cdot\left(\frac{\mathrm{t}_{\mathrm{p}(\mathrm{b})}}{\mathrm{t}_{(\mathrm{b})}}\right)
\end{aligned}
$$

Dabei ist $\mathrm{t}_{(\mathrm{b})}$ [s] die Gesamtzeit für den Vortrieb $\mathrm{b}, \mathrm{t}_{\mathrm{a}(\mathrm{b})}[\mathrm{s}$ ] die Summe der Zeitinkremente in denen das Werkzeug aktiv über die Ortsbrust geführt wurde und $\mathrm{t}_{\mathrm{p}(\mathrm{b})}$ [s] die Summe der Zeitinkremente in denen das Werkzeug passiv über die Ortsbrust geführt wurde.

\subsubsection{Laufweg $s_{c}$}

Der Laufweg $s_{c}[\mathrm{~km}]$ eines Werkzeuges ist definiert als Summe des Laufweges in den beiden Schneidraddrehrichtungen zwischen Ein- und Ausbau des Werkzeuges auf dem Schneidrad.

Die Laufbahn der Abbauwerkzeuge entspricht einer in VTR liegende Helix. Der Spurradius $r_{s}[\mathrm{~mm}]$ des Abbauwerkzeuges gibt den Radius der Helix an, die Penetration $p[\mathrm{~mm} / \mathrm{U}]$ entspricht der Ganghöhe der Helix, während die Tunnelachse der Längsachse der Helix entspricht (Abb. 18). Aus praktischen Gründen wurde für die Berechnung des Laufwegs $s_{c}[\mathrm{~km}]$ die Ganghöhe der Helix vernachlässigt, sodass der Laufweg $\mathrm{s}_{\mathrm{c}}$ vereinfacht mit $\mathrm{Gl}$. 35 berechnet werden kann.

$$
\mathrm{s}_{\mathrm{c}}=\frac{2 \pi}{1000} \cdot \frac{\mathrm{r}_{\mathrm{s}}}{1000} \cdot \sum_{\mathrm{b}_{0}}^{\mathrm{b}_{\mathrm{v}}} \frac{\mathrm{l}_{(\mathrm{b})} \cdot 1000}{\mathrm{p}_{(\mathrm{b})}}
$$

Dabei ist $r_{s}[\mathrm{~mm}]$ der Radius der Spur, $\mathrm{I}_{(\mathrm{b})}[\mathrm{m}]$ die mittlere Breite eines Tübbingrings und $\mathrm{p}_{(\mathrm{b})}[\mathrm{mm} / \mathrm{U}]$ die mittlere Penetration je Vortrieb $b$. Die mittlere Penetration je Vortrieb kann aus den Maschinendaten entnommen werden, $b_{0}$ und $b_{v}$ sind die Ringnummern bei Ein- und Ausbau des Werkzeuges auf dem Schneidrad.

Da die Penetration $\mathrm{p}[\mathrm{mm} / \mathrm{U}]$ im Vergleich zum Spurradius $r_{\mathrm{s}}[\mathrm{mm}]$ der Werkzeuge stets sehr klein ist, ergibt die Vernachlässigung der Ganghöhe der Helix bezogen auf die tatsächliche erreichten Laufwege der Werkzeuge eine vernachlässigbare, systematische Abweichung des Laufwegs um $<0,01 \%$.

Die Berechnung der maßgeblichen Schneidradumdrehungen $\mathrm{n}_{\mathrm{u}(\mathrm{b})}$ während eines einzelnen Vortriebes b erfolgt in der Formel für den Laufweg $\mathrm{s}_{\mathrm{c}}[\mathrm{km}]$ durch den Term in $\mathrm{Gl} .36$ :

$$
\mathrm{n}_{\mathrm{u}(\mathrm{b})}=\frac{\mathrm{l}_{(\mathrm{b})} \cdot 1.000}{\mathrm{p}_{(\mathrm{b})}}
$$

Somit werden alle Schneidradumdrehungen, während derer das Schneidrad nicht in Vortriebsrichtung verschoben wurde, also kein Bodenabbau stattfand, hinreichend genau eliminiert. 
Der Laufwegs $\mathrm{s}_{\mathrm{c}}$ kann analog zur Vortriebsstrecke $\mathrm{I}_{\mathrm{c}}$ anhand der in den Momentanwerten der Betriebsdatenerfassung der TVM enthaltenen Statusangaben für Drehen des Schneidrades in Richtung links bzw. rechts in den Aktiv-Laufweg $s_{c a}$ und den Passiv-Laufweg $s_{c p}$ unterteilt werden. Dabei wird die Drehrichtung nur in sehr seltenen Fällen innerhalb eines Vortriebes b gewechselt, sodass der jeweils erreichte Laufweg vollständig einer der beiden Drehrichtungen zugeordnet werden kann. Damit gelten als Formulierungen für den Aktiv-Laufweg $\mathrm{s}_{\mathrm{ca}}$ (Gl. 37) und Passiv-Laufweg $\mathrm{s}_{\mathrm{cp}}$ (Gl. 38):

$$
\begin{aligned}
& \mathrm{s}_{\mathrm{ca}}=\frac{2 \pi}{1.000} \cdot \frac{\mathrm{r}_{\mathrm{s}}}{1.000} \cdot \sum_{\mathrm{b}_{0}}^{\mathrm{b}_{\mathrm{v}}} \frac{\left(\mathrm{b}_{\mathrm{a}} \cdot \mathrm{l}_{(\mathrm{b})}\right) \cdot 1.000}{\mathrm{p}_{(\mathrm{b})}} \\
& \mathrm{s}_{\mathrm{cp}}=\frac{2 \pi}{1.000} \cdot \frac{\mathrm{r}_{\mathrm{s}}}{1.000} \cdot \sum_{\mathrm{b}_{0}}^{\mathrm{b}_{\mathrm{v}}} \frac{\left(\mathrm{b}_{\mathrm{p}} \cdot \mathrm{l}_{(\mathrm{b})}\right) \cdot 1.000}{\mathrm{p}_{(\mathrm{b})}}
\end{aligned}
$$

Wird innerhalb eines Bohrhubes die Drehrichtung des Schneidrades gewechselt, so kann der jeweilige Anteil der beiden Drehrichtungen über deren zeitlichen Anteil an der Vorschubzeit für den Vortrieb $\mathrm{t}_{(b)}$ ermittelt werden. Die aufgenommenen Zeitintervalle in den Momentanwerten der TVM sind jeweils gleich lang (10 s). Die Bestimmung des Anteils $I_{a(b)} b z w . I_{p(b)}$ an der mittleren Länge $I_{(b)}$ des Vortriebs $b$ in dem ein Abbauwerkzeug in aktiver oder passiver Richtung über die Ortsbrust geführt wurde, kann für betroffene Vortriebe mit Gl. 33 bzw. Gl. 34 (Kap. 5.4.3) ermittelt werden.

\subsubsection{Lösevolumen $v_{c}$}

Die Bestimmung des Lösevolumens $\mathrm{v}_{\mathrm{c}}\left[\mathrm{m}^{3}\right]$ als Standgröße ist für die verschiedenen Werkzeugtypen auf dem Schneidrad grundsätzlich identisch. Das Lösevolumen eines spezifischen Werkzeuges kann vereinfacht als liegender Zylinderkörper beschrieben werden. Die Länge des Zylinderkörpers entspricht dabei analog zur Ermittlung der Vortriebsstrecke $I_{c}[\mathrm{~m}]$ der Wegstrecke, welche die Schildmaschine zwischen dem Vortriebsstand bei Einbau des Werkzeuges und dem Vortriebsstand bei Ausbau des Werkzeuges zurückgelegt hat. Diese wird mit Gl. 30 ermittelt.

Die Wandstärke des Zylinders entspricht der Breite, mit der das Werkzeug in die Ortsbrust eingreift. Diese ergibt sich folglich aus der Differenz des Außenradius $r_{s 2}[\mathrm{~mm}]$ und Innenradius $r_{s 1}[\mathrm{~mm}] \mathrm{zwi}$ schen denen das Werkzeug in die Ortsbrust eingreift. Das Lösevolumen $v_{c}\left[\mathrm{~m}^{3}\right]$ kann damit als Volumen des Zylinderkörpers mit Gl. 39 berechnet werden:

$$
\mathrm{v}_{\mathrm{c}}=\mathrm{l}_{\mathrm{c}} \cdot \frac{\mathrm{r}_{\mathrm{s} 2}^{2}-\mathrm{r}_{\mathrm{s} 1}^{2}}{1.000 \cdot 1.000} \cdot \pi
$$

Diese Methode zur Volumenberechnung vernachlässigt, dass die beiden Kopfflächen des Zylinderkörpers in der Realität nicht senkrecht zu den Seitenflächen stehen, sondern analog zu den Erläuterungen zum Laufweg $\mathrm{s}_{\mathrm{c}}$ einem Gewindegang einer Helix entsprechen, deren Ganghöhe gleich der Penetration $\mathrm{p}[\mathrm{mm} / \mathrm{U}]$ ist. Entsprechend der Erläuterungen in Kap. 5.4.4 sind die sich daraus ergebenden Fehler vernachlässigbar gering.

Die Werkzeuge haben entsprechend der Analyse der Ortsbrustbilder und Verschleißbilder in Kap. 5.1 einen unterschiedlich hohen Anteil am Bodenabbau und sind zum Teil überschneidend und in unterschiedlichem Abstand zum Schneidrad-Stahlbau angeordnet. Entsprechend müssen auch die Lösevolumina aufgeteilt werden, um eine realistisches Lösevolumen $v_{c}$ für ein spezifisches Werkzeug zu erhalten. 
Schneidrollen sind im Regelfall vorlaufend zu den anderen Werkzeugen auf dem Schneidrad angeordnet. Entsprechend der Analyse in Kap. 5.3.2.1 dringt dabei der Schneidring in den Boden ein und hinterlässt eine Furche in der Ortsbrust. Die Tiefe der Furchen entspricht jeweils der Differenz zwischen dem Vorlauf der Schneidrolle $h_{S R}[\mathrm{~mm}]$ über dem Schneidradstahlbau und dem Vorlauf der hinter dem Schneidring angeordneten Schälmesser $h_{S M}[\mathrm{~mm}]$ über dem Stahlbau. Die Breite des Bodenabbaus durch den Schneidring in der Ortsbrust entspricht folglich der Differenz zwischen dem Außenradius $r_{\mathrm{s} 2}$ und Innenradius $r_{\mathrm{s} 1}$ der Furche in der Ortsbrust auf Niveau der nachlaufend angeordneten Schälmesser (Abb. 63).

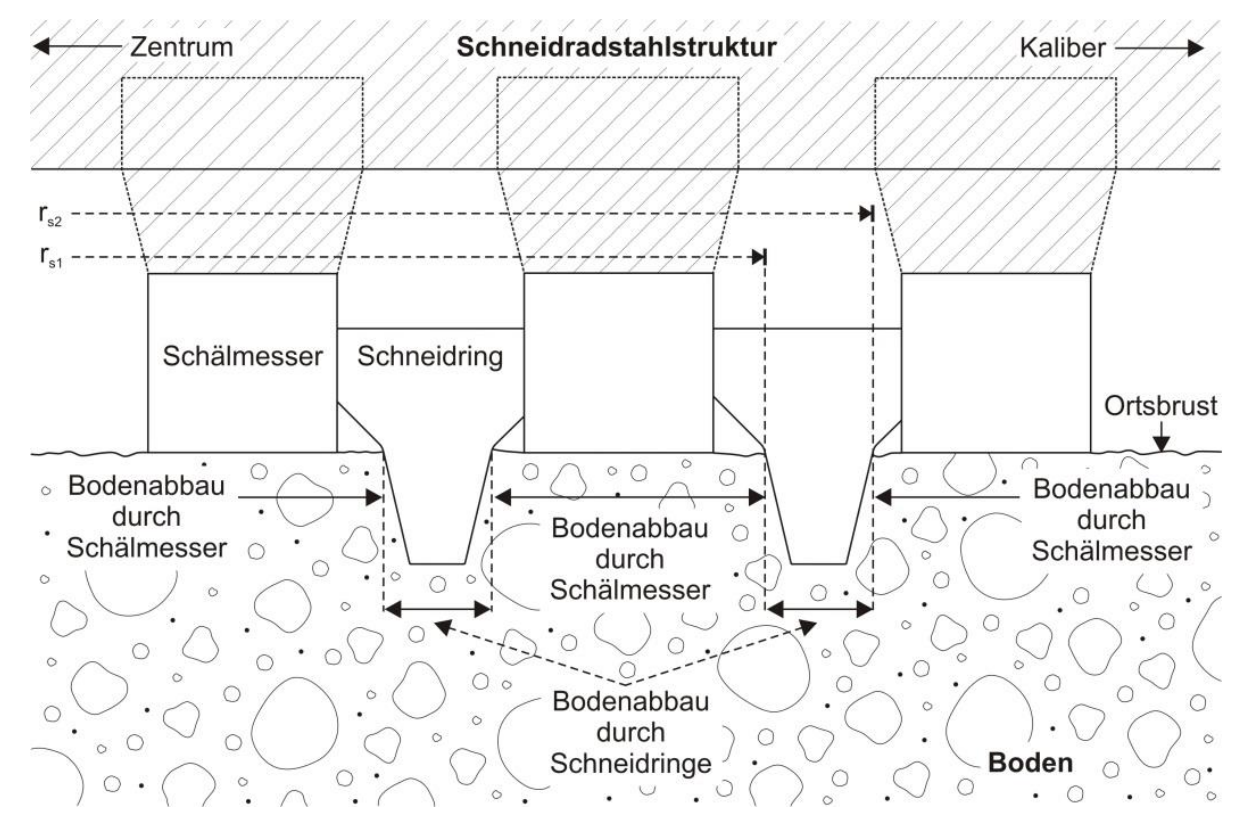

Abb. 63: Bodenabbau durch Schälmesser und Schneidringe bei Furchenbildung durch die Schneidringe in der Ortsbrust.

Schälmesser bauen beim aktiven Einsatz zum primären Bodenabbau ohne vorlaufende Schneidrollen auf Ihrer gesamten Breite Boden in der Ortsbrust ab (Kap. 5.3.4.1). Zur Berechnung des Lösevolumens kann als Außenradius $r_{\mathrm{s} 2}$ und Innenradius $r_{s 1}$ jeweils der Radius der dem Kaliberbereich bzw. dem Schneidradzentrum zugewandten Schälmesserkante eingesetzt werden.

Zu berücksichtigen ist jedoch, dass je Schneidradumdrehung die entsprechende Penetration $\mathrm{p}$ nicht ausschließlich von einem aktiven Schälmesser geleistet wird. Die Penetration teilt sich zwischen aktiv und gleichzeitig passiv im Einsatz befindlichen Schälmessern auf und zwischen den zusätzlichen Schälmessern bei mehrfach besetzten Spuren. Folglich reduziert sich auch das Lösevolumen $v_{c}$ entsprechend dem Anteil an der Penetration $p$ der verschiedenen Schälmesser auf der jeweiligen Spur (Abb. 64).

Die Aufteilung der Penetration $p$ erfolgt dabei nicht nach gleichen Teilen, sondern hängt vom Winkelabstand der Schälmessers zum nächsten in Schneidraddrehrichtung auf derselben Spur vorlaufenden Schälmesser ab. Der Winkelabstand zwischen aktiven und passiven, sowie mehrfach besetzten Schälmessern ist in der Regel nicht gleichmäßig aufgeteilt. Der Winkelabstand zum vorlaufenden Schälmesser muss deshalb unterschieden werden für den aktiven Einsatz des Schälmessers $\delta_{a}\left[{ }^{\circ}\right]$ und den passiven Einsatz $\delta_{p}\left[{ }^{\circ}\right]$. 


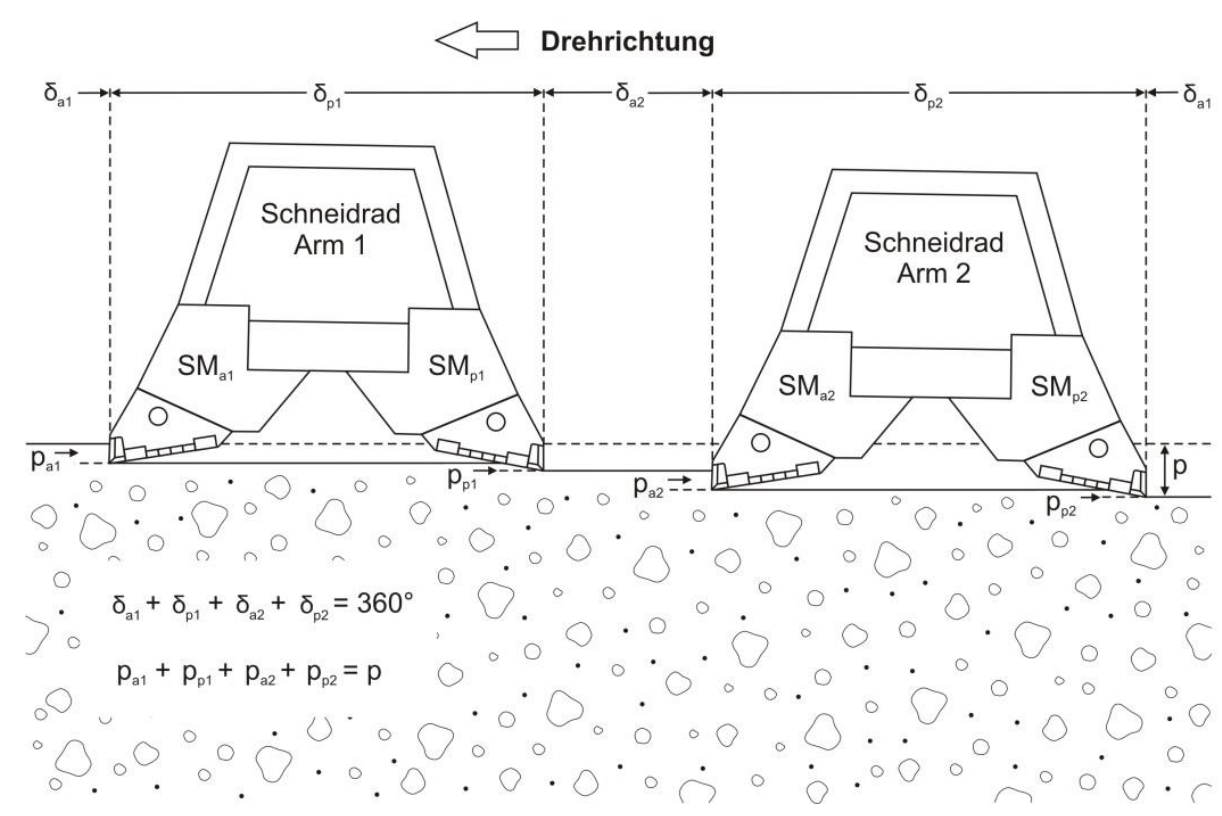

Abb. 64: Aufteilung der Penetration $p$ auf die verschiedenen aktiven und passiven Schälmesser $\left(S_{a} \& \mathrm{SM}_{\mathrm{p}}\right)$ bei mehrfach besetzten Spuren in Abhängigkeit des Winkelabstandes $\delta$ zwischen den Schälmessern.

Der Anteil der Aktiv-Penetration $\mathrm{p}_{\mathrm{a}}[\mathrm{mm} / \mathrm{U}]$ bzw. Passiv-Penetration $\mathrm{p}_{\mathrm{p}}[\mathrm{mm} / \mathrm{U}]$ eines Schälmessers an der Penetration $\mathrm{p}[\mathrm{mm} / \mathrm{U}]$ kann mit dem in Gl. 40 bzw. Gl. 41 bestimmt werden:

$$
\mathrm{p}_{\mathrm{a}}=\frac{\delta_{\mathrm{a}}}{360} \cdot \mathrm{p}
$$

$$
\mathrm{p}_{\mathrm{p}}=\frac{\delta_{\mathrm{p}}}{360} \cdot \mathrm{p}
$$

Entsprechend der Aufteilung des Winkelabstandes können anschließend unter Berücksichtigung der aktiv und passiv zurückgelegten Vortriebsstrecken $I_{c a}$ und $I_{c p}$ (Kap. 5.4.3) mit Gl. 31 bzw. Gl. 32 das Aktiv-Lösevolumen $v_{\mathrm{ca}}$ und Passiv-Lösevolumen $v_{\mathrm{cp}}$ mit Gl. 42 bzw. Gl. 43 errechnet werden:

$$
\mathrm{v}_{\mathrm{ca}}=\mathrm{l}_{\mathrm{ca}} \cdot \frac{\mathrm{r}_{\mathrm{s} 2}^{2}-\mathrm{r}_{\mathrm{s} 1}^{2}}{1000 \cdot 1000} \cdot \pi \cdot \frac{\delta_{\mathrm{a}}}{360}
$$

$$
\mathrm{v}_{\mathrm{cp}}=\mathrm{l}_{\mathrm{cp}} \cdot \frac{\mathrm{r}_{\mathrm{s} 2}^{2}-\mathrm{r}_{\mathrm{s} 1}^{2}}{1000 \cdot 1000} \cdot \pi \cdot \frac{\delta_{\mathrm{p}}}{360}
$$

Das Gesamt-Lösevolumen $v_{c}$ eines Schälmessers ergibt sich damit aus der Summe des aktiven und passiven Anteils mit Gl. 44:

$$
\mathrm{v}_{\mathrm{c}}=\mathrm{v}_{\mathrm{ca}}+\mathrm{v}_{\mathrm{cp}}
$$


Sind die Schälmesserspuren seitlich überlappend angeordnet, muss der überlappenden Bereich entsprechend der oben beschriebenen Methodik durch passende Auswahl von $r_{1}$ und $r_{2}$ separat betrachtet werden. Das gilt auch für Schälmesserspuren, bei denen ein Teil des Schälmessers aufgrund eines vorlaufenden Werkzeuges, z.B. einer Schneidrolle, keinen Kontakt zur Ortsbrust hat und folglich in diesem Teilbereich auch nicht am Bodenabbau teilnimmt. Diese Bereiche müssen durch entsprechende Auswahl von $r_{\mathrm{s} 1}$ und $\mathrm{r}_{\mathrm{s} 2}$ ebenfalls separat berücksichtigt werden.

Durch die beiden zuletzt genannten Faktoren ergeben sich mehrere Lösevolumeninkremente, die zum Lösevolumen $v_{c}$ eines Schälmessers aufsummiert werden müssen. In der Praxis sind häufig drei bis fünf Inkremente je Schälmesserspur notwendig, um das Lösevolumen korrekt zu bestimmen. Decken vorlaufende Werkzeuge, z.B. Stichel, die Ortsbrustfläche vollständig ab und die Schälmesser haben keinen Kontakt mehr zur Ortsbrust (Kap. 5.3.3.1), so leisten die Schälmesser auch keinen Bodenabbau mehr und das Lösevolumen $\mathrm{v}_{\mathrm{c}}$ nimmt den Wert null an.

Räumer nehmen im Lockergestein genauso wie Schälmesser am aktiven und passiven Bodenabbau teil. Die Ermittlung des Lösevolumens $v_{c}$ entspricht der oben dargestellten Methodik für Schälmesser. Allerdings muss zusätzlich die Krümmung im Kaliberbereich berücksichtigt werden. Die Radien $\mathrm{r}_{1}$ und $r_{2}$ werden dazu in der Regel senkrecht auf die Ortsbrustebene projiziert.

Die Berechnung des Lösevolumens $v_{c}$ für Stichel entspricht der Methodik für Schälmesser. Allerdings erfolgt hier keine Aufteilung des Lösevolumens in aktives und passives Lösevolumen $v_{c a}$ und $v_{c p}$, da Stichel üblicherweise für beide Schneidraddrehrichtungen mit einer Schneide ausgestattet sind. Entsprechend leisten die Stichel in beiden Schneidraddrehrichtungen aktiven Bodenabbau.

Die Summe der Lösevolumina $v_{c}$ aller eingesetzten Abbauwerkzeuge über die gesamte Tunnellänge $\mathrm{L}_{\mathrm{TA}}$ entspricht in $\mathrm{Gl} .45$ dem gesamten Ausbruchvolumen $\mathrm{V}_{\mathrm{TA}}$ :

$$
\sum \mathrm{v}_{\mathrm{c}}=\mathrm{L}_{\mathrm{TA}} \cdot \frac{\pi}{4} \cdot \mathrm{D}_{\mathrm{TVM}}^{2}=\mathrm{V}_{\mathrm{TA}}
$$

Im Vergleich zu dieser relativ aufwändigen Methode zur Bestimmung des Lösevolumens sind auch einfachere Schätzmethoden möglich, die jedoch zu ungenaueren und über alle gewechselten Abbauwerkzeuge gemittelten Werten führen. Die einfachste Methode besteht darin, das Ausbruchvolumen $\mathrm{V}_{\mathrm{TA}}$ durch auf die Summe der gewechselten Abbauwerkzeuge $\mathrm{n}_{\mathrm{c}(\mathrm{TA})}$ mit $\mathrm{Gl}$. 46 aufzuteilen:

$$
\mathrm{v}_{\mathrm{c}} \approx \frac{\mathrm{v}_{\mathrm{TA}}}{\mathrm{n}_{\mathrm{c}(\mathrm{TA})}}
$$

Diese Methode kann auch auf einzelne Abschnitte des Vortriebs übertragen werden. Problematisch an dieser Schätzmethode ist jedoch, dass keine differenzierte Betrachtung eines einzelnen Werkzeugtyps möglich ist und andererseits nur ein sehr grober Mittelwert errechnet wird, der für einzelne Werkzeuge zu einer deutlichen Über- oder Unterschätzung des tatsächlichen Lösevolumens führt.

Eine etwas genauere Schätzmethode besteht darin, keine Aufteilungen des Lösevolumens $\mathrm{v}_{\mathrm{c}}$ vorzunehmen. Die relevanten Spurradien $r_{\mathrm{s} 1}$ und $r_{\mathrm{s} 2}$ zur Berechnung des Lösevolumens mit Gl. 39 ergeben sich dann vereinfacht aus:

$$
r_{s 1}=r_{s}-\frac{r_{s}-r_{s-1}}{2}
$$

$$
r_{s 2}=r_{s}+\frac{r_{s+1}-r_{s}}{2}
$$


Diese Methode führt aufgrund der Vernachlässigung des Spurbesatzes $k_{s}$ und der seitlichen Überschneidungen zu einer deutlichen Überschätzung des Lösevolumens $v_{c}$ der einzelnen Werkzeuge. Die Überschätzung wird noch deutlich größer, wenn die Schätzmethode gleichzeitig auf verschiedene Werkzeugtypen angewendet wird, die vor- bzw. nachlaufend zueinander angeordnet sind. Das Verhältnis zwischen den Lösevolumina $v_{c}$ der einzelnen Abbauwerkzeuge und dem Ausbruchvolumen $V_{T A}$ in Gl. 45 geht folglich verloren.

\subsubsection{Betriebsstunden (Standzeit) $t_{c}$}

Die Bestimmung der Betriebsstunden $t_{c}[h]$ als Standgröße ist für alle Werkzeugtypen gleich. Sie wird festgelegt durch die Zeit [h], in welcher die Schildmaschine zwischen der Ringnummer $b_{0}$ bei Einbau des Werkzeuges und der Ringnummer $b_{v}$ bei Ausbau des Werkzeuges durch Drehen des Schneidrades und gleichzeitigen Vorschub Bodenabbau geleistet hat.

In den von der TVM aufgezeichneten Mittelwertdaten wird in den untersuchten Projekten die Vorschubzeit $t_{(b)}[s]$ je Vortrieb b bereits automatisiert zusammengefasst. Die Betriebsstunden $t_{c}[h]$ eines Abbauwerkzeuges zwischen Ein- und Ausbaustationierung $b_{0}$ und $b_{v}$ lassen sich also relativ einfach durch den Zusammenhang in Gl. 49 ermitteln:

$$
t_{c}=\sum_{b_{0}}^{b_{v}} \frac{t_{(b)}}{3.600}
$$

Dabei ist $b_{v}$ die Hubnummer bei Ausbau des Werkzeuges auf dem Schneidrad, $b_{0}$ die Hubnummer bei Einbau des Werkzeuges auf dem Schneidrad und $\mathrm{t}_{(\mathrm{b})}$ die Vorschubzeit je Bohrhub.

Analog zur Vortriebsstrecke $I_{c}$ können auch die Betriebsstunden $t_{c}$ in die beiden Schneidraddrehrichtungen für Schälmesser und Räumer in Aktiv-Betriebsstunden $t_{c a} b z w$. Passiv-Betriebsstunden $t_{c p}$ unterteilt werden. Die Drehrichtung wird nur in sehr seltenen Fällen innerhalb eines Vortriebes b gewechselt, sodass die erreichte Vorschubzeit $t_{(b)}$ vollständig einer der beiden Drehrichtungen zugeordnet werden kann. Die Aktiv-Betriebsstunden $\mathrm{t}_{\mathrm{ca}}[\mathrm{h}]$ und Passiv-Betriebsstunden [h] können mit Gl. 50 bzw. Gl. 51 berechnet werden:

$$
\mathrm{t}_{\mathrm{ca}}=\sum_{\mathrm{b}_{0}}^{\mathrm{b}_{\mathrm{v}}} \frac{\mathrm{t}_{\mathrm{a}(\mathrm{b})}}{3.600}
$$

$$
t_{c p}=\sum_{b_{0}}^{b_{v}} \frac{t_{p(b)}}{3.600}
$$

Wird innerhalb eines Vortriebes $b$ die Schneidraddrehrichtung geändert, können die Aktiv- und PassivBetriebsstunden je Bohrhub aus den Momentanwerten der Betriebsdaten ermittelt werden. Da die aufgenommenen Zeitintervalle jeweils gleich lang sind (10 s) ist die Bestimmung des jeweiligen Anteils durch die Summierung der Zeitintervalle je Drehrichtung möglich. 


\subsubsection{Auswahl der geeigneten Standgröße für die Datenanalyse}

Für die weiteren Versuche und Datenanalyse in der vorliegenden Arbeit wurden für alle vorhandenen Werkzeugwechsel zunächst alle vier angegebenen Standgrößen berechnet. Die Datenanalyse hat jedoch im weiteren Verlauf ergeben, dass der Laufweg $\mathrm{s}_{\mathrm{c}}$ die optimale Standgröße für die weitere Verwendung darstellt.

Die Betrachtungsrichtung bei der Berechnung der Standgröße stimmt beim Laufweg $\mathrm{S}_{\mathrm{c}}$ mit der Bewegungsrichtung des Werkzeuges beim Bodenabbau überein und damit mit der Richtung der Relativbewegung zwischen Boden und Werkzeugoberfläche, die ursächlich zum Materialverlust an der Werkzeugoberfläche beiträgt. Damit werden die Laufwege $\mathrm{s}_{\mathrm{c}}$ von Werkzeugen auf verschiedenen Spuren mit unterschiedlichen Spurradien $r_{s}$ vergleichbar. Der Laufweg $s_{c}$ zeigt vermutlich aus diesen Gründen eine sehr gute Korrelation zur Verschleißrate $c_{v}$ (Kap. 5.5) und zu den analysierten Einflussfaktoren auf die Standgrößen (Kap. 5.5.4). Zusätzlich ist der Laufweg $\mathrm{s}_{\mathrm{c}}$ in der Praxis leicht bestimmbar und kann für die Anwendung in der Vortriebsplanung auch relativ einfach in Vortriebsstrecken umgerechnet werden (Kap. 6.3.1).

Auch beim Lösevolumen $v_{c}$ stimmt die Betrachtungsrichtung bei der Berechnung der Standgröße grundsätzlich mit der Bewegungsrichtung des Abbauwerkzeuges beim Bodenabbau überein, sodass die Laufwege $\mathrm{s}_{\mathrm{c}}$ von Abbauwerkzeuge mit unterschiedlichen Spurradien $\mathrm{r}_{\mathrm{s}}$ vergleichbar werden. Um gute Korrelationen mit der Verschleißrate $c_{v}$ und zu den analysierten Einflussfaktoren auf die Standgrößen zu erhalten, ist jedoch eine im Vergleich deutlich aufwändigere Berechnung notwendig. Das in Kap. 5.4.5 beschriebenen Schätzverfahren ergibt dagegen keine zufriedenstellenden Korrelationen. Zusätzlich stellt die Methodik im Vergleich zum Laufweg $\mathrm{s}_{\mathrm{c}}$ höhere Anforderungen an die Dokumentation im Vortrieb. So ist z.B. zur Ermittlung des Lösevolumens von Schneidrollen eine Messung der Breite der Furchen in der Ortsbrust notwendig, welche die Schneidrollen beim Bodenabbau hinterlassen. Weiterhin ändert sich durch den fortlaufend ansteigenden Verschleißzustand der Werkzeuge und die damit verbundenen Formänderung die Aufteilung des Lösevolumens zwischen den einzelnen Werkzeugen. Abschließend ist zu berücksichtigen, dass aufgrund der Hinweise auf eine Verdichtung der Ortsbrust beim Bodenabbau (Kap. 5.3.2.1) nicht ausgeschlossen werden kann, dass durch den Abbaumechanismus z.B. von Schneidrollen auch eine laterale Verschiebung von Bodenvolumina in der Ortsbrust stattfindet und damit die Aufteilung des Lösevolumens zwischen verschiedenen Werkzeugen und Schneidspuren ungenau wird. Abschließend bedingt das Lösevolumen $v_{c}$ auch eine deutlich aufwändigere Umrechnung in eine entsprechende Vortriebsstrecke für die Vortriebsplanung. Das Lösevolumen wurde deshalb in den weiteren Versuchen und in der Datenanalyse (Kap. 5.5.4) nur eingeschränkt weiter verwendet.

Im Vergleich zum Laufweg $\mathrm{s}_{\mathrm{c}}$ und dem Lösevolumen $\mathrm{v}_{\mathrm{c}}$ liegt die Betrachtungsrichtung bei der Berechnung der Vortriebsstrecke $I_{c}$ senkrecht zur Bewegungsrichtung des eigentlichen Abbauvorgangs. Werkzeugen auf verschiedenen Spuren mit unterschiedlichem Spurradius werden damit bei identischen Einsatzbedingungen automatisch unterschiedliche Beträge für die Vortriebsstrecke $I_{c}$ zugeordnet und sind deshalb nicht vergleichbar. Ein übereinstimmendes Problem ergibt sich bei der Verwendung der Betriebsstunden $t_{c}$. Beide Standgrößen sind damit trotz sehr einfachen Bestimmungsmöglichkeiten weder für die Anwendung in der vorliegenden Datenanalyse, noch für die Vortriebsplanung geeignet. 


\subsection{Versuche zur Verschleißrate $c_{v}$}

\subsubsection{Massenverlust an Schneidringen}

In RP 10 wurden während des Vortriebes in Zusammenarbeit mit der bauausführenden Firma Messungen der Verschleißrate an Schneidringen durchgeführt. Die Messungen wurden innerhalb Homogenbereich (HGB) 1 (321 m) durchgeführt. Für die weitere Auswertung wurden nur Schneidringe berücksichtigt deren Ein- und Ausbauzeitpunkt innerhalb des Homogenbereiches lagen. Der Homogenbereich bestand aus einer im Zentimeterbereich feingeschichteten Wechsellagerung aus stark sandigen Schluffen $\left(U, \mathrm{~s}^{*}\right)(43 \%)$ und stark schluffigen Sanden $\left(\mathrm{S}, \mathrm{u}^{*}\right)(57 \%)$, die eine Feuchtwichte $\mathrm{Y}^{*}$ von $1,80-1,90 \mathrm{~g} / \mathrm{cm}_{3}$ aufwiesen. Abrasivitätskennwerte wurden in diesem Homogenbereich nicht bestimmt. Die Schneidringe leisteten im untersuchten Bereich den primären Bodenabbau.

Jede Schneidrolle wurde in RP 10 mit einer eindeutigen Identifikationsnummer (ID) auf der Stirnfläche der Achse versehen. Alle Schneidrollenwechsel wurden während dem Vortrieb mit Datum, Vortriebsstationierung, Spurnummer sowie ID-Nummer der ein- und ausgebauten Schneidrollen genau dokumentiert. Anschließend wurden während der Wiederaufbereitung der Schneidrollen in der Werkstatt die Schneidringe vom Rollengrundkörper demontiert und mit einer Laborwaage mit einer Genauigkeit von $+/-1 \mathrm{~g}$ gewogen (Abb. 65).

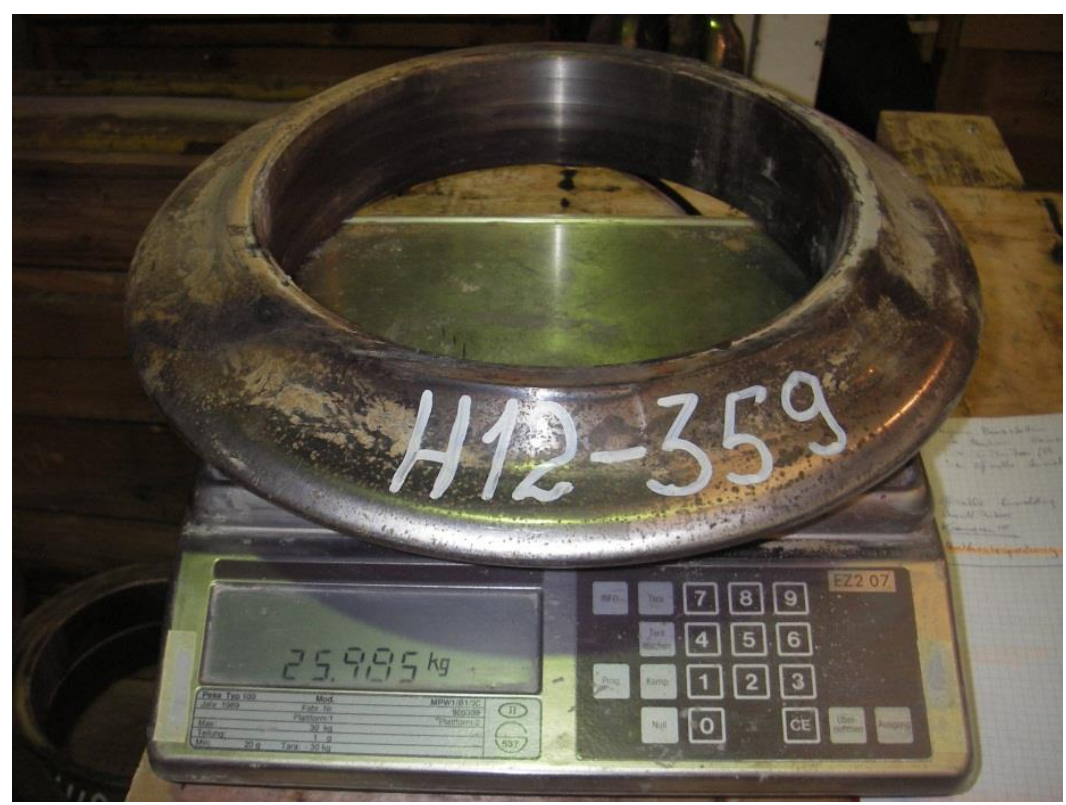

Abb. 65: Wägung eines Schneidrings mit der Laborwaage auf +/- $1 \mathrm{~g}$ genau.

Die Masse der verschlissenen Schneidringe $m_{v}[g]$ kann ins Verhältnis zur Ausgangsmasse $m_{0}[g]$ gesetzt werden und der jeweilige Massenverlust $m_{d}[\mathrm{~g}]$ mit Gl. 25 bestimmt werden (Kap. 5.4.2).

Der Massenverlust wurde anschließend auf den Laufweg $\mathrm{s}_{\mathrm{c}}[\mathrm{km}]$ der Schneidringe bezogen. Es wurden ausschließlich Schneidringe ausgewertet, die während des Vortriebs nicht blockiert waren, also radial gleichmäßigen Abrieb aufwiesen. Da die Schneidringe unabhängig von der Schneidraddrehrichtung aktiven Bodenabbau leisten, kann der Laufweg $\mathrm{s}_{\mathrm{c}}$ mit $\mathrm{Gl}$. 35 (Kap. 5.4.4) bestimmt werden.

Insgesamt ergaben sich 53 Einzelmessungen. Diese sind in Abb. 66 zusammengefasst. Die Verteilung der Messergebnisse in Abb. 66 zeigt einen deutlichen Trend zur linearen Abhängigkeit des Massenverlustes $m_{d}[\mathrm{~g}]$ der Schneidringe vom zugehörigen Laufweg $s_{c}[\mathrm{~km}]$. Dieser ist durch die zusätzlich eingetragene lineare Regressionslinie verdeutlicht. Der zugehörige Korrelationskoeffizient $R$ beträgt $94 \%$. 


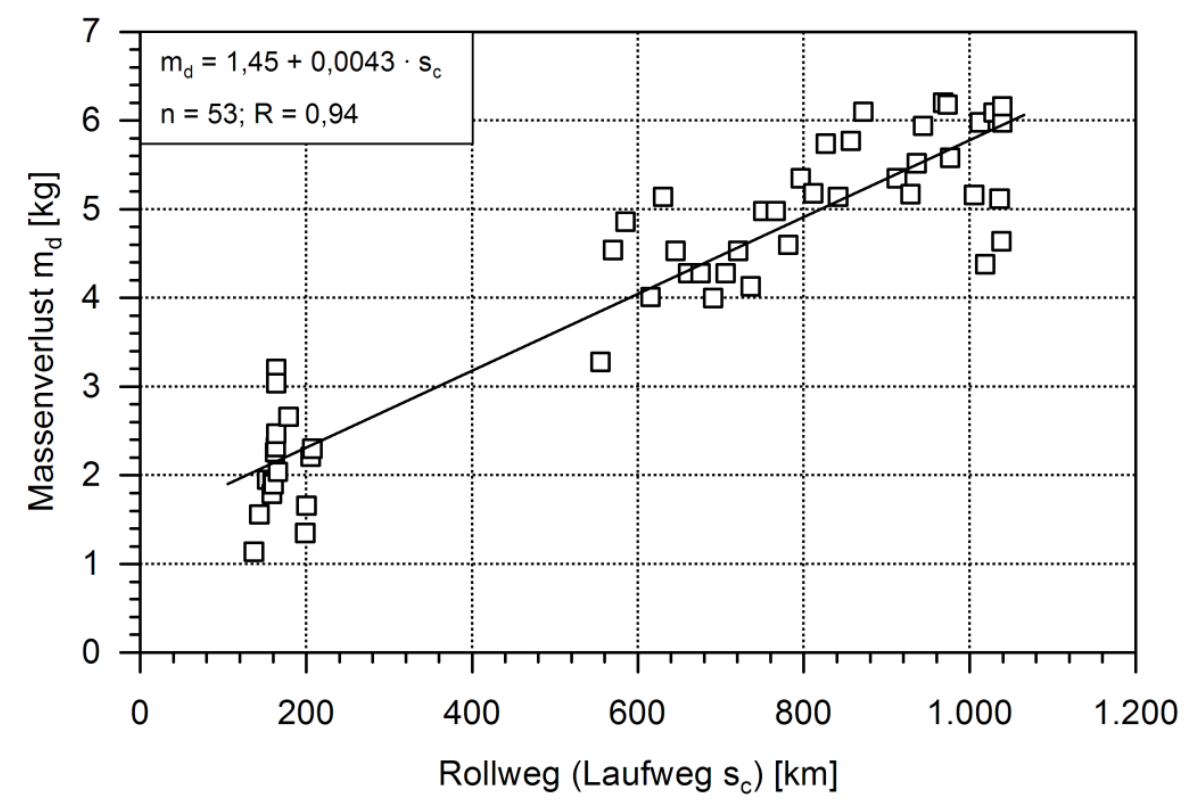

Abb. 66: Massenverlust $m_{d}[\mathrm{~kg}]$ der Schneidringe angetragen gegen den Laufweg $\mathrm{s}_{\mathrm{c}}[\mathrm{km}]$ der Schneidringe. Die Messpunkte zeigen einen deutlichen Trend zur linearen Abhängigkeit des Massenverlustes $m_{d}$ vom Laufweg $s_{c}$. Dieser ist durch die Trendlinie verdeutlicht. Der Korrelationskoeffizient $R$ der zugehörigen linearen Regression beträgt $R=0,94$.

Aus der Steigung der Regressionsgerade in Abb. 66 ergibt sich in diesem HGB für die Schneidringe eine Verschleißrate von:

$$
\mathrm{c}_{\mathrm{v}}=4,3 \frac{\mathrm{g}}{\mathrm{km}}
$$

Das Fehlen von Messpunkten zwischen ca. $200 \mathrm{~km}$ und $550 \mathrm{~km}$ Laufweg wird durch den Umstand verursacht, dass innerhalb des untersuchten Homogenbereiches keine Schneidringe ausgebaut wurden, die diesen Laufweg erreicht haben. Die Schwankungsbreite der Messergebnisse basiert einerseits auf der durch den Fertigungsprozess gegebenen Schwankungsbreite der Ausgangsmasse der Schneidringe $m_{0}$ von $+/-135 \mathrm{~g}$. Andererseits ist die Schwankungsbreite wahrscheinlich auch durch die natürliche Schwankungsbreite des Abriebs beim Bodenabbau und die natürliche Schwankungsbreite in der Zusammensetzung der Schluffe und Sande bedingt. Eine saubere Trennung der beiden Einflussfaktoren ist jedoch auf Basis der vorliegenden Daten nicht möglich. Das Ausgangsgewicht der einzelnen Schneidringe vor dem Test wurde nicht bestimmt, zusätzlich wurden die Bodeneigenschaften nicht in ausreichender Dichte bestimmt, um eine Aussage über die natürliche Schwankungsbreite zu ermöglichen.

\subsubsection{Abrieb an Sticheln}

In Referenzprojekt 5 wurden während des Vortriebes in Zusammenarbeit mit der bauausführenden Firma Messungen der Verschleißrate an Sticheln durchgeführt. Der Vortrieb der TVM erfolgte vorlaufend zur Messung in zwei Teilstrecken, die durch einen Zwischenschacht getrennt sind.

Im Zwischenschacht vor der zweiten Teilstrecke wurden alle Stichel vollständig erneuert. In der anschließenden Teilstrecke bis zum Erreichen der Ortbetonwand des Zielschachtes wurden die Stichel nicht gewechselt. Beim Einfahren in die zweite Teilstrecke wurde zunächst eine 1,20 m dicke Schlitzwand aus Beton der Festigkeitsklasse C25/30 durchfahren. An die Schlitzwand angeschlossen wurde ein ca. $10 \mathrm{~m}$ langer Dichtblock durchfahren, der im HDI Verfahren aus anstehenden sandigen Kiesen hergestellt wurde. Der Dichtblock entsprach ca. einem Bodenbeton der Festigkeitsklasse C8/10. 
Am Zielschacht wurde das Schneidrad ca. $30 \mathrm{~cm}$ in die Ortbetonwand eines bestehenden Bahnhofes eingefahren. Der Ortbeton entsprach einer Festigkeitsklasse von C25/30. Der Durchbruch in den Bahnhof erfolgte aufgrund des dort laufenden U-Bahn Betriebs zunächst nicht.

Die $680 \mathrm{~m}$ lange Teilvortriebsstrecke zwischen Dichtblock des Zwischenschachtes und Ortbetonwand des Zielbahnhofes setzt sich aus zwei geotechnischen Homogenbereichen zusammen.

Beginnend vom Zwischenschacht wurden auf einer Strecke von ca. $220 \mathrm{~m}$ vollflächig mitteldicht bis sehr dicht gelagerte quartäre Kiese (GW, GI, GE) aufgefahren. Die Kiese zeigten eine starke bis sehr starke Wasserdurchlässigkeit und eine große bis sehr große Scherfestigkeit (Bez. n. DIN 18196). Das Kornspektrum der Kiese zeigt gut gerundete Kalkkomponenten. Abrasivitätskennwerte wurden für die Kiese nicht bestimmt.

Ab ca. 220 m steigt von der Sohle her schleifend die Untergrenze der quartären Kiese zu den darunter liegenden tertiären Tonen und Schluffen in den Ausbruchquerschnitt an. Ab ca. $400 \mathrm{~m}$ bis zur Ortbetonwand des Zielbahnhofes nehmen die tertiären Tone und Schluffe den Ausbruchquerschnitt vollflächig ein. Die Tone und Schluffe (TM, TA, UL, UM) zeigten geringe bis sehr geringe Wasserdurchlässigkeit und eine große Scherfestigkeit (Bez. n. DIN 18196). Abrasivitätskennwerte wurden für die Tone und Schluffe nicht bestimmt.

Die Stichel leisteten im untersuchten Bereich den primären Bodenabbau, wurden jedoch aktiv und passiv eingesetzt. Wesentlich für die nachfolgend beschriebenen Messungen ist, dass die Stichel sowohl kurz vor Ausfahren aus dem Dichtblock am Zwischenschacht, als auch beim ersten Kontakt mit der Ortbetonwand des Zielbahnhofes bei Drucklufteinstiegen kontrolliert wurden.

Bei der Kontrolle nach dem Dichtblock wurde kein Verschleiß an den Sticheln festgestellt. Bei der Kontrolle an der Ortbetonwand wurde im Wesentlichen der Verschleißzustand an den Sticheln festgestellt, der auch bei der im Folgenden beschriebenen detaillierten Messung an den Sticheln nach weiteren $30 \mathrm{~cm}$ Vortrieb im Ortbeton dokumentiert wurde. Der Verschleißzustand der Stichel konnte folglich eindeutig auf den Bodenabbau zurückgeführt werden.

Nach Abschluss des Vortriebes in der Ortbetonwand wurde an den Sticheln mit einer elektronischen Schieblehre jeweils die Höhe der Hartmetallzähne an der Schneide auf $+/-0,1 \mathrm{~mm}$ an beiden Seiten der Stichel gemessen (Abb. 67). Die so bestimmte Höhe der Zähne $h_{v}[\mathrm{~mm}]$ kann ins Verhältnis zur Ausgangshöhe $h_{0}[\mathrm{~mm}]$ der Zähne gesetzt werden. Mit Gl. 27 (Kap. 5.4.2) kann der Höhenverlust $h_{d}$ [mm] bestimmt werden.

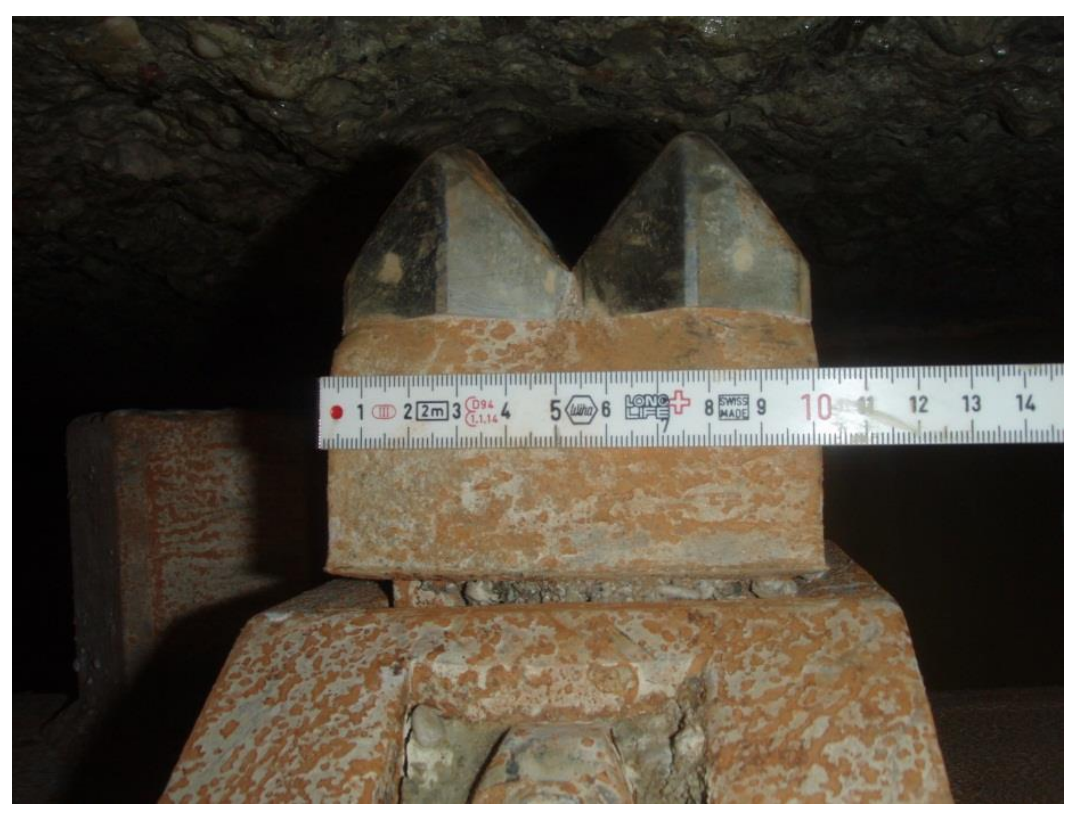

Abb. 67: Stichel bei der Messung in der Ortbetonwand. Der Meterstab dient als Maßstab für das Bild. Die Messung der Höhe der Hartmetallzähne wurde mit einer elektronischen Schieblehre auf $+/-0,1 \mathrm{~mm}$ genau vorgenommen. 
Der Höhenverlust $h_{d}$ wurde anschließend auf den Laufweg $s_{c}[\mathrm{~km}]$ auf Basis von Gl. 35 (Kap. 5.4.4) der Stichel bezogen. Insgesamt ergaben sich 42 Einzelmessungen, diese sind in Abb. 68 zusammengefasst.

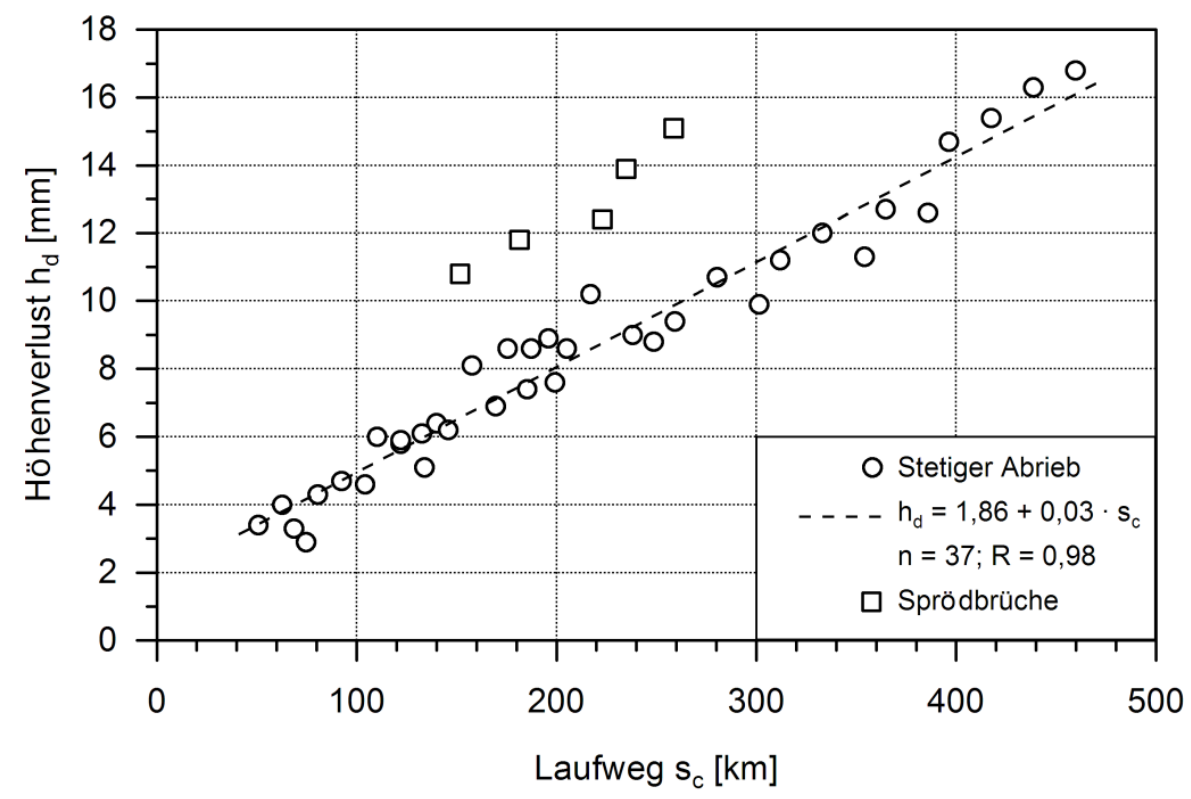

Abb. 68: Höhenverlust $h_{d}[\mathrm{~mm}]$ der Hartmetalleinsätze (Stichel) angetragen gegen den Laufweg $\mathrm{S}_{c}[\mathrm{~km}]$ der Stichel. Die Messpunkte zeigen einen deutlichen Trend zur linearen Abhängigkeit des Höhenverlustes $h_{d}$ vom Laufweg $\mathrm{s}_{\mathrm{c}}$. Dieser ist durch die Trendlinie verdeutlicht. Der zugehörigen Korrelationskoeffizient $\mathrm{R}$ der linearen Regression beträgt $\mathrm{R}=0,98$.

Die Verteilung der Messergebnisse in Abb. 68 zeigt einen deutlichen Trend zu einer linearen Abhängigkeit des Höhenverlustes $h_{d}$ der Stichel vom zugehörigen Laufweg $s_{c}$. Dieser ist durch die zusätzliche eingetragene lineare Regressionslinie verdeutlicht. Der zugehörige Korrelationskoeffizient $R$ beträgt 0,98. An fünf Sticheln wurden Gewaltschäden in Form von Ausbrüchen an den entsprechenden Hartmetallzähnen festgestellt. Diese Messpunkte wurden in der Regression nicht berücksichtigt. Aus der Steigung der Regressionsgerade ergibt sich für die vorliegenden Messungen für die Hartmetallzähne der Stichel eine Verschleißrate $c_{v}$ von $0,03 \mathrm{~mm} / \mathrm{km}$.

Die Ausgangshöhe der Hartmetallzähne $h_{0}$ besitzt bedingt durch den Fertigungsprozess nur eine sehr geringe Schwankungsbreite von ca. $+/-0,15 \mathrm{~mm}$. Die Messmethode mithilfe der elektronischen Schieblehre hat eine Genauigkeit von ca. $+/-0,1 \mathrm{~mm}$. Die in Abb. 68 dargestellte Schwankungsbreite des Höhenverlustes liegt deutlich höher als die Summe der möglichen Abweichungen aus der Ausgangshöhe und der Messmethode. Die Schwankungsbreite kann damit weitgehend auf geologische Einflüsse zurückgeführt werden. Einerseits weisen die beschriebenen quartären Kiese und tertiären Tone und Schluffe in sich jeweils eine deutliche Inhomogenität in der Korngrößenverteilung, Lagerungsdichte und Scherfestigkeit auf. Andererseits ergibt sich aufgrund des schleifenden Übergangs von quartären Kiesen zu tertiären Tonen und Schluffen im Vortrieb für die einzelnen Stichel ein deutlich unterschiedlicher Anteil an aktivem Laufweg in den Kiesen, Tonen und Schluffen. Da der genaue Verlauf des Übergangs zwischen quartären Kiesen und tertiären Tonen und Schluffen im Vortrieb nicht dokumentiert wurde, kann dieser Einflussfaktor nicht aus den dargestellten Messungen gefiltert werden. 


\subsubsection{Massenverlust an Schälmessern}

Im Referenzprojekt 5 wurden zeitgleich zur Messung an den Sticheln auch Messungen zum Massenverlust an den Schälmesser durchgeführt, die für den Vortrieb der ersten Teilstrecke vor dem Zwischenschacht eingesetzt wurden.

Im Zwischenschacht vor der zweiten Teilstrecke wurden alle Schälmesser der ersten Teilstrecke vom Schneidrad demontiert und mit der entsprechenden Arm Nr., Spur Nr. und Drehrichtung beschriftet. So war eine eindeutige Zuordnung des jeweiligen Massenverlustes zur Einbauposition des Schälmessers auf dem Schneidrad möglich. Während der ersten Teilstrecke bis zum Durchbruch in den Zwischenschacht wurden die Schälmesser nicht gewechselt.

Beim Einfahren in die erste Teilstrecke wurde zunächst eine 1,20 m dicke Schlitzwand aus Beton der Festigkeitsklasse C25/30 durchfahren. An die Schlitzwand angeschlossen wurde ein ca. $10 \mathrm{~m}$ langer Dichtblock durchfahren, der im HDI Verfahren aus anstehenden sandigen Kiesen hergestellt wurde. Der Dichtblock entsprach ca. einem Bodenbeton der Festigkeitsklasse C8/10. Beim Einfahren in den Zwischenschacht wurden in umgekehrter Reihenfolge wiederum ein $10 \mathrm{~m}$ langer Dichtblock und eine 1,20 m dicke Schlitzwand durchfahren.

Die $490 \mathrm{~m}$ lange Teilvortriebsstrecke zwischen den beiden Dichtblöcken setzt sich nur aus einem geotechnischen Homogenbereichen zusammen. Aufgefahren wurden volfflächig mitteldicht bis sehr dicht gelagerte quartäre Kiese (GW, GI, GE). Die Kiese zeigten eine starke bis sehr starke Wasserdurchlässigkeit und eine große bis sehr große Scherfestigkeit. Das Kornspektrum der Kiese zeigt im Wesentlichen gut gerundete Kalkkomponenten. Abrasivitätskennwerte wurden für die Kiese nicht bestimmt.

Der primäre Bodenabbau erfolgte über die gesamte Vortriebsstrecke durch die vor den Schälmessern angeordneten Stichel. Die Stichel decken aufgrund ihrer Anordnung die gesamte Ortsbrustfläche ab, sodass die Schälmesser keinen aktiven Bodenabbau geleistet haben und ausschließlich passiv und ohne Kontakt zur Ortsbrust mitgeführt wurden.

Die Wägung der Schälmesser wurden mit einer Laborwaage mit einer Genauigkeit von +/-1,0 g durchgeführt (Abb. 69).

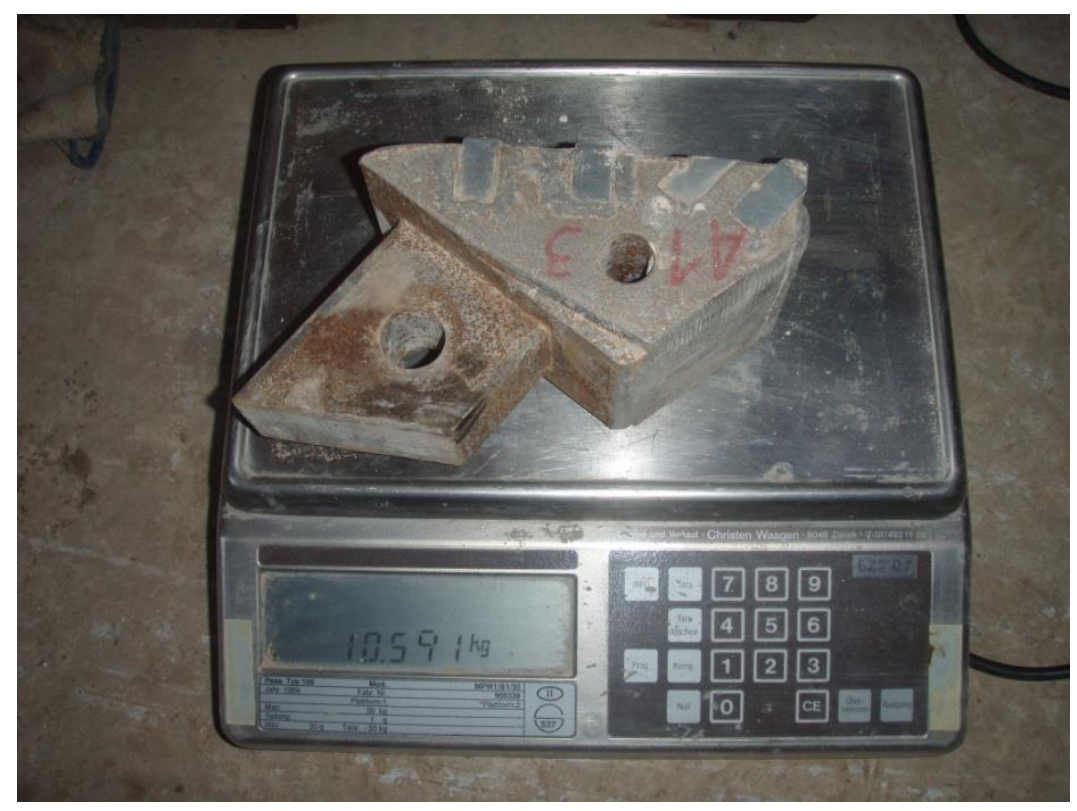

Abb. 69: Wägung eines Schälmessers mit der Laborwaage auf $+/-1 \mathrm{~g}$ genau. 
Die so bestimmte Masse der Schälmesser $m_{v}[g]$ kann ins Verhältnis zur Ausgangsmasse $m_{0}[g]$ gesetzt werden und mit Gl. 25 (Kap. 5.4.2) der jeweilige Massenverlust $m_{d}$ [g] bestimmt werden. Der Massenverlust $m_{d}$ wurde anschließend auf den Laufweg $s_{c}[\mathrm{~km}]$ der Schälmesser aus Gl. 35 (Kap. 5.4.4) bezogen. Insgesamt ergaben sich 64 Einzelmessungen, diese sind in Abb. 70 zusammengefasst.

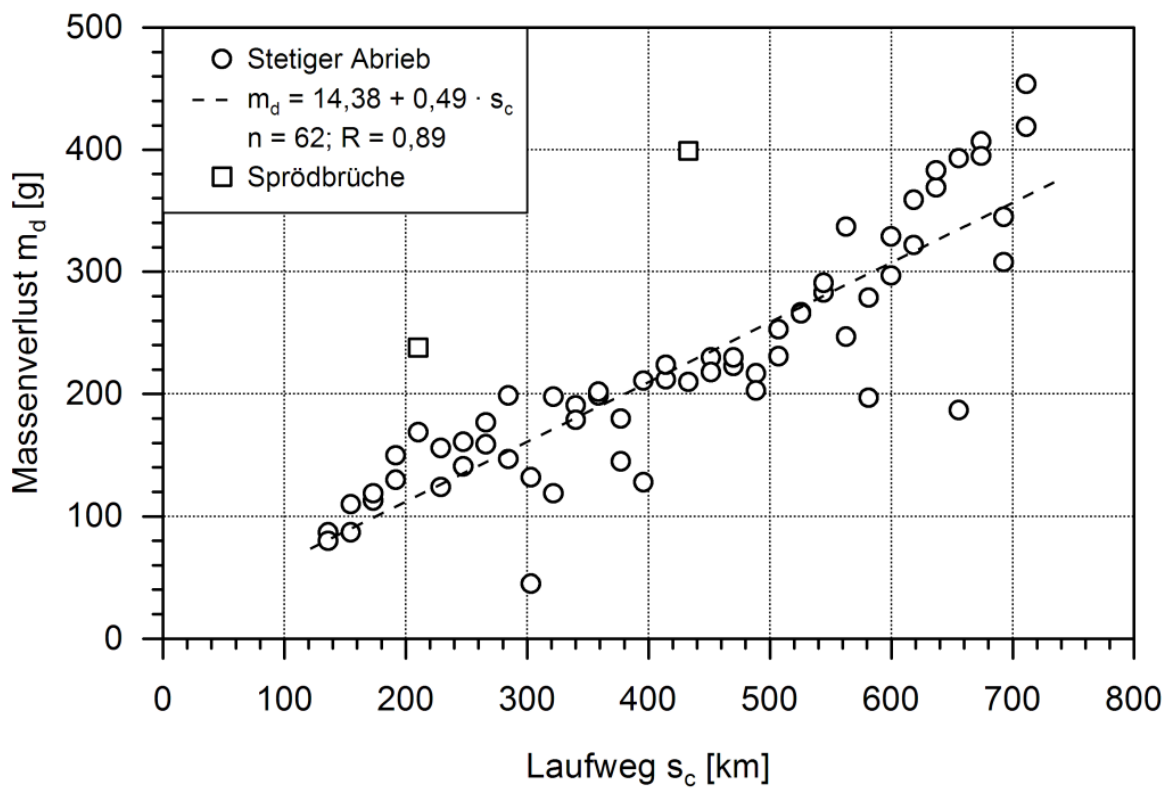

\begin{abstract}
Abb. 70: Massenverlust $m_{d}[g]$ der Schälmesser angetragen gegen den Laufweg $s_{c}[k m]$ der Schälmesser. Die Messpunkte zeigen einen deutlichen Trend zur linearen Abhängigkeit des Massenverlustes $m_{d}$ vom Laufweg $s_{c}$. Dieser ist durch die Trendlinie (gestrichelt) verdeutlicht. Der Korrelationskoeffizient der linearen Regression beträgt $R=89 \%$.
\end{abstract}

Die Verteilung der Messergebnisse in Abb. 70 zeigt einen deutlichen Trend zu einer linearen Abhängigkeit des Massenverlustes $m_{d}$ der Schälmesser vom zugehörigen Laufweg $s_{c}$. Dieser ist durch die zusätzlich eingetrage lineare Regressionslinie verdeutlicht. Der zugehörige Korrelationskoeffizient $R$ beträgt 0,89. In der Regression wurden zwei Messpunkte nicht berücksichtigt, bei denen an den Schälmessern Sprödbrüche an den Hartmetalleinsätzen festgestellt wurden. Aus der Steigung der Regressionsgerade ergibt sich für diesen Homogenbereich für die Schälmesser eine Verschleißrate $c_{v}$ von $0,49 \mathrm{~g} / \mathrm{km}$.

Im Bereich zwischen $300 \mathrm{~km}$ und $500 \mathrm{~km}$ Laufweg zeigt die Verteilung der Messpunkte in Abb. 70 eine deutliche Abstufung. In diesem Bereich steigt der Massenverlust $m_{d}$ im Verhältnis zum Laufweg $\mathrm{s}_{\mathrm{c}}$ deutlich weniger an, als in den Bereichen darunter und darüber. Aufgrund der Versuchsanordnung haben alle in Abb. 70 dargestellten Schälmesser die identische Vortriebsstrecke durchlaufen. Der Unterschied im Laufweg $\mathrm{s}_{\mathrm{c}}$ ergibt sich ausschließlich durch die verschiedenen Spurradien $\mathrm{r}_{\mathrm{s}}$ der Schälmesser. Die Abstufung in der Verteilung der Messpunkte entspricht folglich einem bestimmten Radiusbereich auf dem Schneidrad zwischen den Spurradien $r_{\mathrm{s}}$ von $1.470 \mathrm{~mm}$ bis $2.370 \mathrm{~mm}$. Eine Abweichung in den geotechnischen Verhältnissen in der Ortsbrust oder in der Steuerung des Abbauprozesses für die Schälmesser in diesem engen Bereich als Ursache für die Abweichung der Verschleißrate $c_{v}$ im Vergleich zu den übrigen Schälmessern ist damit ausgeschlossen. Die Auslegung und Anordnung der Schälmesser in diesem Bereich ist ebenfalls identisch mit den übrigen Schälmessern.

Die Ursache für die Abweichung der Verschleißrate $c_{v}$ liegt vermutlich im Materialfluss zwischen Ortsbrust, Schneidrad und Tauchwandöffnung. Im Radiusbereich unterhalb von $1.470 \mathrm{~mm}$ grenzt unmittelbar der eher geschlossene Zentrumsbereich des Schneidrades an. Hier muss der von den Sticheln und dem Zentrumskamm abgebaute Boden zwischen Schneidrad und Ortsbrust nach außen hin zu den Schneidradöffnungen gefördert werden. Durch die Einengung in diesem Bereich erhöht sich ver- 
mutlich die Kontakthäufigkeit zwischen dem bereits abgebauten Boden in der Stützflüssigkeit und den Schälmessern im Vergleich zum weiter außen liegenden Bereich oberhalb von $1.470 \mathrm{~mm}$. Im Radiusbereich oberhalb von $2.370 \mathrm{~mm}$ nimmt die Kontakthäufigkeit zwischen den Schälmessern und dem bereits abgebauten Boden vermutlich erneut zu, weil in diesem Bereich der aus der gesamten Ortsbrustfläche in die Schildsohle sinkende Boden von den Schälmessern in Richtung der Tauchwandöffnung gefördert werden muss. Die erhöhte Kontakthäufigkeit zwischen dem bereits abgebauten Boden und den Schälmessern führt vermutlich in beiden Bereichen zu einer Erhöhung der Verschleißrate $c_{v}$ durch Sekundärverschleiß. Die Abstufung der Verschleißrate $c_{v}$ in Abb. 70 wird also vermutlich durch Unterschiede im Materialfluss in der Abbaukammer verursacht.

Die Schwankungsbreite der Messergebnisse basiert einerseits auf der durch den Fertigungsprozess gegebenen Schwankungsbreite der Ausgangsmasse $m_{0}$ der Schälmesser von $+/-50 \mathrm{~g}$. Andererseits ist die Schwankungsbreite vermutlich auch durch die natürliche Schwankungsbreite des Abriebs beim Bodenabbau, sowie die natürliche Schwankungsbreite in der Zusammensetzung der dicht gelagerten Kiese bedingt. Eine saubere Trennung der beiden Einflussfaktoren ist jedoch auf Basis der vorliegenden Daten nicht möglich. Das Ausgangsgewicht der einzelnen Schneidringe vor dem Test wurde nicht bestimmt wurde, zusätzlich wurden die Bodeneigenschaften nicht in ausreichender Dichte bestimmt, um eine Aussage über die natürliche Schwankungsbreite zu ermöglichen.

\subsubsection{Zusammenfassung}

Die drei Versuche zur Verschleißrate zeigen unabhängig von der angewandten Messmethode in allen drei Fällen eine sehr starke lineare Abhängigkeit vom Laufweg $s_{c}$ mit entsprechend hohen Werten des Korrelationskoeffizienten R von 0,89-0,98. Die drei Einzelversuche decken zudem ein relativ breites Spektrum an Einsatzbedingungen und Verschleißtypen ab. Der Versuch zum Massenverlust an Schneidringen erfasst den Primärverschleiß durch aktiven Bodenabbau, ebenso der Versuch zum Höhenverlust an den Sticheln. Dagegen haben die Schälmesser im Versuch zum Massenverlust $m_{d}$ keinen Kontakt zur Ortsbrust, es wird also der Sekundärverschleiß durch bereits abgebauten Boden erfasst.

Die Versuchsergebnisse legen zusammenfassend nahe, dass unabhängig vom Verschleißtyp der Abrieb an Werkzeugen in Form von Massenverlust oder Längenverlust in Lockergesteinen in guter Näherung direkt proportional zum Laufweg $\mathrm{s}_{\mathrm{c}}$ der Werkzeuge ist.

Die Bestimmung der Verschleißrate erfordert in zukünftigen Projekten zwingend folgende Voraussetzungen, die für die Dokumentation entsprechender Versuche oder Auswertungen von hoher Bedeutung sind:

- Kenntnis über Zeitpunkt oder Ort des Beginns des Messintervalls

- Kenntnis über den genauen Werkzeugzustand am Beginn des Messintervalls (Null-Messung)

- Kenntnis über Zeitpunkt oder Ort des Endes des Messintervalls

- Kenntnis über den genauen Werkzeugzustand am Ende des Messintervalls

- Kenntnis über den erzielten Laufweg im Messintervall (Maschinendaten)

- Möglichst genaue Kenntnis der Anwendungsbedingungen des Werkzeuges im Messintervall

Die Ergebnisse zu den Verschleißbildern von Schneidringen, Schälmessern und Sticheln aus Kap. 5.1 zeigen, dass mit zunehmendem Verschleiß bei allen Werkzeugtypen die Kontaktfläche zur Ortsbrust zum Teil erheblich ansteigt. Demzufolge ist es theoretisch denkbar, dass ab einem gewissen Abrieb$\mathrm{maß}$ z.B. der Massenverlust bereits vor Erreichen der Verschleißgrenze nicht mehr linear, sondern überlinear ansteigt. Der Massenverlust bzw. Höhenverlust wäre damit für diesen Bereich nicht mehr linear vom Laufweg $s_{c}$ der Werkzeuge abhängig. Ein noch deutlicherer Anstieg des Massenverlustes wäre für Schälmesser, Stichel und Räumer auf Basis der Beobachtungen in Kap. 5.1 zu erwarten, sobald der Verschleißschutz durch Hartauftrag oder Hartmetalleinsätze verloren geht. 
Innerhalb der Versuche zum Höhenverlust $h_{d}$ an den Hartmetallzähnen der Stichel und zum Massenverlust $m_{d}$ der Schälmesser lag der absolute Verschleißzustand der Werkzeuge jeweils nur bei ca. $10-20 \%$ der verfügbaren Masse bzw. Höhe bis zur Verschleißgrenze. Hier kann deshalb kein überlinearer Anstieg der Verschleißrate $c_{v}$ dargestellt werden. Bei den Versuchen zum Massenverlust der Schneidringe lag der absolute Verschleißzustand der Ringe zwischen 60\% und $80 \%$ der bis zur Verschleißgrenze verfügbaren Masse. Auch hier kann kein exponentieller Anstieg der Verschleißrate $c_{v}$ mit zunehmendem Laufweg $\mathrm{s}_{\mathrm{c}}$ dargestellt werden.

Die Versuche zum Massenverlust $m_{d}$ der Schälmesser (Kap. 5.5.1) zeigen, dass bei Abbauwerkzeugen, die ausschließlich dem Sekundärverschleiß aufgrund von Kontakt mit bereits abgebautem Boden in der Stützflüssigkeit unterliegen, die Verschleißrate $c_{v}$ innerhalb eines geotechnischen Homogenbereiches nicht für alle Werkzeuge eines Typs auf dem Schneidrad zur gleichen Zeit den gleichen Betrag aufweisen muss. Durch den Materialfluss des abgebauten Bodens von der Ortsbrust zur Tauchwandöffnung können sich in Abhängigkeit der konstruktiven Auslegung des Schneidrads und des Schildes erhebliche Unterschiede ergeben.

Die Auswirkungen des Materialflusses auf die Verschleißrate $c_{v}$ müssen im Verhältnis zum Primärverschleiß der Abbauwerkzuge relativiert werden. Die Schälmesser im vorliegenden Versuch stellen einen Sonderfall dar, weil sie aufgrund der vollflächig vorlaufenden Stichel zu keinem Zeitpunkt direkt in die Ortsbrust eingegriffen haben. Die zugehörigen Stichel zeigen bei identischer Versuchsanordnung (Kap. 5.5.2) keine entsprechende Abstufung der Verschleißrate $c_{v}$ in Abhängigkeit des Spurradius $r_{s}$, obwohl diese neben dem Primärverschleiß aus dem Bodenabbau sehr ähnlichen Verhältnissen für den Sekundärverschleiß unterliegen, wie die Schälmesser. Daraus ergibt sich die Annahme, dass im absoluten Vergleich der Betrag der Verschleißrate $c_{v}$ aus Sekundärverschleiß durch bereits abgebauten Boden in der Stützflüssigkeit deutlich niedriger liegt, als der Betrag aus Primärverschleiß durch Kontakt mit dem Boden in der Ortsbrust. Treten die beiden Verschleißtypen in Kombination auf, werden entsprechende Effekte aus dem Sekundärverschleiß durch den Primärverschleiß überlagert, sodass die Verschleißrate $c_{v}$ für alle Abbauwerkzeuge auf dem Schneidrad unter den gegebenen Versuchsbedingungen annähernd den gleichen Betrag aufweist.

Für die Datenanalyse zu den Standgrößen (Kap. 5.6) der Abbauwerkzeuge beim Einsatz auf Hydroschild TVM in Lockergesteinen und den Aufbau eines empirischen Prognosemodells (Kap. 6) begründen die beschriebenen Ergebnisse zur Verschleißrate $c_{v}$ folgende Annahmen:

- Der Massenverlust $m_{d}[g]$ und der Höhenverlust $h_{d}[\mathrm{~mm}]$ der Abbauwerkzeuge sind unter gleichbleibenden Einsatzbedingungen für alle Werkzeug- und Verschleißtyp linear abhängig vom Laufweg $\mathrm{s}_{\mathrm{c}}[\mathrm{km}]$.

- Die Verschleißrate $c_{v}$ ist unter gleichbleibenden Einsatzbedingungen vom Einbau der Abbauwerkzeuge auf dem Schneidrad bis zum Erreichen der Verschleißgrenze konstant.

- Der Betrag der Verschleißrate $c_{v}$ ist für alle Abbauwerkzeuge des gleichen Typs auf dem Schneidrad zum gleichen Zeitpunkt und unter den gleichen Einsatzbedingungen annähernd identisch. 


\subsection{Einflussfaktoren auf den Laufweg $s_{c}$}

Das Ziel der folgenden Analyse der Zusammenhänge zwischen den Standgrößen der Abbauwerkzeuge, Auslegung der Hydroschild TVM, Boden- und Gebirgseigenschaften und Steuerung des Abbauprozesses ist:

- Identifikation der maßgeblichen Einflussfaktoren auf die Standgrößen der Abbauwerkzeuge.

- Quantifizierung der maßgeblichen Einflussfaktoren.

Die Beschreibung der Ergebnisse ist im Folgenden in die Kategorien an Einflussfaktoren gegliedert, die bereits bei der Beschreibung des bestehenden Wissensstandes (Kap. 4) eingesetzt wurde:

- Auslegung der Hydroschild TVM

- Steuerung des Abbauprozesses

- Boden- und Gebirgseigenschaften

Zuvor erfolgt eine Beschreibung der Aufbereitung der Rohdaten aus den Referenzprojekten für die Datenanalyse.

\subsubsection{Aufbereitung der Rohdaten}

Aus den 10 Referenzprojekten, 13 Hydroschild TVM und 18 Vortrieben (Kap. 5.1) ergibt sich in Summe ein Vortriebsvolumen von 5.592.143 $\mathrm{m}^{3}$. Einen Überblick über die enthaltenen Bodenvolumina, unterteilt nach Bodengruppen entsprechend DIN EN 14688-1 und die darin jeweils enthaltenen Werkzeugwechsel, gibt Tab. 14. In RP 8 konnten ca. 1.250 Schälmesserwechsel nicht dokumentiert werden (Kap. 5.1) und sind in Tab. 14 nicht enthalten, weil sie für die weitere Datenanalyse nicht nutzbar sind.

Zu jedem der 4.607 Werkzeugwechsel wurde in der Folge der Laufweg $\mathrm{s}_{\mathrm{c}}$ berechnet.

Tab. 14: Übersicht über die Rohdaten für die Analyse der Werkzeugstandgrößen aus den Referenzprojekten.

\begin{tabular}{|l|c|c|c|c|c|}
\hline \multicolumn{1}{|c|}{ Bodengruppe } & Volumen & Schneidrollen & Stichel & Schälmesser & Summe \\
\hline \multicolumn{1}{|c|}{ DIN EN 14688-1 } & {$\left[\mathbf{m}^{\mathbf{3}}\right]$} & {$[$ Stk.] } & {$[$ Stk.] } & [Stk.] & [Stk.] \\
\hline Ton \& Schluff & 2.857 .864 & 212 & 12 & 327 & 551 \\
\hline Sand & 1.513 .403 & 238 & 127 & 937 & 1.302 \\
\hline Kies & 1.220 .876 & 1.180 & 238 & 1.336 & 2.754 \\
\hline Summe: & $\mathbf{5 . 5 9 2 . 1 4 3}$ & $\mathbf{1 . 6 3 0}$ & $\mathbf{3 7 7}$ & $\mathbf{2 . 6 0 0}$ & $\mathbf{4 . 6 0 7}$ \\
\hline
\end{tabular}


Um die Rohdaten zu strukturieren und für die Analyse hin auf verschiedene Einflussfaktoren aus den Einsatzbedingungen aufzubereiten wurden folgende Schritte unternommen:

- Zuordnung der Parameter aus der Auslegung der Hydroschild TVM zu den einzelnen Werkzeugwechseln.

- Unterteilung der Vortriebsstrecken in geotechnische Homogenbereiche.

- Zuordnung der Boden- und Gebirgseigenschaften zu den einzelnen Werkzeugwechseln.

- Zuordnung der Parameter aus der Steuerung des Abbauprozesses zu den einzelnen Werkzeugwechseln.

- Elimination von präventiven und schadensbedingten Werkzeugwechseln.

Der zuletzt genannte Punkt erfordert, basierend auf den in Kap. 5.2 dargestellten Ergebnissen zu den Verschleißgrenzen der Abbauwerkzeuge und der Dokumentation in den Referenzprojekten, besondere Aufmerksamkeit, um Vergleichbarkeit der Werkzeugwechseldaten zwischen verschiedenen Referenzprojekten zu erhalten bzw. herzustellen.

\subsubsection{Zuordnung der Auslegung der Hydroschild TVM}

Die in Kap. 5.2.3 genannten Parameter aus der Auslegung der Hydroschild TVM sind in allen Referenzprojekten vollständig vorhanden und konnten problemlos jedem einzelnen Werkzeugwechsel zugeordnet werden.

\subsubsection{Zuordnung der Boden- und Gebirgseigenschaften}

Um den Einfluss der Boden- und Gebirgseigenschaften zu analysieren, müssen jedem Werkzeugwechsel eindeutige geologische und geotechnische Parameter zugeordnet werden.

Die Ergebnisse zu den Verschleißgrenzen der Abbauwerkzeuge und der Dokumentation in Kap. 5.2 werfen dabei Probleme auf. Die Anzahl $\mathrm{n}_{\mathbf{l}(\mathrm{lc})}$ der Abbauwerkzeuginspektionen zwischen Ein- und Ausbau der Abbauwerkzeuge ist nicht hoch genug, um den Zustand jedes einzelnen Werkzeuges bei jedem Wechsel der geotechnischen Rahmenbedingungen festzustellen und zu dokumentieren (Kap. 5.2.1.1). Zusätzlich ist die Dokumentationsdichte im Hinblick auf die tatsächlich angetroffenen geotechnischen Bedingungen in der Ortsbrust (Kap. 5.2.2.3) und den Zustand der Werkzeuge bei Schneidradkontrollen (Kap. 5.2.1.3) sehr niedrig.

Um dennoch eine Zuordnung eindeutiger geologischer und geotechnischer Parameter zu den einzelnen Werkzeugwechseln zu ermöglichen, ist eine Einteilung der Vortriebsstrecken in geotechnischen Homogenbereiche (HGB) unumgänglich. Zur Einteilung der Vortriebsstrecken $L_{T A}$ in die Homogenbereiche wurden auf Basis der geotechnischen Längsschnitte in den Referenzprojekten folgenden Kriterien eingesetzt:

- Isotrope Zusammensetzung der Ortsbrust in Richtung der Tunnelachse (TA). (Gleichbleibender Anteil verschiedener Bodenarten in der Ortsbrust [\%])

- Gleichbleibende Überlagerungshöhe $\mathrm{H}_{\mathrm{TA}}[\mathrm{m}]$ über der Tunnelachse.

- Gleichbleibender Wasserspiegel $\mathrm{W}_{\mathrm{TA}}[\mathrm{m}]$ über der Tunnelachse.

Das Kriterium zum gleichbleibenden Anteil an verschiedenen Bodenarten in der Ortsbrust dient dazu, innerhalb der HGB eindeutige Werte für die in Kap. 5.2.2.3 genannten geotechnischer Parameter zu ermitteln. Die Berechnung erfolgte durch Bildung gewichteter Mittelwerte entsprechend dem prozentualen Flächenanteil der jeweiligen Bodenart in der Ortsbrust. Damit können jedem Werkzeugwechsel die in Tab. 12 angegebenen Parameter in Abhängigkeit von deren Verfügbarkeit in den Referenzprojekten zugeordnet werden. Die beiden Kriterien zur Überlagerung $H_{T A}$ und Wasserspiegel $W_{T A}$ dienen 
dazu, den Spannungszustand des Bodens in der Ortsbrust einzugrenzen, weil dieser nach den Erkenntnissen aus dem bestehenden Wissensstand (Kap. 4.3.1) Einfluss auf den Verschleiß der Abbauwerkzeuge hat.

Voraussetzung für die Berechnung und Zuordnung der geotechnischen Parameter ist, dass die Ringnummern $b_{0}$ und $b_{v}$ bei Ein- und Ausbau der Abbauwerkzeuge auf dem Schneidrad innerhalb eines geotechnischen Homogenbereiches liegen. Die Dokumentation der Werkzeugwechsel in den Referenzprojekten (Kap. 5.2.1.3) erlaubt aufgrund der niedrigen Anzahl an Kontrollen $n_{1(\text { (c) })}$ der Abbauwerkzeuge (Abb. 34) keine Beurteilung des Zustandes der Abbauwerkzeuge an der Grenze zwischen zwei geotechnischen Homogenbereichen. Deshalb kann eine eindeutige Zuordnung der geotechnischen Parameter nur für Abbauwerkzeuge erfolgen, die innerhalb eines Homogenbereichs gewechselt wurden. Abbauwerkzeuge, die zwischen Einbau $b_{0}$ und Ausbau $b_{v}$ mehr als einen geotechnischen Homogenbereich durchlaufen haben, wurden eliminiert und fließen nicht in die weitere Analyse der Einflussfaktoren ein.

In den Referenzprojekten wurden die geotechnischen Bedingungen jeweils anhand der metrischen Stationierung der Tunnelachse erfasst. Die metrische Stationierung bei Ein- und Ausbau der Abbauwerkzeuge wurde aus den Ringnummern $b_{0}$ und $b_{v}$ und der mittleren Breite der Tübbingringe $I_{(b)}$ berechnet. Bei der Zuordnung der geotechnischen Bedingungen zu den Werkzeugwechseln ist deshalb zu berücksichtigen, dass die tatsächliche Stationierung der Ortsbrust beim Bodenabbau und Ein- und Ausbau der Abbauwerkzeuge etwa um die Länge der Schildmaschine $L_{T V M}[\mathrm{~m}]$ in Vortriebsrichtung versetzt liegt (Kap. 5.4.1).

Durch die Festlegung der geotechnischen Homogenbereiche entsteht ein systembedingter Widerspruch bei der Analyse des Einflusses der Boden- und Gebirgseigenschaften auf die Standgrößen der Abbauwerkzeuge. Eine möglichst genaue Analyse der Einflussfaktoren (Kap. 5.6.4) erfordert für die drei genannten Kriterien zur Bildung der Homogenbereiche eine relativ hohe Genauigkeit, um die Schwankungsbreite der enthaltenen geotechnischen Parameter möglichst gering zu halten. Daraus entstehen tendenziell sehr kurze geotechnische Homogenbereiche, sodass die Anzahl an Abbauwerkzeugen, deren Einbau $b_{0}$ und Ausbau $b_{v}$ innerhalb eines Homogenbereiches liegt sehr gering wird. Umgekehrt erfordert die Analyse jedoch auch ein möglichst hohes Kollektiv an Basisdaten, also Abbauwerkzeuge, deren Einbau $b_{0}$ und Ausbau $b_{v}$ innerhalb eines Homogenbereiches liegen. Folglich sind relativ lange geotechnischen Homogenbereiche notwendig. Dazu muss zwangsläufig eine Reduktion der Genauigkeit der Kriterien zur Bildung der geotechnischen Homogenbereiche und eine höhere Schwankungsbreite der geotechnischen Parameter in den Homogenbereichen akzeptiert werden.

Um für die Kriterien zur Einteilung der geotechnischen Homogenbereiche eine Genauigkeit bzw. akzeptierte Schwankungsbreite festzulegen, wurde deshalb eine Sensitivitätsanalyse für die drei Kriterien durchgeführt. Dazu wurde die Genauigkeit der drei Kriterien einzeln schrittweise variiert und das verbleibende Volumen an Abbauwerkzeugwechseln aus den Referenzprojekten erfasst. Die Analyse zeigt, dass innerhalb der Referenzprojekte die Länge der Homogenbereiche und das verbleibende Datenvolumen sehr sensibel auf die Genauigkeit des Anteils der Bodenarten in der Ortsbrust anspricht, während die Überlagerung $\mathrm{H}_{\mathrm{TA}}$ und der Wasserspiegel $\mathrm{W}_{\mathrm{TA}}$ erst bei relativ starker Einschränkung der akzeptierten Schwankungsbreite eine Änderung der Länge der Homogenbereiche und des verbleibenden Datenvolumens bewirken. Ursache dafür ist, dass die Topographie der Geländeoberkante (GOK) und der Verlauf des Wasserspiegels $W_{T A}$ in den Referenzprojekten über weite Strecken sehr geringe Reliefunterschiede aufweisen. Gleichzeitig sind die Steigungsgradienten der Tunnelachsen relativ gering. Die Genauigkeit bei der Bestimmung des Anteils verschiedener Bodenarten in der Ortsbrust hat zudem eine höhere Bedeutung für die Datenanalyse, weil damit die Schwankungsbreite aller in Tab. 12 aufgelisteten Kennwerte gesteuert wird. Die beiden übrigen Kriterien steuern im Vergleich dazu hauptsächlich die Schwankungsbreite des Spannungszustandes des Bodens in der Ortsbrust, also eines einzelnen Einflussfaktors auf die Standgrößen der Abbauwerkzeuge.

Für die Anwendung der Kriterien in den Referenzprojekten wurde deshalb zunächst die akzeptierte Schwankungsbreite des Anteils verschiedener Bodenarten in der Ortsbrust festgelegt. Hier wurde eine Schwankungsbreite von $+/-10 \%$ akzeptiert, wobei $57 \%$ des Datenvolumens der Abbauwerkzeugwechsel verloren gehen. Bei einer weiteren Reduktion der akzeptierten Schwankungsbreite fällt das verbleibende Datenvolumen überproportional schnell gegen null ab. Bei einer weiteren Erhöhung der akzeptierten Schwankungsbreite steigt das verbleibende Datenvolumen unterproportional mit der Schwankungsbreite an. Einen Überblick über diesen Zusammenhang gibt Abb. 71. 


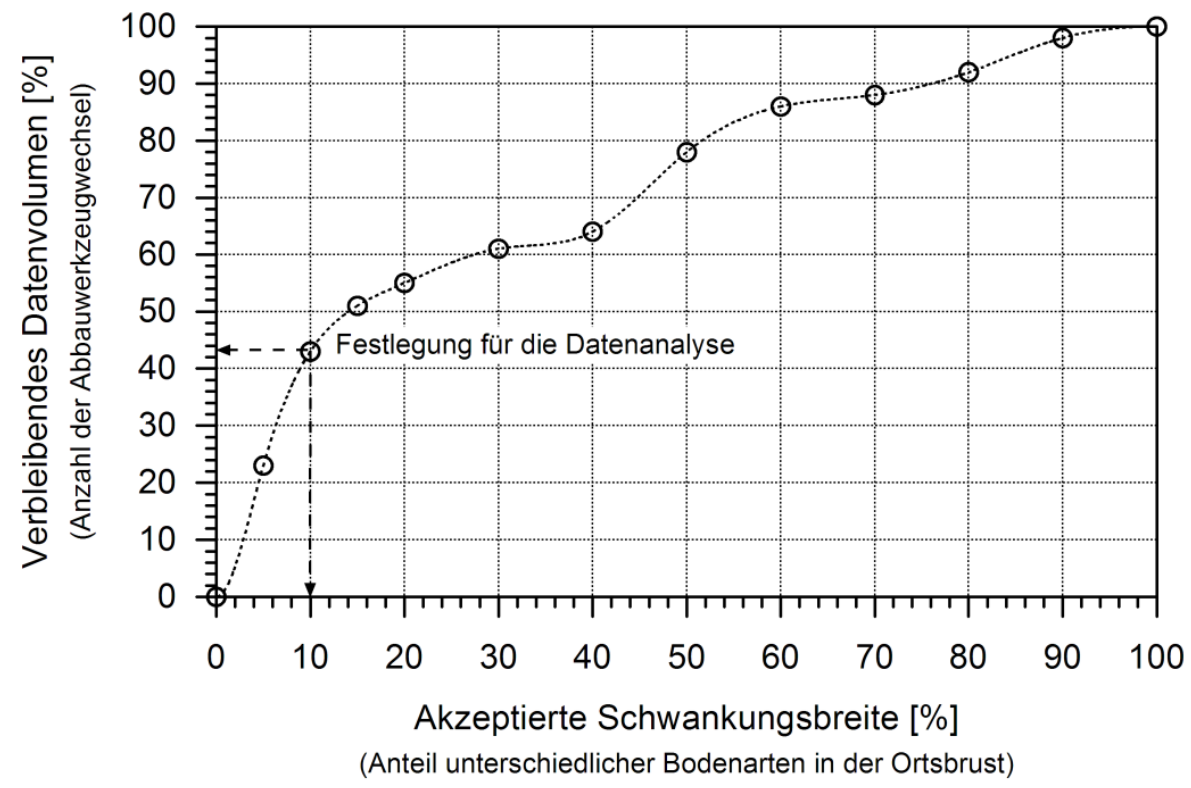

\begin{abstract}
Abb. 71: Anteil des verbleibenden Datenvolumens der Abbauwerkzeugwechsel [\%], in Abhängigkeit der akzeptierten Schwankungsbreite des Anteils [\%] unterschiedlicher Bodenarten in der Ortsbrust bei der Bildung der geotechnischen Homogenbereiche für die Datenanalyse. Für die Datenanalyse wurde eine akzeptierte Schwankungsbreite von $+/-10 \%$ festgelegt, sodass $43 \%$ des Datenvolumens nutzbar sind.
\end{abstract}

Anschließend wurden anhand des Anteils verschiedener Bodenarten in der Ortsbrust 62 vorläufige geotechnische Homogenbereiche gebildet und die zugehörigen Werte für die Überlagerung $\mathrm{H}_{T \mathrm{~A}}$ und den Wasserspiegel $W_{T A}$ als Mittelwerte über die Länge der Homogenbereiche $L_{H G B}$ bestimmt. Die Differenzen in der Überlagerung und dem Wasserspiegel zwischen zwei benachbarten geotechnischen Homogenbereichen ergeben einen Anhaltspunkt für die Festlegung der akzeptierten Schwankungsbreite für die Überlagerung $\mathrm{H}_{\mathrm{TA}}$ und den Wasserspiegel $\mathrm{W}_{\mathrm{TA}}$. Aufgrund des Unterschiedes in der Sensitivität im Vergleich zum Anteil verschiedener Bodenarten in der Ortsbrust sind diese Differenzen zwischen den vorläufigen Homogenbereichen bereits relativ gering. Bei der Überlagerung $\mathrm{H}_{\mathrm{TA}}$ ergeben sich nur bei 4 der 62 Homogenbereiche Differenzen $>5 \mathrm{~m}$ an einer der beiden Grenzen. Der Rest der Differenzen liegt bei $\leq 5 \mathrm{~m}$. Beim Wasserspiegel $W_{T A}$ liegen die verbleibenden Differenzen zwischen den Homogenbereichen durchgehend bei $\leq 5 \mathrm{~m}$. Für beide Kriterien kann deshalb eine Genauigkeit von +/-5 m angesetzt werden, ohne das verbleibende Datenvolumen weiter einzuschränken. Aufgrund der Überlagerung $\mathrm{H}_{\mathrm{TA}}$ ergeben sich lediglich 4 zusätzliche Homogenbereiche, die jedoch nur minimalen Einfluss auf das Datenvolumen haben.

Zusammenfassend wurden also folgende Werte für die akzeptierte Schwankungsbreite bei der Anwendung der Kriterien zur Bildung der geotechnischen Homogenbereiche angesetzt:

- Anteil verschiedener Bodenarten in der Ortsbrust: $+/-10 \%$

- Überlagerung $\mathrm{H}_{\mathrm{TA}}[\mathrm{m}]:+/-5 \mathrm{~m}$

- Wasserspiegel $\mathrm{W}_{\mathrm{TA}}[\mathrm{m}]:+/-5 \mathrm{~m}$

Einen Überblick über die Länge und enthaltene Bodengruppen der 66 resultierenden geotechnischen Homogenbereichen nach DIN EN 14688-1 gibt Abb. 72. 


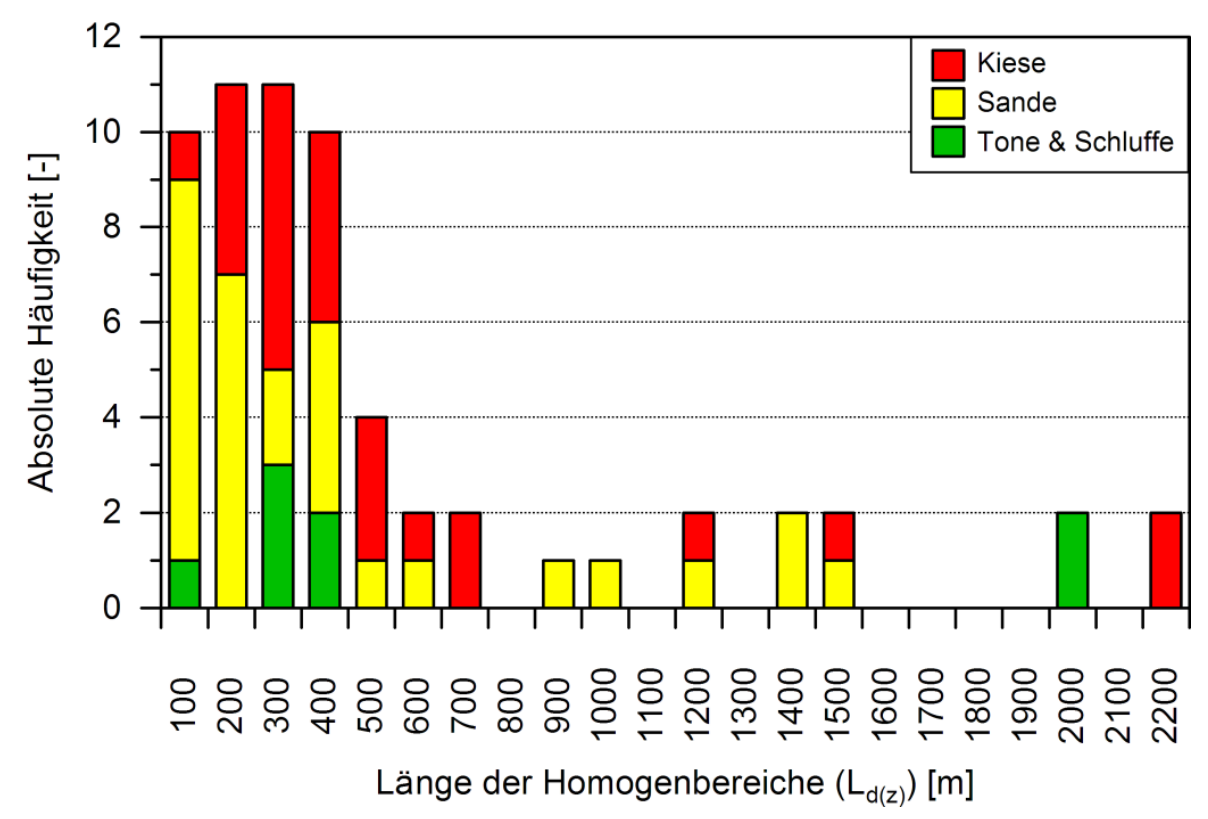

\begin{abstract}
Abb. 72: Verteilung der Länge der geotechnischen Homogenbereiche $L_{d(z)}[\mathrm{m}]$ unterteilt nach Bodengruppen (DIN EN 14688-1). 2 Homogenbereiche in Schluff mit einer Länge von jeweils $7.170 \mathrm{~m}$ sind aufgrund der Übersichtlichkeit nicht im Diagramm dargestellt.
\end{abstract}

In Summe entfallen durch die Festlegung der Homogenbereiche 57\% aller Werkzeugwechsel. Nur $43 \%$ aller Werkzeuge wurden innerhalb eines geotechnischen Homogenbereiches gewechselt. Einen Überblick über die Anzahl der verbleibenden Werkzeugwechsel im Vergleich zu den Rohdaten (Tab. 14) gibt Tab. 15.

Tab. 15: Übersicht über die verbleibende Anzahl an Werkzeugwechseln für die Datenanalyse nach Bildung der geotechnischen Homogenbereiche.

\begin{tabular}{|l|c|c|c|c|c|}
\hline \multicolumn{1}{|c|}{ Bodengruppe } & Volumen & Schneidrollen & Stichel & Schälmesser & Summe \\
\hline \multicolumn{1}{|c|}{ DIN EN 14688-1 } & {$\left[\mathrm{m}^{3}\right.$ ] } & [Stk.] & [Stk.] & [Stk.] & [Stk.] \\
\hline Ton \& Schluff & 2.801 .799 & 48 & 8 & 194 & 242 \\
\hline Sand & 860.431 & 125 & 115 & 287 & 527 \\
\hline Kies & 1.096 .277 & 684 & 58 & 476 & 1.208 \\
\hline Summe: & $\mathbf{4 . 7 5 8 . 5 0 7}$ & $\mathbf{8 5 7}$ & $\mathbf{1 8 1}$ & $\mathbf{9 5 7}$ & $\mathbf{1 . 9 7 7}$ \\
\hline
\end{tabular}

\title{
5.6.1.3 Zuordnung der Parameter aus der Steuerung des Abbauprozesses
}

Für die Datenanalyse müssen jedem Werkzeugwechsel charakteristische Wert für die Steuerung des Abbauprozesses zugeordnet werden. Für die relevanten Betriebsparameter der TVM (Kap. 5.6.3) wurden dazu über die Strecke der geotechnischen Homogenbereiche arithmetische Mittelwerte gebildet und allen Werkzeugwechseln im jeweiligen Homogenbereich zugeordnet.

Analog zur Zuordnung der geotechnischen Bedingungen ist zu beachten, dass die Betriebsdaten der TVM anhand der Ringnummer b erfasst werden. Die tatsächliche Stationierung der Ortsbrust jedoch ca. um die Länge der Schildmaschine $L_{T V M}[\mathrm{~m}]$ in Vortriebsrichtung versetzt liegt (Kap. 5.4.1). Die Wechsel der Abbauwerkzeuge in den Referenzprojekten wurden ebenfalls anhand der Ringnummern $b_{v}$ erfasst, sodass eine Referenzierung mit den Betriebsdaten der TVM problemlos möglich ist. 
Werden die Ringnummern $b_{0}$ und $b_{v}$ bei Ein- und Ausbau der Abbauwerkzeuge zur Referenzierung mit den geotechnischen Bedingungen in eine metrische Vortriebsstationierung umgerechnet, muss die entsprechend Verschiebung auch für die Betriebsdaten der TVM beachtet werden.

Zusätzlich wurde für die geotechnischen Homogenbereich mithilfe von Chi-Quadrat Tests $(\alpha=0,1)$ überprüft, ob die Betriebsparameter innerhalb der Homogenbereiche normalverteilt sind. Die Testergebnisse zeigen, dass sowohl die vom Maschinenfahrer gesteuerten Einstellwerte, wie z.B. Schneidraddrehzahl $U$ und Stützdruck $P_{S F}$, als auch die resultierenden Größen, wie z.B. Schneidraddrehmoment $\mathrm{M}_{\mathrm{TVM}}$ und Schneidradanpresskraft $\mathrm{F}_{\mathrm{SV}}$, in $90 \%$ aller Fälle normalverteilt sind.

Mit zweiseitigen t-Tests $(\alpha=0,1)$ wurde überprüft, ob sich die Homogenbereiche hinsichtlich der Einstellwerte und Reaktionsgrößen signifikant unterscheiden. Die Tests ergaben, dass in 63\% der Homogenbereiche keine signifikante Unterscheidung der Einstellgrößen feststellbar ist. Im Gegensatz dazu ist in $86 \%$ der Fälle ein signifikanter Unterschied in den Reaktionsgrößen nachweisbar. Folglich lassen sich bei einer Änderung der geotechnischen Rahmenbedingungen nicht unmittelbar signifikante Reaktionen in der Steuerung der TVM durch den Schildfahrer nachweisen. Die Reaktionsgrößen der TVM zeigen jedoch signifikante Änderungen bei geänderten geotechnischen Rahmenbedingungen.

Dieses Ergebnis stimmt mit den Ergebnissen von WENDL (2012: 86 ff.) überein, die für zwei Referenzprojekte ebenfalls signifikante Änderungen in Reaktionsgrößen der TVM-Steuerung in Abhängigkeit der geotechnischen Bedingungen nachweist. Für das Schneidraddrehmoment $M_{T V M}$ und die Schneidradanpresskraft $F_{S R}$ können eindeutige Änderung in Abhängigkeit der geotechnischen Bedingungen im Rahmen der vorliegenden Untersuchungen ebenfalls gezeigt werden (Kap. 5.6.4.2). Im Hinblick auf die Steuerung der TVM durch den Schildfahrer weisen diese Testergebnisse darauf hin, dass zunächst versucht wird, den Vortriebsprozess möglichst konstant und auf hoher Vortriebsgeschwindigkeit zu halten. Auf relativ geringe Änderungen in den geotechnischen Bedingungen erfolgt mit Ausnahme des Stützdrucks deshalb zunächst keine unmittelbare Reaktion durch den Schildfahrer. Die Reaktionsgrößen der TVM ändern sich jedoch stetig in Abhängigkeit der geotechnischen Bedingungen. Eine Änderung der Einstellgrößen durch den Schildfahrer erfolgt erst, wenn die Reaktionsgrößen tendenzielle eine ungünstige Entwicklung nehmen oder hohen Spielraum für Optimierungen lassen.

Eine weitere detaillierte Untersuchung zu den statistischen Eigenschaften der TVM-Betriebsdaten erfolgt im Rahmen dieser Arbeit nicht, da diese nicht Kernthema der Arbeit sind. Wichtig für die weiteren Untersuchungen sind die beiden gewonnenen Kernaussagen. Sowohl die Einstellparameter der TVM-Steuerung, als auch die Reaktionsgrößen sind innerhalb der Homogenbereiche normalverteilt. Damit kann für die weiteren Untersuchungen der arithmetische Mittelwert als repräsentativer Wert für die Steuerparameter der TVM innerhalb der Homogenbereiche verwendet werden. Weiterhin wichtig ist der grundsätzliche Nachweis, dass Änderung der geotechnischen Rahmenbedingungen auch Änderung in den Reaktionsgrößen der TVM-Steuerung bedeuten und damit die Einteilung der Homogenbereiche im Hinblick auf die Untersuchung der Werkzeugstandgrößen in sinnvoller Weise erfolgt ist.

\subsubsection{Elimination von präventiven Werkzeugwechseln und Schäden}

Für die Analyse der Einflussfaktoren sind ausschließlich normale Werkzeugwechsel bei Erreichen der Verschleißgrenze des Abbauwerkzeuges (Kap. 5.2.1.1) maßgeblich. Bei präventiven Werkzeugwechseln ist auf Basis des Zustandes bei Ausbau im Verhältnis zur Verschleißgrenze theoretisch eine Abschätzung des Betrags der Standgrößen bei Erreichen der Verschleißgrenze möglich. Werkzeugwechsel aufgrund von Schäden, Bestückungsänderungen oder Werkzeugverlust müssen aus dem Datenpool herausgefiltert werden. Die dazu notwendige Dokumentation aus den Referenzprojekten zum Wechselgrund (Kap. 5.2.1) liegt jedoch nicht vor. Einerseits lagen in den Referenzprojekten für den Großteil der Abbauwerkzeuge keine objektiv messbaren Verschleißgrenzen vor, anhand derer der Zustand der Werkzeuge zum Zeitpunkt des Ausbaus beurteilt werden könnte (Kap. 5.3). Andererseits wurde der Zustand der Abbauwerkzeuge bei Ausbau häufig nur unzureichend dokumentiert (Kap. 5.2.1.3). Folglich ist auf Basis dieser Angaben keine entsprechende Filterung der Werkzeugwechsel möglich. 
Die Filterung der Daten kann jedoch mithilfe der Verteilungsform des Laufwegs $\mathrm{s}_{\mathrm{c}}$ innerhalb der geotechnischen Homogenbereiche vorgenommen werden. Zunächst wurden dazu die Wertekollektive des Laufwegs $s_{c}$ innerhalb der geotechnischen Homogenbereiche mithilfe von Chi-Quadrat Tests auf Normalverteilung überprüft. Dabei ergibt sich, dass mit wenigen Ausnahmen keine Normalverteilung des Laufwegs $\mathrm{s}_{\mathrm{c}}$ in den geotechnischen Homogenbereichen vorliegt.

Anschließend wurde mithilfe von Histogrammen der Verteilungsform der Standgrößen dargestellt. Die so visualisierten Verteilungen der Wertekollektive des Laufwegs $s_{c}$ weisen in Summe drei typische Verteilungsformen auf. Diese können auf Basis der relativ detaillierten Dokumentation in den Referenzprojekten 5 und 9 (Tab. 10) sowie mithilfe theoretischer Überlegungen zu den zu erwartenden Verteilungsformen der Standgrößen interpretiert werden.

\section{Blockverteilungen}

Auffällig sind zunächst Blockverteilungen, bei denen über den gesamten Wertebereich des Laufwegs $\mathrm{S}_{\mathrm{c}}$ die absolute Häufigkeit jeweils gleich hoch ist (Abb. 73). Dieser Verteilungstyp betrifft innerhalb der Referenzprojekte $5 \%$ aller geotechnischen Homogenbereiche.

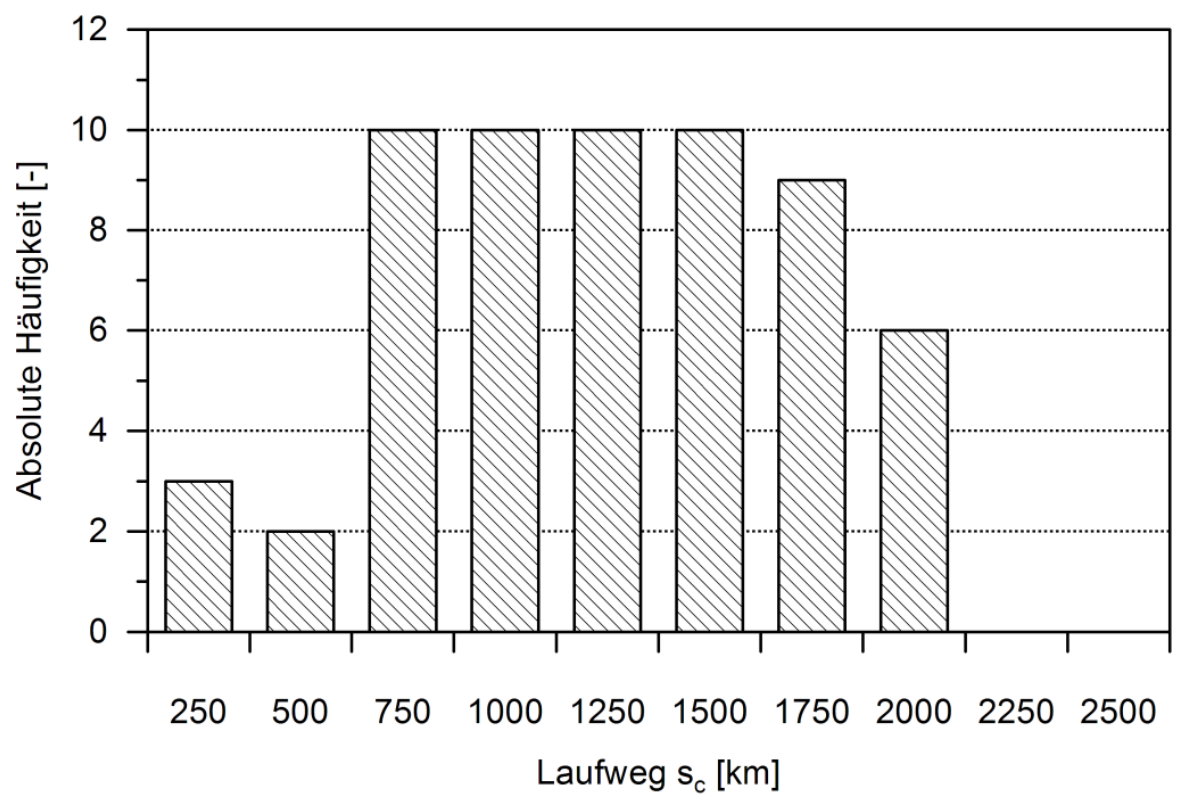

Abb. 73: Beispiel für eine Blockverteilung des Laufwegs $s_{c}$ innerhalb eines geotechnischen Homogenbereiches. Bis auf wenige Abweichungen ergibt sich für jede Kategorie des Laufwegs $\mathrm{s}_{\mathrm{c}}$ die gleiche absolute Häufigkeit.

Die Verteilungsform ergibt sich überwiegend bei sehr kurzen Vortrieben oder bei Vortrieben in denen in regelmäßigen Abständen feste Punkte für Werkzeugwechsel, z.B. Injektionsblöcke vorgesehen waren. Hier wurden jeweils nach Erreichen eines Zwischenschachtes oder Injektionsblocks alle Werkzeuge auf dem Schneidrad unabhängig von ihrem Zustand vollständig gewechselt.

Damit ergibt sich für alle gewechselten Werkzeuge ein übereinstimmender Ein- und Ausbauzeitpunkt $b_{0}$ bzw. $b_{v}$. Entsprechend unterscheiden sich die gewechselten Werkzeuge in der Formel des Laufwegs $s_{c}(G l .35)$ lediglich in den Spurradien $r_{s}$. Für jede Kategorie im Histogramm ergibt sich deshalb die gleiche absolute Häufigkeit. Aus der Blockverteilung lässt sich folglich ohne eine detaillierte Aufnahme des Verschleißzustandes der Werkzeuge kein Modalwert für den Laufweg $\mathrm{s}_{\mathrm{c}, \mathrm{mod}}[\mathrm{km}]$ bei Erreichen der Verschleißgrenze ableiten. Da für die betroffenen Homogenbereiche die Dokumentation der Werkzeugwechsel nicht den notwendigen Detaillierungsgrad aufweist, sind die Werkzeugwechsel daraus nicht für die weitere Analyse nutzbar. 


\title{
Links-steile Verteilungen
}

Der zweite auffällige Verteilungstyp des Laufwegs $s_{c}$ sind links-steile Verteilungen (Abb. 74). Diese ergeben sich innerhalb der Referenzprojekte bei $52 \%$ aller geotechnischen Homogenbereiche. Innerhalb des Wertebereichs von $\mathrm{s}_{\mathrm{c}}$ liegt hier der Modalwert $\mathrm{s}_{\mathrm{c}, \text { mod }}$ in der Darstellung deutlich links bzw. niedriger als der Mittelwert.

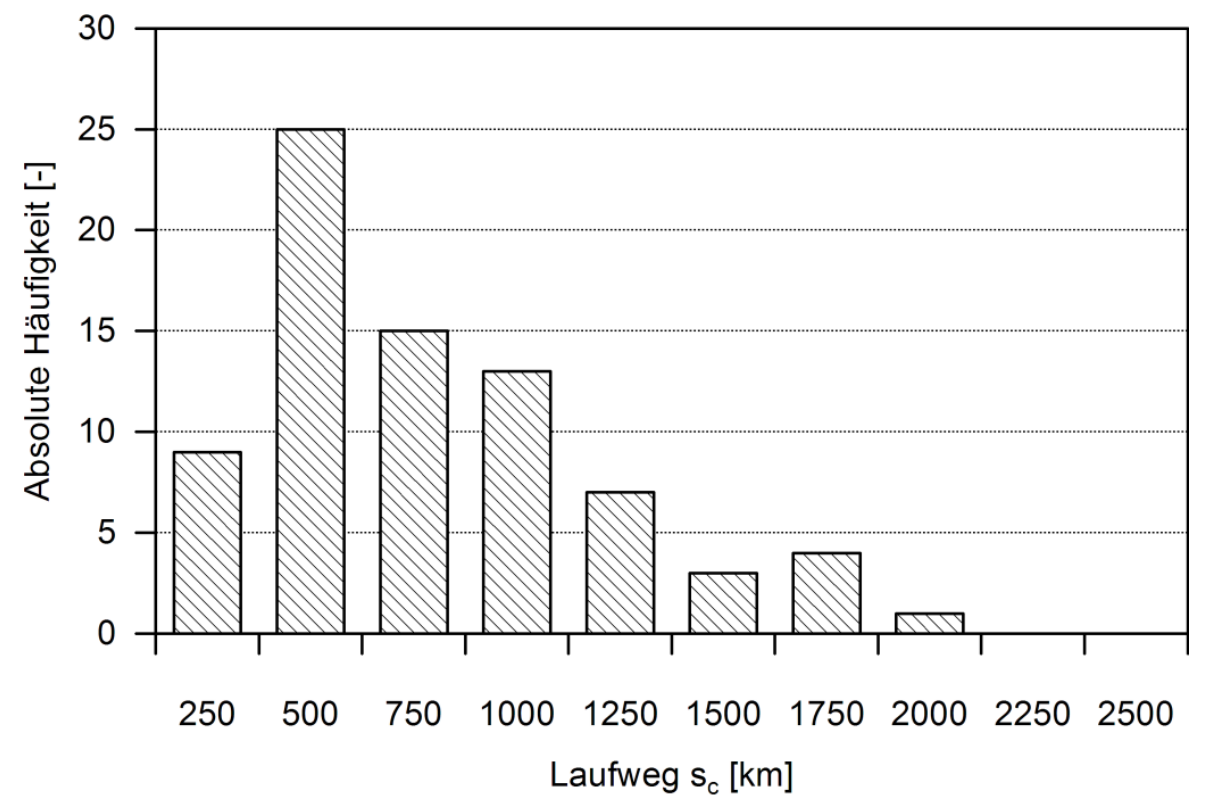

\begin{abstract}
Abb. 74: Beispiel für eine links-steile Verteilung des Laufwegs $s_{c}$ innerhalb eines geotechnischen Homogenbereiches. Der Modelwert des Laufweg $s_{c, \text { mod }}[\mathrm{km}]$ liegt aufgrund des Einflusses von Gewaltschäden durch Steine und Blöcke deutlich links bzw. niedriger als der Mittelwert des Laufweg $\mathrm{s}_{\mathrm{c}}$ und ist nicht charakteristisch für den stetigen Verschleiß der Abbauwerkzeuge.
\end{abstract}

Der Abgleich mit der Dokumentation der Werkzeugwechsel ergibt, dass diese Verteilungsform häufig im Zusammenhang mit einem hohen Anteil an Gewaltschäden aufgrund von Steinen oder Blöcke auftritt. Die Gewaltschäden durch Steine und Blöcke erzwingen in diesen geotechnischen Homogenbereichen vergleichsweise niedrige Intervalle $L_{d(\mathrm{~m})}[\mathrm{m}]$ zwischen den Inspektionen der Abbauwerkzeuge, um die beschädigten Werkzeuge zeitnah zu wechseln. Gleichzeitig wird die Dauer der Einstiege minimiert, um möglichst wenig Stillstandzeit der TVM zu verursachen. Folglich werden überwiegend beschädigte Werkzeuge und solche mit hohem Verschleiß nahe an der Verschleißgrenze gewechselt.

Die Gewaltschäden treten im Wesentlichen wahllos verteilt über alle Spuren auf dem Schneidrad auf, weil die Position in der ein Stein oder Block in der Ortsbrustfläche angetroffen wird, einer Zufallsvariable entspricht, die weitgehend unabhängig von der Position der Werkzeuge auf dem Schneidrad ist.

Die Gewaltschäden traten jeweils deutlich vor Erreichen der Verschleißgrenze der Abbauwerkzeuge auf. Entsprechend nimmt die Wahrscheinlichkeit für Beträge des Laufwegs $\mathrm{s}_{\mathrm{c}} \mathrm{zu}$, die deutlich unterhalb des Laufwegs $s_{c}$ bei Erreichen der Verschleißgrenze liegen. Die Wahrscheinlichkeit, dass Abbauwerkzeuge bis zum Erreichen der Verschleißgrenze auf dem Schneidrad verbleiben, nimmt dagegen im selben Maße ab. Folglich ergeben sich links-steile Verteilungen des Laufwegs $\mathrm{s}_{\mathrm{c}}$.

Der Modalwert des Laufwegs $\mathbf{s}_{c, \text { mod }}$ in Homogenbereichen mit links-steilen Verteilungen wird also im Wesentlichen von der Häufigkeit von Steinen oder Blöcken im Boden bestimmt. Der Modalwert der Verteilung entspricht nicht dem Betrag des Laufwegs $s_{c}$ bei Erreichen der Verschleißgrenze. Eine Ermittlung des charakteristischen Laufwegs $\mathrm{s}_{\mathrm{c}}$ bei Erreichen der Verschleißgrenze ist damit nur mithilfe einer detaillierten Aufnahme des Verschleißzustandes der einzelnen Abbauwerkzeuge möglich. Da in den Referenzprojekten für die betroffenen Homogenbereiche die Dokumentation der Werkzeugwechsel jedoch keinen ausreichenden Detaillierungsgrad aufweist, sind die Werkzeugwechsel aus den links-steilen Verteilungen für die weitere Analyse nicht nutzbar. 


\title{
Rechts-steile Verteilungen
}

Der dritte auffällige Verteilungstyp des Laufwegs $\mathrm{s}_{\mathrm{c}}$ sind rechts-steile Verteilungen (Abb. 75). Dieser Verteilungstyp trifft innerhalb der Referenzprojekte auf $43 \%$ aller geotechnischen Homogenbereiche zu. Innerhalb des Wertebereichs liegt hier der Modalwert im Histogramm deutlich rechts bzw. höher als der Mittelwert.

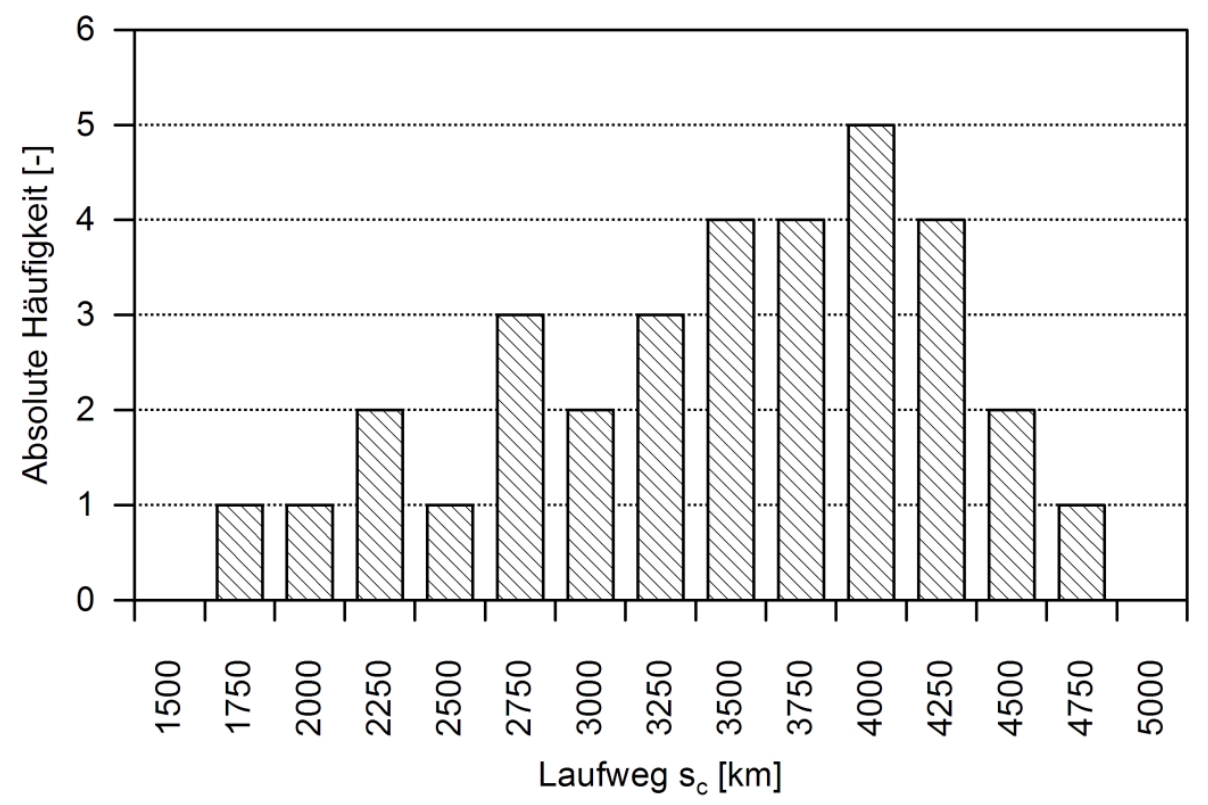

\begin{abstract}
Abb. 75: Beispiel für eine rechts-steile Verteilung des Laufwegs $s_{c}$ innerhalb eines geotechnischen Homogenbereiches. Der Modelwert des Laufweg $\mathrm{s}_{\mathrm{c} \text {,mod }}$ liegt deutlich rechts bzw. höher als der Mittelwert des Laufweg $\mathrm{s}_{\mathrm{c}}$ und ist charakteristisch für den stetigen Verschleiß der Abbauwerkzeuge.
\end{abstract}

Aus der Dokumentation der Werkzeugwechsel ergibt sich, dass diese Verteilungsform häufig in Homogenbereichen vorliegt, in denen ein hoher Anteil der Werkzeuge aufgrund von Primär- und Sekundärverschleiß oder präventiv gewechselt wurde. Gleichzeitig war das Risiko für Gewaltschäden durch Steine und Blöcke gering.

Die rechts-steile Verteilungsform ergibt sich aus dem Bestreben, die Länge der Intervalle $L_{d(m)}[m]$ zwischen den Inspektionen der Abbauwerkzeuge zu maximieren. Die Länge der Intervalle wird dazu im Regelfall so hoch gewählt, dass die Abbauwerkzeuge auf den äußeren Schneidspuren bei der nächsten Kontrolle vollständig verschlissen sind. Um das darauf folgende Intervall wiederum so hoch wie möglich zu setzen, gleichzeitig jedoch das Risiko für Verschleißschäden gering zu halten, werden präventive Werkzeugwechsel auf weiter innen liegenden Spuren notwendig.

Mit dieser Systematik wird der Hauptanteil der Abbauwerkzeuge nahe am Zeitpunkt des Erreichens der Verschleißgrenze gewechselt, bzw. es entsteht eine relativ hohe Wahrscheinlichkeit für Werkzeugwechsel bei Erreichen der Verschleißgrenze. Im Vergleich dazu werden nur sehr wenige Verschleißschäden zugelassen. Diesen wird also eine relativ niedrige Wahrscheinlichkeit zugeordnet. Präventive Werkzeugwechsel werden nur in absolut notwendigem Umfang durchgeführt, sodass innen ebenfalls eine im Vergleich niedrige Wahrscheinlichkeit und absolute Häufigkeit zugeordnet wird. Damit ergeben sich rechts-steile Verteilungen für den Laufweg $\mathrm{s}_{\mathrm{c}}$.

Der Modalwert $\mathrm{s}_{\mathrm{c} \text {,mod }}$ dieser Verteilungen entspricht dem Betrag des Laufwegs $\mathrm{s}_{\mathrm{c}}$ bei Erreichen der Verschleißgrenze der Abbauwerkzeuge, weil diesen Werkzeugwechseln systembedingt die höchste Wahrscheinlichkeit und absolute Häufigkeit zugeordnet wird. Der Modalwert dieser Verteilungen ist damit für die weitere Analyse einsetzbar. 
Zusammenfassend können anhand der Verteilungsform des Laufwegs $\mathrm{s}_{\mathrm{c}}$ diejenigen geotechnischen Homogenbereiche gefiltert werden, in denen eine sichere Bestimmung des Modalwertes für den Laufweg $\mathrm{s}_{\mathrm{c}}$ bei Erreichen der Verschleißgrenze der Abbauwerkzeuge möglich ist. Im Gegenzug bedingt diese Methodik jedoch, dass alle geotechnischen Homogenbereiche, in denen der Laufweg $\mathrm{s}_{\mathrm{c}}$ nicht einer rechts-steilen Verteilung unterliegt, für die weitere Betrachtung entfallen. Eine Übersicht über die verbleibenden Homogenbereiche im Vergleich zu den Rohdaten (Abb. 72) gibt Abb. 76.

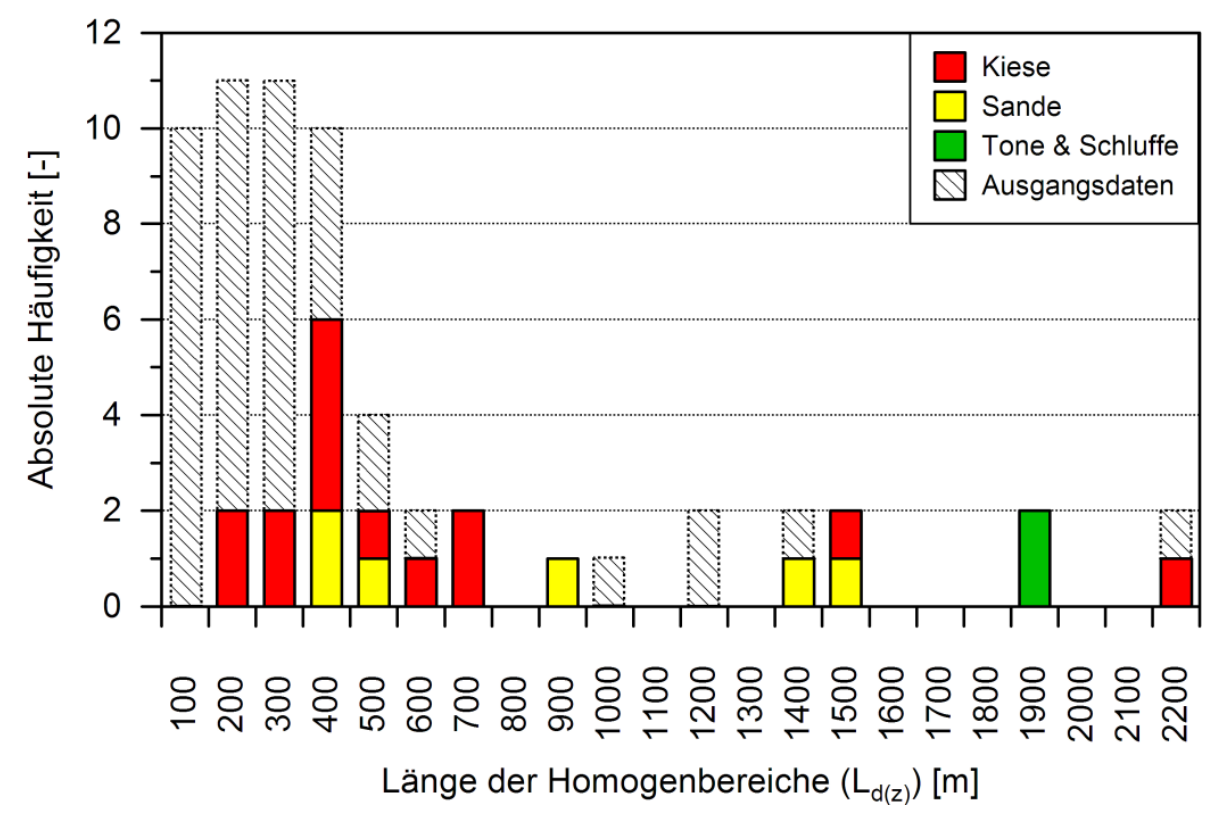

Abb. 76: Verteilung der Länge der geotechnischen Homogenbereiche $L_{d(z)}[\mathrm{m}]$ unterteilt nach Bodengruppen (DIN EN 14688 T1) nach Elimination von präventiven Werkzeugwechseln und Gewaltschäden.

Auch alle darin enthaltenen Werkzeugwechsel entfallen für die weitere Analyse. Abschließend verbleiben im Vergleich zu den Rohdaten (Tab. 14) die in Tab. 16 zusammengefassten Bodenvolumina und Werkzeugwechsel.

Tab. 16: Übersicht über die verbleibende Anzahl an Werkzeugwechseln für die Datenanalyse nach Elimination von präventiven Werkzeugwechseln und Gewaltschäden.

\begin{tabular}{|l|c|c|c|c|c|}
\hline \multicolumn{1}{|c|}{ Bodengruppe } & Volumen & Schneidrollen & Stichel & Schälmesser & Summe \\
\hline \multicolumn{1}{|c|}{ DIN EN 14688-1 } & {$\left[\mathrm{m}^{3}\right]$} & {$[$ Stk.] } & [Stk.] & [Stk.] & [Stk.] \\
\hline Ton \& Schluff & 2.801 .799 & 32 & 8 & 119 & 151 \\
\hline Sand & 617.272 & 125 & 0 & 106 & 231 \\
\hline Kies & 824.986 & 315 & 58 & 325 & 668 \\
\hline Summe: & $\mathbf{4 . 2 4 4 . 0 5 7}$ & $\mathbf{4 7 2}$ & $\mathbf{6 6}$ & $\mathbf{5 5 0}$ & $\mathbf{1 . 0 8 8}$ \\
\hline
\end{tabular}

Für die Analyse der Einflussfaktoren auf die Standgrößen und als geeignete Basisdaten für das nachfolgende Prognosemodell verbleiben also nur $24 \%$ aller Werkzeugwechsel in den Referenzprojekten innerhalb $80 \%$ des untersuchten Ausbruchvolumens. 


\subsubsection{Auslegung der Hydroschild TVM}

Die Auslegung der Abbauwerkzeuge und deren Anordnung auf dem Schneidrad haben Einfluss auf die Standgrößen der Werkzeuge. Nachfolgend sind die wesentlichen Einflussfaktoren dargestellt und soweit wie möglich quantifiziert.

\subsubsection{Höhe der Werkzeuge über dem Schneidradstahlbau}

Die Höhe der Abbauwerkzeuge über dem Schneidradstahlbau hat einen wesentlichen Einfluss auf die Standgrößen der Werkzeuge. Dabei spielt sowohl die absolute Höhe der Werkzeuge über dem Schneidradstahlbau eine Rolle, als auch die daraus resultierenden Höhenunterschiede zwischen den Abbauwerkzeugen.

Der Einfluss der absoluten Höhe der Schneidrollen $h_{S R}$, Schälmesser $h_{S M}$, Stichel $h_{S T}$ und Räumer $h_{R M}$ ist anhand der Projektdaten nicht auf direktem Weg analysierbar. Ursache dafür ist, dass sowohl innerhalb der einzelnen Referenzprojekte, als auch im Vergleich zwischen den Referenzprojekten die absolute Höhe der Abbauwerkzeuge über dem Schneidradstahlbau nicht variiert wird. Innerhalb eines Vortriebes wird die Höhe der Abbauwerkzeuge über dem Schneidradstahlbau nicht verändert, da hierzu umfangreiche Schweißarbeiten am Schneidrad notwendig wären, die in der Vortriebsphase kaum durchführbar sind. Übergreifend über alle Referenzprojekte liegt eine hohe Übereinstimmung in der Auswahl der Höhe der Schneidrollen $h_{S R}$ von $175 \mathrm{~mm}$ und Höhe der Schälmesser $h_{S M}$ von $140 \mathrm{~mm}$ vor. Die beiden Werte sind so aufeinander abgestimmt, dass selbst bei Erreichen der theoretischen Verschleißgrenze der Schneidrollen von $25 \mathrm{~mm}$ radialem Abrieb am Schneidring noch $10 \mathrm{~mm}$ Überstand zu den Schälmessern vorhanden sind. Diese greifen so beim Antreffen von Festgesteinshorizonten oder Blöcken nicht direkt in das Gestein ein und werden nicht beschädigt. Zudem erlauben die beiden Werte die effektive Abfuhr des abgebauten Bodens zwischen Schneidradstahlbau und Ortsbrust zu den Schneidradöffnungen hin.

Deutlich höhere Werte für die absolute Höhe über dem Stahlbau von Sticheln wurden außerhalb der Referenzprojekte zum Teil schon realisiert, um bei sehr grobkörnigen Böden eine bessere Abfuhr der abgebauten Bodenkomponenten zu den Schneidradöffnungen hin zu ermöglichen. Allerdings nehmen mit zunehmender Höhe auch die Probleme zu, die Werkzeuge ausreichend gegen die aus den Schneidkräften resultierenden Momente zu sichern.

Im Gegensatz zur absoluten Höhe der Abbauwerkzeuge über dem Schneidradstahlbau kann der Einfluss der relativen Höhe zwischen den Abbauwerkzeugen durchaus anhand der Projektdaten untersucht werden. Ein erster deutlicher Hinweis auf den Einfluss wird durch das Verschleißbild der Abbauwerkzeuge gegeben (Kap. 5.3). Dieses weist darauf hin, dass die Abriebrate nachlaufender Abbauwerkzeuge umso geringer ausfällt, je größer der Höhenunterschied zu den vorlaufenden Abbauwerkzeugen ist und je flächendeckender die vorlaufenden Abbauwerkzeuge den Boden in der Ortsbrust abbauen.

Dieser Zusammenhang kann in quantitativer Form am Anwendungsfall von Schälmessern in RP 9 in HGB 3 dargestellt werden. Hier wurden die Schälmesser in Kombination mit vorlaufenden Sticheln eingesetzt. HGB 3 ist zusammengesetzt aus $43 \%$ schluffigem Sand und $57 \%$ stark sandigem, schluffigem Kies. Die Stichel deckten dabei mit einer Breite von $100 \mathrm{~mm}$ und einem Spurabstand von $100 \mathrm{~mm}$ die Ortsbrust vollständig ab, sodass die Schälmesser keinen Bodenabbau mehr leisteten. Als Vergleich dazu werden die erreichten Laufwege $s_{c}$ der Schälmesser im gleichen Referenzprojekt in den HGB 2 und 9 herangezogen. Hier wurden die Schälmesser jeweils in Kombination mit vorlaufenden Schneidrollen eingesetzt, sodass die Schälmesser auf einer Breite von ca. $70 \mathrm{~mm}$ zwischen den Spuren der Schneidrollen den primären Bodenabbau leisteten. HGB 2 ist zu $100 \%$ aus den schluffigen Sanden aufgebaut, die in HGB 3 43\% der Ortsbrustfläche einnehmen, während HGB 9 zu 100\% aus stark sandigem, schluffigem Kies aufgebaut ist, der in HGB 3 57\% der Ortsbrustfläche einnimmt. 
In Abb. 77 ist der erreichte Laufweg $s_{c}$ der Schälmesser in den drei Homogenbereichen dargestellt. Beim Abbau der schluffigen Sande in HGB 2 erreichen die Schälmesser einen Laufweg $\mathrm{s}_{\mathrm{c}}$ von $836 \mathrm{~km}$. In den stark sandigen, schluffigen Kiesen in HGB 9 wurde noch ein Laufweg $\mathrm{s}_{\mathrm{c}}$ von $430 \mathrm{~km}$ erreicht. Bei entsprechendem Bodenabbau durch die Schälmesser in HGB 3 kann auf Basis des Anteils der beiden Bodenarten in der Ortsbrust und der erreichten Laufwege in HGB 2 und 9 theoretisch ein Laufweg $\mathrm{s}_{\mathrm{c}}$ in der Größenordnung von $605 \mathrm{~km}$ abgeschätzt werden. Tatsächlich liegt der erreichte Laufweg $\mathrm{s}_{\mathrm{c}}$ der Schälmesser in HGB 3 mit $2.048 \mathrm{~km}$ deutlich höher, weil die Schälmesser aufgrund der vorlaufenden Stichel nicht am Bodenabbau teilgenommen haben.

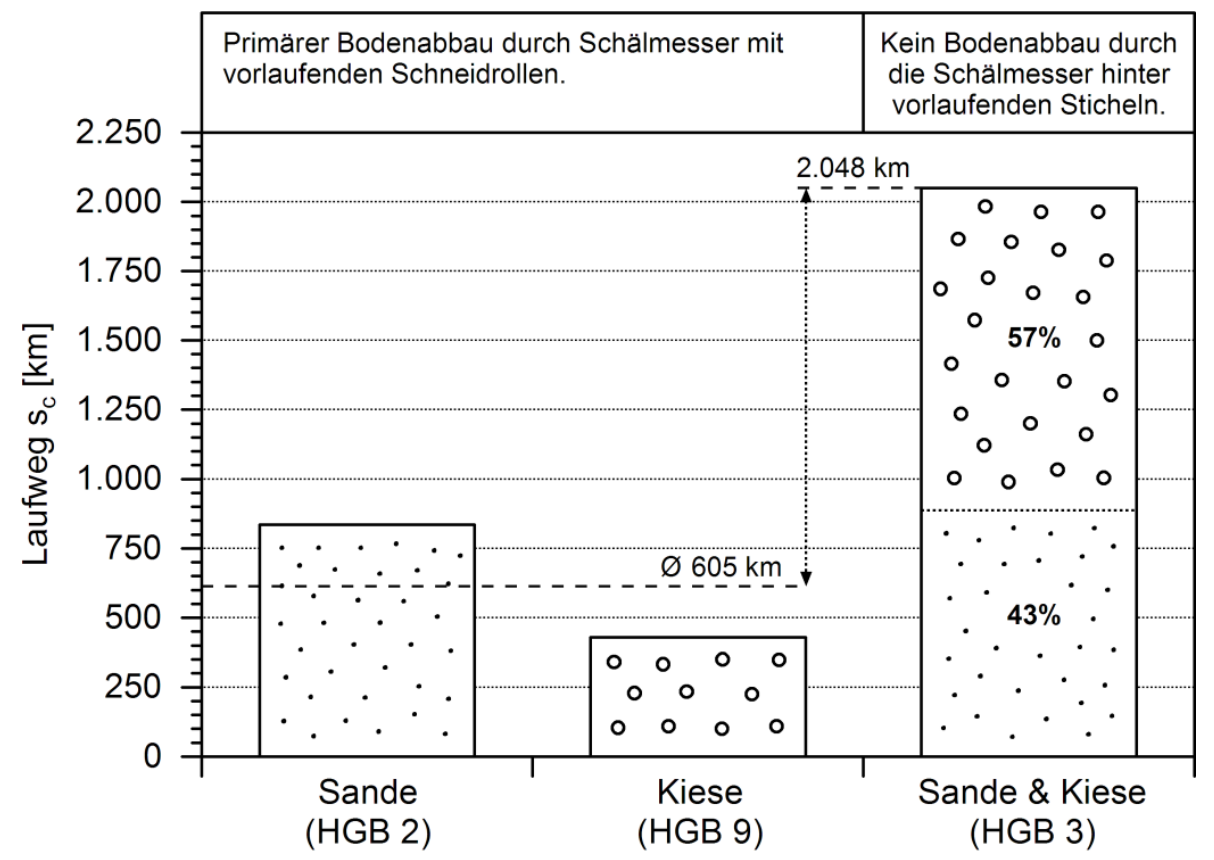

\begin{abstract}
Abb. 77: Vergleich des erreichten Laufwegs $s_{c}$ von Schälmessern bei primärem Bodenabbau zwischen vorlaufend angeordneten Schneidrollen in Sanden (HGB 2) und Kiesen (HGB 9) und ohne Kontakt zur Ortsbrust bzw. Bodenabbau hinter vorlaufend angeordneten Sticheln in identischen Sanden und Kiesen (HGB 3).
\end{abstract}

Anhand dieses Beispiels kann eine grobe Größenordnung für den Einfluss der relativen Höhe zwischen den Werkzeugen abgeschätzt werden. Der Faktor zwischen theoretisch erreichbarem Laufweg $\mathrm{s}_{\mathrm{c}}$ bei vollem Bodenabbau $(600 \mathrm{~km})$ und erreichtem Laufweg $\mathrm{s}_{\mathrm{c}}$, ohne tatsächlichen Kontakt der Schälmesser zur Ortsbrust (2.048 km), beträgt im Vorliegenden Fall 3,4.

Allgemeiner betrachtet gibt das Verhältnis der beiden Zahlen auch einen Hinweis auf das Verhältnis der Verschleißraten $c_{v}$ die einerseits durch den primären Bodenabbau in der Ortsbrust verursacht werden (Primärverschleiß) und andererseits durch den Kontakt mit bereits abgebautem Boden in der Abbaukammer (Sekundärverschleiß). Die Verschleißrate $c_{v}$ aufgrund von Primärverschleiß fällt in diesem Beispiel um ca. Faktor 2,5 höher aus, als die Verschleißrate aufgrund von Sekundärverschleiß.

\title{
5.6.2.2 Spurbesatz $\mathrm{k}_{\mathrm{s}}$
}

Die mehrfache Belegung von Schneidspuren ist innerhalb der Referenzprojekte nur bei Schälmessern üblich. Der Spurbesatz $k_{s}$ hat dort aufgrund seiner Auswirkung auf die Penetration $p_{a}[\mathrm{~mm} / \mathrm{U}]$ der einzelnen Abbauwerkzeuge auf einer Schneidspur einen hohen Einfluss auf die Standgrößen. Die Penetration teilt sich, wie bei der Bestimmung des Lösevolumens $\mathrm{v}_{\mathrm{c}}$ in Kap. 5.4.5 in Gl. 40 und Gl. 41 gezeigt, auf die einzelnen Schälmesser auf einer Schneidspur auf.

Der Einfluss einer Variation des Spurbesatzes $k_{s}$ und der Penetration $p_{a}$ kann mithilfe eines Vergleichs zwischen Schneidspuren mit unterschiedlichem Spurbesatz $k_{s}$ auf dem Schneidrad dargestellt wer- 
den. Hierzu stehen in Summe drei HGB in zwei RP zur Verfügung. Um den Vergleich herzustellen wurden die Werkzeugwechsel innerhalb der Homogenbereiche nach dem zugehörigen Spurbesatz $\mathrm{k}_{\mathrm{s}}$ getrennt. Verglichen wurden jeweils die Modalwerte der daraus entstehenden Verteilungen für den Laufweg $\mathrm{s}_{\mathrm{c}}$ der Schälmesser bei unterschiedlichem Spurbesatz $\mathrm{k}_{\mathrm{s}}$. Zusätzlich ist das Verhältnis zwischen der Penetration $p$ und der auf jedes einzelne Schälmesser entfallenden Aktiv-Penetration $p_{a}$ eingetragen. Die Ergebnisse des Vergleichs sind in Tab. 17 dargestellt.

Tab. 17: Vergleich des Laufwegs $\mathrm{s}_{\mathrm{c}}$ und der prozentualen tatsächlichen Penetration der Schälmesser bei unterschiedlichem Spurbesatz $\mathrm{k}_{\mathrm{s}}$.

\begin{tabular}{|l|c|c|c|c|c|c|}
\hline \multirow{2}{*}{$\begin{array}{l}\text { Referenzprojekt \& } \\
\text { Homogenbereich }\end{array}$} & \multicolumn{2}{|c|}{ Spurbesatz $\mathbf{k}_{\mathbf{s}}$} \\
\cline { 2 - 7 } & $\mathbf{s}_{\mathrm{c}}[\mathrm{km}]$ & $\mathbf{p}_{\mathrm{a}} / \mathbf{p}[\%]$ & $\mathbf{s}_{\mathrm{c}}[\mathbf{k m}]$ & $\mathbf{p}_{\mathrm{a}} / \mathbf{p}[\%]$ & $\mathbf{s}_{\mathrm{c}}[\mathbf{k m}]$ & $\mathbf{p}_{\mathrm{a}} / \mathbf{p}[\%]$ \\
\hline RP 10, HGB 11 & - & - & $250 \mathrm{~km}$ & $49 \%$ & $450 \mathrm{~km}$ & $24 \%$ \\
\hline RP 12, HGB 1 & $125 \mathrm{~km}$ & $97 \%$ & $175 \mathrm{~km}$ & $47 \%$ & - & - \\
\hline RP 12, HGB 2 & $125 \mathrm{~km}$ & $97 \%$ & $175 \mathrm{~km}$ & $47 \%$ & - & - \\
\hline
\end{tabular}

Die Ergebnisse in Tab. 17 zeigen, dass mit steigendem Spurbesatz $k_{s}$ der Laufweg $s_{c}$ ansteigt, während der Anteil der tatsächlichen aktiven Penetration $\mathrm{p}_{\mathrm{a}}$ der Schälmesser an der Penetration $\mathrm{p}$ entsprechend Gl. 40 sinkt. Der absolute Anstieg des Laufwegs $\mathrm{s}_{\mathrm{c}}$ fällt dabei geringer aus als der Anstieg des Spurbesatzes $k_{s}$ bzw. die Abminderung der aktiven Penetration $p_{a}$. Bei einer Verdopplung des Spurbesatzes $k_{s}$ bzw. Halbierung der aktiven Penetration $p_{a}$ nimmt der Laufweg $s_{c}$ jeweils nur um Faktor 1,4 bis $1,8 \mathrm{zu}$. Die Ursache dafür ist vermutlich, dass der Anteil, den der Sekundärverschleiß durch Kontakt mit bereits abgebautem Boden am Gesamtverschleiß der Schälmesser einnimmt, unabhängig vom Spurbesatz $k_{s}$ für alle Schälmesser annähernd gleich hoch ist.

Für Schneidrollen und Stichel kann kein vergleichbarer Zusammenhang hergestellt werden, da die Schneidspuren der Schneidrollen innerhalb der Referenzprojekte nicht mehrfach besetzt sind. Für Räumer ist ein ähnlicher Zusammenhang wie für Schälmesser anzunehmen, hierzu fehlen jedoch die notwendigen Werkzeugwechseldaten für die Analyse.

\subsubsection{Winkelabstand der Abbauwerkzeuge}

Der Winkelabstand zwischen einzelnen Abbauwerkzeugen hat vermutlich Einfluss auf den Laufweg $\mathrm{s}_{\mathrm{c}}$ der Schälmesser und Räumer, da hier die Schneidspuren häufig mehrfach besetzt sind. Allerdings ist der Einfluss mithilfe der vorliegenden Daten nur in Teilen quantifizierbar. Von Bedeutung sind vor allem drei Aspekte:

- Einfluss des Winkelabstandes zwischen den einzelnen Werkzeugen gleicher Orientierung auf die Aufteilung der Penetration (Aktiver Einsatz).

- Einfluss des Winkelabstandes zwischen den einzelnen Werkzeugen entgegengesetzter Orientierung auf den Sekundärverschleiß (Passiver Einsatz).

- Einfluss des Winkelabstandes zwischen den einzelnen Werkzeugen entgegengesetzter Orientierung auf den Materialfluss.

Der Einfluss des Winkelabstandes auf die Aktiv-Penetration einzelner Schälmesser bei mehrfach besetzten Spuren (Kap. 5.6.2.2) ist deutlich darzustellen. Der Winkelabstand der Schälmesser resultiert hier mehr oder weniger aus dem Spurbesatz $k_{s}$, da aus konstruktiver Sicht im Sinne einer gleichmäßigen Verteilung der Penetration $p$ und gleichmäßiger Belastung des Schneidrades möglichst gleiche Winkelabstände zwischen den einzelnen Schälmessern gewählt werden. Die Auswirkungen einer ungleichmäßigen Verteilung der Werkzeuge können anhand der Ergebnisse in Kap. 5.6.2.2 abgeschätzt werden. 
In ähnlicher Weise kann anhand der genannten Ergebnisse zum Einfluss des Spurbesatzes $k_{s}$ und der Penetration $p$ vermutet werden, dass sich der Winkelabstand zwischen zwei Schälmessern oder Räumern entgegengesetzter Orientierung auf den Sekundärverschleiß beim passiven Einsatz auswirkt. Je geringer der Winkelabstand gewählt wird, umso geringer ist die Passiv-Penetration $p_{p}[\mathrm{~mm} / \mathrm{U}]$ des Schälmessers oder Räumers. Folglich reduziert sich theoretisch sowohl der Abrieb aus dem passiven Einsatz, als auch der Materialfluss, der aus dem Freiwinkel des Werkzeuges hin zu den Schneidradöffnungen erfolgen muss. Eine Quantifizierung dieses Zusammenhangs ist jedoch auf Basis der vorliegenden Daten aus den Referenzprojekten nicht möglich.

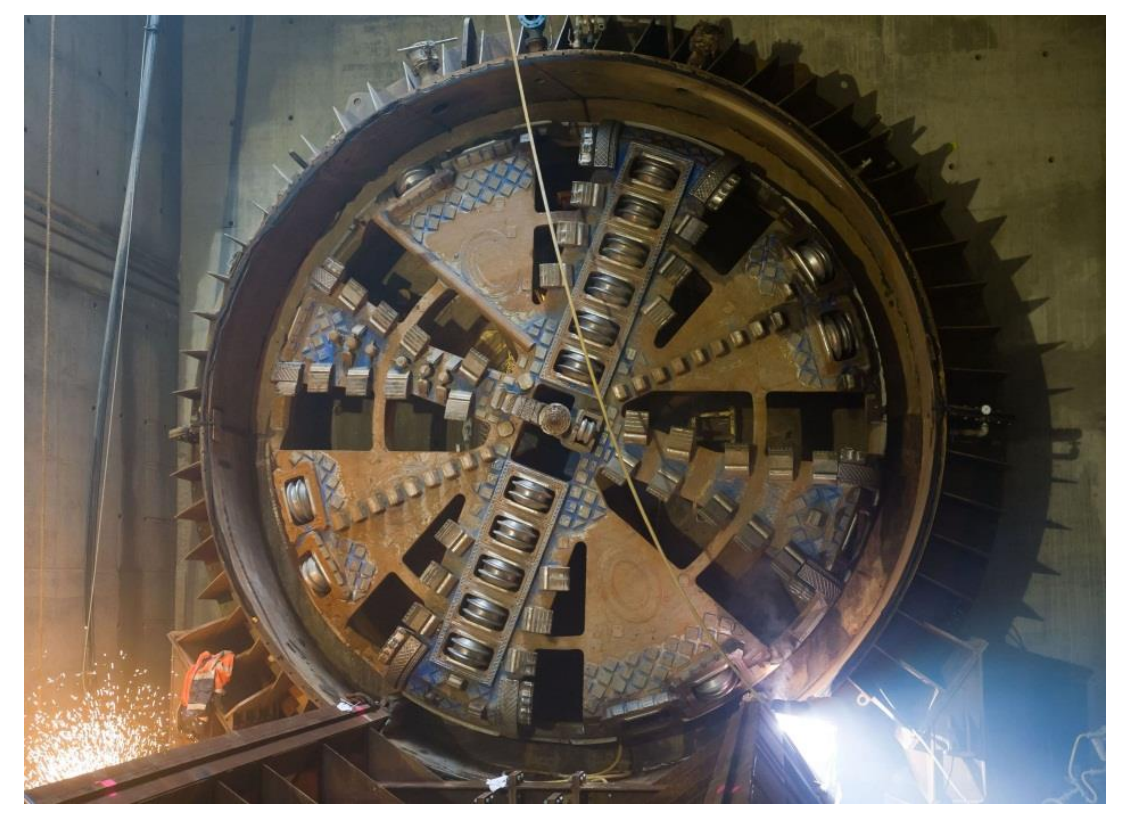

\begin{abstract}
Abb. 78: Frontansicht des Schneidrades einer Hydroschild TVM. Deutlich zu erkennen sind die vier Schneidradarme, auf denen Schälmesser angebracht sind. Diese schließen einen radialen Kanal vom Zentrum zum Kaliberbereich ein. In den beiden annähernd senkrecht stehenden Kanälen sind zwischen den Schälmessern vorlaufende Schneidrollen angeordnet. (Herrenknecht AG)
\end{abstract}

Abschließend hat der Winkelabstand zwischen den Werkzeugen vermutlich auch Einfluss auf den Materialfluss von der Ortsbrust zu den Schneidradöffnungen und damit auf den Sekundärverschleiß der Werkzeuge. Aus dem Verschleißbild in den RP 8, 9, und 10 haben sich hierzu Hinweise ergeben, dass der Sekundärverschleiß an Schälmessern beim passiven Einsatz unter anderem davon abhängt, ob zwischen aktivem und passivem Schälmesser ein vorlaufendes Werkzeug angeordnet ist oder nicht. Die Schälmesser schließen bei der üblichen Anordnung auf den Schneidradarmen eine Art Kanal zwischen Ortsbrust und Schneidradfrontblech ein (Abb. 78).

Werden in diesem Kanal zu den Schälmessern vorlaufende Werkzeuge angeordnet, so muss das von diesen abgebaute Material an den dahinter laufenden passiv eingesetzten Schälmessern vorbei zur nächstgelegenen Schneidradöffnung strömen. Dadurch ergibt sich ein höherer Sekundärverschleiß im Vergleich zu Schälmessern, die auf Schneidradarmen ohne vorlaufende Werkzeuge zwischen den Schälmessern angeordnet sind. Anhand der vorliegenden Daten aus den genannten Referenzprojekten reicht der Detaillierungsgrad der Verschleißdokumentation jedoch nicht aus, um den beschriebenen Effekt des Winkelabstandes auf den Sekundärverschließ quantitativ nachzuweisen. 


\subsubsection{Werkzeuggeometrie}

Die Geometrie der Abbauwerkzeuge wirkt sich einerseits auf die Form des Eingriffs in die Ortsbrust und die resultierenden Schneidkräfte (Kap. 4.2.1) aus. Andererseits bestimmt die Form der Abbauwerkzeuge sowohl den Materialfluss um das Abbauwerkzeug herum, als auch das zur Verfügung stehende Verschleißvolumen. Die Geometrie der Abbauwerkzeuge wirkt sich damit deutlich auf den Laufweg $\mathrm{s}_{\mathrm{c}}$ aus.

Die wesentlichen geometrischen Eigenschaften von Schälmessern sind in diesem Zusammenhang die Breite der Schneide $b_{S M}[\mathrm{~mm}]$, der Schneidwinkel $\beta_{\mathrm{c}}\left[^{\circ}\right]$ und der Freiwinkel $\alpha_{\mathrm{c}}\left[^{\circ}\right]$. Alle drei Einflussfaktoren sind anhand der Daten aus den Referenzprojekten nicht quantifizierbar. Ursache dafür ist, dass die drei genannten Eigenschaften während dem Vortrieb in den Referenzprojekten nicht mehr verändert wurden. Folglich können keine vergleichenden Analysen zwischen unterschiedlichen Auslegungen vorgenommen werden, sodass nur auf die in Kap. 4.2.1 dargestellten Zusammenhänge verwiesen werden kann.

Beim Bodenabbau mit Schneidrollen greift planmäßig nur der Schneidring in den Boden ein. Die wesentlichen geometrischen Eigenschaften, die sich auf den Laufweg $\mathrm{s}_{\mathrm{c}}$ auswirken, sind also der Umfang des Schneidrings in $\mathrm{mm}$, die Form der Schneide und die Breite des Schneidrings $\mathrm{b}_{\mathrm{SR}}[\mathrm{mm}]$.

Die Form der Schneide wurde innerhalb der Referenzprojekte nicht variiert. Weiterhin wurden innerhalb der Referenzprojekte ausschließlich Schneidringe mit einem Außendurchmesser von 17" $(431,8 \mathrm{~mm})$ eingesetzt. Folglich ist keine vergleichende Analyse zum Einfluss des Schneidringdurchmessers möglich. Auf Basis der Untersuchungen zur Verschleißrate $c_{v}$ an Schneidringen in Kap. 5.5.1 kann jedoch indirekt der qualitative Zusammenhang hergestellt werden, dass ein höherer Außendurchmesser und die damit verbundene Erhöhung des verfügbaren Verschleißvolumens auch zu einer Erhöhung des Laufwegs $\mathrm{s}_{\mathrm{c}}$ führt. Der Abrieb (Massenverlust) an Schneidringen ist direkt proportional zum Laufweg $\mathrm{s}_{\mathrm{c}}$. Deshalb ist der Laufweg $\mathrm{s}_{\mathrm{c}}$ als Standgrößen theoretisch auch direkt proportional zum verfügbaren Verschleißvolumen des Schneidrings.

Das verfügbare Verschleißvolumen von Schneidringen wird auch durch die Breite der Schneide $b_{S R}$ [mm] bestimmt. Bei gleichem Außendurchmesser nimmt das verfügbare Verschleißvolumen proportional mit der Schneidenbreite $b_{S R} z u$. Die entsprechende Zunahme des Laufwegs $s_{c}$ kann anhand der Schneidrollen in RP 11 in HGB 1 dargestellt werden.

Hier wurden 1-Ring Schneidrollen eingesetzt, die wahlweise mit Schneidringen mit $19 \mathrm{~mm}$ oder $24 \mathrm{~mm}$ Schneidenbreite bestückt waren. Zusätzlich wurden Monoblockschneidrollen mit einer Schneidenbreite von $22 \mathrm{~mm}$ eingesetzt. Die erreichten Laufwege der Schneidrollen sind in Abb. 79 dargestellt. Mit Schneidringen mit $24 \mathrm{~mm}$ Schneidenbreite wurde ein deutlich höherer Laufweg $\mathrm{s}_{\mathrm{c}}$ von $468 \mathrm{~km}$ erzielt, als mit Schneidringen mit einer Schneidenbreite von $19 \mathrm{~mm}$ und einem Laufweg $\mathrm{s}_{\mathrm{c}}$ von $352 \mathrm{~km}$. Der Unterschied im Laufweg $\mathrm{s}_{\mathrm{c}}$ von $19 \mathrm{~mm}$ zu $24 \mathrm{~mm}$ Schneidringbreite ist mit $32 \%$ dabei annähernd proportional zum Unterschied in der Schneidenbreite bzw. dem verfügbaren Verschleißvolumen von $26 \%$.

Diesem Zusammenhang widerspricht augenscheinlich dem mit den Monoblockschneidrollen mit einer Schneidenbreite von $22 \mathrm{~mm}$ erzielte Laufweg $\mathrm{s}_{\mathrm{c}}$ von $623 \mathrm{~km}$. Dieser liegt deutlich höher als der Laufweg $\mathrm{s}_{\mathrm{c}}$ der 1-Ring Schneidrollen mit $24 \mathrm{~mm}$ Schneidenbreite. Die Ursache hierfür liegt jedoch nicht in der Schneidenbreite, sondern im Aufbau des Monoblocksystems. Im Gegensatz zu 1-Ring Schneidrollen mit austauschbarem Schneidring, sind Schneide und Rollengrundkörper hier aus einem Stück gefertigt. Die Schneide kann theoretisch bis zur vollständigen Einebnung ausgenutzt werden, wohingegen bei Schneidrollen mit wechselbarem Schneidring der Sicherungsring eine zusätzliche Verschleißgrenze darstellt. Die Monoblockschneidrollen haben hier im Vortrieb folglich einen wesentlich höheren Ausnutzungsgrad der Schneide zugelassen, deshalb ergibt sich unabhängig von der Schneidenbreite $b_{S R}$ ein höherer Laufweg $s_{c}$. 


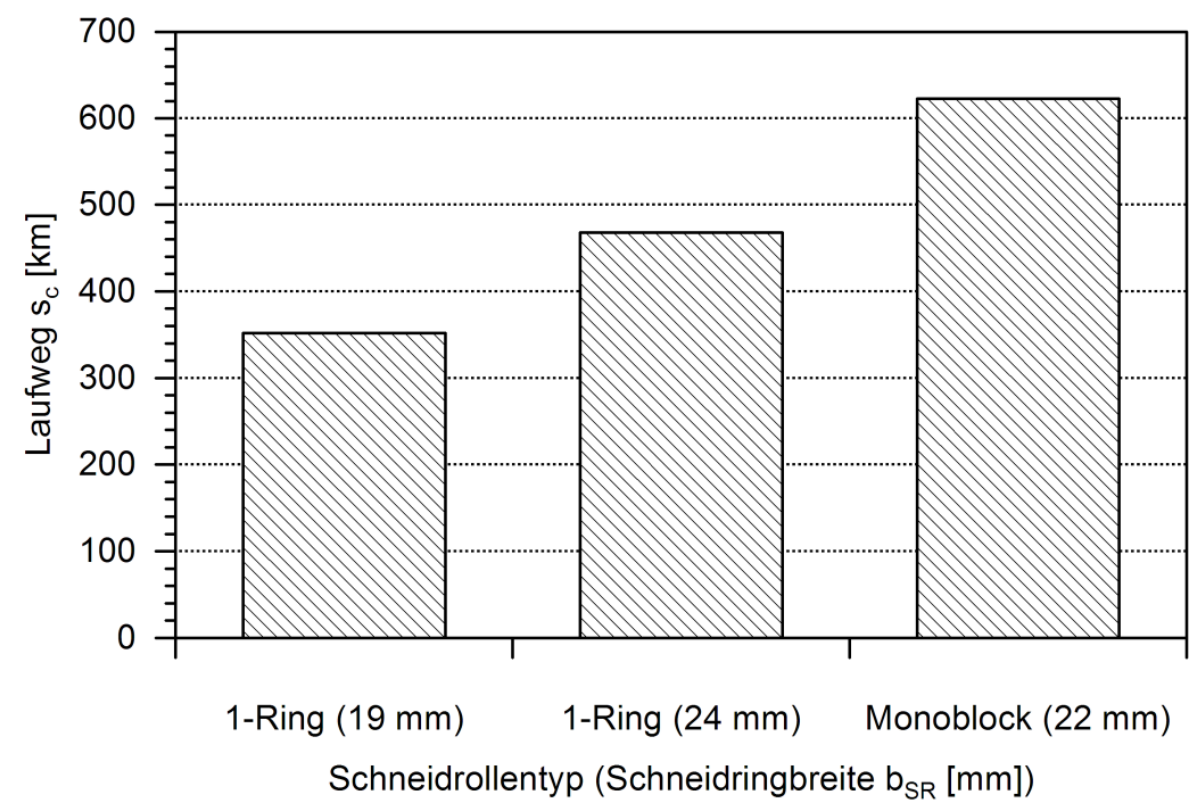

Abb. 79: Vergleich des Laufwegs $s_{c}$ zwischen verschiedenen Schneidrollentypen und verschiedenen Schneidenbreiten $b_{S R}[\mathrm{~mm}]$ in RP 11, HGB 1.

Ergänzend ist darauf hinzuweisen, dass im beschriebenen Referenzprojekt trotz des Vorteils im Laufweg $s_{c}$, Monoblockschneidrollen nicht weiter eingesetzt wurden. Bei der Sanierung von Monoblockschneidrollen muss zwingend die Einheit aus Schneide und Rollengrundkörper ausgetauscht werden. Dazu ist eine vollständige Demontage der Schneidrolle notwendig. Im Gegensatz dazu kann bei den 1-Ring Schneidrollen der Schneidring unabhängig vom Rollengrundkörper ausgetauscht werden. Eine vollständige Demontage der Schneidrolle ist nur notwendig, wenn diese während eines Eingangstests Anzeichen für verschlissene oder defekte Gleitringdichtungen oder Kegelrollenlager zeigt, oder der Rollengrundkörper und die seitlichen Deckel verschlissen sind. Im vorliegenden Referenzprojekt lagen die Kosten für die Sanierung der Monoblockschneidrollen aufgrund des höheren Aufwands zur Demontage und dem höheren Verbrauch an Rollengrundkörpern im Vergleich zu den 1-Ring Schneidrollen so hoch, dass mit dem Einsatz der Monoblockschneidrollen trotz des Vorteils im Laufweg $\mathrm{s}_{\mathrm{c}}$ in Summe kein Kostenvorteil erzielt werden konnte.

\subsubsection{Werkstoffe und Eigenschaften}

Die in den Abbauwerkzeugen eingesetzten Werkstoffe und deren Eigenschaften haben wesentlichen Einfluss auf die Verschleißrate $\mathrm{c}_{\mathrm{v}}$ und den Laufweg $\mathrm{s}_{\mathrm{c}}$. Zu den wichtigsten Werkstoffeigenschaften zählt die Härte (Kap. 4.1.1).

Schneidringe sind aus einem Stück Stahl gefertigt, das durch die nachfolgende mechanische Bearbeitung und Wärmebehandlung über den gesamten Querschnitt homogene Werkstoffeigenschaften aufweist. Die verschleißrelevanten Werkstoffeigenschaften sind also theoretisch selbst bei zunehmendem Abrieb am Schneidring eindeutig bestimmbar. Innerhalb der Referenzprojekte wurde jedoch nur ein Stahltyp für Schneidringe eingesetzt, sodass auf Basis der vorhandenen Daten keine vergleichende Analyse zwischen verschiedenen Stahltypen und Werkstoffeigenschaften durchführbar ist.

Im Gegensatz dazu bestehen Schälmesser aus einem Grundkörper aus Stahl, der in unterschiedlicher Form und Umfang mit Verbundwerkstoffen, Hartmetallen (Wolframcarbide) und schweißbaren Hartaufträgen gegen Verschleiß geschützt wird. Hier sind die verschleißrelevanten Werkstoffeigenschaften für das Abbauwerkzeug nicht mehr eindeutig definierbar, weil einerseits in Abhängigkeit des Verschleißzustandes an verschiedenen Stellen des Schälmessers verschiedenen Werkstoffe maßgeblich sind. Andererseits stellen die Werkstoffe selbst Verbundmaterialien dar. Eine direkte vergleichende Analyse zwischen verschiedenen Werkstoffen, deren Eigenschaften und deren Einfluss auf die Verschleißrate $c_{v}$ und den Laufweg sc ist damit nicht möglich. 
Allerdings lassen sich bei Schälmessern mit unterschiedlicher Ausstattung an Verschleißschutz durchaus Unterschiede in den Standgrößen nachweisen. Der mögliche Einfluss der Werkstoffeigenschaften lässt sich an den Schälmessern in RP 1 in HGB 1 abschätzen. Hier wurden aufgrund anfänglicher Verschleißprobleme beim Vortrieb in Geschiebemergel (toniger, stark schluffiger Sand) vier verschiedene Schälmesservarianten eingesetzt. Die Schälmesser besaßen eine Breite von $250 \mathrm{~mm}$ und waren mit dem in Tab. 18 zusammengefassten Verschleißschutz ausgestattet.

Tab. 18: Verschleißschutz der verschiedenen Schälmesservarianten in Referenzprojekt 1, Homogenbereich 1.

\begin{tabular}{|c|c|c|c|c|}
\hline \multirow{2}{*}{$\begin{array}{l}\text { Verschleiß- } \\
\text { schutz }\end{array}$} & \multicolumn{4}{|c|}{ Schälmesservariante } \\
\hline & 1 & 2 & 3 & 4 \\
\hline Schneide & $\begin{array}{l}\text { Verbundplatte, 1-reihig } \\
\text { (Wolframcarbide in Stahlmatrix } \\
\text { eingestreut, als Verbundplatte } \\
\text { auf den Grundkörper ge- } \\
\text { schweißt) }\end{array}$ & $\begin{array}{l}\text { Wolframcarbid, 1-reihig } \\
\text { (Autogen in eine Stahlleiste } \\
\text { eingelötet. Stahlleiste auf den } \\
\text { Grundkörper geschweißt.) }\end{array}$ & $\begin{array}{l}\text { Wolframcarbid, 2-reihig } \\
\text { (Induktiv in den Grundkörper } \\
\text { eingelötet). }\end{array}$ & $\begin{array}{l}\text { Wolframcarbid, 2-reihig } \\
\text { (Induktiv in den Grundkörper } \\
\text { eingelötet). }\end{array}$ \\
\hline Rücken & $\begin{array}{l}\text { Verbundplatte, 1-reihig } \\
\text { (Wolframcarbide in Stahlmatrix } \\
\text { eingestreut, als Verbundplatte } \\
\text { auf den Grundkörper ge- } \\
\text { schweißt) }\end{array}$ & $\begin{array}{l}\text { Verbundplatte, flächig } \\
\text { (Wolframcarbide in Stahlmatrix } \\
\text { eingestreut, als Verbundplatte } \\
\text { auf den Grundkörper ge- } \\
\text { schweißt) }\end{array}$ & $\begin{array}{l}\text { Wolframcarbid, 3-reihig } \\
\text { (Induktiv in den Grundkörper } \\
\text { eingelötet). }\end{array}$ & $\begin{array}{l}\text { Wolframcarbid, 4-reihig } \\
\text { (Induktiv in den Grundkörper } \\
\text { eingelötet). }\end{array}$ \\
\hline Grundkörper & $\begin{array}{l}\text { Werkzeugstahl } \\
\text { (Unvergütet; HV 162) }\end{array}$ & $\begin{array}{l}\text { Werkzeugstahl } \\
\text { (Unvergütet; HV 162) }\end{array}$ & $\begin{array}{l}\text { Werkzeugstahl } \\
\text { (Vergütet; HV 446) }\end{array}$ & $\begin{array}{l}\text { Werkzeugstahl } \\
\text { (Vergütet; HV 512) }\end{array}$ \\
\hline
\end{tabular}

Die Schälmesservarianten wurden in der angegebenen Reihenfolge auf dem Schneidrad eingesetzt, mit dem Ziel den erreichbaren Laufweg $\mathrm{s}_{\mathrm{c}}$ zu erhöhen. Qualitativ ist dabei von Variante 1 zu Variante 4 eine stetige Steigerung des Verschleißschutzes festzustellen. Die Menge der eingesetzten Verschleißschutzmaterialien wurde erhöht und Materialien (Verbundplatten), die keine zufriedenstellenden Ergebnisse lieferten, durch höherwertige Materialien ersetzt (Wolframcarbid) und zusätzlich die Härte des Grundkörpers erhöht. Die erreichten Laufwege $s_{c}$ der verschiedenen Schälmesservarianten sind in Abb. 80 zusammengefasst.

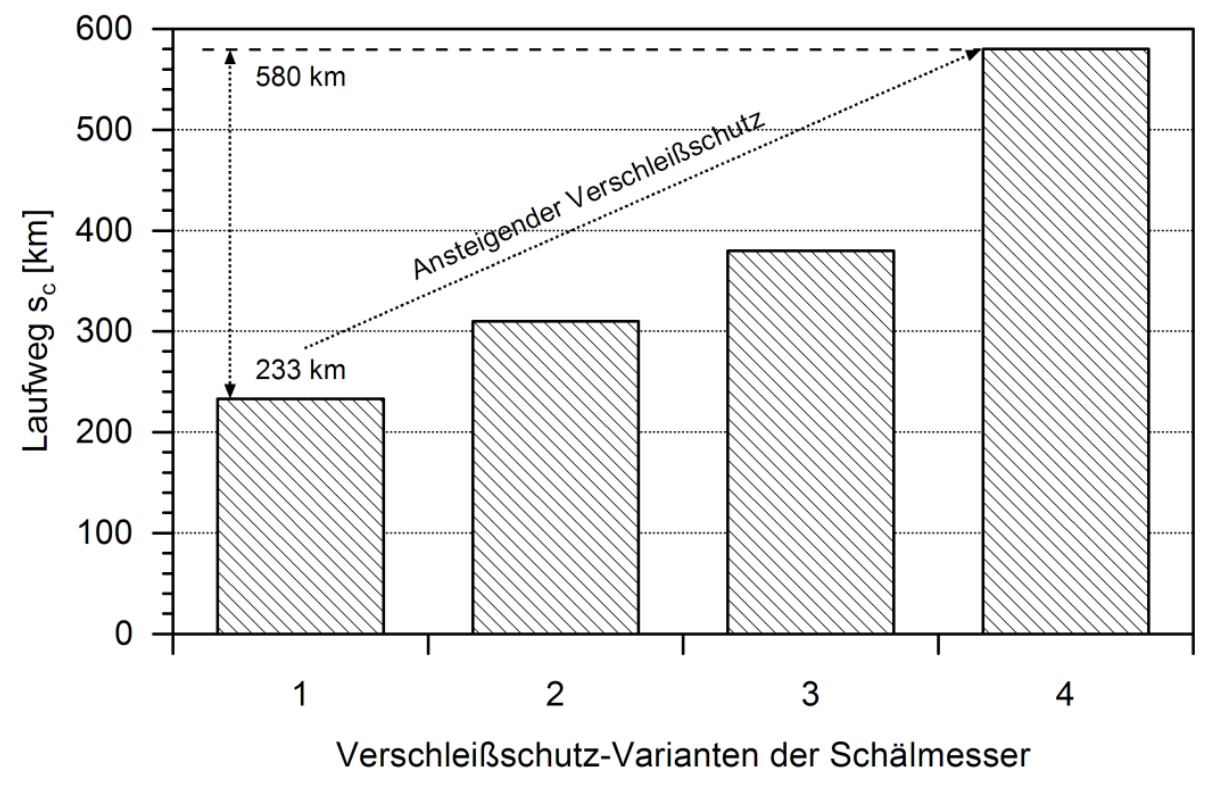

Abb. 80: Vergleich des Laufwegs $s_{c}$ verschiedener Schälmesservarianten mit unterschiedlichem Verschleißschutz. Die Schälmesservarianten sind in Tab. 18 beschrieben. 
Das Balkendiagramm in Abb. 80 zeigt, dass durch die stetige Steigerung des Verschleißschutzes bei ansonsten gleichen Einsatzbedingungen auch eine Steigerung des erreichten Laufweges $\mathrm{s}_{\mathrm{c}}$ von $233 \mathrm{~km}$ (Variante 1) bis $580 \mathrm{~km}$ (Variante 4) bewirkt wurde. Die Rangfolge der erreichten Laufwege $\mathrm{s}_{\mathrm{c}}$ der Varianten 1 bis 4 entspricht der Rangfolge in der Menge und Qualität des Verschleißschutzes.

Dieser Anwendungsfall bestätigt damit den qualitativen Zusammenhang, dass eine Erhöhung des Verschleißschutzes (Menge \& Härte) auch eine Erhöhung des Laufwegs $\mathrm{s}_{\mathrm{c}}$ bewirkt. Quantitativ ist im vorliegenden Fall der Laufweg $\mathrm{s}_{\mathrm{c}}$ des Schälmessers mit dem umfangreichsten Verschleißschutz (Variante 4) um den Faktor 2,5 höher, als der Laufweg $s_{c}$ von Variante 1 mit dem relativ geringsten Verschleißschutz. Diese Spannweite kann in keinem der anderen Referenzprojekte überprüft werden, weil hier keine entsprechend hohe Variation des Verschleißschutzes mehr vorgenommen wird. Die Ausstattung von Schälmessern mit induktiv eingelöteten Hartmetalleinsätzen stellt bereits innerhalb der Referenzprojekte einen Entwicklungsstandard da, der nicht mehr unterschritten wird.

\subsubsection{Materialfluss}

Abschließend sind für die Verschleißrate $c_{v}$ und den Laufweg $s_{c}$ vermutlich auch alle Faktoren der Auslegung der TVM maßgeblich, die den Materialfluss in der Abbaukammer beeinflussen. Ausgangspunkt für die Vermutung ist, dass umso weniger Kontakt zwischen bereits abgebautem Boden und den Abbauwerkzeugen entsteht, je schneller der abgebaute Boden hinter das Schneidrad in die Abbaukammer gelangt und über die Förderleitung in der Sohle abgefördert wird. Die nachfolgenden Faktoren, die den Materialfluss in der Abbaukammer beeinflussen, lassen sich dabei anhand der Referenzprojekte zwar qualitativ erfassen und beschreiben, eine quantitative Abschätzung des Einflusses ist aufgrund mangelnder Vergleichsmöglichkeiten jedoch nicht möglich.

Nach dem Lösen des Bodens durch die Abbauwerkzeuge ist es in erster Linie wichtig, wie schnell die gelösten Bodenteile hinter das Schneidrad gelangen. Entscheidend hierfür ist neben den in Kap. 5.6.2.1 beschriebenen Auswirkungen der Werkzeugbestückung hauptsächlich die Position und Größe der Schneidradöffnungen. Anzunehmen ist, dass umso weniger Kontakt zwischen bereits abgebautem Boden und den Abbauwerkzeugen entsteht, je größer das Schneidradöffnungsverhältnis $\mathrm{O}_{\text {TVM }}$ ist. Folglich wird der Sekundärverscheiß an den Werkzeugen umso geringer, je größer das Schneidradöffnungsverhältnis $\mathrm{O}_{T V M}[\%]$ ist. Dabei ist zu berücksichtigen, dass das Öffnungsverhältnis in der Praxis nicht beliebig groß gewählt werden kann, da einerseits Platz für die Anordnung der Abbauwerkzeuge und andererseits eine ausreichend steife Schneidradstahlstruktur benötigt wird.

Ausschlaggebend ist jedoch nicht allein der absolute Betrag des Schneidradöffnungsverhältnisses, sondern auch die Verteilung der Öffnungen über den Schneidradradius. Hier kann ein direkter Zusammenhang zwischen normalisiertem Materialfluss und dem Verschleiß der Schneidradstahlstruktur hergestellt werden. Dieser Zusammenhang ist vermutlich auch auf den Sekundärverschleiß der Abbauwerkzeuge übertragbar. Dieser steigt in betroffenen Bereichen vermutlich mit steigendem normalisiertem Materialfluss an. Zusätzlich resultiert aus Bereichen mit hohem normalisiertem Materialfluss ein erhöhtes Risiko für Verklebungen, die sich ebenfalls auf den Sekundärverschleiß der Abbauwerkzeuge auswirken oder zum Blockieren von Schneidrollenführen können (Kap. 4.2.2.1). Betroffen sind häufig Bereiche im Zentrum des Schneidrades, da hier ein hoher Anteil der verfügbaren Schneidradfläche für die Abbauwerkzeuge und die von der Rückseite anliegenden Verbindungsstreben der Schneidradbefestigung benötigt wird.

Hinter dem Schneidrad wird der Materialfluss zur Förderleitung primär von den nachfolgenden Faktoren beeinflusst:

- Anzahl und Position der an der Schneidradrückseite angebrachten Rotoren und den an der Druckwand angebrachten Statoren.

- Anzahl, Position und Ausrichtung der Speiseöffnungen für frische Bentonitsuspension in der Druckwand.

- Auslegung des Schildsohlsegmentes. 
Alle genannten Faktoren haben Einfluss darauf, wie schnell die abgebauten Bodenkomponenten zum Einlass der Förderleitung gelangen. Je effektiver hier der Abtransport der abgebauten Bodenkomponenten erfolgt, umso geringer ist die Wahrscheinlichkeit für einen erneuten Kontakt zwischen bereits abgebauten Bodenkomponenten und den Abbauwerkzeugen, sodass sich auch eine positive Auswirkung auf den Sekundärverschleiß ergibt. Über das gesamte Spektrum an Einflussfaktoren auf den Abbauwerkzeugverschleiß betrachtet, ist der Einfluss dieser Faktoren jedoch vermutlich relativ gering. Durch die Rotoren und Statoren, sowie die geeignete Auslegung der Speiseöffnungen für frische Bentonitsuspension wird eine Ansammlung von bereits abgebautem Boden im Sohlbereich des Schildes hinter dem Schneidrad verhindert. Die Auslegung des Sohlsegmentes ist für eine effektive Trennung der Zufuhr von frischer Bentonitsuspension und der Abfuhr der mit abgebautem Boden aufgeladenen Stützflüssigkeit (Kap. 3.2.3) verantwortlich.

\subsubsection{Steuerung des Abbauprozesses}

Die Steuerung des Abbauprozesses hat wesentlichen Einfluss auf die Verschleißrate $c_{v}$ und den Laufweg $\mathrm{s}_{\mathrm{c}}$ der Abbauwerkzeuge. Die vom Schildfahrer bestimmte Einstellwerte (Aktivparameter) (Kap. 3.3.1) sind:

- Schneidraddrehzahl $U\left[\mathrm{~min}^{-1}\right]$

- Vortriebsgeschwindigkeit $\mathrm{V}_{\mathrm{TVM}}[\mathrm{mm} / \mathrm{min}]$

- Druckbegrenzung der Vortriebspressengruppen

- Stützdruck $P_{S F}[b a r]$

- Fördervolumen in $v_{\mathrm{FL}}\left[\mathrm{m}^{3} / \mathrm{h}\right]$

- Speisevolumen in $v_{S L}\left[m^{3} / h\right]$

Aus den Einstellwerten, den geotechnischen Bedingungen in der Ortsbrust und der Auslegung der Hydroschild TVM resultieren die folgenden Passivparameter, die zusätzlich in die Datenanalyse mit einbezogen werden:

- Penetration $\mathrm{p}[\mathrm{mm} / \mathrm{U}]$

- Dichte der Stützflüssigkeit $\rho_{\mathrm{SF}}\left[\mathrm{g} / \mathrm{cm}^{3}\right]$

- Schneidradanpresskraft $F_{S R}[k N]$

- Schneiddrehmoment $\mathrm{M}_{\mathrm{SR}}[\mathrm{kNm}]$

Nachfolgend wird der Einfluss der genannten Größen auf die Verschleißrate $c_{v}$ und den Laufweg $s_{c}$ der Abbauwerkzeuge untersucht und soweit wie möglich anhand der Daten aus den Referenzprojekten quantifiziert.

\subsubsection{Schneidraddrehzahl U}

Der Einfluss der Schneidraddrehzahl $U$ auf die Verschleißrate $c_{v}$ und den Laufweg $s_{c}$ ergibt sich aus der Schneidgeschwindigkeit der Abbauwerkzeuge $v_{s c}[\mathrm{~m} / \mathrm{s}]$. Diese entspricht der Bahngeschwindigkeit der Abbauwerkzeuge auf der Schneidspur und ergibt sich mit Gl. 53 aus dem Spurradius $r_{s}$ und der Schneidraddrehzahl U:

$$
\mathrm{v}_{\mathrm{sc}}=2 \cdot \frac{\mathrm{r}_{\mathrm{s}}}{1000} \cdot \pi \cdot \frac{\mathrm{U}}{60}
$$


Eine direkte Analyse der Auswirkung der Schneidgeschwindigkeit $\mathrm{v}_{\mathrm{sc}}$ auf den Laufweg $\mathrm{s}_{\mathrm{c}}$ ist mit der in Kap. 5.6.1.4 beschriebene Methode zur Filterung der Rohdaten nicht möglich. Die Schneidraddrehzahl $U$ und damit auch die Schneidgeschwindigkeit $v_{s c}$ ist nach den Ergebnissen in Kap. 5.6.1.3 innerhalb der geotechnischen Homogenbereiche normalverteilt. Entsprechend können innerhalb der Referenzprojekte bei gleichbleibenden geotechnischen Bedingungen und TVM-Auslegung keine Bereiche mit signifikant unterschiedlicher Steuerung der Schneidraddrehzahl U ausgegliedert werden. Für eine direkte Analyse des Einflusses der Schneidraddrehzahl U wären folglich Homogenbereiche notwendig, die sich ausschließlich in der Steuerung der Schneidraddrehzahl unterscheiden. Innerhalb der Referenzprojekte liegt jedoch keine entsprechende Möglichkeit für einen Vergleich vor.

Ein alternativer Ansatz besteht darin, die Menge der Werkzeugwechsel, die nach der Filterung der Daten (Kap. 5.6.1.4) innerhalb der Kategorie des Modalwertes liegen, einzeln zu betrachten. Für diese Werkzeugwechsel kann angenommen werden, dass sie bei Erreichen der Verschleißgrenze gewechselt wurden. Der Laufweg $\mathrm{s}_{\mathrm{c}}$ dieser Werkzeuge kann gegen die jeweilige Schneidgeschwindigkeit $\mathrm{v}_{\mathrm{sc}}$ angetragen werden, um so eine mögliche Abhängigkeit darzustellen. Für Schneidrollen sind entsprechende Daten aus RP 7 in HGB 9 in Abb. 81 dargestellt. Die Daten für Schälmesser sind in Abb. 82 ebenfalls aus RP 7 aus den HGB 1 und 11 enthalten.

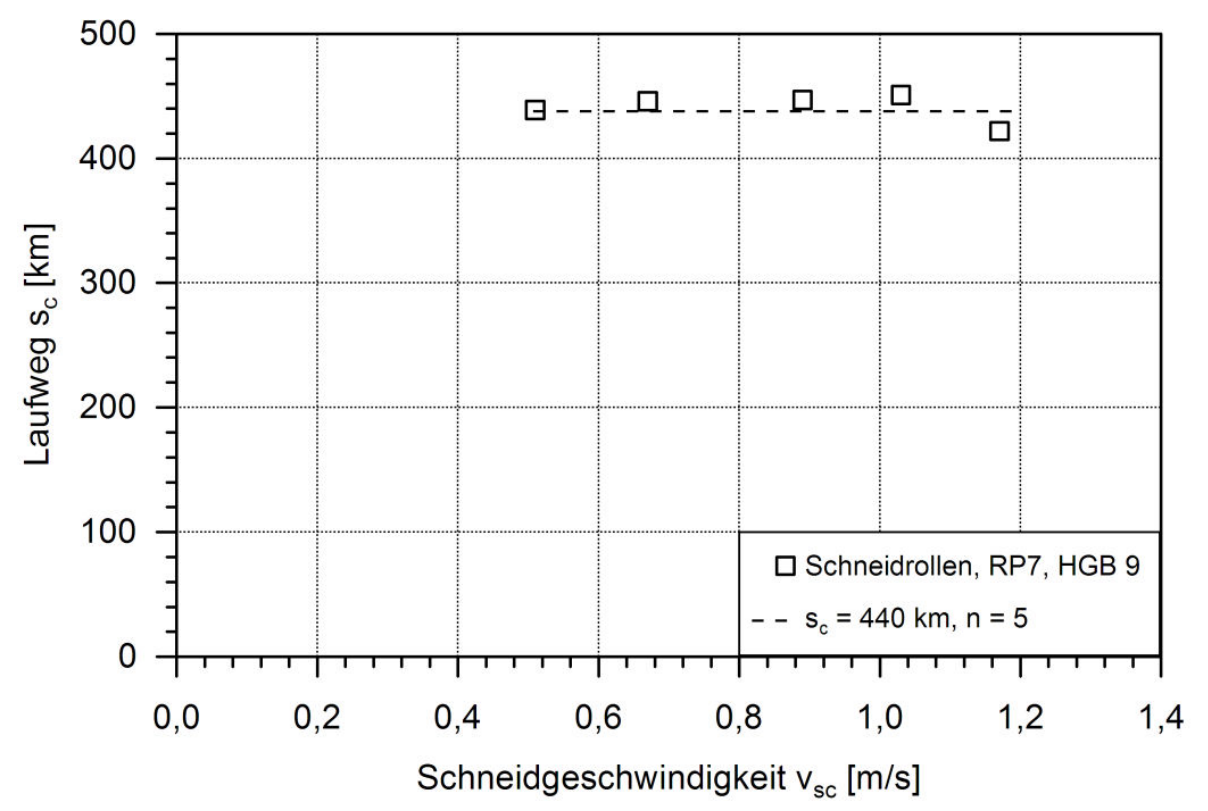

Abb. 81: Im Diagramm ist die Schneidgeschwindigkeit $\mathrm{v}_{\mathrm{sc}}[\mathrm{m} / \mathrm{s}]$ der gewechselten Schneidrollen in RP 7 in HGB 9 gegen den Laufweg $\mathrm{s}_{c}[\mathrm{~km}]$ angetragen. Der Laufweg $\mathrm{s}_{\mathrm{c}}$ liegt unabhängig von der Schneidgeschwindigkeit $v_{s c}$ gleich hoch. Die Schneidgeschwindigkeit $v_{s c}$ hat folglich keinen maßgeblichen Einfluss auf den Laufweg $\mathrm{s}_{\mathrm{c}}$.

Die beiden Diagramme zeigen sowohl für Schneidrollen, als auch für Schälmesser, keinen Trend zu einer Abhängigkeit des Laufwegs $s_{c}$ von der Schneidgeschwindigkeit $v_{s c}$. In beiden Fällen ergeben sich trotz einer breiten Streuung der Schneidgeschwindigkeit $v_{s c}$ keine Änderungen des Laufwegs $\mathrm{s}_{\mathrm{c}}$.

Der fehlende Einfluss der Schneidgeschwindigkeit $v_{s c}$ auf die Verschleißrate $c_{v}$ und den Laufweg $s_{c}$ wird auch durch die Ergebnisse im bestehenden Wissensstand und die Analyse zum Verschleißbild und zur Abbaumechanik untermauert. 


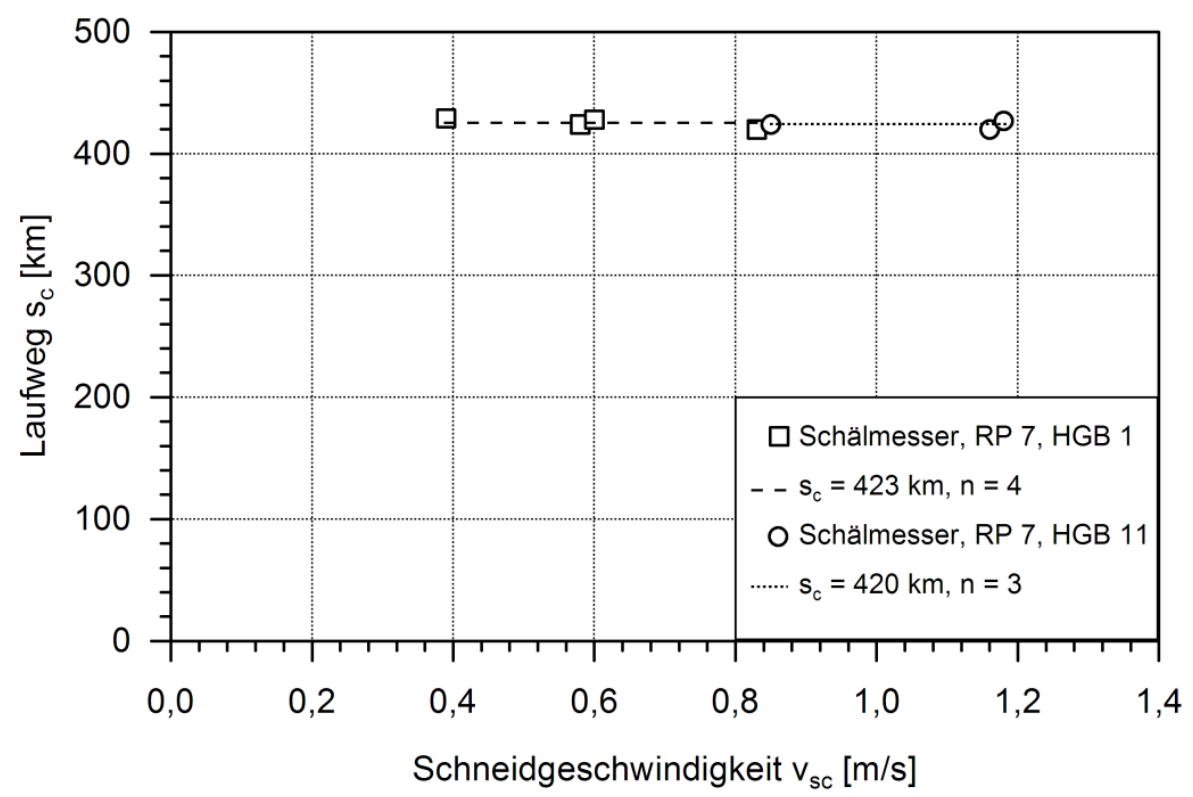

\begin{abstract}
Abb. 82: Im Diagramm ist die Schneidgeschwindigkeit $v_{s c}[\mathrm{~m} / \mathrm{s}]$ der gewechselten Schälmesser in RP 7 in HGB 1 und HGB 11 gegen den Laufweg $\mathrm{s}_{\mathrm{c}}[\mathrm{km}]$ angetragen. Der Laufweg $\mathrm{s}_{\mathrm{c}}$ liegt unabhängig von der Schneidgeschwindigkeit $v_{s c}$ gleich hoch. Die Schneidgeschwindigkeit $v_{s c}$ hat folglich keinen maßgeblichen Einfluss auf den Laufweg $\mathrm{s}_{\mathrm{c}}$.
\end{abstract}

Für Schälmesser nehmen die Schneidkräfte mit zunehmender Schneidgeschwindigkeit $\mathrm{v}_{\mathrm{sc}}$ zu (Kap. 4.2.1). Ursache ist einerseits ein Anstieg der dynamischen Kohäsion $\mathrm{c}_{\mathrm{dyn}}$ im Bereich unterhalb von $0,17 \mathrm{~m} / \mathrm{s}$. Andererseits resultiert der Anstieg der Schneidkräfte mit zunehmender Schneidgeschwindigkeit aus der notwendigen Beschleunigung des im Abbau befindlichen Bodens. Mit steigender Schneidgeschwindigkeit $v_{s c}$ steigen also grundsätzlich die Schneidkräfte an und die daraus resultierenden Kontaktspannungen zwischen Boden und Werkzeugoberfläche. Daraus resultiert folglich vermutlich ein Anstieg der Verschleißrate $c_{v}$ und ein Absinken des Laufwegs $s_{c}$ mit zunehmender Schneidgeschwindigkeit $\mathrm{v}_{\mathrm{sc}}$ im Bereich unterhalb von $0,17 \mathrm{~m} / \mathrm{s}$. In den untersuchten Referenzprojekten liegt die durchschnittliche Schneidgeschwindigkeit $v_{s c}$ der Abbauwerkzeuge mit 0,25-0,90 m/s (Kap. 5.2.2.1) jedoch deutlich höher als $0,17 \mathrm{~m} / \mathrm{s}$. Zusätzlich dominieren in den Referenzprojekten kohäsionslose Bodenarten. Eine Änderung der dynamischen Kohäsion $\mathrm{c}_{\mathrm{dyn}}$ des Bodens mit der Schneidgeschwindigkeit $v_{s c}$ ist in den untersuchten Homogenbereichen folglich nicht zu erwarten, sodass sich kein Einfluss auf die Verschleißrate $c_{v}$ und den Laufweg $s_{c}$ ergibt. Der Anteil der steigenden Beschleunigung des Bodens mit zunehmender Schneidgeschwindigkeit $\mathrm{v}_{\mathrm{sc}}$ ist nach den Ergebnissen in Abb. 83 und Abb. 84 augenscheinlich relativ gering und im Laufweg $\mathrm{s}_{\mathrm{c}}$ nicht nachzuweisen.

Für Schneidrollen liegen keine vergleichbaren modellhaften Untersuchungen vor. Aufgrund der in Kap. 5.3.2.1 beschriebenen Rückschlüsse auf die Abbaumechanik von Schneidrollen in Lockergesteinen lassen sich jedoch sehr ähnliche Zusammenhänge, wie für Schälmesser vermuten. Die durchschnittliche Schneidgeschwindigkeit $v_{s c}$ der Abbauwerkzeuge liegt auch hier mit 0,25-0,90 m/s über dem Bereich, in dem ein Anstieg der dynamischen Kohäsion $c_{d y n}$ mit der Schneidgeschwindigkeit $v_{s c}$ in bindigen Böden zu erwarten ist. Zudem dominieren auch für Schneidrollen kohäsionslose Böden in den Referenzprojekten. Augenscheinlich ergibt sich aus der zunehmenden Beschleunigung des Bodens mit steigender Schneidgeschwindigkeit $\mathrm{v}_{\mathrm{sc}}$ auch für Schneidrollen kein maßgeblicher Anstieg der Spannungen zwischen dem Boden und der Werkzeugoberfläche, der eine Änderung des Laufwegs $\mathrm{s}_{c}$ bewirkt.

Über den Anstieg der Schneidkräfte mit zunehmender Schneidgeschwindigkeit $\mathrm{v}_{\mathrm{sc}}$ hinaus, werfen KöHLER et al. (2011: 46) die Frage nach Kavitation der Stützflüssigkeit im Bereich der Abbauwerkzeuge auf, die bei Implosion der entsprechenden Dampfblasen zu hohen Spannungsspitzen und Verschleiß an der Werkzeugoberfläche führen würde. Dazu ist theoretisch eine lokale Unterschreitung des Sättigungsdampfdrucks der Bentonitsuspension durch den statischen Druck in der Suspension notwendig. Der statische Druck hängt unter anderem stark von der lokalen Fließgeschwindigkeit der Bentonitsuspension in der Abbaukammer ab. Daraus lässt sich die Vermutung ableiten, dass mit zu- 
nehmender Schneidraddrehzahl U die lokalen Fließgeschwindigkeiten in der Abbaukammer ansteigen und damit auch die Wahrscheinlichkeit für Kavitation steigt. Damit würde sich mit steigender Schneidraddrehzahl ein Anstieg der Verschleißrate $c_{v}$ und ein Absinken des Laufwegs $s_{c}$ ergeben. Allgemein sprechen die Größenordnung des Stützdruck $P_{S F}$ und die vergleichsweise hohe Dichte der Stützflüssigkeit $\rho_{\mathrm{SF}}$ jedoch eher gegen das Auftreten von Kavitation in der Abbaukammer, da diese den Sättigungsdampfdruck der Bentonitsuspension deutlich erhöhen. Für eine detaillierte Untersuchung des Sachverhalts wäre hierzu, ähnlich wie für die in Kap. 5.2.2 diskutierte Verweildauer der Bodenkomponenten in der Abbaukammer, eine detaillierte Untersuchung zu den lokalen Fließgeschwindigkeiten und zur Dichteverteilung der Bentonitsuspension in der Abbaukammer notwendig.

Mit ansteigender Schneidgeschwindigkeit $\mathrm{v}_{\mathrm{sc}}$ steigen abschließend auch die Kontaktkräfte und Spannungen zwischen Werkzeugoberfläche und Boden beim Auftreffen auf Hindernisse wie Steine und Blöcke. Damit steigt auch die Wahrscheinlichkeit für Gewaltschäden an. Ein entsprechender Unterschied in der Häufigkeit von Gewaltschäden bei Schneidrollen und Schälmessern zwischen inneren und äußeren Spuren auf dem Schneidrad ist jedoch aufgrund mangelnder Dokumentation der Verschleißbilder in den Referenzprojekten nicht nachweisbar.

Zusammenfassend ist also innerhalb der Referenzprojekte kein maßgeblicher Einfluss der Schneidgeschwindigkeit $\mathrm{v}_{\mathrm{sc}}$ und damit der Schneidraddrehzahl $U$ auf die Verschleißrate $\mathrm{c}_{\mathrm{v}}$ und den Laufweg $\mathrm{s}_{\mathrm{c}}$ nachweisbar. Kritisch anzumerken bleibt, dass einerseits Referenzwerte für Schneidgeschwindigkeiten unterhalb $0,25 \mathrm{~m} / \mathrm{s}$ fehlen. Andererseits fehlen Untersuchungen zur Entstehung und Auswirkung von Kavitation bei Kombinationen hoher Schneidraddrehzahl U, geringem Stützdruck $P_{S F}$ und geringer Dichte $\rho_{\mathrm{SF}}$ der Stützflüssigkeit. Abschließend unterstützen die Ergebnisse jedoch unter den in Kap. 5.6.3.2 angegebenen Einschränkungen die Argumentation für eine penetrationsbetonte Fahrweise der TVM mit tendenziell hohe Penetration $p$ und niedriger Schneidraddrehzahl U.

\subsubsection{Penetration $p$}

Die Penetration $\mathrm{p}[\mathrm{mm} / \mathrm{U}]$ ergibt sich mit Gl. 2 aus der vom Schildfahrer eingestellten Schneidraddrehzahl $U\left[\mathrm{~min}^{-1}\right]$ und der Vortriebsgeschwindigkeit $\mathrm{V}_{\text {TVM }}[\mathrm{mm} / \mathrm{min}]$ (Kap. 3.3.1).

Eine direkte vergleichende Analyse der Auswirkung der Penetration $p$ auf den Laufweg $\mathrm{s}_{\mathrm{c}}$ ist mit der in Kap. 5.6.1.4 beschriebene Methode zur Filterung der Rohdaten nicht möglich. Die Penetration $p$ ist nach den Ergebnissen in Kap. 5.6.1.3 innerhalb der geotechnischen Homogenbereiche normalverteilt. Entsprechend können innerhalb der Referenzprojekte bei gleichbleibenden geotechnischen Bedingungen und TVM-Auslegung keine Bereiche mit signifikant unterschiedlicher Steuerung der Penetration $p$ ausgegliedert werden. Für eine direkte Analyse des Einflusses der Penetration $p$ wären folglich Homogenbereiche notwendig, die sich ausschließlich in der Steuerung der Penetration unterscheiden. Innerhalb der Referenzprojekte liegt jedoch keine entsprechende Möglichkeit für einen Vergleich vor.

Für Schälmesser kann der Einfluss der Penetration auf die Abriebrate $c_{v}$ und den Laufweg $s_{c}$ jedoch indirekt über den Einfluss des Spurbesatzes $k_{s}$ (Kap. 5.6.2.2) nachgewiesen werden. Diese zeigen für eine Verdopplung des Spurbesatzes $\mathrm{k}_{\mathrm{s}}$ bzw. Halbierung der Aktiv-Penetration $\mathrm{p}_{\mathrm{a}}$ jeweils eine Zunahme des Laufwegs $s_{c}$ der Schälmesser um einen Faktor 1,4-1,8. Mit steigender Aktiv-Penetration $p_{a}$ und damit auch allgemein mit steigender Penetration $p$ sinkt also tendenziell der Betrag des Laufwegs $\mathrm{s}_{\mathrm{c}}$.

Der Laufweg $\mathrm{S}_{\mathrm{c}}$ ist folglich unter den vorliegenden Rahmenbedingungen für Schälmesser nicht unabhängig von der Penetration $p$. Der Faktor der Änderung des Laufwegs $s_{c}$ ist jedoch jeweils deutlich geringer als die entsprechende Änderung der Penetration $p$. Das Lösevolumen $v_{c}$ der Schälmesser nimmt tendenziell bei einer Erhöhung der Penetration $p$ zu. Am Beispiel der aus Tab. 17 ermittelten Faktoren für die Änderung des Laufwegs $\mathrm{s}_{\mathrm{c}}$ bewirkt eine Verdopplung der Penetration $\mathrm{p}$ theoretisch eine Zunahme des Lösevolumens $v_{c}$ um ca. 10\% - 40\%. Damit sinkt bei Erhöhung der Penetration $p$ die Anzahl der notwendigen Werkzeugwechsel.

Für Schneidrollen ist kein vergleichbarer Nachweis des Einflusses der Penetration $p$ auf den Laufweg $\mathrm{s}_{\mathrm{c}}$ über den Spurbesatz $\mathrm{k}_{\mathrm{s}}$ möglich, weil in den Referenzprojekten die Schneidspuren der Schneidrollen jeweils nur einfach besetzt sind. Darüber hinaus lässt die Analyse zur Abbaumechanik der Schneidrollen in Lockergesteinen (Kap. 5.3.2.1) den Schluss zu, dass sich aufgrund der nachlaufend angeordneten Schälmesser bei einer Erhöhung der Penetration $p$ die Eindringtiefe des Schneidrings in die Ortsbrust nicht erhöht. Die Kontaktfläche des Schneidrings zum Boden an den Seitenflächen, 
wo auf Basis des Verschleißbildes die höchste Intensität des Abriebs beim Primärverschleiß zu erwarten ist, steigt folglich nicht an. Zwischen dem Boden und der Lauffläche des Schneidrings entsteht durch die Erhöhung der Penetration vermutlich eine Erhöhung der Kontaktspannung. Aufgrund der geringen Relativbewegung zwischen Boden und Lauffläche (Abb. 40) hat diese jedoch kaum Auswirkungen auf die Verschleißrate $c_{v}$ und den Laufweg $s_{c}$. Der Einfluss der Penetration $p$ auf die Verschleißrate $c_{v}$ und den Laufweg $s_{c}$ wird folglich vermutlich durch die Anordnung der Abbauwerkzeuge innerhalb der Referenzprojekte nahezu eliminiert. Offen bleibt, wie sich bei einer von den Referenzprojekten abweichenden Anordnung der Abbauwerkzeuge der Einfluss der Penetration $p$ auf die Verschleißrate $c_{v}$ und den Laufweg $s_{c}$ der Schneidrollen ändert.

Für Stichel und Räumer ist anhand der verfügbaren Basisdaten keine Untersuchung zum Einfluss der Penetration $p$ auf den Laufweg $s_{c}$ analog zu den Schälmessern durchführbar. Die Schneidspuren der Stichel waren in den Referenzprojekten aufgrund des Einbaus in Schneidrollengehäuse durchgehend nur einfach besetzt. Für Räumer liegen keine ausreichenden Werkzeugwechseldaten vor, um entsprechende Vergleiche anzustellen. Aufgrund der hohen Ähnlichkeit in den Verschleißbildern und der Abbaumechanik zwischen Schälmessern, Sticheln und Räumern (Kap. 5.3.3 \& 5.3.5) kann vermutet werden, dass die Penetration $p$ einen sehr ähnlichen Einfluss auf den Laufweg $s_{c}$ von Sticheln und Räumern hat, wie bei Schälmessern. Eine Überprüfung dieser Vermutung ist anhand der gegeben Referenzdaten jedoch nicht möglich.

Aufgrund der Erhöhung des Abbauvolumens $v_{c}$ bei steigender Penetration $p$ für Schälmesser und des geringen Einflusses der Penetration $p$ auf den Laufweg $s_{c}$ der Schneidrollen ist, ähnlich wie im Festgesteinsvortrieb von FRENZEL (2010a: 59) dargestellt, auch beim Vortrieb mit Hydroschild TVM in Lockergesteinen eine penetrationsbetonte Steuerung des Abbauprozesse vorteilhaft. Sie bewirkt tendenziell eine Verringerung der Anzahl $\mathrm{n}_{\mathrm{c}(\mathrm{TA})}$ der Werkzeugwechsel über die Tunnelachse und der dazu benötigten Anzahl an Vortriebsunterbrechungen $n_{1(T A)}$. Voraussetzung zur Nutzung der Vorteile einer penetrationsbetonten Steuerung des Abbauprozesses ist jedoch einerseits, dass keine Hindernisse wie z.B. Steine oder Blöcke angetroffen werden und andererseits die Auslegung der TVM den anfallenden Volumenstrom bewältigen kann. Sind diese Voraussetzungen nicht gegeben, besteht die Gefahr von Gewaltschäden an den Werkzeuge, sowie Verklebungen und hohem Sekundärverschleiß an der Schneidradstruktur, welche die Vorteile der penetrationsbetonten Steuerung deutlich überwiegen können.

In den Referenzprojekten werden in bindigen Böden mit niedriger Konsistenzzahl $\mathrm{I}_{\mathrm{C}}(<0,75)$ hohe Werte für die Penetration $p$ von $20-40 \mathrm{~mm} / \mathrm{U}$ erreicht. Im überwiegenden Anteil der Homogenbereiche in mitteldicht bis dicht gelagerten Sanden und Kiesen ergeben sich dagegen Werte von $10-20 \mathrm{~mm} / \mathrm{U}$. Noch deutlich niedrigere Werte liegen aus Homogenbereichen mit Steinen und Blöcken in der Ortsbrust vor, in denen die Penetration $p$ auf Werte $<10 \mathrm{~mm} / \mathrm{U}$ reduziert wurde, um Schäden an den Abbauwerkzeugen zu vermeiden. Die erreichten Penetrationswerte sind also nicht unabhängig von den Boden- und Gebirgseigenschaften. In Verbindung mit den Ergebnissen zum Einfluss der Boden- und Gebirgseigenschaften auf den Laufweg $\mathrm{s}_{\mathrm{c}}$ (Kap. 5.6.4) kann deshalb ähnlich wie für Festgesteinen von KöPPL et al. (2009: 416) dargestellt vermutet werden, dass sich einzelne in die Penetration $p$ und die Abrasivität des Bodens einfließende Parameter (Reibungswinkel $\varphi^{\prime}$, Kohäsion c', Korngrößenverteilung, Mineralzusammensetzung des Bodens) in Teilen positiv korreliert sind. Damit steigt einerseits die theoretisch mögliche Bandbreite an Ergebnissen bei der Verschleißprognose. Andererseits wirken sich sehr hohe/ungünstige bzw. sehr niedrige/günstige Werte der genannten geotechnischen Parameter doppelt ungünstig bzw. günstig auf den Laufweg $\mathrm{s}_{\mathrm{c}}$ aus.

Abschließend muss in Betracht gezogen werden, dass die Penetration p vermutlich auch Einfluss auf die Verdichtung des Bodens beim Abbau in der Ortsbrust hat (Kap. 4.2.2.1). Eine hohe Penetration $p$ würde sich damit über die Verdichtung des Bodens in der Ortsbrust theoretisch negativ auf den Laufweg $\mathrm{s}_{\mathrm{c}}$ der Abbauwerkzeuge auswirken. Ein entsprechender Nachweis dieser Wirkungskette ist jedoch bisher nicht gegeben. Zudem neigen vermutlich nicht alle Bodenarten in gleicher Weise zur Verdichtung in der Ortsbrust und der Verdichtungsmechanismus ist zudem stark von weiteren Parametern der Steuerung des Abbauprozesses (Stützdruck $P_{\mathrm{SF}}$, Dichte der Stützflüssigkeit $\rho_{\mathrm{SF}}$ ) abhängig. Unter Berücksichtigung der oben beschriebenen Ergebnisse ist ein allgemeiner Zusammenhang zwischen hohen Penetrationswerten, Verdichtung der Ortsbrust und negativer Auswirkung auf den Laufweg $\mathrm{s}_{\mathrm{c}}$ deshalb als unwahrscheinlich anzusehen. 


\subsubsection{Dichte der Stützflüssigkeit $\rho_{S F}$}

Die Dichte der Stützflüssigkeit $\rho_{S F}\left[\mathrm{~g} / \mathrm{cm}^{3}\right]$ resultiert hauptsächlich aus dem Verhältnis zwischen dem vom Schildfahrer eingestellten Förder- und Speisevolumen $v_{F L}$ bzW. $v_{S L}\left[m^{3} / h\right]$ und der Vortriebsgeschwindigkeit $\mathrm{V}_{\mathrm{TVM}}[\mathrm{mm} / \mathrm{min}]$. Die Vortriebsgeschwindigkeit gibt den Volumenstrom des Bodens in die Abbaukammer vor. Von hier aus wird der Boden mit dem durch Förder- und Speisevolumen definierten Volumenstrom der Stützflüssigkeit zur Separationsanlage transportiert. Neben dem Verhältnis der beiden Volumenströme hängt die Dichte der Stützflüssigkeit $\rho_{\mathrm{SF}}$ in der Abbaukammer folglich zusätzlich von der Dichte der eingespeisten Stützflüssigkeit, der Effizienz der Separationsanlage und Durchmischungsvorgängen in der Abbaukammer ab (Kap. 3.2.4).

Ähnlich wie die Penetration $p$ ist auch die Dichte der Stützflüssigkeit $\rho_{\mathrm{SF}}\left[\mathrm{g} / \mathrm{cm}^{3}\right]$ innerhalb der geotechnischen Homogenbereiche normalverteilt (Kap. 5.6.1.3). In den Referenzprojekten können bei gleichbleibenden geotechnischen Bedingungen und TVM-Auslegung keine Bereiche mit signifikant unterschiedlicher Steuerung der Dichte der Stützflüssigkeit $\rho_{\mathrm{SF}}$ ausgegliedert werden. Darüber hinaus ergeben sich zwischen verschiedenen Referenzprojekten oder parallelen Vortrieben innerhalb der Referenzprojekte keine Möglichkeiten für eine vergleichende Analyse des Einflusses der Dichte der Stützflüssigkeit $\rho_{\mathrm{SF}}$ auf den Laufweg $\mathrm{s}_{\mathrm{c}}$. Trotzdem können aus dem bestehenden Wissensstand (Kap. 4.3.3) und den Daten aus den Referenzprojekten drei wichtige qualitative Zusammenhänge zum Einfluss der Dichte der Stützflüssigkeit auf den Laufweg $\mathrm{s}_{\mathrm{c}}$ abgeleitet werden.

Der erste Zusammenhang der Dichte der Stützflüssigkeit mit dem Laufweg $\mathrm{s}_{\mathrm{c}}$ ergibt sich direkt aus dem Anteil der abgebauten Bodenkomponenten in der Stützflüssigkeit. Anhand der Verschleißbilder der Schälmesser und Räumer kann gezeigt werden, dass mit steigendem Anteil an abgebauten Bodenkomponenten in der Stützflüssigkeit die Wahrscheinlichkeit steigt, dass zwischen der Ortsbrust und den Werkzeugen Korn-zu-Korn-Brücken entstehen. Über diese können wesentlich höhere Spannungen zwischen Bodenkomponenten und Werkzeugoberfläche aufgebaut werden, als dies allein durch den Stützdruck $P_{S F}$ und die Relativbewegung zwischen Bodenkomponenten und Werkzeugoberfläche möglich wäre. In diesem Fall ändert sich auch die Belastungsart zwischen den Bodenkomponenten und der Werkzeugoberfläche von eher prallendem Kontakt hin zu eher mahlendem Kontakt. Ein ähnlicher Zusammenhang wurde bereits für Erddruckschilde erarbeitet (Kap. 4.3.1.5). An den Schälmessern und Räumern kann in diesen Fällen hoher Sekundärverschleiß an der Rückenfläche mit Freilegung der Hartmetalleinsätze festgestellt werden (Kap. 5.3.4.2), weil beim passiven Einsatz vermutlich bereits abgebaute Bodenkomponenten im Freiwinkel $\beta_{c}$ angesammelt werden. Für den Verschleiß an den Gleitringdichtungen der Schneidrollen und am Rollengrundkörper ist der Anteil an abgebauten Bodenkomponenten in der Stützflüssigkeit nach den Ergebnissen in Kap. 5.3.2.2 ebenfalls ein maßgeblicher Einflussfaktor.

Die Dichte der Stützflüssigkeit $\rho_{S F}$ wirkt sich also zum einen über die Häufigkeit des Kontaktes zwischen den abgebauten Bodenkomponenten und den Abbauwerkzeugen und zum anderen über die Art des Kontaktes und die damit verbundenen Spannungen zwischen Bodenkomponenten und Werkzeugoberfläche aus. Der Sekundärverschleiß der Abbauwerkzeuge wird vermutlich proportional von der Dichte der Stützflüssigkeit beeinflusst, der Laufweg $\mathrm{s}_{\mathrm{c}}$ sinkt tendenziell mit steigender Dichte der Stützflüssigkeit $\rho_{\mathrm{SF}}$. Als zweiter wesentlicher Zusammenhang aus den Referenzprojekten muss hier jedoch berücksichtigt werden, dass der Einfluss der Dichte der Stützflüssigkeit $\rho_{\mathrm{SF}}$ auf den Sekundärverschleiß der Abbauwerkzeuge vermutlich deutlich mit der Abrasivität der Bodenkomponenten gegenüber der Härte der Werkstoffe in den Abbauwerkzeugen schwankt. Änderungen der Dichte der Stützflüssigkeit wirken sich umso stärker auf den Laufweg $\mathrm{s}_{\mathrm{c}}$ aus, je höher die Abrasivität der Bodenkomponenten gegenüber den eingesetzten Werkstoffen ist.

Abschließend hat die Dichte der Stützflüssigkeit $\rho_{\mathrm{SF}}$ auch Einfluss auf die Entstehung von Verklebungen an den Abbauwerkzeugen und der Schneidradstruktur. Das Risiko für Verklebungen und Verklebungsschäden an den Abbauwerkzeugen steigt mit der Dichte der Stützflüssigkeit an. Bereits bei geringen Anteilen von bindigen Böden mit Verklebungspotential in der Ortsbrust wird deshalb empfohlen, die Austauschrate der Stützflüssigkeit, also Speise- und Fördervolumen, soweit möglich zu maximieren (Kap. 4.2.2.1) und damit deren Dichte $\rho_{\mathrm{SF}} \mathrm{zu}$ minimieren. Hier können bereits geringe Änderungen der Dichte der Stützflüssigkeit hohe Auswirkungen auf die Bildung von Verklebungen und die Entstehung von Verklebungsschäden haben. 


\subsubsection{Stützdruck $P_{S F}$}

Der Stützdruck $P_{S F}$ in der Abbaukammer wird durch den Schildfahrer oder den Schichtingenieur in Abhängigkeit der geotechnischen Rahmenbedingungen eingestellt (Kap. 3.3.1). Der Stützdruck hat also eigentlich den Charakter einer Reaktionsgröße, die den Spannungszustand in der Ortsbrust wiederspiegelt, obwohl der Wert auf der TVM vom Schildfahrer eingestellt wird.

Für den Primärverschleiß der Abbauwerkzeuge beim Bodenabbau in der Ortsbrust gibt es keine Hinweise zum Einfluss des Stützdrucks $P_{\mathrm{SF}}$ auf den Abrieb. Der Abrieb wird nach den Ergebnissen Kap. 5.6.4 unter anderem durch die Kontaktspannungen $\mathrm{T}_{\text {act }}\left[\mathrm{kN} / \mathrm{m}^{2}\right]$ zwischen Boden und Werkzeugoberfläche bestimmt. Der Einfluss des Stützdrucks $P_{S F}$ ist dabei vermutlich gering, da er in gleicher Weise allseitig auf alle beteiligten Systemkomponenten wirkt. Die Kontaktspannungen Tact korrelieren in den Referenzprojekten stark mit der Scherfestigkeit $\mathrm{T}_{\mathrm{c}}$ (Gl. 54) des Bodens in der Ortsbrust (Kap. 5.6.4.2). Die zur Bestimmung von $\mathrm{T}_{\mathrm{c}}$ eingesetzten Basisparameter wie Kohäsion $\mathrm{c}^{c}\left[\mathrm{kN} / \mathrm{m}^{2}\right]$, Reibungswinkel $\varphi^{c}\left[^{\circ}\right]$, Wichte des Bodens $\mathrm{y}\left[\mathrm{kN} / \mathrm{m}^{3}\right]$ und Wichte des Bodens unter Auftrieb $\mathrm{Y}^{\mathrm{c}}\left[\mathrm{kN} / \mathrm{m}^{3}\right]$, Überlagerung $\mathrm{H}_{T A}$ und Wasserspiegel $\mathrm{W}_{\mathrm{TA}}[\mathrm{m}]$ werden in gleicher Weise zur Bestimmung des Stützdrucks $P_{\mathrm{SF}}$ (Kap. 3.2.4) eingesetzt. Denkbar ist deshalb, dass eine scheinbare Korrelation zwischen Stützdruck $\mathrm{P}_{\mathrm{SF}}$ und Laufweg $\mathrm{s}_{\mathrm{c}}$ dargestellt werden kann, weil der Stützdruck mit dem Spannungszustand im Boden korreliert. Diese Korrelation beruht jedoch nicht auf einer direkten Ursache-Wirkung Beziehung zwischen den beiden Größen. Zielführender ist direkte Analyse des Einflusses des Spannungszustandes im Boden auf die Verschleißrate $\mathrm{c}_{\mathrm{v}}$ und den Laufweg $\mathrm{s}_{\mathrm{c}}$ (Kap. 5.6.4.2).

Im Hinblick auf den Sekundärverschleiß weisen bestehende Versuche zum Einfluss der Konsistenz auf den Sekundärverschleiß bei EPB-TVM (Kap.4.3.1.5) darauf hin, dass bei flüssiger Konsistenz des Erdbreis der Umgebungsdruck keinen Einfluss auf den Abrieb an den Abbauwerkzeugen hat. Aufgrund der flüssigen Konsistenz sind diese Ergebnisse überschlägig auf die Verhältnisse in der Abbaukammer bei Hydroschild TVM übertragbar, wobei der Umgebungsdruck dem Stützdruck $P_{S F}$ entspricht. Folglich hat auch hier der Stützdruck $P_{S F}$ keinen Einfluss auf den Sekundärverschleiß der Abbauwerkzeuge.

Zusammenfassend kann auf Basis der genannten theoretischen Überlegungen und des bestehenden Wissensstandes angenommen werden, dass der Stützdruck $P_{S F}$ nur geringen Einfluss auf die Verschleißrate $c_{v}$ und den Laufweg $\mathrm{s}_{\mathrm{c}}$ der Abbauwerkzeuge hat. Innerhalb der Referenzprojekte kann diese Vermutung jedoch nicht durch eine direkte Analyse der Auswirkung des Stützdrucks auf die Verschleißrate $\mathrm{c}_{\mathrm{v}}$ und den Laufweg $\mathrm{s}_{\mathrm{c}}$ überprüft werden. Die in Kap. 5.6.1.2 beschriebenen Kriterien zur Bildung der geotechnischen Homogenbereiche führen aufgrund der Fixierung der Überlagerung $\mathrm{H}_{\mathrm{TA}}[\mathrm{m}]$ und des Wasserspiegels $\mathrm{W}_{\mathrm{TA}}[\mathrm{m}]$ dazu, dass auch der Stützdruck innerhalb der Homogenbereiche nur einer geringen Schwankungsbreite unterliegt. Zusätzlich kann nachgewiesen werden, dass die Werte für den Stützdruck $P_{S F}$ in den geotechnischen Homogenbereichen normalverteilt sind. Folglich können innerhalb der geotechnischen Homogenbereiche keine Bereiche mit signifikant unterschiedlicher Steuerung des Stützdrucks $\mathrm{P}_{\mathrm{SF}}$ ausgegliedert werden. Für eine direkte Analyse des Einflusses des Stützdrucks wären folglich separate Homogenbereiche notwendig, die sich ausschließlich im Stützdruck $P_{S F}$ unterscheiden. Innerhalb der Referenzprojekte liegen jedoch keine entsprechenden Möglichkeiten für eine vergleichende Analyse vor.

Ergänzend ist darauf hinzuweisen, dass weitere sekundäre Einflüsse des Stützdrucks auf den Abbauvorgang und die Verschleißmechanismen denkbar sind. Zum einen ist der Stützdruck $P_{S F}$ eine wesentliche Größe bei der Diskussion um Kavitation im Bereich der Abbauwerkzeuge (Kap. 5.6.3.1). Zum anderen ist auch ein Einfluss des Stützdrucks $P_{\text {SF }}$ auf die Bodenverdichtung in der Ortsbrust beim Bodenabbau denkbar (Kap. 4.2.2.1). Beide Themen sind jedoch nicht Kernthema der vorliegenden Arbeit und werden deshalb nicht weiter untersucht. 


\subsubsection{Schneidradanpresskraft $F_{S R}$ und Schneiddrehmoment $M_{S R}$}

Die Schneidradanpresskraft $F_{S R}[k N]$ wird von den Vortriebspressen (Zylinder) der TVM aufgebracht. In der Vortriebspressenkraft $F_{\text {VTP }}[\mathrm{kN}]$ sind darüber hinaus jedoch auch noch weitere Kraftkomponenten wie z.B. Reibungskräfte aufsummiert. Eine Beschreibung zur überschlägigen Ermittlung der Schneidradanpresskraft $F_{S R}$ aus der Vortriebspressenkraft $F_{V T P}$ ist in Kap. 3.3.1 geben.

Die Vortriebspressengruppen bilden ein volumengesteuertes und druckbegrenztes System. Der Schildfahrer stellt die Vortriebsgeschwindigkeit $\mathrm{V}_{\mathrm{TVM}}[\mathrm{mm} / \mathrm{min}]$ ein und gibt so den Volumenstrom des Öls in die Vortriebspressengruppen vor. Der Öldruck in den Vortriebspressen steigt innerhalb der systemtechnischen Auslegungsgrenze der Hydraulikzylinder auf den notwendigen Wert an, um den vorgegeben Volumenstrom bzw. die eingestellte Vortriebsgeschwindigkeit zu erreichen. Der Betrag der Schneidradanpresskraft $F_{S R}$ in der Vortriebspressenkraft $F_{V T P}$ ist in diesem Anwendungsfall eine Resultierende (Passivparameter) aus den geotechnischen Eigenschaften des Bodens in der Ortsbrust (Eindringwiderstand $\mathrm{W}_{\mathrm{TC}}$ ), der Auslegung der TVM und der Steuerung des Abbauprozesses. DüLLMANN et al. (2013: 624) und HoLLMANN et al. (2013: 639) fassen hierzu die folgenden Einflussfaktoren aus der Auslegung der TVM und Steuerung des Abbauprozesses zusammen:

- Schneidraddurchmesser $\mathrm{D}_{\mathrm{TVM}}[\mathrm{m}]$

- Schneidradöffnungsverhältnis $\mathrm{O}_{\mathrm{TVM}}[\%]$

- Werkzeugbestückung des Schneidrades

- Aktueller Verschleißzustand der Abbauwerkzeuge

- Vortriebsgeschwindigkeit $\mathrm{V}_{\mathrm{TVM}}[\mathrm{mm} / \mathrm{min}]$

- Schneidraddrehzahl $\cup\left[\mathrm{min}^{-1}\right]$

- Verklebungen \& Hindernisse

Zusätzlich kann der Schildfahrer eine Druckbegrenzung für einzelne Vortriebspressengruppen vornehmen, sodass ein Teil des Ölvolumenstroms nicht in Zylindervorschub umgesetzt wird. Damit kann einerseits die Richtung der resultierenden Vortriebspressenkraft $F_{\text {VTP }}$ beeinflusst werden, um die Vortriebsrichtung der Schildmaschine zu steuern. Andererseits wird durch die Druckbegrenzung jedoch auch die Schneidradanpresskraft $F_{S R}$ innerhalb der Vortriebspressenkraft $F_{V T P}$ reduziert. Als Rückkopplung daraus sinkt die Vortriebsgeschwindigkeit $\mathrm{V}_{\mathrm{TVM}}$. In diesem Fall nimmt der Schildfahrer indirekt eine Einstellung der Schneidradanpresskraft $F_{S R}$ vor, sodass diese nach DüLLMANN et al. (2013: 622) auch als Aktivparameter (Einstellwert) interpretiert werden kann.

Analog zur Schneidradanpresskraft $F_{\mathrm{SR}}$ sind auch im Schneidraddrehmoment $\mathrm{M}_{\mathrm{TVM}}[\mathrm{kNm}]$ neben dem Schneiddrehmoment $M_{S R}[\mathrm{kNm}]$ einige weitere Komponenten aufsummiert (Kap.3.3.1). Das Schneiddrehmoment $\mathrm{M}_{\mathrm{SR}}$ ist eine Reaktionsgröße (Passivparameter) aus den geotechnischen Eigenschaften des Bodens (Scherwiderstand $\mathrm{W}_{\mathrm{SC}}$ ) in der Ortsbrust und dem bereits für die Schneidradanpresskraft $\mathrm{F}_{\mathrm{SR}}$ aufgelisteten Satz an Einflussfaktoren aus der Auslegung der TVM und der Steuerung des Abbauprozesses. Die Leistung des Schneidradantriebs wird durch die SPS der TVM (Kap. 3.3.4) innerhalb der systemtechnischen Leistungsgrenzen des Antriebs (Abb. 16) so geregelt, dass die vom Schildfahrer eingestellte Schneidraddrehzahl U [ $\left.\mathrm{min}^{-1}\right]$ erreicht wird.

Sowohl in der Schneidradanpresskraft $F_{S R}$, als auch im Schneiddrehmoment $M_{S R}$ sind die an den einzelnen Abbauwerkzeugen anliegenden Komponenten (Anpresskraft $F_{T C}$ \& Schneidkraft $F_{S C}$ ) der Schneidkraft $F_{C}$ aufsummiert. Bei einer Aufteilung der Schneidradanpresskraft $F_{S R}$ und des Schneiddehmomentes $\mathrm{M}_{\mathrm{SR}}$ auf die einzelnen Abbauwerkzeuge können theoretisch die Kontaktspannungen $\mathrm{T}_{\mathrm{act}}$ $\left[\mathrm{kN} / \mathrm{m}^{2}\right]$ an den Oberflächen der Abbauwerkzeuge beim Bodenabbau abgeschätzt werden. Dies stellen nach dem bestehenden Wissensstand (Kap. 4.1.1.3) einen wichtigen Einflussfaktor auf die Art der auftretenden Verschleißmechanismen und die Verschleißrate $c_{v}$ an den Oberflächen der Abbauwerkzeuge dar. 
Die hierzu geforderte Aufteilung ist mit bestehenden Methoden aus folgenden Gründen nicht möglich:

- Eine rechnerische Abtrennung der Schneidradanpresskraft $F_{S R}$ aus der Kraft an den Schneidradverschiebezylindern $F_{S V}(G l .6)$ oder der Vortriebspressenkraft $F_{V T P}(G l .10)$ ist nicht möglich. Es bestehen keine Berechnungsmöglichkeiten für die Kraft aus mechanischer Stützung $F_{\mathrm{ME}}$ und die Reibkraft $\mathrm{F}_{\mathrm{RA}}$ aus den Zylindern und der Lagerung des Schneidradantriebs, sodass diese nicht von der Schneidradanpresskraft $F_{S R}$ getrennt werden können (Kap. 3.3.1).

- Eine rechnerische Abtrennung des Schneiddrehmomentes $M_{S R}$ aus dem Schneidraddrehmoment $\mathrm{M}_{\mathrm{TVM}}$ ist nicht möglich, weil die darin zusätzlich enthaltenen Komponenten (Gl. 12) rechnerisch nicht ermittelt werden können (Kap. 3.3.1).

- Eine schlüssige Aufteilung der Schneidradanpresskraft $F_{S R}$ und des Schneiddrehmomentes $M_{S R}$ auf die unterschiedlichen Abbauwerkzeugtypen auf dem Schneidrad ist nicht möglich. Mit Ausnahme von Schälmessern existieren keine Modelle oder Methoden, um den jeweiligen Anteil der unterschiedlichen Abbauwerkzeugtypen abzuschätzen.

Die Untersuchung des Einflusses der Kontaktspannungen $T_{\text {act }}\left[\mathrm{kN} / \mathrm{m}^{2}\right]$ auf den Laufweg $\mathrm{s}_{\mathrm{c}}[\mathrm{km}]$ der Abbauwerkzeuge erfolgt deshalb nicht direkten über die Schneidradanpresskraft $F_{S R}$ und das Schneiddrehmoment $M_{S R}$, sondern indirekt über die Scherfestigkeit $\mathrm{T}_{\mathrm{c}}\left[\mathrm{kN} / \mathrm{m}^{2}\right]$ des Bodens in der Ortsbrust auf Höhe der Tunnelachse (Kap. 5.6.4.2). Diese wird als Vergleichswert für die Kontaktspannungen $\mathrm{T}_{\text {act }}$ angesetzt.

\subsubsection{Boden- und Gebirgseigenschaften}

Die Boden- und Gebirgseigenschaften im Vortrieb stellen wesentliche Einflussfaktoren auf die Abriebrate $c_{v}$ und den Laufweg $s_{c}$ dar. Dabei ist der Umfang an beschreibbaren Bodeneigenschaften, die sich vermutlich in Kombination auf die Abriebrate $\mathrm{c}_{v}$ und den Laufweg $\mathrm{S}_{\mathrm{c}}$ auswirken durchaus hoch. Einen Überblick über die theoretisch notwendigen Parameter gibt Tab. 11 während in Tab. 12 der Umfang und die Qualität der tatsächlich erkundeten Parameter in den Referenzprojekten zusammengefasst ist. Daraus ergeben sich für die weitere Analyse zwei zentrale Probleme.

In den Referenzprojekten sind die Bodeneigenschaften nicht vollständig erkundet und zudem häufig in statistisch unzureichender Form angegeben (Kap. 5.2.2.3). Dem gegenüber ist aber für eine umfassende Analyse und saubere Trennung des Einflusses einzelner Bodeneigenschaften auf den Laufweg $\mathrm{s}_{\mathrm{c}}$, z.B. in Form einer mehrfaktoriellen Varianzanalyse, unbedingt die Kenntnis aller genannten Parameter inklusive ihrer statistischen Eigenschaften (Verteilungsform) notwendig.

Alternativ verbleibt die Möglichkeit, nach Homogenbereichen zu suchen, die sich bei ansonsten identischen Bedingungen (Steuerung des Abbauprozesses und Auslegung der TVM) jeweils nur in einer einzigen Bodeneigenschaft unterscheiden. Änderungen des Laufwegs $s_{c}$ wären somit eindeutig auf die Änderung in der jeweiligen Bodeneigenschaft zurückzuführen. Entsprechend vergleichbare Homogenbereiche existieren innerhalb der Referenzprojekte jedoch nicht.

Eine rein empirische Analyse einzelner Bodeneigenschaften im Rahmen der Referenzprojekte ergibt in der Konsequenz keine oder qualitativ schlechte Ergebnisse zum Einfluss auf den Laufweg $\mathrm{s}_{\mathrm{c}}$. Als Beispiel hierzu ist in Abb. 83 der Laufweg $\mathrm{S}_{\mathrm{C}}[\mathrm{km}]$ baugleicher Schälmesser und weitgehend identischer TVM-Auslegung und Steuerung des Abbauprozesses gegen den Äquivalenten Quarzgehalt (ÄQu) [\%] angetragen. 


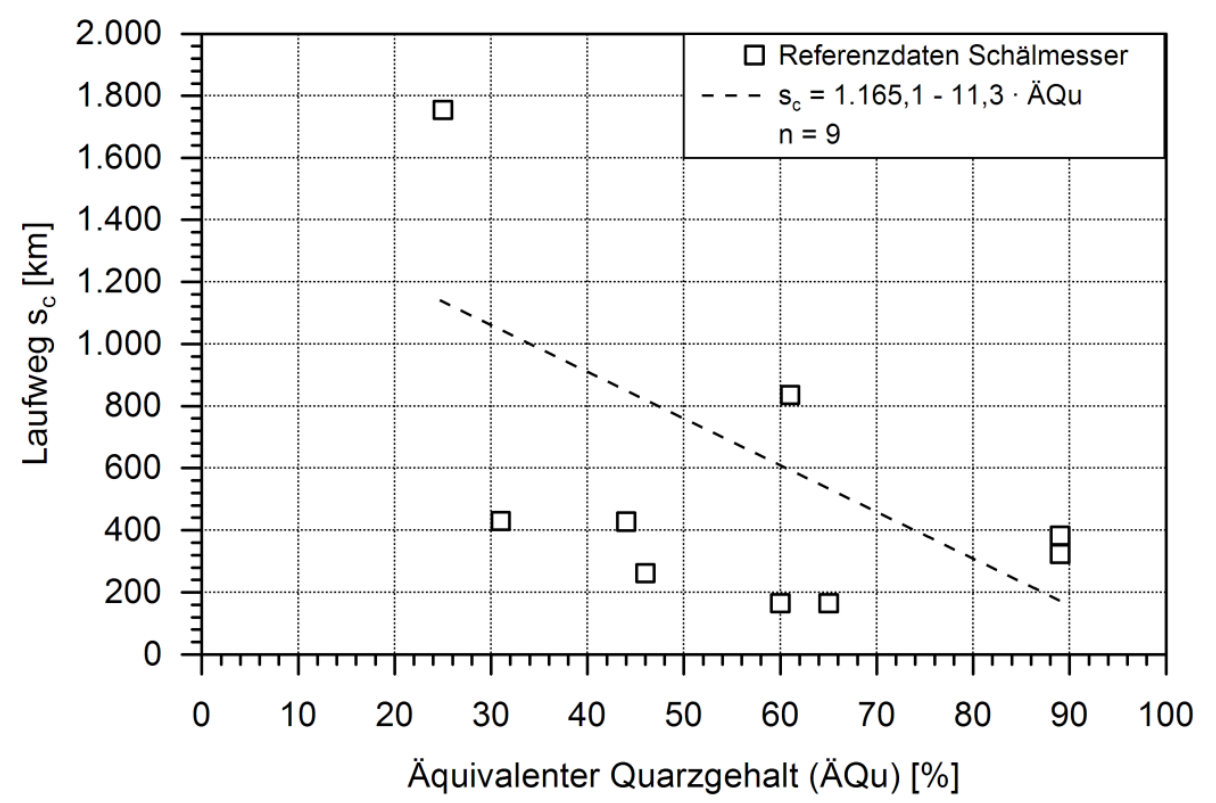

\begin{abstract}
Abb. 83: Laufweg $s_{c}[\mathrm{~km}]$ baugleicher Schälmesser aus verschiedenen Homogenbereichen bei weitgehend identischen TVM-Steuerparametern angetragen gegen den Äquivalenten Quarzgehalt (ÄQu) [\%]. Es ist nur ein schwacher Trend zur Abhängigkeit des Laufwegs $\mathrm{S}_{\mathrm{c}}$ vom Äquivalenten Quarzgehalt (ÄQu) darstellbar.
\end{abstract}

Auf Basis des bestehenden Wissenstandes in Kap. 4.3.1.1 wäre hier ein klarer Zusammenhang zwischen Äquivalentem Quarzgehalt und dem Laufweg $\mathrm{s}_{\mathrm{c}} z \mathrm{z}$ erwarten. Die Verteilung der Datenpunkte in Abb. 83 zeigt zwar qualitativ die erwartete Tendenz zu sinkendem Laufweg $\mathrm{s}_{\mathrm{c}}$ bei steigendem Äquivalentem Quarzgehalt. Die in Abb. 83 eingetragene Regressionskurve ergibt aufgrund der hohen Streubreite der Datenpunkte jedoch keinen statistisch signifikanten Zusammenhang. Ursache dafür ist, dass sich zwischen den Datenpunkten gleichzeitig mit dem Äquivalenten Quarzanteil auch andere maßgebliche Bodeneigenschaften, wie z.B. die Korngrößenverteilung ändern. Ähnliche, jedoch deutlich schlechtere Korrelationen mit dem Laufweg $\mathrm{s}_{\mathrm{c}}$ ergeben sich auch für Indextests, wie z.B. den LCPC-Test, wobei hier das verfügbare Datenvolumen in den Referenzprojekten deutlich niedriger ist.

Um den Einfluss der Bodeneigenschaften auf den Laufweg $\mathrm{s}_{\mathrm{c}}$ trotzdem eingrenzen zu können, wurde in Folge eine prozessorientierte Analyse verschiedener Kombinationen von geotechnischen Parametern durchgeführt und deren Korrelation zum Laufweg $\mathrm{s}_{\mathrm{c}}$ untersucht. Die Analyse basiert auf der grundlegenden Annahme, dass die Abriebrate $c_{v}$ und der Laufweg $s_{c}$ auf Basis des bestehenden Wissensstandes (Kap. 4.1) und der in Kap. 5.1 zusammengefassten Beobachtungen zur Abbaumechanik vermutlich von drei geotechnischen Hauptfaktoren abhängen:

\title{
- Abrasivität der Bodenkomponenten
}

- Kontaktspannung zwischen Boden und Abbauwerkzeug beim Bodenabbau

- Korngrößenverteilung, Kornform und Rundungsgrad der Bodenkomponenten

Folglich wurden Kombinationen verschiedener geotechnischer Kennwerte, welche die genannten drei Hauptfaktoren beschreiben in Form von Indexwerten gebildet und deren Korrelation mit dem Laufweg $\mathrm{s}_{\mathrm{c}}$ aus den Referenzprojekten untersucht. Dabei hängt das Ergebnis der Untersuchung nicht ausschließlich vom tatsächlichen Einfluss der jeweiligen Kombination auf den Laufweg $s_{c}$ ab, sondern zunächst auch von der Bestimmbarkeit der Kennwerte für verschiedene Bodenarten und der Verfügbarkeit der Kennwerte in den Referenzprojekten (Kap. 5.2.2.3). Vor allem die Verfügbarkeit der Kennwerte schränkt die Datenanalyse für den Einfluss der Boden- und Gebirgseigenschaften auf 32 geotechnische Homogenbereiche ein, in denen ein umfangreicher Satz an geotechnischen Parametern bestimmt wurde. Im Detail wurden die nachfolgend beschriebenen geotechnischen Kennwerte die Anwendbarkeit und Verfügbarkeit überprüft. 


\subsubsection{Abrasivität}

Zur Beschreibung der Abrasivität der Bodenkomponenten stehen sowohl direkte Verfahren als auch die in Kap. 4.3.1 beschriebenen Indextests zur Verfügung.

Als Verfahren zur direkten Härtebestimmung einzelner Bodenkomponenten stehen die Ritzhärte nach MOHS (HMO), die Härte nach VICKERS (HV) (DIN 2006a-d), die Härte nach RockWELL (HRC) (DIN 2006e-g) und die absolute Schleifhärte nach RosiwAL (HRW) zur Verfügung. Diese Testverfahren verlangen eine bestimmte Mindestgröße der Probe, sodass die Härte an Bodenkomponenten ca. $<10 \mathrm{~mm}$ nicht bestimmbar ist. Weiterhin kann die Bestimmung ausschließlich an monomineralischen Komponenten erfolgen, sodass der bei weitem überwiegende Anteil der Bodenkomponenten nicht berücksichtigt werden kann. Eine statistisch saubere Zusammenfassung der Härten der einzelnen Bodenkomponenten zur Abrasivität des Bodens ist mit diesen Verfahren folglich nur schwer bzw. für den überwiegenden Anteil der Bodenarten nicht möglich. Zusätzlich wurden die genannten Kennwerte in den Referenzprojekten nicht bestimmt und entfallen damit für die vorliegende Untersuchung.

Der Cerchar Abrasivitäts-Index (CAI) und der LCPC-Versuch bilden als Indextests aufgrund der Standardisierung der Härte des Prüfstiftes bzw. Prüfflügels durch den jeweiligen Kennwert im Prinzip die Abrasivität einzelner Bodenkomponenten bzw. einer Bodenprobe ab. Für die vorliegende Untersuchung ergeben sich für beide Testverfahren jedoch ähnliche Probleme, wie für die Verfahren zur direkten Härtebestimmung.

Der CAI kann aufgrund der notwendigen Teststrecke nur an einzelnen Bodenkomponenten deutlich $>10 \mathrm{~mm}$ bestimmt werden. Für kleinere Bodenkomponenten ist kein CAI-Wert bestimmbar. Eine statistisch saubere Zusammenfassung der Härten der einzelnen Bodenkomponenten zur Abrasivität des Bodens ist mit diesen Verfahren folglich nur schwer bzw. für den überwiegenden Anteil der Bodenarten nicht möglich. Innerhalb der Referenzprojekte wurde der CAI-Wert nur an wenigen Bodenproben bestimmt, dabei fehlen zusätzlich Angaben, mit welcher Methode die Ergebnisse der Einzeltests zu einem für den gesamten Boden repräsentativen Wert verrechnet wurden. Der CAI-Wert entfällt damit aufgrund der Einschränkung in der Bestimmbarkeit und der vorhandenen Datenmenge für die vorliegende Untersuchung.

Der LCPC Abrasivitäts-Koeffizient (LAK) wird, deutlich durch die Korngröße und Kornform der Bodenkomponenten beeinflusst (Kap. 4.3.1.2). Der Versuch bildet folglich nicht ausschließlich die Abrasivität der Bodenprobe ab, sondern integriert weitere geotechnische Eigenschaften. Zusätzlich erfordern die Geometrie der Versuchseinrichtung und die Prüfvorschrift für den Versuch einen relativ engen Korngrößenbereich von 4,0-6,3 mm für die Versuchsdurchführung. Bodenkomponenten $>6,3 \mathrm{~mm}$ können durch Brechen, z.B. mit einem Backenbrecher, auf die notwendige Korngröße zerkleinert werden. Dabei wird jedoch wiederum die Kornform der Bodenkomponenten verändert. Bodenkomponenten $<4,0 \mathrm{~mm}$ entfallen für den Test vollständig. Damit ist auch der LAK nicht für alle Bodenarten bestimmbar. Zusätzlich wurde der LAK nur in wenigen Fällen in den Referenzprojekten bestimmt, sodass nur eine geringe Datenmenge zur Verfügung steht. Der LAK entfällt damit aufgrund der zusätzlichen Einflüsse, den Einschränkungen in der Bestimmbarkeit und der vorhandenen Datenmenge für die vorliegende Untersuchung.

Der Äquivalente Quarzgehalt (ÄQu) [\%] bildet durch die Normierung der absoluten Mineralhärte auf die Härte von Quarz ebenfalls die Abrasivität der Bodenkomponenten ab. Außerhalb des prozentualen Anteils der verschiedenen Minerale integriert der Äquivalente Quarzgehalt keine weiteren Eigenschaften der Bodenkomponenten. Der Kennwert kann entweder an Bodenkomponenten ca. $>5 \mathrm{~cm}$ mithilfe des Pointcounter-Verfahrens an Gesteinsdünnschliffen bestimmt werden oder an pulverisierten Proben durch Röntgendiffraktometrie (RDA). Bei der Bestimmung mithilfe des Pointcounter-Verfahrens muss der ÄQu der einzelnen Bodenkomponenten in gewichteter Form zum entsprechenden Wert für den Boden zusammengefasst werden. Bei Bestimmung des ÄQu durch RDA wird bei entsprechender Probenauswahl und -vorbereitung direkt der ÄQu des Bodens erfasst. Dadurch ergeben sich für die Bestimmung des ÄQu keine Einschränkungen hinsichtlich der Größe der Bodenkomponenten bzw. der Bodenarten. Zusätzlich wurde der Äquivalente Quarzgehalt in den Referenzprojekten nahezu durchgehend bestimmt. Der ÄQu [\%] wurde deshalb für die vorliegende Untersuchung als Kennwert für die Abrasivität der Bodenproben berücksichtigt.

Die Eignung der beschriebenen Verfahren für die Beschreibung der Härte der Bodenkomponenten in der vorliegenden Datenanalyse ist zusammenfassend in Tab. 19 dargestellt. 
Tab. 19: Eignung der verschiedenen Verfahren zur Bestimmung der Härte der Bodenkomponenten bzw. der Abrasivität einer Bodenprobe für die vorliegende Datenanalyse und das zu entwickelnde Prognosemodell auf Basis der Bestimmbarkeit (Einschränkungen durch die Testverfahren) und Verfügbarkeit der Kennwerte in den Referenzprojekten.

\begin{tabular}{|c|c|c|c|c|}
\hline \multicolumn{2}{|r|}{ Härtebestimmung } & Bestimmbarkeit & Verfügbarkeit & Eignung \\
\hline \multirow{4}{*}{ 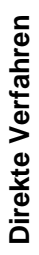 } & Ritzhärte n. MoHs & \multirow{4}{*}{$\begin{array}{l}\text { Nur an monomineralischen } \\
\text { Bodenkomponenten be- } \\
\text { stimmbar. } \\
\text { Mindestgröße der Probe ca. } \\
>10 \mathrm{~mm} \text {. }\end{array}$} & \multirow{4}{*}{$\begin{array}{l}\text { In den Referenzprojek- } \\
\text { ten durchgehend nicht } \\
\text { bestimmt. }\end{array}$} & \multirow{4}{*}{$\begin{array}{l}\text { Für die Datenanalyse } \\
\text { ungeeignet. } \\
\text { Für das Prognosemodell } \\
\text { aufgrund der Bestimm- } \\
\text { barkeit nicht geeignet. }\end{array}$} \\
\hline & Härte n. VICKERS & & & \\
\hline & Härte n. ROcKWELL & & & \\
\hline & Schleifhärte n. RosIWAL & & & \\
\hline & Abrasivitätsbestimmung & Bestimmbarkeit & Verfügbarkeit & Eignung \\
\hline \multirow{3}{*}{ 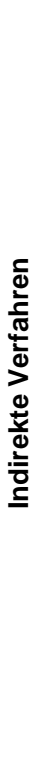 } & $\begin{array}{l}\text { CERCHAR Abrasivitäts Index } \\
(\mathrm{CAI})\end{array}$ & $\begin{array}{l}\text { Mindestgröße der Probe } \\
>10 \mathrm{~mm} .\end{array}$ & $\begin{array}{l}\text { In } 5 \text { von } 10 \text { Referenz- } \\
\text { projekten bestimmt. } \\
\text { Unzureichende Ver- } \\
\text { suchsanzahl. }\end{array}$ & $\begin{array}{l}\text { Für die Datenanalyse } \\
\text { aufgrund der Datenmenge } \\
\text { nicht geeignet. } \\
\text { Für das Prognosemodell } \\
\text { bei grobkörnigen Böden } \\
\text { und hoher Versuchs- } \\
\text { anzahl eingeschränkt } \\
\text { geeignet. }\end{array}$ \\
\hline & LCPC-Test (LAK) & $\begin{array}{l}\text { Maximale Größe der Bo- } \\
\text { denkomponenten: } 6,3 \text { mm. }\end{array}$ & $\begin{array}{l}\text { In } 5 \text { von } 10 \text { Referenz- } \\
\text { projekten bestimmt. } \\
\text { Überwiegend unzu- } \\
\text { reichende Versuchsan- } \\
\text { zahl. }\end{array}$ & $\begin{array}{l}\text { Für die Datenanalyse } \\
\text { aufgrund Datenmenge } \\
\text { nicht geeignet. } \\
\text { Für das Prognosemodell } \\
\text { bei Böden mit maximaler } \\
\text { Korngröße: }<6,3 \mathrm{~mm} \\
\text { eingeschränkt geeignet. }\end{array}$ \\
\hline & $\begin{array}{l}\text { Äquivalenter Quarzgehalt } \\
\text { (ÄQu) }\end{array}$ & $\begin{array}{l}\text { Für alle Bodenarten be- } \\
\text { stimmbar. }\end{array}$ & $\begin{array}{l}\text { In allen Referenz- } \\
\text { projekten bestimmt. } \\
\text { Nahezu vollständig für } \\
\text { alle Bodenarten. }\end{array}$ & $\begin{array}{l}\text { Für die Datenanalyse } \\
\text { geeignet. } \\
\text { Für das Prognosemodell } \\
\text { geeignet. }\end{array}$ \\
\hline
\end{tabular}

Weitere Indextests, die ähnlich wie der LAK oder der ÄQu, die Abrasivität des Bodens abbilden (Kap. 4.3.1.5), wurden nicht berücksichtigt. Diese sind einerseits mit sehr ähnlichen Problemen im Hinblick auf die Bestimmbarkeit für verschiedene Bodenarten behaftet und wurden andererseits durchgehend in keinem der Referenzprojekte angewendet.

\subsubsection{Kontaktspannungen}

Die Kontaktspannung $T_{\text {act }}$ zwischen Boden und Werkzeugoberfläche beim Bodenabbau sind für Schälmesser bei horizontalem Bodenabbau ohne Umgebungsdruck (Stützdruck) theoretisch mit einer Modellrechnung (Kap. 4.2.1) bestimmbar. Als Eingangsparameter dazu werden dynamische Scherparameter (Reibungswinkel $\varphi_{\text {dyn }} \&$ Kohäsion $c_{\text {dyn }}$ ) benötigt. Diese wurden in den Referenzprojekten nicht bestimmt. Für Schneidrollen existiert zusätzlich kein entsprechendes Modell, das eine dreidimensionale Betrachtung des Abbauvorgangs ermöglicht. Damit ist eine einheitliche Ermittlung der Kontaktspannungen $\mathrm{T}_{\mathrm{act}}$ auf diesem Weg nicht möglich.

Eine weitere Möglichkeit zur überschlägigen Bestimmung der Kontaktspannung besteht theoretisch in der Aufteilung der Schneidkräfte aus der Schneidradanpresskraft $F_{S R}$ und dem Schneiddrehmoment $M_{S R}$ auf die einzelnen Abbauwerkzeuge. Daraus ist auf Basis der Werkzeuggeometrie eine Abschätzung der Kontaktspannungen einzelner Abbauwerkzeuge denkbar. Analog zur theoretischen Berechnung sind jedoch auch hier keine ausreichenden Anhaltspunkte für das tatsächliche Verhältnis der 
Schneidkräfte der einzelnen Abbauwerkzeuge und Werkzeugtypen auf dem Schneidrad gegeben. Entsprechend ist auch hier keine ausreichend zuverlässige Ermittlung der Kontaktspannung möglich.

Um dennoch die Größenordnung der Kontaktspannung $T_{\text {act }}\left[\mathrm{kN} / \mathrm{m}^{2}\right]$ zwischen den Werkzeugoberflächen und dem Boden abzuschätzen, wurden als Alternative zu den beiden genannten Möglichkeiten für jeden Homogenbereich die Scherfestigkeit $\mathrm{T}_{\mathrm{c}}$ des Bodens im Moment des Bodenabbaus auf Basis der statischen Scherparameter und einer Betrachtung mit dem Bruchkriterium nach MOHR-COULOMB geschätzt:

$$
\tau_{\mathrm{c}}=\mathrm{c}^{\prime}+\sigma_{\mathrm{n}} \cdot \tan \varphi^{\prime}
$$

In Gl. 54 ist c' die Kohäsion [kN/m²] und $\varphi^{\prime}$ der Reibungswinkel [ ${ }^{\circ}$, die jeweils aus der Vorerkundung zu den Referenzprojekten bekannt sind (Tab. 12), $\sigma_{n}\left[\mathrm{kN} / \mathrm{m}^{2}\right]$ ist die jeweilige Normalspannung.

Durch die Verwendung der statischen Scherparameter ergibt sich dabei zunächst ein systematischer Fehler. Für bindige Böden bestehen Unterschiede zwischen statischer und dynamischer Kohäsion (Kap. 5.2.2.1), während der Reibungswinkel unabhängig von der Schergeschwindigkeit konstant bleibt. Die Kohäsion nimmt dabei mit steigender Schergeschwindigkeit bis zu einem Wert von $10 \mathrm{~m} / \mathrm{min}(0,17 \mathrm{~m} / \mathrm{s})$ deutlich zu. Bei noch höheren Schergeschwindigkeiten ist keine Änderung der Kohäsion mehr festzustellen. Für nahezu kohäsionslose gemischtkörnige und rollige Bodenarten liegen dagegen keine Untersuchungen zu den dynamischen Scherparametern im entsprechenden Geschwindigkeitsbereich vor.

Im Vergleich dazu liegt die Schneidgeschwindigkeit $\mathrm{v}_{\mathrm{sc}}$ der Abbauwerkzeuge in den untersuchten Homogenbereichen bei durchschnittlich 0,25 - 0,90 m/s. Zusätzlich dominieren in den untersuchten Homogenbereichen kohäsionsarme gemischtkörnige und rollige Böden. Für diese Bodenarten liegen zwar im bestehenden Wissensstand keine Untersuchungen zur Geschwindigkeitsabhängigkeit der Scherparameter vor, jedoch ist auf Basis der oben beschriebenen Zusammenhänge zu vermuten, dass sich keine wesentlichen Änderungen des Reibungswinkels $\varphi_{\text {dyn }}$ im Vergleich zu $\varphi^{\prime}$ ergeben. Für die, untergeordnet in den untersuchten Homogenbereichen enthaltenen, bindigen Böden wird durch die tatsächliche Kohäsion $\mathrm{c}_{\text {dyn }}$ durch die Verwendung der quasistatisch ermittelten Kohäsion c' deutlich unterschätzt. Vorteilhaft ist in diesem Zusammenhang jedoch, dass der Reibungsanteil der Scherfestigkeit $\mathrm{T}_{\mathrm{c}}$ in den untersuchten Homogenbereichen dem Anteil aus der Kohäsion aufgrund der hohen Überlagerungen $\mathrm{H}_{\mathrm{TA}}$ von 12,5 - 48,0 m deutlich überwiegt. Dadurch verringert sich der Einfluss des systematischen Fehlers im Anteil der Kohäsion auf das Gesamtergebnis der Scherfestigkeit $\mathrm{T}_{\mathrm{c}}$ deutlich. Insgesamt ist im vorliegenden Fall also vermutlich nur ein geringer Einfluss der systematischen Fehler durch die Verwendung der statischen Scherparameter in Gl. 54 gegeben.

Ein deutlich größeres Problem für die vorliegende Anwendung stellt die Definition der Normalspannung $\sigma_{n}$ in Gl. 54 dar. Selbst bei deutlich vereinfachter Betrachtung des Abbauvorgangs ist weder für Schälmesser, noch für Schneidrollen eine eindeutige und für den Abrieb am Werkzeug maßgebliche Definition der in Gl. 54 zu Grunde liegenden Betrachtungsebene möglich.

Der für den Wechsel einer Schneidrolle maßgebliche Abrieb am Schneidring tritt auf Basis der Ergebnisse in Kap. 5.3.2.1 beim Bodenabbau sowohl seitlich am Schneidring, als auch in radialer Richtung auf. Die zugehörigen Schervorgänge im Boden und die entsprechenden Spannungszustände können zwar grundsätzlich in einer übereinstimmenden Ebene dargestellt werden. Allerdings erfolgt der zugehörige Schervorgang in unterschiedlicher Richtung. Damit kann keine eindeutige maßgebliche Normalspannung $\sigma_{n}$ definiert werden.

Für die Anwendung auf Schälmesser ergibt sich ein ähnliches Problem. Hier tritt der für den Wechsel eines Schälmessers maßgebliche Abrieb sowohl an der Frontfläche, als auch an der Rückenfläche des Schälmessers auf (Kap. 5.3.3). Auf Basis der von BERETITSCH (1992: 110) beschriebenen Bruchkörperform ist auch hier keine vereinfachte Betrachtung des Spannungszustandes in einer Ebene möglich, die eine eindeutige Festlegung der Normalspannung $\sigma_{\mathrm{n}}$ erlaubt. 
Zusätzlich ist der bestehende Spannungszustand im Korngerüst des Bodens zu berücksichtigen. Während der Drehung der Werkzeuge mit dem Schneidrad ändert sich die Richtung der Schervorgänge beim Bodenabbau im Vergleich zur Richtung der bestehenden Hauptspannungsrichtungen. Diese sind theoretisch aus den im Ausgangszustand an der Ortsbrust überschlägig anliegenden Spannungskomponenten bestimmbar:

- $\sigma_{z z}:$ Vertikale Hauptnormalspannung parallel zur Ortsbrust

- $\sigma_{y y}$ : Horizontale Hauptnormalspannung senkrecht zur Ortsbrust

- $\sigma_{x x}$ : Horizontale Hauptnormalspannung parallel zur Ortsbrust

Der Betrag der vertikalen Spannungskomponente $\sigma_{z z}\left[\mathrm{kN} / \mathrm{m}^{2}\right]$ entspricht dabei den effektiven Spannungen auf Höhe der Tunnelachse TA. Diese wurden jeweils aus dem Schichtmodell in den Homogenbereichen mit Gl. 55 berechnet:

$$
\sigma_{\mathrm{zz}}=\sum_{1}^{\mathrm{i}} \gamma_{\mathrm{i}} \cdot \mathrm{H}_{\mathrm{i}}
$$

Der Index i entspricht hier den verschiedenen Schichten im Schichtmodell. Für die Wichte $\gamma_{i}$ wird entsprechend der Lage der Schicht ober- oder unterhalb des Wasserspiegels $W_{T A}$ die Feuchtwichte $Y$ oder die Wichte unter Auftrieb $\mathrm{Y}^{\mathrm{k}}\left[\mathrm{kN} / \mathrm{m}^{3}\right]$ eingesetzt. Der Wert $\mathrm{H}_{\mathrm{i}}[\mathrm{m}]$ gibt die Mächtigkeit der Schicht an. Die Summe aller $\mathrm{H}_{\mathrm{i}}$ muss damit der Überlagerung $\mathrm{H}_{\mathrm{TA}}[\mathrm{m}]$ auf Höhe der Tunnelachse entsprechen.

Zusätzliche Auflasten, z.B. durch vorhandene Bebauung, müssen gegebenenfalls zusätzlich berücksichtigt werden. Der Betrag der horizontalen Spannungskomponente $\sigma_{x x}\left[\mathrm{kN} / \mathrm{m}^{2}\right]$ parallel zur Ortsbrust kann über den Erdruhedruckbeiwert $\mathrm{k}_{0}$ aus $\sigma_{\mathrm{zz}}$ abgeschätzt werden:

$$
\sigma_{\mathrm{xx}} \approx \sigma_{\mathrm{zz}} \cdot \mathrm{k}_{0}=\sigma_{\mathrm{zz}} \cdot\left(1-\sin \varphi^{\prime}\right)
$$

Der Betrag der horizontalen Spannungskomponente $\sigma_{y y}\left[\mathrm{kN} / \mathrm{m}^{2}\right]$ senkrecht zur Ortsbrustfläche entspricht mindestens dem Betrag, der sich aus Gl. 56 für $\sigma_{x x}$ ergibt. Im Vortrieb ergeben sich durch die Stützung der Ortsbrust und den Abbauvorgang jedoch zusätzliche Einflüsse. Der tatsächliche Spannungsbetrag, der aus dem Stützdruck $P_{S F}$ über den Filterkuchen an der Ortsbrust und die Strömungskräfte beim Einströmen der Stützflüssigkeit in die Ortsbrust auf das Korngerüst übertragen wird, liegt im Regelfall etwas höher als der Wert aus Gl. 56. Der maximale Betrag von $\sigma_{x x}$ entspricht bei vollständiger Versiegelung der Ortsbrust dem Stützdruck $P_{\mathrm{SF}}$.

$$
\sigma_{\mathrm{xx}} \leq \sigma_{\mathrm{yy}} \leq \mathrm{P}_{\mathrm{SF}}
$$

Der Porenwasserdruck u [kN/m²] entspricht beim Bodenabbau in der Penetrationstiefe der Werkzeuge etwa dem Stützdruck $\mathrm{P}_{\mathrm{SF}}$. Messungen des Porenwasserdrucks in wassergesättigten Sanden mit einer Durchlässigkeit von $10^{-4} \mathrm{~m} / \mathrm{s}$ und systematische Schwankungen des Wasserspiegels $W_{T A}$ in der Umgebung der Ortsbrust im Regelvortrieb in wassergesättigten Sanden und Kiesen bis zu einer Durchlässigkeit von $1 \cdot 10^{-6} \mathrm{~m} / \mathrm{s}$ bestätigen diese Annahme (Kap. 3.2.4). Die Durchlässigkeits-beiwerte in den betrachteten Homogenbereichen liegen mit einer Ausnahme ebenfalls bei $\geq 10^{-6} \mathrm{~m} / \mathrm{s}$. Die Ausnhame bildet ein HGB in dem stark tonige, sandige Schluffe aufgefahren wurden. Hier liegt der Durchlässigkeitswert im Bereich von $10^{-9} \mathrm{~m} / \mathrm{s}$. Obige Annahme wurde auch für diesen HGB beibehalten, obwohl in bindigen Böden vermutlich ein stärkerer Druckgradient vom Stützdruck in der Abbaukam- 
mer hin zum natürlichen Porenwasserdruck im Boden hinter der Ortsbrust herrscht. Der Porenwasserdruck ist damit vermutlich bereits in der Penetrationstiefe der Werkzeuge niedriger als der Stützdruck.

Zusammenfassend kann aufgrund der genannten Ursachen auf analytischem Weg aus den tatsächlichen Schervorgängen beim Bodenabbau keine eindeutige und für alle Werkzeugtypen geeignete Normalspannung $\sigma_{n}$ zur Verwendung im Bruchkriterium nach MOHR-CoULOMB in Gl. 54 festgelegt werden. Eine detaillierte Aufarbeitung zu den tatsächlichen Kontaktspannungen Tact für Schneidrollen und Schälmesser erfordert Untersuchungen analog zu den bisher untersuchten Modellen (Kap. 4.2.1). Diese sind aufgrund des Umfangs nicht Bestandteil der vorliegenden Arbeit.

Zur weiteren Analyse des Einflusses der Bodeneigenschaften auf die Standgrößen wurde deshalb für die Anwendung in Gl. 54 die vereinfachte Annahme getroffen, dass die Normalspannung $\sigma_{n}$ proportional zur primären vertikalen Hauptnormalspannung $\sigma_{z z}$ in der Ortsbrust ist:

$$
\sigma_{\mathrm{n}} \sim \sigma_{\mathrm{zz}}
$$

Mit steigendem vertikalem Spannungsanteil $\sigma_{z z}$ ergibt sich folglich aus Gl. 54 eine steigende Scherfestigkeit $\mathrm{T}_{\mathrm{c}}$ des Bodens im Moment des Abbaus. Da in den Referenzprojekten überwiegend Böden mit geringer Kohäsion bei hoher Überlagerung $\mathrm{H}_{\mathrm{TA}}$ aufgefahren werden, ist auch die Scherfestigkeit $\mathrm{T}_{\mathrm{c}}$ überschlägig proportional zur primären vertikalen Hauptnormalspannung $\sigma_{z z}$ (Gl. 59):

$$
\tau_{\mathrm{c}} \sim \sigma_{\mathrm{zz}}
$$

Für die weitere Untersuchung des Einflusses der Bodeneigenschaften wurde darauf aufbauend angenommen, dass die Scherfestigkeit $\mathrm{T}_{\mathrm{c}}$ proportional zu den tatsächlichen Kontaktspannungen $\mathrm{T}_{\mathrm{act}}$ ist:

$$
\tau_{\mathrm{c}} \sim \tau_{\mathrm{act}}
$$

Diese Annahme kann anhand der Betriebsdaten der TVM in den RP überprüft werden. Das Schneiddrehmoment $M_{S R}$ und die Schneidradanpresskraft $F_{S R}$ stellen die Summe der resultierenden Schneidkräfte $F_{\mathrm{TC}}$ bzw. $F_{\mathrm{SC}}$ der Abbauwerkzeuge parallel und senkrecht zur Ortsbrust dar (Kap. 3.3.1) und bilden die Kontaktspannungen $\mathrm{T}_{\mathrm{act}}$ an den Werkzeugoberflächen beim Bodenabbau ab:

$$
\begin{gathered}
\mathrm{M}_{\mathrm{SR}} \sim \tau_{\mathrm{act}} \\
\mathrm{F}_{\mathrm{SR}} \sim \tau_{\mathrm{act}}
\end{gathered}
$$

Wenn die Annahme in Gl. 60 zutrifft und die mit Gl. 54 abgeschätzte Scherfestigkeit $\mathrm{T}_{\mathrm{c}}$ des Bodens in der Ortsbrust proportional zu den Kontaktspannungen $\mathrm{T}_{\text {act }}$ an den Abbauwerkzeugen ist, müsste sich folglich auch eine Korrelation zwischen dem Schneiddrehmoment $\mathrm{M}_{\mathrm{SR}}$, der Schneidradanpresskraft $\mathrm{F}_{\mathrm{SR}}$ und der Scherfestigkeit $\mathrm{T}_{\mathrm{C}}$ ergeben:

$$
\mathrm{M}_{\mathrm{SR}} \sim \tau_{\mathrm{c}}
$$

$$
\mathrm{F}_{\mathrm{SR}} \sim \tau_{\mathrm{c}}
$$


Zur Darstellung der Korrelationen in Gl. 63 und Gl. 64 können das Schneiddrehmoment $M_{S R}$ und die Schneidradanpresskraft $F_{S R}$ theoretisch mit Gl. 6 bzw. Gl. 12 (Kap. 3.3.1) aus im Vortrieb gemessenen Werten für das Schneidraddrehmoment $\mathrm{M}_{\mathrm{TVM}}$ und der Kraft an der Schneidradverschiebung $\mathrm{F}_{\mathrm{SV}}$ berechnet werden. Im Hinblick auf das Schneidraddrehmoment $\mathrm{M}_{\mathrm{TVM}}$ ist jedoch keine Berechnung der dazu notwendigen Anteile aus dem Eigendrehmoment des Schneidrades $M_{E S}$, den Rührverlusten $M_{R S}$ und den Reibungsverlusten $\mathrm{M}_{\mathrm{ME}}$ möglich. Für die weitere Analyse wurde deshalb das unkorrigierte Schneidraddrehmoment $\mathrm{M}_{\mathrm{TVM}}[\mathrm{kNm}]$ eingesetzt. Von der Kraft an den Verschiebezylindern $\mathrm{F}_{\mathrm{SV}}$ kann mithilfe von Gl. 6 und dem im Vortrieb aufgezeichneten Stützdruck $P_{S F}$ die Stützkraft $F_{S B}[k N]$ aus der Fläche der Schneidradbefestigung abgetrennt werden. Eine rechnerische Ermittlung der Kraft aus mechanischer Stützung zwischen Schneidrad und Ortsbrust $F_{M E}$ und der Reibungskraft $F_{R A}$ ist jedoch nicht möglich. Für die weitere Analyse wurde für die Schneidradanpresskraft $F_{S R}$ deshalb die Differenz aus der Kraft an den Verschiebezylindern $F_{S V}$ und der Stützkraft $F_{S B}$ eingesetzt.

In den Referenzprojekten liegen so aus den geotechnischen Homogenbereich jeweils Wertepaare für die Scherfestigkeit $T_{c}$, das Schneiddrehmoment $M_{S R}$ und die Schneidradanpresskraft $F_{S R}$ vor, die zur Untersuchung der angenommenen Korrelationen in Gl. 63 und Gl. 64 herangezogen werden können. DüLLMANN et al. (2013: 624) und HOLLMANN et al. (2013: 639) weisen hierzu darauf hin, dass die Schneidradanpresskraft $F_{S R}$ und das Schneiddrehmoment $M_{S R}$ neben den geotechnischen Bedingungen zusätzlich von weiteren Faktoren abhängig sind:

- Schneidraddurchmesser $\mathrm{D}_{\mathrm{TVM}}[\mathrm{m}]$

- Schneidradöffnungsverhältnis $\mathrm{O}_{T V M}[\%]$

- Werkzeugbestückung des Schneidrades

- Verschleißzustand der Abbauwerkzeuge

- Penetration $\mathrm{p}[\mathrm{mm} / \mathrm{U}]$

- Verklebungen \& Hindernisse

Die genannten Faktoren unterscheiden sich in den Referenzprojekten zwischen den betrachteten Maschinen (Tab. 8) und geotechnischen Homogenbereichen erheblich. HolLMANN et al. (2013: 12) schlagen für ähnliche Untersuchungen vor, gezielt einzelne Bereiche zu vergleichen, in denen sich jeweils nur einer der genannten Einflussfaktoren verändert. Übertragen auf die vorliegende Fragestellung wären also geotechnische Homogenbereiche notwendig, zwischen denen sich ausschließlich die Scherfestigkeit $T_{C}$, das Schneiddrehmoment $M_{S R}$ und die Schneidradanpresskraft $F_{S R}$ unterscheiden. Gleichzeitig müssten alle weiteren genannten Parameter konstant sein. Entsprechende Vergleichsmöglichkeiten liegen jedoch innerhalb der Referenzprojekte selbst bei Betrachtung einzelner Vortriebe nicht vor, weil sich insbesondere die Penetration $\mathrm{p}$ zwischen den geotechnischen Homogenbereichen häufig signifikant unterscheidet (Kap. 5.6.1.3). Um das vorhandene Datenvolumen über die Referenzprojekte hinweg zur Untersuchung der Korrelationen in Gl. 63 und Gl. 64 nutzen zu können, wurde deshalb ein Ansatz entwickelt, um das Schneiddrehmoment $M_{S R}$ und die Schneidradanpresskraft $F_{S R}$ auf eine Teil der von DüLLMANN et al. (2013: 624) genannten Faktoren zu normieren.

Aus dem Schneiddrehmoment $M_{S R}[k N m]$ ergibt sich das normierte Schneiddrehmoment $M_{\text {norm }}[k N m]$ unter Berücksichtigung des Schneidraddurchmessers $D_{\text {TVM }}[\mathrm{m}]$ und der Penetration $\mathrm{p}[\mathrm{mm} / \mathrm{U}]$ aus Gl. 65:

$$
M_{\text {norm }}=M_{S R} \cdot \frac{1}{D_{\mathrm{TVM}}^{2} \cdot \frac{\pi}{4}} \cdot \frac{1}{p}
$$


Die normierte Schneidradanpresskraft $F_{\text {norm }}$ wird analog mit Gl. 66 unter Berücksichtigung des Schneidraddurchmessers $D_{\text {TVM }}[\mathrm{m}]$ und der Penetration $\mathrm{p}[\mathrm{mm} / \mathrm{U}]$ aus der Schneidradanpresskraft $F_{\mathrm{SR}}$ $[\mathrm{kN}]$ berechnet:

$$
\mathrm{F}_{\text {norm }}=\mathrm{F}_{\mathrm{SR}} \cdot \frac{1}{\mathrm{D}_{\mathrm{TVM}}^{2} \cdot \frac{\pi}{4}} \cdot \frac{1}{\mathrm{p}}
$$

Die Normierung bezieht in beiden Anwendungsfällen den Schneidraddurchmesser $D_{\text {TVM }}$ und die Penetration $p$ ein. Das Schneidradöffnungsverhältnis $\mathrm{O}_{\text {TVM }}[\%]$ wurde in der Normierung nicht berücksichtigt, weil dieses in den betrachteten Referenzprojekten nur eine relativ geringen Schwankungsbreite von $26-31 \%$ besitzt. Das normierte Schneiddrehmoment $\mathrm{M}_{\text {norm }}[\mathrm{kNm}]$ und die normierte Schneidradanpresskraft $F_{\text {norm }}[\mathrm{kN}]$ können anschließend gegen die Scherfestigkeit $\mathrm{T}_{\mathrm{c}}\left[\mathrm{kN} / \mathrm{m}^{2}\right]$ angetragen werden. Dabei werden die Daten nach verschiedenen Schneidradtypen und Werkzeugbestückungsvarianten unterschieden. Zusätzlich sind Datensätze mit Hinweisen auf Verklebungen in bindigen Böden separat ausgewiesen. Der tatsächliche Verschleißzustand der Abbauwerkzeuge kann aufgrund mangelnder Quantifizierungsmöglichkeiten und der Datenverfügbarkeit nicht in die Normierung mit einbezogen werden und bleibt damit unberücksichtigt. Die Daten für die normierte Schneidradanpresskraft $F_{\text {norm }}$ sind in Abb. 84 dargestellt. Die Daten für das normierte Schneiddrehmoment $M_{\text {norm }}$ sind in Abb. 84 angegeben.

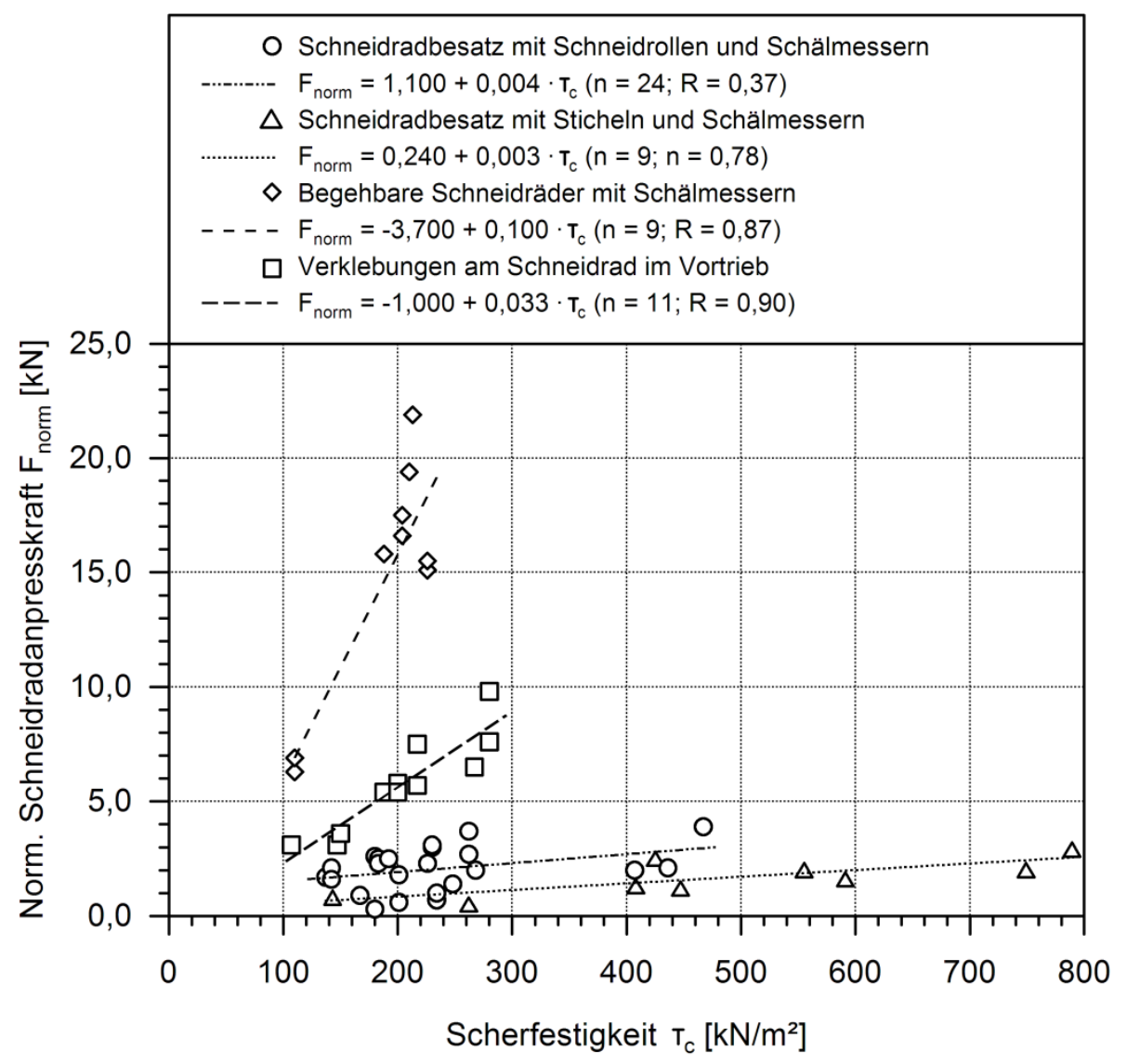

Abb. 84: Korrelation der normierten Schneidradanpresskraft $F_{\text {norm }}[\mathrm{kN}]$ mit der Scherfestigkeit $\mathrm{T}_{\mathrm{c}}\left[\mathrm{kN} / \mathrm{m}^{2}\right]$ des Bodens in der Ortsbrust für unterschiedlichen Schneidradtypen und Bestückungsvarianten mit Schneidrollen und Sticheln. 


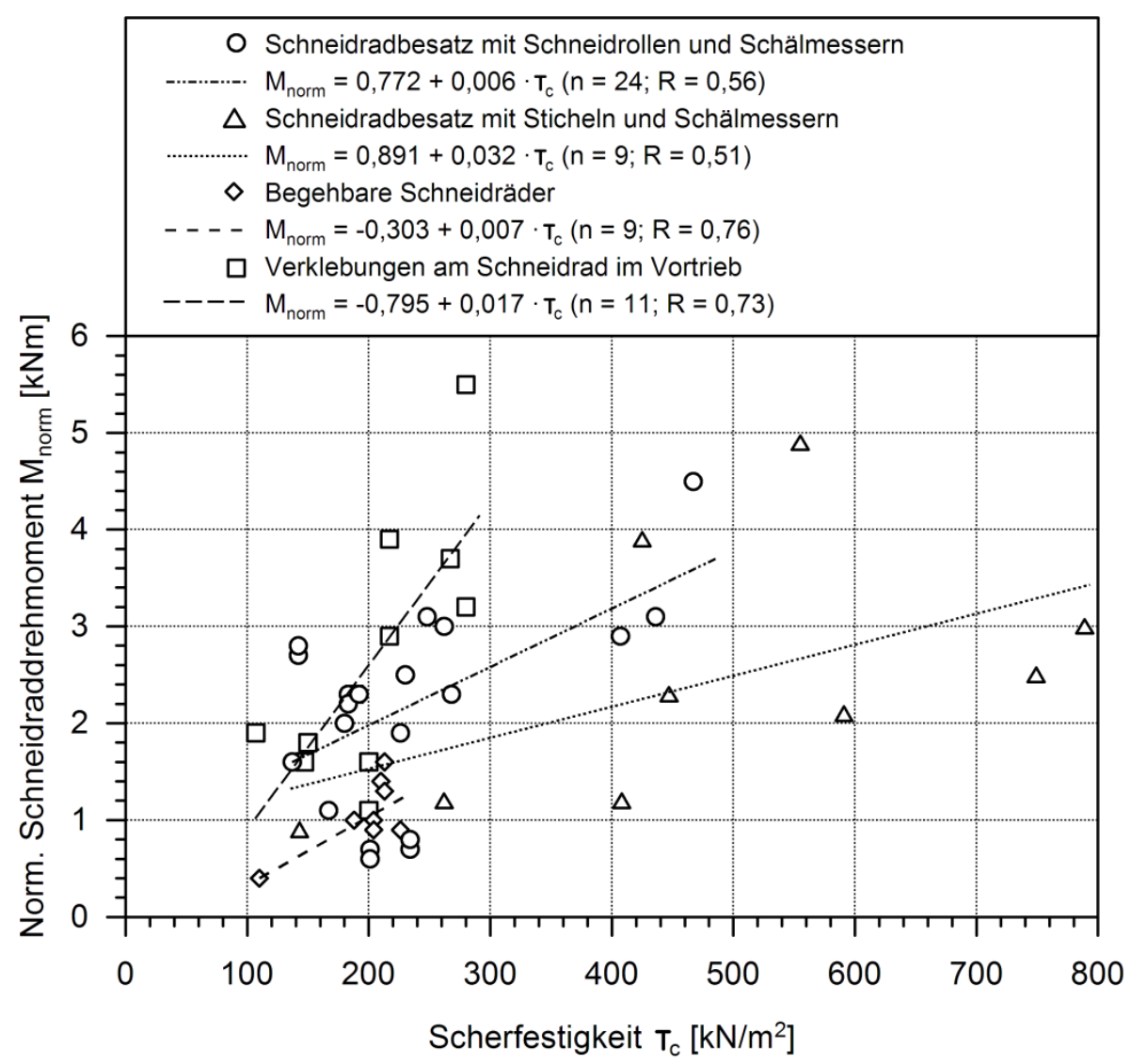

Abb. 85: Korrelation des normierten Schneiddrehmomentes $M_{\text {norm }}[k N m]$ mit der Scherfestigkeit $T_{c}\left[k N / m^{2}\right]$ des Bodens in der Ortsbrust für unterschiedlichen Schneidradtypen und Bestückungsvarianten mit Schneidrollen und Sticheln.

Die Verteilung der Datenpunkte in Abb. 84 und Abb. 85 zeigt für die vier dargestellten Schneidradund Werkzeugbestückungsvarianten jeweils einen Trend zu steigender normierter Schneidradanpresskraft $F_{\text {norm }}$ bzw. steigendem normiertem Schneiddrehmoment $M_{\text {norm }}$ mit steigender Scherfestigkeit $T_{c}$. Zwischen den angetragenen Größen ergeben sich überwiegend mittlere bis hohe Werte für den Korrelationskoeffizienten $\mathrm{R}$ von 0,37 - 0,90. In Abb. 84 und Abb. 85 wurde deshalb eine lineare Interpretation des Zusammenhangs zwischen der Scherfestigkeit $\mathrm{T}_{\mathrm{c}}$ und normierter Schneidradanpresskraft $F_{\text {norm }}$ bzw. normiertem Schneiddrehmoment $M_{\text {norm }}$ vorgenommen. Die zugehörigen Regressionslinien sind in Abb. 84 und Abb. 85 eingetragen. Die Ursachen für die zum Teil hohe Schwankungsbreite innerhalb der dargestellten Datensätze sind einerseits die fehlenden Möglichkeiten zur vollständigen Abtrennung der Schneidradanpresskraft $F_{S R}$ und des Schneiddrehmomentes $M_{S R}$ aus den gemessenen Werten für die Kraft an den Schneidradverschiebezylindern $F_{S V}$ und dem gemessenen Schneidraddrehmoment $\mathrm{M}_{\mathrm{TVM}}$. Andererseits wird ein Teil der Schwankungsbreite vermutlich auch durch den tatsächlichen Verschleißzustand der Abbauwerkzeuge verursacht, der in der Normierung in Gl. 65 und Gl. 66 nicht berücksichtigt werden konnte.

Der Trend $z u$ steigender normierter Schneidradanpresskraft $F_{\text {norm }}$ bzw. steigendem normiertem Schneiddrehmoment $\mathrm{M}_{\text {norm }}$ mit steigender Scherfestigkeit $\mathrm{T}_{\mathrm{c}}$. entspricht qualitativ dem erwarteten $\mathrm{Zu}$ sammenhang zwischen den Scherparametern des Bodens in der Ortsbrust, den Schneidkräften $\mathrm{F}_{\mathrm{TC}}$ bzw. $F_{S C}$ (Kap. 4.2.1) und der Schneidradanpresskraft $F_{S R}$ bzw. dem Schneiddrehmoment $M_{S R}$ (Kap. 3.3.1). Die Linearität des Zusammenhangs zwischen der Scherfestigkeit $\mathrm{T}_{\mathrm{c}}$ und der normierter Schneidradanpresskraft $F_{\text {norm }}$ bzw. dem normierten Schneiddrehmoment $M_{\text {norm }}$ bestätigen unabhängig von der Abbauwerkzeugbestückung des Schneidrades und der Schneidradauslegung die in Gl. 63 und Gl. 64 getroffene Annahme der Proportionalität zwischen der Scherfestigkeit $T_{c}$ und der Schneidradanpresskraft $\mathrm{F}_{\mathrm{SR}}$ und dem Schneiddrehmoment $\mathrm{M}_{\mathrm{SR}}$. 
Aufgrund der in Gl. 61 und Gl. 62 formulierten Proportionalität zwischen Schneidradanpresskraft $F_{S R}$ bzw. Schneiddrehmoment $M_{S R}$ und der Kontaktspannungen $T_{a c t}$ kann daraus geschlossen werden, dass auch eine Proportionalität zwischen der Scherfestigkeit $\mathrm{T}_{\mathrm{c}}$ des Bodens in der Ortsbrust und den Spannungen $T_{\text {act }}$ an den Werkzeugoberflächen besteht. Die Annahme in Gl. 60 kann über die dargestellten Zusammenhänge also bestätigt werden. Die Scherfestigkeit $\mathrm{T}_{\mathrm{c}}$ wird deshalb im Folgenden als indikativer Vergleichswert für die Spannungen $T_{\text {act }}$ an den Werkzeugoberflächen angesetzt. Ein quantitativer Rückschluss aus der Scherfestigkeit $\mathrm{T}_{\mathrm{c}}$ auf den absoluten Betrag der Spannungen $\mathrm{T}_{\mathrm{act}}$ ist jedoch nicht möglich.

Darüber hinaus ergeben die Datensätze Abb. 84 und Abb. 85 weitere Hinweise zum Abbauvorgang. Für Abbauwerkzeugbestückungen mit Schneidrollen und Sticheln steigt sowohl die normierte Schneidradanpresskraft $F_{\text {norm }}$, als auch das normierte Schneiddrehmoment $M_{\text {norm }}$ im annähernd selben $\mathrm{Maß}$ mit der Scherfestigkeit an. Die Werte für Abbauwerkzeugbestückungen mit Schneidrollen liegen dabei jedoch konstant höher als die Werte für Bestückungen mit Sticheln. Beim Bodenabbau mit Sticheln entstehen also unabhängig von der Scherfestigkeit $T_{c}$ des Bodens vermutlich geringere Schneidkräfte $F_{\mathrm{TC}}$ und $\mathrm{F}_{\mathrm{SC}}$ als beim Abbau mit Schneidrollen. Diese Differenz sollte in zukünftigen Projekten als zusätzliches Argument in die konkrete Entscheidung zum Einsatz von Sticheln oder Schneidrollen einbezogen werden.

Bei Bestückung des Schneidrades mit Schneidrollen liegen die Werte für die normierte Schneidradanpresskraft $F_{\text {norm }}$ und das normierte Schneiddrehmoment $M_{\text {norm }}$ in Bereichen mit Verklebungen am Schneidrad deutlich über den vergleichbaren Werten in Bereichen ohne Verklebungen. Die Daten belegen folglich den negativen Einfluss von Verklebungen auf den Vortrieb (Kap. 4.2.2.1).

Die Daten für die normierte Schneidradanpresskraft $F_{\text {norm }}$ und das normierte Schneiddrehmoment $M_{\text {norm }}$ für begehbare Schneidräder unterscheiden sich maßgeblich von den Daten für normale Schneidräder mit Schneidrollen oder Sticheln. Die normierte Schneidradanpresskraft $F_{\text {norm }}$ liegt bei begehbaren Schneidrädern deutlich höher als bei normalen Schneidrädern und steigt auch mit der Scherfestigkeit $\mathrm{T}_{\mathrm{c}}$ deutlich stärker an. Das normierte Schneiddrehmoment $\mathrm{M}_{\text {norm }}$ liegt bei begehbaren Schneidrädern deutlich niedriger als bei normalen Schneidrädern und steigt annähernd im selben Maß mit der Scherfestigkeit an. Diese Unterschiede werden vermutlich durch die konstruktive Auslegung des Schneidrades und des Schneidradantriebes verursacht. Der Zugang zu den Schneidradarmen bedingt einen vergleichsweise hohen Durchmesser des Schneidradantriebs. Die begehbaren Schneidradarme führen zu einer deutlich abweichenden Verteilung der Schneidradöffnungen. Gleichzeitig sind die betrachteten Schneidräder ausschließlich mit Schälmessern ohne vorlaufende Schneidrollen oder Stichel ausgerüstet. Diese Faktoren können in Summe die Unterschiede zu normalen Schneidrädern vermutlich erklären. Eine detaillierte Untersuchung ist jedoch nicht Ziel der vorliegenden Arbeit und wird deshalb nicht weiter verfolgt.

\subsubsection{Korngrößenverteilung, Kornform und Rundungsgrad}

Der Korngrößenverteilung des Bodens wird in nahezu allen bisherigen Untersuchungen zu den Einflussfaktoren auf den Abrieb an den Abbauwerkzeugen genannt (Tab. 4). Die Kornform- und der Kornrundungsgrad werden mit direktem Bezug zum Abrieb an den Abbauwerkzeugen von SCHOLZ \& WENDL (2010: 3513) angeführt. Die Bedeutung der Korngrößenverteilung und des Kornrundungsgrades für den Abrieb an den Abbauwerkzeugen wird zusätzlich durch die Abhängigkeit des LAK [g/t] vom Korndurchmesser $D_{50}[\mathrm{~mm}]$ und dem Rundungsgrad untermauert. Ähnliche Abhängigkeiten der Einschätzung der Abrasivität des Bodens von der Korngröße und Kornrundungsgrad ergeben sich auch für die weiteren beschriebenen Indextests (Kap. 4.3.1.5).

Bei der Untersuchung des Einflusses der Boden- und Gebirgseigenschaften auf den Laufweg $\mathrm{s}_{\mathrm{c}}$ müssen deshalb theoretisch alle drei genannten Kenngrößen berücksichtigt werden:

- Korngrößenverteilung

- Kornform

- Kornrundungsgrad 
Die Korngrößenverteilung wurde innerhalb der Referenzprojekte durchgehend in allen Projekten für die relevanten Bodenarten bestimmt und ist damit für die Analyse verfügbar (Tab. 12). Im Gegensatz dazu wurden Kornform und Kornrundungsgrad nur in zwei der Referenzprojekte bestimmt. Die beiden Parameter sind folglich für die Analyse nicht nutzbar. Die Untersuchung des Einflusses auf den Laufweg $s_{c}$ beschränkt sich deshalb im Folgenden auf die Korngrößenverteilung. Für zukünftige Projekte sind Kornform und Kornrundungsgrad jedoch in den Empfehlungen für die geotechnische Vorerkundung und Dokumentation (Tab. 11) enthalten.

Die Korngrößenverteilung ist nach DIN ISO TS 17892-4 eindeutig bestimmbar. Für die vorliegende Untersuchung ist die Korngröße durch einen einzelnen Kennwert zu beschreiben. Üblich sind dazu Angaben von Korndurchnmessern wie dem $D_{60}[\mathrm{~mm}]$ oder $D_{10}[\mathrm{~mm}]$, welche die Korngröße angeben, bei der die Kornsummenkurve die $60 \%$ bzw. 10\%-Linie überschreitet. Daraus lassen sich sowohl die dimensionslose Ungleichkörnigkeitszahl $\mathrm{C}_{u}$, als auch die Krümmungszahl $\mathrm{C}_{\mathrm{c}}$ errechnen. Für den $\mathrm{Ab}$ rieb an den Abbauwerkzeugen ist jedoch eher die absolute Korngröße von Bedeutung. Ein deutlicher Hinweis darauf ist die Abhängigkeit des LAK [g/t] vom $D_{50}[\mathrm{~mm}]$.

Für die vorliegende Analyse wurde auf Basis der oben genannten Ergebnisse der $D_{60}[\mathrm{~mm}]$ zur Beschreibung der Korngrößenverteilung eingesetzt, weil dieser für die Bestimmung der Krümmungszahl $\mathrm{C}_{\mathrm{c}}$ und der Ungleichkörnigkeitszahl $\mathrm{C}_{\mathrm{u}}$ ohnehin in der Vorerkundung erfasst werden muss.

\subsubsection{Entwicklung des Soil Abrasivity Index (SAl)}

Zusammenfassend wurden für die weitere Analyse des Einflusses der Boden- und Gebirgseigenschaften auf den Laufweg $\mathrm{s}_{\mathrm{c}}$ folgende Parameter ausgewählt:

- Abrasivität der Bodenkomponenten: Äquivalenter Quarzgehalt (ÄQu) [\%]

- Kontaktspannungen: Scherfestigkeit $\mathrm{T}_{\mathrm{c}}\left[\mathrm{kN} / \mathrm{m}^{2}\right]$

- Korngrößenverteilung: Korndurchmesser $D_{60}[\mathrm{~mm}]$

Um aus den drei genannten Parametern einen Index zu entwickeln, der mit dem Laufweg $\mathrm{s}_{\mathrm{c}}$ der $\mathrm{Ab}$ bauwerkzeuge korreliert werden kann, wurde zunächst die Bandbreite der auftretenden Werte innerhalb der Referenzprojekte, sowie die theoretisch in natürlichen Grenzen mögliche Bandbreite untersucht.

Für den Äquivalenten Quarzgehalt (ÄQu) liegt die Untergrenze der natürlich möglichen Bandbreite bei $0 \%$. Die Obergrenze ist weniger klar definiert. Für klastische Lockergesteine ist theoretisch ein $\ddot{A} Q u$ größer als $100 \%$ denkbar, weil einige, in der Erdkruste als Nebenbestandteile auftretende, Minerale wie z.B. Zirkon oder Topas eine absolute Schleifhärte n. RosIWAL deutlich >100 (Quarz) besitzen (Tab. 5). In seltenen Fällen können diese Minerale in klastischen Sedimenten in zum Teil abbauwürdiger Konzentration angereichert sein. Solche Anreicherungen spielen jedoch aufgrund der Seltenheit in der Praxis für den Vortrieb mit Hydroschild TVM keine Rolle. Quarz ist dagegen in der Erdkruste sehr häufig, sodass die praktisch realisierbare Obergrenze für den ÄQu vermutlich nahe an $100 \%$ liegt. 
Innerhalb der untersuchten Homogenbereiche liegt der erreichte ÄQu zwischen min. 25\% und max. 89\%. Die Verteilung des ÄQu in den untersuchten Homogenbereichen ist in Abb. 86 angegeben.

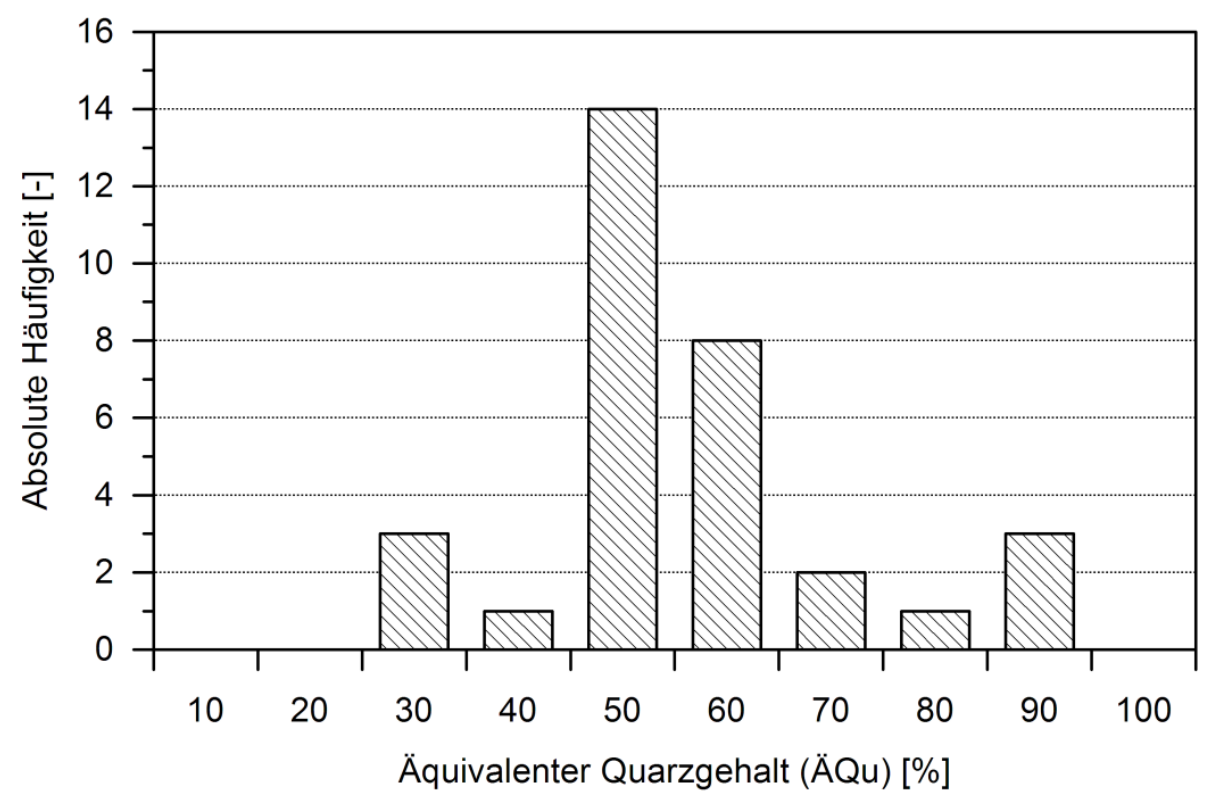

Abb. 86: Verteilung des Äquivalenten Quarzgehalts (ÄQu) [\%] in den 32 untersuchten geotechnischen Homogenbereichen.

Für die Scherfestigkeit $\mathrm{T}_{\mathrm{c}}\left[\mathrm{kN} / \mathrm{m}^{2}\right]$ sind die natürlich erreichbaren Grenzen deutlich schwerer anzugeben, da hierzu theoretische Anwendungsfälle als Berechnungsgrundlage definiert werden müssen, die etwa die aktuellen Anwendungsgrenzen von Hydroschild TVM wiedergeben. Als Untergrenze kann theoretisch ein Vortrieb in vollständig wassergesättigtem und kohäsionslosem Boden angenommen werden, bei dem die Oberkante des Ausbruchdurchmessers der Geländeoberkante (GOK) entspricht. Die Überlagerung $\mathrm{H}_{\mathrm{TA}}[\mathrm{m}]$ sinkt damit auf $50 \%$ des Schneidraddurchmessers $\mathrm{D}_{\mathrm{TVM}}$. Unter Annahme eines Reibungswinkels $\varphi^{\prime}$ von $25^{\circ}$, einer Wichte unter Auftrieb $\gamma^{\prime}$ von $10 \mathrm{kN} / \mathrm{m}^{3}$ und einem Ausbruchdurchmesser $D_{\text {TVM }}$ von 6,0 m ergibt sich daraus aus Gl. 54 und Gl. 55 für die Scherfestigkeit $T_{c}$ ein Minimalwert von ca. $13 \mathrm{kN} / \mathrm{m}^{2}$. Als Obergrenze können z.B. wassergesättigte überkonsolidierte glaziale Sedimente mit einer Kohäsion von ca. $10 \mathrm{kN} / \mathrm{m}^{2}$ und einem Reibungswinkel von $35^{\circ}$ angesetzt werden. Bei einer Überlagerung von $\mathrm{H}_{\mathrm{TA}}$ von $100 \mathrm{~m}$, einem Wasserspiegel $\mathrm{W}_{\mathrm{TA}}$ von $30 \mathrm{~m}$ und einer Wich-

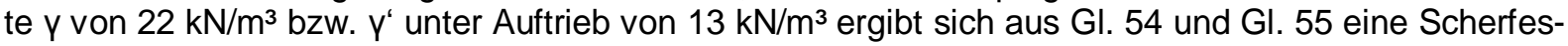
tigkeit $\mathrm{T}_{\mathrm{c}}$ von ca. $1.360 \mathrm{kN} / \mathrm{m}^{2}$. In den untersuchten Homogenbereichen ergibt sich eine Scherfestigkeit $\mathrm{T}_{\mathrm{c}}$ von $\min .107 \mathrm{kN} / \mathrm{m}^{2}$ und max. $789 \mathrm{kN} / \mathrm{m}^{2}$. Die Verteilung der Scherfestigkeit $\mathrm{T}_{\mathrm{c}}$ in den untersuchten Homogenbereichen ist in Abb. 87 angegeben. 


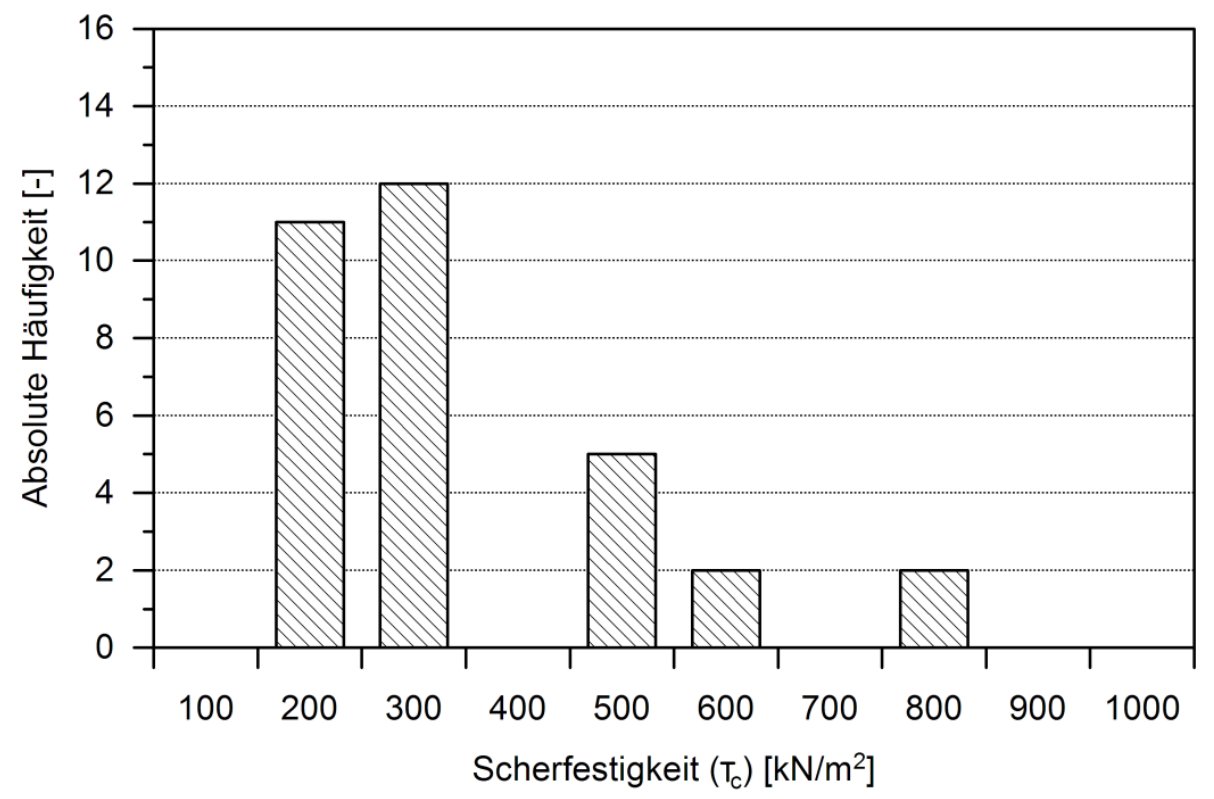

Abb. 87: Verteilung der Scherfestigkeit $\mathrm{T}_{\mathrm{c}}\left[\mathrm{kN} / \mathrm{m}^{2}\right]$ in den 32 untersuchten geotechnischen Homogenbereichen.

Für die Korngröße $D_{60}[\mathrm{~mm}]$ zur Beschreibung der Korngröße lassen sich die natürlich erreichbaren Grenzen deutlich leichter abschätzen. Die Untergrenze wird vermutlich in reinen Tonen erreicht. Hier kann der minimale $D_{60}$ mit ca. $0,001 \mathrm{~mm}$ abgeschätzt werden. Eine realistische Obergrenze für den $D_{60}$ lässt sich mit ca. $63 \mathrm{~mm}$ angeben. Das entspricht bereits sehr grobkörnigen Lockergesteinen, wie z.B. hochenergetischen, fluviatilen Sedimenten. Noch gröbere Lockergesteine bleiben damit zwar unberücksichtigt, spielen jedoch aufgrund ihrer Seltenheit auch nur eine untergeordnete Rolle für die vorliegende Untersuchung. Innerhalb der untersuchten Homogenbereiche liegt der erreichte $D_{60}$ bei min. 0,028 mm und max. 12,9 mm. Die Verteilung des $D_{60}$ in den untersuchten Homogenbereichen ist in Abb. 88 angegeben.

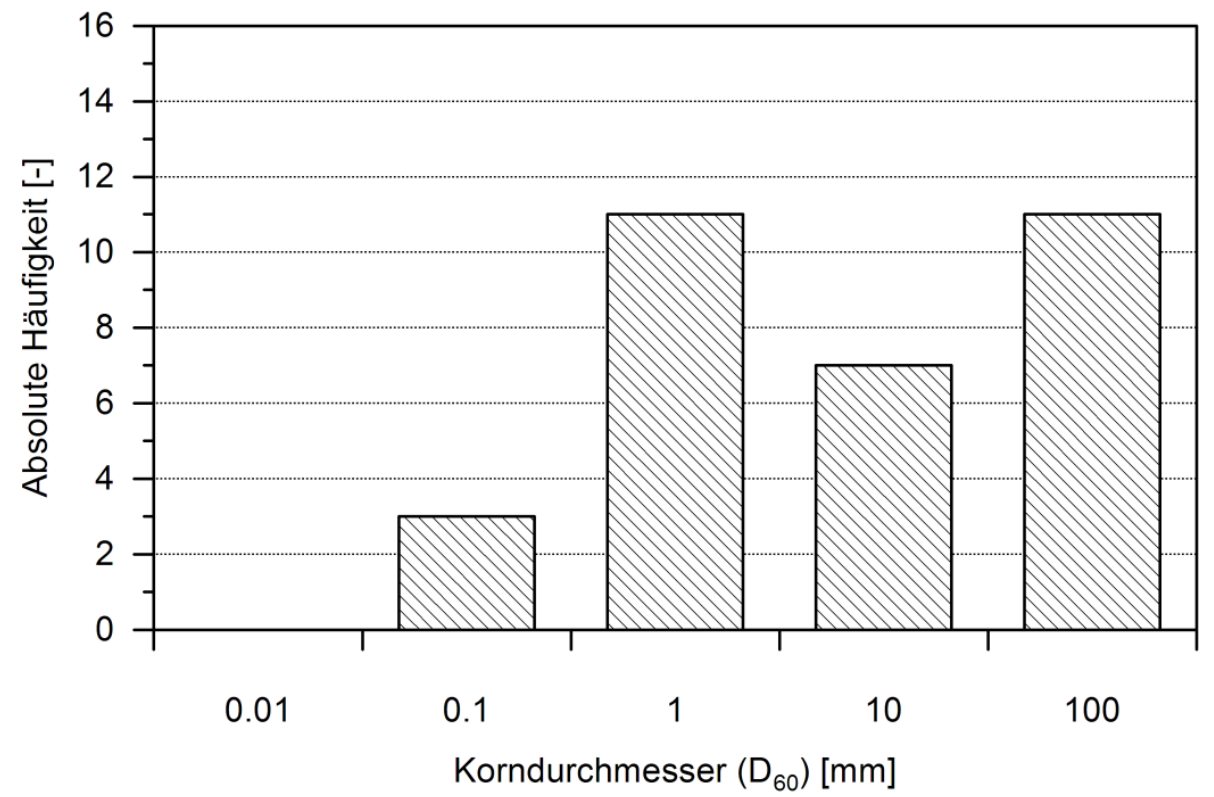

Abb. 88: Verteilung des Korndurchmessers $D_{60}[\mathrm{~mm}]$ in den 32 untersuchten geotechnischen Homogenbereichen. 
Zusammenfassend sind die natürlich erreichbaren Grenzen, sowie die in den Referenzprojekten realisierten Werte für den Äquivalenten Quarzgehalt [\%], die Scherfestigkeit $T_{c}\left[\mathrm{kN} / \mathrm{m}^{2}\right]$ und den Korndurchmesser $\mathrm{D}_{60}[\mathrm{~mm}]$ in Tab. 20 dargestellt.

Tab. 20: Vergleich der theoretisch möglichen Bandbreite von Äquivalentem Quarzgehalt (ÄQu) [\%], Scherfestigkeit $\mathrm{T}_{\mathrm{c}}\left[\mathrm{kN} / \mathrm{m}^{2}\right]$, Korndurchmesser $\mathrm{D}_{60}[\mathrm{~mm}]$ und der entsprechenden Bandbreite in den untersuchten Homogenbereichen (HGB).

\begin{tabular}{|l|c|c|}
\hline \multicolumn{1}{|c|}{ Kennwert } & Mögliche Bandbreite & Bandbreite in den HGB \\
\hline Äquivalenter Quarzgehalt (ÄQu) [\%] & $0-100 \%$ & $25-89 \%$ \\
\hline Scherfestigkeit $\mathrm{T}_{\mathrm{c}}[\mathrm{MPa}]$ & $13 \mathrm{kN} / \mathrm{m}^{2}-1.360 \mathrm{kN} / \mathrm{m}^{2}$ & $107 \mathrm{kN} / \mathrm{m}^{2}-789 \mathrm{kN} / \mathrm{m}^{2}$ \\
\hline Korndurchmesser $\mathrm{d}_{60} \mathrm{~mm}$ & $0,001 \mathrm{~mm}-63 \mathrm{~mm}$ & $0,028 \mathrm{~mm}-15,1 \mathrm{~mm}$ \\
\hline
\end{tabular}

Um die drei Kennwerte zu einem Indexwert für die Abrasivität des Bodens zu kombinieren, wurde zunächst eine einfache Multiplikation der drei Basiskennwerte in Betracht gezogen. Der Äquivalente Quarzgehalt hat im Vergleich zur Scherfestigkeit $\mathrm{T}_{\mathrm{c}}$ und dem Korndurchmesser $\mathrm{D}_{60}$ jedoch vermutlich einen höheren Einfluss auf den Laufweg $s_{c}$, weil der ÄQu sowohl den Primärverschleiß beim Bodenabbau beeinflusst, als auch für den Sekundärverschleiß durch den bereits abgebauten Boden in der Stützflüssigkeit von hoher Bedeutung ist. Dem gegenüber hat die Scherfestigkeit $\mathrm{T}_{\mathrm{c}}$ keinen und die Korngröße $D_{60}$ vermutlich geringeren Einfluss auf den Sekundärverschleiß durch den bereits abgebauten Boden. Eine Erhöhung des Einflusses des Äquivalenten Quarzgehalts im Vergleich zur Scherfestigkeit $T_{c}$ und der Korngröße $D_{60}$ in der Formulierung des Indexwertes erscheint deshalb sinnvoll. Der Äquivalente Quarzgehalt fließt dazu als Quadrat in den Indexwert in Gl. 67 ein, wobei sich aufgrund der Verwendung des ÄQu als Dezimalzahl durch Quadrieren keine Erhöhung der möglichen Schwankungsbreite (Tab. 20) ergibt.

Der so errechnete Indexwert für die Abrasivität des Bodens wurde abschließend als „Soil Abrasivity Index" (SAI) benannt. Die formale Berechnung des SAI ist definiert als:

$$
\mathrm{SAI}=\left(\frac{\mathrm{A} Q \mathrm{u}}{100}\right)^{2} \cdot \tau_{\mathrm{c}} \cdot \mathrm{D}_{60}
$$

Der SAI weist theoretisch die Dimension $\mathrm{N} / \mathrm{m}$ auf. Aufgrund des vollständig empirischen Charakters des Indexwertes sollte dieser jedoch als dimensionslos betrachtet werden. Auf Basis der möglichen Bandbreiten der Eingangswerte (Tab. 20) kann der SAI nach Gl. 67 theoretische Werte zwischen 0 und ca. 85.000 annehmen. Dem gegenüber ergeben sich aus den Referenzprojekten Werte zwischen 1 und 1.772. Die tatsächliche Wahrscheinlichkeitsverteilung des Äquivalenten Quarzgehalts ÄQu, der Scherfestigkeit $\mathrm{T}_{\mathrm{c}}$ und der Korngröße $\mathrm{D}_{60}$ über die in Tab. 20 angegeben Intervalle ist unbekannt. Folglich kann für den SAI keine exakte Wahrscheinlichkeitsverteilung bestimmt werden, anhand derer bestimmt werden könnte, welches Quantil des SAI durch die Projektdaten zwischen den Werten 1 und 1.772 abgedeckt wird.

Nimmt man für die drei Eingangsparameter überschlägig eine Wahrscheinlichkeitsverteilung an, kann daraus durch empirische Simulation auch die annähernde Wahrscheinlichkeitsverteilung des SAI bestimmt werden. Für den Äquivalenten Quarzgehalt (ÄQu) und die Scherfestigkeit $\mathrm{T}_{\mathrm{c}}$ wurde eine Normalverteilung angenommen, deren Mittelwert jeweils bei 50\% des in Tab. 20 angegebenen Intervalls liegt. Die Standardabweichung $\sigma$ wurde so gewählt, dass an der Obergrenze des Intervalls ein Wert von 0,99 für die kumulative Wahrscheinlichkeit erreicht wird. Die Standardabweichung $\sigma$ für ÄQu beträgt $21 \%$, für die Scherfestigkeit $288 \mathrm{kN} / \mathrm{m}^{2}$. Der Mittelwert der Korngröße $\mathrm{D}_{60}$ liegt unter realen Bedingungen vermutlich nicht bei $50 \%$ des in Tab. 20 angegebenen Intervalls. Der Wert für $\mathrm{D}_{60}$ läge hier bei $31,5 \mathrm{~mm}$. Dieser Wert liegt einerseits vermutlich deutlich zu hoch und würde bei Annahme einer Normalverteilung extrem grobkörnige Böden überrepräsentieren. Andererseits werden HydroschildTVM verfahrensbedingt tendenziell eher in gemischt- und grobkörnigen Böden eingesetzt. 
Die Annahme einer Normalverteilung für die Korngröße $D_{60}$ trifft hier also vermutlich nicht zu. Die reale Wahrscheinlichkeitsverteilung der Korngröße $D_{60}$ wird wird zutreffender durch eine links-steile Verteilung abgebildet, deren Modalwert in der Nähe der Grenze zwischen Sand und Kies bei ca. 2 mm liegt. Die Form der Verteilung wurde so gewählt, dass an der Untergrenze des Intervalls in Tab. 20 ein Wert für die kumulative Wahrscheinlichkeit von 0,01 und an der Obergrenze bei $63 \mathrm{~mm}$ ein Wert von 0,99 vorliegt.

Anschließend wurde für jede der drei Eingangsgrößen entsprechend der gewählten Wahrscheinlichkeitsverteilung 10.000 Zufallszahlen generiert und mit Gl. 67 zum Soil Abrasivity Index (SAI) verrechnet. Die resultierende Wahrscheinlichkeitsverteilung des SAI ist in Abb. 89 dargestellt.

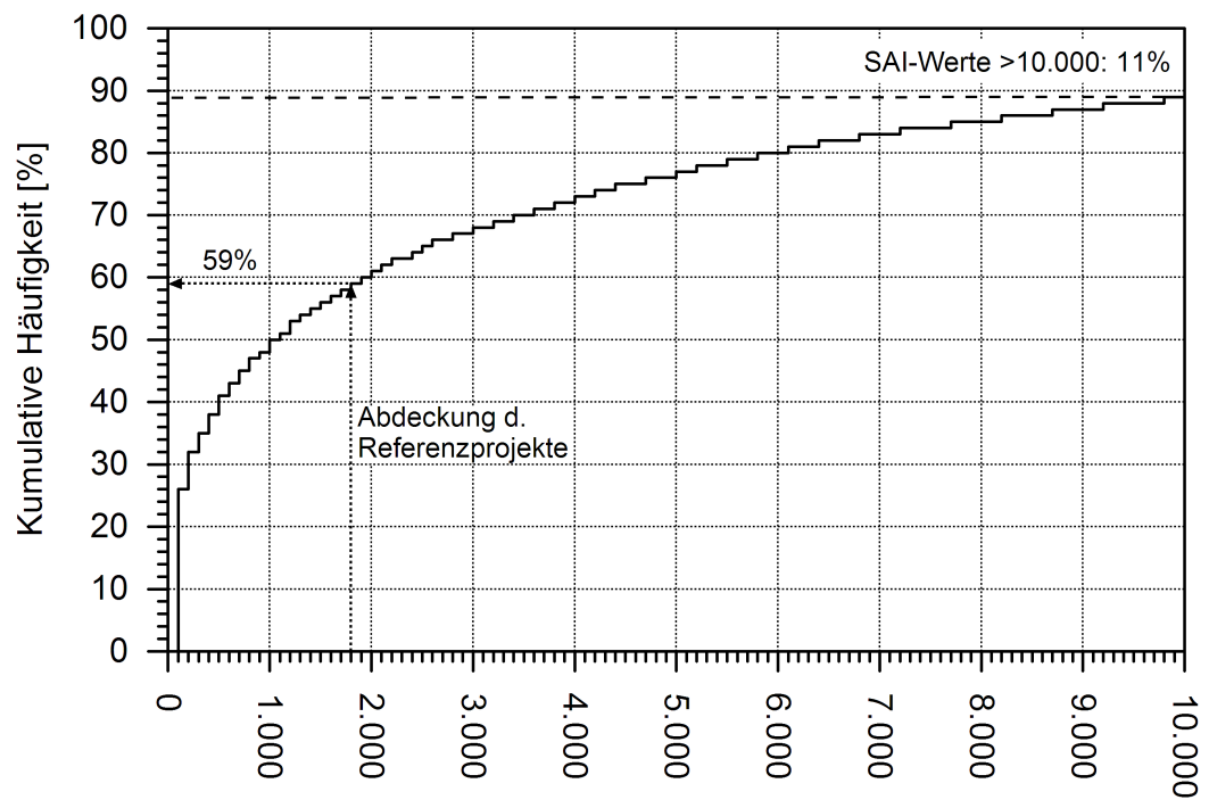

Soil Abrasivity Index (SAI) [-]

Abb. 89: Theoretische, kumulative Häufigkeitsverteilung des Soil Abrasivity Index (SAI) [-]. Die Referenzprojekte decken ca. $59 \%$ aller möglichen Werte für den Soil Abrasivity Index ab.

Die Anzahl der generierten Zufallszahlen stellt einen Kompromiss zwischen der erforderlichen Datenmenge und der zur Verfügung stehenden Rechenkapazität dar. Die Anzahl von 10.000 Kombinationen ist im Vergleich zur Anzahl und Bandbreite der Eingangswerte relativ gering, sodass sich in Abb. 89 keine saubere stetige Verteilungsform ergibt. Zusätzlich geht diese Methode zur Annäherung der Wahrscheinlichkeitsverteilung des SAI davon aus, dass die drei Eingangsgrößen unabhängig voneinander sind. Diese Annahme trifft vermutlich nicht vollständig zu, weil z.B. Abhängigkeiten zwischen der Korngröße $D_{60}$ und der Scherfestigkeit $T_{c}$ denkbar sind. Für die vorliegende Fragestellung ist die Anzahl der Zufallskombinationen und die Annahme der Unabhängigkeit jedoch ausreichend.

In der in Abb. 89 dargestellten Wahrscheinlichkeitsverteilung deckt der Bereich des SAI in den Referenzprojekten von 1 - 1.770 ein Quantil von ca. 59\% ab. Die Daten aus den Referenzprojekten bilden folglich einen hohen Anteil an real denkbaren Einsatzbedingungen von Hydroschild-TVM ab. 


\subsubsection{Korrelation von Soil Abrasivity Index (SAI) und Laufweg $\left(s_{c}\right)$}

Die Korrelation des Soil Abrasivity Index (SAI) [-] mit dem Laufweg $\mathrm{s}_{\mathrm{c}}[\mathrm{km}]$ der Abbauwerkzeuge wurde anschließend anhand von Schneidrollen, Schälmessern und Sticheln aus den vorliegenden Homogenbereichen untersucht. Dazu wurden zunächst sowohl für alle Werkzeugtypen Homogenbereiche ausgewählt, in denen möglichst übereinstimmende Rahmenbedingungen hinsichtlich der Auslegung der TVM und der Steuerung des Abbauprozesses vorliegen.

Für Schneidrollen wurden ausschließlich Homogenbereiche berücksichtigt, in denen die Schneidrollen folgenden Kriterien entsprechen:

- 17 Zoll 1-Ring Schneidrollen

- Schneidringe aus hochlegiertem Werkzeugstahl mit einer Härte von 57 HRC

Zusätzlich wurde die Breite der Schneidringe anhand der Ergebnisse in Kap. 5.6.2.4 auf $19 \mathrm{~mm}$ normiert, weil dieser Wert in den Referenzprojekten am häufigsten eingesetzt wurde. Die Datenanalyse zeigt, dass sich der Laufweg $\mathrm{s}_{\mathrm{c}}[\mathrm{km}]$ proportional mit der Breite der Schneidringe $\mathrm{b}_{\mathrm{SR}}[\mathrm{mm}]$ ändert. Die Normierung der Schneidringbreite $b_{\mathrm{SR}}$ kann folglich durch einen Korrekturfaktor vorgenommen werden, der sich aus dem Verhältnis der tatsächlichen Schneidringbreite $b_{S R}[\mathrm{~mm}]$ zum Referenzwert von 19 mm mit Gl. 68 ergibt:

$$
\mathrm{f}_{\mathrm{b}}=\frac{\mathrm{b}_{\mathrm{SR}}}{19 \mathrm{~mm}}
$$

Wird in Gl. 68 eine Schneidringbreite größer als $19 \mathrm{~mm}$ eingesetzt, nimmt der Korrekturfaktor $\mathrm{f}_{\mathrm{b}}$ einen Wert größer als eins an und der Laufweg $\mathrm{s}_{\mathrm{c}}$ wird im Verhältnis nach oben korrigiert. Wird in Gl. 68 eine Schneidringbreite kleiner als $19 \mathrm{~mm}$ eingesetzt, nimmt der Korrekturfaktor $\mathrm{f}_{\mathrm{b}}$ einen Wert kleiner als 1 an und der Laufweg $\mathrm{s}_{\mathrm{c}}$ wird im Verhältnis nach unten korrigiert.

Für die Auswahl der Datensätze für Schälmesser wurden die folgenden Kriterien angesetzt:

- Verschleißschutz von mindestens einer durchgehenden Schneide aus Hartmetall und $30 \%$ Abdeckung der Rückenfläche durch Hartmetalleinsätze

- Sekundärer Bodenabbau in Kombination mit vorlaufenden Schneidrollen

Der Laufweg $\mathrm{s}_{\mathrm{c}}$ für Schälmesser muss zusätzlich mithilfe der Ergebnisse in Kap. 5.6.3.2 auf die Penetration $p$ normiert werden. Als Referenzwert für die Normierung wurde der arithmetische Mittelwert der Penetration $p$ über alle in den Referenzprojekten enthaltenen Homogenbereiche gewählt. Dieser beträgt $16 \mathrm{~mm} / \mathrm{U}$. Zusätzlich muss bei der Normierung der tatsächliche Spurbesatz $\mathrm{k}_{\mathrm{s}}$ berücksichtigt werden, weil dieser Einfluss auf die tatsächliche Aktiv-Penetration $p_{a}[\mathrm{~mm} / \mathrm{U}]$ des einzelnen Schälmessers hat. Der exakte Wert für $p_{a}$ wurde anhand des Winkelabstandes $\delta_{a}$ der Schälmesser auf dem Schneidrad mit Gl. 40 bestimmt (Kap.5.4.5).

Für die Normierung wurde auf Basis der Ergebnisse in Tab. 17 jeweils eine durchschnittliche Zunahme des Laufwegs $\mathrm{s}_{\mathrm{c}}$ um den Faktor 1,6 bei Halbierung der Penetration $\mathrm{p}_{\mathrm{a}}$ angenommen. Daraus ergibt sich für Schälmesser aus Gl. 69 der Korrekturfaktor $\mathrm{f}_{\mathrm{p}}$ für die Penetration $\mathrm{p}_{\mathrm{a}}[\mathrm{mm} / \mathrm{U}]$ :

$$
f_{p}=\frac{1}{1,6^{\log _{0,5}\left(\frac{16}{p_{a}}\right)}}
$$


KÖPPL \& THURO (2013a: 57) und KÖPPL \& THURO (2013b: 1741) unterteilen diesen Schritt in zwei separate Korrekturen für die Penetration $p[\mathrm{~mm} / \mathrm{U}]$ und den Spurbesatz $\mathrm{k}_{\mathrm{s}}[-]$. Beide Faktoren bewirken indirekt eine Korrektur des Laufwegs $s_{c}$ um die tatsächliche Penetration $p_{a}$ der einzelnen Schälmesser, weil die Penetration $p$ in Abhängigkeit des Spurbesatzes $k_{s}$ und des Winkelabstandes auf diese aufgeteilt wird (Kap. 5.4.5). Die beiden Korrekturfaktoren können folglich bei direkter Berücksichtigung der Penetration $\mathrm{p}_{\mathrm{a}}$ zusammengefasst werden.

Der Term im Nenner von Gl. 69 ergibt für einen Penetration $p_{a}$ von $16 \mathrm{~mm} / \mathrm{U}$ den Wert 1, sodass der Korrekturfaktor $f_{p}$ in Summe den Wert 1 annimmt und der Laufweg $s_{c}$ nicht korrigiert wird. Für Penetrationswerte kleiner als $16 \mathrm{~mm} / \mathrm{U}$ nimmt der Exponent im Nenner von Gl. 69 einen negativen Wert an. Für den Nenner ergibt sich so ein Wert kleiner 1 , der Korrekturfaktor $f_{p}$ wird größer als 1 und der Laufweg $\mathrm{s}_{\mathrm{c}}$ wird nach oben korrigiert. Für Penetrationswerte größer als $16 \mathrm{~mm} / \mathrm{U}$ nimmt der Exponent im Nenner von Gl. 69 einen positiven Wert an. Für den Nenner ergibt sich so ein Wert größer 1. Der Korrekturfaktor $\mathrm{f}_{\mathrm{p}}$ wird kleiner als 1 und der Laufweg $\mathrm{s}_{\mathrm{c}}$ wird nach unten korrigiert.

Für Stichel wurden folgende Einschränkungen bei der Auswahl der Datensätze vorgenommen:

- Verschleißschutz von mindestens einer durchgehenden Schneide aus Hartmetall und $30 \%$ Abdeckung der Rückenfläche durch Hartmetalleinsätze

- Primärer Bodenabbau ohne Kombination mit Schneidrollen auf vorlaufenden oder angrenzenden Spuren

Analog zu den Schälmessern muss auch für Stichel der Laufweg $s_{c}$ mit Gl. 69 um die AktivPenetration $\mathrm{p}_{\mathrm{a}}$ korrigiert werden. Der Spurbesatz $\mathrm{k}_{\mathrm{s}}$ der Stichel liegt in den Referenzprojekten durchgehend bei 1 , sodass die Aktiv-Penetration $p_{a}[\mathrm{~mm} / \mathrm{U}]$ der Stichel der Penetration $p[\mathrm{~mm} / \mathrm{U}]$ entspricht.

Die Werte für den Laufweg $s_{c}$ wurden abschließend für die drei Werkzeugtypen gegen den Soil Abrasivity Index (SAI) angetragen. Für Schneidrollen legt die Verteilung der Datenpunkte in Abb. 90 einen Zusammenhang zwischen Laufweg $s_{c}$ und dem Soil Abrasivity Index in Form einer Exponentialfunktion nahe. Diese ist als Regressionslinie in Abb. 90 dargestellt.

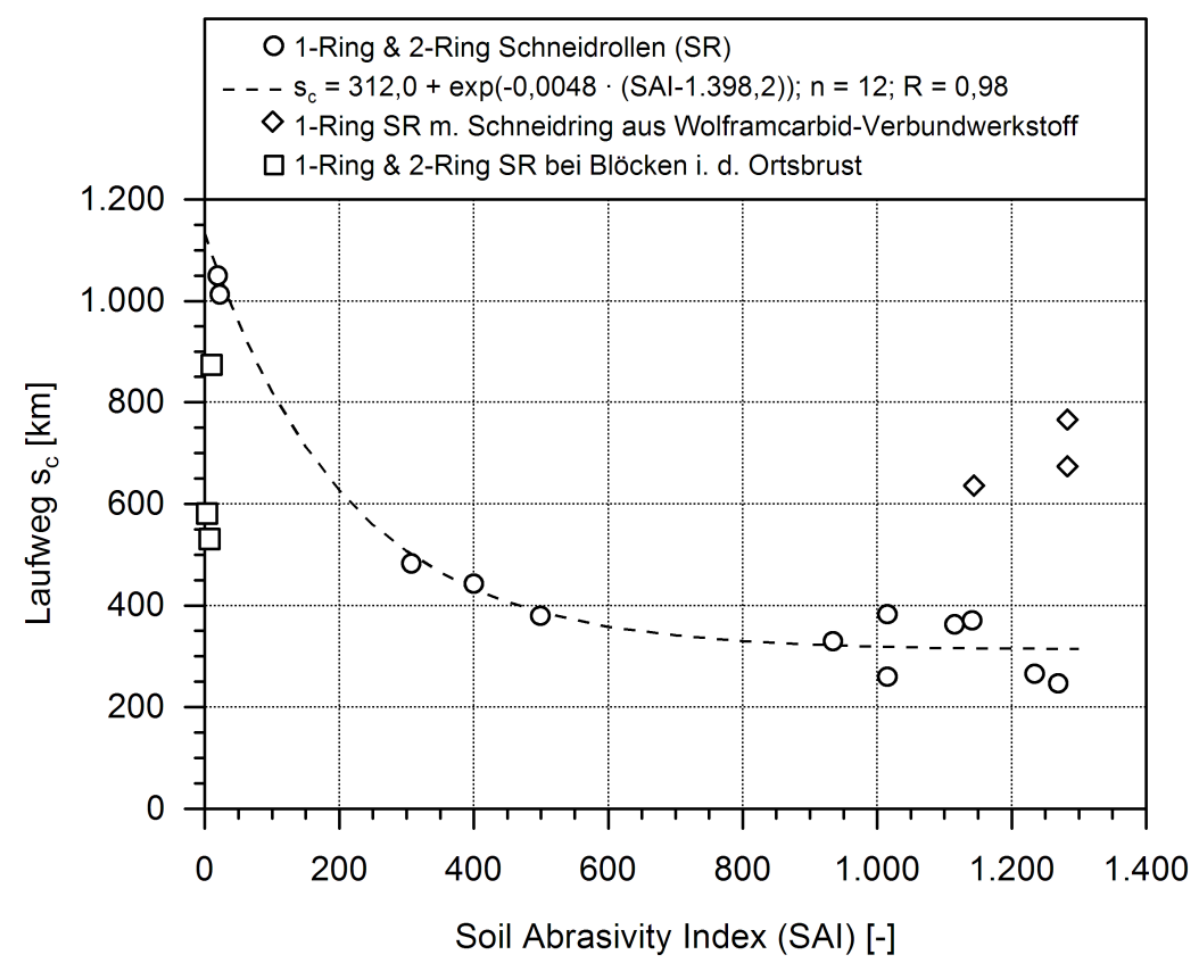

Abb. 90: Korrelation des Laufwegs $\mathrm{s}_{\mathrm{c}}[\mathrm{km}]$ von Schneidrollen mit dem Soil Abrasivity Index (SAI) [-]. Die Verteilung der Datenpunkte legt einen Zusammenhang zwischen dem Laufweg $\mathrm{s}_{\mathrm{c}}$ und dem SAI in Form einer Exponentialfunktion nahe. Diese ist als Regressionslinie in das Diagramm eingetragen. 
Die Kurvengleichung für die Trendlinie, mit der in Abb. 90 der Zusammenhang zwischen Soil Abrasivity Index (SAl) und Laufweg $\mathrm{s}_{\mathrm{c}}$ von Schneidrollen beschrieben wird, lautet:

$$
\mathrm{s}_{\mathrm{c}}=312,0+\exp (-0,0048 \cdot(\mathrm{SAI}-1.398,2))
$$

Die exponentielle Regression ergibt für Schneidrollen einen Korrelationskoeffizienten $\mathrm{R}$ zwischen Soil Abrasivity Index (SAl) und Laufweg $\mathrm{s}_{\mathrm{c}}$ von 0,98 . Es besteht folglich ein sehr starker Zusammenhang zwischen den beiden Größen.

Zusätzlich sind in Abb. 90 zwei Datenpunkte eingetragen, die Homogenbereiche mit Blöcken in der Ortsbrust darstellen. Der Laufweg $\mathrm{s}_{\mathrm{c}}$ liegt hier im Verhältnis zu den Datenpunkten ohne Blöcke deutlich niedriger, weil in diesen Bereichen ein hoher Anteil der Schneidrollen aufgrund von Schäden gewechselt wurde. Diese traten jeweils vor Erreichen der Verschleißgrenze der Schneidrollen auf.

Daneben sind drei Datenpunkte eingetragen, in denen Schneidringe mit einer Schneide aus einem Wolframcarbid-Verbundwerkstoff eingesetzt wurden. Der Laufweg $\mathrm{s}_{\mathrm{c}}$ dieser Schneidrollen liegt deutlich höher als die vergleichbaren Datenpunkte für Schneidringe aus hochlegiertem Stahl mit 57 HRC. Ursache für die Differenz ist die Erhöhung der Härte des Schneidrings, die zu einer geringeren Verschleißrate $c_{v}$ führt.

Der Laufweg $\mathrm{S}_{\mathrm{c}}$ für Schälmesser ist in Abb. 91 gegen den Soil Abrasivity Index angetragen. Die Verteilung der Datenpunkte in Abb. 91 legt wiederum einen Zusammenhang zwischen Laufweg $\mathrm{s}_{\mathrm{c}}$ und dem Soil Abrasivity Index in Form einer Exponentialfunktion nahe. Diese ist als Regressionslinie in Abb. 91 dargestellt.

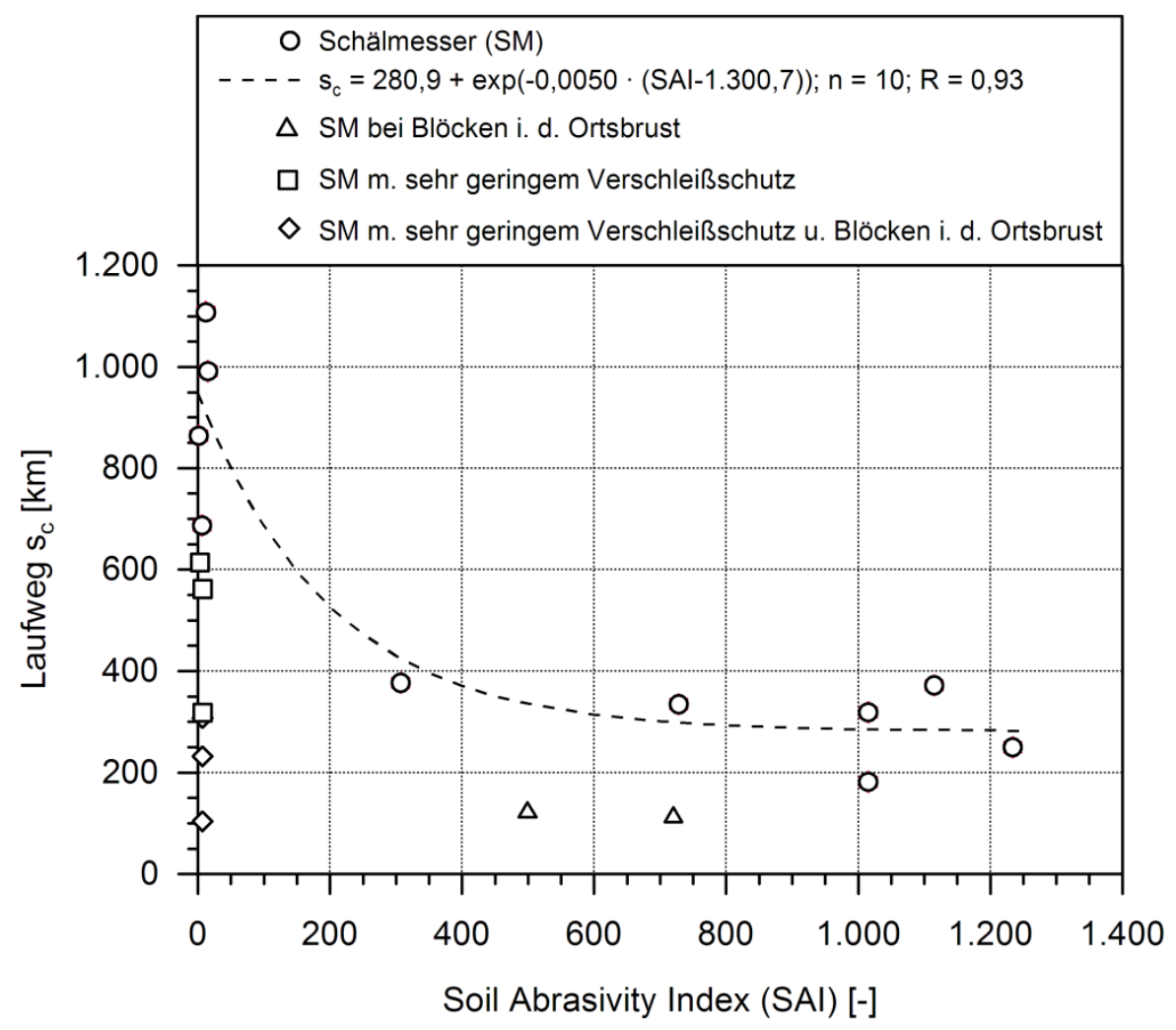

Abb. 91: Korrelation des Laufwegs $\mathrm{s}_{c}[\mathrm{~km}]$ von Schälmessern mit dem Soil Abrasivity Index (SAI) [-]. Die Verteilung der Datenpunkte legt einen Zusammenhang zwischen dem Laufweg $\mathrm{s}_{\mathrm{c}}$ und dem SAI in Form einer Exponentialfunktion nahe. Diese ist als Regressionslinie in das Diagramm eingetragen. 
Die Kurvengleichung für die Trendlinie, mit der in Abb. 91 der Zusammenhang zwischen Soil Abrasivity Index (SAl) und Laufweg $\mathrm{S}_{\mathrm{c}}$ von Schälmessern beschrieben wird, lautet:

$$
\mathrm{s}_{\mathrm{c}}=280,9+\exp (-0,0050 \cdot(\mathrm{SAI}-1.300,7))
$$

Die exponentielle Regression ergibt für Schälmesser einen Korrelationskoeffizienten R zwischen Soil Abrasivity Index (SAl) und Laufweg $\mathrm{s}_{\mathrm{c}}$ von 0,93 . Es besteht folglich ein sehr starker Zusammenhang zwischen den beiden Größen.

Zusätzlich sind in Abb. 91 fünf Datenpunkte für Schälmesser eingetragen, die in Bereichen mit Blöcken in der Ortsbrust eingesetzt wurden. Trotz der vorlaufend angeordneten Schneidrollen wurde hier ein hoher Anteil der Schälmesser aufgrund von Schäden gewechselt. Der Laufweg $s_{c}$ liegt deshalb deutlich niedriger als vergleichbare Datenpunkte aus Bereichen ohne Blöcke in der Ortsbrust. Daneben sind drei weitere Datenpunkte eingetragen, bei denen zusätzlich zu Blöcken in der Ortsbrust auch ein relativ geringer Verschleißschutz eingesetzt wurde. Der Laufweg $s_{c}$ liegt hier nochmals niedriger, als bei den vergleichbaren Datenpunkten für Schälmesser mit stärkerem Verschleißschutz. Ursache dafür ist, dass hier ein noch höherer Anteil der Schälmesser aufgrund von Schäden vor Erreichen der Verschleißgrenze gewechselt wurde.

Daneben sind zwei Datenpunkte von Schälmessern eingetragen bei denen lediglich ein im Vergleich geringer Verschleißschutz eingesetzt wurde. Auch hier liegt der Laufweg $\mathrm{s}_{\mathrm{c}}$ niedriger als bei vergleichbaren Datenpunkten für Schälmesser mit einer durchgehenden Schneide aus Hartmetall und mindestens $30 \%$ Abdeckung der Rückenfläche mit Hartmetalleinsätzen. Hier trat aufgrund des geringeren Verschleißschutzes eine deutlich höhere Verschleißrate $c_{v}$ auf, sodass die Verschleißgrenze der Schälmesser im Vergleich eher erreicht wurde.

Drei weitere Datenpunkte für Schälmesser, die mit vorlaufenden Sticheln eingesetzt wurden, sind in Abb. 91 aus Gründen der Übersichtlichkeit nicht eingetragen. Die Werte für den Laufweg $\mathrm{s}_{\mathrm{c}}$ liegen hier für alle drei Datenpunkte zwischen $5.000 \mathrm{~km}$ und $6.000 \mathrm{~km}$, also im Vergleich zu den Datenpunkten für Schälmesser mit vorlaufenden Schneidrollen extrem hoch. Ursache dafür ist, dass die Stichel jeweils die vollständige Ortsbrustfläche abdecken. Die Schälmesser nehmen so nicht am Bodenabbau teil und haben keinen Kontakt zum Boden in der Ortsbrust. Der Verschleiß der Schälmesser wurde hier folglich ausschließlich durch den bereits abgebauten Boden in der Stützflüssigkeit verursacht.

Der Laufweg $\mathrm{S}_{\mathrm{c}}$ für Stichel ist in Abb. 92 gegen den Soil Abrasivity Index angetragen. In Summe liegen für Stichel nur 5 Datenpunkte vor, die zusätzlich nicht gleichmäßig über die Bandbreite des Soil Abrasivity Index verteilt sind. Allein anhand dieser Datenpunkte kann keine Aussage über die Form eines möglichen Zusammenhangs zwischen Laufweg $\mathrm{S}_{\mathrm{c}}$ der Stichel und dem SAl getroffen werden.

Die Ergebnisse zu den Verschleiß- und Schadensarten (Kap.5.3.3) weisen darauf hin, dass der primäre Bodenabbau an der Ortsbrust und die Verschleißmechanismen bei Sticheln sehr ähnlich verlaufen, wie bei Schälmessern. Die beiden Werkzeugtypen unterscheiden sich in der Geometrie des Eingriffs in die Ortsbrust und im Verschleißschutz häufig nur geringfügig. In Abb. 92 ist deshalb zusätzlich zu den Datenpunkten der Stichel die bereits in Abb. 91 abgebildete Regressionskurve zwischen dem Laufweg $\mathrm{s}_{\mathrm{c}}$ der Schälmesser und dem Soil Abrasivity Index dargestellt. Die Datenpunkte für die Stichel liegen in dieser Darstellung sehr nahe an der Regressionskurve der Schälmesser. Die Ähnlichkeit der beiden Werkzeugtypen kann also auch an dieser Stelle gezeigt werden. Diese lässt die Vermutung zu, dass auch für Stichel ein sehr ähnlicher Zusammenhang zwischen Laufweg $\mathrm{s}_{\mathrm{c}}$ und dem SAI besteht, wie für Schälmesser. Eine eigene Regressionskurve kann aufgrund der geringen Anzahl der Datenpunkte jedoch nicht abgeleitet werden. 


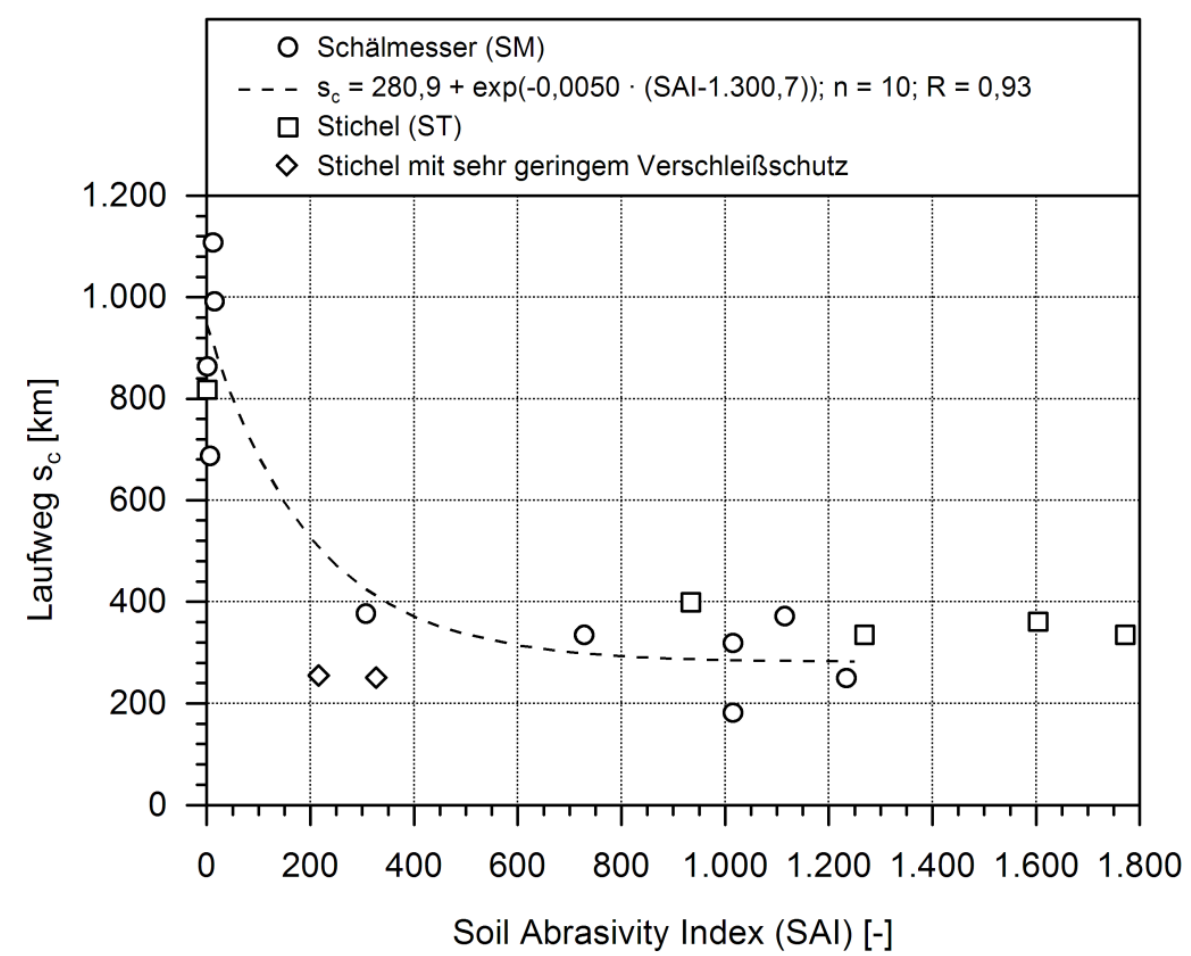

\begin{abstract}
Abb. 92: Korrelation des Laufwegs $\mathrm{s}_{\mathrm{c}}[\mathrm{km}]$ von Sticheln mit dem Soil Abrasivity Index (SAI) [-]. Zusätzlich sind als Orientierung die Datenpunkte und die Regressionskurve für Schälmesser eingetragen. Aufgrund der Lage und der geringen Anzahl an Datenpunkten für Stichel $(n=5)$ ist die Ableitung einer eigenen Regressionskurve nicht sinnvoll. Die Datenpunkte für Stichel liegen jedoch sehr nahe an der Regressionskurve für Schälmesser, sodass für Stichel ein ähnlicher Zusammenhang zwischen dem Laufweg $\mathrm{s}_{\mathrm{c}}$ und dem SAI vermutet werden kann.
\end{abstract}

Der grundsätzliche Trend, der durch den Verlauf der Regressionskurven für Schneidrollen und Schälmesser aufgezeigt wird, entspricht dem erwarteten Zusammenhang zwischen dem Soil Abrasivity Index (SAl) und dem Laufweg $\mathrm{s}_{\mathrm{c}}$ der Abbauwerkzeuge. Mit steigendem Produkt aus Gehalt an verschleißrelevanten Mineralen (Äquivalenter Quarzgehalt), Kontaktspannungen zwischen Werkzeugoberfläche und Boden (Scherfestigkeit $\left.T_{c}\right)$ und Korngröße $\left(D_{60}\right)$ sinkt der erreichte Laufweg $S_{c}$ der $A b$ bauwerkzeuge deutlich, bzw. nimmt die Abriebrate $c_{v}$ deutlich zu.

Die Art des Zusammenhangs zwischen dem Soil Abrasivity Index und dem Laufweg $\mathrm{s}_{\mathrm{c}}$ in Form einer Exponentialfunktion ist auf Basis bestehender Erkenntnisse zum Abbauwerkzeugverschleiß in Lockergesteinen nicht abschließend zu bewerten. Einerseits liegen überhaupt nur sehr wenige Untersuchungen zu diesem Zusammenhang vor (Kap. 4.4), andererseits fehlt in bisherigen Betrachtungen die Berücksichtigung der Kontaktspannungen zwischen Werkzeugoberfläche und Boden vollständig. Im Ansatz vergleichbar sind die vorläufigen Ergebnisse der Untersuchungen von JACOBSEN ET AL. (2013a: 113), die einen relativ ähnlichen, exponentiellen Zusammenhang zwischen den Resultaten des SAT-Test für die Bodenabrasivität und dem Lösevolumens $\mathrm{v}_{\mathrm{c}}$ der Abbauwerkzeuge herstellen. Weiterhin entspricht der vorliegende Zusammenhang in Form einer Potenzfunktion in zwei wesentlichen Kernpunkten bisherigen grundlegenden Ergebnissen zur Korrelation der Verschleißrate $\mathrm{C}_{\mathrm{v}}$ und der Abrasivität von Gesteinen. FRENZEL (2010a: 47) weist unter Einbeziehung älterer Untersuchungen und auf Basis relativ großer Datenmengen deutlich auf Nicht-Linearität und Stetigkeit dieses grundlegenden Zusammenhangs hin (Kap. 4.1.2). Die mit Gl. 70 und Gl. 71 beschriebenen Exponentialfunktion zur Korrelation des Soil Abrasivity Index SAI und dem Laufweg $\mathrm{S}_{\mathrm{c}}$ entspricht diesen Kriterien.

Auf Basis der oben dargestellten Daten und Zusammenhänge müssen im Hinblick auf eine allgemeine Aussage zum Einfluss der Bodeneigenschaften auf den Abbauwerkzeugverschleiß in Lockergesteinen und im Hinblick auf die Verwendung der Daten für das nachfolgende Prognosemodell (Kap. 6) noch die folgende Punkte berücksichtigt werden. 
Sowohl bei Schneidrollen, als auch bei Schälmessern, decken die vorhandenen Daten übereinstimmend ca. 59\% der theoretisch möglichen Bandbreite des SAI ab. Die Datenpunkte liegen alle unterhalb eines SAI-Wertes von 1.770. Die Abdeckung ist also zwar relativ hoch, aber auf eine Seite der möglichen Bandbreite beschränkt. Eine Überprüfung der Zusammenhangshypothese zwischen Soil Abrasivity Index und Laufweg $\mathrm{s}_{\mathrm{c}}$ für SAI-Werte größer 1.770 war im Rahmen dieser Arbeit nicht möglich. Die vorhandenen Datenpunkte bilden in dieser Hinsicht den aktuellen Anwendungsschwerpunkt von Hydroschild TVM in Lockergesteinen nach. Sollte zukünftig aufgrund geänderter Projektanforderungen eine deutliche Verschiebung des Anwendungsschwerpunktes hin zu höheren SAI-Werten erfolgen, ist eine Revision der Korrelationen zwingend notwendig. Eine entsprechende Verschiebung könnte sich langfristig hauptsächlich durch den Einsatz der Hydroschild TVM unter immer größer werdenden Überlagerungen $\mathrm{H}_{\mathrm{TA}}$ einstellen.

Abschließend kann auf Basis der dargestellten Daten die ursprüngliche Annahme bestätigt werden, dass die Abrasivität von Lockergesteinen im Hinblick auf den Verschleiß von Abbauwerkzeugen im Vortrieb mit Hydroschild TVM ein Produkt aus dem Anteil an verschleißrelevanten Mineralen, der Korngrößenverteilung und der Kontaktspannungen zwischen Werkzeugoberfläche und den Bodenkomponenten ist. Der SAI stellt im Rahmen der vorliegenden Daten eine geeignete und aussagekräftige Methode zur Beschreibung dieses Produktes dar. Für die weitere Entwicklung des Indexwertes und Erhöhung der Aussagekraft sind insbesondere folgende Ansatzpunkte wichtig:

- Erweiterung der Datenbasis durch detaillierte Dokumentation in neuen Referenzprojekten

- Integration der Kornform und des Rundungsrades in den Soil Abrasivity Index

- Detaillierte Untersuchungen zu den Kontaktspannungen zwischen dem Boden in der Ortsbrust und den Werkzeugoberflächen. 


\section{Empirische Verschleißprognose}

Die Ergebnisse aus der Analyse der Verschleißtypen und -grenzen (Kap. 5.3), den Versuchen zur Verschleißrate $c_{v}$ (Kap. 5.5) und der Datenanalyse zu den Einflussfaktoren auf den Laufweg $\mathrm{s}_{\mathrm{c}}$ der Abbauwerkzeuge (Kap. 5.6), ermöglichen die Ableitung eines empirischen Prognosemodells für den Verschleiß der Abbauwerkzeuge beim Vortrieb mit Hydroschild TVM in Lockergesteinen.

Im Kern des Prognosemodells steht die empirische Korrelation des Laufwegs $\mathrm{s}_{\mathrm{c}}$ mit dem Soil Abrasivity Index (SAI). Diese kann auf Basis der geotechnischen Bedingungen und unter anschließender Korrektur der quantifizierbaren Einflussfaktoren aus der Auslegung der TVM und der Steuerung des Abbauprozesses zur Prognose des Laufwegs $\mathrm{S}_{\mathrm{c}}$ genutzt werden. Die Verschleißrate $\mathrm{c}_{v}$ ist innerhalb der Verschleißgrenzen der Abbauwerkzeuge konstant. Aus dem Laufweg $s_{c}$ können also entsprechend der Zielsetzung des Modells (Kap. 2.4.1) sowohl die erreichbaren Vortriebsstrecken zwischen den notwendigen Vortriebsunterbrechungen für Werkzeugwechsel, als auch die Anzahl der zu wechselnden Abbauwerkzeuge geschätzt werden.

Die Nutzung der Korrelation zwischen dem Laufweg $\mathrm{s}_{\mathrm{c}}$ und dem SAl und die Annahme einer konstanten Verschleißrate $c_{v}$ innerhalb der Verschleißgrenzen bedingen eine Einschränkung des Prognosemodells auf Abbauwerkzeuge, die aufgrund von Primär- und Sekundärverschleiß ausgetauscht werden müssen. Effekte durch Schäden an den Abbauwerkzeugen wurden sowohl in der Analyse der Einflussfaktoren auf den Laufweg $s_{c}$, als auch in den Versuchen zur Verschleißrate $c_{v}$ eliminiert. Schäden an den Abbauwerkzeugen, die durch Steine oder Blöcke in der Ortsbrust, Verklebungen oder andere Hindernisse im Vortrieb entstehen, werden durch das Prognosemodell nicht abgedeckt.

Die Architektur des Prognosemodells und der Prognoseprozess sind entsprechend der Anforderungen (Kap. 2.4.2) so gestaltet, dass sich aufgrund der Beschränkung auf Einflussfaktoren mit hohem Einfluss eine Reduktion der Komplexität im Vergleich zum realen System ergibt. Die Gliederung des Prognosemodells in Sektionen ist so angelegt, dass sie eine zukünftige Erweiterung und Anpassung des Prognosemodells erlaubt. Eine detaillierte Beschreibung der Modellsektionen, des Prognoseprozesses und der dazu erforderlichen Datengrundlage ist in Kap. 6.1 gegeben.

Abschließend erfolgt die Validierung des Prognosemodells auf Basis der in Kap. 2.4.4 beschriebenen Methodik unter Berücksichtigung der kritischen Faktoren (Kap. 2.4.3) innerhalb des Modells. Eine detaillierte Beschreibung der Ergebnisse der Validierung ist in Kap. 6.6 gegeben.

\subsection{Modellarchitektur}

\subsubsection{Basisdaten für den Prognoseprozess}

Die für den Prognoseprozess notwendigen Basisdaten richten sich nach den Empfehlungen zur geotechnischen Vorerkundung für Vortriebe mit Hydroschild TVM in Lockergesteinen (Kap. 5.2.2.1), den notwendigen Basisdaten aus der TVM Auslegung (Kap. 5.2.3) und aus der Steuerung des Abbauprozesses (Kap. 5.2.4). Während die umfassende Bestimmung dieser Basisdaten die Grundlage für eine möglichst realistische Verschleißprognose und auch für zukünftige Untersuchungen bildet, fasst die nachfolgende Beschreibung die Minimalanforderungen an die Basisdaten zur Durchführung des Prognoseprozesses zusammen.

\subsubsection{TVM-Auslegung}

Aus der TVM-Auslegung sind für die Durchführung des Prognoseprozesses mindestens die folgenden Basisdaten notwendig:

- Ausbruchdurchmesser $D_{\mathrm{TVM}}[\mathrm{m}]$

- Spurbesatz $\mathrm{k}_{\mathrm{s}}[-]$ für alle Werkzeugtypen

- Schneidringbreite $b_{S R}[\mathrm{~mm}]$ (Schneidradbesatz mit Schneidrollen) 
Zusätzlich sind für den Abgleich der TVM-Auslegung mit den im Prognosemodell nicht quantifizierbaren Einflussfaktoren (Kap. 6.2.3.3) die folgenden Basisdaten notwendig:

- Beschreibung des Verschleißschutzes aller Abbauwerkzeuge auf dem Schneidrad.

- Höhe über Schneidradstahlbau $h_{S R}, h_{S T}, h_{S M}, h_{R M}[m m]$.

- Winkelabstand der Abbauwerkzeuge $\delta_{\mathrm{a}}$ und $\delta_{\mathrm{p}}\left[^{\circ}\right]$.

- Beschreibung der Werkzeuggeometrie aller Abbauwerkzeuge auf dem Schneidrad.

- Anzahl der Rotoren (Schneidrad) und Statoren (Tauchwand).

- Schneidradöffnungsverhältnis $\mathrm{O}_{\mathrm{TVM}}[\%]$.

\subsubsection{Geotechnische Parameter}

Zu jeder geotechnischen Einheit, die im Verlauf der Tunnelachse in der Ortsbrust angeschnitten wird, oder den Tunnelquerschnitt überlagert sind mindestens folgende Kennwerte notwendig:

- Korngrößenverteilung

- Feuchtwichte y $\left[\mathrm{kN} / \mathrm{m}^{3}\right]$ und Wichte unter Auftrieb $\mathrm{y}^{\mathrm{c}}\left[\mathrm{kN} / \mathrm{m}^{3}\right]$

- Kohäsion c' [kN/m²]

- Reibungswinkel $\varphi^{\circ}\left[^{\circ}\right]$

- $\quad$ Äquivalenter Quarzgehalt ÄQu [\%]

- Konsistenzzahl IC (bindige Böden)

- Plastizitätszahl $I_{\mathrm{P}}[\%]$ (bindige Böden)

- Kornform

- Kornrundungsgrad

Zusätzlich ist die Verteilung der geotechnischen Einheiten entlang der Tunnelachse von entscheidender Bedeutung, die in einem geotechnischen Längsschnitt dargestellt wird. Für den Prognoseprozess müssen im Längsschnitt mindestens folgende Informationen enthalten sein:

- Verteilung der Bodenarten im Ausbruchquerschnitt und in der Überlagerung entlang der Tunnelachse.

- Überlagerung $\mathrm{H}_{\mathrm{TA}}[\mathrm{m}]$.

- Wasserspiegel $\mathrm{W}_{\mathrm{TA}}[\mathrm{m}]$.

- Lage, Art und Eigenschaften künstlicher Hindernisse im Boden.

- Lage, Art und Eigenschaften der Bebauung im Bereich der Tunnelachse. 
Die Anwendung des Prognoseprozesses erfordert aufbauend auf die Verteilung der geotechnischen Einheiten die Einteilung der Vortriebsstrecke in geotechnische Homogenbereiche (HGB). Zur Bildung der Homogenbereiche können die in Kap. 5.6.1.2 genannten Kriterien herangezogen werden:

- Isotrope Zusammensetzung der Ortsbrust in Richtung der Tunnelachse (Gleichbleibender Anteil verschiedener Bodenarten in der Ortsbrust [\%]).

- Gleichbleibende Überlagerungshöhe $\mathrm{H}_{\mathrm{TA}}[\mathrm{m}]$ über der Tunnelachse.

- Gleichbleibender Wasserspiegel $\mathrm{W}_{\mathrm{TA}}[\mathrm{m}]$ über der Tunnelachse.

Dabei werden folgende Schwankungsbreiten akzeptiert:

- Anteil verschiedener geologischer Einheiten in der Ortsbrust: $+/-10 \%$

- Überlagerungshöhe $\mathrm{H}_{\mathrm{T}}$ in $\mathrm{m}:+/-5 \mathrm{~m}$

- Wasserspiegel $\mathrm{W}_{\mathrm{TA}}$ in $\mathrm{m}:+/-5 \mathrm{~m}$

\subsubsection{Steuerung Abbauprozess}

Aus der Steuerung des Abbauprozesses ist für die Durchführung des Prognoseprozesses mindestens die Festlegung eines Erwartungswerts für die Penetration $p_{e}[\mathrm{~mm} / \mathrm{U}]$ für jeden HGB notwendig. Der Erwartungswert für die Penetration $p_{\mathrm{e}}$ muss geschätzt werden, weil für Hydroschild TVM in Lockergesteinen kein entsprechendes Prognosemodell zur Verfügung steht. Aus den Referenzprojekten können hierzu die in Tab. 21 (Kap. 6.2.3.2) zusammengefassten Richtwerte abgeleitet werden.

Zusätzlich sind für den Abgleich der Steuerung des Abbauprozesses mit den im Prognosemodell nicht quantifizierbaren Einflussfaktoren (Kap. 6.2.3.3) die folgenden Basisdaten für jeden HGB notwendig:

- Erwartungswert für die Schneidraddrehzahl $U\left[\mathrm{~min}^{-1}\right]$

- Erwartungswert für fir Vortriebsgeschwindigkeit $\mathrm{V}_{\mathrm{TVM}}[\mathrm{mm} / \mathrm{min}]$

- Erwartungswert für die Dichte der Stützflüssigkeit $\rho_{S F}\left[g / m^{3}\right]$

- Erwartungswert für den Stützdruck $P_{S F}[b a r]$

\subsubsection{Modellsektionen}

Ausgehend von den Basisdaten (Kap. 6.1.1) besteht das Prognosemodell aus vier Sektionen:

- Sektion 1: Schätzung des Erwartungswertes für den Laufwegs $\mathrm{s}_{c, \mathrm{e}}[\mathrm{km}]$ für jeden HGB.

- Sektion 2: Schätzung der Erwartungswerte für die Stationierung $\mathrm{L}_{\mathrm{l}, \mathrm{e}}[\mathrm{m}]$ bzw. $\mathrm{b}_{\mathrm{l}, \mathrm{e}}$ der Vortriebsunterbrechungen I für Werkzeugwechsel.

- Sektion 3: Schätzung der Erwartungswerte für die Anzahl der Werkzeugwechsel $n_{c, e}$ je Vortriebsunterbrechung I bzw. über die Tunnelachse (TA).

- Sektion 4: Baubetriebliche Anpassung der Verschleißprognose.

Die Sektionen enthalten jeweils bis zu 6 Prozessschritte (Kap. 6.1.3), die nach jeder Sektion zu einem Teilergebnis führen. Die Sektionen sind linear angeordnet und bauen jeweils auf die Teilergebnisse aus den vorlaufenden Sektionen auf (Kap. 6.1.3). Der Aufbau und die Gliederung der Sektionen gibt die Möglichkeit für zukünftige Erweiterungen oder Anpassungen des Prognosemodells, die in der strukturellen Validierung des Prognosemodells (Kap. 6.6.1) detailliert beschrieben werden. 


\subsubsection{Prognoseprozess}

Für den Prognoseprozess werden sowohl die geotechnischen Homogenbereiche HGB, als auch die Vortriebunterbrechungen I mit einem ab Vortriebsbeginn ansteigenden ganzzahligen Index durchnummeriert. Für die Homogenbereiche (HGB) wird der Index z verwendet, für die Vortriebsunterbrechungen I der Index m. Alle weiteren Größen im Prognoseprozess, die sich auf einen bestimmten Homogenbereich $\mathrm{HGB}_{(z)}$ beziehen werden ebenfalls mit dem Index $\mathrm{z}$ versehen. Ebenso werden alle Größen im Prognoseprozess, die sich auf eine bestimmte Vortriebsunterbrechung $\mathrm{I}_{(\mathrm{m})}$ beziehen mit dem Index m versehen.

Eine detaillierte Gliederung der Prozessschritte innerhalb der 4 Modellsektionen (Kap. 6.1.2) ist in Abb. 93 dargestellt. Für die Verschleißprognose werden ausgehend von den Basisdaten (Kap. 6.1.1) die einzelnen Prozessschritte in der in Abb. 93 dargestellten Reihenfolge abgearbeitet.

In Sektion 1 wird aus den Basisdaten mithilfe der Ergebnisse zu den Einflussfaktoren auf den Laufweg $\mathrm{s}_{\mathrm{c}}$ für jedes einzelne Abbauwerkzeug auf dem Schneidrad in jedem geotechnischen Homogenbereich ein Erwartungswert für den Laufweg $\mathrm{s}_{\mathrm{c}, \mathrm{e}(\mathrm{z})}[\mathrm{km}]$ geschätzt.

Der Erwartungswert für den Laufweg $\mathrm{s}_{\mathrm{c}, \mathrm{e}(\mathrm{z})}[\mathrm{km}]$ wird in Sektion 2 als Eingangswert eingesetzt. Zusätzlich sind die Länge der geotechnischen Homogenbereiche $L_{d(z)}[\mathrm{m}]$, die Stationierung der Grenzen zwischen den geotechnischen Homogenbereichen $L_{H G B(z)}[\mathrm{m}]$ und der Erwartungswert für die Penetration $p_{e(z)}[\mathrm{mm} / \mathrm{U}]$ in jedem geotechnischen Homogenbereich notwendig. Daraus werden in Sektion 2 als Teilergebnis die geplanten Vortriebsstrecken $L_{d, e(m)}[\mathrm{m}]$ zwischen den Vortriebsunterbrechungen $I_{(m)}$ bestimmt. Innerhalb der Vortriebsstrecken $L_{d, e(m)}$ können mehrere $\mathrm{HGB}_{(z)}$ angefahren bzw. durchfahren werden, in denen unterschiedliche Erwartungswerte für den Laufweg $\mathrm{s}_{\mathrm{c}, \mathrm{e}(\mathrm{z})}$ und die Penetration $p_{e(z)}$ gelten. Die Bestimmung der Vortriebsstrecken $L_{d, e(m)}$ erfordert deshalb einen iterativen Prozess zum Abgleich mit den Vortriebsstrecken $\mathrm{L}_{\mathrm{d}(\mathrm{z})}$ in den $\mathrm{HGB}_{(\mathrm{z})}$. Aus den aneinander gereihten Vortriebsstrecken $L_{d, e(m)}$ resultieren die geplanten Stationierung $L_{1, e(m)}[m]$ der Vortriebsunterbrechungen $I_{(m)}$ für Werkzeugwechsel. Somit steht auch die Anzahl $n_{1, e(T A)}$ der Vortriebsunterbrechungen $I_{(m)}$ entlang der Tunnelachse fest. Anschließend wird für jedes einzelne Abbauwerkzeug der kumulierte Laufweg $\mathrm{s}_{\mathrm{c}, \mathrm{e}(\mathrm{m})}$ und Ausnutzungsgrad $\mathrm{e}_{\mathrm{c}, \mathrm{e}(\mathrm{m})}$ an den Vortriebsunterbrechungen $\mathrm{I}_{(\mathrm{m})}$ geschätzt.

In Sektion 3 werden der Erwartungswert für den Laufweg $\mathrm{s}_{\mathrm{c}, \mathrm{e}(\mathrm{z})}[\mathrm{km}]$, die Stationierung $\mathrm{L}_{\mathrm{l}, \mathrm{e}(\mathrm{m})}[\mathrm{m}] \mathrm{der}$ Vortriebsunterbrechungen $I_{(m)}$ und der kumulierte Ausnutzungsgrad $e_{c, e(m)}$ der Abbauwerkzeuge an den Vortriebsunterbrechungen $I_{(m)}$ eingesetzt. Daraus die Anzahl der notwendigen Werkzeugwechsel $\mathrm{n}_{\mathrm{c}, \mathrm{e}(\mathrm{m})}$ je Vortriebsunterbrechung $\mathrm{I}_{(\mathrm{m})}$ ermittelt. Als Summe über alle Vortriebsunterbrechungen $\mathrm{I}_{(\mathrm{m})}$ wird so auch der Erwartungswert für die Anzahl $n_{c, e(T A)}$ der notwendigen Werkzeugwechsel über die Tunnelachse festgelegt.

In Sektion 4 werden an den geplanten Stationierung $L_{l, e(m)}$ der Vortriebsunterbrechungen $I_{(m)}$ die Rahmenbedingungen für den Zugang zur Abbaukammer für Werkzeugwechsel ermittelt. Durch gezielte Variation der Basisdaten, der Werkzeugwechselstrategie oder die Planung von Zusatzmaßnah wird die Stationierung $L_{l, e(m)}$ der Vortriebsunterbrechungen $I_{(m)}$ optimiert. Bei der Optimierung können verschiedene Projektziele im Hinblick auf die Vortriebsdauer, die Verschleißkosten oder die Planungssicherheit berücksichtigt werden. Die Schätzung der Verschleißkosten, der Vortriebsdauer bzw. Vortriebsgeschwindigkeit und die Bestimmung der Zugangsmöglichkeiten zur Abbaukammer, z.B. durch Arbeiten unter Druckluft, in Sektion 4 sind in hohem Maße projektspezifisch und nicht Kernthema der vorliegenden Arbeit. Es erfolgt deshalb keine detaillierte Untersuchung und Beschreibung zur Schätzmethodik.

Der Prognoseprozess enthält folglich zwei inneinander liegende Iterationszyklen, in denen Gruppen von Prozessschritt mehrfach wiederholt werde, um ein realistisches Prognoseergebnis zu erhalten:

- Iterative Bestimmung der Vortriebsstrecken $\mathrm{L}_{\mathrm{d}, \mathrm{e}(\mathrm{m})}[\mathrm{m}]$ zwischen den Vortriebsunterbrechungen $\mathrm{I}_{(\mathrm{m})}$ unter Berücksichtigung der geotechnischen Homogenbereiche $\mathrm{HGB}_{(\mathrm{z})}$ (Kap. 6.3.4).

- Iterative Anpassung der Stationierungen $\mathrm{L}_{\mathrm{l}, \mathrm{e}(\mathrm{m})}$ der Vortriebsunterbrechungen $\mathrm{I}_{(\mathrm{m})}$ an die Rahmenbedingungen im Projekt für den Zugang zur Abbaukammer oder Bildung von verschiedenen Vortriebsszenarien zur empirischen Ermittlung der möglichen Bandbreite der Prognoseergebnisse (Kap. 6.5). 
Als Ergebnis des Prognoseprozesses wird durch den Bearbeiter ein Planungsszenario festgelegt (Kap. 6.5.3). Dieses setzt sich aus den Basisdaten, den geschätzten Erwartungswerten für Laufweg $\mathrm{s}_{\mathrm{c}, \mathrm{e}(\mathrm{z})}$, die Stationierungen $\mathrm{L}_{\mathrm{l}, \mathrm{e}(\mathrm{m})}$ und Anzahl $\mathrm{n}_{\mathrm{l}, \mathrm{e}(\mathrm{TA})}$ der Vortriebsunterbrechungen $\mathrm{I}_{(\mathrm{m})}$, der Anzahl der Werkzeugwechsel $n_{c, e(m)}$ bzw. $n_{c, e(T A)}$ je Vortriebsunterbrechung $I_{(m)}$ und über die Tunnelachse TA und der Beschreibung von eventuellen Zusatzmaßnahmen für den Zugang zur Abbaukammer zusammen.

Das Planungsszenario und alle darin enthaltnenen Daten und Annahmen müssen im Vortrieb nachverfolgt werden, weil aufgrund der zahlreichen Einflussfaktoren und Anpassungsmöglichkeiten im Prognoseprozess Änderungen in der Vortriebsphase im Vergleich zum bestehenden Planungsszenario durchaus häufig sind (Kap. 6.5.3.2). Das Planungsszenario wird so in der Vortriebsphase auf Basis der anfallenden Daten und Erfahrungen permanent validiert und für die verbleibende Vortriebsstrecke angepasst. Die mittel und langfristigen Auswirkungen der Änderungen im Planungsszenario können so frühzeitig bestimmt werden und entsprechende Maßnahmen abgeleitet werden.

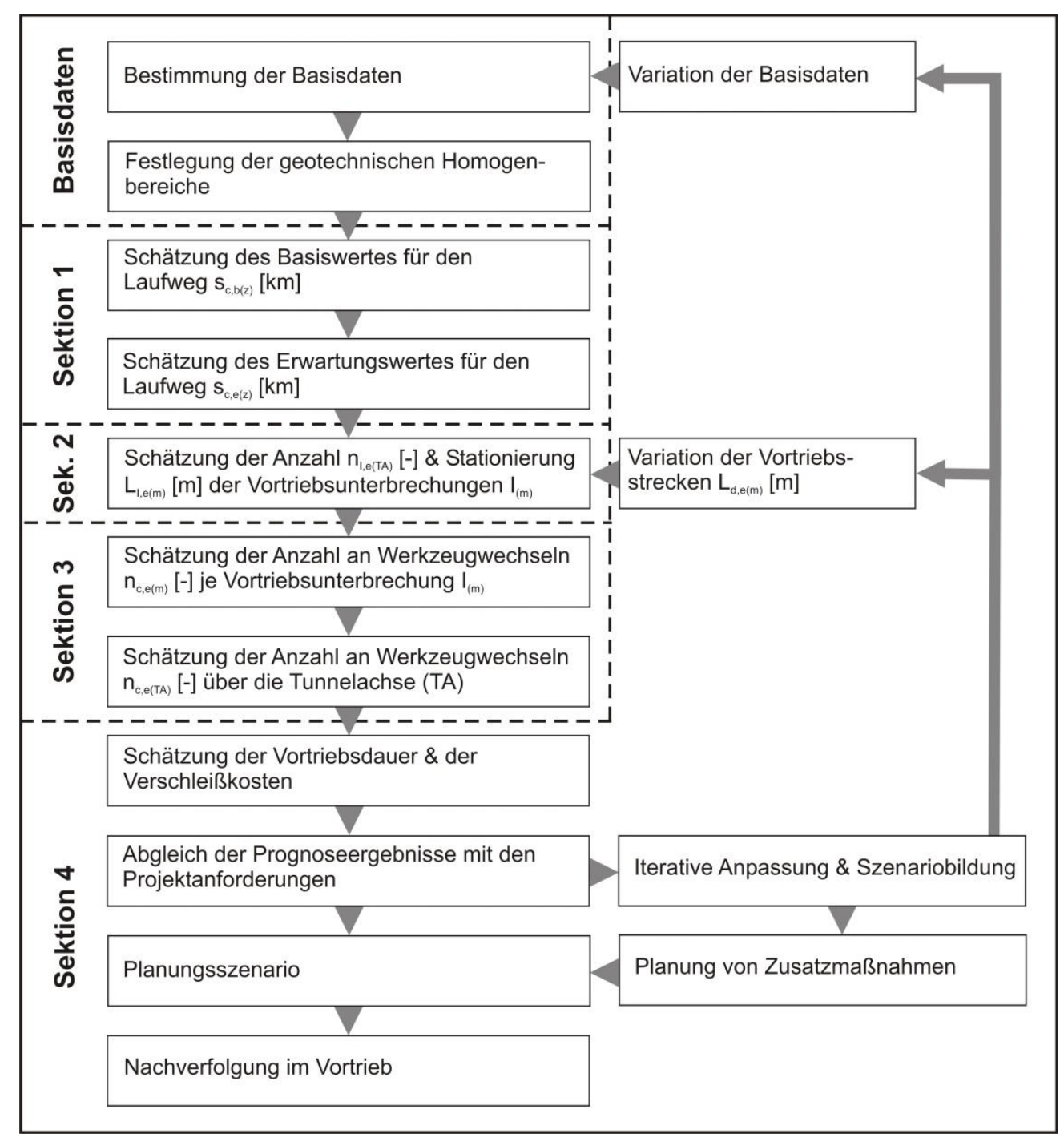

Abb. 93: Sektionen und Prozessschritte des Prognosemodells. 


\subsection{Schätzung des Laufwegs $s_{c}$}

\subsubsection{Anwendung}

Die Schätzung des Laufwegs $\mathrm{s}_{\mathrm{c}}$ basiert auf der Korrelation zwischen den geotechnischen Parametern, die im SAI zusammengefasst sind und dem Laufweg $S_{c}[k m]$ von Schneidrollen, Schälmessern und Sticheln. Die Schätzung wird für jeden $\mathrm{HGB}_{(z)}$ separat durchgeführt.

Ausgehend von dieser Korrelation ergibt sich ein Basiswert für den Laufweg $\mathrm{s}_{\mathrm{c}, \mathrm{b}(\mathrm{z})}[\mathrm{km}]$, der bedingt durch die Varianz der Eingangsparameter auch selbst einer bestimmten Varianz unterliegt. Die Varianz der Eingangsparameter ist aufgrund der fehlenden Untersuchung in der Vorerkundung der Referenzprojekte (Kap. 5.2.2.3) nicht bestimmbar, sodass auch die Varianz des Basiswertes für den Laufweg $\mathrm{S}_{\mathrm{c}, \mathrm{b}(\mathrm{z})}$ nicht ermittelt werden kann. Eine allgemeine Untersuchung der Varianzfortpflanzung im Prognosemodell ist folglich nicht möglich. Im Prognoseprozess (Abb. 93) ist deshalb ein Arbeitsschritt zur empirischen Untersuchung der Auswirkungen einer Variation der Eingangsparameter integriert.

Zur Ermittlung des Erwartungswertes für den Laufweg $\mathrm{s}_{\mathrm{c}, \mathrm{e}(\mathrm{z})}$, der im Prognoseprozess weiter verwendet wird, muss der Basiswert $\mathrm{s}_{\mathrm{c}, \mathrm{b}(\mathrm{z})}$ anschließend um die bekannten und quantifizierbaren Einflussfaktoren korrigiert werden.

Nicht quantifizierbare Einflussfaktoren können im Prognosemodell nicht berücksichtigt werden. Die Grenzen des Wertebereichs dieser Faktoren in den Referenzprojekten bilden folglich Anwendungsgrenzen für das Prognosemodell. Außerhalb dieser Grenzen kann zwar eine qualitative Tendenz zum Einfluss dieser Faktoren angegeben werden. Eine quantitative Bestimmung ist jedoch nicht möglich.

\subsubsection{Basiswert des Laufwegs $\mathrm{s}_{\mathrm{c}, \mathrm{b}(\mathrm{z})}$}

Die Prognose des Basiswertes für den Laufwegs $\mathrm{s}_{\mathrm{c}, \mathrm{b}(\mathrm{z})}[\mathrm{km}]$ erfolgt für Schneidrollen, Stichel und Schälmesser anhand der in Gl. 70 und Gl. 71 beschriebenen Korrelationen zwischen dem SAI und dem Laufweg $\mathrm{s}_{\mathrm{c}}$.

Der dimensionslose $\mathrm{SAl}_{(z)}$ ergibt sich für jeden $\mathrm{HGB}_{(\mathrm{z})}$ übereinstimmend mit $\mathrm{Gl} .67$ aus $\mathrm{Gl} .72$ :

$$
\operatorname{SAI}_{(\mathrm{z})}=\left(\frac{\mathrm{ÄQu}_{(\mathrm{z})}}{100}\right)^{2} \cdot \tau_{\mathrm{c}(\mathrm{z})} \cdot \mathrm{D}_{60(\mathrm{z})}
$$

Die Scherfestigkeit $\mathrm{T}_{\mathrm{c}(\mathrm{z})}\left[\mathrm{kN} / \mathrm{m}^{2}\right]$ im betrachteten $\mathrm{HGB}_{(\mathrm{z})}$ ergibt sich in Übereinstimmung mit $\mathrm{Gl} .54$ aus:

$$
\tau_{\mathrm{c}(\mathrm{z})}=\mathrm{c}_{(\mathrm{z})}^{\prime}+\sigma_{\mathrm{n}(\mathrm{z})} \cdot \tan \varphi_{(\mathrm{z})}^{\prime}
$$

Die in Gl. 73 eingesetzte Normalspannung $\sigma_{n(z)}\left[\mathrm{kN} / \mathrm{m}^{2}\right]$ wird analog zu Gl. 55 als Betrag der effektiven vertikalen Spannungen im Boden auf Höhe der Tunnelachse TA mit Gl. 74 ermittelt:

$$
\sigma_{\mathrm{n}(\mathrm{z})}=\sum_{1}^{\mathrm{i}} \gamma_{\mathrm{i}} \cdot \mathrm{H}_{\mathrm{i}}
$$


Der Index i entspricht hier den verschiedenen Schichten im Schichtmodell. Für die Wichte $\gamma_{i}$ wird entsprechend der Lage der Schicht ober- oder unterhalb des Wasserspiegels $W_{T A}$ jeweils die Feuchtwichte $\mathrm{y}$ oder die Wichte unter Auftrieb $\mathrm{y}^{\prime}\left[\mathrm{kN} / \mathrm{m}^{3}\right]$ eingesetzt. Der Wert $\mathrm{H}_{\mathrm{i}}[\mathrm{m}]$ gibt die Mächtigkeit der Schichten oberhalb der Tunnelachse an.

Aus dem $\mathrm{SAl}_{(\mathrm{z})}$ nach Gl. 72 kann damit der Basiswert für den Laufweg $\mathrm{s}_{\mathrm{c}, \mathrm{b}(\mathrm{z})}[\mathrm{km}]$ für Schneidrollen ermittelt werden aus:

$$
\mathrm{s}_{\mathrm{c}, \mathrm{b}(\mathrm{z})}=312,0+\exp \left(-0,0048 \cdot\left(\mathrm{SAI}_{(\mathrm{z})}-1.398,2\right)\right)
$$

Für Schälmesser ergibt sich der Basiswert für den Laufweg $\mathrm{s}_{\mathrm{c}, \mathrm{b}(\mathrm{z})}[\mathrm{km}]$ aus dem $\mathrm{SAl}_{(\mathrm{z})}$ entsprechend aus:

$$
\mathrm{s}_{\mathrm{c}, \mathrm{b}(\mathrm{z})}=280,9+\exp \left(-0,0050 \cdot\left(\mathrm{SAI}_{(\mathrm{z})}-1.300,7\right)\right)
$$

Die Korrelation in Gl. 76 kann aufgrund der Ähnlichkeit der Werkzeuge, deren Verschleißschutz und des Ausbruchmechanismuses (Kap. 5.3) auch zur Schätzung des Basiswertes des Laufwegs $\mathrm{S}_{\mathrm{c}, \mathrm{b}(\mathrm{z})}$ für Stichel und Räumer angenommen werden. Der entsprechend ermittelte Basiswert $\mathrm{s}_{\mathrm{c}, \mathrm{b}(\mathrm{z})}$ muss jedoch im Vortrieb mit deutlich erhöhter Aufmerksamkeit verifiziert werden (Kap. 6.6.4). Eine quantitative Überprüfung dieser Annahme ist auf Basis der Daten aus den Referenzprojekten nicht möglich.

\subsubsection{Erwartungswert des Laufwegs $\mathrm{s}_{\mathrm{c}, \mathrm{e}(\mathrm{z})}$}

\subsubsection{Korrektur der Werkzeuggeometrie}

Für Schneidrollen muss auf Basis der Ergebnisse in Kap. 5.6.2.4 eine Korrektur für die Breite der Schneide des Schneidrings $b_{S R}[\mathrm{~mm}]$ vorgenommen werden. Für die Korrelation des $\mathrm{SAl}_{(\mathrm{z})}$ mit dem Laufwegs $\mathrm{S}_{\mathrm{c}(\mathrm{z})}$ in $\mathrm{Gl} .70$ wurde die Schneidringbreite auf $19 \mathrm{~mm}$ normiert. Die Datenanalyse zeigt, dass sich der Laufweg $\mathrm{s}_{\mathrm{c}}[\mathrm{km}]$ proportional mit der Breite der Schneidringe $\mathrm{b}_{\mathrm{SR}}[\mathrm{mm}]$ ändert. Der Basiswert des Laufwegs $\mathrm{S}_{\mathrm{c}, \mathrm{b}(\mathrm{z})}$ aus GI. 75 kann folglich übereinstimmend durch einen Faktor korrigiert werden, der sich aus dem Verhältnis der tatsächlichen Schneidringbreite $b_{S R}[\mathrm{~mm}]$ zum Referenzwert von $19 \mathrm{~mm}$ aus Gl. 77 ergibt:

$$
f_{b(z)}=\frac{b_{S R}}{19}
$$

Für Schälmesser, Räumer und Stichel ist auf Basis der Ergebnisse in Kap. 5.6.2.4 keine Quantifizierung des Einflusses der Breite der Schneide $b_{S M}$ bzw. $b_{S T}$ und $b_{R M}[\mathrm{~mm}]$, des Schneidwinkels $\beta_{\mathrm{c}}\left[{ }^{\circ}\right]$ und des Freiwinkels $\alpha_{c}\left[^{\circ}\right]$ möglich. Entsprechend kann für diese Einflüsse kein Korrekturfaktor abgeleitet werden. Die genannten Faktoren werden deshalb als nicht-quantifizierbare Einflüsse in Kap. 6.2.3.3 behandelt.

Bei der Anwendung von Schälmessern hinter vorlaufenden Sticheln mit vollständiger Abdeckung der Ortsbrust konnte in der Datenanalyse (Kap. 5.6.2.1) aufgrund des fehlenden Kontaktes der Schälmesser mit dem Boden in der Ortsbrust ein mittlerer Anstieg des Laufweg $\mathrm{s}_{\mathrm{c}}$ um den Faktor 3,4 festgestellt werden. Dieser Einflussfaktor kann in der Verschleißprognose bei entsprechenden Schneidradbestückungen mit dem Korrekturfaktor $\mathrm{f}_{\mathrm{h}(\mathrm{z})}$ aus $\mathrm{Gl}$. 78 berücksichtigt werden:

$$
f_{h(z)}=3,4
$$


Decken die vorlaufenden Stichel die Ortsbrust nicht vollständig ab, geht der positive Einfluss der Stichel auf den Laufweg $s_{c}$ der Schälmesser verloren. Eine teilweise Reduktion des Korrekturfaktors $f_{h(z)}$ im Verhältnis zur Abdeckung der Ortsbrust durch die Stichel ist deshalb nicht zulässig.

Der Korrekturfaktor $\mathrm{f}_{h(z)}$ ist aufgrund der Ähnlichkeit in der Anwendung auch für Räumer mit vorlaufenden Sticheln einsetzbar. Hier ist aufgrund der gekrümmten Schneidkontur eine separate Überprüfung der Abdeckung der Ortsbrust durch die Stichel notwendig. Für Stichel entfällt der Korrekturfaktor $\mathrm{f}_{\mathrm{h}(\mathrm{z})}$ bzw. nimmt den Wert 1 an, sodass sich kein Einfluss auf den Laufweg $\mathrm{s}_{\mathrm{c}}$ ergibt.

\subsubsection{Korrektur der Penetration $\mathrm{p}$}

Für Schälmesser, Räumer und Stichel muss der Basiswert des Laufwegs $\mathrm{s}_{\mathrm{c}, \mathrm{b}(\mathrm{z})}$ aufgrund der Ergebnisse in Kap. 5.6.3.2 um die Aktiv-Penetration $p_{a}$ korrigiert werden. Für die Korrelation des SAl mit dem Laufweg $s_{c}$ in Gl. 71 wurde eine Normierung der Aktiv-Penetration $p_{a}$ auf den arithmetischen Mittelwert der Penetration $p$ in den Referenzprojekten von $16 \mathrm{~mm} / \mathrm{U}$ vorgenommen. Dazu wurde aus den Ergebnissen in Kap. 5.6.3.2 der Korrekturfaktor $f_{p}$ (Gl. 69) entwickelt. Dieser ist umgekehrt auch für die Korrektur des Basiswertes für den Laufweg $\mathrm{s}_{\mathrm{c}, \mathrm{b}(\mathrm{z})}$ einsetzbar:

$$
f_{p(z)}=\frac{1}{1,6^{\log _{0,5}\left(\frac{16}{p_{a, e(z)}}\right)}}
$$

Zur Ermittlung von $f_{p(z)}$ mit Gl. 79 ist folglich ein Erwartungswert für die Aktiv-Penetration $p_{a, e(z)}[\mathrm{mm} / \mathrm{U}]$ im $\mathrm{HGB}_{(z)}$ notwendig. Bis dato bestehen keine Prognosemodelle für die Penetration $\mathrm{p}$ beim Vortrieb mit Hydroschild TVM in Lockergesteinen. Hauptproblem ist, dass die Penetration p im Gegensatz zu Festgesteinsvortrieben keine direkte Funktion der Gesteinsfestigkeit und Antriebsauslegung der TVM ist, sondern noch von zahlreichen weiteren Faktoren abhängt. Ein entsprechendes Modell ist nicht Kernziel der vorliegenden Arbeit. Aus den Referenzprojekten können jedoch die in Tab. 21 zusammengefassten Erfahrungswerte für die Penetration $p$ abgeleitet werden. Diese können als Richtwerte zur Ermittlung des Erwartungswertes für die Aktiv-Penetration $p_{a, e(z)}$ eingesetzt werden.

Tab. 21: Erfahrungswerte für die Penetration $p$ aus den Referenzprojekten.

\begin{tabular}{|l|c|}
\hline Bodenart & Richtwert Penetration p \\
\hline Böden mit Stein- \& Blockgehalt & $<10 \mathrm{~mm}$ \\
\hline Grob- \& gemischtkörnige Böden & $10-20 \mathrm{~mm}$ \\
\hline Bindige Böden & $20-40 \mathrm{~mm}$ \\
\hline
\end{tabular}

Zur Verwendung in Gl. 79 muss der Erwartungswert Penetration $p_{e(z)}[\mathrm{mm} / \mathrm{U}]$ in die Aktiv-Penetration $\mathrm{p}_{\mathrm{a}, \mathrm{e}(\mathrm{z})}$ der einzelnen Abbauwerkzeuge aufgeteilt werden. Bei mehrfach besetzten Schneidspuren erfolgt die Aufteilung anhand des Winkelabstandes $\delta_{a}\left[{ }^{\circ}\right]$ in Aktiv-Richtung (Kap. 5.4.5) zwischen den Abbauwerkzeugen auf einer Schneidspur. Der Erwartungswert für die Aktivpenetration $p_{a, e(z)}[\mathrm{mm} / \mathrm{U}]$ im $\mathrm{HGB}_{(\mathrm{z})}$ ergibt sich analog zu Gl. 40 aus Gl. 80:

$$
\mathrm{p}_{\mathrm{a}, \mathrm{e}(\mathrm{z})}=\frac{\delta_{\mathrm{a}}}{360} \cdot \mathrm{p}_{\mathrm{e}(\mathrm{z})}
$$

Für Schneidrollen ist auf Basis der Ergebnisse in Kap. 5.6.3.2 keine Korrektur des Erwartungswertes um die Penetration $\mathrm{p}$ notwendig. 


\subsubsection{Nicht quantifizierbare Einflussfaktoren}

Die Schwankungsbreite aller Einflussfaktoren auf den Laufweg $\mathrm{s}_{c}$, die in der Datenanalyse (Kap. 5.6) nicht quantifiziert werden konnten, wurden bei der Untersuchung zur Korrelation des SAI mit dem Laufweg $s_{c}$ nicht korrigiert. Umgekehrt können diese Faktoren auch bei der Schätzung des Erwartungswertes für den Laufweg $\mathrm{s}_{\mathrm{c}, \mathrm{e}(\mathrm{z})}$ nicht in quantitativer Form berücksichtigt werden.

$\mathrm{Zu}$ beachten ist jedoch, dass die Wertebereiche dieser Faktoren in den Referenzprojekten implizit auch der Korrelation des Laufwegs $\mathrm{S}_{\mathrm{c}}$ mit dem SAl in Gl. 75 und Gl. 76 im Prognosemodell zu Grunde liegen. Die Grenzen dieser Wertebereiche bilden folglich Anwendungsgrenzen für das Prognosemodell. Zusätzlich zu den in Tab. 22 genannten Einschränkungen wurden bei der Untersuchung der Korrelationen zwischen dem SAl und dem Laufweg $S_{c}$ für die verschiedenen Werkzeugtypen in Kap. 5.6.4.5 weitere Einschränkungen gemacht. Für Schneidrollen sind dies:

- 17 Zoll 1-Ring Schneidrollen

- Schneidringe aus hochlegiertem Werkzeugstahl mit einer Härte von 57 HRC

Für die Auswahl der Datensätze für Schälmesser wurden die folgenden Kriterien angesetzt:

- Verschleißschutz von mindestens einer durchgehenden Schneide aus Hartmetall und $30 \%$ Abdeckung der Rückenfläche durch Hartmetalleinsätze

- Sekundärer Bodenabbau in Kombination mit vorlaufenden Schneidrollen

Für Stichel wurden folgende Einschränkungen bei der Auswahl der Datensätze vorgenommen:

- Verschleißschutz von mindestens einer durchgehenden Schneide aus Hartmetall und $30 \%$ Abdeckung der Rückenfläche durch Hartmetalleinsätze

- Primärer Bodenabbau ohne Kombination mit Schneidrollen auf vorlaufenden oder angrenzenden Spuren

Um bei der Verschleißprognose für neue Projekte einen entsprechenden Abgleich zu ermöglichen ist in Tab. 22 der Wertebereich, sowie der vermutete Einfluss der nicht quantifizierbaren Faktoren in den Basisdaten des Prognosemodells dargestellt. Bei einer entsprechenden Abweichung von dem in Tab. 22 gegebenen Wertebereich in einem neuen Projekt verliert das Prognosemodell streng betrachtet seine Gültigkeit. Für einige der Faktoren kann jedoch zumindest eine qualitative Tendenz im Hinblick auf den Laufweg $\mathrm{s}_{\mathrm{c}}$ abgeleitet werden. 
Tab. 22: Zusammenfassung der nicht quantifizierbaren Einflussfaktoren auf den Laufweg $s_{c}$, deren vermutete Auswirkung und qualitative Tendenz in Bezug auf den Laufweg $\mathrm{s}_{\mathrm{c}}$ und der zugehörige Wertebereich in den Basisdaten aus den Referenzprojekten.

\begin{tabular}{|c|c|c|c|}
\hline $\begin{array}{l}\text { Nicht quantifizierbare Einfluss- } \\
\text { faktoren auf den Laufweg } s_{c}\end{array}$ & $\begin{array}{l}\text { Vermutl. Auswirkung } \\
\text { a. d. Laufweg } s_{c}\end{array}$ & $\begin{array}{c}\text { Vermutete qualitative } \\
\text { Tendenz } \\
\end{array}$ & $\begin{array}{l}\text { Wertebereich in den } \\
\text { Referenzdaten }\end{array}$ \\
\hline \multicolumn{4}{|l|}{ Auslegung der Hydroschild TVM } \\
\hline Schneidradöffnungsverhältnis $\left(\mathrm{O}_{\mathrm{TVM}}\right)$ [\%] & Hoch & Proportional & $28,4-31,0 \%$ \\
\hline Anzahl Rotoren \& Statoren [-] & Niedrig & Nicht qualifizierbar & $2-6$ \\
\hline $\begin{array}{l}\text { Schneidrollen Höhe über Schneidrad- } \\
\text { stahlbau }\left(\mathrm{h}_{\mathrm{SR}}\right)[\mathrm{mm}]\end{array}$ & Niedrig & Nicht qualifizierbar & $175 \mathrm{~mm}$ \\
\hline $\begin{array}{l}\text { Stichel Höhe über Schneidradstahlbau } \\
\left(\mathrm{h}_{\mathrm{ST}}\right)[\mathrm{mm}]\end{array}$ & Niedrig & Nicht qualifizierbar & $175-180 \mathrm{~mm}$ \\
\hline $\begin{array}{l}\text { Schälmesser Höhe über Schneidrad- } \\
\text { stahlbau }\left(h_{S M}\right)[\mathrm{mm}]\end{array}$ & Niedrig & Nicht qualifizierbar & $135-140 \mathrm{~mm}$ \\
\hline Winkelabstand $\delta_{a}\left[{ }^{\circ}\right]$ & Niedrig & Nicht qualifizierbar & $25^{\circ}-180^{\circ}$ \\
\hline Winkelabstand $\delta_{p}\left[^{\circ}\right]$ & Niedrig & Nicht qualifizierbar & $0^{\circ}-10^{\circ}$ \\
\hline Schälmesser Schneidwinkel $\left(\alpha_{c}\right)\left[^{\circ}\right]$ & Niedrig & Nicht qualifizierbar & $75^{\circ}-90^{\circ}$ \\
\hline Schälmesser Freiwinkel $\left(\beta_{c}\right)\left[^{\circ}\right]$ & Niedrig & Nicht qualifizierbar & $0^{\circ}-22^{\circ}$ \\
\hline Verschleißschutz Schälmesserschneide & Hoch & Proportional & $\begin{array}{l}\text { Hartmetall (WC), } \\
\text { durchgehend. }\end{array}$ \\
\hline $\begin{array}{l}\text { Verschleißschutz Schälmesser- } \\
\text { rückenfläche }\end{array}$ & Hoch & Proportional & $\begin{array}{l}\text { Hartmetall (WC), } \\
\text { min. } 30 \% \text { Abdeckung }\end{array}$ \\
\hline Verschleißschutz Schneidringe & Hoch & Proportional & $\begin{array}{l}\text { Hochleg. Stahl, durch- } \\
\text { gehärtet: } 57 \text { HRC }\end{array}$ \\
\hline \multicolumn{4}{|l|}{ Steuerung des Abbauprozesses } \\
\hline Dichte der Stützflüssigkeit $\left(\rho_{\mathrm{SF}}\right)\left[\mathrm{g} / \mathrm{cm}^{3}\right]$ & Mittel & $\begin{array}{l}\text { Umgekehrt } \\
\text { proportional }\end{array}$ & $1,15-1,37 \mathrm{~g} / \mathrm{cm}^{3}$ \\
\hline Stützdruck $\left(P_{S F}\right)[$ bar $]$ & Niedrig & $\begin{array}{l}\text { Umgekehrt } \\
\text { proportional }\end{array}$ & $0,9-3,7$ bar \\
\hline \multicolumn{4}{|l|}{ Geotechnische Parameter } \\
\hline Kornform & Mittel & Nicht qualifizierbar & flach - kubisch \\
\hline Kornrundungsgrad & Mittel & Proportional & $\begin{array}{l}\text { angerundet bis } \\
\text { gut gerundet }\end{array}$ \\
\hline
\end{tabular}

Aufgrund des hohen vermuteten Einflusses und im Hinblick auf zukünftige Untersuchungen werden für den Verschleißschutz der Abbauwerkzeuge und das Schneidringöffnungsverhältnis $\mathrm{O}_{\text {TVM }}[\%]$ im Prognosemodell bereits folgende Korrekturfaktoren eingefügt:

- Korrekturfaktor für den Verschleißschutz $f_{v}$

- Korrekturfaktor für das Schneidradöffnungsverhältnis $f_{\circ}$

Bis zu einer Quantifizierung der beiden Einflussfaktoren dienen diese als Platzhalter und tragen den Wert 1, sodass sie vorläufig auf das Ergebnis der Schätzung des Erwartungswertes für den Laufweg $\mathrm{s}_{\mathrm{c}, \mathrm{e}(\mathrm{z})}$ keinen Einfluss haben.

Die Kornform und der Kornrundungsgrad sollten im Hinblick auf zukünftige Untersuchungen in den SAI eingearbeitet werden (Kap. 7.2.3). 


\subsubsection{Berechnung des Erwartungswertes für den Laufweg $s_{c, e(z)}$}

Der Erwartungswert für den Laufweg $\mathrm{s}_{\mathrm{c}, \mathrm{e}(\mathrm{z})}$ ergibt sich allgemein aus der Multiplikation des Basiswertes für den Laufweg $\mathrm{S}_{\mathrm{c}, \mathrm{b}(\mathrm{z})}$ mit den Korrekturfaktoren für die Werkzeuggeometrie (Kap. 6.2.3.1), die AktivPenetration $\mathrm{p}_{\mathrm{a}, \mathrm{e}(\mathrm{z})}$ (Kap. 6.2.3.2) und bisher nicht quantifizierbaren Einflussfaktoren (Kap. 6.2.3.3).

Für Schneidrollen ist die Korrektur des Basiswertes $\mathrm{s}_{\mathrm{c}, \mathrm{b}(\mathrm{z})}$ um folgende Faktoren notwendig:

- Schneidringbreite $b_{S R}[\mathrm{~mm}]$ durch den Faktor $f_{b}$

- Verschleißschutz durch den Faktor $f_{v}$

- Schneidradöffnungsverhältnis $\mathrm{O}_{\text {TVM }}[\%]$ durch den Faktor $f_{\circ}$

Die Faktoren $f_{p}$ und $f_{o}$ zur Korrektur des Verschleißschutzes und des Schneidradöffnungsverhältnisses tragen vorerst den Wert 1, da sie in der vorliegenden Arbeit nicht ausreichend quantifiziert werden konnten. Der Erwartungswert für den Laufweg $\mathrm{S}_{\mathrm{c}, \mathrm{e}(\mathrm{z})}[\mathrm{km}]$ für Schneidrollen ergibt sich folglich aus Gl. 81:

$$
s_{c, e(z)}=s_{c, b(z)} \cdot f_{b(z)} \cdot f_{v(z)} \cdot f_{o(z)}
$$

Für Schälmesser, Stichel und Räumer ist die Korrektur des Basiswertes $\mathrm{s}_{\mathrm{c}, \mathrm{b}(\mathrm{z})}$ um folgende Faktoren notwendig:

- Aktivpenetration $\mathrm{p}_{\mathrm{a}, \mathrm{e}(\mathrm{z})}[\mathrm{mm} / \mathrm{U}]$ durch den Faktor $\mathrm{f}_{\mathrm{p}(\mathrm{z})}$

- Verschleißschutz durch den Faktor $f_{v(z)}$

- Schneidradöffnungsverhältnis $\mathrm{O}_{\mathrm{TVM}}[\%]$ durch den Faktor $\mathrm{f}_{\mathrm{o(z)}}$

- Wechselwirkung von Schälmessern und Räumern mit vorlaufenden Sticheln durch den Faktor $f_{h(z)}$

Die Faktoren $f_{p(z)}$ und $f_{o(z)}$ zur Korrektur des Verschleißschutzes und des Schneidradöffnungsverhältnisses tragen vorerst wiederum den Wert 1 . Der Erwartungswert für den Laufweg $s_{c, e(z)}[k m]$ für Schälmesser, Stichel und Räumer ergibt sich folglich aus GI. 82:

$$
s_{c, e(z)}=s_{c, b(z)} \cdot f_{p(z)} \cdot f_{v(z)} \cdot f_{o(z)} \cdot f_{h(z)}
$$

Der Erwartungswert für den Laufweg $\mathrm{s}_{\mathrm{c}, \mathrm{e}(\mathrm{z})}[\mathrm{km}]$ wird im Folgenden für die Schätzung der Intervalle $L_{\max (m)} z$ wischen den Vortriebsunterbrechungen $I_{(m)}$ für Werkzeugwechsel und der Anzahl der Werkzeugwechsel $n_{c, e(m)}$ eingesetzt. 


\subsection{Prognose der Vortriebsunterbrechungen I für Werkzeugwechsel}

\subsubsection{Grundlegende Zusammenhänge}

Die erwartete Anzahl $n_{\mathrm{l}, \mathrm{e}(\mathrm{TA})}$ und die Stationierung $\mathrm{L}_{\mathrm{l}, \mathrm{e}(\mathrm{m})}[\mathrm{m}]$ bzW. $\mathrm{b}_{\mathrm{l}, \mathrm{e}(\mathrm{m})}[-]$ der Vortriebsunterbrechungen $I_{(m)}$ für verschleißbedingte Werkzeugwechsel entlang der Tunnelachse hängen entscheidend von der Vortriebsstrecke $L_{d, e(m)}[m]$ zwischen den Vortriebsunterbrechungen $I_{(m)}$ ab. Durch konsekutive Aneinanderreihung der Vortriebsstrecken $L_{d, e(m)}$ von Vortriebsbeginn an, kann sowohl die erwartete Stationierung $L_{l, e(m)}$ bzw. $b_{l, e(m)}$, als auch die Anzahl der Vortriebsunterbrechungen $n_{l, e(T A)}$ bestimmt werden.

Die tatsächlich erreichten Vortriebsstrecken $L_{1(m)}$ im Vortrieb hängen neben dem Laufweg $S_{c}$ vom tatsächlichen Verschleißzustand der Abbauwerkzeuge, der Länge $L_{d(z)}[\mathrm{m}]$ und Abfolge der $\mathrm{HGB}_{(\mathrm{z})}$ und einer Reihe weicher Faktoren und Entscheidungen ab. Diese werden der Reihe nach im Prognoseprozess berücksichtigt. Als Ausgangspunkt für die Prognose der Vortriebsstrecken $L_{d, e(m)}$ werden zunächst idealisierte Bedingungen angenommen, sodass die Vortriebsstrecke $L_{d, e(m)}$ ausschließlich vom Erwartungswert für den Laufweg $\mathrm{s}_{\mathrm{c}, \mathrm{e}(\mathrm{z})}[\mathrm{km}]$ und dem Erwartungswert für die Penetration $\mathrm{p}_{\mathrm{e}(\mathrm{z})}$ $[\mathrm{mm} / \mathrm{U}]$ abhängt. Die idealisierten Bedingungen umfassen:

- Vollständig neuer Besatz des Schneidrades mit unverschlissenen Abbauwerkzeugen

- Betrachtung innerhalb eines unendlich langen Homogenbereichs $\mathrm{HGB}_{(\mathrm{z})}$

- Einhaltung der Verschleißgrenzen der Abbauwerkzeuge

- Keine Einschränkung der Vortriebsstrecke $L_{d, e(m)}$ durch andere Faktoren

Zur Bestimmung von $L_{d, e(m)}$ wird zunächst aus dem Erwartungswert für den Laufweg $s_{c, e(z)}[k m]$, dem Erwartungswert für die Penetration $p_{e(z)}[\mathrm{mm} / \mathrm{U}]$ und dem Spurradius $r_{\mathrm{s}}[\mathrm{mm}]$ für jedes einzelne $A b-$ bauwerkzeug auf dem Schneidrad der Erwartungswert der maximal erreichbaren Vortriebsstrecke $L_{c(m)}[m]$ zwischen den Vortriebsunterbrechungen $I_{(m)}$ bis zum Erreichen der Verschleißgrenzen mit Gl. 83 berechnet:

$$
\mathrm{L}_{\mathrm{c}(\mathrm{m})}=\frac{\mathrm{s}_{\mathrm{c}, \mathrm{e}(\mathrm{z})} \cdot \mathrm{p}_{\mathrm{e}(\mathrm{z})} \cdot 1000}{2 \cdot \pi \cdot \mathrm{r}_{\mathrm{s}}}
$$

Zu beachten ist dabei, dass in Gl. 83 für die unterschiedlichen Werkzeugtypen der jeweils zugehörige Erwartungswert $\mathrm{s}_{\mathrm{c}, \mathrm{e}(\mathrm{z})}$ aus Gl. 75 oder Gl. 76 (Kap. 6.2.3.4) eingesetzt werden muss. Umgekehrt gilt für alle Abbauwerkzeuge auf dem Schneidrad der gleiche Erwartungswert für die Penetration $\mathrm{p}_{\mathrm{e}(\mathrm{z})}[\mathrm{mm} / \mathrm{U}]$ (Tab. 21). Die Vortriebsstrecke $L_{d, e(m)}$ bis zur nächsten Vortriebsunterbrechung $I_{(m)}$ kann nur maximal so hoch gewählt werden, bis das erste Abbauwerkzeug auf dem Schneidrad die Verschleißgrenze erreicht. Zur Bestimmung des Maximalwertes $L_{\max (\mathrm{m})}[\mathrm{m}]$ muss also aus der Menge an Werten von $L_{c(m)}[\mathrm{m}]$ aller Abbauwerkzeuge auf dem Schneidrad der Minimalwert mit Gl. 84 ermittelt werden:

$$
\mathrm{L}_{\max (\mathrm{m})}=\min \left(\mathrm{L}_{\mathrm{c}(\mathrm{m})}\right)
$$

Unabhängig vom Werkzeugtyp liegt $L_{\max (\mathrm{m})}$ häufig bei Abbauwerkzeugen im Kaliberbereich des Schneidrads, weil die Abbauwerkzeuge hier aufgrund des im Vergleich hohen Spurradius $r_{s}$ je Schneidradumdrehung den höchsten Laufweg zurücklegen. Im Vortrieb muss als Erwartungswert der Vortriebsstrecken $L_{d, e(m)} z$ wischen den Vortriebsunterbrechungen $I_{(m)}$ nicht zwingend der mit Gl. 83 und Gl. 84 bestimmte Maximalwert $L_{\max (m)}$ angesetzt werden. 
Für $L_{d, e(m)}$ kann mit Gl. 85 auch jeder beliebige Wert kleiner als $L_{\max (m)}$ ausgewählt werden, ohne dass die Verschleißgrenze eines Abbauwerkzeuges erreicht oder überschritten wird:

$$
\mathrm{L}_{\mathrm{d}, \mathrm{e}(\mathrm{m})} \leq \mathrm{L}_{\max (\mathrm{m})}
$$

Diese Variationsmöglichkeit ist für den Algorithmus zur Anpassung der Verschleißprognose an die Umgebungsbedingungen im Projekt ein entscheidender Ansatzpunkt.

Innerhalb der geplanten Vortriebsstrecke $L_{d, e(m)}[m]$ legt jedes Abbauwerkzeug auf dem Schneidrad einen definierten Laufweg $S_{c d, e(m)}$ zurück. Dieser kann mithilfe des Erwartungswertes für die Penetration $p_{e(z)}[\mathrm{mm} / \mathrm{U}]$ und dem Spurradius $r_{s}[\mathrm{~mm}]$ mit Gl. 86 berechnet werden:

$$
\mathrm{s}_{\mathrm{cd}, \mathrm{e}(\mathrm{m})}=\frac{\mathrm{L}_{\mathrm{d}, \mathrm{e}(\mathrm{m})} \cdot 2 \cdot \pi \cdot \mathrm{r}_{\mathrm{s}}}{\mathrm{p}_{\mathrm{e}(\mathrm{z})} \cdot 1000}
$$

Der erwartete partielle Laufweg $\mathrm{s}_{\mathrm{cd}, \mathrm{e}(\mathrm{m})}[\mathrm{km}] \mathrm{kann}$ in Beziehung zum Erwartungswert des Laufwegs $\mathrm{S}_{\mathrm{c}, \mathrm{e}(\mathrm{z})}[\mathrm{km}]$ gesetzt werden, sodass sich aus Gl. 87 der Erwartungswert für den partiellen Ausnutzungsgrad $\mathrm{e}_{\mathrm{cd}, \mathrm{e}(\mathrm{m})}[\%]$ der einzelnen Abbauwerkzeuge innerhalb der Vortriebsstrecke $L_{d, e(m)}$ ergibt:

$$
\mathrm{e}_{\mathrm{cd}, \mathrm{e}(\mathrm{m})}=\frac{\mathrm{s}_{\mathrm{c}, \mathrm{e}(\mathrm{m})}}{\mathrm{s}_{\mathrm{c}, \mathrm{e}(\mathrm{z})}}
$$

Die dargestellten Zusammenhänge zur Prognose der Vortriebsstrecke $L_{d, e(m)}$ und zur Bestimmung des partiellen Laufwegs $S_{c d, e(m)}$ und des Ausnutzungsgrades $e_{c d, e(m)}$ werden im Folgenden Schritt für Schritt um folgende Punkte erweitert:

- Verkettung der Vortriebsstrecken $\mathrm{L}_{\mathrm{d}, \mathrm{e}(\mathrm{m})}[\mathrm{m}]$ (Kap. 6.3.2)

- Berücksichtigung der Abfolge unterschiedlicher geotechnischer Homogenbereiche $\mathrm{HGB}_{(\mathrm{z})}$ im Projekt (Kap. 6.3.3).

- Algorithmus zur Anpassung der Verschleißprognose an die Umgebungsbedingungen im Projekt (Kap. 6.5).

Die eingangs beschriebenen idealisierten Bedingungen werden in diesen Schritten abgebaut und die Verschleißprognose soweit wie möglich an ein realistisches Vortriebsszenario angepasst.

\subsubsection{Verkettung der Vortriebsstrecken $L_{d, e(m)}$}

Um die erwartete Anzahl $n_{1(T A)}$ und Stationierung $L_{1, e(m)}$ bzw. $b_{1, e(m)}$ der verschleißbedingten Vortriebsunterbrechungen $I_{(m)}$ für Werkzeugwechsel entlang der Tunnelachse zu ermitteln, müssen die Vortriebsstrecken $L_{d, e(m)}$ von Vortriebsbeginn an aneinandergereiht werden. Die Summe aller Vortriebsstrecken $L_{d, e(m)}[m]$ muss folglich der Tunnellänge $L_{T A}[m]$ entsprechen (Gl. 88):

$$
\mathrm{L}_{\mathrm{TA}}=\sum_{1}^{\mathrm{m}} \mathrm{L}_{\mathrm{d}, \mathrm{e}(\mathrm{m})}
$$


An den Knotenpunkten zwischen den Vortriebsstrecken $L_{d, e(m)}$ liegen jeweils die Vortriebsunterbrechungen $I_{(m)}$ für Werkzeugwechsel. Die kumulierte Vortriebsstrecke $L_{l, e(m)}[m]$ kann an jeder beliebigen Vortriebsunterbrechung $I_{(m)}$ aus der Summe der davor liegenden Vortriebsstrecken $L_{d, e(m)}[m]$ mit Gl. 89 bestimmt werden:

$$
\mathrm{L}_{\mathrm{I}, \mathrm{e}(\mathrm{m})}=\sum_{1}^{\mathrm{m}} \mathrm{L}_{\mathrm{d}, \mathrm{e}(\mathrm{m})}
$$

Der Fall in Gl. 88 ist folglich ein Spezialfall von Gl. 89, wobei in Gl. 88 der Durchbruch am Ende des Tunnels der letzten Vortriebsunterbrechung entspricht, sodass die kumulierte Vortriebsstrecke $L_{l, e(m)}$ in diesem Fall der Tunnellänge $L_{T A}$ entspricht.

Die kumulierte Vortriebsstrecke $\mathrm{L}_{\mathrm{l}, \mathrm{e}(\mathrm{m})}[\mathrm{m}]$ gibt die metrische Stationierung der Vortriebsunterbrechungen $\mathrm{I}_{(\mathrm{m})}$ an, bzw. kann ohne weiteres in diese umgerechnet werden, falls der Nullpunkt der Tunnelkilometrierung nicht an der Anschlagwand des Tunnels liegt. Zur Umrechnung der kumulierten Vortriebsstrecke $\mathrm{L}_{\mathrm{l}, \mathrm{e}(\mathrm{m})}$ in die zugehörigen Ringnummern $\mathrm{b}_{\mathrm{l}, \mathrm{e}(\mathrm{m})}$ im Vortrieb anhand der mittleren Breite der Tübbingringe $\mathrm{I}_{(\mathrm{b})}[\mathrm{m}]$ muss in Gl. 90 die Länge der Schildmaschine $\mathrm{L}_{\text {TVM }}[\mathrm{m}]$ berücksichtigt werden, weil die Ortsbrust ca. um die Länge der Schildmaschine in Vortriebsrichtung versetzt zum Einbaupunkt der Tübbingringe liegt:

$$
\mathrm{b}_{\mathrm{I}, \mathrm{e}(\mathrm{m})}=\frac{\mathrm{L}_{\mathrm{I}, \mathrm{e}(\mathrm{m})}-\mathrm{L}_{\mathrm{TVM}}}{\mathrm{l}_{(\mathrm{b})}}
$$

Zusätzlich müssen projektspezifische Verschiebungen der Ringnummern berücksichtigt werden, falls die Zählung der Ringnummern nicht an der Anschlagwand des Tunnels beginnt.

Ähnlich wie die kumulierte Vortriebsstrecke $L_{l, e(m)}$ kann der kumulierte Laufweg $s_{c, e(m)}$ an der Vortriebsunterbrechung $\mathrm{I}_{(\mathrm{m})}$ der einzelnen Abbauwerkzeuge durch Summierung aller bereits durchlaufenen partiellen Laufwege $\mathrm{S}_{\mathrm{cd}, \mathrm{e}(\mathrm{m})}$ mit Gl. 91 ermittelt werden. Als Beginn der Summierung wird hier die Ringnummer $b_{0}$ bei Einbau der Abbauwerkzeuge angesetzt:

$$
\mathrm{s}_{\mathrm{c}, \mathrm{e}(\mathrm{m})}=\sum_{\mathrm{b}_{0}}^{\mathrm{b}_{\mathrm{I}, \mathrm{e}(\mathrm{m})}} \mathrm{s}_{\mathrm{cd}, \mathrm{e}(\mathrm{m})}
$$

Analog zu Gl. 91 kann auch der kumulierte Ausnutzungsgrad $e_{c, e(m)}$ an den Vortriebsunterbrechungen $I_{(m)}$ durch Summierung der partiellen Ausnutzungsgrade $e_{c d, e(m)}$ berechnet werden:

$$
\mathrm{e}_{\mathrm{c}, \mathrm{e}(\mathrm{m})}=\sum_{\mathrm{b}_{0}}^{\mathrm{b}_{\mathrm{I}, \mathrm{e}(\mathrm{m})}} \mathrm{e}_{\mathrm{cd}, \mathrm{e}(\mathrm{m})}
$$

Ausgehend von einer beliebigen aktuellen Vortriebsunterbrechung $I_{(m)}$ kann die maximal erreichbare Vortriebsstrecke $L_{\max (m+1)}$ wieder mit Gl. 83 und Gl. 84 (Kap. 6.3.1) berechnet werden. Die Berechnung in Gl. 83 geht von einem vollständig neuen Besatz des Schneidrades mit unverschlissnenen Abbauwerkzeugen aus. 
Diese Annahme wird zunächst beibehalten, obwohl der kumulierte Ausnutzungsgrad $e_{c, e(m)}$ der Abbauwerkzeuge bei der Vortriebsunterbrechung $I_{(m)}$ aus GI. 92 bekannt ist. Aus Gl. 83 ergibt sich folglich die maximal erreichbare Vortriebsstrecke $L_{\max (m+1)}$ bis zur Vortriebsunterbrechung $I_{(m+1)}$.

Analog zu Gl. 85 gilt auch für die Vortriebsstrecke $L_{d, e(m+1)}$, dass sie kleiner oder gleich dem Maximalwert $L_{\max (m+1)}$ aus $G$ l. 84 ausgewählt werden kann:

$$
\mathrm{L}_{\mathrm{d}, \mathrm{e}(\mathrm{m}+1)} \leq \mathrm{L}_{\max (\mathrm{m}+1)}
$$

Die kumulierte Vortriebsstrecke $L_{\mathrm{l}, \mathrm{e}(\mathrm{m}+1)}$ bzw. Stationierung der folgenden Vortriebsunterbrechung $\mathrm{I}_{(\mathrm{m}+1)}$ kann folglich aus der Summe der kumulierten Vortriebsstrecke $L_{l, e(m)}$ der aktuellen Vortriebsunterbrechung $I_{(m)}$ und der geplanten Vortriebsstrecke $L_{d, e(m+1)}$ mit $G l .94$ berechnet werden:

$$
\mathrm{L}_{\mathrm{I}, \mathrm{e}(\mathrm{m}+1)}=\mathrm{L}_{\mathrm{I}, \mathrm{e}(\mathrm{m})}+\mathrm{L}_{\mathrm{d}, \mathrm{e}(\mathrm{m}+1)}
$$

Der erwartete partielle Laufweg der Abbauwerkzeuge $\mathrm{s}_{\mathrm{cd}, \mathrm{e}(\mathrm{m}+1)}[\mathrm{km}]$ in der geplanten Vortriebsstrecke $\mathrm{L}_{\mathrm{d}, \mathrm{e}(\mathrm{m}+1)}[\mathrm{m}]$ kann analog zu Gl. 86 für jedes einzelne Abbauwerkzeug unter Berücksichtigung des Spurradius $r_{s}[\mathrm{~mm}]$ und dem Erwartungswert für die Penetration $p_{e(z)}[\mathrm{mm} / \mathrm{U}]$ berechnet werden:

$$
\mathrm{s}_{\mathrm{cd}, \mathrm{e}(\mathrm{m}+1)}=\frac{\mathrm{L}_{\mathrm{d}, \mathrm{e}(\mathrm{m}+1)} \cdot 2 \cdot \pi \cdot \mathrm{r}_{\mathrm{s}}}{\mathrm{p}_{\mathrm{e}(\mathrm{z})} \cdot 1000}
$$

Der partielle Ausnutzungsgrad $e_{c d, e(m+1)}$ ergibt sich analog zu Gl. 87 aus dem Verhältnis des partiellen Laufwegs $\mathrm{s}_{\mathrm{cd}, \mathrm{e}(\mathrm{m}+1)}[\mathrm{km}]$ zum Erwartungswert des Laufwegs $\mathrm{s}_{\mathrm{c}, \mathrm{e}(\mathrm{z})}[\mathrm{km}]$ :

$$
\mathrm{e}_{\mathrm{cd}, \mathrm{e}(\mathrm{m}+1)}=\frac{\mathrm{s}_{\mathrm{cd}, \mathrm{e}(\mathrm{m}+1)}}{\mathrm{s}_{\mathrm{c}, \mathrm{e}(\mathrm{z})}}
$$

Abschließend kann aus dem vorliegenden kumulierten Laufweg $\mathrm{s}_{\mathrm{c}, \mathrm{e}(\mathrm{m})}$ an der aktuellen Vortriebsunterbrechung $I_{(m)}$ und dem geplanten partiellen Laufweg $s_{c d, e(m+1)}(G I .86)$ in der Vortriebsstrecke $L_{d, e(m+1)}$ bis zur nächsten Vortriebsunterbrechung $I_{(m+1)}$ auch der kumulierte Laufweg $\mathrm{s}_{c, e(m+1)}$ an der Vortriebsunterbrechung $\mathrm{I}_{(\mathrm{m}+1)}$ mit Gl. 97 berechnet werden:

$$
\mathrm{s}_{\mathrm{c}, \mathrm{e}(\mathrm{m}+1)}=\mathrm{s}_{\mathrm{c}, \mathrm{e}(\mathrm{m})}+\mathrm{s}_{\mathrm{cd}, \mathrm{e}(\mathrm{m}+1)}
$$

Analog zur Berechnung des erwarteten kumulierten Laufwegs $\mathrm{s}_{\mathrm{c}, \mathrm{e}(\mathrm{m}+1)}$ an der Vortriebsunterbrechung $I_{(m+1)}$ mit Gl. 98 kann auch der Erwartungswert des kumulierten Ausnutzungsgrades $e_{c, e(m+1)}$ an dieser Vortriebsunterbrechung berechnet werden:

$$
\mathrm{e}_{c, \mathrm{e}(\mathrm{m}+1)}=\mathrm{e}_{c, \mathrm{e}(\mathrm{m})}+\mathrm{e}_{\mathrm{cd}, \mathrm{e}(\mathrm{m}+1)}
$$


Ausgehend von der Vortriebsunterbrechung $I_{(m)}$ ist die Auswahl der Vortriebsstrecke $L_{d, e(m+1)}$ in $G$ l. 98 bis zur nächsten Vortriebsunterbrechung $\mathrm{I}_{(\mathrm{m}+1)}$ zunächst ohne Berücksichtigung des kumulierten Ausnutzungsgrads $e_{c, e(m)}$ der Abbauwerkzeuge erfolgt. Der kumulierte Ausnutzungsgrad der Abbauwerkzeuge an der Vortriebsunterbrechung $\mathrm{I}_{(\mathrm{m}+1)}$ kann folglich Werte $>1$ annehmen. Das bedeutet, dass für diese Abbauwerkzeuge bis zur Vortriebsunterbrechung $I_{(m+1)}$ die Verschleißgrenze überschritten werden würde.

Der geplante kumulierte Ausnutzungsgrad $e_{c, e(m+1)}$ muss zur Einhaltung der Verschleißgrenzen also als Kriterium für die Werkzeugwechsel in der Vortriebsunterbrechung $I_{(m)}$ herangezogen werden (Kap. 6.4). Für Abbauwerkzeuge, die während der Vortriebsunterbrechung $I_{(m)}$ gegen neue, unverschlissene Abbauwerkzeuge ausgetauscht werden, nimmt der Ausnutzungsgrad $\mathrm{e}_{\mathrm{c}, \mathrm{e}(\mathrm{m})}$ dann den Wert 0 an, weil bei der Berechnung von $e_{c, e(m)}$ mit Gl. 92 die Ringnummer bei Einbau $b_{0}$ der Stationierung $\mathrm{b}_{\mathrm{l}, \mathrm{e}(\mathrm{m})}$ der Vortriebsunterbrechung $\mathrm{I}_{(\mathrm{m})}$ entspricht.

Die Formulierung des Prognosemodells gibt an dieser Stelle folglich der Auswahl der Vortriebsstrecke $\mathrm{L}_{\mathrm{d}, \mathrm{e}(\mathrm{m}+1)}[\mathrm{m}]$ Vorrang vor der Bestimmung der notwendigen Anzahl an Werkzeugwechseln. Diese Vorgehensweise entspricht einerseits der eingangs in der Arbeit festgellten hohen Bedeutung der Anzahl und Positionierung der Vortriebsunterbrechungen $I_{(m)}$ für die Vortriebsleistung, Verschleißkosten und Verfahrenssicherheit (Kap. 1.2) im Vergleich zur Anzahl der Werkzeugwechsel. Andererseits ermöglicht diese Vorgehensweise einen flexiblen Anpassungsprozess (Kap. 6.5) der Vortriebsstrecken $L_{d, e(m)}$ an die Umgebungsbedingungen im Projekt.

\subsubsection{Verkettung der geotechnischen Homogenbereiche $\mathrm{HGB}_{(z)}$}

Die Auswahl der Vortriebsstrecken $\mathrm{L}_{\mathrm{d}, \mathrm{e}(\mathrm{m})}$ in $\mathrm{Gl} .85$ bzw. $\mathrm{L}_{\mathrm{d}, \mathrm{e}(\mathrm{m}+1)}$ in $\mathrm{Gl} .93$ basiert auf der idealisierten Annahme, dass der Vortrieb vollständig innerhalb eines geotechnischen Homogenbereichs $\mathrm{HGB}_{(\mathrm{z})}$ liegt. Tatsächlich werden in den Vortrieben häufig mehrere aufeinanderfolgende geotechnische Homogenbereiche durchfahren. Die Längen $L_{d(z)}[m]$ der geotechnischen Homogenbereiche ergeben in Summe die Länge der Tunnelachse $L_{T A}[m]$ :

$$
\mathrm{L}_{\mathrm{TA}}=\sum_{1}^{\mathrm{z}} \mathrm{L}_{\mathrm{d}(\mathrm{z})}
$$

Ähnlich wie die Vortriebsstrecken $L_{d, e(m)}$ können auch die geotechnischen Homogenbereiche bzw. deren Längen $L_{d(z)}[\mathrm{m}]$ von Vortriebsbeginn an miteinander verkettet werden. Die kumulierte Vortriebsstrecke $\mathrm{L}_{\mathrm{HGB}(\mathrm{z})}[\mathrm{m}]$ nach Durchfahren eines beliebigen Homogenbereichs $\mathrm{HGB}_{(\mathrm{z})}$ ergibt sich analog zu Gl. 89 aus der Summe der Längen $L_{d(z)}$ aller vorlaufend bereits durchfahrenen Homogenbereiche:

$$
\mathrm{L}_{\mathrm{HGB}(\mathrm{z})}=\sum_{1}^{\mathrm{z}} \mathrm{L}_{\mathrm{d}(\mathrm{z})}
$$

Der Fall in Gl. 99 ist folglich wiederum ein Spezialfall von Gl. 100, wobei in Gl. 99 der Homogenbereich $\mathrm{HGB}_{(\mathrm{z})}$ mit dem Durchbruch am Ende des Tunnels endet, sodass die kumulierte Vortriebsstrecke $\mathrm{L}_{\mathrm{HGB}(\mathrm{z})}$ in diesem Fall der Tunnellänge $\mathrm{L}_{\mathrm{TA}}$ entspricht.

Die kumulierte Vortriebsstrecke $\mathrm{L}_{\mathrm{HGB}(\mathrm{z})}[\mathrm{m}]$ gibt die metrische Stationierung der Grenzen zwischen den geotechnischen Homogenbereichen $\mathrm{HGB}_{(z)}$ und $\mathrm{HGB}_{(z+1)}$ an, bzw. kann ohne weiteres in diese umgerechnet werden, falls der Nullpunkt der Tunnelkilometrierung nicht an der Anschlagwand des Tunnels liegt. 
Zur Umrechnung der kumulierten Vortriebsstrecke $\mathrm{L}_{\mathrm{HGB}(\mathrm{z})}$ in die zugehörigen Ringnummern $\mathrm{b}_{\mathrm{HGB}(\mathrm{z})} \mathrm{im}$ Vortrieb anhand der mittleren Breite der Tübbingringe $\mathrm{I}_{(\mathrm{b})}[\mathrm{m}]$ muss die Länge der Schildmaschine $\mathrm{L}_{\text {TVM }}$ [m] in Gl. 101 berücksichtigt werden, weil die Ortsbrust ca. um die Länge der Schildmaschine in Vortriebsrichtung versetzt zum Einbaupunkt der Tübbingringe liegt:

$$
\mathrm{b}_{\mathrm{HGB}(\mathrm{z})}=\frac{\mathrm{L}_{\mathrm{HGB}(\mathrm{z})}-\mathrm{L}_{\mathrm{TVM}}}{\mathrm{l}_{(\mathrm{b})}}
$$

Zusätzlich müssen projektspezifische Verschiebungen der Ringnummern berücksichtigt werden, falls die Zählung der Ringnummern nicht an der Anschlagwand des Tunnels beginnt.

\subsubsection{Abgleich der Vortriebsstrecken $L_{d, e(m)}$ und Homogenbereiche $H_{G B} B_{(z)}$}

Aufgrund der Änderung der geotechnischen Parameter ergeben sich in den Homogenbereichen $\mathrm{HGB}_{(\mathrm{z})}$ aus Gl. 72 (Kap. 6.2.2) unterschiedliche Werte für den $\mathrm{SAl}_{(\mathrm{z})}$, sodass bereits aus Gl. 75 bzw. Gl. 76 unterschiedliche Basiswerte für den Laufweg $\mathrm{S}_{\mathrm{c}, \mathrm{b}(z)}$ resultieren. Zusätzlich können auch Änderung der Steuerung des Abbauprozesses, z.B. der Penetration $p_{e(z)}$, oder Änderung der Auslegung der TVM, z.B. der Werkzeugbestückung, in Gl. 81 und Gl. 82 zu weiteren Unterschieden zwischen den Erwartungswerten für den Laufweg $\mathrm{S}_{\mathrm{c}, \mathrm{e}(\mathrm{z})}$ in den geotechnischen Homogenbereichen beitragen. Die geotechnischen Homogenbereiche $\mathrm{HGB}_{(z)}$ müssen folglich bei der Prognose der Vortriebsunterbrechungen $I_{(m)}$ (Kap. 6.3) aufgrund der Unterschiede im Erwartungswert für den Laufweg $\mathrm{s}_{\mathrm{c}, \mathrm{e}(\mathrm{z})}$ berücksichtigt werden.

Die Auswahl der Vortriebsstrecken $L_{d, e(m+1)}$ zwischen den aufeinanderfolgenden Vortriebsunterbrechungen $I_{(m)}$ und $I_{(m+1)}$ mit Gl. 85 geht davon aus, dass $I_{(m)}$ und $I_{(m+1)}$ im gleichen geotechnischen Homogenbereich $\mathrm{HGB}_{(z)}$ liegen. Bei mehreren aufeinanderfolgenden geotechnischen Homogenbereichen $\mathrm{HGB}_{(1-z)}$ treten jedoch zwangsläufig Vortriebsstrecken $L_{d, e(m)}$ auf, innerhalb derer eine Grenze zwischen zwei $\mathrm{HGB}_{(z)}$ durchfahren wird. Diese können erfasst werden, indem an den Vortriebsunterbrechungen $I_{(m)}$ die geplante kumulierte Vortriebsstrecke $L_{1, e(m+1)}[m]$ an der folgenden Vortriebsunterbrechung $\mathrm{I}_{(\mathrm{m}+1)}$ mit der kumulierten Vortriebsstrecke $\mathrm{L}_{\mathrm{HGB}(\mathrm{z})}$ der geotechnischen Homogenbereichen abgeglichen wird.

Für den Abgleich ist es zunächst notwendig, den Vortriebsunterbrechungen $\mathrm{I}_{(\mathrm{m})}$ als zweiten Index die Nummer des Homogenbereichs $\mathrm{HGB}_{(z)}$ zuzuordnen, indem sie zum Liegen kommen. Diese Indizierung ergibt also die Vortriebsunterbrechungen $\mathrm{I}_{(\mathrm{m} ; \mathrm{z})}$ im Homogenbereich $\mathrm{HGB}_{(\mathrm{z})}$. Die Prognose der Anzahl $\mathrm{n}_{\mathrm{l}, \mathrm{e}(\mathrm{TA})}$ und Stationierung $\mathrm{L}_{\mathrm{l}, \mathrm{e}(\mathrm{m})}$ bzw. $\mathrm{b}_{\mathrm{l}, \mathrm{e}(\mathrm{m})}$ der verschleißbedingten Vortriebsunterbrechungen $I_{(m, z)}$ muss aufgrund des Abgleichs als iterativer Prozess formuliert werden. Dieser dient zur Annäherung der maximal erreichbaren Vortriebsstrecke $L_{\max (m+1)}$ zwischen den Vortriebsunterbrechungen $I_{(m)}$ und $\mathrm{I}_{(\mathrm{m}+1)}$. Die Anzahl der Wiederholungen des Rechenprozesses bzw. der Iterationen zur Annäherung von $L_{\max (m+1)}$ wird im Rechenprozess mit dem Index $n$ versehen.

\section{Schritt 1:}

\section{a) 1. Iteration}

An einer aktuellen Vortriebsunterbrechung $I_{(z ; m)}$ wird mit Gl. 83 und Gl. 84 (Kap. 6.3.1) zunächst die maximal erreichbare Vortriebsstrecke $L_{\max (m+1)}[\mathrm{m}]$ bis zur folgenden Vortriebsunterbrechung bestimmt. In Gl. 83 werden dabei Erwartungswerte für den Laufweg $\mathrm{s}_{\mathrm{c}, \mathrm{e}(\mathrm{z})}$ und die Penetration $\mathrm{p}_{\mathrm{e}(\mathrm{z})}$ im $\mathrm{HGB}_{(\mathrm{z})}$ eingesetzt, indem auch die Vortriebsunterbrechung $\mathrm{I}_{(\mathrm{m} ; \mathrm{z})}$ liegt. Die Berechnung geht also davon aus, dass die Vortriebsstrecke $L_{\max (m+1)}$ vollständig im $\mathrm{HGB}_{(\mathrm{z})}$ verläuft. Folglich würde die nächste folgende Vortriebsunterbrechung den Index $I_{(m+1 ; z)}$ tragen. 
b) n-te Iteration

Die Berechnung der maximal erreichbaren Vortriebsstrecke $L_{\max (m+1)}$ wird in $n$ separat zu betrachtende Teilstücke aufgeteilt. Der ganzzahlige Index $\mathrm{n}$ gibt die Anzahl der Iterationen bzw. Wiederholungen des Rechenprozesses zwischen Schritt 1 und Schritt 4 an. Die erste Durchführung des Rechenprozesses, also die 1. Iteration, ist separat beschrieben. Der Index $\mathrm{n}$ kann deshalb im Folgenden nur Werte $\mathrm{n}>1$ annehmen.

Das erste Teilstück der maximal erreichbaren Vortriebsstrecke $L_{\max (m+1)}[\mathrm{m}]$ bildet die Vortriebsstrecke $L_{\max (m+1 ; z)}[m]$, die ausgehend von der Vortriebsunterbrechung $I_{(m ; z)}$ noch im Homogenbereich $\mathrm{HGB}_{(\mathrm{z})}$ aufzufahren ist (Gl. 102):

$$
\mathrm{L}_{\max (\mathrm{m}+1 ; \mathrm{z})}=\mathrm{L}_{\mathrm{HGB}(\mathrm{z})}-\mathrm{L}_{\mathrm{I}, \mathrm{e}(\mathrm{m})}
$$

Innerhalb des ersten Teilstücks legt jedes Abbauwerkzeug auf dem Schneidrad den partiellen Laufweg $\mathrm{S}_{\mathrm{cd}, \mathrm{e}(\mathrm{m}+1 ; \mathrm{z})}[\mathrm{km}]$ zurück, der analog zu Gl. 86 (Kap. 6.3.2) mit Gl. 103 berechnet wird:

$$
\mathrm{s}_{\mathrm{cd}, \mathrm{e}(\mathrm{m}+1 ; \mathrm{z})}=\frac{\mathrm{L}_{\max (\mathrm{m}+1 ; \mathrm{z})} \cdot 2 \cdot \pi \cdot \mathrm{r}_{\mathrm{s}}}{\mathrm{p}_{\mathrm{e}(\mathrm{z})} \cdot 1.000}
$$

Aus dem Laufweg $s_{c d, e(m+1 ; z)}$ kann mit Bezug auf den Erwartungswert für den Laufweg $s_{c, e(z)}$ im Homogenbereich $\mathrm{HGB}_{(z)}$ der partielle Ausnutzungsgrad $\mathrm{e}_{\mathrm{cd}, \mathrm{e}(\mathrm{m}+1 ; \mathrm{z})}$ der einzelnen Abbauwerkzeuge an der Grenze zwischen den Homogenbereichen $\mathrm{HGB}_{(z)}$ und $\mathrm{HGB}_{(z+1)}$ nach Durchlaufen von $L_{\max (m+1 ; z)}$ mit Gl. 104 berechnet werden:

$$
\mathrm{e}_{\mathrm{cd}, \mathrm{e}(\mathrm{m}+1 ; \mathrm{z})}=\frac{\mathrm{s}_{\mathrm{cd}, \mathrm{e}(\mathrm{m}+1 ; \mathrm{z})}}{\mathrm{s}_{\mathrm{c}, \mathrm{e}(\mathrm{z})}}
$$

Zwischen dem ersten und dem letzten Teilstück der maximal erreichbaren Vortriebsstrecke $\mathrm{L}_{\max (m+1)}$ liegen $\mathrm{n}-2$ geotechnische Homogenbereiche von $\mathrm{HGB}_{(z+1)}$ bis $\mathrm{HGB}_{(z+(n-1))}$, die ohne Vortriebsunterbrechung vollständig durchfahren werden. Innerhalb dieser Homogenbereiche legt jedes Abbauwerkzeug auf dem Schneidrad den partiellen Laufweg $S_{c d, e(m+1 ; z+n)}[k m]$ zurück, der analog zu Gl. 103 berechnet werden kann:

$$
\mathrm{s}_{\mathrm{cd}, \mathrm{e}(\mathrm{m}+1 ; \mathrm{z}+\mathrm{n})}=\frac{\mathrm{L}_{\mathrm{d}(\mathrm{z}+\mathrm{n})} \cdot 2 \cdot \pi \cdot \mathrm{r}_{\mathrm{s}}}{\mathrm{p}_{\mathrm{e}(\mathrm{z}+\mathrm{n})} \cdot 1.000}
$$

Da die geotechnischen Homogenbereiche $\mathrm{HGB}_{(z)}$ vollständig durchfahren werden, entspricht in Gl. 105 die Länge der geotechnischen Homogenbereiche $L_{d(z+n)}$ der maximal erreichbaren Vortriebsstrecke $L_{(\max ; z+n)}[\mathrm{m}]$. Aus dem Laufweg $\mathrm{S}_{\mathrm{cd}, \mathrm{e}(\mathrm{m}+1 ; z+n)}$ kann mit Bezug auf den Erwartungswert für den Laufweg $S_{c, e(z+n)}$ im Homogenbereich $\mathrm{HGB}_{(z+n)}$ der partielle Ausnutzungsgrad $e_{c d, e(m+1 ; z+n)}$ der einzelnen Abbauwerkzeuge beim Durchlaufen von $L_{d(z+n)}$ berechnet werden:

$$
\mathrm{e}_{\mathrm{cd}, \mathrm{e}(\mathrm{m}+1 ; \mathrm{z}+\mathrm{n})}=\frac{\mathrm{s}_{\mathrm{cd}, \mathrm{e}(\mathrm{m}+1 ; \mathrm{z}+\mathrm{n})}}{\mathrm{s}_{\mathrm{c}, \mathrm{e}(\mathrm{z}+\mathrm{n})}}
$$


Das letzte Teilstück der maximal erreichbaren Vortriebsstrecke $L_{l, m a x(m+1)}$ wird analog zu Gl. 83 und Gl. 84 (Kap. 6.3.1) berechnet. Hierbei muss zusätzlich der bestehende Ausnutzungsgrad der Abbauwerkzeuge aus den zuvor durchlaufenen Homogenbereichen $\mathrm{HGB}_{(z)}$ bis $\mathrm{HGB}_{(z+(n-1))}$ berücksichtigt werden. Aus dem Erwartungswert für den Laufweg $s_{c, e(z+n)}[k m]$, der Penetration $p_{e(z+n)}$ $[\mathrm{mm} / \mathrm{U}]$ und dem Spurradius $r_{\mathrm{s}}[\mathrm{mm}]$ wird für jedes einzelne Abbauwerkzeug auf dem Schneidrad die maximal erreichbare Vortriebsstrecke $L_{c(m+1 ; z+n)}[\mathrm{m}]$ zwischen der Grenze zwischen den Homogenbereichen $\mathrm{HGB}_{(z+(n-1))}$ und $\mathrm{HGB}_{(z+n)}$ bis zum Erreichen der Verschleißgrenzen mit Gl. 107 berechnet:

$$
\mathrm{L}_{\mathrm{c}(\mathrm{m}+1 ; \mathrm{z}+\mathrm{n})}=\mathrm{s}_{\mathrm{c}, \mathrm{e}(\mathrm{z}+\mathrm{n})} \cdot\left(1-\sum_{\mathrm{z}=1}^{\mathrm{n}-1} \mathrm{e}_{\mathrm{cd}, \mathrm{e}(\mathrm{m}+1 ; \mathrm{z}+\mathrm{n})}\right) \cdot \mathrm{p}_{\mathrm{e}(\mathrm{z}+\mathrm{n})} \cdot \frac{1.000}{2 \cdot \pi \cdot \mathrm{r}_{\mathrm{s}}}
$$

Zur Bestimmung von $L_{\max (m+1 ; z+n)}[m]$ muss anschließend aus der Menge an Werten von $L_{c(m+1 ; z+n)}$ [m] aller Abbauwerkzeuge auf dem Schneidrad mit Gl. 108 der Minimalwert ermittelt werden:

$$
\mathrm{L}_{\max (\mathrm{m}+1 ; \mathrm{z}+\mathrm{n})}=\min \left(\mathrm{L}_{\mathrm{c}(\mathrm{m}+1 ; \mathrm{z}+\mathrm{n})}\right)
$$

Innerhalb des letzten Teilstücks $L_{\max (m+1 ; z+n)}$ legt jedes Abbauwerkzeug auf dem Schneidrad den partiellen Laufweg $S_{c d, e(m+1 ; z+n)}[k m]$ zurück, der mit Gl. 109 berechnet wird:

$$
\mathrm{s}_{\mathrm{cd}, \mathrm{e}(\mathrm{m}+1 ; \mathrm{z}+\mathrm{n})}=\frac{\mathrm{L}_{\max (\mathrm{m}+1 ; \mathrm{z}+\mathrm{n})} \cdot 2 \cdot \pi \cdot \mathrm{r}_{\mathrm{s}}}{\mathrm{p}_{\mathrm{e}(\mathrm{z}+\mathrm{n})} \cdot 1.000}
$$

Aus dem erwarteten partiellen Laufweg $s_{c d, e(m+1 ; z+n)}$ kann mit Bezug auf den Erwartungswert für den Laufweg $S_{c, e(z+n)}$ im Homogenbereich $\mathrm{HGB}_{(z+n)}$ der partielle Ausnutzungsgrad $e_{c d, e(m+1 ; z+n)}$ der einzelnen Abbauwerkzeuge beim Durchlaufen von $L_{\max (m+1 ; z+n)}$ mit $G l .110$ berechnet werden:

$$
\mathrm{e}_{\mathrm{cd}, \mathrm{e}(\mathrm{m}+1 ; \mathrm{z}+\mathrm{n})}=\frac{\mathrm{s}_{\mathrm{cd}, \mathrm{e}(\mathrm{m}+1 ; \mathrm{z}+\mathrm{n})}}{\mathrm{s}_{\mathrm{c}, \mathrm{e}(\mathrm{z}+\mathrm{n})}}
$$

Die maximal erreichbare Vortriebsstrecke $L_{\max (m+1)}[\mathrm{m}]$ zwischen den aufeinanderfolgenden Vortriebsunterbrechungen $I_{(m ; z)}$ und $I_{(m+1 ; z+n)}$ in den aufeinanderfolgenden Homogenbereichen $\mathrm{HGB}_{(\mathrm{z})}$ bis $\mathrm{HGB}_{(z+n)}$ ergibt sich aus der Summierung der $n$ Teilstücke mit Gl. 111:

$$
\mathrm{L}_{\max (m+1)}=\sum_{1}^{\mathrm{n}} \mathrm{L}_{\max (m+1 ; \mathrm{z}+\mathrm{n})}
$$




\section{Schritt 2:}

Der Maximalwert der erreichbaren Vortriebsstrecke $L_{\max (m+1)}[\mathrm{m}]$ wird anschließend in Gl. 112 zur Berechnung der erwarteten Stationierung $L_{1, e(m+1)}[m]$ der Vortriebsunterbrechung $I_{(m+1 ; z+n)}$ eingesetzt:

$$
\mathrm{L}_{\mathrm{I}, \mathrm{e}(\mathrm{m}+1)}=\mathrm{L}_{\mathrm{I}, \mathrm{e}(\mathrm{m})}+\mathrm{L}_{\max (\mathrm{m}+1)}
$$

\section{Schritt 3:}

\section{a) 1. Iteration}

Mit GI. 100 (Kap. 6.3.3) wird die kumulierte Vortriebsstrecke LHGB(z) $_{\text {[m] }}$ bis zum Endes des geotechnischen Homogenbereichs $\mathrm{HGB}_{(z)}$ berechnet, in dem die Vortriebsunterbrechung $\mathrm{I}_{(\mathrm{m} ; \mathrm{z})}$ liegt.

b) n-te Iteration

Mit Gl. 100 (Kap. 6.3.3) wird die kumulierte Vortriebsstrecke $L_{H G B(z+n)}$ bis zum Endes des geotechnischen Homogenbereichs $\mathrm{HGB}_{(\mathrm{z}+\mathrm{n})}$ berechnet.

\section{Schritt 4:}

\section{a) 1. Iteration}

Die kumulierte Vortriebsstrecke $\mathrm{L}_{\mathrm{l}, \mathrm{e}(\mathrm{m}+1)}[\mathrm{m}]$ aus Schritt 2 (Gl. 112) wird mit der kumulierten Vortriebsstrecke $L_{H G B(z)}[\mathrm{m}]$ aus Schritt 3 verglichen. Der Vergleich kann zwei Ergebnisfälle liefern:

$$
\begin{aligned}
& \mathrm{L}_{\mathrm{I}, \mathrm{e}(\mathrm{m}+1)} \leq \mathrm{L}_{\mathrm{HGB}(\mathrm{z})} \\
& \mathrm{L}_{\mathrm{I}, \mathrm{e}(\mathrm{m}+1)}>\mathrm{L}_{\mathrm{HGB}(\mathrm{z})}
\end{aligned}
$$

Wenn Gl. 113 gilt, wird innerhalb von $L_{\max (m+1)}$ die Grenze zwischen den Homogenbereichen $\mathrm{HGB}_{(\mathrm{z})}$ und $\mathrm{HGB}_{(\mathrm{z}+1)}$ nicht überschritten. Die aufeinander folgenden Vortriebsunterbrechungen $\mathrm{I}_{(\mathrm{m} ; \mathrm{z})}$ und $\mathrm{I}_{(\mathrm{m}+1 ; z)}$ liegen also im gleichen Homogenbereich $\mathrm{HGB}_{(\mathrm{z})}$. Die Vortriebsstrecke $\mathrm{L}_{\max (\mathrm{m}+1)}$ kann also ohne weitere Rechenschritte in die Prognose übernommen werden. Der iterative Prozess wird mit Schritt 5 fortgesetzt.

Wenn Gl. 114 gilt, wird innerhalb von $L_{\max (m+1)}$ die Grenze zwischen $\mathrm{HGB}_{(z)}$ und $\mathrm{HGB}_{(\mathrm{z}+1)}$ überschritten. Im HGB $\mathrm{HG}_{(z+1)}$ gilt ein anderer Erwartungswerte für den Laufweg $\mathrm{S}_{\mathrm{c}, \mathrm{e}(\mathrm{z}+1)}$ und die Penetration $p_{e(z+1)}$. Die Berechnung des Maximalwerts der Vortriebsstrecke $L_{\max (m+1)}$ muss ab dem 1 . Schritt wiederholt und in zwei Teile aufgeteilt werden. 
b) n-te Iteration

Die erwartete kumulierte Vortriebsstrecke bzw. Stationierung $L_{l, e(m+1)}[\mathrm{m}]$ aus Schritt 2 (Gl. 112) wird mit der kumulierten Vortriebsstrecke $L_{H G B(z+n)}[m]$ aus Schritt 3 verglichen. Der Vergleich kann zwei Ergebnisfälle liefern:

$$
\begin{aligned}
& \mathrm{L}_{\mathrm{I}, \mathrm{e}(\mathrm{m}+1)} \leq \mathrm{L}_{\mathrm{HGB}(\mathrm{z}+\mathrm{n})} \\
& \mathrm{L}_{\mathrm{I}, \mathrm{e}(\mathrm{m}+1)}>\mathrm{L}_{\mathrm{HGB}(\mathrm{z}+\mathrm{n})}
\end{aligned}
$$

Wenn Gl. 115 gilt, wird innerhalb von $L_{\max (m+1)}$ die Grenze zwischen den Homogenbereichen $\mathrm{HGB}_{(z+n)}$ und $\mathrm{HGB}_{(z+(n+1))}$ nicht überschritten. Die Vortriebsunterbrechungen $\mathrm{I}_{(m+1 ; z+n)}$ liegt also im Homogenbereich $\mathrm{HGB}_{(z+n)}$. Die Vortriebsstrecke $L_{\max (m+1)}$ kann also ohne weitere Iteration übernommen werden. Der Prozess wird mit Schritt 5 fortgesetzt.

Wenn Gl. 116 gilt, wird innerhalb von $L_{\max (m+1)}$ die Grenze zwischen den Homogenbereichen $\mathrm{HGB}_{(z+n)}$ und $\mathrm{HGB}_{(z+(n+1))}$ überschritten. Im Homogenbereich $\mathrm{HGB}_{(z+(n+1))}$ gilt ein anderer Erwartungswert für den Laufweg $\mathrm{S}_{\mathrm{c}, \mathrm{e}(\mathrm{z}+(n+1))}$ und die Penetration $\mathrm{p}_{\mathrm{e}(\mathrm{z}+(\mathrm{n}+1))}$. Um diese zu berücksichtigen, muss der Maximalwert der Vortriebsstrecke $L_{\max (m+1)}$ mit der Iteration $n+1$ ab Schritt 1 weiter angenähert werden.

\section{Schritt 5:}

Analog zu (Gl. 86) (Kap. 6.3.1) wird der Erwartungswert der Vortriebsstrecke $L_{d, e(m+1)}[m]$ kleiner oder gleich dem Maximalwert $L_{\max (m+1)}$ aus Schritt 1 (Gl. 111) ausgewählt:

$$
\mathrm{L}_{\mathrm{d}, \mathrm{e}(\mathrm{m}+1)} \leq \mathrm{L}_{\max (\mathrm{m}+1)}
$$

\section{Schritt 6:}

Aus der ausgewählten Vortriebsstrecke $L_{d, e(m+1)}[\mathrm{m}]$ aus $\mathrm{Gl} .117$ in Schritt 5 und der kumulierten Vortriebsstrecke $L_{1, e(m)}[m]$ an der aktuellen Vortriebsunterbrechung $I_{(m ; z)}$ wird mit $G$ l. 118 analog zu Gl. 112 die geplante kumulierte Vortriebsstrecke $L_{l, e(m+1)}$ berechnet:

$$
\mathrm{L}_{\mathrm{I}, \mathrm{e}(\mathrm{m}+1)}=\mathrm{L}_{\mathrm{I}, \mathrm{e}(\mathrm{m})}+\mathrm{L}_{\mathrm{d}, \mathrm{e}(\mathrm{m}+1)}
$$

Anschließend wird anhand der kumulierten Vortriebsstrecke $\mathrm{L}_{\mathrm{l}, \mathrm{e}(\mathrm{m}+1)}[\mathrm{m}]$ durch Abgleich mit den kumulierten Vortriebsstrecken $\mathrm{L}_{\mathrm{HGB}(z)}$ an den Grenzen zwischen den geotechnischen Homogenbereichen bestimmt, in welchem Homogenbereich die Vortriebsunterbrechung $I_{(m+1)}$ liegt. In Abhängigkeit davon, welcher Wert für $L_{d, e(m+1)}$ in Gl. 117 in Schritt 5 im Verhältnis $z u L_{\max (m+1)}$ ausgewählt wurde, können dies die Homogenbereiche $\mathrm{HGB}_{(z)}$ bis $\mathrm{HGB}_{(\mathrm{z}+\mathrm{n})}$ sein.

Um im nachfolgenden Schritten 7 Verwechslungen mit der Nummerierung der Iterationen durch den Index $\mathrm{n}$ zu vermeiden, wird der Homogenbereich in dem die Vortriebsunterbrechung $\mathrm{I}_{(\mathrm{m}+1)}$ eingeordnet wird als $\mathrm{HGB}_{(\mathrm{z}+\mathrm{k})}$ bezeichnet. Für die ganzzahlige Variable k muss folglich Gl. $119 \mathrm{gel}-$ ten:

$$
0 \leq \mathrm{k} \leq \mathrm{n}
$$


Die Vortriebsunterbrechung $I_{(m+1 ; z+k)}$ muss folglich zwischen der Untergrenze $L_{H G B(z+(k-1))}$ und der Obergrenze $\mathrm{L}_{\mathrm{HGB}(z+\mathrm{k})}$ der geotechnischen Homogenbereich $\mathrm{HGB}_{(z+k)}$ liegen. Für die kumulierte Vortriebsstrecke $L_{l, e(m+1)}$ an der Vortriebsunterbrechung $I_{(m+1 ; z+k)}$ gilt also Gl. 120:

$$
\mathrm{L}_{\mathrm{HGB}(\mathrm{z}+(\mathrm{k}-1))}<\mathrm{L}_{\mathrm{I}, \mathrm{e}(\mathrm{m}+1)} \leq \mathrm{L}_{\mathrm{HGB}(\mathrm{z}+\mathrm{k})}
$$

\section{Schritt 7:}

In Schritt 7 werden der erwartete kumulierte Laufweg $\mathrm{s}_{\mathrm{c}, \mathrm{e}(\mathrm{m}+1)}$ und der kumulierte Ausnutzungsgrad $\mathrm{e}_{\mathrm{c}, \mathrm{e}(\mathrm{m}+1)}$ an der Vortriebunterbrechung $\mathrm{I}_{(\mathrm{m}+1 ; z+\mathrm{k})}$ berechnet. Ähnlich wie die Berechnung der maximal erreichbaren Vortriebsstrecke $L_{\max (m+1)}$ in der $n$-ten Iteration in Schritt 1 muss die erwartete Vortriebsstrecke $L_{d, e(m+1)}$ dazu in $k+1$ separat $z u$ betrachtende Teilstücke $L_{d, e(m+1 ; z+k)}$ aufgeteilt werden. Für diese Teilstücke wird jeweils der partielle Laufweg $s_{c d, e(m+1 ; z+k)}$ und der partielle Ausnutzungsgrad $e_{c d, e(m+1 ; z+k)}$ berechnet. Die kumulierten Werte für den Laufweg $s_{c, e(m+1)}$ und den Ausnutzungsgrad $e_{c, e(m+1)}$ werden abschließend durch Summierung der Teilstücke generiert.

Das erste Teilstück $L_{d, e(m+1 ; z)}[m]$ der erwarteten Vortriebsstrecke $L_{d, e(m+1)}$ ergibt sich aus der Vortriebsstrecke, die ausgehend von der Vortriebsunterbrechung $\mathrm{I}_{(\mathrm{m} ; \mathrm{z})}$ noch im $\mathrm{HGB}_{(\mathrm{z})}$ aufzufahren ist mit Gl. 121:

$$
\mathrm{L}_{\mathrm{d}, \mathrm{e}(\mathrm{m}+1 ; \mathrm{z})}=\mathrm{L}_{\mathrm{HGB}(\mathrm{z})}-\mathrm{L}_{\mathrm{I}, \mathrm{e}(\mathrm{m})}
$$

Innerhalb des ersten Teilstücks $L_{d, e(m+1 ; z)}$ legt jedes Abbauwerkzeug auf dem Schneidrad den erwarteten partiellen Laufweg $S_{c d, e(m+1 ; z)}[k m]$ zurück (Gl. 122):

$$
\mathrm{s}_{\mathrm{cd}, \mathrm{e}(\mathrm{m}+1 ; \mathrm{z})}=\frac{\mathrm{L}_{\mathrm{d}, \mathrm{e}(\mathrm{m}+1 ; \mathrm{z})} \cdot 2 \cdot \pi \cdot \mathrm{r}_{\mathrm{s}}}{\mathrm{p}_{\mathrm{e}(\mathrm{z})} \cdot 1.000}
$$

Aus dem Erwartungswert des partiellen Laufwegs $s_{c d, e(m+1 ; z)}$ kann mit Bezug auf den Erwartungswert für den Laufweg $\mathrm{s}_{\mathrm{c}, \mathrm{e}(\mathrm{z})}$ im Homogenbereich $\mathrm{HGB}_{(\mathrm{z})}$ der erwartete partielle Ausnutzungsgrad $\mathrm{e}_{\mathrm{cd}, \mathrm{e}(\mathrm{m}+1 ; \mathrm{z})}$ der einzelnen Abbauwerkzeuge mit Gl. 123 berechnet werden:

$$
\mathrm{e}_{\mathrm{cd}, \mathrm{e}(\mathrm{m}+1 ; \mathrm{z})}=\frac{\mathrm{s}_{\mathrm{cd}, \mathrm{e}(\mathrm{m}+1 ; \mathrm{z})}}{\mathrm{s}_{\mathrm{c}, \mathrm{e}(\mathrm{z})}}
$$

Zwischen dem ersten und dem letzten Teilstück der Vortriebsstrecke $L_{d, e(m+1)}$ liegen maximal k-2 geotechnische Homogenbereiche von $\mathrm{HGB}_{(z+1)}$ bis $\mathrm{HGB}_{(z+(k-1))}$, die ohne Vortriebsunterbrechung vollständig durchfahren werden. Innerhalb dieser Homogenbereiche legt jedes Abbauwerkzeug auf dem Schneidrad den erwarteten partiellen Laufweg $\mathrm{s}_{\mathrm{cd}, e(\mathrm{~m}+1 ; z+k)}[\mathrm{km}]$ zurück, der mit Gl. 124 berechnet werden kann:

$$
\mathrm{s}_{\mathrm{cd}, \mathrm{e}(\mathrm{m}+1 ; \mathrm{z}+\mathrm{k})}=\frac{\mathrm{L}_{\mathrm{HGB}(\mathrm{z}+\mathrm{k})} \cdot 2 \cdot \pi \cdot \mathrm{r}_{\mathrm{s}}}{\mathrm{p}_{\mathrm{e}(\mathrm{z}+\mathrm{k})} \cdot 1.000}
$$


Aus dem Erwartungswert des partiellen Laufwegs $\mathrm{S}_{\mathrm{cd}, \mathrm{e}(\mathrm{m}+1 ; z+\mathrm{k})}$ wird mit Bezug auf den Erwartungswert für den Laufweg $\mathrm{s}_{\mathrm{c}, \mathrm{e}(\mathrm{z}+\mathrm{k})}$ im Homogenbereich $\mathrm{HGB}_{(\mathrm{z}+\mathrm{k})}$ der Erwartungswert des partiellen Ausnutzungsgrads $\mathrm{e}_{\mathrm{cd}, \mathrm{e}(\mathrm{m}+1 ; \mathrm{z}+\mathrm{k})}$ der einzelnen Abbauwerkzeuge mit Gl. 125 berechnet:

$$
\mathrm{e}_{\mathrm{cd}, \mathrm{e}(\mathrm{m}+1 ; \mathrm{z}+\mathrm{k})}=\frac{\mathrm{s}_{\mathrm{c}(\mathrm{m}+1 ; \mathrm{z}+\mathrm{k})}}{\mathrm{s}_{\mathrm{c}, \mathrm{e}(\mathrm{z}+\mathrm{k})}}
$$

Das letzte Teilstück $L_{d, e(m+1 ; z+k)}$ der Vortriebsstrecke $L_{d, e(m+1)}$ ergibt sich aus der Vortriebsstrecke, die ausgehend von der Grenze $L_{H G B(z+(k-1))}$ zwischen den Homogenbereichen $\operatorname{HGB}_{(z+(k-1))}$ und $\mathrm{HGB}_{(z+k)}$ bis zur Vortriebsunterbrechung $I_{(m+1 ; z+k)}$ an der kumulierten Vortriebsstrecke $L_{1, e(m+1)}$ im Homogenbereich $\mathrm{HGB}_{(\mathrm{z}+\mathrm{k})}$ aufgefahren wird (Gl. 126):

$$
\mathrm{L}_{\mathrm{d}, \mathrm{e}(\mathrm{m}+1 ; \mathrm{z}+\mathrm{k})}=\mathrm{L}_{\mathrm{I}, \mathrm{e}(\mathrm{m}+1)}-\mathrm{L}_{\mathrm{HGB}(\mathrm{z}+(\mathrm{k}-1))}
$$

Innerhalb des letzten Teilstücks $L_{d, e(m+1 ; z+k)}$ legt jedes Abbauwerkzeug auf dem Schneidrad den erwarteten partiellen Laufweg $\mathrm{s}_{\mathrm{cd}, \mathrm{e}(\mathrm{m}+1 ; z+\mathrm{k})}[\mathrm{km}]$ aus $\mathrm{Gl} .127$ zurück:

$$
\mathrm{s}_{\mathrm{d}, \mathrm{ec}(\mathrm{m}+1 ; \mathrm{z}+\mathrm{k})}=\frac{\mathrm{L}_{\mathrm{I}(\mathrm{m}+1 ; \mathrm{z}+\mathrm{k})} \cdot 2 \cdot \pi \cdot \mathrm{r}_{\mathrm{s}}}{\mathrm{p}_{\mathrm{e}(\mathrm{z}+\mathrm{k})} \cdot 1.000}
$$

Aus dem Erwartungswert des Laufwegs $s_{c d, e(m+1 ; z+k)}$ wird mit Bezug auf den Erwartungswert für den Laufweg $S_{c d, e(z+k)}$ im Homogenbereich $\mathrm{HGB}_{(z+k)}$ der erwartete partielle Ausnutzungsgrad $e_{c d, e(m+1 ; z+k)}$ der einzelnen Abbauwerkzeuge mit Gl. 128 berechnet:

$$
\mathrm{e}_{\mathrm{cd}(\mathrm{m}+1 ; \mathrm{z}+\mathrm{k})}=\frac{\mathrm{s}_{\mathrm{cd}, \mathrm{e}(\mathrm{m}+1 ; \mathrm{z}+\mathrm{k})}}{\mathrm{s}_{\mathrm{c}, \mathrm{e}(\mathrm{z}+\mathrm{k})}}
$$

Der Erwartungswert des partiellen Laufwegs $S_{c d, e(m+1)}[k m]$ in der Vortriebsstrecke $L_{d, e(m+1)}$ wird durch Summierung der k-Teilstücke aus Gl. 122, Gl. 124 und Gl. 127 berechnet:

$$
\mathrm{s}_{\mathrm{cd}, \mathrm{e}(\mathrm{m}+1)}=\sum_{1}^{\mathrm{k}} \mathrm{s}_{\mathrm{cd}, \mathrm{e}(\mathrm{m}+1 ; \mathrm{z}+\mathrm{k})}
$$

Der partielle Ausnutzungsgrad $e_{c d, e(m+1)}[\%]$ in der Vortriebsstrecke $L_{d, e(m+1)}$ wird analog durch Summierung der Teilstücke aus Gl. 123, Gl. 124 und Gl. 127 berechnet:

$$
\mathrm{e}_{\mathrm{cd}, \mathrm{e}(\mathrm{m}+1)}=\sum_{1}^{\mathrm{k}} \mathrm{e}_{\mathrm{c}(\mathrm{m}+1 ; \mathrm{z}+\mathrm{k})}
$$


Der Erwartungswert des kumulierten Laufwegs $\mathrm{s}_{\mathrm{c}, \mathrm{e}(\mathrm{m}+1)}[\mathrm{km}]$ wird mit $\mathrm{Gl} .131$ berechnet:

$$
\mathrm{s}_{\mathrm{c}, \mathrm{e}(\mathrm{m}+1)}=\mathrm{s}_{\mathrm{c}, \mathrm{e}(\mathrm{m})}+\mathrm{s}_{\mathrm{cd}, \mathrm{e}(\mathrm{m}+1)}
$$

Der Erwartungswert des kumulierte Ausnutzungsgrads $e_{c, e(m+1)}[k m]$ wird mit Gl. 132 berechnet:

$$
\mathrm{e}_{\mathrm{c}, \mathrm{e}(\mathrm{m}+1)}=\mathrm{e}_{\mathrm{c}, \mathrm{e}(\mathrm{m})}+\mathrm{e}_{\mathrm{cd}, \mathrm{e}(\mathrm{m}+1)}
$$

\section{Schritt 8:}

Die Anzahl $n_{1(T A)}$ der Vortriebsunterbrechungen $I_{(m ; z)}$ für Werkzeugwechsel über die Tunnellänge $\mathrm{L}_{\mathrm{TA}}$ kann durch Summierung der Vortriebsunterbrechungen $\mathrm{I}_{(\mathrm{m} ; \mathrm{z})}$ bestimmt werden. Die Anzahl der Vortriebsunterbrechungen $n_{1(T A)}$ entspricht folglich auch dem Maximalwert des Index $\mathrm{m}$. In beiden Fällen ist zu beachten, dass im Prognoseprozess bei der Bestimmung der Vortriebsstrecken $L_{d, e(m)}$ zwischen den Vortriebsunterbrechungen $I_{(m)}$ auch der Durchbruch am Ende des Tunnels als Vortriebsunterbrechung behandelt wird. Die Anzahl $n_{l(T A)}$ der Vortriebsunterbrechungen $I_{(m)}$ innerhalb der Tunnellänge $L_{T A}$ nach denen der Vortrieb fortgesetzt wird, ist also jeweils um 1 geringer als der Maximalwert des Index m (Gl. 133):

$$
\mathrm{n}_{\mathrm{I}(\mathrm{TA})}=\sum_{\mathrm{TA}} \mathrm{I}_{(\mathrm{m})}-1=\mathrm{m}-1
$$

Der iterative Prozess zur Bestimmung der Vortriebsunterbrechung $I_{(m+1 ; z+k)}$ und der zugehörigen Erwartungswerte für den kumulierten Laufweg $\mathrm{s}_{\mathrm{c}, \mathrm{e}(\mathrm{m}+1)}$ und den Ausnutzungsgrad $\mathrm{e}_{\mathrm{c}, \mathrm{e}(\mathrm{m}+1)}$ ist damit abgeschlossen.

Die Anzahl $n$ der Iterationen hängt stark von der Länge $\mathrm{L}_{H G B(z)}$ der geotechnischen Homogenbereiche im zu bearbeitenden Projekt ab. In Projekten mit relativ niedriger Länge der geotechnischen Homogenbereiche ergibt sich eine höhere Anzahl an Iterationen, weil zwischen den Vortriebsunterbrechungen eine höhere Anzahl an Homogenbereichen durchfahren werden kann. Umgekehrt liegt die Anzahl der Iterationen in Projekten mit relativ langen geotechnischen Homogenbereichen relativ niedrig, weil die Vortriebsunterbrechungen häufiger innerhalb eines geotechnischen Homogenbereichs liegen.

Innerhalb der RP und der darin enthaltenen Verteilung der Länge der $\mathrm{HGB}_{(z)}$ (Kap. 5.6.1.2) waren bei der Validierung des Prognosemodells maximal 3 Iterationen der Schritte 1-4 notwendig. 


\subsection{Prognose der Anzahl der Werkzeugwechsel $\mathbf{n}_{\mathrm{c}}$}

In der Formulierung des Prognosemodells hat die Auswahl des Erwartungswertes für die Vortriebsstrecke $L_{d, e(m+1)}[m]$ Vorrang vor der Bestimmung der notwendigen Anzahl an Werkzeugwechseln. Durch den in Kap. 6.3.4 ausführlich beschriebenen Prozess zur Berechnung der maximal erreichbaren Vortriebsstrecke $L_{\max (m+1)}$ und die Auswahl der Vortriebsstrecke $L_{d, e(m+1)}$ wird sichergestellt, dass $z$ wischen den Vortriebsunterbrechungen $I_{(m)}$ und $I_{(m+1)}$ die Verschleißgrenze der Abbauwerkzeuge auf dem Schneidrad nicht überschritten wird.

Die Berechnung der maximal erreichbaren Vortriebsstrecke $L_{\max (m+1)}$ geht zunächst immer von neuen, unverschlissenen Abbauwerkzeugen aus. Die Abbauwerkzeuge besitzen jedoch an der Vortriebsunterbrechung $\mathrm{I}_{(\mathrm{m})}$ bereits den bestehenden kumulierten Ausnutzungsgrad $\mathrm{e}_{\mathrm{c}, \mathrm{e}(\mathrm{m})}$. In Abhängigkeit der Auswahl der geplanten Vortriebsstrecke $L_{d, e(m+1)}$ kleiner oder gleich der maximal erreichbaren Vortriebsstrecke in Gl. 117 (Kap. 6.3.4) kann so der kumulierte Ausnutzungsgrad $e_{c, e(m+1)}$ in $G$ l. 132 an der Vortriebsunterbrechung $\mathrm{I}_{(\mathrm{m}+1)}$ für einzelne Abbauwerkzeuge planmäßig Werte $>1$ annehmen. Diese Abbauwerkzeuge müssen an der Vortriebsunterbrechung $\mathrm{I}_{(\mathrm{m})}$ folglich gewechselt werden, um die ausgewählte Vortriebsstrecke $L_{d, e(m+1)}$ ohne planmäßige Überschreitung der Verschleißgrenze zu erreichen.

Der planmäßige Ausnutzungsgrad $e_{c, e(m+1)}$ stellt folglich die Kenngröße dar, mit deren Hilfe ein klares Kriterium für einen Werkzeugwechsel in der Vortriebsunterbrechung $\mathrm{I}_{(\mathrm{m})}$ formuliert werden kann (Gl. 134):

Der Wechsel eines Abbauwerkzeuges bei einer Vortriebsunterbrechung $I_{(m)}$ ist notwendig, wenn der erwartete kumulierte Ausnutzungsgrad $e_{c, e(m+1)}$ an der nächsten geplanten Vortriebsunterbrechung $I_{(m+1)}$ einen Wert $>1$ annimmt und damit die Verschleißgrenze des Abbauwerkzeuges überschritten wird:

$$
\mathrm{e}_{\mathrm{c}, \mathrm{e}(\mathrm{m}+1)}>1
$$

Die Bestimmung der notwendigen Werkzeugwechsel in einer Vortriebsunterbrechung $I_{(m)}$ ist folglich funktional mit der Auswahl der geplanten Vortriebsstrecke $L_{d, e(m+1)}$ bis zur nächsten folgenden Vortriebsunterbrechung $I_{(m+1)}$ verbunden. Für die Planung und Ausführung des Vortriebs bedeutet das:

- Die Bestimmung der Abbauwerkzeuge $c$, die während einer Vortriebsunterbrechung $I_{(m)}$ gewechselt werden müssen, ist nur sinnvoll möglich, wenn zuvor der Erwartungswert für die Vortriebsstrecke $L_{d, e(m+1)}$ bis zur nächsten folgenden Vortriebsunterbrechung $I_{(m+1)}$ festgelegt wird.

- Die geplante Vortriebsstrecke $L_{d, e(m+1)}$ wird nur ohne Überschreitung von Verschleißgrenzen erreicht, wenn die dazu notwendigen Werkzeugwechsel in der davor liegenden Vortriebsunterbrechung $I_{(m)}$ konsequent ausgeführt werden.

- Jede Änderung der geplanten Vortriebsstrecke $L_{d, e(m+1)}$ bewirkt eine Änderung in der Anzahl der notwendigen Werkzeugwechsel $n_{c, e(m)}$ in der Vortriebsunterbrechung $I_{(m)}$ und umgekehrt.

- Wenn die notwendigen Werkzeugwechsel $n_{c, e(m)}$ in der Vortriebsunterbrechung $I_{(m)}$ ausgeführt und der Vortrieb der geplanten Strecke $L_{d, e(m+1)}$ begonnen wurden, kann die geplante Strecke $L_{d, e(m+1)}$ nicht nachträglich verlängert werden. Dazu wären entweder zusätzliche Werkzeugwechsel notwendig oder es wird das Risiko einer Überschreitung der Verschleißgrenzen und möglicher Folgeschäden eingegangen. Eine Verkürzung der geplanten Vortriebsstrecke $L_{d, e(m+1)}$ ist dagegen jederzeit möglich.

Der tatsächlich im Vortrieb erwartete Laufweg $\mathrm{s}_{\mathrm{cv}, \mathrm{e}(\mathrm{m})}[\mathrm{km}]$ der Abbauwerkzeuge zwischen Einbau und Ausbau auf dem Schneidrad entspricht dem kumulierten Laufweg $\mathrm{S}_{\mathrm{c}, \mathrm{e}(\mathrm{m})}$, an der Vortriebsunterbrechung $\mathrm{I}_{(\mathrm{m})}$, an der das Abbauwerkzeug auf dem Schneidrad ausgebaut wurden. 
Der Laufweg $\mathrm{s}_{\mathrm{cv}, \mathrm{e}(\mathrm{m})}$ kann folglich als Summe aller partiellen Laufwege $\mathrm{s}_{\mathrm{cd}, \mathrm{e}(\mathrm{m})} \mathrm{zwischen}$ den Vortriebsunterbrechungen bei Ein- und Ausbau des Abbauwerkzeuges formuliert werden (GI. 135). Die Ringnummern $b_{0}$ und $b_{v}$ bei Ein- und Ausbau des Abbauwerkzeuges entsprechen der Stationierung bzw. den Ringnummern $\mathrm{b}_{\mathrm{l}, \mathrm{e}(\mathrm{m})}$ der zugehörigen Vortriebsunterbrechungen $\mathrm{I}_{(\mathrm{m})}$ aus $\mathrm{Gl} .90$ (Kap. 6.3.2):

$$
\mathrm{s}_{\mathrm{cv}, \mathrm{e}(\mathrm{m})}=\sum_{\mathrm{b}_{0}}^{\mathrm{b}_{\mathrm{v}}} \mathrm{s}_{\mathrm{cd}, \mathrm{e}(\mathrm{m})}
$$

Der tatsächliche Ausnutzungsgrad $\mathrm{e}_{\mathrm{cv}, \mathrm{e}}[\%]$ der Abbauwerkzeuge bei Ausbau auf dem Schneidrad wird aus der Summe aller partiellen Ausnutzungsgrade $e_{c d, e(m)} z$ wischen Einbau $b_{0}$ und Ausbau $b_{v}$ mit Gl. 136 berechnet:

$$
\mathrm{e}_{\mathrm{cv}, \mathrm{e}(\mathrm{m})}=\sum_{\mathrm{b}_{0}}^{\mathrm{b}_{\mathrm{v}}} \mathrm{e}_{\mathrm{cd}, \mathrm{e}(\mathrm{m})}
$$

Der Erwartungswert für die Anzahl $\mathrm{n}_{\mathrm{c}, \mathrm{e}(\mathrm{m})}$ der zu wechselnden Abbauwerkzeuge $\mathrm{c}$ in der Vortriebsunterbrechung $I_{(m)}$ wird durch Summierung aller Abbauwerkzeuge mit Gl. 137 ermittelt, für die bei $I_{(m)}$ aufgrund der geplanten Vortriebsstrecke $L_{d, e(m+1)}$ und des kumulierten Ausnutzungsgrades $e_{c, e(m+1)}$ das Wechselkriterium in Gl. 134 gilt:

$$
\mathrm{n}_{\mathrm{c}, \mathrm{e}(\mathrm{m})}=\sum_{\mathrm{c}} \mathrm{e}_{\mathrm{c}, \mathrm{e}(\mathrm{m}+1)}>1
$$

Der Erwartungswert für die Anzahl $\mathrm{n}_{\mathrm{c}, \mathrm{e}(\mathrm{TA})}$ der zu wechselnden Abbauwerkzeuge $\mathrm{c}$ über die Tunnellänge $L_{T A}$ wird durch Summieren der Anzahl der Werkzeugwechsel $n_{c, e(m)}$ in den Vortriebsunterbrechungen $I_{(m)}$ mit Gl. 138 bestimmt:

$$
\mathrm{n}_{\mathrm{c}, \mathrm{e}(\mathrm{TA})}=\sum_{1}^{\mathrm{m}} \mathrm{n}_{\mathrm{c}, \mathrm{e}(\mathrm{m})}
$$




\subsection{Baubetriebliche Anpassung der Verschleißprognose}

Das Prognosemodell für die Anzahl $n_{I(T A)}$ und die Stationierung $L_{l_{l, e}(m)}$ der Vortriebsunterbrechungen $I_{(m)}$ beinhaltet als vierten Hauptblock (Kap.6.1.3) einen Anpassungsalgorithmus. Dieser ist aus zwei Gründen notwendig:

- In der Prognose der Stationierung $\mathrm{L}_{\mathrm{l}, \mathrm{e}(\mathrm{m})}[\mathrm{m}]$ der Vortriebsunterbrechungen $\mathrm{I}_{(\mathrm{m})}$ werden die Rahmenbedingungen im Projekt für den Zugang zur Abbaukammer nicht berücksichtigt. Diese sind jedoch entscheidend dafür, ob und mit welcher Methode die Vortriebsunterbrechungen $\mathrm{I}_{(\mathrm{m})}$ an den erwarteten Stationierungen $\mathrm{L}_{\mathrm{l}, \mathrm{e}(\mathrm{m})}[\mathrm{m}]$ bzw. $\mathrm{b}_{\mathrm{l}, \mathrm{e}(\mathrm{m})}[-]$ durchgeführt werden können.

- Die Ergebnisse der Datenanalyse erlauben keine Untersuchung der Fortpflanzung der Varianzen der Eingangsparameter im Prognosemodell. Die mögliche Bandbreite der Prognoseergebnisse ist jedoch eine wichtige Eingangsgröße für die Vortriebs- und Projektplanung.

Der Algorithmus beschreibt deshalb die geeigneten Ansatzpunkte im Prognoseprozess und die Methodik für:

- Baubetriebliche Anpassung der erwarteten Stationierungen $L_{l, e(m)}[m]$ der Vortriebsunterbrechungen $\mathrm{I}_{(\mathrm{m})}$ an die Rahmenbedingungen im Projekt für den Zugang zur Abbaukammer.

- Bildung von Szenarien zur empirischen Ermittlung der möglichen Bandbreite der Prognoseergebnisse.

Beide Ziele basieren auf denselben Ansatzpunkten zur Variation verschiedener Parameter im Prognoseprozess, sodass diese im Folgenden gemeinsam beschrieben werden (Kap. 6.5.2).

Die Anpassung der erwarteten Stationierungen $\mathrm{L}_{\mathrm{l}, \mathrm{e}(\mathrm{m})}$ der Vortriebsunterbrechungen $\mathrm{I}_{(\mathrm{m})}$ an die Rahmenbedingungen im Projekt für den Zugang zur Abbaukammer ist zwingender Bestandteil der Verschleißprognose, weil ohne diesen Schritt die Aussagekraft des Prognoseergebnisses sehr gering ist, bzw. extreme Abweichungen zwischen dem Prognoseergebnis und dem tatsächlichen Vortriebsablauf wahrscheinlich sind.

Die Bildung verschiedener Szenarien ist nicht zwingend notwendig, aber vor allem bei steigender Komplexität von Projekten mit herausfordernden Rahmenbedingungen zu empfehlen. Für die Bestimmung der möglichen Bandbreite der Prognoseergebnisse genügt theoretisch die Bildung eines Worst-Case und Best-Case Szenarios zusätzlich zum Planungsszenario. Diese lassen jedoch keine Aussage über die Wahrscheinlichkeitsverteilung der Prognoseergebnisse zu. Wenn ausreichend viele Szenarien untersucht werden, ähnelt die beschriebene Methode im Ansatz einer Monte-CarloSimulation. Diese Methode wird z.B. von FRENZEL (2010a: 55) für die Verschleißprognose für Schneidrollen in Festgesteinen vorgeschlagen. SANDER \& SPIEGEL (2011:67) schlagen einen identischen Ansatz für die übergreifende Kalkulation von Bauvorhaben vor.

\subsubsection{Prozessübersicht}

Die bisher beschriebenen Schritte des Prognoseprozesses (Kap. 6.1.3) liefern als Zwischenergebnis ein Teilszenario, das die folgenden Informationen enthält:

- Basisdatensatz der Verschleißprognose (Kap. 6.1.1)

- $\quad$ Erwartungswert für den Laufweg $\mathrm{s}_{\mathrm{c}, \mathrm{e}(\mathrm{z})}[\mathrm{km}]($ Kap. 6.2.3)

- Anzahl $n_{1(T A)}$ und Stationierung $b_{1, e(m)}$ bzw. $L_{1, e(m)}[m]$ der Vortriebsunterbrechungen $I_{(m)}$ für Werkzugwechsel (Kap. 6.3)

- Anzahl $\mathrm{n}_{\mathrm{c}, \mathrm{e}(\mathrm{m})}$ der Werkzeugwechsel (Kap. 6.4) 
Diese Informationen sind sowohl für die Anpassung der erwarteten Stationierungen $\mathrm{L}_{\mathrm{l}, \mathrm{e}(\mathrm{m})}$ der Vortriebsunterbrechungen $I_{(m)}$ an die Rahmenbedingungen im Projekt für den Zugang zur Abbaukammer, als auch für die empirische Ermittlung der möglichen Bandbreite der Prognoseergebnisse durch Bildung verschiedener Szenarien ausreichend.

Um konkreteren Angaben zum Einfluss des Verschleißes der Abbauwerkzeuge in der Vortriebsplanung, der Angebotskalkulation und der Auslegung der TVM (Kap. 1.2) zu ermöglichen, wird empfohlen, auf Basis des Teilszenarios zusätzlich Schätzungen vorzunehmen für:

- Vortriebsleistung bzw. Vortriebsdauer

- Verschleißkosten

Die Schätzung der Vortriebsleistung und der Verschleißkosten ist nicht Kernthema der vorliegenden Arbeit, sodass keine detaillierte Untersuchung und Beschreibung zur Schätzmethodik erfolgt. Der Einfluss der Vortriebsunterbrechungen $I_{(m)}$ und der Anzahl der Werkzeugwechsel $n_{c, e(m)}$ auf die Vortriebsdauer ergibt sich aus der dazu notwendigen Stillstandzeit der TVM. Die Schätzung der Verschleißkosten umfasst minimal die Materialkosten für den Ersatz der verschlissenen Abbauwerkzeuge. Tatsächlich sind mit der Ausführung der Werkzeugwechsel, der Bereitstellung und evtl. Sanierung von Abbauwerkzeugen weitere projektspezifische Kostenpositionen verbunden, die in die Schätzung mit einbezogen werden können. Ein Überblick über die möglichen Positionen gibt Tab. 23.

Tab. 23: Gliederung der TVM-Verschleißkosten nach Kosten für Abbauwerkzeuge und Wechselkosten (Ausführung der Werkzeugwechsel).

\begin{tabular}{|c|c|c|c|c|c|c|}
\hline \multicolumn{7}{|c|}{ TVM-Verschleißkosten $\mathrm{K}_{\mathrm{v}}$ für Abbauwerkzeuge } \\
\hline \multicolumn{4}{|c|}{ Wechselkosten } & \multicolumn{3}{|c|}{ Abbauwerkzeugkosten } \\
\hline $\begin{array}{c}\text { Präventive } \\
\text { Maßnahmen }\end{array}$ & $\begin{array}{c}\text { Zusatzmaß- } \\
\text { nahmen }\end{array}$ & $\begin{array}{l}\text { Stillstand- } \\
\text { kosten }\end{array}$ & $\begin{array}{l}\text { Personal- } \\
\text { kosten }\end{array}$ & $\begin{array}{c}\text { Material- } \\
\text { kosten }\end{array}$ & $\begin{array}{l}\text { Bereitstel- } \\
\text { lungskosten }\end{array}$ & $\begin{array}{l}\text { Sanierungs- } \\
\text { kosten }\end{array}$ \\
\hline $\begin{array}{l}\text { Betriebsdaten- } \\
\text { überwachung } \\
\text { Verschleiß- } \\
\text { erkennung } \\
\text { Metall- } \\
\text { abscheider }\end{array}$ & $\begin{array}{l}\text { Injektionen } \\
\text { Zusätzliche } \\
\text { Auflasten } \\
\text { Mixgas- } \\
\text { anlage } \\
\text { Sättigungs- } \\
\text { tauchen }\end{array}$ & $\begin{array}{l}\text { Zeitgebun- } \\
\text { dene Fix- } \\
\text { kosten d. } \\
\text { Baustelle }\end{array}$ & $\begin{array}{l}\text { Personal } \\
\text { (Ausführung } \\
\text { Werkzeug- } \\
\text { wechsel) }\end{array}$ & $\begin{array}{l}\text { Abbauwerk- } \\
\text { zeuge } \\
\text { Ersatzteile }\end{array}$ & $\begin{array}{l}\text { Transporte } \\
\text { Zölle } \\
\text { Steuern } \\
\text { Lagerhaltung } \\
\text { Finanzierung } \\
\text { Abschreibung }\end{array}$ & $\begin{array}{l}\text { Werkstatt } \\
\text { Personal } \\
\text { (Sanierung) }\end{array}$ \\
\hline
\end{tabular}

Der Basisdatensatz, die Erwartungswerte für den Laufweg $\mathrm{s}_{\mathrm{c}, \mathrm{e}(\mathrm{z})}[\mathrm{km}]$, die Anzahl $\mathrm{n}_{\mathrm{l}(\mathrm{TA})}$ und Stationierung $b_{1, e(m)}$ bzw. $L_{l, e(m)}[m]$ der Vortriebsunterbrechungen $I_{(m)}$ für Werkzeugwechsel, die Anzahl $n_{c, e(m)}$ der Werkzeugwechsel und die geschätzte Vortriebsleistung und Verschleißkosten ergeben zusammen ein Planungsszenario.

Das Planungsszenario muss anschließend im Hinblick mit den gegebenen Anforderungen im Projekt abgeglichen werden. Die Anforderungen beziehen sich insbesondere auf folgende Punkte:

- Vortriebsleistung bzw. Vortriebsdauer

- Verschleißkosten

- Verfahrenssicherheit

Vortriebsdauer und Verschleißkosten sind quantitativ gut erfassbar, sodass entsprechend harte Zielgrößen bzw. Anforderungen in einem Projekt definiert werden können. Der Abgleich der Anforderungen im Projekt hinsichtlich der Verfahrenssicherheit bezieht sich primär darauf, Vortriebsunterbrechungen $\mathrm{I}_{(\mathrm{m})}$ in Bereichen zu vermeiden, in denen der Zugang zur Abbaukammer aufgrund der geo- 
technischen Rahmenbedingungen nur eingeschränkt, mit Zusatzmaßnahmen oder gar nicht möglich ist. Folglich müssen die Rahmenbedingungen für den Zugang zur Abbaukammer an jeder geplanten Stationierung $\mathrm{L}_{\mathrm{l}, \mathrm{e}(\mathrm{m})}$ einer Vortriebsunterbrechung $\mathrm{I}_{(\mathrm{m})}$ entlang der Tunnelachse überprüft werden.

Auf Basis der Prüfung können anschließend gezielte Variationen der Verschleißprognose zur Anpassung der Stationierung $b_{1, e(m)}$ bzW. $L_{l, e(m)}$ der Vortriebsunterbrechungen $I_{(m)}$, zur Verringerung der Anzahl an Vortriebsunterbrechungen $n_{1(T A)}$ oder der Anzahl an Werkzeugwechseln $n_{c(T A)}$ und zur Bildung von verschiedenen Szenarien vorgenommen werden. Die Ansatzpunkte für die Variationen in der Verschleißprognose sind:

- Variation der Basisdaten (Kap. 6.1.1)

- Variation der Werkzeugwechselstrategie bei der Auswahl der Vortriebsstrecken $L_{d, e(m)} z w i-$ schen den Vortriebsunterbrechungen $I_{(m)}$ in Gl. 117 (Kap. 6.3.4).

- Planung von Zusatzmaßnahmen zur Durchführung der Vortriebsunterbrechungen $I_{(m)}$ an den geplanten Stationierungen $\mathrm{L}_{\mathrm{l}, \mathrm{e}(\mathrm{m})}$ oder zur Begrenzung von Risiken.

Die Verschleißprognose wird anschließend mit allen vorgenommenen Variationen erneut durchgeführt, sodass ein verändertes Planungsszenario entsteht. Durch schrittweise Anpassung und erneute Iteration der Verschleißprognose kann so ein möglichst realistisches Planungsszenario für den Vortrieb erarbeitet werden. Mit Ausnahme der Planung von Zusatzmaßnahmen wirkt sich jede der genannten Variationsmöglichkeiten auf die Stationierung $L_{1, e(m)}$ aller Vortriebsunterbrechungen $I_{(m)}$ aus. $\mathrm{Zu}$ Gunsten einer zielgerichteten Vorgehensweise sind deshalb eher kleine Anpassungsschritte zu empfehlen, die eine eher hohe Anzahl an Iterationen notwendig machen. Zusätzlich hängt die Anzahl der Iterationen von der Komplexität der Rahmenbedingungen im Projekt und der Anzahl an erforderlichen Szenarien ab.

Abschließend muss eine klare Festlegung getroffen werden, welches Szenario bei der Auslegung der TVM und der Ausführung des Vortriebs verfolgt wird. Das festgelegte Planungsszenario muss im Vortrieb konsequent nachverfolgt werden, um die Annahmen darin zu validieren (Kap. 6.5.3.2).

\subsubsection{Anpassung und Szenariobildung}

Die im Folgenden beschriebenen Ansatzpunkte zur Variation der Verschleißprognose können sowohl zur Anpassung der Stationierung $b_{1, e(m)}$ bzW. $L_{l, e(m)}$ der Vortriebsunterbrechungen $I_{(m)}$, zur Verringerung $\operatorname{der}$ Anzahl an Vortriebsunterbrechungen $n_{1(T A)}$ oder der Anzahl an Werkzeugwechseln $n_{c(T A)}$, als auch zur Bildung von verschiedenen Szenarien eingesetzt werden. Die Ansatzpunkte sind:

- Basisdaten der Verschleißprognose (Kap. 6.5.2.1)

- Auswahl der Vortriebsstrecken $L_{d, e(m)}[m]$ zwischen den Vortriebsunterbrechungen $I_{(m)}$ (Kap. 6.5.2.2) (Werkzeugwechselstrategie).

- Planung von Zusatzmaßnahmen zur Durchführung der Vortriebsunterbrechungen $I_{(m)}$ an den geplanten Stationierungen oder zur Begrenzung von Risiken (Kap. 6.5.2.3).

Die verschiedenen Ansatzpunkte und deren tendenzielle Auswirkung auf das Prognoseergebnis sind zum Teil komplex miteinander verbunden und mit Ausnahme der Planung von Zusatzmaßnahmen wirkt sich jede der genannten Variationsmöglichkeiten auf die Stationierung $\mathrm{L}_{\mathrm{I}, \mathrm{e}(\mathrm{m})}$ aller Vortriebsunterbrechungen $I_{(m)}$ aus. Deshalb sind sowohl bei der Anpassung der Verschleißprognose, als auch bei der Bildung verschiedener Szenarien eher kleine Anpassungsschritte zu empfehlen, um eine zielgerichtete Vorgehensweise sicherzustellen. Bei der Variation der verschiedenen Parameter sollte darüber hinaus klar unterschieden werden nach:

- Parameter, die einer vorhandenen natürlichen Schwankungsbreite unterliegen.

- Parameter, die frei aktiv beeinflusst und variiert werden können. 
Innerhalb der Verschleißprognose unterliegen vornehmlich die geotechnischen Parameter einer natürlichen Schwankungsbreite. Diese muss für die Verschleißprognose als gegeben hingenommen werden und kann mit Ausnahme von z.B. einer Trassenänderung, Bodenaustausch oder -verbesserung nicht verändert werden. Die Schwankungsbreite dieser Parameter sollte also zwingend in der Verschleißprognose berücksichtigt werden. Im Gegensatz dazu können die Parameter der Auslegung der TVM und der Steuerung des Abbauprozesses innerhalb bestimmter Grenzen durch den Bearbeiter der Verschleißprognose frei variiert werden. Sie unterliegen tendenziell keiner bzw. nur einer vernachlässigbar geringen natürlichen Schwankungsbreite. Für diese Parameter ist zusätzlich eine Unterscheidung wichtig nach:

- Parameter, die zum Zeitpunkt der Prognose noch frei variiert werden können.

- Parameter, die zum Zeitpunkt der Prognose bereits festgelegt sind.

Innerhalb der Verschleißprognose ist die Auslegung der TVM z.B. nur so lange frei variierbar, bis die TVM hergestellt wurde und damit die wesentlichen Auslegungsparameter fixiert sind. Die Möglichkeiten zur Variation dieser Parameter auf der Baustelle in der Vortriebsphase sind folglich auf ein Minimum eingeschränkt. Die Parameter der Steuerung des Abbauprozesses können dagegen bis in die Vortriebsphase hinein noch in relativ weiten Grenzen variiert werden.

\subsubsection{Variation der Basisdaten}

\section{Geotechnische Parameter}

Innerhalb der geotechnischen Basisdaten fließen die nachfolgenden Parameter direkt in den Prognoseprozess ein. Sie unterliegen einer natürlichen Schwankungsbreite, die in der geotechnischen Vorerkundung ermittelt werden kann. Sie können jedoch auch zur Bildung verschiedener Szenarien aktiv vom Bearbeiter variiert werden:

- Überlagerung $\mathrm{H}_{\mathrm{TA}}[\mathrm{m}]$

- Wasserspiegel $\mathrm{W}_{\mathrm{TA}}[\mathrm{m}]$

- Verteilung verschiedenen Bodenarten in der Ortsbrust [\%]

- Feuchtwichte des Bodens y $\left[\mathrm{kN} / \mathrm{m}^{2}\right]$

- Wichte des Bodens unter Auftrieb y` $\left[\mathrm{kN} / \mathrm{m}^{2}\right]$

- Reibungswinkel in $\varphi^{\prime}\left[{ }^{\circ}\right]$

- Kohäsion c` $\left[\mathrm{kN} / \mathrm{m}^{2}\right]$

- $\quad$ Äquivalenter Quarzgehalt ÄQu [\%]

- Korngröße $D_{60}[\mathrm{~mm}]$

Die Variation der Überlagerung $\mathrm{H}_{T A}$, des Wasserspiegels $\mathrm{W}_{\mathrm{TA}}$ und der Verteilung verschiedener Bodenarten in der Ortsbrust wirkt sich zunächst auf die Bildung der geotechnischen Homogenbereiche entlang der Tunnelachse aus.

Die Variation der aufgelisteten Parameter führt darüber hinaus über die Berechnung der Scherfestigkeit $\mathrm{T}_{\mathrm{c}}$ (Gl. 73) und die Berechnung des Soil Abrasivity Index (Gl. 72) zu einer Änderung des Basiswertes des Laufwegs $s_{c, b(z)}$. Diese pflanzt sich über den Erwartungswert für den Laufweg $s_{c, e(z)}$ in die Schätzung der Stationierung $b_{1, e(m)}$ bzw. $L_{l, e(m)}$ und Anzahl $n_{1(T A)}$ aller Vortriebsunterbrechungen $I_{(m)}$ fort (Kap. 6.3.4). Folglich ergibt sich auch eine Änderung der notwendigen Anzahl an Werkzeugwechseln $\mathrm{n}_{\mathrm{c}, \mathrm{e}(\mathrm{m})}$ während der Vortriebsunterbrechungen. 
Die Variation der geotechnischen Parameter muss theoretisch innerhalb der einzelnen Bodeneinheiten betrachtet werden, weil diese aus stochastischer Sicht weitgehend unabhängig voneinander sind. Das bedeutet, dass zur Bildung verschiedener Szenarien nicht alle Bodeneinheiten in gleichem Maße und in gleicher Richtung variiert werden müssen.

Von der Variation der geotechnischen Parameter sind alle Abbauwerkzeuge auf dem Schneidrad gleichermaßen betroffen. Als qualitative Tendenz kann aus dem Prognoseprozess für die Überlagerung $\mathrm{H}_{T A}$, die Feuchtwichte $\gamma$, den Reibungswinkel in $\varphi^{\prime}$, die Kohäsion c', den Äquivalenten Quarzgehalt $\ddot{A} Q u$ und die Korngröße $D_{60}$ deshalb abgeleitet werden:

- Je höher diese Parameter liegen, umso niedriger werden der Basiswert für den Laufweg $\mathrm{s}_{\mathrm{c}, \mathrm{b}(\mathrm{z})}$ und die maximal erreichbare Vortriebsstrecke $L_{\max (m)} z$ wischen den Vortriebsunterbrechungen $I_{(m)}$. Umgekehrt steigt die Anzahl $n_{1(T A)}$ der Vortriebsunterbrechungen $I_{(m)}$ und die Anzahl $n_{c(T A)}$ der notwendigen Werkzeugwechsel an.

- Je niedriger diese Parameter liegen, umso höher werden der Basiswert für den Laufweg $\mathrm{s}_{\mathrm{c}, \mathrm{b}(\mathrm{z})}$ und die maximal erreichbare Vortriebsstrecke $L_{\max (m)}$ zwischen den Vortriebsunterbrechungen $I_{(m)}$. Umgekehrt sinkt die Anzahl $n_{(\text {(TA) }}$ der Vortriebsunterbrechungen $I_{(m)}$ und die Anzahl $n_{c(T A)}$ der notwendigen Werkzeugwechsel.

Zusätzlich zum Einfluss der aufgelisteten geotechnischen Parameter auf die Bildung der geotechnischen Homogenbereiche und den Soil Abrasivity Index (SAI) haben die geotechnischen Parameter auch Einfluss auf die Schätzung der Eintrittswahrscheinlichkeit und Schadensintensität an den Abbauwerkzeugen bei Steinen und Blöcken im Boden oder bei Verklebungen. Hier sind auf Basis des bestehenden Wissenstandes (Kap. 4.2) hauptsächlich die folgenden Parameter von Bedeutung:

- Korngröße von Steinen oder Blöcken im Boden [mm]

- Einaxiale Druckfestigkeit (UCS) [MPa] der Steine oder Blöcke

- Plastizitätszahl IP [\%] bindiger Böden

- Konsistenzzahl $\mathrm{I}_{\mathrm{C}}$ bindiger Böden

Für die Variation dieser Parameter gelten dieselben grundsätzlichen Zusammenhänge im Hinblick auf die Unabhängigkeit der Bodeneinheiten und Parameter innerhalb der Bodeneinheiten. Die Auswirkungen können aber innerhalb des vorgestellten Prognosemodells nicht quantitativ ermittelt werden.

\section{Auslegung der TVM}

Aus der Auslegung der TVM fließen die nachfolgenden Parameter direkt in den Prognoseprozess ein. Sie können zur Anpassung der Prognose der Vortriebsunterbrechungen $I_{(m)}$ an die Rahmenbedingungen im Projekt für den Zugang zur Abbaukammer, als auch für die Bildung verschiedener Szenarien aktiv vom Bearbeiter variiert werden:

- $\quad$ Spurbesatz $\mathrm{k}_{\mathrm{s}}$

- Werkzeuggeometrie

Eine uneingeschränkte Variation der beiden Parameter ist nur solange möglich, bis die Auslegung der TVM für die Herstellung fixiert wird. Eine Änderung des Spurbesatzes $k_{s}$ ist im Vortrieb nur noch eingeschränkt möglich. Durch den Ausbau einzelner Abbauwerkzeuge kann $k_{s}$ im Vortrieb zum Teil noch verringert werden. Der Einbau zusätzlicher Halter für Abbauwerkzeuge ist jedoch kaum noch möglich und erfordert aufwändige Schweißarbeiten am Schneidrad. Die Geometrie der Abbauwerkzeuge kann auch im Vortrieb noch in Grenzen variiert werden, weil die zugehörigen Halter häufig den Einbau einer hohen Bandbreite an verschiedenen Abbauwerkzeugvarianten zulassen (Kap. 3.2.1). 
Die Variation des Spurbesatzes $\mathrm{k}_{\mathrm{s}}$ und der Werkzeuggeometrie führt über die Korrekturfaktoren für die Aktiv-Penetration $p_{a, e(z)}$ (Gl. 79) und die Werkzeuggeometrie $f_{b}$ (Gl. 77) zu einer Änderung des Erwartungswertes des Laufweg $s_{c, e(z)}$. Diese pflanzt sich in die Schätzung der Stationierung $b_{1, e(m)} b z w . L_{l, e(m)}$ und Anzahl $n_{\text {I(TA) }}$ der Vortriebsunterbrechungen $I_{(m)}$ fort (Kap. 6.3.4). Folglich ergibt sich auch eine Änderung der notwendigen Anzahl an Werkzeugwechseln $\mathrm{n}_{\mathrm{c}, \mathrm{e}(\mathrm{m})}$ in den Vortriebsunterbrechungen.

Die Beschreibung der Auswirkungen der Variation der beiden Parameter auf das Prognoseergebnis ist nur in der Gesamtbetrachtung aller Abbauwerkzeugtypen auf dem Schneidrad möglich. Aus dem Prognoseprozess können folgende qualitativen Tendenzen abgeleitet werden:

- Die Variation des Spurbesatz $k_{s}$ wirkt sich über die Aktiv-Penetration $p_{a, e(z)}[\mathrm{mm} / \mathrm{U}]$ der Schälmesser und den Korrekturfaktor $f_{p}$ auf den Erwartungswert des Laufwegs $\mathrm{s}_{\mathrm{c}, \mathrm{e}(\mathrm{z})}$ aus. Je höher der Spurbesatz $k_{s}$ gewählt wird, umso geringer wird die Aktiv-Penetration $p_{a, e(z)}$, umso höher wird der Korrekturfaktor $f_{p}$ und der Erwartungswert des Laufwegs $s_{c, e(z)}$. Eine Erhöhung der maximal erreichbaren Vortriebsstrecke $L_{\max (m)}$ ergibt sich nur dann, wenn vor der Variation des Spurbesatzes $k_{s}$ die maximal erreichbare Vortriebsstrecke $L_{c(m)}$ eines einzelnen Schälmessers die Begrenzung der maximal erreichbaren Vortriebsstrecke $L_{\max (m)}$ in Gl. 84 war. Die Anzahl der notwendigen Werkzeugwechsel $\mathrm{n}_{\mathrm{c}(\mathrm{TA})}$ sinkt unabhängig davon aufgrund der Erhöhung des

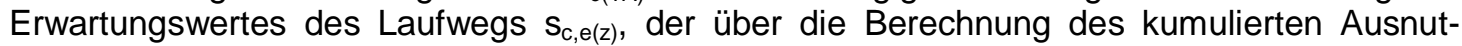
zungsgrades $e_{c, e(m+1)}(G l .132)$ in das Kriterium für Werkzeugwechsel (Gl. 134) einfließt.

- Die Variation der Schneidringbreite $b_{S R}[\mathrm{~mm}]$ wirkt sich über den Korrekturfaktor $\mathrm{f}_{\mathrm{b}}(\mathrm{Gl}$. 77) auf den Erwartungswert für den Laufweg $\mathrm{S}_{\mathrm{c}, \mathrm{e}(\mathrm{z})}$ der Schneidrollen aus. Je höher die Schneidringbreite $b_{S R}$ gewählt wird, umso höher wird der Korrekturfaktor $f_{b}$ und letztlich auch der Erwartungswert für den Laufweg $\mathrm{S}_{\mathrm{c}, \mathrm{e}(\mathrm{z})}$ (Gl. 81) der Schneidrollen. Eine Erhöhung der maximal erreichbaren Vortriebsstrecke $L_{\max (m)}$ ergibt sich daraus nur dann, wenn vor der Variation der Schneidringbreite $b_{S R}$ die maximal erreichbare Vortriebsstrecke $L_{c(m)}$ einer einzelnen Schneidrolle in Gl. 84 maßgeblich für die Begrenzung der maximal erreichbaren Vortriebsstrecke $\mathrm{L}_{\max (\mathrm{m})}$ war. Die Anzahl der notwendigen Werkzeugwechsel $\mathrm{n}_{\mathrm{c}(\mathrm{TA})}$ sinkt unabhängig davon aufgrund der Erhöhung des Erwartungswertes für den Laufweg $\mathrm{s}_{\mathrm{c}, \mathrm{e}(\mathrm{z})}$, der über die Berechnung des kumulierten Ausnutzungsgrades $e_{c, e(m+1)}(G l .132)$ in das Kriterium für Werkzeugwechsel (Gl. 134) einfließt.

Darüber hinaus sind im Modell bereits Korrekturfaktoren für den Verschleißschutz der Abbauwerkzeuge und das Schneidradöffnungsverhältnis enthalten. Beide konnten in der Datenanalyse als maßgebliche Einflussfaktoren auf den Laufweg $\mathrm{s}_{\mathrm{c}}$ der Abbauwerkzeuge qualifiziert, jedoch nicht quantifiziert werden (Kap. 6.2.3.3). Die Gültigkeit des Prognosemodells ist deshalb auf die in den Referenzprojekten vorliegende Bandbreite der beiden Faktoren (Tab. 22) beschränkt (Kap.6.2.3.3). Außerhalb dieser Bandbreite können die qualitative Tendenz der Auswirkung einer Variation der beiden Faktoren wie folgt beschrieben werden:

- Durch eine Erhöhung des Verschleißschutzes über das in Tab. 22 beschriebene Maß hinaus kann bei Schälmessern, Sticheln und Räumern zum Teil eine deutliche Erhöhung des Laufwegs $s_{c}$ erzielt werden. Bei Schneidrollen ist die Erhöhung des Verschleißschutzes aus technischen Gründen deutlich schwieriger. Der Aufwand für die Erhöhung des Verschleißschutzes muss jeweils im Verhältnis zu den dazu erzielten Vorteilen im Vortrieb betrachtet werden. Eine Erhöhung der maximal erreichbaren Vortriebsstrecke $L_{\max (\mathrm{m})}$ ergibt sich nur dann, wenn vor der Variation des Verschleißschutzes die maximal erreichbare Vortriebsstrecke $L_{c(m)}$ durch ein einzelnes Abbauwerkzeug des betroffenen Typs in Gl. 84 maßgeblich für die Begrenzung der maximal erreichbaren Vortriebsstrecke $L_{\max (m)}$ war. Die Anzahl der notwendigen Werkzeugwechsel $\mathrm{n}_{\mathrm{c}(\mathrm{TA})}$ sinkt unabhängig davon aufgrund der Erhöhung des Erwartungswertes für den Laufweg $\mathrm{s}_{\mathrm{c}, \mathrm{e}(\mathrm{z})}$, der über die Berechnung des kumulierten Ausnutzungsgrades $\mathrm{e}_{\mathrm{c}, \mathrm{e}(\mathrm{m}+1)}$ (Gl. 132) in das Kriterium für Werkzeugwechsel (Gl. 134) einfließt.

- Das Schneidradöffnungsverhältnis $O_{T V M}[\%]$ beeinflusst den Materialfluss von der Ortsbrust hinter das Schneidrad. Die isolierte Optimierung des Schneidradöffnungsverhältnisses über die in Tab. 22 angegeben Werte hinaus hat teilweise nur geringe Auswirkungen auf den Sekundärverschleiß der Abbauwerkzeuge. Im Vordergrund steht die Optimierung der gesamten Materialflusskette zwischen Ortsbrust und Förderleitung. 
Neben den beschriebenen Maßnahmen sind weitere Anpassungen der Auslegung der TVM denkbar, die keinen Einfluss auf das Prognoseergebnis haben, jedoch zur Nachverfolgung des Verschleißzustandes der Abbauwerkzeuge im Vortrieb und zur frühzeitigen Erkennung von Schäden an den Abbauwerkzeugen beitragen können. Dazu zählen:

- Einbau von Verschleißerkennungssystemen in den Abbauwerkzeugen oder den Abbauwerkzeughaltern bzw. Gehäusen.

- Einbau eines Metallabscheiders in der Separationsanlage bzw. Überwachung und Dokumentation von Metallteilen auf den groben Siebstufen der Separationsanlage.

\section{Steuerung des Abbauprozesses}

Aus der Steuerung des Abbauprozesses fließt nur der Erwartungswert für die Penetration $p_{e(z)}[\mathrm{mm} / \mathrm{U}]$ direkt in den Prognoseprozess ein. Dieser kann zur Anpassung der Prognose der Vortriebsunterbrechungen $\mathrm{I}_{(\mathrm{m})}$ an die Rahmenbedingungen im Projekt für den Zugang zur Abbaukammer, als auch für die Bildung verschiedener Szenarien aktiv vom Bearbeiter variiert werden.

Die Variation der Penetration $p_{e(z)}$ ist bis in die Vortriebsphase eines Projektes hinein möglich. Als Anhaltspunkte dienen einerseits die in Tab. 21 zusammengefassten Erfahrungswerte, andererseits müssen bei der Variation der Penetration $p_{\mathrm{e}(z)}$ Einschränkungen aufgrund von Steinen und Blöcken oder anderen Hindernissen in der Ortsbrust berücksichtigt werden (Kap. 5.6.3.2).

Aus dem Prognoseprozess können folgende qualitative Tendenzen für die Auswirkung einer Variation der Penetration $\mathrm{p}_{\mathrm{e}(\mathrm{z})}$ abgeleitet werden:

- Die Variation des Erwartungswertes für die Penetration $p_{e(z)}$ wirkt sich über den Korrekturfaktor $\mathrm{f}_{\mathrm{p}}\left(\mathrm{Gl}\right.$. 79) auf den Erwartungswert für den Laufweg $\mathrm{s}_{\mathrm{c}, \mathrm{e}(\mathrm{z})}$ von Schälmessern aus. Je höher die Penetration $p_{e(z)}$ gewählt wird, umso höher wird die Aktivpenetration $p_{a, e(z)}$, umso geringer wird der Korrekturfaktor $f_{p}$ und der Erwartungswert für den Laufweg $s_{c, e(z)}(G l .81)$. Je niedriger der Erwartungswert für den Laufweg $\mathrm{s}_{\mathrm{c}, \mathrm{e}(\mathrm{z})}$ gewählt wird, umso niedriger wird die maximal erreichbare Vortriebsstrecke $L_{c(m)}$ der einzelnen Schälmesser.

- Die Variation des Erwartungswertes für die Penetration $p_{\mathrm{e}(z)}$ wirkt sich in Gl. 83 zusätzlich auf die Bestimmung der maximal erreichbaren Vortriebsstrecke $L_{c(m)}$ der einzelnen Schälmesser aus. Je höher die Penetration $p_{\mathrm{e}(z)}$ gewählt wird, umso höher wird die maximal erreichbare Vortriebsstrecke $L_{c(m)}$ der einzelnen Schälmesser.

Die beiden beschriebenen Auswirkungen einer Variation der Penetration $p_{\mathrm{e}(z)}$ sind im Hinblick auf die maximal erreichbare Vortriebsstrecke $L_{\max (m)}$ und die notwendige Anzahl an Werkzeugwechseln $n_{c(T A)}$ also qualitativ gegenläufig. Quantitativ bewirkt eine Erhöhung der Penetration $p_{\mathrm{e}(\mathrm{z})} z$.B. um den Faktor 2 über den Korrekturfaktor $f_{p}$ (Gl. 79) eine Reduktion des Erwartungswertes für den Laufweg $s_{c, e(z)} u m$ den Faktor 1,6. Dieser pflanzt sich direkt in die Berechnung der maximal erreichbaren Vortriebsstrecke $L_{c(m)}$ der einzelnen Schälmesser (Gl. 83) fort. In der Berechnung in Gl. 83 fließt die um den Faktor 2 erhöhte Penetration $p_{\mathrm{e}(z)}$ jedoch auch direkt im Zähler ein. Aus quantitativer Sicht ergibt sich aus Gl. 83 durch die Erhöhung der Penetration $p_{\mathrm{e}(z)}$ deshalb trotz der Verringerung des Erwartungswertes für den Laufweg $s_{c, e(z)}$ eine Erhöhung der maximal erreichbaren Vortriebsstrecke $L_{c(m)}$ der einzelnen Schälmesser.

Darüber hinaus konnten in der Datenanalyse die Dichte der Stützflüssigkeit $\rho_{S F}\left[\mathrm{~g} / \mathrm{cm}^{3}\right]$ und der Stützdruck $P_{S F}$ [bar] als Einflussfaktoren auf den Laufweg $S_{c}$ der Abbauwerkzeuge qualifiziert, jedoch nicht quantifiziert werden (Kap. 6.2.3.3). Die Gültigkeit des Prognosemodells ist deshalb auf die in den Referenzprojekten vorliegende Bandbreite der beiden Faktoren (Tab. 22) beschränkt (Kap.6.2.3.3). 
Außerhalb dieser Bandbreite kann die qualitative Tendenz der Auswirkung einer Variation der beiden Faktoren wie folgt beschrieben werden:

- $\quad$ Eine Erhöhung der Dichte der Stützflüssigkeit $\rho_{\mathrm{SF}}\left[\mathrm{g} / \mathrm{cm}^{3}\right]$ über den in Tab. 22 angegebenen Wertebereich hinaus führt tendenziell zu steigendem Sekundärverschleiß an den Abbauwerkzeugen, weil die Kontakthäufigkeit zwischen den Abbauwerkzeugen und bereits abgebauten Bodenteilen in der Stützflüssigkeit ansteigt. Der Erwartungswert für den Laufweg $\mathrm{s}_{\mathrm{c}, \mathrm{e}(\mathrm{z})}$ der Abbauwerkzeuge nimmt deshalb tendenziell ab, sodass sich eine Verringerung der maximal erreichbaren Vortriebsstrecken $L_{\max (m)}$ und eine Erhöhung der Anzahl an Werkzeugwechseln $\mathrm{n}_{\mathrm{c}(\mathrm{TA})}$ ergibt. Der Einfluss der Dichte der Stützflüssigkeit $\rho_{\mathrm{SF}}\left[\mathrm{g} / \mathrm{cm}^{3}\right]$ auf den Erwartungswert für den Laufweg $\mathrm{s}_{\mathrm{c}, \mathrm{e}(\mathrm{z})}$ ist auf Basis der Analyse in Kap. 5.6.3.3 als mittel einzuschätzen.

- Der Stützdruck $P_{S F}$ [bar] hat auf Basis der Analyse in Kap. 5.6.3.4 nur sehr niedrigen Einfluss auf den Erwartungswert des Laufwegs $\mathrm{s}_{\mathrm{c}, \mathrm{e}(\mathrm{z}) \text {. Eine detaillierte Untersuchung anhand der Daten }}$ aus den Referenzprojekten konnte jedoch nicht erfolgen. Bei einer Erhöhung des Stützdrucks $P_{S F}$ über den in Tab. 22 angegebenen Wertebereich hinaus sollte deshalb im Rahmen des Prognosemodells von einer leichten Verringerung des Erwartungswertes für den Laufweg $\mathrm{s}_{\mathrm{c}, \mathrm{e}(\mathrm{z})}$ der Abbauwerkzeuge ausgegangen werden. Tendenziell ergeben sich daraus eine Verringerung der maximal erreichbaren Vortriebsstrecken $L_{\max (m)}$ und eine Erhöhung der Anzahl an Werkzeugwechseln $\mathrm{n}_{\mathrm{c}(\mathrm{TA})}$.

\subsubsection{Werkzeugwechselstrategie}

Die Bestimmung der Vortriebsstrecke $L_{d, e(m+1)}$ in GI. 93 zwischen den Vortriebsunterbrechungen $I_{(m)}$ und $I_{(m+1)}$ erlaubt dem Bearbeiter der Verschleißprognose die freie Auswahl der Stationierung der Vortriebsunterbrechung $I_{(m+1)}$ innerhalb der Vortriebsstrecke $L_{\max (m+1)}$. Die Vortriebsunterbrechungen können mit dieser Anpassungsmöglichkeit z.B. gezielt vor Bereiche gelegt werden, in denen der Zugang zur Abbaukammer aufgrund der gegebenen Rahmenbedingungen nur schwer möglich ist bzw. vermieden werden soll. Die Auswahl der Vortriebsstrecke $L_{d, e(m+1)}$ in $G l .93$ zwischen den Vortriebsunterbrechungen $\mathrm{I}_{(\mathrm{m})}$ und $\mathrm{I}_{(\mathrm{m}+1)}$ stellt also eine Variationsmöglichkeit in der Verschleißprognose dar, mit der die Stationierung $L_{1, e(m)}$ bzW. $b_{1, e(m)}$ an die Umgebungsbedingungen für den Zugang zur Abbaukammer angepasst werden kann, mit der aber auch verschiedene Szenarien gebildet werden können. Diese Variationsmöglichkeit wird unter dem Begriff Werkzeugwechselstrategie zusammengefasst.

Die tendenziellen Auswirkungen einer Variation der Vortriebsstrecke $L_{d, e(m+1)}$ in $G l .93$ können wie folgt zusammengefasst werden:

- Wird in Gl. 93 für $L_{d, e(m+1)}$ ein Wert gleich oder ähnlich hoch wie $L_{\max (m+1)}$ gewählt, wird über die Tunnelachse die Anzahl der Vortriebsunterbrechungen $n_{I(T A)}$ für Werkzeugwechsel minimiert. Umgekehrt ist dazu ein im Vergleich hoher Anteil an präventiven Werkzeugwechseln notwendig, sodass die Anzahl der notwendigen Werkzeugwechsel $\mathrm{n}_{\mathrm{c}(\mathrm{TA})}$ planmäßig maximiert wird.

- Je geringer der Wert für $L_{d, e(m+1)}$ im Verhältnis zu $L_{\max (m+1)}$ in $G l .93$ gewählt wird, umso mehr erhöht sich die Anzahl der notwendigen Vortriebsunterbrechungen $n_{\text {I(TA) }}$ für Werkzeugwechsel über die Tunnelachse. Umgekehrt dazu nimmt die Anzahl der notwendigen Werkzeugwechsel $\mathrm{n}_{\mathrm{c}(\mathrm{TA})}$ über die Tunnelachse tendenziell ab, weil die Anzahl der präventiven Wechsel sinkt.

Die Auswahl der Vortriebsstrecke $L_{d, e(m+1)}$ kann bis in die Vortriebsphase hinein geändert werden. Jede Änderung der Vortriebsstrecke $L_{d, e(m+1)}$ pflanzt sich in die Stationierung $L_{\mathrm{l}, e(\mathrm{~m}+1)}$ bzW. $b_{\mathrm{l}, \mathrm{e}(\mathrm{m}+1)}$ aller folgenden Vortriebsunterbrechungen fort. Die Auswirkungen müssen folglich immer über die gesamte Tunnelachse betrachtet werden. Wurde der Vortrieb von einer Vortriebsunterbrechung $\mathrm{I}_{(\mathrm{m})}$ aus in Richtung der folgenden Vortriebsunterbrechung $I_{(m+1)}$ begonnen, ist theoretisch immer noch eine Verkürzung der zuvor ausgewählten Vortriebsstrecke $L_{d, e(m+1)}$ möglich. Eine Erhöhung ist in diesem Fall nicht mehr möglich, ohne dass die Verschleißgrenze einzelner Abbauwerkzeuge überschritten wird.

In diesem Zusammenhang ist abschließend nochmals auf die in Kap. 6.4 erläuterten funktionalen Zusammenhänge zwischen der Anzahl der notwendigen Werkzeugwechsel $\mathrm{n}_{\mathrm{c}, \mathrm{e}(\mathrm{m})}$ innerhalb einer Vortriebsunterbrechung $I_{(m)}$ und der bis zur nächsten Vortriebsunterbrechung $I_{(m+1)}$ festgelegten Vortriebsstrecke $L_{d, e(m+1)}$ hinzuweisen. 


\subsubsection{Zusatzmaßnahmen}

In Abhängigkeit der geotechnischen Bedingungen in einem Projekt können Fälle auftreten, in denen mit den bisher beschriebenen Ansätzen zur Variation der Basisdaten und der Werkzeugwechselstrategie Vortriebsunterbrechungen $\mathrm{I}_{(\mathrm{m})}$ in Bereichen mit eingeschränkten Zugangsmöglichkeiten zur Abbaukammer nicht vollständig vermieden werden können. Die Einschränkung des Zugangs zur Abbaukammer ergibt sich häufig durch Probleme mit:

- Stabilität der Ortsbrust.

- $\quad$ Sicherheit gegen Ausbläser.

- Druckluftarbeiten in der Abbaukammer bei Stützdruck $P_{S F}>3,6$ bar

Für Vortriebsunterbrechungen $\mathrm{I}_{(\mathrm{m})}$ in solchen Bereichen, müssen für die Durchführung der Werkzeugwechsel entsprechende Zusatzmaßnahmen vorgesehen werden. Das Ziel der Maßnahmen ist es, die Umgebungsbedingungen so zu ändern, dass die Durchführung der Werkzeugwechsel deutlich erleichtert, bzw. überhaupt erst möglich wird. Dazu sind grundsätzlich folgende Möglichkeiten gegeben:

- Erhöhung der Stabilität der Ortsbrust.

- Erhöhung der Sicherheit gegen Ausbläser.

- Ausrüstung der TVM für Druckluftarbeiten in der Abbaukammer bei Stützdruck $P_{S F}>3,6$ bar

Die nachfolgende Beschreibung gibt einen Überblick über die Bandbreite der möglichen Maßnahmen. Die Ausgestaltung und Anwendbarkeit der Maßnahmen im Vortrieb ist stark von den Bedingungen im Projekt abhängig. Eine detaillierte Beschreibung ist deshalb nicht Bestandteil der vorliegenden Arbeit. Weiterführende Informationen geben z.B. KolYMBAS (2011: 357) und STEIN (200: 503).

Die Stabilität der Ortsbrust kann theoretisch mit folgenden Maßnahmenkategorien beeinflusst werden:

- Injektionen des Bodenkörpers aus dem Schild der TVM heraus. Dazu müssen bereits in der Designphase die entsprechenden Einrichtungen in der Auslegung der TVM berücksichtigt werden. Eine Nachrüstung im Vortrieb ist nicht mehr möglich.

- Injektionen des Bodenkörpers von der Geländeoberfläche aus. Diese können theoretisch noch bis in die Vortriebsphase hinein nachträglich angeordnet werden. Eine frühzeitige Planung innerhalb des Projektes ist jedoch zu empfehlen.

- Herstellung von Bohrpfählen oder Schlitzwandkörpern im Bereich des geplanten Drucklufteinstiegs von der Geländeoberfläche aus. Diese können theoretisch noch bis in die Vortriebsphase hinein nachträglich angeordnet werden. Eine frühzeitige Planung innerhalb des Projektes ist jedoch zu empfehlen.

- Gefrieren des Bodenkörpers im Bereich der geplanten Vortriebsunterbrechung.

Die genannten Maßnahmen haben auch Einfluss auf die Sicherheit gegen Ausbläser im Bereich der Vortriebsunterbrechung. Die Hauptmaßnahmen zur Erhöhung Sicherheit gegen Ausbläser konzentrieren sich jedoch auf das Verhältnis zwischen den vertikalen Spannungen im Boden aus der Überlagerung und dem Stützdruck $P_{S F}$. Als geeignete Maßnahme zur Erhöhung der Sicherheit gegen Ausbläser werden deshalb beschrieben:

- Einbringen zusätzlicher Auflasten durch Aufschüttung der Geländeoberkante oder der Gewässersohle im Bereich des geplanten Drucklufteinstiegs.

- Abdichtung der Ortsbrust durch Anpassung der Stützflüssigkeit oder Zugabe von Additiven. 
Die Durchführung von Arbeiten unter Druckluft in der Abbaukammer bei $>3,6$ bar Stützdruck erfordert ebenfalls Zusatzmaßnahmen. Diese lassen sich auf Basis der Methodik für Drucklufteinstiege (Kap. 3.3.3) wie folgt zusammenfassen:

- Auslegung der TVM für Druckluftarbeiten bei >3,6 bar Stützdruck mit einer entsprechenden Mischgasanlage.

- $\quad$ Auslegung der TVM für die Anwendung der Sättigungstechnik bei >3,6 bar Stützdruck, z.B. durch Berücksichtigung der Transportwege und Anschlussmöglichkeiten für mobile Druckkammern, sowie entsprechende zusätzliche Versorgungseinrichtungen auf der Baustelleneinrichtungsfläche.

Die genannten Maßnahmen müssen ebenfalls bereits in der Designphase der TVM berücksichtigt werden, da eine nachträgliche Umrüstung im Vortrieb kaum mehr möglich ist.

\subsubsection{Planungsszenario}

Am Ende des Prognoseprozesses (Abb. 93) ist die Festlegung eines Planungsszenarios durch den Bearbeiter der Verschleißprognose notwendig. Der Inhalt des Planungsszenarios muss in der Vortriebsphase nachverfolgt werden. Nachfolgend sind sowohl der notwendige Inhalt, als auch die Nachverfolgung im Vortrieb zusammengefasst.

\subsubsection{Inhalt}

Das Planungsszenario umfasst alle Basisdaten, die in die Verschleißprognose eingegangen sind, die daraus gewonnene Werkzeugwechselstrategie und Angaben über geplante Maßnahmen aus dem Anpassungsprozess. Zusammenfassend bilden also folgende Daten den Inhalt des Planungsszenarios:

- Basisdaten der TVM-Auslegung (Kap. 6.1.1.1)

- Basisdaten für die geotechnischen Parameter und Einteilung der geotechnischen Homogenbereiche (Kap. 6.1.1).

- Basisdaten der Steuerung des Abbauprozesses (Kap. 6.1.1.3)

- $\quad$ Erwartungswerte für die Laufwege $\mathrm{s}_{\mathrm{c}, \mathrm{e}(\mathrm{z})}[\mathrm{km}]$ (Kap. 6.2.3.4)

- Stationierung $L_{1, e(m)}[m]$ bzw. $b_{1, e(m)}$ der Vortriebsunterbrechungen $I_{(m)}$ (Kap. 6.3.4)

- Anzahl der Werkzeugwechsel $\mathrm{n}_{\mathrm{c}, \mathrm{e}(\mathrm{m})}$ (Kap. 6.4)

- $\quad$ Angaben zu Zusatzmaßnahmen (Kap. 6.5.2.3)

\subsubsection{Nachverfolgung im Vortrieb}

Das Planungsszenario muss in der Vortriebsphase des Projektes konsequent nachverfolgt werden. Änderungen der Basisdaten im Vergleich zu den für die Verschleißprognose angesetzten Werten pflanzen sich in die Verschleißprognose fort und können hier zu einer Anpassung der Schätzungen für den Laufweg $\mathrm{s}_{\mathrm{c}, \mathrm{e}(\mathrm{z})}$ und die maximale erreichbaren Vortriebsstrecken $L_{\max (\mathrm{m})}$ führen.

Folglich kann eine Anpassung der Auswahl der Vortriebsstrecken $L_{d, e(m)}$ und der Stationierung der Vortriebsunterbrechungen $\mathrm{L}_{\mathrm{l}, \mathrm{e}(\mathrm{m})}$ bzw. $\mathrm{b}_{\mathrm{l}, \mathrm{e}(\mathrm{m})}$ notwendig werden. Eine Änderung der Stationierung der Vortriebsunterbrechungen kann sich jedoch auch durch Entscheidungen in der Vortriebsphase zur Änderung der Vortriebsstrecken $L_{d, e(m)}$ ohne Bezug zu einer Änderung der Basisdaten ergeben. 
Unabhängig davon muss als Folge einer Änderung der Stationierung der Vortriebsunterbrechungen $\mathrm{I}_{(\mathrm{m})}$ erneut der Anpassungsalgorithmus durchgeführt werden, um die Stationierungen erneut an die Rahmenbedingungen im Projekt für den Zugang zur Abbaukammer anzupassen. Ergebnis daraus ist ein aktualisiertes Planungsszenario, sodass sich bei wiederholten Änderungen ein rollierender Anpassungsprozess ergibt.

Aufgrund der zahlreichen Einflussfaktoren und deren möglicher Schwankungsbreite, sowie der umfangreichen Anpassungsmöglichkeiten der Verschleißprognose sind Änderungen im Vergleich zum bestehenden Planungsszenario in der Vortriebsphase durchaus häufig. Die Nachverfolgung des Planungsszenarios hat also folgende aufeinander aufbauende Ziele:

- Überprüfung der Basisdaten

- Überprüfung der in der Verschleißprognose getroffenen Annahmen und Schätzungen

- Rollierende Überarbeitung der Vortriebsplanung

Dazu ist ein Mindestaufwand an Dokumentation im Vortrieb notwendig. Diese richtet sich grundsätzlich nach den Anforderungen in Kap. 5.2 und umfasst die folgenden Felder:

- Dokumentation der Abbauwerkzeugwechsel (Kap. 5.2.1)

- Dokumentation der angetroffenen geotechnischen Verhältnisse (Kap. 5.2.2)

- Dokumentation der TVM-Auslegung (Kap. 5.2.3)

- Dokumentation der TVM-Betriebsdaten (Kap. 5.2.4)

Die Zusammenführung der genannten Daten ist aufgrund des hohen Datenvolumens zum Teil manuell nicht mehr möglich. Entsprechend werden hier zunehmend spezialisierte Datenbanksysteme eingesetzt, die eine einfache Analyse und Visualisierung der Daten erlauben. Ein Beispiel und Beschreibung zu einem solchen Datenbanksystem gibt www-006.

Aufbauend auf die Dokumentation müssen alle im Prognoseprozess getroffenen Annahmen und Schätzungen überprüft werden. Dies gilt insbesondere für die Parameter mit hohem Einfluss auf die Stationierung $\mathrm{L}_{\mathrm{l}, \mathrm{e}(\mathrm{m})}$ bzw. $\mathrm{b}_{\mathrm{l}, \mathrm{e}(\mathrm{m})}$ der Vortriebsunterbrechungen $\mathrm{I}_{(\mathrm{m})}$ und die Anzahl der Werkzeugwechsel $\mathrm{n}_{\mathrm{c}, \mathrm{e}(\mathrm{m})}$ :

- Überlagerung $\mathrm{H}_{\mathrm{TA}}$ und Wasserspiegel $\mathrm{W}_{\mathrm{TA}}[\mathrm{m}]$

- Verteilung der Bodenarten in der Ortsbrust entlang der Tunnelachse

- Korngrößenverteilung der Bodenarten

- Äquivalenter Quarzgehalt (ÄQu) [\%] der Bodenarten

- Verhältnis der tatsächlichen Penetration $\mathrm{p}_{(z)}$ zum geplanten Erwartungswert $\mathrm{p}_{\mathrm{e}(\mathrm{z})}[\mathrm{mm} / \mathrm{U}]$

- $\quad$ Dichte der Stützflüssigkeit in der Abbaukammer $\rho_{\mathrm{SF}}\left[\mathrm{g} / \mathrm{cm}^{3}\right]$

- Verhältnis des tatsächlichen Laufwegs $\mathrm{s}_{\mathrm{cv}(\mathrm{z})}[\mathrm{km}]$ zum geplanten Erwartungswert $\mathrm{s}_{\mathrm{cv}, \mathrm{e}(\mathrm{z})}[\mathrm{km}]$

- Verhältnis der tatsächlichen Vortriebsstrecken $L_{d(m)}[m]$ im Vergleich zu den geplanten Vortriebsstrecken $\mathrm{L}_{\mathrm{d}, \mathrm{e}(\mathrm{m})}[\mathrm{m}]$

Auf Basis der Überprüfung kann eine rollierende Anpassung des Planungsszenarios vorgenommen werden. Das bedeutet, dass jede Änderung der genannten Parameter im Vortrieb gegenüber den geplanten Werten als neue Grundlage in den Prognoseprozess aufgenommen und die Vortriebsplanung für die verbleibende Vortriebsstrecke angepasst wird. 


\subsection{Validierung des Prognosemodells}

\subsubsection{Strukturelle Validierung}

Aus struktureller Sicht beschreibt das Modell zunächst aufgrund der Beschränkung der Datenanalyse auf Primär- und Sekundärverschleiß der Abbauwerkzeuge bzw. Filterung der Werkzeugwechsel aufgrund von Schäden nur einen Teil des realen Systems. Die Mechanismen, die zu Schäden an den Abbauwerkzeugen führen und die zugehörigen Verschleißbilder sind zwar in der Analyse und Beschreibung der Verschleißtypen und Verschleißgrenzen (Kap. 5.3) enthalten. In der Analyse der Einflussfaktoren auf den Laufweg $\mathrm{s}_{\mathrm{c}}$ der Abbauwerkzeuge (Kap. 5.6) werden bei der Aufbereitung der Rohdaten (Kap. 5.6.1) Werkzeugwechsel aufgrund von Schäden durch Steine, Blöcke, künstliche Hindernisse oder Verklebungen eliminiert.

Folglich erfasst auch das empirische Prognosemodell die Mechanismen und Auswirkungen von Schäden an den Abbauwerkzeugen durch Steine, Blöcke oder künstliche Hindernisse in der Ortsbrust und Verklebungen nicht. Zur Beschreibung der Mechanismen, die zu Schäden an den Abbauwerkzeugen führen, sind weitere Untersuchungen notwendig. Theoretisch führen Schäden an einzelnen Abbauwerkzeugen zu einem abrupten Funktionsverlust, sodass unmittelbar eine Vortriebsunterbrechung für Werkzeugwechsel notwendig ist. Folglich verkürzen sich die maximal erreichbaren Vortriebsstrecken $L_{\max (m)}$ im Vergleich zu reinem Primär- und Sekundärverschleiß zum Teil deutlich und die Anzahl der notwendigen Werkzeugwechsel $\mathrm{n}_{\mathrm{c}(\mathrm{m})}$ steigt im Verhältnis an.

Bei Verklebungen liegt aufgrund des von FRENZEL \& BABENDEREDE (2011: 41) beschriebenen Verhältnisses zwischen abgebautem Bodenvolumen und verfügbarer Öffnungsfläche des Schneidrades die Wahrscheinlichkeit für Schäden an den Schneidrollen im Schneidradzentrum tendenziell höher als in den übrigen Bereichen. Schäden durch Steine oder Blöcke zeigen dagegen augenscheinlich eine zufällige Verteilung über die Schneidradfläche bzw. den Radius $\mathrm{R}_{\text {TVM }}$. Ort und Intensität der Schäden hängen hier hauptsächlich davon ab, wo in der Ortsbrustfläche ein Stein oder Block angetroffen wird, ob bzw. wie weit durch die eingesetzten Abbauwerkzeuge eine Zerkleinerung in der Ortsbrust gelingt und wie schnell die verbleibenden Bruchstücke aufgrund der Verteilung der Schneidradöffnungen und deren Größe im Verhältnis zur Korngröße der Bruchstücke hinter das Schneidrad transportiert werden (Kap. 5.3).

Die Beschreibung dieser komplexen Kette an Mechanismen erfordert probabilistische Methoden, um die Eintrittswahrscheinlichkeit und Intensität von Schäden an einzelnen Abbauwerkzeugen zu quantifizieren. Eine zukünftige Integration der Schadensmechanismen in das Prognosemodell kann darauf aufbauend mit folgenden Schritten erfolgen:

- Einführung zusätzlicher Prozessschritte in der Schätzung der Vortriebsstrecken $\mathrm{L}_{\mathrm{I}, \mathrm{e}(\mathrm{m})}$, um diese bei vorliegenden Risiken für Schäden zu reduzieren und so zusätzliche Vortriebsunterbrechungen $\mathrm{I}_{(\mathrm{m})}$ zu berücksichtigen.

- Einführung zusätzlicher Prozessschritte zur Schätzung der zusätzlichen Anzahl der Werkzeugwechsel $\mathrm{n}_{\mathrm{c}, \mathrm{e}(\mathrm{m})}$ aufgrund von Schäden.

Der Anpassungsmechanismus zur Bildung verschiedener Vortriebsszenarien (Kap. 6.5.2) erlaubt bereits in der vorliegenden Form auch die Berücksichtigung von Risiken durch Schäden an den Abbauwerkzeugen. Die Szenarien für die möglichen Auswirkungen von Schäden können hier vermutlich mithilfe der Eintrittswahrscheinlichkeiten und geschätzten Intensitäten bzw. Auswirkungen auch quantitativ beschrieben werden. 
Das System aus Einflussfaktoren auf den Primär- und Sekundärverschleiß wird durch das Prognosemodell und die darin enthaltenen Einflussfaktoren vollständig erfasst. Zu berücksichtigen sind jedoch folgende Einschränkungen:

- In den Prognoseprozess fließen nur die quantifizierbaren Einflussfaktoren direkt ein. Die nicht quantifizierbaren Einflussfaktoren beeinflussen den Prognoseprozess nur insofern, dass ihre Wertebereiche (Tab. 22) Anwendungsgrenzen für das Prognosemodell bilden.

- Die Kontaktspannung $T_{a c t}\left[\mathrm{kN} / \mathrm{m}^{2}\right]$ im Prognosemodell wird mithilfe der Scherfestigkeit $T_{c}$ $\left[\mathrm{kN} / \mathrm{m}^{2}\right]$ des Bodens in der Ortsbrust als Bezugsgröße beschrieben.

- Für Räumer und Stichel begründet die Datenanalyse die Annahme, dass für diese Abbauwerkzeugtypen der identische Satz an Einflussfaktoren relevant ist, wie für Schälmesser. Eine Überprüfung dieser Annahme ist aufgrund des geringen, verfügbaren Datenvolumens für die beiden Werkzeugtypen nicht möglich.

Um die genannten Einschränkung bei der Abbildung der Systemelemente im Prognosemodell zu beheben, sind folgende Schritte denkbar:

- Untersuchung der bisher nicht quantifizierbaren Einflussfaktoren und Entwicklung zugehöriger Korrekturfaktoren für den Basiswert des Laufwegs $\mathrm{s}_{\mathrm{c}, \mathrm{b}(\mathrm{z}) \text {. }}$.

- Entwicklung direkter Berechnungsmöglichkeiten für die Kontaktspannungen $T_{\text {act }}\left[\mathrm{kN} / \mathrm{m}^{2}\right] \mathrm{zwi}$ schen den Werkzeugoberflächen und dem Boden aus der Schneidradanpresskraft $F_{S R}[k N]$ und dem Schneiddrehmoment $\mathrm{M}_{\mathrm{SR}}[\mathrm{kNm}]$.

- Untersuchung der Einflussfaktoren auf den Laufweg $s_{c}$ von Räumern und Sticheln. Ableitung einer eigenen Korrelationen zwischen dem Laufweg $\mathrm{s}_{\mathrm{c}}$ und dem SAI, sowie der zugehörigen Korrekturfaktoren für den Basiswert des Laufwegs $\mathrm{s}_{\mathrm{c}, \mathrm{b}(\mathrm{z})}$ für Stichel und Räumer.

Zur Anwendung des Prognosemodells ist die Schätzung eines Erwartungswertes für die Penetration $\mathrm{p}_{\mathrm{e}(z)}[\mathrm{mm} / \mathrm{U}]$ notwendig. Hierzu liegen bisher nur die Anhaltspunkte aus den Referenzprojekten vor. Folglich ist eine Erweiterung des Prognosemodells um ein Teilmodell zur Prognose der Penetration $p_{e(z)}$ in Abhängigkeit der Auslegung der TVM und der geotechnischen Bedingungen denkbar. Das Teilmodell kann als eigene Sektion vor Sektion 1 eingegliedert werden. Der Erwartungswert für die Penetration $p_{e(z)}[\mathrm{mm} / \mathrm{U}]$ stünde so in der darauf folgenden Sektion für die Schätzung des Erwartungswertes für den Laufweg $\mathrm{s}_{\mathrm{c}, \mathrm{e}(\mathrm{z})}[\mathrm{km}]$ zur Verfügung.

\subsubsection{Deskriptive Validierung}

Die deskriptive Validierung des Prognosemodells erfolgt anhand der Referenzprojekte (RP). Dazu wird das Prognosemodell auf die Basisdaten aus den RP angewendet um einen Abgleich der Ergebnisse mit den tatsächlichen Werten aus dem Vortrieb in den RP zu ermöglichen. Der Abgleich erfolgt anhand der drei kritischen Faktoren im Prognosemodell (Kap.2.4.4):

- Länge der Vortriebsstrecken $L_{d, e(m)}$ zwischen den Vortriebsunterbrechungen $I_{(m)}$ für Werkzeugwechsel (Kap. 6.3.4).

- $\quad$ Laufweg $\mathrm{s}_{\mathrm{cv}, \mathrm{e}(\mathrm{z})}$ der Abbauwerkzeuge (Kap. 6.6.2.2).

- Anzahl der Werkzeugwechsel über die Tunnelachse $n_{c, e(T A)}$ (Kap. 6.6.2.2).

Die Rahmenbedingungen und projektspezifischen Entscheidungen für den Zugang zur Abbaukammer in den Referenzprojekten sind nicht in ausreichendem Maß bekannt. Deshalb liegt einerseits keine Entscheidungsgrundlage zur Auswahl der Vortriebsstrecken $L_{d, e(m)}$ bzw. $L_{d, e(m+1)}$ in $G l .112$ (Kap. 6.3.4) vor, andererseits kann keine baubetriebliche Anpassung (Kap. 6.5) der Stationierung $L_{l, e(m)}$ der Vortriebsunterbrechungen $I_{(m)}$ vorgenommen werden. 
In der Anwendung des Prognosemodells auf die RP wurden in Gl. 112 deshalb für den Erwartungswert der Vortriebsstrecken $L_{1, e(m+1)}$ die maximal erreichbaren Vortriebsstrecken $L_{\max (m)}$ aus $G$ l. 111 angesetzt. Die so gewonnen Erwartungswerte für $L_{d, e(m+1)}$ können anschließend mit den tatsächlich erreichten Vortriebsstrecken $L_{d(m)}$ aus den RP verglichen werden. Aufgrund der fehlenden Anpassung in Gl. 112 ist dabei zu erwarten, dass die Werte für $L_{d, e(m)}$ aus dem Prognosemodell tendenziell höher liegen als die tatsächlich in den RP erreichten Werte $L_{d(m)}$.

Für die Untersuchung des Laufwegs $\mathrm{s}_{\mathrm{cv}, \mathrm{e}(\mathrm{z})}$, sowie für die Untersuchung der Anzahl der Werkzeugwechsel $\mathrm{n}_{\mathrm{c}, \mathrm{e}(\mathrm{TA})}$ wurde das Prognosemodell in ähnlicher Weise auf die Basisdaten der RP angewendet. Hier wurde die im Vortrieb getroffene Auswahl der Vortriebsstrecken $L_{d(m)}$ und baubetriebliche Anpassung der Stationierung $\mathrm{L}_{(\mathrm{m})}$ der Vortriebsunterbrechungen $\mathrm{I}_{(\mathrm{m})}$ in das Prognosemodell übernommen. Dazu wurden in Gl. 112 (Kap. 6.3.4) für den Erwartungswert der Vortriebsstrecken $L_{d, e(m)}$ die tatsächlich erreichten Vortriebsstrecken $L_{d(m)}$ aus den RP eingesetzt. Dadurch entfällt im Prognoseprozess (Kap.6.3.4) die iterative Anpassung der Vortriebsstrecken $L_{l, e(m)}$ an die Länge der geotechnischen Homogenbereiche. Für die Berechnung der partiellen Laufwege der Abbauwerkzeuge in Gl. 122, Gl. 124 \& Gl. 126 wurde die in den RP erreichte Penetration $\mathrm{p}_{(z)}[\mathrm{mm} / \mathrm{U}]$ eingesetzt.

Die Werkzeugwechsel im Prognosemodell wurden mit dem Kriterium in Gl. 134 angesetzt, sodass auch präventive Wechsel berücksichtigt sind. Analog zur Datenanalyse (Kap.5.6.1.4) wurden anschließend für jeden Werkzeugtyp und HGB Modalwerte für die Laufwege $\mathrm{s}_{\mathrm{cv}(\mathrm{m})}$ und $\mathrm{s}_{\mathrm{cv}, \mathrm{e}(\mathrm{m})}$ gebildet. Im Unterschied zur Datenanalyse wurden hierbei alle Werkzeugwechsel aus den RP berücksichtigt, auch solche aufgrund von Schäden und präventiven Bestückungswechseln. Die beiden Modalwerte können so direkt verglichen werden und die Auswirkungen von Schäden dargestellt werden.

Mit dieser Methode kann auch die Anzahl der Werkzeugwechsel durch einen direkten Vergleich der Prognoseergebnisse mit den Daten aus den RP validiert werden. Es sind jedoch drei unterschiedliche Betrachtungsweisen notwendig, um mögliche Abweichungen zwischen dem Prognoseergebnis und den Werten aus den RP zu erkennen und zu erklären:

- Vergleich der Anzahl der Werkzeugwechsel $\mathrm{n}_{\mathrm{c}, \mathrm{e}(\mathrm{TA})}$ bzw. $\mathrm{n}_{\mathrm{c}(\mathrm{TA})}$ über die Tunnelachse

- Vergleich der Anzahl der Werkzeugwechsel $n_{c, e(m)}$ je Vortriebsunterbrechung $I_{(m)}$ kumuliert über die Tunnelachse

- Verteilung der Anzahl der Werkzeugwechsel $\mathrm{n}_{\mathrm{c}, \mathrm{e}(\mathrm{s})}$ je Schneidspur.

Aufgrund der durchgehenden Anwendung des Werkzeugwechselkriteriums in Gl. 134 in der Verschleißprognose beinhalten die Ergebnisse zur Anzahl der Werkzeugwechsel $n_{c(T A)}$ ausschließlich verschleißbedingte Werkzeugwechsel. Folglich entstehen zwischen den Ergebnissen der Verschleißprognose und den Werten aus den RP in folgenden Fällen zwangsläufig Abweichungen:

- Homogenbereiche oder Vortriebsunterbrechungen in den RP, in denen ein hoher Anteil der Werkzeugwechsel aufgrund von Gewaltschäden vorgenommen wurde. Hier wird der Laufweg $\mathrm{S}_{\mathrm{cv}(\mathrm{m})}$ der Abbauwerkzeuge aus den RP durch die Werte aus der Verschleißprognose tendenziell überschätzt und die Anzahl der Werkzeugwechsel $\mathrm{n}_{\mathrm{c}(\mathrm{m})}$ unterschätzt.

- Homogenbereiche in den RP, in denen eine oder mehrere Vortriebsstrecken $L_{d(m)}$ höher gewählt wurden, als die maximal erreichbaren Vortriebsstrecken $L_{\max (\mathrm{m})}$ aus dem Prognosemodell. Hier wird aufgrund der festgelegten Vortriebsstrecken $L_{d(m)}$ aus den RP im Prognosemodell trotz des Kriteriums in Gl. 134 ein Teil der Werkzeugwechsel erst bei einem Ausnutzungsgrad größer 1 vorgenommen. Die Verschleißprognose enthält also Werkzeugwechsel, die in der Theorie als Verschleißschäden zu bezeichnen sind. Folglich kann hier der Modalwert des Laufwegs $\mathrm{s}_{\mathrm{cv}(\mathrm{z})}$ der in der Verschleißprognose ausgeführten Werkzeugwechsel höher liegen, als der ursprünglich geschätzte Erwartungswert $\mathrm{s}_{\mathrm{cv}, \mathrm{e}(\mathrm{z}) \text {. }}$.

- In einigen RP wurden planmäßige Wechsel der Schneidradbestückung vorgenommen, um die Abbauwerkzeuge an stark wechselnde geotechnische Bedingungen anzupassen. Diese wurden so in der Verschleißprognose nicht umgesetzt. Folglich überschätzten die Prognoseergebnisse an diesen Stellen die Laufwege $\mathrm{s}_{\mathrm{cv}(\mathrm{m})}$ der Abbauwerkzeuge aus den RP, wohingegen die Anzahl der Werkzeugwechsel $\mathrm{n}_{\mathrm{c}(\mathrm{m})}$ unterschätzt wird. 
Die hieraus entstehenden Abweichungen können in den Ergebnissen der Validierung sowohl am Laufweg $\mathrm{s}_{\mathrm{cv}(\mathrm{m})}$, als auch an der Anzahl der Werkzeugwechsel $\mathrm{n}_{\mathrm{c}(\mathrm{m})}$ aufgezeigt und erklärt werden. Es erfolgt eine Unterteilung der Abweichungen in drei Kategorien:

- Modellbedingte Abweichungen

- Schadensbedingte Abweichungen

- Abweichungen aufgrund einer Überschreitung der geschätzten maximal erreichbaren Vortriebsstrecken $L_{\max (m)}(G l .111)$ durch die tatsächlich realisierten Vortriebsstrecken $L_{d(m)}$.

Für die deskriptive Validierung wurden aus den verfügbaren Referenzprojekten (Tab. 8) abschließend folgende fünf Vortriebe zur Bearbeitung ausgewählt, um bei vertretbarem Rechenaufwand eine möglichst hohe Bandbreite an TVM-Durchmessern, Bestückungsvarianten und geotechnischen Bedingungen abzudecken:

- Referenzprojekt 3, TVM 4, Vortrieb 5

- Referenzprojekt 6, TVM 9, Vortrieb 13

- Referenzprojekt 7, TVM 10, Vortrieb 14

- Referenzprojekt 9, TVM 12, Vortrieb 16

- Referenzprojekt 10, TVM 13, Vortrieb 17

Die Vortriebe wurden in der Verschleißprognose jeweils vollständig vom Start des Tunnels bis zum Durchbruch betrachtet. 


\subsubsection{Vortriebsstrecken $L_{d(m)}$}

Die Vortriebsstrecken $L_{d(m)}$ aus den Referenzprojekten sind in Abb. 94 den Erwartungswerten für die Vortriebsstrecken $L_{d, e(m)}$ aus der Verschleißprognose gegenübergestellt. Dazu wird der Wertebereich von $L_{d(m)}$ und $L_{d, e(m)}$ um den arithmetischen Mittelwert über alle Einzelwerte aus den Referenzprojekten und der Verschleißprognose dargestellt.

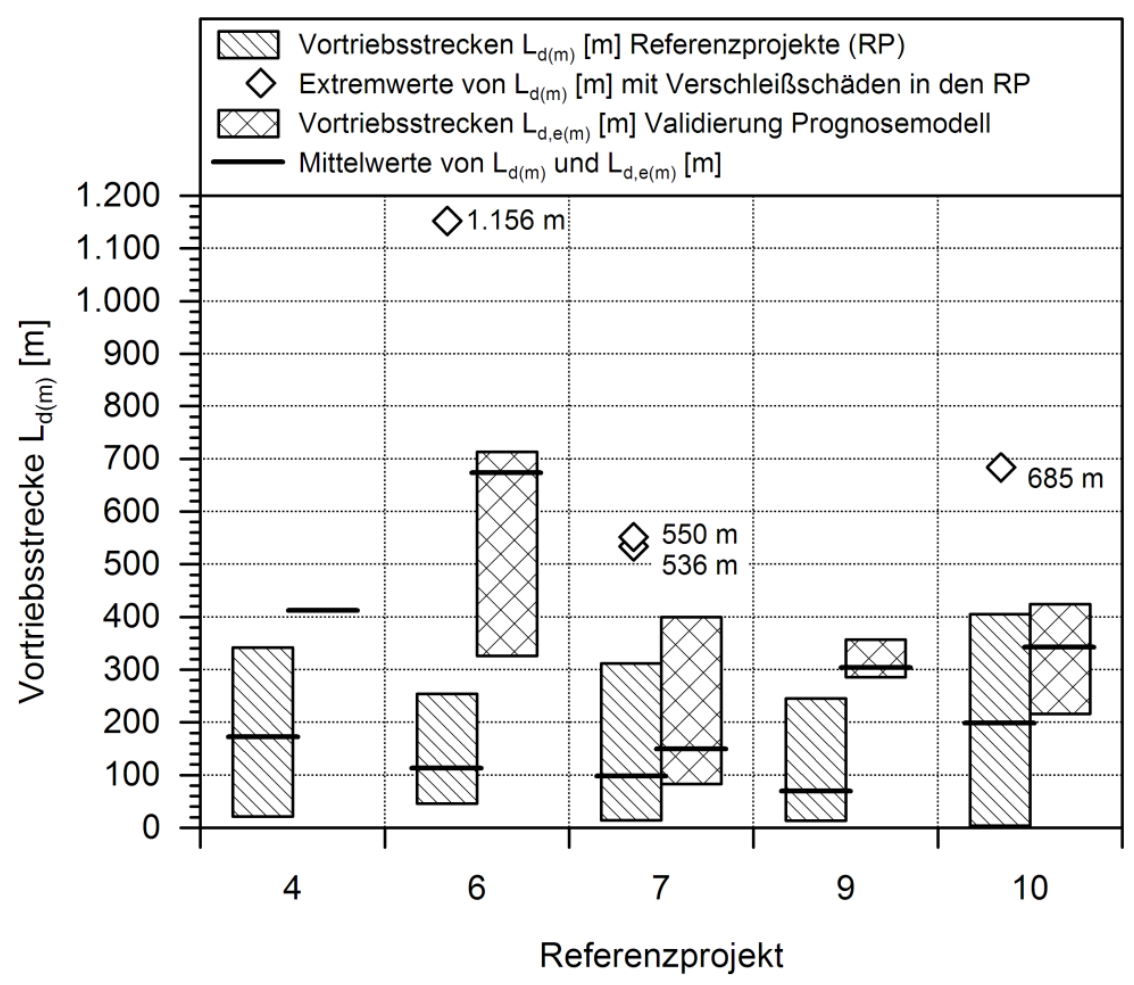

Abb. 94: Vergleich der Vortriebsstrecken $L_{d(\mathrm{~m})}[\mathrm{m}]$ aus den Referenzprojekten mit den Erwartungswerten für die Vortriebsstrecken $L_{d, e(m)}[m]$ aus der Validierung des Prognosemodells.

Die Darstellung der Daten in Abb. 94 zeigt, dass die Mittelwerte über die Vortriebsstrecken $L_{d, e(m)}$ aus der Verschleißprognose jeweils deutlich höher liegen, als die zugehörigen Mittelwerte aus den Referenzprojekten. Die Hauptursache hierfür ist die fehlende baubetriebliche Anpassung der Vortriebsstrecken $L_{d, e(m)}$ in der Verschleißprognose (Kap. 6.6.2). Die auffallend hohe Differenz zwischen dem Mittelwert über die Vortriebsstrecken $L_{d, e(m)}$ aus der Verschleißprognose und $L_{d(m)}$ in Referenzprojekt 7 kann durch einen rigiden Werkzeugwechselplan (Kap. 6.6.2.2) erklärt werden, für den im Vortrieb im Verhältnis sehr kurze Vortriebsstrecken $\mathrm{L}_{\mathrm{d}(\mathrm{m})}$ ausgewählt wurden.

Die Differenz zwischen den mittleren Vortriebsstrecken wirkt sich auch auf das Verhältnis zwischen der Anzahl der Vortriebsunterbrechungen über die Tunnelachse in den Referenzprojekten $n_{\text {I(TA) }}$ und in der Verschleißprognose $n_{\mathrm{l}, \mathrm{e}(\mathrm{TA})}$ aus. Die Anzahl der Vortriebsunterbrechungen in der Verschleißprognose $n_{l, e(T A)}$ liegt hier umgekehrt deutlich niedriger als in den Referenzprojekten.

Die in Abb. 94 argestellte Bandreite der Werte für die Vortriebsstrecken $L_{d(m)}$ aus den Referenzprojekten und $L_{d, e(m)}$ aus der Verschleißprognose zeigt für die Maximalwerte eine ähnliche Abweichung, wie für die Mittelwerte. Die Maximalwerte aus der Verschleißprognose liegen durchgehend höher als die Maximalwerte aus den Referenzprojekten. Die absoluten Differenzen unterscheiden sich jedoch deutlich im Vergleich zu den Mittelwerten. Die Ursache dafür ist eine stark unterschiedliche Ausnutzung der theoretisch maximal erreichbaren Vortriebsstrecken $L_{\max (m)}$ aus der Verschleißprognose durch die in den Referenzprojekten ausgewählten Vortriebsstrecken $L_{d(m)}$.

Die Minimalwerte für die Vortriebsstrecken aus den Referenzprojekten $L_{d(m)}$ in Abb. 94 liegen ebenfalls durchgehend niedriger als die Minimalwerte für die Vortriebsstrecken $L_{d, e(m)}$ aus der Verschleißprog- 
nose. Die absoluten Differenzen unterscheiden sich jedoch wiederum deutlich im Vergleich zu den Mittelwerten und den Maximalwerten. Die Ursache dafür sind die unterschiedlichen Auswirkungen von Schäden an den Abbauwerkzeugen durch Steine und Blöcke in der Ortsbrust oder Verklebungen, die in den Referenzprojekten in einzelnen Homogenbereichen zum Teil zu sehr niedrigen Werten für die Vortriebsstrecken $L_{d(m)}$ geführt haben (Kap. 6.6.2.2).

Für Referenzprojekt 3 ist in Abb. 94 keine Bandbreite für die Vortriebsstrecken $\mathrm{L}_{\mathrm{l}, \mathrm{e}(\mathrm{m})}$ aus der Verschleißprognose dargestellt. Der Vortrieb verläuft hier vollständig innerhalb eines geotechnischen Homogenbereichs, sodass sich in der Verschleißprognose nur ein Erwartungswert für die Vortriebsstrecken $L_{d, e(m)}$ ergibt. Die absoluten Differenzen zwischen den Vortriebsstrecken $L_{d(m)}$ und $L_{d, e(m)}$ zeigen sowohl für die Mittelwerte, als auch für die Minimal- und Maximalwerte eine hohe Schwankungsbreite zwischen den Referenzprojekten. Dies verdeutlicht, dass für die baubetriebliche Anpassung der Vortriebsstrecken $L_{d, e(m)}$, aber auch zur Berücksichtigung von Schäden keine pauschale Abminderung der maximal erreichbaren Vortriebsstrecken $L_{\max (m)}$ möglich ist. Die baubetriebliche Anpassung muss stets projektspezifisch durch den in Kap. 6.5 beschriebenen Algorithmus erfolgen. Für die Berücksichtigung von Schäden sind weitere Untersuchungen notwendig.

Zusätzlich zur Bandbreite der Werte für die Vortriebsstrecken aus den Referenzprojekten und der Verschleißprognose sind in Abb. 94 in den Referenzprojekten 6,7 und 10 einzelne Extremwerte für die Vortriebsstrecken $L_{d(m)}$ eingetragen. Hier wurden in den Referenzprojekten deutliche Verschleißschäden an den Abbauwerkzeugen und zum Teil auch an der Schneidradstahlstruktur festgestellt, die durch umfangreiche Reparaturen im Vortrieb behoben werden mussten. Die hier erreichten Werte von $L_{d(m)}$ liegen um 38 - $62 \%$ höher, als die errechneten Maximalwerte $L_{\max (m)}$ aus der Verschleißprognose. In der vorliegenden Validierung wurde in der Verschleißprognose für die Erwartungswerte der Vortriebsstrecken $L_{d, e(m)}$ in Gl. 93 jeweils die theoretisch maximal erreichbare Vortriebsstecke $L_{\max (m)}$ eingesetzt (Kap. 6.6.2). Die in Abb. 94 dargestellten Verschleißschäden bei den Extremwerten für $L_{d(m)}$ in den Referenzprojekten können also als starker Hinweis darauf gewertet werden, dass die in der Verschleißprognose ermittelten maximal erreichbaren Vortriebsstrecken $L_{\max (m)}$ die Verschleißgrenzen der Abbauwerkzeuge tatsächlich in zutreffender Form berücksichtigen. Durch die Einhaltung der maximal erreichbaren Vortriebsstrecken $L_{\max (m)}$ aus der Verschleißprognose können folglich Verschleißschäden an den Abbauwerkzeugen und der Schneidradstahlstruktur vermieden werden.

\subsubsection{Laufweg $s_{\mathrm{cv}(\mathrm{m})}$}

Der Vergleich zwischen den Prognoseergebnissen und den Werten aus den Referenzprojekten wird im Folgenden aus Gründen der Übersichtlichkeit anhand von Referenzprojekt 7 erläutert. Aufgrund relativ langer Homogenbereiche und einer hohen Variation der Werkzeugbestückung können hier verschiedene Effekte und Abweichungen gut dargestellt werden. Die Ergebnisse aus den übrigen Referenzprojekten aus der Validierung werden im Anschluss daran als Übersicht zusammengefasst.

Der Vergleich der Laufwege $\mathrm{s}_{\mathrm{cv}, \mathrm{e}(\mathrm{m})}$ und $\mathrm{s}_{\mathrm{cv}(\mathrm{m})}$ für RP 7 ist untergliedert nach geotechnischen Homogenbereichen in Tab. 24 dargestellt. Die Abweichung $d_{s c}[\%]$ des prognostizierten Laufweg $s_{c v, e(m)}$ vom tatsächlichen Laufweg $\mathrm{s}_{\mathrm{cv}(\mathrm{m})}$ in RP 7 wurde dazu mit Gl. 139 berechnet:

$$
\mathrm{d}_{\mathrm{sc}}=\frac{\left(\mathrm{s}_{\mathrm{cv}, \mathrm{e}(\mathrm{m})}-\mathrm{s}_{\mathrm{cv}(\mathrm{m})}\right)}{\mathrm{s}_{\mathrm{cv}(\mathrm{m})}}
$$

Im Falle einer Überschätzung von $\mathrm{s}_{\mathrm{cv}(\mathrm{m})}$ durch das Prognosemodell nimmt $\mathrm{d}_{\mathrm{sc}}$ einen positiven Wert an. Bei einer Unterschätzung von $\mathrm{s}_{\mathrm{cv}(\mathrm{m})}$ durch das Prognosemodell ergibt $\mathrm{Gl}$. 139 für $\mathrm{d}_{\mathrm{sc}}$ einen negativen Prozentwert. 
Tab. 24: Vergleich der tatsächlich erreichten Laufwege $\mathrm{s}_{\mathrm{cv}(\mathrm{m})}$ aus Referenzprojekt 7 mit den geschätzten Laufwegen $\mathrm{s}_{\mathrm{cv}, \mathrm{e}(\mathrm{m})}$ bei Ausbau der Abbauwerkzeuge aus dem Prognosemodell-

\begin{tabular}{|c|c|c|c|c|c|c|c|c|c|}
\hline \multirow{2}{*}{ HGB } & \multicolumn{3}{|c|}{ Schneidrollen } & \multicolumn{3}{c|}{ Stichel } & \multicolumn{3}{c|}{ Schälmesser } \\
\cline { 2 - 11 } & $\mathbf{S}_{\mathbf{c v}(\mathbf{m})}[\mathbf{k m}]$ & $\mathbf{S}_{\mathrm{cv}, \mathbf{e}(\mathbf{m})}[\mathbf{k m}]$ & $\mathbf{d}_{\mathbf{s c}}[\%]$ & $\mathbf{S}_{\mathbf{c v}(\mathbf{m})}[\mathbf{k m}]$ & $\mathbf{S}_{\mathbf{c v}, \mathbf{e}(\mathbf{m})}[\mathbf{k m}]$ & $\mathbf{d}_{\mathbf{s c}}[\%]$ & $\mathbf{S}_{\mathbf{c v}(\mathbf{m})}[\mathbf{k m}]$ & $\mathbf{S}_{\mathrm{cv}, \mathbf{e}(\mathbf{m})}[\mathbf{k m}]$ & $\mathbf{d}_{\mathbf{s c}}[\%]$ \\
\hline $\mathbf{1}$ & 1.013 & 1.013 & $0 \%$ & - & - & - & - & - & - \\
\hline $\mathbf{2}$ & 1.050 & 870 & $-17 \%$ & - & - & - & 2.120 & 1.720 & $-19 \%$ \\
\hline $\mathbf{3}$ & - & - & - & 913 & 1.063 & $16 \%$ & 2.620 & 2.500 & $-5 \%$ \\
\hline $\mathbf{4}$ & - & - & - & 660 & 780 & $18 \%$ & 2.250 & 2.070 & $-8 \%$ \\
\hline $\mathbf{5}$ & - & - & - & 300 & 350 & $17 \%$ & 1.300 & 1.300 & $0 \%$ \\
\hline $\mathbf{6}$ & - & - & - & 677 & 773 & $14 \%$ & 2.042 & 2.082 & $2 \%$ \\
\hline $\mathbf{7}$ & - & - & - & 360 & 690 & $92 \%$ & 2.262 & 2.137 & $-6 \%$ \\
\hline $\mathbf{8}$ & 638 & 638 & $0 \%$ & - & - & - & 513 & 1.537 & $197 \%$ \\
\hline $\mathbf{9}$ & 450 & 390 & $-13 \%$ & - & - & - & 587 & 823 & $40 \%$ \\
\hline $\mathbf{1 0}$ & - & - & - & 473 & 968 & $105 \%$ & 1.120 & 1.760 & $57 \%$ \\
\hline $\mathbf{1 1}$ & - & - & - & 360 & 960 & $167 \%$ & 420 & 1.540 & $267 \%$ \\
\hline
\end{tabular}

Die Daten zum Laufweg $\mathrm{S}_{\mathrm{cv}(\mathrm{m})}$ aus den Referenzprojekten und $\mathrm{s}_{\mathrm{cv}, \mathrm{e}(\mathrm{m})}$ aus der Verschleißprognose in Tab. 24 können anhand der Werkzeugwechseldaten und Verschleißbilder aus dem Referenzprojekt interpretiert werden.

In den Homogenbereichen 1 und 3 - 6 wurden die Werkzeuge überwiegend aufgrund von Primär- und Sekundärverschleiß gewechselt. Ebenso die Schälmesser in HGB 7 und die Schneidrollen in den HGB 8 und 9. Hier können die Werte für den Laufweg $\mathrm{s}_{\mathrm{cv}(\mathrm{m})}$ und $\mathrm{s}_{\mathrm{cv}, \mathrm{e}(\mathrm{m})}$ direkt verglichen werden, um modellbedingte Abweichungen zu erfassen. Für Schneidrollen ergibt sich eine Abweichung des prognostizierten Laufweg $\mathrm{s}_{\mathrm{cv}, \mathrm{e}(\mathrm{m})}$ von den Werten für $\mathrm{s}_{\mathrm{cv}(\mathrm{m})}$ aus dem Referenzprojekten von $-13 \%$ bis $0 \%$. Für Stichel überschätzt das Prognosemodell den Laufweg $\mathrm{s}_{\mathrm{cv}(\mathrm{m})}$ aus den Referenzprojekten dagegen um $14-18 \%$. Die Abweichungen des Laufwegs $\mathrm{S}_{\mathrm{cv}, \mathrm{e}(\mathrm{m})}$ der Schälmesser von den Werten für $\mathrm{S}_{\mathrm{cv}(\mathrm{m})}$ aus den Referenzprojekten betragen $-8 \%$ bis $2 \%$.

In HGB 2 liegt der Laufweg $\mathrm{S}_{\mathrm{cv}, \mathrm{e}(\mathrm{m})}$ aus der Verschleißprognose bei Schneidrollen $17 \%$ und bei Schälmessern $19 \%$ unter dem Laufweg $\mathrm{S}_{\mathrm{cv}(\mathrm{m})}$ aus dem Referenzprojekt. Ursache hierfür ist die Überschreitung der maximal erreichbaren Vortriebsstrecke $L_{\max (\mathrm{m})}$ durch den Extremwert von $550 \mathrm{~m}$ für $\mathrm{L}_{d(\mathrm{~m})}$ (Abb. 94). Diese hatte im Vortrieb Verschleißschäden an den Abbauwerkzeugen zur Folge, die sich auch in der deutlichen Überschreitung des prognostizierten Laufwegs $\mathrm{s}_{\mathrm{cv}, \mathrm{e}(\mathrm{m})}$ durch den realen Laufweg $\mathrm{S}_{\mathrm{cv}(\mathrm{m})}$ wiederspiegeln.

In den HGB 8 bis 11 liegt der Laufweg $\mathrm{s}_{\mathrm{cv}, \mathrm{e}(\mathrm{m})}$ für Schälmesser aus der Verschleißprognose um 40 bis $267 \%$ höher als der Laufweg $\mathrm{s}_{\mathrm{cv}(\mathrm{m})}$ aus dem Referenzprojekt. Ursache für diese Abweichung sind Schäden an den Schälmessern im Vortrieb, die durch Steine und Blöcke in der Ortsbrust verursacht wurden. Diese bewirken Werkzeugwechsel deutlich vor Erreichen der Verschleißgrenze der Schälmesser. Das Prognosemodell beinhaltet in der vorliegenden Form keine Möglichkeit solche Auswirkungen von Schäden quantitativ zu ermitteln und zu berücksichtigen. Der Laufweg $\mathrm{s}_{\mathrm{cv}, \mathrm{e}(\mathrm{m})}$ aus dem Prognosemodell überschätzt deshalb in diesen Homogenbereichen den tatsächlichen Laufweg $\mathrm{S}_{\mathrm{cv}(\mathrm{m})}$ deutlich. Dieser Zusammenhang führt auch für die Stichel in den Homogenbereichen 7, 10 und 11 zu einer Überschätzung des Laufwegs $\mathrm{s}_{\mathrm{cv}(\mathrm{m})}$ durch das Prognosemodell um 92 bis 167\%. 
Innerhalb der vier weiteren RP 3, 6 und 9, die zur Validierung herangezogen wurden, weisen die Abweichungen zwischen $\mathrm{s}_{\mathrm{cv}(\mathrm{m})}$ und $\mathrm{s}_{\mathrm{cv}, \mathrm{e}(\mathrm{m})}$ ähnliche Tendenzen und Beträge auf (Tab. 25). Die modellbedingten Abweichungen für Schneidrollen liegen bei $0 \%$ bis $-5 \%$. Für Schälmesser konnte nur in einem Homogenbereiche eine modellbedingte Abweichung von -5\% ermittelt werden. Eine Ausnahme bilden die modellbedingten Abweichungen für Schälmesser in den HGB 2 bis 4 in RP 9 . Hier wurden Schälmesser mit sehr geringem Verschleißschutz eingesetzt. Dieser liegt vom Umfang her deutlich niedriger als die in Kap. 6.2.3.3 bzw. Tab. 22 angegeben Einsatzgrenzen des Prognosemodells aufgrund nicht quantifizierbarer Einflussfaktoren. Das Prognosemodell überschätzt den tatsächlichen Laufweg $\mathrm{S}_{\mathrm{cv}(\mathrm{m})}$ hier deshalb um 64 bis $84 \%$.

Tab. 25.: Abweichungen $d_{s c}[\%]$ (Gl. 139) des geschätzten Laufwegs $s_{c v, e(m)}[k m]$ aus dem Prognosemodell vom tatsächlichen Laufweg $S_{\mathrm{cv}(\mathrm{m})}[\mathrm{km}]$ in den RP 3, 6 und 9. Die Abweichungen können neben modellbedingten Einflüssen zum Teil auf Verschleißschäden und präventive Bestückungswechsel in den RP zurückgeführt werden.

\begin{tabular}{|c|c|c|c|c|c|c|}
\hline \multirow{3}{*}{ 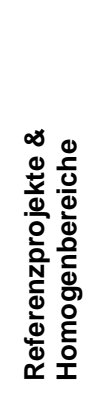 } & \multicolumn{2}{|c|}{ Schneidrollen } & \multirow{2}{*}{ 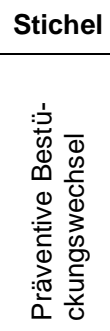 } & \multicolumn{3}{|c|}{ Schälmesser } \\
\hline & 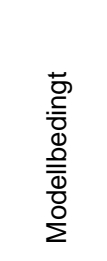 & 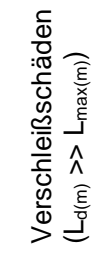 & & 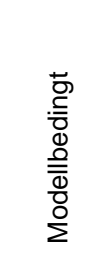 & 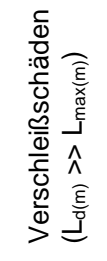 & 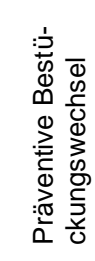 \\
\hline & $\mathbf{d}_{\mathrm{sc}}[\%]$ & $\mathbf{d}_{\mathrm{sc}}[\%]$ & $d_{s c}[\%]$ & $\mathbf{d}_{\mathrm{sc}}[\%]$ & $\mathbf{d}_{\mathrm{sc}}[\%]$ & $\mathbf{d}_{\mathrm{sc}}[\%]$ \\
\hline \multicolumn{7}{|l|}{ RP 3} \\
\hline HGB 1 & $0 \%$ & - & - & - & $-73 \%$ & - \\
\hline \multicolumn{7}{|l|}{ RP 6} \\
\hline HGB 1 & - & - & - & - & $-53 \%$ & - \\
\hline HGB 2 & - & - & - & - & - & $72 \%$ \\
\hline HGB 3 & - & - & - & - & - & $89 \%$ \\
\hline HGB 4 & - & - & - & - & - & $89 \%$ \\
\hline HGB 5 & - & - & - & - & - & $93 \%$ \\
\hline \multicolumn{7}{|l|}{ RP 9} \\
\hline HGB 1 & - & - & - & - & - & - \\
\hline HGB 2 & $-2 \%$ & - & - & $64 \%$ & - & - \\
\hline HGB 3 & $-5 \%$ & - & - & $84 \%$ & - & - \\
\hline HGB 4 & $-2 \%$ & - & - & $78 \%$ & - & - \\
\hline \multicolumn{7}{|l|}{ RP 10} \\
\hline HGB 1 & - & - & - & - & - & - \\
\hline HGB 2 & - & $-32 \%$ & - & - & - & - \\
\hline HGB 3 & - & - & - & - & - & - \\
\hline HGB 4 & - & - & $80 \%$ & $-5 \%$ & - & - \\
\hline
\end{tabular}

Daneben treten in den weiteren RP auch Abweichungen zwischen dem prognostizierten Laufweg $\mathrm{s}_{\mathrm{cv}, \mathrm{e}(\mathrm{m})}$ und dem tatsächlichen Laufweg $\mathrm{s}_{\mathrm{cv}(\mathrm{m})}$ aufgrund von Verschleißschäden auf. Das Prognosemodell unterschätzt den tatsächlichen Laufweg $\mathrm{S}_{\mathrm{cv}(\mathrm{m})}$ der Abbauwerkzeuge in den betroffenen Homogenbereichen deutlich. Hier wurden in den RP zum Teil Vortriebsstrecken $L_{d(\mathrm{~m})}$ angesetzt, die höher liegen, als die maximal erreichbaren Vortriebsstrecken $L_{\max (m)}$ aus dem Prognosemodell. 
Die Abweichungen betragen hier für Schneidrollen -32\% und für Schälmesser -53\%. Eine Ausnahme bildet die Abweichung von $-78 \%$ in HGB 1 in RP 3 . Hier wurden im Vortrieb im Vergleich zur maximal erreichbaren Vortriebsstrecke $L_{\max (m)}$ aus dem Prognosemodell relativ kurze Vortriebsstrecken $L_{d(m)}$ angesetzt (Abb. 94). Während der Vortriebsunterbrechungen wurden jedoch trotz erheblicher Überschreitung der Verschleißgrenzen der Schälmesser kaum Werkzeugwechsel durchgeführt, sodass die Abweichung hier auf eine mangelnde Anzahl an Werkzeugwechseln zurückzuführen ist.

Im Falle von präventiven Bestückungswechseln überschätzt das Prognosemodell den tatsächlichen Laufweg $\mathrm{s}_{\mathrm{cv}(\mathrm{m})}$ der Abbauwerkzeuge deutlich. In RP 10 wurden die Stichel in HGB 4 zum Teil mit relativ geringem Abrieb ausgebaut, um die Werkzeugbestückung des Schneidrades an wechselnde Bodenbedingungen in der Ortsbrust anzupassen. Die Überschätzung von $\mathrm{s}_{\mathrm{cv}(\mathrm{m})}$ durch das Prognosemodell liegt hier bei $80 \%$. In RP 6 wurde nach einem anfänglichen Verschleißschaden in HGB 1 ein strikter Werkzeugwechselplan eingeführt, der ein hohes Maß an Sicherheit gegenüber weiteren Verschleißschäden zum Ziel hatte. Folglich wurde eine hohe Anzahl an präventiven Schälmesserwechseln deutlich vor Erreichen der Verschleißgrenze durchgeführt. Das Prognosemodell überschätzt den tatsächlich erreichten Laufweg $\mathrm{s}_{\mathrm{cv}(\mathrm{m})}$ hier in HGB 2 bis 5 um 72 bis $93 \%$.

\subsubsection{Anzahl der Werkzeugwechsel}

\section{Anzahl der Werkzeugwechsel $\mathbf{n}_{\mathrm{c}(\mathrm{m})}$ bzw. $\mathbf{n}_{\mathrm{c}, \mathrm{e}(\mathrm{m})}$ je Vortriebsunterbrechung $\mathrm{I}_{(\mathrm{m})}$}

Zusätzlich zum Laufweg $\mathrm{s}_{\mathrm{cv}(\mathrm{m})}$ ist besonders eine zuverlässige Schätzung der Anzahl der notwendigen Werkzeugwechsel $\mathrm{n}_{c(\mathrm{~m})}$ in den Vortriebsunterbrechungen durch die prognostizierte Anzahl $\mathrm{n}_{c, e(m)}$ von hoher Bedeutung. Für die Vortriebsplanung und aus wirtschaftlicher Sicht ist dazu eine kumulative Betrachtung der Anzahl der Werkzeugwechsel $n_{c(m)}$ bzw. $n_{c, e(m)}$ in den Vortriebsunterbrechungen $I_{(m)}$ entlang der Tunnelachse sinnvoll. Diese ist in Abb. 95 für Schneidrollen und Stichel und in Abb. 96 für Schälmesser in RP 7 dargestellt. Die Darstellung für Schneidrollen und Stichel erfolgt hier in summierter Form, weil die beiden Werkzeugtypen im vorliegenden Fall in denselben Gehäusen auf dem Schneidrad befestigt wurden. Je Schneidspur kann also entweder eine Schneidrolle oder ein Stichel eingesetzt werden.

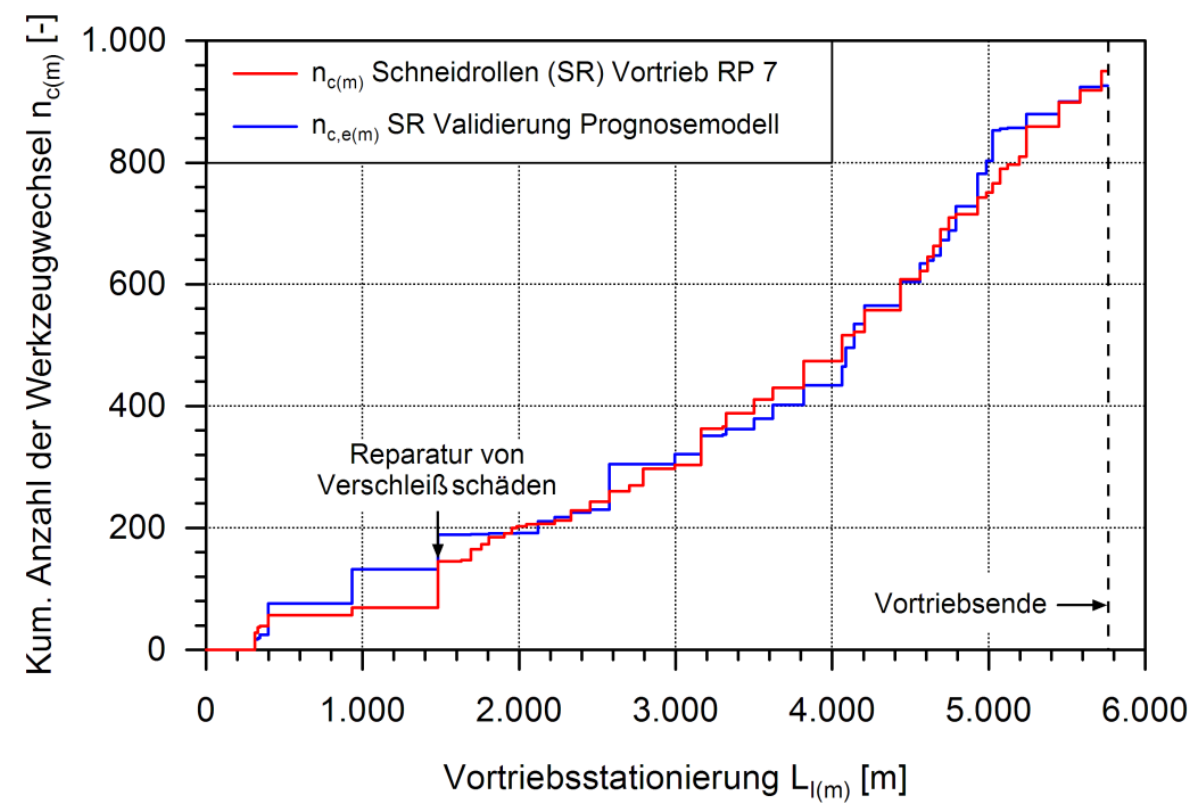

Abb. 95: Kumulierte Darstellung der Anzahl der Werkzeugwechsel $n_{c(m)}$ je Vortriebsunterbrechung $I_{(m)}$ über die Vortriebsstationierung $L_{1(m)}$ aus RP 7 (rot) gegenüber der geschätzten Anzahl an Werkzeugwechsel $n_{c, e(m)}$ aus dem Prognosemodell (blau) für Schneidrollen und Stichel. 


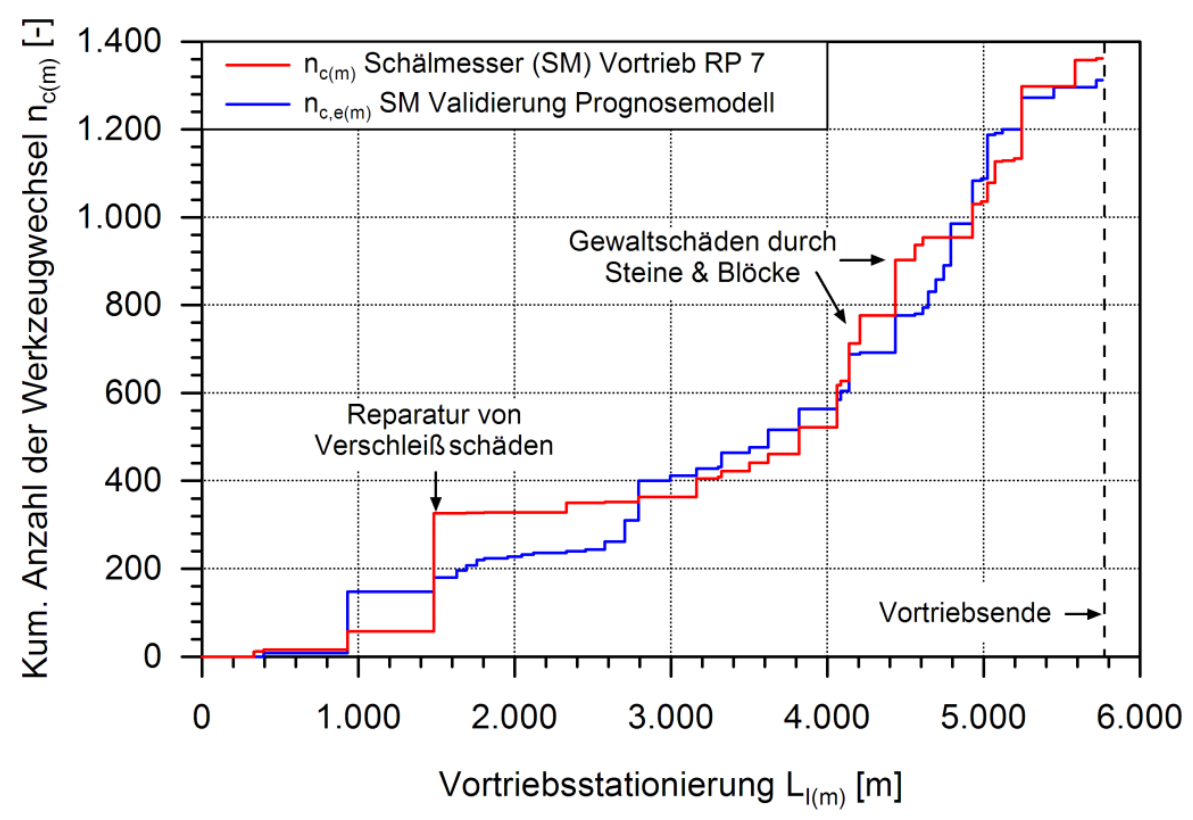

Abb. 96: Kumulierte Darstellung der Anzahl der Werkzeugwechsel $n_{c(m)}$ je Vortriebsunterbrechung $I_{(m)}$ über die Vortriebsstationierung $L_{1(m)}$ aus RP 7 (rot) gegenüber der geschätzten Anzahl an Werkzeugwechsel $n_{c, e(m)}$ aus dem Prognosemodell (blau) für Schälmesser.

Der kumulierte Verlauf der Anzahl der Werkzeugwechsel $\mathrm{n}_{\mathrm{c}(\mathrm{m})}$ und $\mathrm{n}_{\mathrm{c}, \mathrm{e}(\mathrm{m})}$ in den Vortriebsunterbrechungen $I_{(m)}$ entlang der Tunnelachse in Abb. 95 und Abb. 96 entspricht für alle drei Werkzeugtypen den Ergebnissen aus den bisherigen Analysen zu den Vortriebsstrecken $L_{d(m)}$ und $L_{d, e(m)}$ bzw. zu den

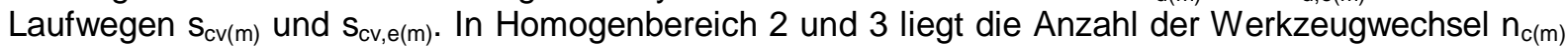
im Vortrieb zunächst deutlich niedriger als die prognostizierte Anzahl $n_{c, e(m)}$. Im Vortrieb wurde hier aufeinanderfolgend zwei sehr hohe Wert von $536 \mathrm{~m}$ und $550 \mathrm{~m}$ für die Vortriebsstrecke $L_{d(\mathrm{~m})}$ angesetzt (Abb. 94). Diese liegen in HGB 2 deutlich höher als die prognostizierte maximal erreichbare Vortriebsstrecke $L_{\max (m)}$ von $400 \mathrm{~m}$ und in HGB 3 etwas niedriger als $L_{\max (m)}$ mit $654 \mathrm{~m}$. Die Überschreitung der maximal erreichbaren Vortriebsstrecke $L_{\max (m)}$ in HGB 2 bewirkt einerseits die Abweichung des prognostizierten Laufwegs $\mathrm{s}_{\mathrm{cv}, \mathrm{e}(\mathrm{m})}$ vom tatsächlichen Laufwegs $\mathrm{s}_{\mathrm{cv}(\mathrm{m})}$ (Tab. 24). Andererseits entstehen daraus im Vortrieb Verschleißschäden an den Abbauwerkzeugen und zum Teil an den Werkzeughaltern, die bei einem vollständigen Wechsel der Werkzeugbestückung des Schneidrades in HGB 3 bei Stationierung $1.482 \mathrm{~m}$ behoben wurden. Die Anzahl der Werkzeugwechsel $\mathrm{n}_{\mathrm{c}(\mathrm{m})}$ steigt an dieser Stationierung folglich sprunghaft, für Schälmesser sogar über die prognostizierte Anzahl $\mathrm{n}_{\mathrm{c}, \mathrm{e}(\mathrm{m})}$ hinaus, an.

In den Homogenbereichen 8 bis $11(4.062-5.760 \mathrm{~m})$ wurden im Vortrieb zum Teil Schäden an den Abbauwerkzeugen aufgrund von Steinen und Blöcken in der Ortsbrust festgestellt. Der Laufweg $\mathrm{s}_{\mathrm{cv}(\mathrm{m})}$ liegt deshalb deutlich niedriger als der Laufweg $\mathrm{s}_{\mathrm{cv}, \mathrm{e}(\mathrm{m})}$ aus der Verschleißprognose (Tab. 24). Diese Abweichung pflanzt sich jedoch nur in den Vortriebsunterbrechungen im Bereich von $4.062-4.744 \mathrm{~m}$ deutlich in die Anzahl $\mathrm{n}_{\mathrm{c}(\mathrm{m})}$ der Werkzeugwechsel von Schälmessern fort. Hier werden aufgrund von Gewaltschäden im Vortrieb in sieben aufeinanderfolgenden Vortriebsunterbrechungen $88 \%$ mehr Schälmesser gewechselt $\left(\mathrm{n}_{\mathrm{c}(\mathrm{m})}\right)$, als durch das Prognosemodell geschätzt $\left(\mathrm{n}_{\mathrm{c}, \mathrm{e}(\mathrm{m})}\right)$. In den verbleibenden Strecken in den HGB 8 bis 11, sowie bei Schneidrollen und Sticheln erhöht sich die Anzahl der Werkzeugwechsel $n_{c(m)}$ im Vortrieb aufgrund von Gewaltschäden nur geringfügig. Die Abweichungen der prognostizierten Anzahl der Werkzeugwechsel $\mathrm{n}_{\mathrm{c}, \mathrm{e}(\mathrm{m})}$ von der tatsächlichen Anzahl der Werkzeugwechsel $n_{c(m)}$ liegen hier so niedrig, dass sie die im Folgenden beschriebenen modellbedingten Abweichungen nicht überschreiten.

In den übrigen Vortriebsbereichen ohne Einflüsse durch Verschleiß- und Gewaltschäden liegen die modellbedingten Abweichungen der prognostizierten Anzahl der Werkzeugwechsel $n_{c, e(m)}$ von der tatsächlichen Anzahl an Werkzeugwechseln $\mathrm{n}_{\mathrm{c}(\mathrm{m})}$ je Vortriebsunterbrechung $\mathrm{I}_{(\mathrm{m})}$ für Schneidrollen und Stichel bei $-10 \%$ bis $13 \%$ und bei Schälmessern bei $-17 \%$ bis $12 \%$. Diese Abweichungen stehen in gutem Verhältnis zu den modellbedingten Abweichungen von $-13 \%$ bis $18 \%$ zwischen den Laufwegen $\mathrm{s}_{\mathrm{cv}, \mathrm{e}(\mathrm{m})}$ und $\mathrm{s}_{\mathrm{cv}(\mathrm{m})}$. Diese pflanzen sich schlüssig in die Anzahl der Werkzeugwechsel $\mathrm{n}_{\mathrm{c}, \mathrm{e}(\mathrm{m})}$ fort. 
In den vier weiteren RP 3, 6, 9 und 10 ergeben sich die in Tab. 26 zusammengefassten Abweichungen $d_{n c}[\%]$ zwischen der geschätzten Anzahl an Werkzeugwechseln $n_{c, e(m)}$ aus dem Prognosemodell und der tatsächlichen Anzahl an Werkzeugwechseln $\mathrm{n}_{\mathrm{c}(\mathrm{m})}$ aus den Referenzprojekten. Der Betrag der Abweichungen $d_{n c}$ [\%] wurde dazu analog zu Gl. 139 (Kap. 6.6.2.2) aus der Anzahl der Werkzeugwechsel $\mathrm{n}_{\mathrm{c}(\mathrm{m})}$ und $\mathrm{n}_{\mathrm{c}, \mathrm{e}(\mathrm{m})}$ berechnet.

Tab. 26: Abweichungen $d_{n c}[\%]$ der geschätzten Anzahl an Werkzeugwechseln $n_{c, e(m)}[-]$ aus dem Prognosemodell von der tatsächlichen Anzahl an Werkzeugwechseln $\mathrm{n}_{\mathrm{c}(\mathrm{m})}[-]$ in den Referenzprojekten. Die Abweichungen können neben modellbedingten Einflüssen zum Teil auf Verschleißschäden und präventive Bestückungswechsel in den Referenzprojekten zurückgeführt werden.

\begin{tabular}{|c|c|c|c|c|c|c|c|}
\hline \multirow{3}{*}{ 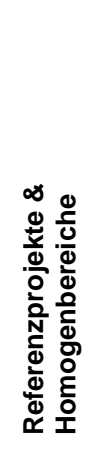 } & \multicolumn{3}{|c|}{ Schneidrollen } & \multirow[b]{2}{*}{ 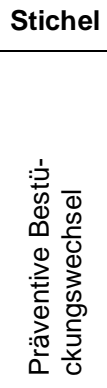 } & \multicolumn{3}{|c|}{ Schälmesser } \\
\hline & 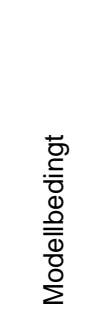 & 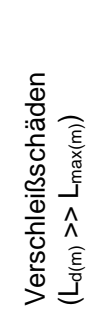 & 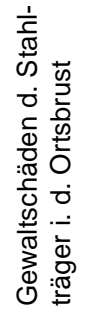 & & 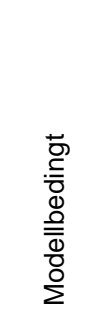 & 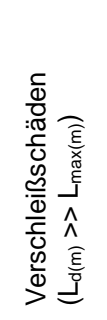 & 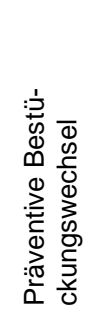 \\
\hline & $\mathbf{d}_{\mathrm{nc}}[\%]$ & $d_{n c}[\%]$ & $\mathbf{d}_{\mathrm{nc}}$ [\%] & $\mathbf{d}_{\mathrm{nc}}$ [\%] & $\mathbf{d}_{\mathrm{nc}}[\%]$ & $\mathbf{d}_{\mathrm{nc}}[\%]$ & $\mathrm{d}_{\mathrm{nc}}[\%]$ \\
\hline \multicolumn{8}{|l|}{ RP 3} \\
\hline HGB 1 & $9 \%$ & - & $75 \%$ & - & - & $220 \%$ & - \\
\hline \multicolumn{8}{|l|}{ RP 6} \\
\hline HGB 1 & - & - & - & - & - & $91 \%$ & - \\
\hline HGB 2 & - & - & - & - & - & - & $-9 \%$ \\
\hline HGB 3 & - & - & - & - & - & - & $-76 \%$ \\
\hline HGB 4 & - & - & - & - & - & - & $-87 \%$ \\
\hline HGB 5 & - & - & - & - & - & - & $-43 \%$ \\
\hline \multicolumn{8}{|l|}{ RP 9} \\
\hline HGB 1 & - & - & - & - & - & - & - \\
\hline HGB 2 & $2 \%$ & - & - & - & $-100 \%$ & - & - \\
\hline HGB 3 & $5 \%$ & - & - & - & $-85 \%$ & - & - \\
\hline HGB 4 & $6 \%$ & - & - & - & $-50 \%$ & - & - \\
\hline \multicolumn{8}{|l|}{ RP 10} \\
\hline HGB 1 & - & - & - & - & - & - & - \\
\hline HGB 2 & - & $16 \%$ & - & - & - & - & - \\
\hline HGB 3 & - & - & - & - & - & - & - \\
\hline HGB 4 & - & - & - & $-88 \%$ & $-14 \%$ & - & - \\
\hline
\end{tabular}

Die modellbedingten Abweichungen zwischen der geschätzten Anzahl an Werkzeugwechseln $\mathrm{n}_{\mathrm{c}, \mathrm{e}(\mathrm{m})}$ aus dem Prognosemodell und der tatsächlichen Anzahl an Werkzeugwechseln $n_{c(m)}$ für Schneidrollen liegen bei 2 bis $9 \%$. Diese Abweichungen stehen in gutem Verhältnis zu den ermittelten Abweichungen zwischen den Laufwegen $\mathrm{s}_{\mathrm{cv}, \mathrm{e}(\mathrm{m})}$ und $\mathrm{s}_{\mathrm{cv}(\mathrm{m})}$ (Tab. 25). In RP 3 wurde hierzu in HGB 1 ein nahezu vollständiger Bestückungswechsel der Schneidrollen (75\%) aufgrund von Gewaltschäden durch einen Stahlträger in der Ortsbrust herausgerechnet und in Tab. 26 separat ausgewiesen. Für Schälmesser liegt die modellbedingte Abweichung in RP 10 in HGB 4 bei $-14 \%$. 
Die modellbedingten Abweichungen zwischen dem geschätzten Laufweg $\mathrm{s}_{\mathrm{cv}, \mathrm{e}(\mathrm{m})}$ und dem tatsächlichen Laufweg $\mathrm{S}_{\mathrm{cv}(\mathrm{m})}$ für Schälmesser in den HGB 2 - 4 in RP 9 aufgrund des sehr geringen Verschleißschutzes der Schälmesser (Tab. 25) setzen sich konsequent in die Anzahl der Werkzeugwechsel fort. Das Prognosemodell unterschätzt die tatsächliche Anzahl an Werkzeugwechseln $\mathrm{n}_{\mathrm{c}(\mathrm{m})}$ in diesen Homogenbereichen um $-50 \%$ bis $-100 \%$.

Auch die Abweichungen zwischen dem prognostizierten Laufweg $\mathrm{s}_{\mathrm{cv}, \mathrm{e}(\mathrm{m})}$ und dem tatsächlichen Laufweg $\mathrm{S}_{\mathrm{cv}(\mathrm{m})}$ aufgrund von Verschleißschäden pflanzen sich schlüssig in die Anzahl der Werkzeugwechsel fort. In RP 3, HGB 1 und RP 6, HGB 1 überschätzt das Prognosemodell die Anzahl der Werkzeugwechsel $\mathrm{n}_{\mathrm{c}(\mathrm{m})}$ aufgrund der Überschreitung der Verschleißgrenzen in den Referenzprojekten für Schälmesser um 91 bis $220 \%$. Die Anzahl der Werkzeugwechsel $\mathrm{n}_{c(\mathrm{~m})}$ für Schneidrollen in RP 10, HGB 2 wird durch das Prognosemodell um 16\% überschätzt.

Im Falle von präventiven Bestückungswechseln der Abbauwerkzeuge im Vortrieb unterschätzt das Prognosemodell die tatsächliche Anzahl an Werkzeugwechseln $\mathrm{n}_{\mathrm{c}(\mathrm{m})}$ als Konsequenz aus der entsprechenden Überschätzung des Laufwegs $\mathrm{S}_{\mathrm{cv}(\mathrm{m})}$ in Tab. 25 (Kap. 6.6.2.2). Der Betrag der Abweichungen $\mathrm{d}_{\mathrm{nc}}$ [\%] aufgrund des Wechsel der Abbauwerkzeuge vor Erreichen der Verschleißgrenzen erreicht in RP 6 HGB 2 - 4 für Schälmesser Werte von -9\% bis -87. Für Stichel liegt der Betrag in RP 10, HGB 4 bei $-88 \%$.

\section{Anzahl der Werkzeugwechsel $\mathbf{n}_{\mathrm{c}(\mathrm{TA})}$ über die Tunnelachse (TA):}

In Summe über die Tunnelachse unterschätzt in Referenzprojekt 7 die prognostizierte Anzahl der Werkzeugwechsel $n_{c, e(T A)}$ die tatsächliche Anzahl der Werkzeugwechsel $n_{c(T A)}$ im Vortrieb für Schälmesser um $3 \%$ und für Schneidrollen um $4 \%$.

In den übrigen RP 3, 6, 9 und 10 liegt diese Abweichung der prognostizierten Werkzeugwechsel über die Tunnelachse $n_{c, e(T A)}$ von der tatsächlichen Anzahl an Werkzeugwechseln $n_{c(T A)} z u m$ Teil deutlich höher. Die Abweichungen können hier einerseits auf eine hohe Anzahl an schadensbedingten Werkzeugwechseln zurückgeführt werden, die durch das Prognosemodell nicht erfasst werden. Andererseits wurden in den Referenzprojekten zum Teil rigide Pläne für präventive Werkzeugwechsel oder vollständige Wechsel des Schneidradbesatzes ausgeführt, um die Abbauwerkzeuge an stark variierende geotechnische Bedingungen anzupassen (Tab. 27). Diese wurden bei der Prognose ebenfalls nicht berücksichtigt. Beide Ursachen wirken sich aufgrund der zum Teil deutlich kürzeren Tunnellänge $\mathrm{L}_{T A}[\mathrm{~m}]$ stärker als Abweichungen zwischen der Anzahl der Werkzeugwechsel $\mathrm{n}_{\mathrm{c}(\mathrm{m})}$ und $\mathrm{n}_{\mathrm{c}, \mathrm{e}(\mathrm{m})}$ aus.

Tab. 27: Vergleich der tatsächlichen Anzahl $n_{c(T A)}$ an Werkzeugwechseln aus den Referenzprojekten (RP) und geschätzte Anzahl $n_{c, e(T A)}$ der Werkzeugwechsel über den gesamten Vortrieb bzw. Länge der Tunnelachse (TA). Die Abweichungen sind nach Schneidrollen (SR), Sticheln (ST) und Schälmessern (SM) untergliedert. Die Abweichungen $d_{n c}[\%]$ spiegeln die unterschiedlichen Einflüsse aus Verschleißschäden, Gewaltschäden und präventiven Bestückungswechseln auf die Anzahl der Werkzeugwechsel wieder.

\begin{tabular}{|c|c|c|c|c|c|c|}
\hline \multirow{2}{*}{$\mathbf{R P}$} & \multicolumn{2}{|c|}{$\mathbf{n}_{\mathbf{c}(\mathrm{TA})}[-]$} & \multicolumn{2}{c|}{$\mathbf{n}_{\mathrm{c}, \mathrm{e} \text { (TA) }}[-]$} & \multicolumn{2}{c|}{$\mathbf{d}_{\mathrm{nc}}[\%]$} \\
\cline { 2 - 7 } & $\mathbf{S R} \& \mathbf{S T}$ & $\mathbf{S M}$ & $\mathbf{S R} \& \mathbf{S T}$ & $\mathbf{S M}$ & $\mathbf{S R} \& \mathbf{S T}$ & $\mathbf{S M}$ \\
\hline $\mathbf{3}$ & 80 & 114 & 53 & 516 & $-51 \%$ & $353 \%$ \\
\hline $\mathbf{6}$ & - & 190 & - & 142 & - & $-25 \%$ \\
\hline $\mathbf{9}$ & 44 & 143 & 46 & 88 & $4 \%$ & $-38 \%$ \\
\hline $\mathbf{1 0}$ & 128 & 149 & 148 & 136 & $14 \%$ & $-9 \%$ \\
\hline
\end{tabular}

In RP 3 wirken sich die Gewaltschäden an den Schneidrollen aufgrund eines Stahlträgers in der Ortsbrust und die Verschleißschäden an den Schälmessern (Tab. 26) auch auf die Abweichung zwischen der Anzahl der Werkzeugwechseln über die Tunnelachse $\mathrm{n}_{\mathrm{c}(\mathrm{TA})}$ bzw. $\mathrm{n}_{\mathrm{c}, \mathrm{e}(\mathrm{TA})}$ aus. Das Prognosemodell unterschätzt hier die tatsächliche Anzahl an Werkzeugwechseln für Schneidrollen aufgrund des Gewaltschadens um $-51 \%$. Die Anzahl der Werkzeugwechsel für Schälmesser wird dagegen aufgrund der zugelassenen Verschleißschäden um 353\% überschätzt (Tab. 27). 
In RP 6 kompensieren sich die Auswirkungen des Verschleißschadens zum Teil mit der erhöhten Anzahl an präventiven Werkzeugwechseln aufgrund eines rigiden Werkzeugwechselplans. Die Abweichung der Anzahl der Werkzeugwechsel über die Tunnelachse $\mathrm{n}_{\mathrm{c}(\mathrm{TA})}$ wird deshalb durch die Anzahl der Werkzeugwechsel $\mathrm{n}_{\mathrm{c}, \mathrm{e}(\mathrm{TA})}$ aus dem Prognosemodell nur um -25\% unterschätzt (Tab. 27). Dieser Betrag ist aufgrund der Kompensation der unterschiedlichen Auswirkungen deutlich geringer als die Beträge der Abweichungen in den einzelnen HGB (Tab. 26).

In RP 9 pflanzen sich die modellbedingten Abweichungen bei Schneidrollen, die bereits bei den Laufwegen $s_{\mathrm{cv}(\mathrm{m})}$ und $\mathrm{s}_{\mathrm{cv}, \mathrm{e}(\mathrm{m})}$ (Kap. 6.6.2.2) und bei der Anzahl der Werkzeugwechsel $n_{\mathrm{c}(\mathrm{m})}$ bzw. $n_{\mathrm{c}, \mathrm{e}(\mathrm{m})}$ in den einzelnen Vortriebsunterbrechungen (Tab. 26) festgestellt wurden, schlüssig in die Anzahl der Werkzeugwechsel über die Tunnelachse $n_{c(T A)}$ bzw. $n_{c, e(T A)}$ fort. Die Anzahl der Werkzeugwechsel $\mathrm{n}_{\mathrm{c}, \mathrm{e}(\mathrm{TA})}$ aus dem Prognosemodell überschätzt die tatsächliche Anzahl an Werkzeugwechseln hier um 4\%. Der geringe Verschleißschutz der Schälmesser in RP 9 wirkt sich ebenfalls auf die Anzahl der Werkzeugwechsel über die Tunnelachse aus. Die geschätzte Anzahl der Werkzeugwechseln $\mathrm{n}_{\mathrm{c}, \mathrm{e}(\mathrm{TA})}$ liegt hier aufgrund der impliziten Annahme eines höherwertigen Verschleißschutzes im Prognosemodell um -38\% niedriger, als die tatsächliche Anzahl der Werkzeugwechsel $\mathrm{n}_{\mathrm{c}(\mathrm{TA})}$ über die Tunnelachse.

Analog zu den Schälmessen in RP 6 findet auch bei Schneidrollen und Sticheln in RP 10 eine teilweise Kompensation zwischen Verschleißschäden und präventiven Bestückungswechseln statt. Die geschätzte Anzahl der Werkzeugwechsel $\mathrm{n}_{\mathrm{c}, \mathrm{e}(\mathrm{TA})}$ überschätzt hier die tatsächliche Anzahl an Werkzeugwechseln $n_{c(T A)}$ über die Tunnelachse um 14\%. Die modellbedingten Abweichungen bei Schälmessern bei den Laufwegen $\mathrm{s}_{\mathrm{cv}(\mathrm{m})}$ und $\mathrm{s}_{\mathrm{cv}, \mathrm{e}(\mathrm{m})}$ (Kap. 6.6.2.2) und bei der Anzahl der Werkzeugwechsel $\mathrm{n}_{\mathrm{c}(\mathrm{m})} \mathrm{bzw}$. $\mathrm{n}_{c, \mathrm{e}(\mathrm{m})}$ in den einzelnen Vortriebsunterbrechungen (Tab. 26) pflanzen sich auch hier schlüssig in die Anzahl der Werkzeugwechsel über die Tunnelachse $n_{c(T A)}$ bzw. $n_{c, e(T A)}$ fort. Das Prognosemodell unterschätzt hier die tatsächliche Anzahl der Werkzeugwechsel $n_{c(T A)}$ über die Tunnelachse um $-9 \%$.

\section{Anzahl der Werkzeugwechsel $\mathbf{n}_{\mathrm{c}(\mathrm{s})}$ je Schneidspur:}

Aus technischer Sicht spielt zusätzlich die Verteilung der Werkzeugwechsel über die einzelnen Schneidspuren eine hohe Rolle. Diese sind aus Referenzprojekt 7 für Schneidrollen und Stichel in Abb. 97 sowie für Schälmesser in Abb. 98 dargestellt.

Die Verteilung der Werkzeugwechsel je Schneidspur für Stichel und Schneidrollen (Abb. 97) weist über alle Schneidspuren eine hohe Ähnlichkeit zwischen der prognostizierten Anzahl $n_{c, e(s)}$ und der tatsächlichen Anzahl an Werkzeugwechseln $\mathrm{n}_{\mathrm{c}(\mathrm{s})}$ je Schneidspur auf.

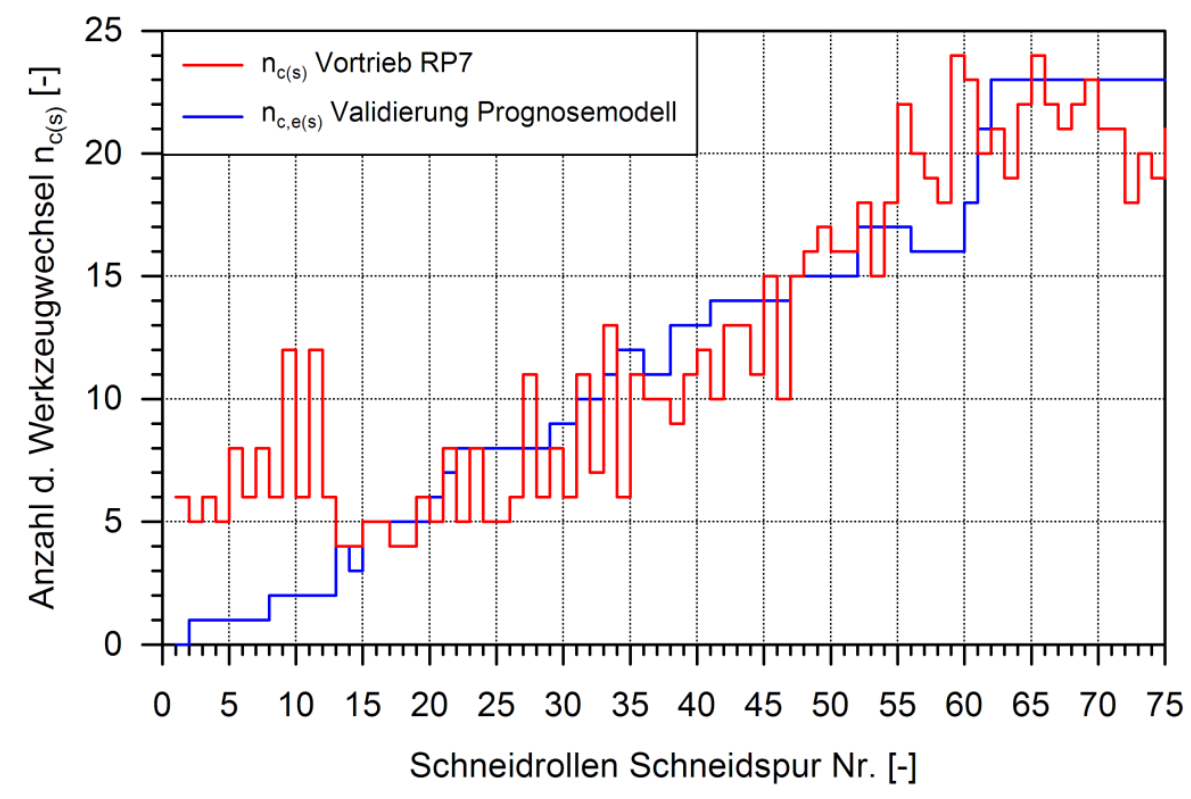

Abb. 97: Darstellung der Anzahl der Werkzeugwechsel $\mathrm{n}_{\mathrm{c}(\mathrm{s})}$ je Schneidspur aus RP 7 (rot) gegenüber der geschätzten Anzahl an Werkzeugwechsel $\mathrm{n}_{\mathrm{c}, \mathrm{e}(\mathrm{m})}$ aus dem Prognosemodell (blau) für Schälmesser. 
Die auffälligste Abweichung ist auf den Schneidspuren 1 bis 12 zu erkennen. Hier liegt die tatsächliche Anzahl der Werkzeugwechsel $n_{c(s)}$ deutlich höher als die Anzahl der Werkzeugwechsel $n_{c, e(s)}$ in der Verschleißprognose. Ursache hierfür sind Verklebungen im Zentrum des Schneidrads, die im Vortrieb bei bindigen Böden in der Ortsbrust auftraten. Die Verklebungen führten zum Blockieren der Schneidrollen im Zentrum und einseitigem Abrieb an den Schneidringen, sodass diese Schneidrollen vorzeitig ausgetauscht werden mussten. Dieser Schadensmechanismus wird vom Prognosemodell nicht erfasst.

Auf den übrigen Schneidspuren liegen die Abweichungen zwischen der prognostizierten Anzahl der Werkzeugwechsel und der tatsächlichen Anzahl an Werkzeugwechseln häufig innerhalb eines $\mathrm{Be}$ reichs von $+/-25 \%$. Auf einzelnen Schneidspuren ergeben sich jedoch Abweichungen von $-33 \%$ bis $100 \%$. Diese können mehrere mögliche Ursachen haben. Einerseits ist denkbar, dass diese Abweichungen durch besonders günstigen bzw. ungünstigen Materialfluss in einigen Bereichen des Schneidrades entstehen. Dieser Faktor konnte im Rahmen der Datenanalyse zum Prognosemodell nicht quantifiziert werden. Andererseits ist auch eine Beeinflussung benachbarter Spuren denkbar, die zu einer Erhöhung bzw. Verringerung der Verschleißrate $c_{v}$ auf einzelnen Spuren führt. Zusätzlich sind nicht alle Schneidspuren für die Inspektion und den Wechsel der Abbauwerkzeuge gleichermaßen zugänglich. So ist denkbar, dass einzelne Spuren im Vortrieb häufiger bzw. weniger häufig kontrolliert werden und so auch mehr bzw. weniger präventive Werkzeugwechsel durchgeführt werden. Das Prognosemodell führt diesen Faktoren gegenüber zu einer theoretischen Glättung der Anzahl der Werkzeugwechsel über die Schneidspuren.

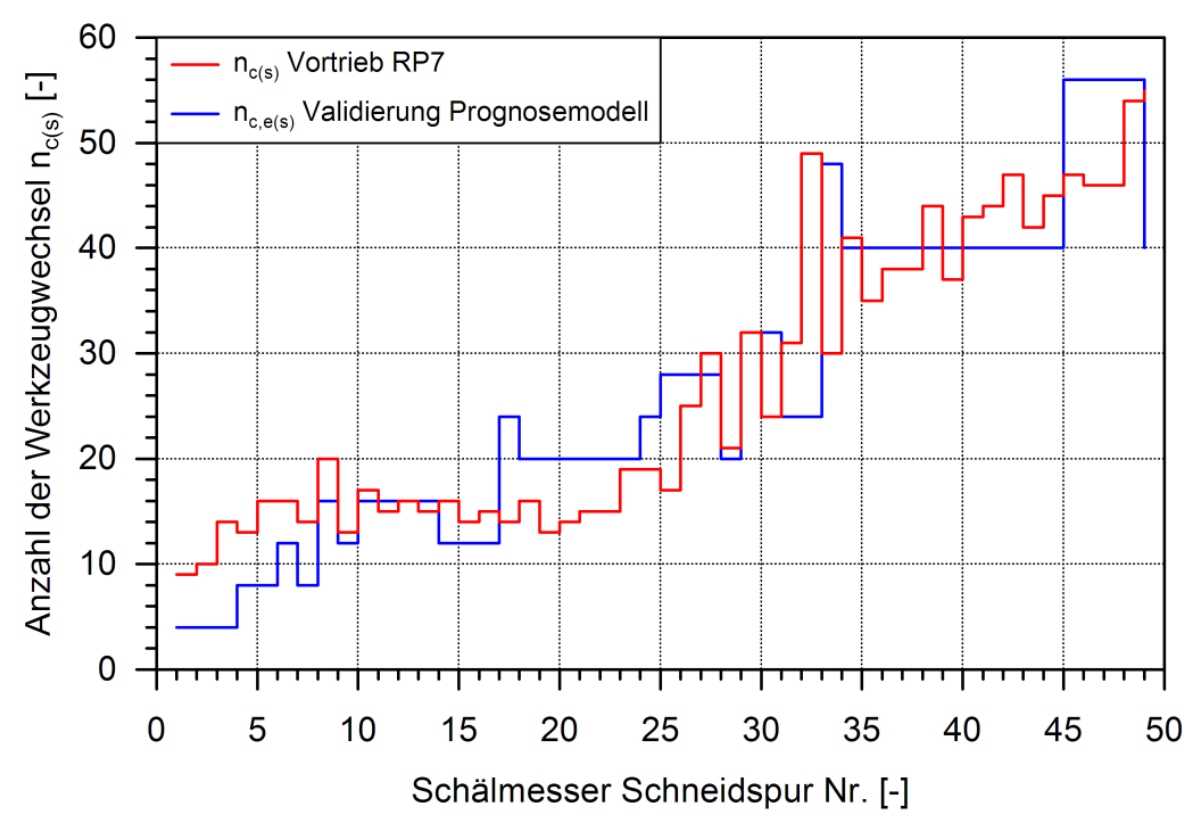

Abb. 98: Darstellung der Anzahl der Werkzeugwechsel $\mathrm{n}_{\mathrm{c}(\mathrm{s})}$ je Schneidspur aus RP 7 (rot) gegenüber der geschätzten Anzahl an Werkzeugwechsel $\mathrm{n}_{\mathrm{c}, \mathrm{e}(\mathrm{m})}$ aus dem Prognosemodell (blau) für Schälmesser.

Die Verteilung der Werkzeugwechsel je Schneidspur für Schälmesser (Abb. 98) weist über alle Schneidspuren hinweg ebenfalls eine hohe Ähnlichkeit zwischen der prognostizierten Anzahl $n_{c, e(s)}$ und der tatsächlichen Anzahl an Werkzeugwechseln $\mathrm{n}_{\mathrm{c}(\mathrm{s})}$ auf.

Die Abweichungen zwischen der prognostizierten Anzahl der Werkzeugwechsel $n_{c, e(s)}$ und der tatsächlichen Anzahl an Werkzeugwechseln $\mathrm{n}_{\mathrm{c}(\mathrm{s})}$ liegen hier über alle Schneidspuren hinweg häufig innerhalb eines Bereichs von $+/-30 \%$. Auf einzelnen Schneidspuren ergeben sich jedoch Abweichungen von $71 \%$ bis $71 \%$. Diese basieren auf den gleichen möglichen Ursachen wie die spurbezogenen Abweichungen bei Schneidrollen und Sticheln. Das Prognosemodell führt diesen Faktoren gegenüber wiederum zu einer theoretischen Glättung der Anzahl der Werkzeugwechsel über die Schneidspuren. 
In den übrigen RP 3, 6, 9 und 10 liegen die absoluten Abweichungen zwischen der geschätzten Anzahl an Werkzeugwechseln $n_{c, e(s)}$ und der tatsächlichen Anzahl an Werkzeugwechseln $n_{c(s)}$ je Schneidspur zum Teil noch wesentlich höher als in RP 7. Zu den bereits bei RP 7 beschriebenen möglichen Ursachen für die Abweichungen kommen hier noch die beschriebenen Verschleißschäden, Gewaltschäden und präventive Bestückungswechsel im Vortrieb als Einflussfaktoren hinzu. In Tab. 28 sind deshalb die mittleren Abweichungen über alle Schneidspuren für Schneidrollen, Stichel und Schälmesser je Referenzprojekt zusammengefasst. Diese stimmen gut mit den bisherigen Ergebnissen zu den Laufwegen $\mathrm{s}_{\mathrm{cv}(\mathrm{m})}$ und $\mathrm{s}_{\mathrm{cv}, \mathrm{e}(\mathrm{m})}$ und der Anzahl der Werkzeugwechsel $\mathrm{n}_{\mathrm{c}(\mathrm{m})}$ bzw. $\mathrm{n}_{\mathrm{c}, \mathrm{e}(\mathrm{m})}$ je Vortriebsunterbrechung und der Anzahl der Werkzeugwechsel $n_{c(T A)}$ bzw. $n_{c, e(T A)}$ über die Tunnelachse überein.

Tab. 28: Mittlere Abweichung $d_{n c}[\%]$ der geschätzten Anzahl der Werkzeugwechsel $n_{c, e(s)}$ je Schneidspur aus dem Prognosemodell von der tatsächlichen Anzahl an Werkzeugwechseln $\mathrm{n}_{\mathrm{c}(\mathrm{s})}$ je Schneidspur in den Referenzprojekten (RP). Die Daten sind nach Schneidrollen (SR), Sticheln (ST) und Schälmessern (SM) untergliedert.

\begin{tabular}{|c|c|c|}
\hline \multirow{2}{*}{ RP } & \multicolumn{2}{|c|}{$\mathbf{d}_{\mathrm{nc}}[\%]$} \\
\cline { 2 - 3 } & SR \& ST & SM \\
\hline $\mathbf{3}$ & $-8 \%$ & $536 \%$ \\
\hline $\mathbf{6}$ & - & $-9 \%$ \\
\hline $\mathbf{9}$ & $8 \%$ & $-24 \%$ \\
\hline $\mathbf{1 0}$ & $14 \%$ & $-4 \%$ \\
\hline
\end{tabular}

In RP 3 wird die Anzahl der Werkzeugwechsel je Schneidspur $n_{c(s)}$ für Schneidrollen durch die Anzahl der Werkzeugwechsel $\mathrm{n}_{\mathrm{c}, \mathrm{e}(\mathrm{s})}$ aus dem Prognosemodell aufgrund des Gewaltschadens im Vortrieb um durchschnittlich $-8 \%$ unterschätzt. Die Anzahl der Werkzeugwechsel je Schneidspur $n_{c(s)}$ für Schälmesser wird durch die Anzahl der Werkzeugwechsel $\mathrm{n}_{\mathrm{c}, \mathrm{e}(\mathrm{s})}$ aus dem Prognosemodell aufgrund der Verschleißschäden im Vortrieb um durchschnittlich 536\% überschätzt. Die Größenordnung der mittleren Abweichungen $d_{n c}[\%]$ steht hier in gutem Verhältnis zu den Abweichungen in der Anzahl der Werkzeugwechsel $\mathrm{n}_{\mathrm{c}(\mathrm{TA})}$ bzw. $\mathrm{n}_{\mathrm{c}, \mathrm{e}(\mathrm{TA})}$ über die Tunnelachse (Tab. 27).

In RP 6 ist analog zur Anzahl der Werkzeugwechsel $n_{c(T A)}$ bzW. $n_{c, e(T A)}$ auch in der Anzahl der Werkzeugwechsel je Schneidspur $\mathrm{n}_{\mathrm{c}(\mathrm{s})}$ bzw. $\mathrm{n}_{\mathrm{c}, \mathrm{e}(\mathrm{s})}$ eine Kompensation zwischen den Einflüssen aus Verschleißschäden und präventiven Werkzeugwechseln festzustellen. Die geschätzte Anzahl der Werkzeugwechsel je Schneidspur $\mathrm{n}_{\mathrm{c}, \mathrm{e}(\mathrm{s})}$ aus dem Prognosemodell unterschätzt die Anzahl der Werkzeugwechsel je Schneidspur $\mathrm{n}_{\mathrm{c}(\mathrm{s})}$ hier um -9\% (Tab. 28).

In RP 9 wirken sich die bereits beschriebenen modellbedingten Abweichungen bei Schneidrollen durchgängig auf die Anzahl der Werkzeugwechsel je Schneidspur $\mathrm{n}_{\mathrm{c}(\mathrm{s})}$ bzw. $\mathrm{n}_{\mathrm{c}, \mathrm{e}(\mathrm{s})}$ aus. Die Anzahl der Werkzeugwechsel $\mathrm{n}_{\mathrm{c}, \mathrm{e}(\mathrm{s})}$ aus dem Prognosemodell überschätzt die tatsächliche Anzahl an Werkzeugwechseln $\mathrm{n}_{\mathrm{c}(\mathrm{s})}$ je Schneidspur um 8\%. Der Einfluss des geringen Verschleißschutzes der Schälmesser setzt sich auch in der Anzahl der Werkzeugwechsel je Schneidspur fort. Die geschätzte Anzahl der Werkzeugwechsel $\mathrm{n}_{\mathrm{c}, \mathrm{e}(\mathrm{s})}$ liegt hier im Prognosemodell durchschnittlich um -24\% niedriger als die tatsächliche Anzahl der Werkzeugwechsel $\mathrm{n}_{\mathrm{c}(\mathrm{s})}$ je Schneidspur.

In RP 10 setzt sich die Kompensation zwischen Verschleißschäden und präventiven Bestückungswechseln bei Schneidrollen und Sticheln durchgängig in die Anzahl der Werkzeugwechsel je Schneidspur $n_{c(s)}$ bzw. $n_{c, e(s)}$ fort. Die geschätzte Anzahl der Werkzeugwechsel $n_{c, e(s)}$ überschätzt hier die tatsächliche Anzahl an Werkzeugwechseln $\mathrm{n}_{\mathrm{c}(\mathrm{s})}$ je Schneidspur durchschnittlich um 14\% (Tab. 28). Die bereits beschriebenen modellbedingten Abweichungen bei Schälmessern setzen sich ebenfalls schlüssig in die Anzahl der Werkzeugwechsel je Schneidspur $n_{c(s)}$ bzw. $n_{c, e(s)}$ fort. Das Prognosemodell unterschätzt hier die Anzahl der Werkzeugwechsel $n_{c(s)}$ über die Tunnelachse um $-4 \%$. 


\subsubsection{Prognostische Validierung}

Aufgrund des eingeschränkten Zeitrahmens und mangelndem Zugang zu geeigneten Projekten konnte im Rahmen der vorliegenden Arbeit keine Validierung des Prognosemodells an laufenden Projekten durchgeführt werden. Der Ansatz zur Validierung des Prognosemodells in einem realen Vortrieb kann jedoch wie folgt zusammengefasst werden.

Ein echter „Blindtest“ des Prognosemodells gegen einen realen Vortrieb erscheint aufgrund der aufeinander aufbauenden Schätzung des Erwartungswertes für den Laufweg $\mathrm{s}_{\mathrm{c}, \mathrm{e}(\mathrm{z})}$, der Vortriebsstrecken $L_{d, e(m)}$ und der notwendigen Werkzeugwechsel $n_{c, e(m)}$ und aufgrund der zahlreichen Einflussfaktoren im Prognosemodell ungeeignet, um mögliche Probleme zu identifizieren.

Eine zielführende Vorgehensweise besteht wahrscheinlich darin, zunächst in einigen Projekten die Erwartungswerte des Laufwegs $\mathrm{s}_{\mathrm{c}, \mathrm{e}(\mathrm{z})}$ aus Gl. 81 und Gl. 82 durch genaue Aufnahmen der TVMBetriebsdaten, der geotechnischen Bedingungen und detaillierte Verschleißmessungen zu validieren. Dazu sollten Projekte ausgewählt werden, die keine zusätzlichen Risiken aufgrund von Steinen oder Blöcken im Boden und bindigen Böden mit Potential für Verklebungen aufweisen. Die Vortriebsstrecken $L_{d, e(m)}(G l .93)$ sollten im ersten Schritt deutlich niedriger als die maximal erreichbaren Vortriebsstrecken $L_{\max (\mathrm{m})}\left(\mathrm{Gl}\right.$. 111) angesetzt werden, z.B. bei 25 bis $50 \%$ von $L_{\max (m)}$. Das Risiko für eine Überschätzung der Vortriebsstrecken $L_{d(m)}$ aufgrund einer Überschätzung des Laufwegs $S_{c(z)}$ und damit verbundene Verschleißschäden kann so weitgehend reduziert werden.

Darauf aufbauend kann das Prognosemodell im nächsten Schritt auf einen realen Vortrieb angewendet werden, in dem die geplanten Vortriebsstrecken $L_{d, e(m)}$, Vortriebsunterbrechungen $I_{(m)}$ und Werkzeugwechsel $n_{c, e(m)}$ ohne Einschränkungen geplant und soweit wie möglich umgesetzt werden. Hier sollte auch eine rollierende Anpassung der Verschleißprognose im Vortrieb stattfindet. Mit diesem Schritt können die Schätzungen der Vortriebsstrecken $L_{d(m)}$, der notwendigen Anzahl an Werkzeugwechseln $\mathrm{n}_{\mathrm{c}(\mathrm{m})}$ und der baubetriebliche Anpassungsprozess validiert werden.

\subsubsection{Prognosegenauigkeit und kritische Faktoren}

Die deskriptive Validierung des Prognosemodells kann im Hinblick auf die modellbedingte Abweichungen zwischen den Prognoseergebnissen und den tatsächlichen Daten aus den Vortrieben wie folgt zusammengefasst werden.

- Die Verschleißgrenzen der Abbauwerkzeuge werden durch die in der Verschleißprognose ermittelten maximal erreichbaren Vortriebsstrecken $L_{\max (m)}$ zutreffend beschrieben.

- Die maximalen modellbedingten Abweichungen zwischen dem geschätzte Laufweg $\mathrm{s}_{\mathrm{cv}, \mathrm{e}(\mathrm{m})}$ der Abbauwerkzeuge zwischen Ein- und Ausbau auf dem Schneidrad und dem tatsächlichen Laufweg $\mathrm{S}_{\mathrm{cv}(\mathrm{m})}$ aus den RP betragen innerhalb der Bandbreite an enthaltenen geotechnischen Bedingungen für Schneidrollen $-13 \%$ bis $0 \%$, für Sticheln $14 \%$ bis $18 \%$ und für Schälmesser $8 \%$ bis $2 \%$.

- Die maximalen modellbedingten Abweichungen zwischen der geschätzten Anzahl an Werkzeugwechseln $n_{c, e(m)}$ in den Vortriebsunterbrechungen $I_{(m)}$ aus dem Prognosemodell und der tatsächlichen Anzahl an Werkzeugwechseln $n_{c(m)}$ in den Referenzprojekten liegen innerhalb der Bandbreite der enthaltenen geotechnischen Bedingungen für Schneidrollen und Stichel bei $-10 \%$ bis $13 \%$ und für Schälmesser bei $-17 \%$ bis $+12 \%$.

- Abweichungen in der Schätzung des Laufwegs $\mathrm{s}_{\mathrm{cv}(\mathrm{m})}$ durch den Erwartungswert $\mathrm{s}_{\mathrm{cv}, \mathrm{e}(\mathrm{m})}$ sind in allen fünf validierten Referenzprojekten schlüssig mit Abweichungen des Erwartungswertes $\mathrm{n}_{c, \mathrm{e}(\mathrm{m})}$ von der tatsächlichen Anzahl der Werkzeugwechsel $\mathrm{n}_{\mathrm{c}(\mathrm{m})}$ verbunden. Eine Überschätzung des Laufwegs $\mathrm{s}_{\mathrm{cv}(\mathrm{m})}$ durch den Erwartungswert $\mathrm{s}_{\mathrm{cv}, \mathrm{e}(\mathrm{m})}$ hat stets eine Unterschätzung der Anzahl an Werkzeugwechseln $\mathrm{n}_{\mathrm{c}(\mathrm{m})}$ durch den Erwartungswert $\mathrm{n}_{\mathrm{c}, \mathrm{e}(\mathrm{m})}$ zur Folge. Umgekehrt hat eine Unterschätzung des Laufweg $s_{\mathrm{cv}(\mathrm{m})}$ durch den Erwartungswert $\mathrm{s}_{\mathrm{cv}, \mathrm{e}(\mathrm{m})}$ stets eine Überschätzung der Anzahl an Werkzeugwechseln $\mathrm{n}_{\mathrm{c}(\mathrm{m})}$ durch den Erwartungswert $\mathrm{n}_{\mathrm{c}, \mathrm{e}(\mathrm{m})}$ zur Folge. 
In der Erläuterung der Methodik (Kap. 2.4.3) zur Erarbeitung des Prognosemodells wurden im Hinblick auf die Ziele der vorliegenden Arbeit (Kap 1.3) die nachfolgenden kritischen Faktoren erarbeitet, die durch das Prognosemodell eingenhalten werden müssen. Folgende Fehleinschätzungen durch das Prognosemodell sind kritisch:

- Überschätzung der Vortriebsstrecken $L_{\max (m)}$

- Überschätzung des Laufweg $\mathrm{s}_{\mathrm{cv}(\mathrm{m})}$

- Unterschätzung der Anzahl der Werkzeugwechsel $\mathrm{n}_{\mathrm{c}(\mathrm{m})}$

Der Abgleich der Ergebnisse der Validierung mit den kritischen Faktoren zeigt im Hinblick auf die Vortriebsstrecken $L_{\max (m)}$, dass die Anforderung an das Prognosemodell eingehalten wird. Die tatsächlich maximal erreichbaren Vortriebsstrecken $L_{\max (m)}$ werden durch das Prognosemodell nicht überschätzt. Durch die Einhaltung der maximalen Vortriebsstrecken $L_{\max (m)}$ aus dem Prognosemodell können Verschleißschäden an den Abbauwerkzeugen und Folgeschäden an deren Haltern vermieden werden.

Für den Laufweg $\mathrm{s}_{\mathrm{cv}(\mathrm{m})}$ zeigt die deskriptive Validierung, dass dieser durch den Erwartungswert $\mathrm{s}_{\mathrm{cv}, \mathrm{e}(\mathrm{m})}$ aus dem Prognosemodell in Abhängigkeit des Abbauwerkzeugtyps um bis zu 18\% überschätzt wird. Die Abweichungen zwischen den beiden Werten pflanzen sich in Form einer Unterschätzung der notwendigen Anzahl an Werkzeugwechseln $n_{c(m)}$ durch den Erwartungswert $n_{c, e(m)}$ um bis zu $-17 \%$ fort. Im Hinblick auf den Laufweg $\mathrm{s}_{\mathrm{c}(\mathrm{m})}$ und die Anzahl der Werkzeugwechsel $\mathrm{n}_{\mathrm{c}(\mathrm{m})}$ kann das Prognosemodell die genannten kritischen Faktoren folglich nur mit einer möglichen Abweichung von ca. $18 \%(\operatorname{scv}(\mathrm{m}))$ bzw. $-17 \%$ einhalten $\left(\mathrm{n}_{\mathrm{c}(\mathrm{m})}\right)$.

In Projekten, in denen die Wahrscheinlichkeit für eine Über- bzw. Unterschätzung des Laufwegs $\mathrm{S}_{\mathrm{cv}(\mathrm{m})}$ bzw. der Anzahl an Werkzeugwechsel $n_{c(m)}$ um die genannten Beträge reduziert oder ausgeschlossen werden soll, muss folglich eine Korrektur der Werte erfolgen. Die für die wirtschaftlichen Auswirkungen auf das Projekt maßgebliche Größe ist letztlich die Anzahl der Werkzeugwechsel $n_{c(m)}$ in den Vortriebsunterbrechungen $\mathrm{I}_{(\mathrm{m})}$. Der Erwartungswert $\mathrm{n}_{\mathrm{c}, \mathrm{e}(\mathrm{m})}$ kann ohne weitere Wechselwirkungen im Modell unmittelbar nach der Schätzung mit Gl. 137 (Kap. 6.4) angepasst werden. Zu empfehlen ist hierzu, die Anzahl der Werkzeugwechsel $\mathrm{n}_{\mathrm{c}, \mathrm{e}(\mathrm{m})}$ aus Gl. 137 mit einem Sicherheitsbeiwert $\mathrm{V}_{\mathrm{nc}}[-]$ zu multiplizieren (Gl. 140). Dieser sollte in Abhängigkeit der Anforderungen in der Projektplanung mindestens einen Betrag von 1,2 bis 1,4 aufweisen, kann jedoch theoretisch auch höher angesetzt werden. Die korrigierte Anzahl an Werkzeugwechseln $\mathrm{n}_{\mathrm{c}, \mathrm{y}(\mathrm{m})}$ fließt so auch weiter in die Schätzung der Vortriebsdauer und der Verschleißkosten (Tab. 23) mit ein.

$$
\mathrm{n}_{\mathrm{c}, \gamma(\mathrm{m})}=\mathrm{n}_{\mathrm{c}, \mathrm{e}(\mathrm{m})} \cdot \gamma_{\mathrm{nc}}
$$

Die Korrektur des Erwartungswertes für die Anzahl der Werkzeugwechsel $\mathrm{n}_{\mathrm{c}, \mathrm{e}(\mathrm{m})}$ mit Gl. 140 wirkt sich aufgrund der Kopplung der beiden Größen im Modell auf den Erwartungswert für den Laufweg $\mathrm{s}_{\mathrm{cv}, \mathrm{e}(\mathrm{m})}$ der Abbauwerkzeuge zwischen Ein- und Ausbau auf dem Schneidrad aus. Dieser muss folglich mit dem Sicherheitsbeiwert $Y_{s c}$ [-] korrigiert werden (Gl. 142). Der Sicherheitsbeiwert $Y_{s c}$ ergibt sich mit Gl. 141 aus dem Kehrwert von $Y_{n c}$ :

$$
\gamma_{\mathrm{sc}}=\frac{1}{\gamma_{\mathrm{nc}}}
$$

$$
\mathrm{s}_{\mathrm{cv}, \gamma(\mathrm{m})}=\mathrm{s}_{\mathrm{c}, \mathrm{e}(\mathrm{m})} \cdot \gamma_{\mathrm{sc}}
$$


In speziellen Anwendungsfällen, z.B. zum rechnerischen Nachweis der Erreichbarkeit einer bestimmten Vortriebsstrecke $L_{\max (m)}$ zwischen zwei Vortriebsunterbrechungen $I_{(m)}$ für Werkzeugwechsel, kann auch hier in Abhängigkeit der Anforderungen an die Projektplanung die Anwendung eines Sicherheitsfaktors erforderlich sein. Der Erwartungswert für die maximal erreichbare Vortriebsstrecke $L_{\max (m)}$ kann hierzu direkt bei der Schätzung mit Gl. 111 (Kap. 6.3.4) mit dem Sicherheitsbeiwert YL [-] multipliziert werden:

$$
\mathrm{L}_{\max , \gamma(\mathrm{m}+1)}=\sum_{1}^{\mathrm{n}} \mathrm{L}_{\max (m+1 ; \mathrm{z}+\mathrm{n})} \cdot \gamma_{\mathrm{L}}
$$

Der Sicherheitsbeiwert YL kann in Gl. 143 grundsätzlich nur Werte $<1$ annehmen, sodass sich eine Reduktion des Erwartungswertes für die maximal erreichbare Vortriebsstrecke $L_{\max (m)}$ ergibt. Der genaue Betrag des Sicherheitsbeiwertes YL muss in Abhängigkeit der Anforderungen an die Projektplanung durch den Planer bestimmt werden. Die Korrektur der maximal erreichbaren Vortriebsstrecke $\mathrm{L}_{\max (\mathrm{m})}$ mit Gl. 111 hat im Prognosemodell keine weitere Auswirkung auf die Prognosegenauigkeit des

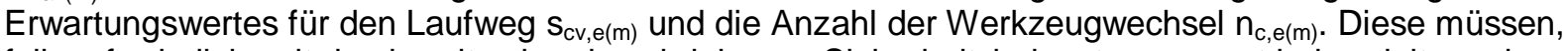
falls erforderlich, mit den bereits oben beschriebenen Sicherheitsbeiwerten separat behandelt werden.

Die strukturelle und deskriptive Validierung des Prognosemodells zeigen darüber hinaus auf, dass zusätzlich aufgrund folgender Faktoren hohe Abweichungen zwischen den Prognoseergebnissen und den tatsächlichen Werten im Vortrieb auftreten können:

- Gewaltschäden durch Steine oder Blöcke und Hindernisse in der Ortsbrust

- Verklebungsschäden

- Nicht quantifizierbare Einflussfaktoren

- Fehlende baubetriebliche Anpassung

Gewaltschäden und Schäden durch Verklebungen sind im Prognosemodell nicht berücksichtigt (Kap. 6.6.1). Für diese Einflussfaktoren fehlen bisher geeignete Prognosemethoden. Die Auswirkungen können einerseits anhand der Minimalwerte der Vortriebsstrecken $L_{d(\mathrm{~m})}$ aus den Referenzprojekten im Vergleich zu den Minimalwerten aus dem Prognosemodel dargestellt werden (Abb. 94). Die deskriptive Validierung zeigt hier, dass die Schäden zu Werten für die Vortriebsstrecken $L_{d(m)}$ führen, die um ca. Faktor 5 bis 20 niedriger liegen, als die zugehörigen Erwartungswerte $L_{d, e(m)}$ aus dem Prognosemodell. Die Auswirkungen können andererseits auch an der Anzahl der Werkzeugwechsel $\mathrm{n}_{\mathrm{c}(\mathrm{m})}$ gezeigt werden. Durch die Schäden liegt die Anzahl der Werkzeugwechsel $\mathrm{n}_{\mathrm{c}(\mathrm{m})}$ in den Referenzprojekten in einigen Vortriebsunterbrechungen $I_{(m)}$ um 20 bis $90 \%$ höher, als die Schätzung durch den Erwartungswert $\mathrm{n}_{\mathrm{c}, \mathrm{e}(\mathrm{m})}$ (Kap. 6.6.2.3). Die Schäden wirken sich zusammenfassend durch eine erhebliche Verkürzung der Vortriebsstrecken $L_{d(m)}$ und eine starke Erhöhung der Anzahl der Werkzeugwechsel $n_{c(m)}$ doppelt negativ auf den Vortrieb aus und stellen im Hinblick auf die kritischen Faktoren ein erhebliches Risiko dar.

Die hohe Schwankungsbreite der Auswirkungen insbesondere auf die Vortriebsstrecken $L_{d(m)}$ zeigt, dass im Falle von Steinen oder Blöcken im Boden, oder einem Potential für Verklebungen in bindigen Böden keine pauschale Abminderung der Vortriebsstrecken $L_{d, e(m)}$ bzw. Erhöhung der Anzahl der Werkzeugwechsel $\mathrm{n}_{\mathrm{c}, \mathrm{e}(\mathrm{m})}$ möglich ist. In der dargestellten Schwankungsbreite ist darüber hinaus nicht berücksichtigt, dass in den Referenzprojekten nicht jeder Stein und Block in der Ortsbrust bzw. jede bindige Bodeneinheit mit Verklebungspotential zuverlässig zu entsprechenden Schäden geführt hat. Die tatsächliche Wahrscheinlichkeit für Schäden bei Steinen oder Blöcken in der Ortsbrust oder vorhandenem Verklebungspotential ist aus den vorliegenden Daten nicht ermittelbar.

Bis zu einer detaillierten Untersuchung der Schadensmechanismen und der Erarbeitung einer quantitativen Prognosemethode ist zu empfehlen, im Fall von Steinen oder Blöcken in der Ortsbrust oder vorhandenem Verklebungspotential die Auswirkungen und mögliche Risiken einer starken Verkürzung 
der Vortriebsstrecken $L_{d(m)}$ und Erhöhung der Anzahl der Werkzeugwechsel $n_{c(m)}$ gezielt in Form von Worst-Case Szenarien abzuschätzen. Diese können mit dem baubetrieblichen Anpassungsalgorithmus (Kap. 6.6.4) erarbeitet werden, indem die Vortriebsstrecken $L_{d, e(m+1)}$ aus Gl. 93 schrittweise bis auf den angegebenen Umfang verringert werden und die Anzahl der Werkzeugwechsel $\mathrm{n}_{\mathrm{c}, \mathrm{e}(\mathrm{m})}$ aus Gl. 135 analog bis auf den angegebenen Umfang erhöht wird.

Die deskriptive Validierung in RP 9 zeigt, dass auch bei einer Abweichung von den Anwendungsgrenzen des Prognosemodells (Kap. 6.2.3.3) in Bezug auf einen der nicht quantifizierbaren Einflussfaktoren (Tab. 22) hohe Abweichungen zwischen den Erwartungswerten für die Vortriebsstrecken $L_{d, e(m)}$ (Abb. 94), den Laufweg $\mathrm{s}_{\mathrm{cv}, \mathrm{e}(\mathrm{m})}$ (Tab. 25), die Anzahl an Werkzeugwechseln $\mathrm{n}_{\mathrm{c}, \mathrm{e}(\mathrm{m})}(\mathrm{Tab} .26)$ und den tatsächlichen Werten im Vortrieb entstehen. Nicht quantifizierbare Einflussfaktoren mit besonders hohen Auswirkungen sind vermutlich (Tab. 22):

- Verschleißschutz der Abbauwerkzeuge

- Schneidradöffnungsverhältnis $\mathrm{O}_{\mathrm{TVM}}[\%]$

Beide Faktoren wirken sich proportional auf den Erwartungswert für den Laufweg $\mathrm{s}_{\mathrm{c}, \mathrm{e}(\mathrm{z})}$ in den geotechnischen Homogenbereichen und den Erwartungswert $\mathrm{s}_{\mathrm{cv}, \mathrm{e}(\mathrm{m})}$ zwischen Ein- und Ausbau der Abbauwerkzeuge auf dem Schneidrad aus (Kap. 6.2.3.3). Bei einer Verringerung des Verschleißschutzes oder des Schneidradöffnungsverhältnisses $\mathrm{O}_{\text {TVM }}$ unter den in Tab. 22 angegebenen Wertebereich führt folglich zu einer Überschätzung der maximal erreichbaren Vortriebsstrecken $L_{\max (m)}$ und einer Unterschätzung der Anzahl an Werkzeugwechseln $n_{c(m)}$ durch den Erwartungswert $n_{c, e(m)}$ aus dem Prognosemodell. Die beiden Faktoren stellen folglich im Hinblick auf die kritischen Faktoren ein deutliches Risiko dar.

Bis zur möglichen Erweiterung der Anwendungsgrenzen des Prognosemodells ist zu empfehlen, im Fall von Abweichungen von den Anwendungsgrenzen des Prognosemodells in einem Projekt, die Auswirkungen und mögliche Risiken einer starken Verkürzung der Vortriebsstrecken $L_{1}(m)$ und Erhöhung der Anzahl der Werkzeugwechsel $\mathrm{n}_{\mathrm{c}(\mathrm{m})}$ gezielt in Form von Worst-Case Szenarien abzuschätzen. Diese können wiederum mit dem baubetrieblichen Anpassungsalgorithmus (Kap. 6.6.4) erarbeitet werden, indem die Vortriebsstrecken $L_{d, e(m+1)}$ aus $G$ l. 93 schrittweise verringert werden und die Anzahl der Werkzeugwechsel $n_{c, e(m)}$ aus Gl. 135 analog erhöht wird. Als bisher einziger Anhaltspunkt für die Größenordnung der Anpassung dienen die Abweichungen in RP 9. Die Vortriebsstrecken $L_{d(m)}$ liegen hier um bis zu Faktor 20 niedriger als die maximal erreichbaren Vortriebsstrecken $L_{\max ,(\mathrm{m})}$ aus dem Prognosemodell. Die Anzahl der Werkzeugwechsel $n_{c(m)}$ liegt um bis zu 100\% höher als die Erwartungswerte aus dem Prognosemodell.

Abschließend kann auch eine fehlende baubetriebliche Anpassung der Verschleißprognose auf die Rahmenbedingungen im Projekt für den Zugang zur Abbaukammer und die Abfolge unterschiedlicher geotechnischen Homogenbereiche zu hohen Abweichungen zwischen den Erwartungswerten für die Vortriebsstrecken $L_{d, e(m)}$, den Laufweg $s_{c v, e(m)}$, die Anzahl an Werkzeugwechseln $n_{c, e(m)}$ und den tatsächlichen Werten im Vortrieb entstehen. Häufige vernachlässigte Einflussfaktoren sind:

- Präventiver Einbau von Sticheln anstatt von Schneidrollen zum effektiveren Bodenabbau in langen Vortriebsstrecken ohne Steine oder Blöcke im Boden. Der Einbau der Stichel wirkt sich bei vollflächiger Abdeckung der Ortsbrust zusätzlich stark positiv auf den Erwartungswert für den Laufweg der Schälmesser $\mathrm{s}_{\mathrm{cv}, \mathrm{e}(\mathrm{z})}$ aus (Kap. 6.2.3.1).

- Präventiver Einbau von Schneidrollen anstatt von Sticheln zum Durchfahren von Vortriebstrecken mit Steinen oder Blöcken im Boden oder zum Einfahren in Schächte mit Betonwänden oder Dichtblöcken mit einer Festigkeit ca. >20 MPa.

- Ersatzloser Ausbau von Abbauwerkzeugen bzw. Einbau von Abbauwerkzeugen in zuvor ungenutzte Halter oder Gehäuse, um die Schneidradbestückung auf den Vortrieb in Böden mit Verklebungspotential anzupassen (Optimierung Penetration $\mathrm{p}_{\mathrm{a}}[\mathrm{mm}]$ \& Schneidradöffnungsverhältnis $\mathrm{O}_{\mathrm{TVM}}[\%]$ ) (Kap. 4.2.2.1).

- Präventive Werkzeugwechsel in Schächten oder Dichtblöcken zwischen zwei Vortriebsstrecken bei vereinfachtem Zugang zur Abbaukammer unter atmosphärischen Bedingungen. 
Alle genannten Faktoren können sowohl eine Erhöhung, als auch eine Verringerung der Vortriebsstrecken $L_{d(m)}$ und Anzahl der Werkzeugwechsel $n_{c(m)}$ im Vergleich zu den Erwartungswerten $L_{d, e(m)}$ und $\mathrm{n}_{\mathrm{c}, \mathrm{e}(\mathrm{m})}$ aus dem Prognosemodell bewirken. Die absoluten Auswirkungen können dabei insbesondere bei eher kurzen Vortrieben sehr hoch sein, weil z.B. die Anzahl der Werkzeugwechsel bei einem einmaligen präventiven Austausch der Schneidrollen gegen Stichel im Verhältnis zur geschätzten Gesamtanzahl der verschleißbedingten Werkzeugwechsel $\mathrm{n}_{\mathrm{c}, \mathrm{e}(\mathrm{TA})}$ über die Tunnelachse bereits sehr hoch sein kann.

Sofern in einem Projekt Unsicherheit in Bezug auf einen der genannten Faktoren besteht, können die möglichen Auswirkungen auf die Vortriebsdauer und die Verschleißkosten wiederum durch Bildung verschiedener Szenarien mit dem Anpassungsalgorithmus (Kap. 6.6.4) abgeschätzt werden. Die beschriebenen Zusammenhänge weisen zusammenfassend darauf hin, dass die baubetriebliche Anpassung der Verschleißprognose ein zwingend erforderlicher Bestandteil des Prognosemodells bzw. des Prognoseprozesses ist, der nicht durch pauschale Anpassungsfaktoren ersetzt oder zu Gunsten einer Vereinfachung vollständig entfallen darf. 


\section{Schlussbetrachtung und Ausblick}

\subsection{Zusammenfassung der zentralen Ergebnisse}

Das Ziel der vorliegenden Arbeit war die Analyse der Zusammenhänge zwischen den Standgrößen der Abbauwerkzeuge, Auslegung der TVM (Gerätschaft), Steuerung des Abbauprozesses (Baubetrieb) und den Boden- und Gebirgseigenschaften (Geotechnik) für Hydroschild TVM in Lockergesteinen. Darauf aufbauend sollte ein Prognosemodell für den Verschleiß der Abbauwerkzeuge entwickelt werden. Das Prognosemodell sollte eine zuverlässige Schätzung der Standgrößen der Abbauwerkzeuge, der notwendige Anzahl an verschleißbedingten Werkzeugwechseln und der dazu notwendigen Anzahl und Stationierung der Vortriebsunterbrechungen für Hydroschild TVM in Lockergesteinen ermöglichen. So sollten mit der vorliegenden Arbeit der Kenntnisstand und die Prognosemöglichkeiten im Hinblick auf den Verschleiß der Abbauwerkzeuge beim Vortrieb mit Hydroschild TVM in Lockergesteinen signifikant erhöht werden, um eine maßgebliche Verbesserung der Planungsgrundlage für die Vortriebsdauer, die Verschleißkosten und zur Einschätzung der Verfahrenssicherheit zu bewirken.

\subsubsection{Folgerungen aus dem bisherigen Wissensstand}

Die ausführliche Recherche des bestehenden Wissensstandes zur Abrasivität von Lockergesteinen und deren Auswirkung in Form von Verschleiß an den Abbauwerkzeugen beim Vortrieb mit Hydroschild TVM in Lockergesteinen (Kap. 4) zeigt, dass zahlreiche Erkenntnisse zu den mikroskopischen Verschleißmechanismen an Metalloberflächen bei der Interaktion mit abrasiven Bodenpartikel vorliegen (Kap. 4.1). Die makroskopischen Auswirkungen dieser Mechanismen auf die Abbaumechanik und das Verschleißbild der Abbauwerkzeuge (Kap. 4.2) werden zum Teil in theoretischen Modellen und Laborversuchen erfasst, die eingeschränkt auf Hydroschild TVM übertragbar sind. Diese werden durch eine begrenzte Anzahl an veröffentlichten Vortriebserfahrungen ergänzt. Die hohe Bandbreite an Einflussfaktoren auf den Verschleiß der Abbauwerkzeuge wird für die Boden- und Gebirgseigenschaften in Form einer hohen Anzahl an Indexversuchen zur Erfassung der Abrasivität von Lockergesteinen untersucht. Zu den Einflussfaktoren aus der Auslegung der Hydroschild TVM und der Steuerung des Abbauprozesses liegen dagegen nur in geringem Umfang Erkenntnisse vor. Dies ist zugleich die Hauptursache für die bisher fehlende Übertragbarkeit der Abrasivitätskennwerte für Lockergesteine in quantitative Prognosemodelle für den Verschleiß der Abbauwerkzeuge beim Vortrieb mit Hydroschild TVM in Lockergesteinen (Kap. 4.4).

\subsubsection{Eregbnisse aus der Analyse der 18 untersuchten Vortriebe}

Als Grundlage für die Analyse der Zusammenhänge zwischen den Boden- und Gebirgseigenschaften, der Auslegung der Hydroschild TVM, der Steuerung des Abbauprozesses und dem Verschleiß der Abbauwerkzeuge konnten in der Datenrecherche 10 Referenzprojekte mit 13 Hydroschild TVM in 18 Vortrieben erarbeitet werden. Die Daten zur Auslegung der TVM wurden aus der Dokumentation des Maschinenherstellers entnommen. Die Daten zur Steuerung des Abbauprozesses wurden aus der Betriebsdatenerfassung der TVM gewonnen. Die geotechnischen Daten stammen überwiegend aus den Berichten und Gutachten zur Vorerkundung in den Referenzprojekten. Die enthaltenen Hydroschild TVM umfassen eine Bandbreite an Schneidraddurchmessern $D_{\text {TVM }}$ von 6,3 bis 15,4 m. In Summe wurde in den Referenzprojekten dabei auf $45.4 \mathrm{~km}$ Vortriebsstrecke ein Ausbruchvolumen von 5.592.143 $\mathrm{m}^{3}$ erzielt. Für die empirische, prozessorientierte Datenanalyse konnten innerhalb der Referenzprojekte in Summe 4.607 Werkzeugwechsel für Schneidrollen, Stichel und Schälmesser dokumentiert werden (Kap. 5.1).

Für die Datenanalyse wurden zunächst Art, Umfang und Detaillierungsgrad der Dokumentation der Werkzeugwechsel, der geotechnischen Bedingungen im Vortrieb, der Auslegung der TVM und der Steuerung des Abbauprozesses untersucht. Daraus wurden neue Standardanforderungen für die Vortriebsdokumentation für Hydroschild TVM in Lockergesteinen entwickelt (Kap. 5.2). Anschließend wurde als Grundlage für die Datenanalyse eine neue Definitione der Verschleiß- und Schadensarten für Hydroschild TVM in Lockergesteinen entwickelt (Kap. 5.3). 
Diese werden begrifflich nach deren Ort an den Abbauwerkzeugen oder der Schneidradstahlstruktur unterschieden und nach der jeweiligen Verschleiß- und Schadensursache getrennt. Die definierten Verschleiß- und Schadensarten und die zugehörigen Verschleiß- und Ortsbrustbilder werden anhand der Referenzprojekte für die unterschiedlichen Abbauwerkzeugtypen detailliert beschrieben. Aus der Beschreibung können einerseits Rückschlüsse auf die Abbaumechanik im Lockergestein gezogen werden. Andererseits wurden daraus für die verschiedenen Abbauwerkzeugtypen klare Verschleißgrenzen bei der Anwendung in Lockergesteinen entwickelt.

Die Verschleißgrenzen stellen die Grundlage für die Berechnung der Standgrößen der Abbauwerkzeuge dar. Als Standgrößen für Abbauwerkzeuge auf Hydroschild TVM in Lockergesteinen stehen die Vortriebsstrecke (Standstrecke) [m] in Richtung der Tunnelachse, der Laufweg (Standweg) [km] entlang der Schneidspur des Abbauwerkzeuges, das Ausbruchvolumen (Standvolumen) [ $\left.\mathrm{m}^{3}\right]$ und die Betriebsstunden (Standzeit) [h] zur Verfügung. Für die Anwendung in Lockergesteinen und in der Datenanalyse wurde der Laufweg $\mathrm{s}_{\mathrm{c}}[\mathrm{km}]$ als geeignete Standgröße festgelegt. Die Betrachtungsrichtung bei der Berechnung des Laufwegs $\mathrm{s}_{\mathrm{c}}$ stimmt mit der Bewegungsrichtung des Werkzeuges beim Bodenabbau überein. Damit werden die Laufwege $s_{c}$ von Werkzeugen auf verschiedenen Schneidspuren mit unterschiedlichen Spurradien $r_{s}[\mathrm{~mm}]$ direkt vergleichbar. Zusätzlich ist der Laufweg $s_{c}[\mathrm{~km}]$ in der Praxis leicht bestimmbar und kann für die Anwendung in der Vortriebsplanung relativ einfach in Vortriebsstrecken umgerechnet werden.

Als weitere Grundlage für die Datenanalyse wurden Baustellenversuche zur Untersuchung der Verschleißrate $c_{v}$ an den Abbauwerkzeugen durchgeführt (Kap. 5.5). Die Verschleißrate $c_{v}$ gibt den durch Verschleiß verursachten Massen-, Volumen- oder Längenverlust eines Abbauwerkzeuges im Verhältnis zum Laufweg $\mathrm{s}_{\mathrm{c}}[\mathrm{km}]$ an. Die Ergebnisse der Baustellenversuche lassen für die weitere Datenanalyse die Annahme zu, dass der Massenverlust und der Längenverlust der Abbauwerkzeuge unter gleichbleibenden Einsatzbedingungen für alle Abbauwerkzeug- und Verschleißtypen linear abhängig vom Laufweg $\mathrm{s}_{\mathrm{c}}$ sind. Die Verschleißrate $\mathrm{c}_{\mathrm{v}}$ ist unter gleichbleibenden Einsatzbedingungen vom Einbau der Abbauwerkzeuge auf dem Schneidrad bis zum Erreichen der Verschleißgrenze konstant. Der Betrag der Verschleißrate $c_{v}$ ist für alle Abbauwerkzeuge des gleichen Typs auf dem Schneidrad zum gleichen Zeitpunkt und unter den gleichen Einsatzbedingungen annähernd identisch.

Die empirische Analyse der Daten im Hinblick auf die Einflussfaktoren auf den Laufweg $\mathrm{s}_{\mathrm{c}}$ der Abbauwerkzeuge wird mit vergleichenden Methoden durchgeführt (Kap. 5.6). Im Vordergrund steht dabei die weitgehende Normierung der unterschiedlichen Einflussfaktoren, um einzelne Faktoren oder Faktorenkombinationen gezielt durch Regressionsanalysen untersuchen zu können. Dazu bieten sich z.B. Vergleiche des Laufwegs $\mathrm{s}_{\mathrm{c}}[\mathrm{km}]$ zwischen unterschiedlichen geotechnischen Bedingungen ohne Änderung der Maschinenauslegung und Steuerung des Abbauprozesses an. Eine detaillierte Analyse der Daten mit statistischen Methoden, wie z.B. einer mehrfaktoriellen Varianzanalyse, ist aufgrund einer hohe Bandbreite an unterschiedlichen Datentypen, unklarer oder fehlender Angaben zu den statistischen Eigenschaften der Basisdaten und stark unterschiedlicher Dokumentationsdichten in den Referenzprojekten, nicht möglich.

Für die Datenanalyse müssen jedem ausgewechselten Abbauwerkzeug charakteristische Werte für die Einflussfaktoren aus der Auslegung der Hydroschild TVM, der Steuerung des Abbauprozesses und den Boden- und Gebirgseigenschaften zugeordnet werden. Dazu ist die Bildung geotechnischer Homogenbereiche notwendig, die anhand der Verteilung der Bodenarten in der Ortsbrust, der Überlagerung und des Wasserspiegels über der Tunnelachse gegliedert werden. In Summe ergeben sich in den Referenzprojekten 62 Homogenbereiche. Für die die Zuordnung der Werte der Einflussfaktoren aus der Steuerung des Abbauprozesses wurden Mittelwerte über die Strecken der geotechnischen Homogenbereiche gebildet. Die Werte der Einflussfaktoren aus der Steuerung des Abbauprozesses sind innerhalb der geotechnischen Homogenbereiche in über $90 \%$ der untersuchten Fälle normalverteilt. Für Einflussfaktoren, die in der Steuerung der TVM Reaktionsgrößen (Passivparameter) sind, können in $86 \%$ der Fälle statistisch signifikante Unterschiede zwischen benachbarten, geotechnischen Homogenbereichen dargestellt werden. Für Einflussfaktoren, die Einstellwerte (Aktivparameter) des Schildfahrers sind, kann dies nur in 63\% der Fälle nachgewiesen werden. In der Datenanalyse werden ausschließlich Abbauwerkzeuge berücksichtigt, die innerhalb eines geotechnischen Homogenbereiches ein- und ausgebaut wurden, sodass eindeutige Werte für die Einflussfaktoren aus der Steuerung des Abbauprozesses und den Boden- und Gebirgseigenschaften zugeordnet werden können. Hierdurch entfallen $57 \%$ aller Werkzeugwechsel für die Datenanalyse. 
Der Fokus der Datenanalyse liegt auf Werkzeugwechseln, die auf Primär- und Sekundärverschleiß zurückzuführen sind. Werkzeugwechsel aufgrund von Verschleißschäden (Überschreitung der Verschleißgrenzen), Gewaltschäden durch Steine, Blöcke oder Hindernisse in der Ortsbrust oder Schäden durch Verklebungen entstanden, wurden anhand der Werkzeugwechseldokumentation in den Referenzprojekten und der Häufigkeitsverteilung des Laufwegs $s_{c}$ in den geotechnischen Homogenbereichen eliminiert. Die Anzahl der verfügbaren Werkzeugwechsel für die Datenanalyse wird durch diesen Schritt weiter reduziert. Abschließend verbleiben für die Datenanalyse $23 \%$ aller Werkzeugwechsel, denen eindeutige Werte für die Einflussfaktoren zugeordnet werden können und für die Schäden als Wechselgrund zuverlässig ausgeschlossen werden können.

Die Datenanalyse ergibt den in Tab. 29 zusammengefassten Satz an Einflussfaktoren. Auf Basis der Daten aus den Referenzprojekten können nicht alle Einflussfaktoren quantifiziert werden.

Eine rein empirische Analyse des Einflusses einzelner Faktoren aus den Boden- und Gebirgseigenschaften auf den Laufweg $\mathrm{s}_{\mathrm{c}}$ ergibt keine oder qualitativ sehr schlechte Korrelationen mit Laufweg $\mathrm{s}_{\mathrm{c}}$. Der Laufweg $\mathrm{s}_{\mathrm{c}}$ wird vielmehr von einer Kombination an geotechnischen Parametern bestimmt:

- Abrasivität der Bodenkomponenten

- Kontaktspannung zwischen Boden und Abbauwerkzeug beim Bodenabbau

- Korngrößenverteilung, Kornform und Rundungsgrad der Bodenkomponenten

Zur Beschreibung der Abrasivität der Bodenkomponenten wurde aufgrund der spezifischen Datenverfügbarkeit in den Referenzprojekten der Äquivalente Quarzgehalt (ÄQU) [\%] herangezogen. Die Kontaktspannungen $\mathrm{T}_{\mathrm{act}}\left[\mathrm{kN} / \mathrm{m}^{2}\right]$ zwischen Boden und Abbauwerkzeugen beim Bodenabbau können theoretisch durch Aufteilung der Schneidradanpresskraft $F_{S R}$ und des Schneiddrehmomentes $M_{S R}$ auf die einzelnen Abbauwerkzeuge ermittelt werden, weil in der Schneidradanpresskraft $F_{S R}$ und im Schneiddrehmoment $M_{S R}$ die an den einzelnen Abbauwerkzeugen anliegenden Schneidkräfte aufsummiert sind. Die geforderte Aufteilung ist jedoch mit dem bestehenden Wissensstand und den verfügbaren Rechenmethoden nicht möglich, weil keine scharfe rechnerische Abtrennung der Schneidradanpresskraft $F_{S R}$ und des Schneiddrehmomentes $M_{S R}$ aus der Vortriebspressenkraft $F_{V T P}$ und dem Schneidraddrehmoment $\mathrm{M}_{\mathrm{TVM}}$ möglich ist. Zudem fehlen Methoden zur schlüssigen Aufteilung der Schneidradanpresskraft $F_{S R}$ und des Schneiddrehmomentes $M_{S R}$ auf die unterschiedlichen Abbauwerkzeugtypen auf dem Schneidrad. Die Untersuchung des Einflusses der Kontaktspannungen $T_{\text {act }}\left[\mathrm{kN} / \mathrm{m}^{2}\right]$ auf den Laufweg $\mathrm{s}_{\mathrm{c}}[\mathrm{km}]$ der Abbauwerkzeuge erfolgt deshalb nicht direkten über die Schneidradanpresskraft $F_{S R}$ und das Schneiddrehmoment $M_{S R}$, sondern indirekt über die Scherfestigkeit $\mathrm{T}_{\mathrm{c}}\left[\mathrm{kN} / \mathrm{m}^{2}\right]$ des Bodens in der Ortsbrust auf Höhe der Tunnelachse. Diese wird mit dem MoHRCOULOMB Kriterium berechnet und als indikativer Vergleichswert für die Kontaktspannungen $T_{\text {act }}$ angesetzt. Die Kornform und der Kornrundungsgrad der Bodenkomponenten wurden in den Referenzprojekten nur sehr selten bestimmt. Die Korngrößenverteilung der Bodenkomponenten fließt deshalb als einziger Formparameter mit dem Korndurchmesser $\mathrm{D}_{60}[\mathrm{~mm}]$ in die Datenanalyse ein.

Der Äquivalente Quarzgehalt (ÄQU) [\%], die Scherfestigkeit $T_{c}\left[k N / m^{2}\right]$ und der Korndurchmesser $D_{60}$ $[\mathrm{mm}]$ werden für die Datenanalyse in gewichteter Form im neu eingeführten Soil Abrasivity Index (SAl) [-] zusammengefasst. Der SAl zeigt sowohl für Schneidrollen, als auch für Schälmesser sehr starke Korrelationen $(R=0,93-0,98)$ mit dem Laufweg $s_{c}$ in Form von Exponentialfunktionen. Für Stichel kann auf Basis der wenigen vorhandenen Datenpunkte ein sehr ähnlicher Zusammenhang zwischen SAI und Laufweg $S_{c}$ angenommen werden wie für Schälmesser. Für Räumer liegen keine Werkzeugwechseldaten vor. Aufgrund der Ähnlichkeit in der Auslegung und Abbaumechanik zu Schälmessern, kann jedoch auch hier ein ähnlicher Zusammenhang wie für Schälmesser angenommen werden.

Der Soil Abrasivity Index (SAI) kann theoretisch Werte von 0 bis ca. 10.000 annehmen. Für diesen Wertebereich kann mithilfe von geschätzten Wahrscheinlichkeitsverteilungen der drei Eingangsgrößen eine theoretische Häufigkeitsverteilung des SAI ermittelt werden. Die Werte für den SAI in den Referenzprojekten von 1 bis 1.770 decken ein Quantil von ca. 59\% der theoretischen Häufigkeitsverteilung $\mathrm{ab}$. 
Tab. 29: Zusammenfassung der wichtigsten Einflussfaktoren auf den Laufweg $\mathrm{s}_{\mathrm{c}}[\mathrm{km}]$ aus der Datenanalyse (Kap. 5.6). Die Kontaktspannungen $T_{\text {act }}\left[\mathrm{kN} / \mathrm{m}^{2}\right]$ können bisher nicht direkt bestimmt werden, deshalb ist kein Wertebereich für Tact angegeben. Für die Datenanalyse wurde $T_{a c t}$ durch die Scherfestigkeit $T_{c}\left[\mathrm{kN} / \mathrm{m}^{2}\right]$ des Bodens in der Ortsbrust auf Höhe der Tunnelachse als indikativer Vergleichswert angesetzt. Auf diesem Weg kann der Einfluss von $\mathrm{T}_{\mathrm{act}}$ auf den Laufweg $\mathrm{s}_{\mathrm{c}}$ indirekt quantifiziert werden.

\begin{tabular}{|c|c|c|}
\hline Einflussfaktoren auf den Laufweg $s_{c}$ & Wertebereich in den Referenzdaten & Quantifizierung \\
\hline \multicolumn{3}{|l|}{ Auslegung der TVM } \\
\hline Schneidradöffnungsverhältnis $\left(\mathrm{O}_{\mathrm{TVM}}\right)[\%]$ & $28,4-31,0 \%$ & nein \\
\hline Anzahl der Rotoren \& Statoren [-] & $2-6$ & nein \\
\hline Schneidrollen Höhe über Schneidradstahlbau $\left(\mathrm{h}_{\mathrm{SR}}\right)[\mathrm{mm}]$ & 175 & nein \\
\hline Stichel Höhe über Schneidradstahlbau ( $\mathrm{h}_{\mathrm{ST}}$ ) [mm] & $175-180$ & nein \\
\hline Schälmesser Höhe über Schneidradstahlbau $\left(\mathrm{h}_{\mathrm{SM}}\right)[\mathrm{mm}]$ & $135-140$ & nein \\
\hline Spurbesatz $\left(k_{s}\right)[-]$ & $2-8$ & ja \\
\hline Winkelabstand $\delta_{a}\left[{ }^{\circ}\right]$ & $25-180$ & ja \\
\hline Winkelabstand $\delta_{p}\left[{ }^{\circ}\right]$ & $0-10$ & ja \\
\hline Schälmesser Schneidwinkel $\left(\alpha_{c}\right)\left[{ }^{\circ}\right]$ & $75-90$ & nein \\
\hline Schälmesser Freiwinkel $\left(\beta_{c}\right)\left[{ }^{\circ}\right]$ & $0-22$ & nein \\
\hline Schneidringbreite $\left(\mathrm{b}_{\mathrm{SR}}\right)[\mathrm{mm}]$ : & $19-24$ & ja \\
\hline Schneidringdurchmesser ( $\left.d_{S R}\right)[\mathrm{mm}]:$ & $432\left(17^{\prime \prime}\right)$ & nein \\
\hline Verschleißschutz Schälmesserschneide & Hartmetall (WC), durchgehend. & nein \\
\hline Verschleißschutz Schälmesserrückenfläche & Hartmetall (WC), min. 30\% Abdeckung & nein \\
\hline Verschleißschutz Schneidringe & Hochleg. Stahl, durchgehärtet: 57 HRC & nein \\
\hline \multicolumn{3}{|l|}{ Steuerung Abbauprozess } \\
\hline Schneidgeschwindigkeit $\left(\mathrm{v}_{\mathrm{sc}}\right)[\mathrm{m} / \mathrm{s}]$ & $0,25-0,90$ & ja \\
\hline Penetration $(p)[\mathrm{mm} / \mathrm{U}]$ & $4,7-43,9$ & ja \\
\hline Schneidradanpresskraft $\left(F_{\mathrm{SR}}\right)[\mathrm{kN}]$ & $1.605-111.438$ & nein \\
\hline Schneiddrehmoment $\left(\mathrm{M}_{\mathrm{SR}}\right)[\mathrm{kNm}]$ & $260-8.050$ & nein \\
\hline Kontaktspannungen $\left(\mathrm{T}_{\mathrm{act}}\right)\left[\mathrm{kN} / \mathrm{m}^{2}\right]$ & - & ja \\
\hline Dichte der Stützflüssigkeit ( $\left.\rho_{\mathrm{SF}}\right)\left[\mathrm{g} / \mathrm{cm}^{3}\right]$ & $1,15-1,37 \mathrm{~g} / \mathrm{cm}^{3}$ & nein \\
\hline Stützdruck $\left(P_{\mathrm{SF}}\right)[\mathrm{bar}]$ & $0,9-3,7$ bar & nein \\
\hline \multicolumn{3}{|l|}{ Geotechnische Parameter } \\
\hline Überlagerung $\left(\mathrm{H}_{\mathrm{TA}}\right)[\mathrm{m}]$ & $12,5-48,0$ & ja \\
\hline Wasserspiegel $\left(\mathrm{W}_{\mathrm{TA}}\right)[\mathrm{m}]$ & $0,0-45,0$ & ja \\
\hline Feuchtwichte $(\mathrm{v})\left[\mathrm{kN} / \mathrm{m}^{3}\right]$ & $18,0-21,0$ & ja \\
\hline Wichte unter Auftrieb $\left(\gamma^{\prime}\right)\left[\mathrm{kN} / \mathrm{m}^{3}\right]$ & $8,6-13,7$ & ja \\
\hline Äquivalenter Quarzgehalt (ÄQu) [\%] & $25,1-89,3$ & ja \\
\hline Reibungswinkel $\left(\varphi^{\prime}\right)\left[^{\circ}\right]$ & $15-44$ & ja \\
\hline Kohäsion (c') [kN/m²] & $0-42$ & ja \\
\hline Scherfestigkeit $\left(\mathrm{T}_{\mathrm{c}}\right)\left[\mathrm{kN} / \mathrm{m}^{2}\right]$ & $107-789$ & ja \\
\hline Korndurchmesser $\left(\mathrm{D}_{60}\right)[\mathrm{mm}]$ & $0,01-15$ & ja \\
\hline Kornform & flach - kubisch & nein \\
\hline Kornrundungsgrad & angerundet bis gut gerundet & nein \\
\hline
\end{tabular}




\subsubsection{Empirische Verschleißprognose}

Die starke Korrelation des Soil Abrasivity Index (SAl) mit dem Laufweg $\mathrm{s}_{\mathrm{c}}$ kann als Ausgangspunkt zur Entwicklung eines empirischen Prognosemodells genutzt werden (Kap. 6). Die Ziele des Modells sind:

- Schätzung des Laufwegs $\mathrm{s}_{\mathrm{c}}[\mathrm{km}]$ der Abbauwerkzeuge.

- Schätzung der Stationierung $\mathrm{L}_{(\mathrm{m})}[\mathrm{m}]$ und Anzahl $\mathrm{n}_{\mathrm{I}(\mathrm{TA})}[-]$ der Vortriebsunterbrechungen $\mathrm{I}_{(\mathrm{m})}$ für verschleißbedingte Werkzeugwechsel.

- Schätzung der Anzahl $\mathrm{n}_{\mathrm{c}(\mathrm{m})}$ verschleißbedingter Werkzeugwechsel.

- Baubetriebliche Anpassung der Verschleißprognose an die geotechnischen Bedingungen für den Zugang zur Abbaukammer und projektspezifische Ziele.

Die Architektur des Prognosemodells (Kap. 6.1) ist hierzu so angelegt, dass zunächst anhand der geotechnischen Basisdaten Homogenbereiche entlang der Tunnelachse gebildet werden. Für jeden Homogenbereich wird anschließend ein Erwartungswert für den Laufweg $\mathrm{s}_{\mathrm{c}, \mathrm{e}(\mathrm{z})}[\mathrm{km}]$ der einzelnen Abbauwerkzeuge auf dem Schneidrad geschätzt. Aus dem Erwartungswert des Laufwegs $\mathrm{s}_{\mathrm{c}, \mathrm{e}(\mathrm{z})}$ können die geplanten Vortriebsstrecken $L_{d, e(m)} z$ wischen den Vortriebsunterbrechungen $I_{(m)}$ ermittelt werden. Die Anzahl der verschleißbedingten Werkzeugwechsel $\mathrm{n}_{\mathrm{c}(\mathrm{m})}[-]$ wird mithilfe des Ausnutzungsgrades $e_{c, e(m)}[\%]$ der Abbauwerkzeuge an den aufeinanderfolgenden Vortriebsunterbrechungen $I_{(m)}$ bestimmt. Abschließend ist eine baubetriebliche Überprüfung und gegebenenfalls eine Anpassung der Stationierung $\mathrm{L}_{\mathrm{l}, \mathrm{e}(\mathrm{m})}$ der Vortriebsunterbrechungen $\mathrm{I}_{(\mathrm{m})}$ im Hinblick auf die Bedingungen für den Zugang zur Abbaukammer notwendig.

Für die Schätzung des Erwartungswertes des Laufwegs $s_{c, e(z)}$ der Abbauwerkzeuge (Kap. 6.2) wird zunächst für jeden geotechnischen Homogenbereich anhand der Basisdaten der Soil Abrasivity Index (SAI) bestimmt. Mit dem SAI kann aus den Korrelationen mit dem Laufweg $s_{c}$ für jeden Abbauwerkzeugtyp der Ausgangswert für den Laufweg $\mathrm{s}_{\mathrm{c}, \mathrm{b}(\mathrm{z})}$ abgelesen werden. Dieser wird anschließend um die bekannten und quantifizierbaren Einflussfaktoren aus der Auslegung der TVM und der Steuerung des Abbauprozesses korrigiert. Hieraus ergibt sich für jedes einzelne Abbauwerkzeug auf dem Schneidrad ein spezifischer Erwartungswert für den Laufweg $\mathrm{s}_{\mathrm{c}, \mathrm{e}(\mathrm{z})}[\mathrm{km}]$. Die Wertebereiche der nicht quantifizierbaren Einflussfaktoren in den Referenzprojekten stellen Anwendungsgrenze des Prognosemodells dar.

Aus dem Erwartungswert des Laufwegs $\mathrm{s}_{\mathrm{c}, \mathrm{e}(\mathrm{z})}[\mathrm{km}]$ wird für jedes einzelne Abbauwerkzeug auf dem Schneidrad die maximal erreichbare Vortriebsstrecke $L_{c(\mathrm{~m})}[\mathrm{m}]$ bis zum Erreichen der Verschleißgrenze geschätzt (Kap. 6.3). Das Abbauwerkzeug mit dem niedrigsten Wert für $\mathrm{L}_{c(\mathrm{~m})}$ auf dem Schneidrad gibt die maximal erreichbare Vortriebsstrecke $L_{\max (m)}[\mathrm{m}]$ der TVM bis zum Erreichen der Verschleißgrenze des ersten Abbauwerkzeuges vor. Der Erwartungswert für die Vortriebsstrecken $L_{d, e(m)}[m]$ zwischen den Vortriebsunterbrechungen $I_{(m)}$ kann beliebig kleiner oder gleich der maximal erreichbaren Vortriebsstrecke $L_{\max (m)}$ gewählt werden. Zusätzlich ist ein Abgleich der Vortriebsstrecken $L_{d, e(m)}$ mit den geotechnischen Homogenbereichen notwendig, weil jeweils ein abweichender Erwartungswert für den Laufweg $S_{c, e(z)}$ zur Schätzung von $L_{d, e(m)}$ eingesetzt werden muss. Die Vortriebsstrecken $L_{d, e(m)}$ [m] ergeben durch Verkettung von Vortriebsbeginn an die metrische Stationierung $L_{1, e(m)}[m]$ der Vortriebsunterbrechungen $\mathrm{I}_{(\mathrm{m})}$ und deren Anzahl $\mathrm{n}_{1(\mathrm{TA})}$ über die Tunnelachse.

Die Berechnung der maximal erreichbaren Vortriebsstrecke $L_{\max (m)}$ geht von neuen, unverschlissenen Abbauwerkzeugen aus. Die Abbauwerkzeuge besitzen jedoch an der Vortriebsunterbrechung $I_{(m)}$ bereits den bestehenden kumulierten Ausnutzungsgrad $e_{c, e(m)}$. In Abhängigkeit der Auswahl der geplanten Vortriebsstrecke $L_{d, e(m+1)}$ kleiner oder gleich der maximal erreichbaren Vortriebsstrecke $L_{\max (m+1)}$ kann so der kumulierte Ausnutzungsgrad $e_{c, e(m+1)}$ an der Vortriebsunterbrechung $I_{(m+1)}$ für einzelne Abbauwerkzeuge planmäßig Werte $>1$ bzw. 100\% annehmen. Diese Abbauwerkzeuge müssen an der Vortriebsunterbrechung $I_{(m)}$ folglich gewechselt werden, um die ausgewählte Vortriebsstrecke $L_{d, e(m+1)}$ ohne planmäßige Überschreitung der Verschleißgrenze zu erreichen. Der Erwartungswert für die Anzahl $n_{c, e(m)}$ der zu wechselnden Abbauwerkzeuge $c$ in der Vortriebsunterbrechung $I_{(m)}$ wird durch Summierung aller Abbauwerkzeuge ermittelt werden, für die bei $I_{(m)}$ aufgrund der geplanten Vortriebsstrecke $L_{d, e(m+1)}$ und des kumulierten Ausnutzungsgrades $e_{c, e(m+1)}$ das beschriebene Wechselkriterium gilt. 
Als vierten Teil beinhaltet das Prognosemodell einen Anpassungsalgorithmus, um in der Planung der Stationierungen $\mathrm{L}_{\mathrm{l}, \mathrm{e}(\mathrm{m})}$ der Vortriebsunterbrechungen $\mathrm{I}_{(\mathrm{m})}$ die Rahmenbedingungen im Projekt für den Zugang zur Abbaukammer zu berücksichtigt. Diese sind entscheidend dafür, ob und mit welcher Methode die Vortriebsunterbrechungen $\mathrm{I}_{(\mathrm{m})}$ an den geplanten Stationierungen $\mathrm{L}_{\mathrm{l}, \mathrm{e}(\mathrm{m})}$ durchgeführt werden können. Der Algorithmus erlaubt eine iterative Anpassung der Stationierungen $\mathrm{L}_{\mathrm{l}, \mathrm{e}(\mathrm{m})}$ durch Variation der Vortriebsstrecken $L_{d, e(m)}$ innerhalb der Grenzen der maximal erreichbaren Vortriebsstrecken $L_{\max (m)}$ oder durch Variation der Basisdaten, indem z.B. die Auslegung der TVM angepasst wird. Die Anpassungen haben jeweils Auswirkungen auf die Anzahl der Vortriebsunterbrechungen $n_{1, e(T A)}$ und die Anzahl der Werkzeugwechsel $\mathrm{n}_{\mathrm{c}, \mathrm{e}(\mathrm{m})}$. Das Ergebnis der iterativen Anpassung ist mindestens ein Planungsszenario, das als Grundlage zur Schätzung der Vortriebsdauer und Verschleißkosten dient. Der Anpassungsalgorithmus kann zusätzlich auch dazu genutzt werden, um die Fortpflanzung z.B. der Varianz der geotechnischen Basisdaten im Prognosemodell zu untersuchen.

Die deskriptive Validierung des Prognosemodells (Kap. 6.6) ergibt, dass die Verschleißgrenzen der Abbauwerkzeuge durch die im Modell ermittelten maximal erreichbaren Vortriebsstrecken $L_{\max (m)} z u-$ treffend beschrieben werden. Die maximalen, modellbedingten Abweichungen zwischen dem geschätzten Laufweg $\mathrm{s}_{\mathrm{cv}, \mathrm{e}(\mathrm{m})}$ der Abbauwerkzeuge zwischen Ein- und Ausbau auf dem Schneidrad aus dem Modell und dem tatsächlichen Laufweg $\mathrm{S}_{\mathrm{cv}(\mathrm{m})}$ der Abbauwerkzeuge in den Referenzprojekten betragen $-13 \%$ bis $18 \%$. Abweichungen in der Schätzung des Laufwegs $\mathrm{S}_{\mathrm{cv}}(\mathrm{m})$ durch den Erwartungswert $\mathrm{s}_{\mathrm{cv}, \mathrm{e}(\mathrm{m})}$ sind schlüssig mit modellbedingten Abweichungen des Erwartungswertes der Anzahl an Werkzeugwechseln $n_{c, e(m)}$ von der tatsächlichen Anzahl der Werkzeugwechsel $n_{c(m)}$ verbunden. Diese betragen maximal $-17 \%$ bis $18 \%$. In Projekten, in denen die Wahrscheinlichkeit für eine Über- bzw. Unterschätzung der Anzahl an Werkzeugwechsel $n_{c(m)}$ um die genannten Beträge reduziert oder ausgeschlossen werden soll oder die Wahrscheinlichkeit für eine Überschätzung der maximal erreichbaren Vortriebsstrecken $L_{\max (m)}$ durch das Modell noch weiter verringert werden soll, ist eine Korrektur der Prognoseergebnisse mit Sicherheitsbeiwerten möglich.

Aus struktureller Sicht fehlt im Modell eine Schätzung der Auswirkungen von Schäden an den Abbauwerkzeugen durch Steine, Blöcke, Hindernisse oder Verklebungen. Die Vortriebsstrecken $L_{d, e(m)}$ aus dem Modell können die tatsächlichen Vortriebsstrecken $L_{d(m)}$ in diesen Fällen um Faktor 5 bis 20 überschätzen. Gleichzeitig liegt die Anzahl der Werkzeugwechsel $n_{c(m)}$ hier um 20 bis $90 \%$ über der geschätzten Anzahl $n_{c, e(m)}$ aus dem Modell. Die hohe Bandbreite der möglichen Abweichungen lässt keine pauschale Abminderung bzw. Erhöhung der Prognoseergebnisse für betroffene geotechnische Homogenbereiche zu. Die möglichen Abweichungen und Risiken können zutreffender z.B. als WorstCase-Szenario mit dem baubetrieblichen Anpassungsalgorithmus erarbeitet werden, indem die Vortriebsstrecken $L_{d, e(m)}$ schrittweise bis auf den angegebenen Umfang verringert werden und die Anzahl der Werkzeugwechsel $\mathrm{n}_{\mathrm{c}, \mathrm{e}(\mathrm{m})}$ analog erhöht wird.

Zusammenfassend erfüllen die erarbeiteten Zusammenhänge zwischen den Boden- und Gebirgseigenschaften, der Auslegung der Hydroschild TVM, der Steuerung des Abbauprozesses und dem Laufweg $\mathrm{s}_{\mathrm{c}}$ der Abbauwerkzeuge, die eingangs als Ziel gesetzte Erhöhung des Kenntnisstandes im Hinblick auf den Verschleiß der Abbauwerkzeuge beim Vortrieb mit Hydroschild TVM in Lockergesteinen. Die neu entwickelten Standards für die Erkundung und Dokumentation im Vortrieb erlauben eine bessere Anpassung der Aktivitäten spezifisch für Hydroschild TVM in Lockergesteinen. Das aus den Zusammenhängen erarbeitete Prognosemodell ermöglicht zuverlässige Schätzungen der Anzahl der verschleißbedingten Werkzeugwechsel und der dazu notwendigen Anzahl und Stationierung der Vortriebsunterbrechungen. Diese Informationen können einerseits genutzt werden, um eine zuverlässige Planung der Vortriebsdauer und Schätzung der Verschleißkosten zu erreichen. Andererseits können durch die vorlaufende Planung der Stationierung der Vortriebsunterbrechungen gezielt kritische Bereiche vermieden werden, in denen der Zugang zur Abbaukammer aufgrund der geotechnischen Rahmenbedingungen kritisch ist oder nur mit aufwändigen Zusatzmaßnahmen ermöglicht werden kann. Die erarbeiteten Erkenntnisse und das Prognosemodell tragen so auch zu einer Erhöhung der Planungs- und Verfahrenssicherheit für Hydroschild TVM in Lockergesteinen bei. 


\subsection{Diskussion und weiterführende Fragen}

\subsubsection{Vorerkundung und Dokumentation}

Die Analyse zur Dokumentation im Vortrieb zeigt deutliche Probleme bei der Erfassung des Zustandes der Abbauwerkzeuge und der tatsächlichen geotechnischen Bedingungen auf (Kap. 5.2).

Der Verschleißzustand der Abbauwerkzeuge wird zwischen Ein- und Ausbau auf dem Schneidrad durchschnittlich nur 2 bis 3 Mal bei einer Werkzeuginspektion in einer Vortriebsunterbrechung erfasst. Während der Inspektion steht der Bearbeiter häufig unter Zeitdruck, um die Stillstandszeit der TVM zu minimieren. Gleichzeitig lagen bisher keine klar definierten Verschleißgrenzen für die unterschiedlichen Werkzeugtypen in Lockergesteinen vor. Die Beurteilung des Verschleißzustandes und die Entscheidung für einen Werkzeugwechsel hängen folglich sehr stark von der bestehenden Erfahrung und dem subjektiven Eindruck des Bearbeiters ab. Die schriftliche und bildliche Zustandsdokumentation der Abbauwerkzeuge ist in den Referenzprojekten häufig lückenhaft. Zusätzlich ist denkbar, dass aus den genannten Gründen eine unmerkliche Fokussierung der Dokumentation des Verschleißzustandes auf sehr auffällige Verschleißphänomene in leicht zugänglichen Bereichen des Schneidrades stattfindet, während undeutlichere Verschleißphänomene in schwerer zugänglichen Bereichen nicht erfasst werden.

Der subjektive Einfluss des Bearbeiters bei der Zustandsbeurteilung der Abbauwerkzeuge im Vortrieb wird vermutlich nie vollständig auszuschließen sein. Die in der vorliegenden Arbeit entwickelte Definition unterschiedlicher Verschleiß- und Schadenstypen, die Verschleißgrenzen für die unterschiedlichen Abbauwerkzeugtypen (Kap. 5.3) in Lockergesteinen und das vorgestellte Prognosemodell (Kap. 6) können in Zukunft jedoch stark zur Vereinheitlichung der Dokumentation beitragen und als Leitfaden für den Bearbeiter dienen. Die Umgebungsbedingungen in der Abbaukammer und der gegebene Zeitdruck bei der Inspektion der Abbauwerkzeuge und dem Werkzeugwechsel wird dadurch jedoch nicht behoben. Ein Ansatz zur Verbesserung der Dokumentationsmöglichkeiten ist hier die Unterstützung des Bearbeiters durch ein elektronisches System. Dieses könnte einerseits bei der Erfassung der Position der Abbauwerkzeuge auf dem Schneidrad (Stationierung, Arm Nr., Spur Nr., Drehrichtung) helfen und andererseits über die Erfassung einer Werkzeug-ID-Nummer helfen, den Zustand und Wechsel der Abbauwerkzeuge mit zugehörigen Bilddaten und geotechnischen Daten in einem Datenbanksystem zu verbinden. Zusätzlich ist auch die weitere Entwicklung von automatisierten Verschleißerkennungssystemen denkbar, wie sie z.B. von WILLIS et al. (2011:68) für Schneidrollen vorgestellt werden, die eine ständige Überwachung des Verschleißzustandes ohne Zugang zur Abbaukammer ermöglichen. Bisher verfügbare Systeme liefern hier nur für einzelne Schneidspuren Alarmsignale, sobald ein definierter Verschleißzustand erreicht wird.

Die Dokumentation der geotechnischen Verhältnisse im Vortrieb unterliegt ähnlichen Einschränkungen wie die Dokumentation des Verschleißzustandes der Abbauwerkzeuge. Im Vergleich zum Wechsel der Abbauwerkzeuge wird der geotechnischen Dokumentation jedoch häufig eine niedrigere Priorität eingeräumt, sodass die verfügbare Zeit noch weiter eingeschränkt wird. Die Häufigkeit der Vortriebsunterbrechungen entlang der Tunnelstrecke ist im Vergleich zur Varianz der geotechnischen Bedingungen zum Teil sehr niedrig. Gleichzeitig ist während der Vortriebsunterbrechungen häufig nur ein Bruchteil der Ortsbrustfläche zugänglich. WENDL (2012) beschreibt hierzu einen Ansatz, wie mithilfe direkter Methoden in den Vortriebsunterbrechungen und indirekter Methoden, z.B. mit dem abgebauten Bodenmaterials aus der Separationsanlage, eine aussagekräftige Dokumentation der geotechnischen Verhältnisse im Vortrieb aufgebaut werden kann. Zusätzlich fehlt hier bisher eine durchgängigen Untersuchungen zum Materialfluss von der Ortsbrust in die Abbaukammer, durch die Förderleitung bis in die Separationsanlage. Innerhalb der Förderstrecke kommt es zu einer Durchmischung verschiedener Bodenarten aus der Ortsbrust und vermutlich in Abhängigkeit der Tunnellänge auch zu einer erheblichen Verschiebung der Korngrößenverteilung. In Kombination mit den eingeschränkten Zugangsmöglichkeiten zur Ortsbrust können Fehleinschätzungen der tatsächlichen geotechnischen Verhältnisse in der Ortsbrust letztlich nicht vollständig ausgeschlossen werden. Hierzu fehlen bisher detaillierte Untersuchungen mit einer ausreichend hohen Datenbasis.

Aus den Referenzprojekten liegen aufgrund der beschriebenen Einschränkungen bei der Dokumentation nur in sehr geringem Umfang geotechnische Daten aus den Vortrieben vor. Die Datenanalyse wurde deshalb weitgehend auf den Daten aus den geotechnischen Gutachten zur Vorerkundung der Projekte aufgebaut. Folglich kann nicht vollständig ausgeschlossen werden, dass in der Datenanalyse 
ein Versatz zwischen den prognostizierten geotechnischen Bedingungen und den tatsächlichen Bedingungen im Vortrieb vorliegt. Zusätzlich weist der Umfang an erkundeten geotechnischen Parametern in der Vorerkundung zwischen den Referenzprojekten große Unterschiede auf und es werden häufig keine verwertbaren Angaben zu den statistischen Verteilungseigenschaften der einzelnen geotechnischen Parameter angegeben. Als Folge daraus ist ein hoher Anteil der verfügbaren geotechnischen Parameter in den Referenzprojekten nicht durchgängig und einheitlich bestimmt, sodass diese für die Datenanalyse nicht genutzt werden können. Die mangelnden Angaben und Untersuchungen der statistischen Verteilungseigenschaften verhindern in der Datenanalyse die Anwendung weiterführender statistischer Methoden.

Die vorliegende Arbeit bietet mit dem neu entwickelten Standard an geotechnischen Kennwerten für die Erkundung und Vortriebsdokumentation einen guten Anhaltspunkt für zukünftige Projekte. Zu den realen statistischen Verteilungseigenschaften der verschiedenen Parameter innerhalb eines als homogen angenommenen Bodenkörpers liegen jedoch bisher kaum Erkenntnisse vor, sodass keine Empfehlungen zum Umfang der Vorerkundung abgeleitet werden können. Hierzu sind grundlegende Untersuchungen zu den statistischen Verteilungseigenschaften der unterschiedlichen geotechnischen Parameter und deren Korrelationen in Lockergesteinen notwendig.

\subsubsection{Datenanalyse}

In der Datenanalyse führen die genannten Probleme in der Dokumentation zunächst dazu, dass der beschriebene Katalog an Verschleiß- und Schadensbildern (Kap. 5.3) unter Umständen nicht vollständig ist. Darüber hinaus müssen bei der Aufbereitung der Daten (Kap. 5.6) trotz der subjektiven Einflüsse einheitliche Verschleißgrenzen in allen untersuchten Referenzprojekten angenommen werden. Aufgrund der geringen Dokumentationsdichte ist bei der Fokussierung der Analyse auf den Primärund Sekundärverschleiß der Abbauwerkzeuge eine sehr starke Filterung der Daten notwendig, sodass letztlich nur $23 \%$ der Werkzeugwechseldaten für die Datenanalyse zuverlässig als charakteristisch für den Primär- und Sekundärverschleiß der Abbauwerkzeuge identifiziert werden können. Die Probleme bei der Dokumentation der Werkzeugwechsel pflanzen sich also nicht nur qualitativ in die Beschreibung der Verschleißtypen fort, sondern auch quantitativ in die Datenanalyse und das darauf aufgebaute Prognosemodell. In Summe wird so von einem im Verhältnis relativ geringen Anteil der Daten auf eine vergleichsweise hohe Anzahl an Einflussfaktoren auf den Laufweg $\mathrm{s}_{\mathrm{c}}$ geschlossen. Folglich ist auch hier nicht auszuschließen, dass es noch Einflussfaktoren auf den Laufweg $\mathrm{s}_{\mathrm{c}}$ gibt, die aufgrund der strikten Filterung der Daten aus den Referenzprojekten nicht erfasst wurden.

Einen Anhaltspunkt zur Beurteilung der Erfassung der Einflussfaktoren auf den Laufweg $\mathrm{s}_{\mathrm{c}}$ in der Datenanalyse sind die Ergebnisse aus der deskriptiven Validierung des Prognosemodells (Kap. 6.6). Das Prognosemodell erlaubt eine gute Nachbildung der Anzahl $n_{1(T A)}$ und Stationierung $L_{1(\mathrm{~m})}$ der Vortriebsunterbrechungen $\mathrm{I}_{(\mathrm{m})}$ und der Anzahl der Werkzeugwechsel $\mathrm{n}_{\mathrm{c}(\mathrm{m})}$ in den Referenzprojekten, sodass angenommen werden kann, dass in der Datenanalyse die maßgeblichen Einflussfaktoren erfasst wurden. Andererseits weisen die nicht quantifizierbaren Einflussfaktoren (Tab. 22) auf den Laufweg $\mathrm{s}_{\mathrm{c}}$ über alle Referenzprojekte durchgehend eine relativ geringe Schwankungsbreite auf, z.B. aufgrund einer sehr ähnlichen Auslegung der Schneidräder und des Förderkreislaufs. Die Basisdaten und das Prognosemodell bilden folglich ein relativ homogenes Maschinencluster ab. Um eine breitere Anwendbarkeit des Prognosemodells zu erreichen, ist folglich die Quantifizierung bisher nicht nur qualitativ erfassbarer Faktoren notwendig. Aus der in Tab. 29 dargestellten Zusammenfassung haben vermutlich die folgenden nicht-quantifizierbaren Faktoren hohen Einfluss auf den Laufweg $\mathrm{s}_{\mathrm{c}}$ :

- Verschleißschutz der Abbauwerkzeuge

- Schneidradöffnungsverhältnis und Verteilung der Öffnungen über den Schneidradradius.

- Kornform und Kornrundungsgrad der Bodenkomponenten

In Bezug auf den Verschleißschutz besteht bei Schälmessern, Sticheln und Räumern das Problem, die verschiedenen Elemente des Verschleißschutzes quantitativ zusammenzufassen. Eine Möglichkeit kann z.B. die Erfassung der Abdeckung der Werkzeugoberflächen mit Hartaufträgen oder Hartmetalleinsätzen sein. Zusätzlich sind das verfügbare Verschleißvolumen und die jeweiligen Werkstoffeigenschafen zu berücksichtigen. 
Hierzu fehlen bisher unter anderem aufgrund der extrem hohen Vielfalt an Auslegungen und Werkstoffeigenschaften geeignete Methoden. Bisherige Untersuchungen beschränken sich deshalb auf Baustellentests, die zwar eine Aussage zur Auswirkung bestimmter Auslegungsunterschiede auf den Laufweg $\mathrm{s}_{\mathrm{c}}$ ergeben, jedoch auf den vorliegenden Satz an Umgebungsbedingungen beschränkt sind. Die in der Datenanalyse getroffene Einschränkung des Verschleißschutzes auf ein bestimmtes Mindestmaß stellt hier nur eine unbefriedigende Lösung dar, weil das Prognosemodell so auf Abbauwerkzeuge mit relativ hohem Verschleißschutz eingeschränkt wird. Aus wirtschaftlicher Sicht können unter bestimmten Bedingungen jedoch auch Abbauwerkzeuge mit niedrigerem Verschleißschutz sinnvoll eingesetzt werden.

Der Einfluss des Schneidradöffnungsverhältnisses und der Verteilung der Öffnungen über den Schneidradradius auf den Materialfluss des abgebauten Bodens von der Ortsbrust hinter das Schneidrad und den Sekundärverschleiß der Abbauwerkzeuge wurde bereits in einzelnen Projekten nachgewiesen. Zusätzlich geben auch die Untersuchungen zur Verschleißrate einen Hinweis auf diesen Einflussfaktor (Kap. 5.5). Mithilfe des normalisierten Materialflusses bzw. dem Verhältnis aus abgebautem Bodenvolumen und Schneidradöffnungsfläche, bezogen auf den Schneidradradius, kann dieser Einflussfaktor zumindest quantitativ erfasst werden. Ähnlich wie bei der Dichte der Stützflüssigkeit hängen die tatsächlichen Auswirkungen auf den Sekundärverschleiß der Abbauwerkzeuge und den Laufweg $\mathrm{s}_{\mathrm{c}}$ jedoch vermutlich stark von der Abrasivität der Bodenkomponenten ab. Eine Untersuchung mithilfe von Verschleißmessungen in unterschiedlichen Bedingungen könnte hierzu detailliertere Ergebnisse liefern.

Der Einfluss von Kornform und Kornrundungsgrad kann anhand der Indextests zur Abrasivität von Böden eindeutig nachgewiesen werden. In den Referenzprojekten fehlen jedoch die notwendigen Daten für eine Untersuchung, um den Einfluss auch direkt am Laufweg $\mathrm{s}_{\mathrm{c}}$ der Abbauwerkzeuge nachzuweisen. Beide Kennwerte sind folglich im neu entwickelten Standard für die geotechnische Vorerkundung und Dokumentation für Projekte mit Hydroschild TVM in Lockergesteinen aufgenommen. Aufgrund der bereits beschriebenen Probleme bei der Probennahme an der Ortsbrust ist auch eine Erfassung der Kornform und des Rundungsgrades in der Separationsanlage denkbar, um hohe Volumina an repräsentativen Daten zu erhalten. Dazu ist vorlaufend die bereits beschriebene Untersuchung des Materialflusses von der Ortsbrust bis zur Separationsanlage und die damit verbundene Verschiebung der Korngrößenverteilung notwendig. Für die Erfassung der Kornform und des Rundungsgrades sind zusätzlich zunehmend photogrammetrische Verfahren verfügbar, die eine Erfassung der beiden Parameter nahezu in Echtzeit z.B. auf einem Förderband oder dem Materialabwurf einer Siebstufe ermöglichen.

Die Bearbeitung der nicht quantifizierbaren Einflussfaktoren hängt übergreifend davon ab, dass geeignete Referenzprojekte vorhanden sind, in denen die zugehörigen Daten dokumentiert werden können. Vor allem für Stichel und Räumer sind im Verhältnis zu Schneidrollen und Schälmessern noch deutlich mehr Werkzeugwechseldaten notwendig. Anhand dieser Daten kann die für das Prognosemodell im Folgenden getroffene Annahme validiert werden, dass für Stichel und Räumer ein sehr ähnlicher Zusammenhang zwischen SAI und Laufweg $\mathrm{s}_{\mathrm{c}}$, sowie ein übereinstimmender Satz an Einflussfaktoren zutrifft.

Neben den nicht quantifizierbaren Einflussfaktoren kann in der Datenanalyse die Kontaktspannung $T_{\text {act }}$ $\left[\mathrm{kN} / \mathrm{m}^{2}\right]$ an der Oberfläche der Abbauwerkzeuge beim Bodenabbau nur mit der Scherfestigkeit $\mathrm{T}_{\mathrm{c}}$ $\left[\mathrm{kN} / \mathrm{m}^{2}\right]$ des Bodens als indikativer Vergleichswert für die Kontaktspannung $T_{\text {act }}$ untersucht werden. Dieser Ansatz kann zwar überschlägig mithilfe des normierten Schneiddrehmomentes $M_{\text {norm }}[\mathrm{kNm}]$ und der normierten Schneidradanpresskraft $F_{\text {norm }}[\mathrm{kN}]$ validiert werden. Dennoch sind für detaillierte Untersuchungen zum Einfluss der Kontaktspannung $T_{\text {act }}$ auf den Laufweg $\mathrm{s}_{\mathrm{c}}$ direktere Bestimmungsmethoden für die Kontaktspannung $T_{\text {act }}$ notwendig. Hierzu sind vor allem direkte Messungen der Schneidkräfte an einzelnen Abbauwerkzeugen denkbar. Entsprechende Messsysteme befinden sich bei verschiedenen Maschinenherstellern in Entwicklung, sind jedoch aufgrund der Umgebungsbedingungen in der Abbaukammer und dem eingeschränkten Zugang bisher mit Problemen bei der Datenaufnahme und der Übertragung der Daten aus der Abbaukammer behaftet. Die Messung der tatsächlichen Schneidkräfte kann langfristig dazu beitragen, diese anhand der Geometrie der Abbauwerkzeuge in die Kontaktspannungen $T_{\text {act }} z u$ übersetzen. So können vermutlich auch geeignete Methoden entwickelt werden, um die Schneidradanpresskraft $F_{S R}[k N]$ und das Schneiddrehmoment $M_{S R}[k N m]$ aus der Betriebsdatenerfassung der TVM auf die unterschiedlichen Abbauwerkzeugtypen und einzelne Abbauwerkzeuge auf dem Schneidrad aufzuteilen, um wiederum die anliegenden Kontaktspannungen $T_{\text {act }} z u$ erfassen. 
In der Datenanalyse konnten die folgenden Einflussfaktoren auf den Laufweg $\mathrm{s}_{\mathrm{c}}$ nicht untersucht werden:

- Mikroskopische Verschleißprozesse an den Werkzeugoberflächen

- Auswirkungen von Schäden durch Steine und Blöcke im Boden oder Verklebungen

Die Untersuchung der mikroskopischen Verschleißprozesse an der Oberfläche der Abbauwerkzeuge (Kap. 4.1) kann vermutlich dazu beitragen, die Korrelation zwischen dem Soil Abrasivity Index (SAI) und dem Laufweg $\mathrm{s}_{\mathrm{c}}$ besser zu verstehen. In bisherigen Veröffentlichungen wird davon ausgegangen, dass Abrasion der dominierende Verschleißmechanismus beim Abbau von Lockergesteinen ist. Sowohl bei Schneidrollen, als auch bei Schälmessern, Räumern und Sticheln ist jedoch zwischen SAIWerten von 250 bis 500 eine relativ starke Änderung der Korrelation zwischen SAI und Laufweg $\mathrm{S}_{\mathrm{c}}$ festzustellen. Dieser Übergang kann vermutlich mit einer stetigen Änderung der beteiligten Verschleißmechanismen an der Werkzeugoberfläche erklärt werden. Oberhalb dieses Bereichs erfolgt der Abrieb an der Oberfläche hauptsächlich durch Abrasion. Unterhalb dieses Bereichs verliert die Abrasion vermutlich etwas an Bedeutung, während Verschleißmechanismen mit weniger intensivem Materialabtrag, z.B. Adhäsion und Oberflächenermüdung, an Einfluss gewinnen. Die mikroskopischen Verschleißprozesse können in zukünftigen Projekten zur Untersuchung dieser Vermutung durch Aufnahmen der verschlissenen Oberflächen von Abbauwerkzeugen mit starken Lichtmikroskopen oder Rasterelektronenmikroskopen abgebildet werden.

Schäden an den Abbauwerkzeugen aufgrund von Steinen und Blöcken im Boden oder durch Verklebungen in bindigen Böden wurden in der Fokussierung der Datenanalyse auf Primär- und Sekundärverschleiß ausgeschlossen. Die Beschreibung der Verschleiß- und Schadenstypen (Kap. 5.3) liefert erste Erkenntnisse zu den Schadensmechanismen. Die deskriptive Validierung des Prognosemodells (Kap. 6.6.2) zeigt in den betroffenen Homogenbereichen einen hohen Einfluss der Schadensmechanismen auf den Vortrieb durch eine erhebliche Verkürzung der erreichbaren Vortriebsstrecken $L_{\max (m)}$ [m] und eine Erhöhung der Anzahl der Werkzeugwechsel $n_{c(m)}$ in den Vortriebsunterbrechungen $I_{(m)}$. Eine detaillierte Untersuchung der Schadensmechanismen und der Auswirkungen auf den Vortrieb erscheint deshalb zur zukünftigen Vervollständigung der Datenanalyse erforderlich. Aus der vorliegenden Analyse können erste Ansatzpunkte zur quantitativen Erfassung der Auswirkungen von Schäden abgeleitet werden.

Schäden durch Steine und Blöcke im Boden stellen eine Kette an Ereignissen dar, die bei entsprechender Untersuchung vermutlich mit probabilistischen Methoden in den folgenden Schritten beschrieben werden kann. Zunächst ist die Frage zu klären wie zuverlässig mit den in der Vorerkundung eingesetzten Methoden der reale Anteil an Steinen und Blöcken im Boden quantifiziert werden kann. Im nächsten Schritt kann daraus mithilfe des abzubauenden Bodenvolumens in der betroffenen Bodeneinheit die Eintrittswahrscheinlichkeit für das Antreffen eines Steins oder Blocks bzw. die wahrscheinliche Anzahl an Steinen und Blöcken in der Ortsbrust abgeschätzt werden. Die Verteilung der Steine oder Blöcke in der angeschnittenen Ortsbrustfläche entspricht in den überwiegenden Fällen einer Zufallsverteilung. Die Häufigkeit der Kontakte zwischen einem Abbauwerkzeug und einem Stein oder Block in der Ortsbrust hängt zusätzlich davon ab, wie weit diese in Abhängigkeit der Bodeneigenschaften und Werkzeugbestückung in der Ortsbrust abgebaut werden können und wie schnell die Steine, Blöcke oder verbleibende Bruchstücke aufgrund des Größenverhältnisses zu den Schneidradöffnungen hinter das Schneidrad transportiert werden können, oder vor dem Schneidrad mitgewältzt werden. Diese Vorgänge können ebenfalls mit verschiedenen Eintrittswahrscheinlichkeiten belegt werden. Abschließend ist zu klären, wie häufig der Kontakt eines Abbauwerkzeuges mit einem Stein oder Block in der Ortsbrust tatsächlich zu einem Schaden führt. Hierzu können durch genaue Aufnahmen des abgebauten Bodens und dem Schadensbild an den Abbauwerkzeugen ebenfalls Eintrittswahrscheinlichkeiten ermittelt werden. Die Ereigniskette vom Antreffen eines Steins oder Blocks bis zum Schaden an einem Abbauwerkzeug kann so vermutlich durchgehend mit Wahrscheinlichkeitsverteilungen beschrieben werden. So kann für jedes einzelne Abbauwerkzeug auf dem Schneidrad eine Eintrittswahrscheinlichkeit für einen Schaden innerhalb einer vorgegebenen Vortriebsstrecke definiert werden, die auch im empirischen Prognosemodell (Kap. 7.2.3) weiter verarbeitet werden kann. 
Für Schäden durch Verklebungen ist ein ähnlicher Ansatz zur probabilistischen Beschreibung denkbar. Das Potential für Verklebungen in Abhängigkeit der Bodeneigenschaften kann mit bestehenden Methoden qualitativ beschrieben werden (Kap. 4.2.2.1). Eine quantitative Beschreibung der Eintrittswahrscheinlichkeit für Verklebungen könnte vermutlich unter Einbeziehung des normalisierten Materialflusses erfolgen, da Verklebungen überwiegend in Schneidradbereichen angetroffen werden, in denen relativ wenig Öffnungsfläche zur Verfügung steht, bzw. ein hoher normalisierter Materialfluss notwendig ist. Ähnlich wie bei Schäden durch Steine und Blöcke führt nicht jede Verklebung am Schneidrad zu Schäden an den Abbauwerkzeugen. Entsprechend sind auch hierzu genaue Aufnahmen in betroffenen Vortrieben notwendig, um letztlich für jedes einzelne Abbauwerkzeug auf dem Schneidrad eine Eintrittswahrscheinlichkeit für einen Schaden innerhalb einer vorgegebenen Vortriebsstrecke zu schätzen, die wiederum im empirischen Prognosemodell (Kap. 7.2.3) weiter verarbeitet werden kann.

\subsubsection{Empirisches Prognosemodell}

Das bestehende empirische Prognosemodell konnte im Rahmen der vorliegenden Arbeit aus struktureller und deskriptiver Sicht validiert werden (Kap. 6.6). Die prognostische Validierung des Modells im Hinblick auf den Verschleiß der Abbauwerkzeuge in zukünftigen Projekten konnte im Rahmen der vorliegenden Arbeit aufgrund des eingeschränkten Zeitrahmens und mangelndem Zugang zu geeigneten Projekten nicht durchgeführt werden. Ein geeigneter Ansatz hierzu ist in Kap. 6.6.3 beschrieben.

Die weitere Entwicklung des bestehenden Prognosemodells kann in Zukunft ausdrücklich auf drei Ansatzpunkte abzielen:

- Erweiterung um eigene Schätzkurven für Stichel und Räumer

- Integration bisher nicht quantifizierbarer Einflussfaktoren

- Erweiterung um Schäden aufgrund von Steinen, Blöcken und Verklebungen

Die Erweiterung des Prognosemodells um eigene Schätzkurven für den Laufweg $\mathrm{s}_{\mathrm{c}}$ in Abhängigkeit des Soil Abrasivity Index (SAI) hängt in hohem Maß von der Dokumentation entsprechender Daten in zukünttigen Projekten ab. Anhand dieser kann die bisherige Vermutung der hohen Ähnlichkeit der Korrelation zwischen Laufweg $\mathrm{S}_{\mathrm{c}}$ und SAI bei Schälmessern, Räumern und Sticheln validiert und gegebenenfalls überarbeitet werden.

Mithilfe der Ergebnisse der Datenanalyse können in Zukunft vermutlich weitere bisher nur qualitativ erfassbare Einflussfaktoren auf den Laufweg $\mathrm{s}_{\mathrm{c}}$ quantifiziert werden. Zur Integration dieser Faktoren sind in Kap. 6.6.1 bereits entsprechende Ansatzpunkte beschrieben. Für das Schneidradöffnungsverhältnis und den Verschleißschutz der Abbauwerkzeuge sind im Prognosemodell bereits Korrekturfaktoren verankert, die jedoch bis zur Quantifizierung der beiden Faktoren ohne Auswirkung auf das Prognoseergebnis bleiben. Kornform und Rundungsgrad können analog zum Korndurchmesser $D_{60}$ $[\mathrm{mm}]$ in den Soil Abrasivity Index (SAI) integriert werden.

Bemerkenswert ist in diesem Zusammenhang, dass etablierte Indextests, wie z.B. der LCPC Test (LAK), aber auch der SAT-Test (SAT), jeweils starke Korrelationen mit dem Äquivalenten Quarzgehalt (ÄQU) [\%], der Korngrößenverteilung bzw. dem Korndurchmesser $D_{50}[\mathrm{~mm}]$ und dem Kornrundungsgrad aufweisen (Kap. 4.3.1). Für die Entwicklung des Prognosemodells steht damit theoretisch eine alternative Entwicklungsrichtung offen, sofern für einen der Indextests empirisch nachgewiesen werden kann, dass die Indexwerte für verschiedene Lockergesteine mit ausreichend hoher Genauigkeit aus dem Äquivalenten Quarzgehalt (ÄQu), dem Korndurchmesser $\left(D_{50} 0 . D_{60}\right)$ und dem Kornrundungsgrad geschätzt werden können. Für diesen Fall ist denkbar, dass der Soil Abrasivity Index (SAI) aus der Scherfestigkeit des Bodens $\mathrm{T}_{c}\left[\mathrm{kN} / \mathrm{m}^{2}\right]$ in der Ortsbrust auf Höhe der Tunnelachse bzw. den tatsächlichen Kontaktspannungen $T_{a c t}\left[\mathrm{kN} / \mathrm{m}^{2}\right]$ und dem Indexwert für die Abrasivität des Bodens zusammengesetzt werden kann, bzw. durch diese Variante ersetzt werden kann. Der Basiswert für den Laufweg $\mathrm{s}_{\mathrm{c}, \mathrm{b}(\mathrm{z})}[\mathrm{km}] \mathrm{im}$ Prognosemodell (Kap. 6.2) könnte dann, ausgehend von einer möglichen Korrelation der SAI-Variante mit dem Laufweg $\mathrm{s}_{c}$, geschätzt werden. Die übrige darauf aufbauende Modellstruktur und der Prognoseprozess (Kap. 6.1) behalten im Fall einer solchen Änderung ihre Funktion. 
In der vorliegenden Arbeit konnte dieser Weg aufgrund des mangelnden Datenvolumens zum LCPCTest und dem SAT-Test in den RP nicht überprüft werden. Zusätzlich zum bisher fehlenden Datenvolumen, sind bei den bestehenden Testverfahren (Kap. 4.3.1) noch weitere Untersuchungen in Bezug auf das Probenvolumen zur Bearbeitung grobkörniger Lockergesteine notwendig. Die Testapparaturen weisen Einschränkungen im Hinblick auf die Größe der Bodenkomponenten auf, sodass grobkörnige Lockergesteine zum Teil für die Durchführung der Tests gebrochen werden müssen. Dabei wird wiederum der Kornrundungsgrad als maßgeblicher Einflussfaktor auf die Abrasivität verändert. Dem entgegen steht ein hoher Aufwand zur Probengewinnung und zur Durchführung der Test, sowie ein starker Anstieg der benötigten Probenmenge und der Größe der Testapparatur, wenn die Testverfahren auch für grobkörnige Lockergesteine mit Korndurchmessern bis $63 \mathrm{~mm}$ (Grobkies) erweitert werden sollen. Um das notwendige Datenvolumen bei einer überschaubaren Anzahl an Projekten mit Hydroschild TVM zu generieren, ist darüber hinaus mittelfristig eine internationale Standardisierung einer der Indextestvarianten für die Abrasivität von Lockergesteinen zu empfehlen.

Abschließend sind für die uneingeschränkte Anwendung des Prognosemodells, wie eingangs beschrieben, Methoden notwendig, um die Auswirkungen von Schäden durch Steine, Blöcke und Verklebungen in das Prognosemodell zu integrieren. Sofern z.B. mithilfe der in Kap. 7.2.2 beschriebenen Ansätze eine quantitative Beschreibung der Auswirkungen auf den Laufweg $\mathrm{s}_{\mathrm{c}}$ gelingt, können diese im Prognosemodell in der Schätzung des Erwartungswertes für den Laufweg $\mathrm{s}_{\mathrm{c}, \mathrm{e}(\mathrm{z})}$ in betroffenen Homogenbereichen in Form von Korrekturfaktoren erfasst werden (Kap. 6.2). Eine Abminderung des Erwartungswertes für den Laufweg $\mathrm{S}_{\mathrm{c}, \mathrm{e}(\mathrm{z})}$ durch einen Korrekturfaktor für Schäden wirkt sich in der Schätzung der Vortriebsstrecken $L_{d, e(m)}$ zwischen Vortriebsunterbrechungen $I_{(m)}$ in Form einer erheblichen Verkürzung der maximal erreichbaren Vortriebsstrecken $L_{\max (m)}$ aus. Die Anzahl der notwendigen Werkzeugwechsel $\mathrm{n}_{\mathrm{c}(\mathrm{m})}$ steigt im Gegenzug stark an. Die Auswirkungen von Schäden an den Abbauwerkzeugen können auch in der deskriptiven Validierung des Prognosemodells (Kap. 6.6.2) gezeigt werden. Die Abweichungen zwischen den geschätzten Vortriebsstrecken $L_{d, e(m)}$ und der Anzahl an Werkzeugwechseln $\mathrm{n}_{\mathrm{c}, \mathrm{e}(\mathrm{m})}$ von den tatsächlichen Werten weisen hier eine so hohe Varianz auf, dass keine pauschalen Korrekturfaktoren abgeleitet werden können.

Die Untersuchung der Auswirkungen von Schäden durch Steine oder Blöcke ist in der bestehenden Form des Prognosemodells daher eher mithilfe von verschiedenen Szenarien für die Auswirkungen der Schäden zu empfehlen (Kap. 6.5.2). Die Daten aus den Referenzprojekten und aus der deskriptiven Validierung bieten hierzu grobe Anhaltspunkte zur quantitativen Einschätzung der Auswirkungen der Schäden (Kap. 6.6.4). Die erreichbare Länge der Vortriebsstrecken $L_{d(\mathrm{~m})}$ zwischen den Vortriebsunterbrechungen sinkt auf Grund der Schäden deutlich. Als Erfahrungswert aus den RP kann hierzu mit Bezug auf die maximal erreichbaren Vortriebsstrecken $L_{\max (m)}$ (Gl. 83) aus dem Prognosemodell für die reduzierten Vortriebsstrecken $L_{d,(m)}$ bei Schäden an den Abbauwerkzeugen der Wertebereich in Gl. 144 geschätzt werden.

$$
\mathrm{L}_{\max (\mathrm{m})} \cdot 0,05<\mathrm{L}_{\mathrm{d}(\mathrm{m})}<\mathrm{L}_{\max (\mathrm{m})} \cdot 0,2
$$

Der Erwartungswert für die Vortriebsstrecken $L_{d, e(m)}$ sollte in betroffenen Homogenbereichen bei der Bildung eines Szenarios mit Schäden an den Abbauwerkzeugen entsprechend reduziert werden. Ein ähnlicher Wertebereich kann auch für die Anzahl der Werkzeugwechsel $n_{c(m)}$ bei Schäden an den Abbauwerkzeugen mit Bezug auf den Erwartungswert der Anzahl der Werkzeugwechsel $n_{c, e(m)}$ eingegerenzt werden (Gl. 145).

$$
\mathrm{n}_{\mathrm{c}, \mathrm{e}(\mathrm{m})} \cdot 1,2<\mathrm{n}_{\mathrm{c}(\mathrm{m})}<\mathrm{n}_{\mathrm{c}, \mathrm{e}(\mathrm{m})} \cdot 2
$$

Der Erwartunsgwert für die Anzahl der Werkzeugwechsel $n_{c, e(m)}$ sollte folglich bei der Bildung von Szenarien mit Schäden an den Abbauwerkzeugen entsprechend erhöht werden. Die tatsächliche Größenordnung der Auswirkungen der Schäden ergibt sich erst aus der Kombination der erhöhten Anzahl an Vortriebsunterbrechungen $I_{(m)}$ und der darin jeweils ansteigenden Anzahl an Werkzeugwechseln $\mathrm{n}_{\mathrm{c}(\mathrm{m})}$. 
Ähnlich wie die Untersuchung der Auswirkungen von Schäden auf die Vortriebsstrecken $L_{d, e(m)}$ und die Anzahl der Werkzeugwechsel $\mathrm{n}_{\mathrm{c}, \mathrm{e}(\mathrm{m})}$ kann bisher auch die Auswirkung der natürlichen Schwankungsbreite von geotechnischen Parametern nur empirisch durch Ausarbeitung verschiedener Vortriebsszenarien mit dem baubetrieblichen Anpassungsalgorithmus im Prognosemodell (Kap. 6.5) analysiert werden. Zusätzlich fehlen hierzu in der Erkundung der geotechnischen Verhältnisse in den Referenzprojekten häufig die notwendigen Angaben zu den statistischen Verteilungseigenschaften der geotechnischen Parameter (Kap. 7.2.1). Zum besseren Verständnis der Wechselwirkungen im Prognosemodell ist eine detaillierte Sensitivitätsanalyse denkbar, in der die Auswirkungen der Schwankungsbreite aller Eingangsparameter auf die Vortriebsstrecken $L_{d, e(m)}$ und die Anzahl der Werkzeugwechsel $\mathrm{n}_{\mathrm{c}, \mathrm{e}(\mathrm{m})}$ anhand von deren statistischen Verteilungseigenschaften untersucht werden. Mithilfe dieser Analyse kann die erforderliche Genauigkeit bei der Bestimmung der geotechnischen Parameter für die Vorerkundung und die Dokumentation im Vortrieb mit Hydroschild TVM in Lockergesteinen vermutlich besser eingeschätzt werden.

Die praktische Anwendung des Prognosemodells mithilfe von gängigen Tabellenkalkulationsprogrammen ist aufgrund der iterativen Prozessschritte (Kap. 6.1.3) bei der Bestimmung der maximal erreichbaren Vortriebstrecken $L_{\max (m)}$ und in der baubetrieblichen Anpassung des Prognosemodells relativ aufwändig. Gleichzeitig muss jedes einzelne Abbauwerkzeug auf dem Schneidrad durch den vollständigen Prognoseprozess verfolgt werden. Zur Reduktion des Aufwands sind zwei Ansätze gegeben. Einerseits ist die Umsetzung des Prognosemodells als Software denkbar, die unter Umständen auch mit dem für die Dokumentation der Werkzeugwechsel und der geotechnischen Bedingungen vorgeschlagenen Datenbanksystem (Kap. 7.2.1) verbunden werden kann. Andererseits ist die Entwicklung von überschlägigen Schätzmethoden für die wesentlichen Größen im Prognosemodell wie der Vortriebstrecken $L_{l, e(m)}$ und der Anzahl der Werkzeugwechsel $n_{c(m)}$ denkbar. Die Reduktion des Aufwandes für die Verschleißprognose führt mit diesen Methoden jedoch vermutlich auch zu einer deutlichen Reduktion der Prognosegenauigkeit und zu höheren Risiken für erhebliche Abweichungen der Daten im Vortrieb von den Prognoseergebnissen.

Beim Vortrieb in Lockergesteinen mit Erddruckschilden (EPB TVM) bestehen sehr ähnliche Herausforderungen (Kap. 1.2) im Hinblick auf die Planung der Vortriebsunterbrechungen und der Werkzeugwechsel, sowie deren mögliche Auswirkungen auf Vortriebsdauer, Verschleißkosten und Verfahrenssicherheit wie beim Vortrieb mit Hydroschild TVM in Lockergesteinen. Die Struktur und der Prognoseprozess des Prognosemodells für den Primär- und Sekundärverschleiß der Abbauwerkzeuge bei Hydroschild TVM in Lockergesteinen sind vermutlich auch auf EPB TVM übertragbar. Allerdings muss für EPB TVM der Satz an Einflussfaktoren auf den Laufweg $\mathrm{S}_{\mathrm{c}}$ separat untersucht werden. Die Ergebnisse für Hydroschild TVM hierzu und insbesondere die Korrelationen des Soil Abrasivity Index (SAI) mit dem Laufweg $\mathrm{s}_{\mathrm{c}}$ sind nicht übertragbar. Die notwendigen Anpassungen im Prognosemodell konzentrieren sich deshalb wahrscheinlich auf die Schätzung des Erwartungswertes des Laufwegs $\mathrm{s}_{\mathrm{c}, \mathrm{e}(\mathrm{z})}$ für EPB TVM in Lockergesteinen in Sektion 1. Die Prozessschritte in den Sektionen 2-4 sind unabhängig vom Maschinentyp und daher theoretisch auch auf EPB TVM in Lockergesteinen übertragbar.

Abschließend ist eine Integration des vorgestellten Prognosemodells in übergreifende Simulationsmodelle für die logistischen Abläufe auf der TVM bzw. zwischen TVM und Baustelleneinrichtungsfläche denkbar. Entsprechende Modelle werden z.B. von SADRI et al. (2012: 555) beschrieben. Die Anordnung der Vortriebsunterbrechungen $I_{(m)}$ für Werkzeugwechsel entlang der Tunnelachse (TA) stellt hier eine wichtige Eingangsgröße zur Bestimmung der Vortriebsdauer dar. 


\subsection{Anwendung des Prognosemodells in der Praxis}

Das empirische Prognosemodell für den Primär- und Sekundärverschleiß der Abbauwerkzeuge bei Vortrieben mit Hydroschild TVM in Lockergesteinen ist in der vorgestellten Fassung nicht auf bestehende Indextests für die Abrasivität des Bodens, wie z.B. den LCPC-Test, angewiesen. Im neu entwickelten Standard zur Vorerkundung geotechnischer Parameter für Projekte mit Hydroschild TVM in Lockergesteine (Tab. 11) und für die Dokumentation im Vortrieb (Kap. 5.2.2) ist der LCPC-Test aus folgenden Gründen dennoch als Kennwert enthalten:

- Der LCPC-Test kann im Vertragsverhältnis zwischen AG (Bauherr) und AN (Bauunternehmung) wie bisher als Kennwert für die Abrasivität des Bodens genutzt werden.

- Durch die weitere Anwendung des LCPC-Tests kann das notwendige Datenvolumen generiert werden, um den in Kap. 7.2.2 angesprochenen alternativen Weg zur Schätzung des Erwartungswertes für den Laufweg $\mathrm{s}_{\mathrm{c}, \mathrm{e}(\mathrm{z})} \mathrm{zu}$ untersuchen.

Die Indextests für die Abrasivität weisen regional stark unterschiedliche Verbreitung und Akzeptanz auf. Zusätzlich ist z.B. der modifizierte LCPC-Test nach DRUCKER (2011b: 222) mittlerweile in eine nationale Norm in Österreich aufgenommen. Bis zu der in Kap.7.2.2 empfohlenen internationalen Standardisierung eines Indexwertes für die Abrasivität des Bodens können folglich anstatt des LCPCTests auch andere Indexversuche (Kap. 4.3.1) zum Einsatz kommen.

In der Angebotskalkulation und Planung von Vortrieben mit Hydroschild TVM kann der AN (Bauunternehmung) das Modell zur Schätzung der Anzahl der Werkzeugwechsel $n_{c(T A)}$ und der Anzahl $n_{\text {I(TA) }}$ und Stationierung der Vortriebsunterbrechungen $I_{(m)}$ als Grundlage zur Bestimmung der Vortriebsdauer und Verschleißkosten nutzen. In dieser Projektphase verfügt der AN häufig noch nicht über die detaillierte Auslegung der TVM. Zusätzlich müssen Betriebsparameter der TVM geschätzt werden:

- Erwartungswert für die Vortriebsgeschwindigkeit $\mathrm{V}_{\mathrm{TVM}}[\mathrm{mm} / \mathrm{min}]$

- Erwartungswert für die Schneidraddrehzahl U [min $\left.{ }_{-1}\right]$

- Erwartungswert für die Penetration $\mathrm{p}_{\mathrm{e}}[\mathrm{mm} / \mathrm{U}]$

- Erwartungswert für die Dichte der Stützflüssigkeit $\rho_{S F}\left[g / m^{3}\right]$

- Erwartungswert für den Stützdruck $P_{S F}[b a r]$

Die Bearbeitung der Verschleißprognose in der Angebotskalkulation und der Planungsphase des Vortriebs erfordert folglich die Zusammenarbeit zwischen dem AN und dem Maschinenhersteller.

Selbst bei vollständiger Verfügbarkeit aller Basisdaten für das Prognosemodell, verbleiben die in Kap. 6.6.4 beschriebenen Risiken für eine Unterschätzung der Anzahl der Werkzeugwechsel $n_{c(T A)}$ und eine Überschätzung der Vortriebsstrecken $L_{d(m)}$ aufgrund der Prognosegenauigkeit des Modells. Diese können aus vertraglicher Sicht im Regelfall nicht an den AG (Bauherr) übertragen werden. Das Prognosemodell erlaubt dem AN dennoch diese Risiken im Verhältnis zum AG zu bearbeiten:

- Die baubetriebliche Anpassung im Modell (Kap.6.5) erlaubt die Erarbeitung verschiedener Vortriebsszenarien. Hiermit können die Auswirkungen z.B. von Fehleinschätzungen der Betriebsparameter der TVM, verschiedener Auslegungsvarianten der TVM oder der Varianz geotechnischer Kennwerte überprüft werden. So kann neben dem Planungsszenario und den darin enthaltenen Verschleißkosten auch deren mögliche Bandbreite bei günstiger oder ungünstiger Entwicklung ermittelt werden. Die Chancen und Risiken, die mit einem bestimmten Planwert für die Verschleißkosten in das Angebot des AN einfließen, können so besser eingeschätzt werden.

- Die Erwartungswerte für die Anzahl der Werkzeugwechsel $n_{c, e(m)}$ und die Vortriebsstrecken $\mathrm{L}_{\mathrm{d}, \mathrm{e}(\mathrm{m})}$ zwischen den Vortriebsunterbrechungen $\mathrm{I}_{(\mathrm{m})}$ können mit Sicherheitsbeiwerten beaufschlagt bzw. abgemindert werden (Kap. 6.6.4). 
Beide Ansätze führen theoretisch eher zur Berücksichtigung von zu hohen, als zu niedrigen Planwerten für die Verschleißkosten und die Vortriebsdauer, woraus sich tendenziell ein Nachteil für den AN im Bieterverfahren ergibt. Deshalb werden vom AN häufig auch gegenüber dem Maschinenhersteller folgende Maßnahmen zur Reduktion der Verschleißkosten bzw. der Risiken für eine Überschreitung des Planwertes angesetzt:

- Abtrennung der vertraglichen Vereinbarungen zur Lieferung und Sanierung der Abbauwerkzeuge vom Werkliefervertrag für die TVM. Die Preise und Lieferbedingungen für die Abbauwerkzeuge können dann nachrangig zur TVM diskutiert werden, sobald die Auslegung der TVM im Detail feststeht.

- Teilweiser Transfer des Risikos für eine Überschreitung des Erwartungswertes für die Anzahl der Werkzeugwechsel $n_{c(T A)}$ und der Verschleißkosten an den Maschinenlieferanten durch Pauschal- oder Laufmetervereinbarungen zur Lieferung der Abbauwerkzeuge.

Der TVM-Lieferant (TL) hat in diesem Verhältnis seinerseits die Möglichkeit, die Chancen und Risiken, die mit einer solchen Vereinbarung verbunden sind, deutlich besser einzuschätzen. Zusätzlich kann der TL durch eine intensive Begleitung des Vortriebs und eine stetige Optimierung der Auslegung der TVM und der Steuerung des Abbauprozesses in enger Zusammenarbeit mit dem AN seinen Projekterfolg steigern.

Für die Vortriebsphase ist entscheidend, dass als Ergebnis der Verschleißprognose ein Planungsszenario festgelegt wird (Kap. 6.5.3), das neben den Basisdaten für die Verschleißprognose als zentrale Angaben die Stationierung $L_{l, e(m)}[m]$ der Vortriebsunterbrechungen $I_{(m)}$, die Anzahl der Werkzeugwechsel $\mathrm{n}_{\mathrm{c}(\mathrm{m})}$ und geplante Zusatzmaßnahmen enthält.

Dieses Szenario muss im Vortrieb nachverfolgt und rollierend angepasst werden. Dazu ist ein Mindestaufwand an Dokumentation und Abstimmung erforderlich:

- Dokumentation der Werkzeugwechsel (Kap. 5.2.1)

- Dokumentation der angetroffenen geotechnischen Verhältnisse (Kap. 5.2.2)

- Dokumentation der Auslegung der Hydroschild TVM (Kap. 5.2.3)

- Dokumentation der Steuerung des Abbauprozesses (Kap. 5.2.4)

- Regelmäßige Abstimmung des AN und des AG über die angetroffenen geotechnischen Verhältnisse und mögliche Auswirkungen auf die Anordnung der Vortriebsunterbrechungen $I_{(m)}$.

- Regelmäßige Abstimmung des AN mit dem Maschinenlieferanten zur Optimierung der Steuerung des Abbauprozesses und der Auslegung der TVM.

Die erwarteten Verschleißkosten $K_{v, e}$ können mithilfe der Stationierung $L_{l, e(m)}$ der geplanten Vortriebsunterbrechungen $\mathrm{I}_{(\mathrm{m})}$ und verschiedener Verteilungsschlüssel für die nicht ortsabhängigen Anteile der Verschleißkosten (Tab. 23) als kumulierte Kurve in Abhängigkeit der Vortriebsstationierung dargestellt werden. Auf Basis der laufenden Dokumentation und der rollierenden Anpassung des Planungsszenarios erhält der AN so die Möglichkeit eine Kostentrendanalyse (KTA) für die Verschleißkosten im Vortrieb mitzuführen. Die tatsächliche Entwicklung der Verschleißkosten $K_{v}$ kann so direkt gegen die erwarteten Verschleißkosten $\mathrm{K}_{\mathrm{v}, \mathrm{e}}$ angetragen werden. Die Auswirkungen von Abweichungen zwischen prognostizierten und tatsächlichen geotechnischen Verhältnissen, sowie Auswirkungen von Anpassungen der Steuerung des Abbauprozesses oder der Auslegung der TVM auf die Verschleißkosten $\mathrm{K}_{\mathrm{v}}$ können so frühzeitig erkannt und gegebenenfalls bearbeitet werden.

Das Prognosemodell bietet hier für alle Beteiligten eine erhebliche Verbesserung der Diskussionsgrundlage für die Steuerung des Vortriebsablaufs und wird abschließend auch der eingangs (Kap. 1.3) gestellten Forderung nach der Möglichkeit zur Berücksichtigung unterschiedlicher Projektziele im Hinblick auf die Kostenentwicklung, Vortriebsdauer und Begrenzung von Risiken gerecht. 


\section{Literaturverzeichnis}

Al-AmeEn, S. I. \& WALLER, M. D. (1994): The influence of rock strength and abrasive mineral content on the Cerchar Abrasivity Index. - Engineering Geology, 36: 293 - 301, Amsterdam (Elsevier).

Anagnostou, G. \& Kovari, K. (1996): Face stability in slurry and EPB shield tunneling. - In: MaIR, R. J. \& TAYLOR, R. N. (Hrsg.): Proceedings of the Symposium on Geotechnical Aspects of Underground Construction in Soft Ground, S. 379 - 384, London (Balkema).

BABENDERERDE, L. (2000): Schwachstellen beim Betrieben von Vortriebsmaschinen mit flüssigkeitsgestützter Ortsbrust: Ursachen, Folgen und Maßnahmen zur Behebung. - In: STUDIENGESELLSCHAFT FÜR UNTERIRDISCHE VERKEHRSANLAGEN E.V. (2000) (Hrsg.): Unterirdisches Bauen 2000: Herausforderungen und Entwicklungspotentiale, S. 78 - 83, Düsseldorf (Alba Fachverlag).

BABENDERERDE, L. (2003): TBM mit Slurry- bzw. Erddruckstützung - Einsatzbereiche und Zuverlässigkeitsanalyse. - Felsbau, 21: 3 - 6, Essen (VGE).

BABENDERERDE, S. \& HolZHÄUSER, J. (1999): Betriebszustand Druckluftstützung beim Hydroschild. - In: DEUTSCHE GESELLSCHAFT FÜR GEOTECHNIK (DGGT) (Hrsg.): Taschenbuch für den Tunnelbau 2000, S. 231 - 252, Essen (VGE).

BARZegari, G., UROMEIHY, A. \& ZhAO, J. (2013): A newly developed soil abrasion testing method for tunnelling using shield machines. - Quarterly Journal of Engineering Geology and Hydrogeology, 46, 1: 63 - 74, London (The Geological Society).

BeretitsCh, F. (1992): Kräftespiel im System Schneidwerkzeug - Boden. - IMB Institut für Maschinenwesen und Baubetrieb, Reihe F, 41: 158 S., Karlsruhe (Universität Fridericina).

BezulJen, A. \& TALmon, A. M. (2006): Calculation models based on monitoring during tunnel construction. - Tunneling and Underground Space Technology, 21: 380 - 381, Amsterdam (Elsevier).

BüCHI, E., MAthiER, J. F. \& WYSS, C. (1995): Gesteinsabrasivität - ein bedeutender Kostenfaktor beim mechanischen Abbau von Fest- und Lockergesteinen. - tunnel, 5: 38 - 45, Gütersloh (Bauverlag).

BuRger, W. (2006): Schneidräder für Lockergestein: Prinzipien und Auslegungsaspekte. - tunnel, 2 : 8 - 19, Gütersloh (Bauverlag).

CERChAR - Centre d`Etudes et Recherches de Charbonnages de France (1986): The Cerchar Abrasivenessindex.- 12 S., Verneuil.

DEUTSCHER Ausschuss für UNTERIRDISCHES BAUEN (DAUB) (2010): Empfehlung zur Auswahl von Tunnelvortriebsmaschinen. - In: DEUTSCHE GESELLSCHAFT FÜR GEOTECHNIK (DGGT) (Hrsg.): Taschenbuch für den Tunnelbau 2011, S. 231 - 299, Essen (VGE).

Dowden, P. B. \& Robinson, R. A. (2001): Coping with boulders in soft ground TBM tunneling. - In: HansmiRe, F. \& GauRING, B. (Hrsg.): RETC Proceedings 2001, S. 961 - 977, Littelton (Society for Mining, Metallurgy and Exploration Inc.). 
DrUCKER, P. (2011a): Aussagekraft des LCPC-Abrasivitätskoeffizienten am Beispiel eines rezenten Donauschotters. - Geomechanics and Tunnelling, Volume 4, 6: 681 - 691, Berlin (Ernst \& Sohn).

DRUCKER, P. (2011b): Abrasivität von Lockergesteinen und der Werkzeugverschleiß im Tief- und Tunnelbau. - Österreichische Ingenieur- und Architekten-Zeitschrift, Jahrgang 156, 1-12: 219 225, Wien (Österreichischer Ingenieur- und Architekten-Verein).

Düllmann, J., Hollmann, F., Thewes, M. \& Alber, M. (2013): Analysis of Soil-Machine-Interactions (Part 1): Processing of TBM-Machine-Data and Extraction of Excavation-specific Data. Proceedings of the Third International Conference on Computational Methods in Tunnelling and Subsurface Engineering, S. 621 - 634, Freiburg (Aedificatio Publishers).

Düllmann, J., AlbeR, M. \& PlinningeR, R., J. (2014): Bewertung der Abrasivität von Lockergesteinen mit Indexverfahren und herkömmlichen Bodenkennwerten. - Geomechanics and Tunnelling, Volume 7, 1: 87 - 97, Berlin (Ernst \& Sohn).

FRENZEL, C. (2010a): Verschleißkostenprognose für Schneidrollen bei maschinellen Tunnelvortrieben in Festgesteinen. - Münchner Geowissenschaftliche Abhandlungen, Reihe B, Ingenieurgeologie Hydrogeologie Geothermie,15: 64 S., München (Verlag Dr. Friedrich Pfeil).

FRENZEL, C. (2010b): Werkzeugverschleiß bei Tunnelvortriebsmaschinen. - In: DeUTSCHE GeselLSCHAFT FÜR GEOTECHNIK (DGGT) (Hrsg.): Taschenbuch für den Tunnelbau 2011, S. 301 336, Essen (VGE ).

Frenzel, C. \& BABEndererde, T. (2011): Tool wear in TBM tunnelling. - Tunnelling Journal, April/Mai 2011: 36 - 42, Maidstone (TSG Media).

GFT - Gesellschaft F. Tribologie E.V. (2002): Arbeitsblatt 7 - Tribologie. - 52 S., Aachen (Gesellschaft für Tribologie e.V.)

Gharahbagh, E. A., Rostami, J. \& Palomino, A. M. (2011): New soil abrasion testing method for soft ground tunneling applications. - Tunneling and Underground Space Technology, 26: 604 613, Amsterdam (Elsevier).

GIRMSCHEID, G. (2004): Tunnelbohrmaschinen - Vortriebsmethoden und Logistik. - In: BERGMEISTER, K. \& WÖRNER, J. D. (Hrsg.): Beton-Kalender 2005, S. 119 - 256, Berlin (Ernst \& Sohn).

GIRMSCHEID, G. (2008): Baubetrieb und Bauverfahren im Tunnelbau. - 2. Auflage, 697 S., Berlin (Ernst \& Sohn).

GÖRTZ, O. (2002): Entwicklung der Tübbingauskleidung im Tunnelbau. - 108 S., München (GRIN Verlag).

Goss, C. M. (2002): Predicting boulder cutting in soft ground tunneling. - In: OzDEMIR, L. (Hrsg.): North American Tunneling: Proceedings 2002, S. 37 - 46, Rotterdam (Balkema).

GRÜBL, F. \& SPEIER, L. (2013): Maschinelle Tunnelvortriebe mit optimierter Stützdrucksteuerung.- tunnel, 2: 46 - 54, Gütersloh (Bauverlag). 
GWILDIS, U., SASS, I., GILBERT, M. (2011): Einfluss der Bodenabrasivität beim maschinellen Tunnelbau. - In: TiedemAnN, J. (Hrsg.): Tagungsband 18. Tagung für Ingenieurgeologie, S. 193 - 199, Berlin (DGGT).

HERRENKNECHT, M. \& BÄPPLER, K. (2007): Compressed air work with tunnel boring machines. - In: BARTÁK, J., HRDina, I., ROMAnCOV, G., Zlámal, J. (Hrsg.): Underground Space - the $4^{\text {th }}$ Dimension of Metropoises, S. 1175 - 1181, London (Taylor \& Francis).

HollmanN, F., Thewes, M. (2011): Bewertung der Neigung zur Ausbildung von Verklebungen und zum Anfall von gelöstem Feinkorn bei Schildvortrieben im Lockergestein. - In: TIEDEMANN, J. (Hrsg.): Tagungsband 18. Tagung für Ingenieurgeologie, S. 237 - 244, Berlin (DGGT).

Hollmann, F., TheWES, M. (2012): Bewertung der Neigung zur Verklebungsbildung und zur Feinkornfreisetzung bei Schildvortrieben. - Geomechanics and Tunnelling, 5, 5: 574 - 580, Berlin (Ernst \& Sohn).

Hollmann, F., Düllmann, J., Thewes, M. \& Alber, M. (2013): Analysis of Soil-Machine-Interactions (Part 2): Influences on the Excavation-specific Data of TBM-Machine Data. - Proceedings of the Third International Conference on Computational Methods in Tunnelling and Subsurface Engineering, S. 635 - 648, Freiburg (Aedificatio Publishers).

HOLZHÄUSER J. \& NILSEN B. (2006): Abrasivität von Lockergestein bei Tunnelvortrieben - Aktuelle Erfahrungen aus Baupraxis und Entwicklung eines neuen Laborversuchs. - In: DEUTSCHE GESELLSCHAFT FÜR GEOTECHNIK (DGGT) (Hrsg.): Vorträge der Baugrundtagung 2006 in Bremen, S. 169 - 176, Essen (VGE).

HOLZHÄUSER J., MAYER, C. \& HUNT, S. W. (2007): Erfahrungen bei Tunnelvortrieben im Lockergestein und im Fels bei sehr hohem Grundwasserdruck. - Tiefbau, 6, 199: 379 - 385, Berlin (Erich Schmidt Verlag GmbH \& Co.).

JAKOBSEn P. D., DAHL, F. (2010): Soil abrasion in TBM tunnelling. - In: ECKERT, L. R., FOWLER, M. E. \& SMITHSON, M. F. JR. (Hrsg.): Proceedings of the 11th KTA International Symposium on Mechanized Tunneling Technology, S. 55 - 63, Seoul (KTA).

Jakobsen P. D., LangmaACK, L., DahL, F. \& Breivik, T. (2012): Predicting the abrasivity of in-situ like soils. - Tunnels \& Tunnelling International, Juni 2012, 6: 36 - 38, San Francisco (World Market Intelligence).

Jakobsen P. D., Bruland, A. \& DaHL, F. (2013a): Review and assessment of the NTNU/SINTEF Soil Abrasion Test (SAT ${ }^{\mathrm{TM}}$ ) for determination of abrasiveness of soil and soft ground. - Tunneling and Underground Space Technology, 37: 107 - 114, Amsterdam (Elsevier).

Jakobsen P. D., LANGMAaCK, L., DahL, F. \& Breivik, T. (2013b): Development of the Soft Ground Abrasion Tester (SGAT) to predict TBM tool wear, torque and thrust. - Tunneling and Underground Space Technology, 38: 398 - 408, Amsterdam (Elsevier).

JANCSECZ, S. \& STEINER, W. (1994): Face Support for large Mixshield in heterogeneous ground conditions. - In: Institution of Mining and Metallurgy, British TunNelling SOCIETy (Hrsg.): Tunneling '94: Papers presented at the seventh international symposium Tunneling '94, S. 531 - 550, London (Chapman \& Hall). 
KÄSLING, H., ThIELE, I. \& ThURO, K. (2007): Abrasivitätsuntersuchungen mit dem Cerchar-Test - Eine Evaluierung der Versuchsbedingungen. - In: OTTO, F. (Hrsg.): Tagungsband 16. Tagung für Ingenieurgeologie, S. 229 235, Bochum (Technische Fachhochschule Georg Agricola).

KäSLING H. \& THURO, K. (2010a): Determining abrasivity of rock and soil in the laboratory. - In: WILLIAMS, A. L., PINCHES, G. M., ChIN, C. Y., MCMORRAN, T. J. \& MASSEY, C.I. (Hrsg.): Geologically Active: Proceedings of the 11th IAEG Congress, S. 1973 - 1980, London (CRC Press).

KÄSLING H. \& THURO, K. (2010b): Betsimmung der Gesteinsabrasivität - Versuchstechnik \& Anwendung. - In: DEUTSCHE GeSELLSCHAFT Für GEOTECHNIK (DGGT) (Hrsg.): Vorträge der Baugrundtagung 2010 in München, S. 233 - 240, Essen (VGE).

KÄSLING H. \& THURO, K. (2010c): Determining rock abrasivity in the laboratory. - In: ZHAO, J., LABIOUSE, V., DUDT, J-P., MATHIER, J-F. (Hrsg.): Proceedings of the European Rock Mechanics Symposium EUROCK 2010, S. 425 - 428, Amsterdam (CRC Press).

Kieffer, S. D., Leelasukseree, C. \& Mustoe, G. G. W. (2008): Behaviour of disc cutters in boulder laden ground. - In: ROACH, F., KRITZER, M. R., OfiARA, D. \& TOWNSEND, B. F. (Hrsg.): North American Tunneling: 2008 Proceedings, S. 129 - 136, Littelton (Society for Mining, Metallurgy and Exploration Inc.).

KÖHLER, M., MAIDL, U. \& MARTAK, L. (2011): Abrasivität und Werkzeugverschleiß beim Schildvortrieb im Lockergestein. Geomechanics and Tunnelling, Volume 4, 1: 36 53, Berlin (Ernst \& Sohn).

KöPPL, F., FrenZEL, C., \& ThURO, K. (2009): Statistische Modellierung von Gesteinsparametern für die Leistungs- und Verschleißprognose bei TBM Vortrieben. - In: SchWERTER, R. (Hrsg.): Tagungsband der 17. Tagung für Ingenieurgeologie, S. 415 - 417, Zittau (DGGT).

KÖPPL, F., \& THURO, K. (2013a): Verschleißprognose für Mix-Schild TVM in Lockergesteinen. - In: THURO, K (Hrsg.): Tagungsband der 19. Tagung für Ingenieurgeologie, S. 55 - 60, München (DGGT).

KÖPPL, F., \& THURO, K. (2013b): Cutting tool wear and management of wear related risks for MixShield TBM in soft ground. - Proceedings of the $18^{\text {th }}$ International Conference on Soil Mechanics and Geotechnical Engineering, S. 1739 - 1742, Paris (Presse du Pont).

KolYMBAS, D. (2011): Geotechnik - Bodenmechanik, Grundbau und Tunnelbau. - 3. Auflage, 595 S., Berlin (Springer Verlag).

KRAUteR, D. (2008): When boulders attack! - Roller Cutters in Soft Ground. - Tunnel Business Magazine, Februar 2008, 2: 22 - 23, Brecksville, (Benjamin Media).

Maidl, B., HeRRENKNeCht, M. \& ANHeuser, L. (1995): Maschineller Tunnelbau im Schildvortrieb. - 1. Auflage, $471 \mathrm{~S}$., Berlin (Ernst \& Sohn).

MAIDL, B. (2004): Handbuch des Tunnel- und Stollenbaus, Band 1: Konstruktionen \& Verfahren. 3. Auflage: 422 S., Essen (VGE).

Maidl, B., HerRenknecht, M., Maidl, U. \& Wehrmeyer, G. (2011): Maschineller Tunnelbau im Schildvortrieb. - 2. Auflage, 492 S., Berlin (Ernst \& Sohn). 
McKeYS, E. \& ALI, O. S. (1977): The cutting of soil by narrow blades. - Journal of Terramechanics, Volume 14, 2: 43 - 58 London (Pergamon Press).

MiedemA, S. A. (1989): On the cutting forces in saturated sand of a seagoing cutter suction dredger. In: VAN DAM, V. L. \& Wilson, J. R. (Hrsg.): Proceedings of the $12^{\text {th }}$ World Dredging Congress, S. 332 - 352, Virginia (Western Dredging Association).

MiedemA, S. A. (1992): New developments of cutting theories with respect to dredging. - In: van Dam, V. L. (Hrsg.): Proceedings of the $13^{\text {th }}$ World Dredging Congress, S. $618-636$, Bombay (World Organisation of Dredging Associations).

MiedemA, S. A. (1995): Production estimation based on cutting theories for cutting water saturated sand. - In: VAN DAM, V. L. (Hrsg.): Proceedings of the $14^{\text {th }}$ World Dredging Congress, S. 225 - 275, Delft (Central Dredging Association CEDA).

NAgel, F. StAscheit, J. \& MeschKe, G. (2010): Prozessorientierte numerische Simulation schildgestützter Tunnelvortriebe in Lockerböden. - Geomechanics and Tunnelling, Volume 3, 3: 268 282, Berlin (Ernst \& Sohn).

Nilsen, B., Dahl, F., Holzhäuser, J., Raleigh, P. (2006a): Abrasivity of soils in TBM tunneling. - Tunnels \& Tunneling International, März 2006, 3: 36 - 38, San Francisco (World Market Intelligence).

Nilsen, B., Dahl, F., Holzhäuser, J., Raleigh, P. (2006b): Abrasivity testing for rock and soils. - Tunnels \& Tunneling International, April 2006: 47 - 49, San Francisco (World Market Intelligence).

Nilsen, B., Dahl, F., Holzhäuser, J., Raleigh, P. (2006c): SAT: NTNU`s new soil abrasion test. - Tunnels \& Tunneling International, Mai 2006: 43 - 45, San Francisco (World Market Intelligence).

Nilsen, B., Dahl, F., Holzhäuser, J., Raleigh, P. (2007): New test methodology for estimating the abrasiveness of soils for TBM tunneling. - In: TRAYLOR, M. T. \&TOWNSEND, J. W. (Hrsg.): RETC 2007 proceedings, S. 104 - 116, Littelton (Society for Mining, Metallurgy and Exploration).

OECD Research Group on Wear of Engineering Materials (1969): Friction, Wear and Lubrication Tribology Glossary of Terms and Definitions, 56 S., Paris (OECD).

PlinNingeR, R. J. (2002): Klassifizierung und Prognose von Werkzeugverschleiß bei konventionellen Gebirgslösungsverfahren im Festgestein. - Münchner Geologische Hefte, Reihe B, Angewandte Geologie, 17: 146 S., München (Verlag Dr. Friedrich Pfeil).

PlinNingeR, R. J., KÄSLING, H. \& THURO, K. (2004): Wear prediction in hard rock excavation using the CERCHAR Abrasivness Index (CAI).- In: SCHUBERT, H. (Hrsg.): Rock engineering - theory and practice,S.599 - 604, Essen (VGE).

ReHM, U. \& Wehrmeyer, G. (2008): Maschinenkonzept für den maschinellen Tunnelvortrieb unter schwierigsten Bedingungen im Unterinntal/A. - tunnel, 1: 32 - 39, Gütersloh (Bauverlag).

RosiwaL, A. (1896): Neue Untersuchungsergebnisse über die Härte von Mineralien und Gesteinen. Verhandlg. d. k. k. geol. R.-A., S. 475 - 491, Wien. 
RosiwAL, A. (1916): Neuere Ergebnisse der Härtebestimmung von Mineralien und Gesteinen. Ein absolutes Maß der Härte spröder Körper. - Verhandlg. d. k .k. geol. R.-A., S. 117 - 147, Wien.

RostAMI, J. (1997): Development of a force estimation model for rock fragmentation with disc cutters through theoretical modeling and physical measurement of crushed zone pressure. - Unveröff. Diss., 382 S., Golden (Colorado School of Mines).

Rostaml J. (2005): CAl testing and its implications. - Tunnels \& Tunneling International, October: 43 45, San Francisco (World Market Intelligence).

Rostami J., OzdemiR, L., Bruland, A., \& DaHL, F. (2005): Review of issues related to cerchar abrasivity testing and their implications on geotechnical investigations and cutter cost estimates. - In: HUTTON, J. D. (Hrsg.): RETC 2005 proceedings, S. 738 - 751, Littelton (Society for Mining, Metallurgy and Exploration).

SADRI, K., Rahm, T., Duhme, J. R., KöNIG, M. \& TheWes, M. (2012): Prozesssimulation von maschinellen Tunnelvortrieben. - Schriftenreihe der Forschungsvereinigung Bau- und Baustoffmaschinen e.V. (FVB), 44: 551 - 570, Dresden (FVB).

SANDER, P. \& SpIEgel, M. (2011): Der Trugschluss der exakten Zahl Fortschrittlichere Wege zur Kosten- und Risikoanalyse. - bau aktuell, März 2011: 65 - 69, Wien (Linde Verlag).

SchimatZeK, J. \& KNATZ, H. (1970): Der Einfluss des Gesteinsaufbaus auf die Schnittgeschwindigkeit und den Meißelverschleiß von Streckenvortriebsmaschinen. - Glückauf, 106: 274 - 278, Essen (VGE).

SchimazeK, J. \& KNATZ H. (1976): Die Beurteilung der Bearbeitbarkeit von Gesteinen durch Schneidund Rollenbohrwerkzeuge. - Erzmetall, Band 29, 3: 113 - 119, Stuttgart (Riederer Verlag).

SCHLICKI, G. (1989): Adhäsion im Boden-Werkzeug System.- IMB Institut für Maschinenwesen und Baubetrieb, Reihe F, 39: 126 S., Karlsruhe (Universität Fridericina).

ScholZ, M. \& WendL, K. (2010): Geological aspects of slurry shield drives. - In: WilLIAMS, A. L., Pinches, G.M., Chin, C.Y., McMorRan, T.J. \& MASSEY, C.I. (Hrsg.): Geologically Active: Proceedings of the 11th IAEG Congress, S. 3507 - 3513, London (CRC Press).

SCHOLZ, M. \& WENDL, K. (2011): Vortriebsbedingte Verdichtung der Ortsbrust bei Mixschildvortrieben. In: TIEDEMANN, J. (Hrsg.): Tagungsband 18. Tagung für Ingenieurgeologie, S. 229 - 234, Berlin (DGGT).

SCHWARZ, J., SCHMIDT, J., MAIDL, R. \& HANDKE, D. (2006): Stützdruckberechnung beim Hydroschildvortrieb - Stand der Technik, dargestellt am City-Tunnel-Leipzig. - In: VOGT, N. (Hrsg.): Beiträge zum 5. Geotechnik-Tag in München - Geotechnik im Verkehrswegebau, S. 117 - 136, München (Zentrum Geotechnik).

SMITH, K. (2011): High pressure interventions. - Tunnelling Journal, Dezember 2011/Januar 2012: 10 18, Maidstone (TSG Media Ltd.).

STEIN, D. (2003): Grabenloser Leitungsbau. - 1. Auflage: 1121 S., Berlin (Ernst \& Sohn). 
Stroppel, T. (1953): Zur Systematik der Technologie des Schneidens. - Grundlagen der Landtechnik, 5:120 - 134, Düsseldorf (VDI Verlag).

THEWES, M. (1999): Adhäsion von Tonböden beim Tunnelvortrieb mit Flüssigkeitsschilden. - Berichte aus Bodenmechanik und Grundbau der Bergischen Universität Wuppertal, FB Bauingenieurwesen, 21: 190 S., Aachen (Shaker Verlag).

THEWES, M. (2003): Adhäsion von Tonböden beim Vortrieb mit Flüssigkeitsschilden. - Geotechnik, 26. Jahrgang, 4: 253 - 261, Berlin (Erst \& Sohn).

THEWES, M. \& BURGER, W. (2003): Verklebungen beim Schildvortrieb in Tonformationen - Erkennen und Begrenzen technischer und vertraglicher Risiken. - Forschung + Praxis, 40: 182 - 187, Gütersloh (Bauverlag).

THEWES, M (2004): Verklebungen beim Schildvortrieb. - In: DEUTSCHE GESELLSCHAFT FÜR GEOTECHNIK (DGGT) (Hrsg.): Taschenbuch für den Tunnelbau 2005, S. 89 - 116, Essen (VGE).

THURO, K. (2002): Geologisch-felsmechanische Grundlagen der Gebirgslösung im Tunnelbau. Münchner Geologische Hefte, Reihe B, Angewandte Geologie, 18: 160 S., München (Verlag Dr. Friedrich Pfeil).

THURO, K., SINGER, J., KÄSLING, H., BAUER, M. (2006): Abrasivitätsuntersuchungen an Lockergesteinen im Hinblick auf die Gebirgslösung. - In: DEUTSCHE GESELLSCHAFT FÜR GEOTECHNIK (DGGT) (Hrsg.): Vorträge der Baugrundtagung 2006 in Bremen, S. 283 - 290, Essen (VGE).

ThuRO, K., SingeR, J., KÄsLING, H., BAUER, M. (2007): Determining abrasivity with the LCPC Test.-In: Eberhardt, E., Stead, D., MorRISOn, T. (Hrsg.): Proceedings of the 1st Canada - U.S. Rock Mechanics Symposium, S. 827 - 834, Vancouver (Taylor \& Francis).

ThURO, K. \& KÄsLING, H. (2009a): Klassifikation der Abrasivität von Boden und Fels. - Geomechanik und Tunnelbau, 2: 179 - 188, Berlin (Ernst \& Sohn).

THURO, K. \& KÄSLING, H. (2009b): Klassifikation der Abrasivität von Locker- und Festgesteinen - Minimierung des Untergrundrisikos. - In: SCHWERTER, R. (Hrsg.): Tagungsband der 17. Tagung für Ingenieurgeologie mit Forum für junge Ingenieurgeologen, S. 137 - 142, Zittau-Görlitz (DGGT).

TUCKER, M., E. (2011): Sedimentary Petrology. An introduction to the origin of sedimentary rocks. 3. Auflage, 251 S., Oxford (Blackwell).

UETZ, H. (1982): Einfluss der Befeuchtung auf den Abrasivgleitverschleiß. - Aufbereitungstechnik, 10, Gütersloh (Bauverlag).

UETZ, H. (1986): Abrasion und Erosion - Grundlagen, Betriebliche Erfahrungen, Verminderung. 829 S., München/Wien (Carl Hanser Verlag).

VAlANTIN, A. (1973): Test Cerchar pour la mesure de la dureté et de l'abrasivitédes roches. - In: Annexe de l'exposée présenté aux Journées de Information „Techniques de creusement", Luxemburg. 
VERHOEF, P. N. W. (1997): Wear of rock cutting tools: Implications for the site investigation of rock dredging projects. - 327 S., Rotterdam (A. A. Balkema).

VAN OS, A. G. \& VON LEUSSEN, W. (1987): Basic research on cutting forces in saturated sand. - Journal of Geotechnical Engineering, Volume 113, 12: S. 1501 1516, New York (American Society of Civil Engineers ASCE).

WEH, M., ZWICK, O. \& ZIEGLER, M. (2009a): Maschinenvortrieb in verklebungsanfälligem Baugrund, Teil 1. - tunnel, 1: 25 - 37, Gütersloh (Bauverlag).

WEH, M., ZWICK, O. \& ZIEGLER, M. (2009b): Maschinenvortrieb in verklebungsanfälligem Baugrund, Teil 2. - tunnel, 2: 18 - 28, Gütersloh (Bauverlag).

WENDL, K. \& SCHOLZ, M. (2009): Die Schildvortriebe in den BEG-Baulosen H3-4 und H8 im Unterinntal - Eine Herausforderung für die ingenieurgeologische Dokumentation. - In: SCHWERTER, R. (Hrsg.): Tagungsband der 17. Tagung für Ingenieurgeologie mit Forum für junge Ingenieurgeologen, S. 443 - 446, Zittau-Görlitz (DGGT).

WeNDL, K., Scholz, M., \& THURO, K. (2010): A new approach to engineering geological documentation of slurry shield drives. - In: Williams, A. L. , PInCHES, G. M., ChIn, C. Y., McMorRan, T. J. \& MASSEY, C.I. (Hrsg.): Geologically Active: Proceedings of the 11th IAEG Congress, S. 3827 3834, London (CRC Press).

WENDL, K. (2011): Möglichkeiten und Grenzen der ingenieurgeologischen Dokumentation von Hydroschildvortrieben - Erkenntnisse aus den Vortrieben im Unterinntal. - In: Tagungsband der 31. Baugrundtagung in München, S. 127 - 134, München (DGGT).

WENDL, K. \& THURO K. (2011): Auswirkung von Hydroschildvortrieben auf den Grundwasserkörper am Bespiel von zwei Vortrieben in Tirol / Österreich. - In: TiedemanN, J. (Hrsg.): Tagungsband 18. Tagung für Ingenieurgeologie, S. 221 - 227, Berlin (DGGT).

WENDL, K. (2012): Ingenieurgeologische Vortriebsdokumentation und Auswertung von Hydroschildvortrieben. - Münchner geowissenschaftliche Abhandlungen, Reihe B, Ingenieurgeologie, Hydrogeologie, Geothermie, 18: 148 S., München (Verlag Dr. Friedrich Pfeil).

WENDL, K., ScholZ, M. \& THURO, K. (2012): Charakterisierung der ingenieurgeologische Vortriebsdokumentation von Hydroschildvortrieben am Beispiel der Baulose H3-4 und H8 im Unterinntal. - Geotechnik, 36. Jahrgang, 3: 168 - 176, Berlin (Ernst \& Sohn).

WEST, G. (1989): Technical Note - Rock Abrasiveness Testing for Tunnelling. - International Journal of Rock Mechanics and Mining Sciences, Volume 26, 2: 151 - 160, Amsterdam (Elsevier).

WILMS, J. (1995): Zum Einfluss der Eigenschaften des Stützmediums auf das Verschleißverhalten eines Erddruckschildes. - In: KUHNE, V. (Hrsg.): Mitteilungen aus dem Fachgebiet Baubetrieb und Bauwirtschaft, 12: 183 S., Essen (Universität Essen).

Willis, D., ShanahAN, A. \& Box, Z. (2011): Rollenmeißel - Fernüberwachung im Tunnelbau. - tunnel, 8: 68 - 75, Gütersloh (Bauverlag).

ZUM GAHR, K. H. (1987): Microstructure and wear of materials. - Tribology series, 10: 560 S., Amsterdam (Elsevier). 


\section{Zitierte Normen:}

AFNOR - Association Francaise de Normalisation (1990): NF P18-579: Granulats: Essai d’abrasivité et de broyabilité. - Paris (AFNOR).

AFNOR - Association Francaise de NoRmalisation (2000): NF P94-430-1: Détermination du pouvoir abra-sive d'une roche - Partie 1: Essai de rayure avec une pointe. - Paris (AFNOR).

ASTM - AMERICAN SOCIETY FOR Testing AND Materials (2001): ASTM G75-01: Standard Test Method for Determination of Slurry Abrasivity (Miller Number) and Slurry Abrasion Response of Materials (SAR Number). - Berlin (Beuth).

DIN - DeUTSChes INSTITUT FÜR NORMUng (1979): DIN 50320: 1979-12: Verschleiß; Begriffe; Systemanalyse von Verschleißvorgängen; Gliederung des Verschleißgebietes. - 8 S., ohne Nachfolgedokument im Nov. 1997 zurückgezogen, Berlin (Beuth).

DIN - DeUtsches INSTITUt Für NoRmung (1981): DIN 6583: 1981-09: Begriffe der Zerspanungstechnik; Standbegriffe. - 64 S., Berlin (Beuth).

DIN - DeUTSChES INSTITUT FÜR NORMUNG (1996): DIN 18126: 1996-11: Baugrund, Untersuchung von Bodenproben - Bestimmung der Dichte nicht bindiger Böden bei lockerster und dichtester Lagerung. - 9 S., Berlin (Beuth).

DIN - DeUtSches INSTITUT FÜR NORMUNG (1998): DIN 18130-1: 1998-05: Baugrund - Untersuchung von Bodenproben; Bestimmung des Wasserdurchlässigkeitsbeiwerts - Teil 1: Laborversuche. - 19 S., Berlin (Beuth).

DIN - DEUTSChES INSTITUT FÜR NORMUNG (2005): DIN ISO/TS 17892-2: 2005-01: Geotechnische Erkundung und Untersuchung - Laborversuche an Bodenproben - Teil 2: Bestimmung der Dichte von feinkörnigem Boden. - 14 S., Berlin (Beuth).

DIN - DEUTSChES INSTITUT FÜR NORMUNG (2005): DIN ISO/TS 17892-3: 2005-01: Geotechnische Erkundung und Untersuchung - Laborversuche an Bodenproben - Teil 3: Bestimmung der Korndichte, Pyknometerverfahren. - 10 S., Berlin (Beuth).

DIN - DEUTSCHES INSTITUT FÜR NORMUNG (2005): DIN ISO/TS 17892-4: 2005-01: Geotechnische Erkundung und Untersuchung - Laborversuche an Bodenproben - Teil 4: Bestimmung der Korngrößenverteilung. - 26 S., Berlin (Beuth).

DIN - DeUTSCHES INSTITUT FÜR NORMUNG (2005): DIN ISO/TS 17892-12: 2005-01: Geotechnische Erkundung und Untersuchung - Laborversuche an Bodenproben - Teil 12: Bestimmung der Zustandsgrenzen. - 14 S., Berlin (Beuth).

DIN - DeUTSCHES INSTITUT FÜR NORMUNG (2006a): DIN EN ISO 6507-1: 2006-03: Metallische Werkstoffe - Härteprüfung nach Vickers - Teil 1: Prüfverfahren. - 23 S., Berlin (Beuth).

DIN - DeUTSChEs INSTITUT FÜR NORMUNG (2006b): DIN EN ISO 6507-2: 2006-03: Metallische Werkstoffe - Härteprüfung nach Vickers - Teil 2: Prüfung und Kalibrierung der Prüfmaschine. - 19 S., Berlin (Beuth). 
DIN - DEUTSChES INSTITUT FÜR NORMUNG (2006c): DIN EN ISO 6507-3: 2006-03: Metallische Werkstoffe - Härteprüfung nach Vickers - Teil 3: Kalibrierung von Härtevergleichsplatten. - 12 S., Berlin (Beuth).

DIN - DEUTSChES INSTITUT FÜR NORMUNG (2006d): DIN EN ISO 6507-4: 2006-03: Metallische Werkstoffe - Härteprüfung nach Vickers - Teil 4: Tabellen zur Bestimmung der Härtewerte. - 51 S., Berlin (Beuth).

DIN - DEUTSChEs INSTITUT FÜR NORMUNG (2006e): DIN EN ISO 6508-1: 2006-03: Metallische Werkstoffe - Härteprüfung nach Rockwell - Teil 1: Prüfverfahren. - 27 S., Berlin (Beuth).

DIN - DeUTSches INSTITUT FÜR NORMUng (2006f): DIN EN ISO 6508-2: 2006-03: Metallische Werkstoffe - Härteprüfung nach Rockwell - Teil 2: Prüfung und Kalibrierung der Prüfmaschine. - 21 S., Berlin (Beuth).

DIN - DeUTSChEs INSTITUT FÜR NORMUNG (2006g): DIN EN ISO 6508-3: 2006-03: Metallische Werkstoffe - Härteprüfung nach Rockwell - Teil 3: Kalibrierung von Härtevergleichsplatten. - 15 S., Berlin (Beuth).

DIN - DeUtsches INSTITUT FÜR NORMUNG (2008): DIN EN 1097-8: 2009: Prüfverfahren für mechanische und physikalische Eigenschaften von Gesteinskörnungen - Teil 8: Bestimmung des Polierwertes. - 30 S., Berlin (Beuth).

DIN - DEUTSChES INSTITUT FÜR NORMUNG (2010): DIN 18137-1: 2010-07: Baugrund, Untersuchung von Bodenproben - Bestimmung der Scherfestigkeit - Teil 1: Begriffe und grundsätzliche Versuchsbedingungen. - 32 S., Berlin (Beuth).

DIN - DEUTSChES INSTITUT FÜR NORMUNG (2010): DIN 18137-2: 2011-04: Baugrund, Untersuchung von Bodenproben - Bestimmung der Scherfestigkeit - Teil 2: Triaxialversuch.- 48 S., Berlin (Beuth).

DIN - DeUtSches INSTITUT FÜR NORMUNG (2011a): DIN 4085: 2011-05: Baugrund - Berechnung des Erddrucks. - 56 S., Berlin (Beuth).

DIN - DeUTSches INSTITUT FÜR NORMUNG (2011b): DIN 18196: 2011-05: Erd- und Grundbau - Bodenklassifikation für bautechnische Zwecke. - 9 S., Berlin (Beuth).

DIN - DeUTSches INSTITUT FÜR NORMUNG (2011c): DIN EN ISO 14688-1: 2011-06: Geotechnische Erkundung und Untersuchung - Benennung, Beschreibung und Klassifizierung von Boden Teil 1: Benennung und Beschreibung. - 16 S., Berlin (Beuth).

DIN - DeUtSches INSTITUT FÜR NORMUNG (2011d): DIN EN ISO 14688-2: 2011-06: Geotechnische Erkundung und Untersuchung - Benennung, Beschreibung und Klassifizierung von Boden Teil 2: Grundlagen für Bodenklassifizierung. - $16 \mathrm{~S}$., Berlin (Beuth).

DIN - DEUTSCHES INSTITUT FÜR NORMUNG (2012): DIN 18127: 2012-09: Baugrund, Untersuchung von Bodenproben - Proctorversuch. - 32 S., Berlin (Beuth).

ISRM INTERNATIONAL SOCIETY FOR ROCK MEChANICS (1978): Suggested Methods for deternmining tensile strength of rock materials. - Commission on Standardization of Laboratory and Field Tests, Int. J. Rock Mech. Min. Sci. \& Geomech. Abstr., Vol. 15, 3, S. 99 - 103, Amsterdam, (Elsevier). 


\section{Zitierte Gesetzestexte:}

BGBI. I S. 1909: Druckluftverordnung vom 4. Oktober 1972 (BGBI. I S.1909), die zuletzt durch Artikel 6 der Verordnung vom 18. Dezember 2008 (BGBI. I S. 2768) geändert worden ist.

\section{Internetzitate:}

www-01: URL: http://www.wf-ingbau.de/unternehmen/historie/ab-1975/1988.html; abgerufen am 13.02.2013.

www-02: URL: http://www.herrenknecht.de/projekte/projektsuche-detailansicht.html; abgerufen am 13.02.2013.

www-03: URL: http://www.herrenknecht.de/verfahren-technologie/maschinentechnik/mixschild.html; abgerufen am 13.02.2013

www-04: URL: http://www.herrenknecht.de/verfahren-technologie/geotechnik.html; abgerufen am 13.02.2013

www-05: URL: http://www.herrenknecht.de/verfahren-technologie/ausbauverfahren/tuebbingausbau.html; abgerufen am 13.02.2013.

www-06: URL: http://www.e6.com/wps/wcm/connect/E6_Content_EN/Home/Materials+and+products/ Tungsten+carbide+products; abgerufen am 14.02.2013.

www-07: URL: http://www.tribo.de/i1/Hartmetall/Hartmetall_Eigenschaften.aspx; abgerufen am 14.02.2012.

www-08: URL: http://www.castolin.com/sites/default/files/ckfinder/files/de-Verschleissbestandige _Werkstoffe_Kobalt_Hartlegierungen.pdf; abgerufen am 14.02.2013.

www-09: URL: http://www.hardox.com/Global/HARDOX/Brochures/en/011_SSAB_plate_welding _UK.pdf; abgerufen am 15.02.2013.

www-10: URL: http://www.lcpc.fr; abgerufen am 25.02.2013. 


\section{Anhang: Abkürzungen \& Formelzeichen}

\begin{tabular}{|c|c|c|c|}
\hline Begriff & Symbol & Einheit & Bemerkung \\
\hline \multicolumn{4}{|l|}{ Allgemeine Abkürzungen } \\
\hline Abbildung & Abb. & - & Abbildungsbeschriftung und Verweise auf Abbildungen im Text. \\
\hline Tabelle & Tab. & - & Tabellenbeschriftung und Verweise auf Tabellen im Text. \\
\hline Gleichung & Gl. & - & Gleichungsnummerierung und Verweise auf Gleichungen im Text. \\
\hline Kapitel & Kap. & - & Kapitelverweise im Text. \\
\hline \multicolumn{4}{|c|}{ Mathematische Größen \& Kennwerte } \\
\hline Korrelationskoeffizient & $\mathrm{R}$ & $\%$ & $\begin{array}{l}\text { Größe zur Bestimmung der Stärke eines positiven oder negativen Zusammen- } \\
\text { hangs zwischen zwei Zufallsvariablen. }\end{array}$ \\
\hline Bestimmtheitsmaß & $\mathrm{R}^{2}$ & - & $\begin{array}{l}\text { Größe zur Beschreibung des Anteils der Streuung einer abhängigen Variablen, } \\
\text { der in einer Regressionsanalyse durch eine unabhängige Variable erklärt } \\
\text { werden kann. }\end{array}$ \\
\hline Standardabweichung & $\sigma$ & - & Streuung der Werte einer Zufallsvariable um den Erwartungswert (Mittelwert). \\
\hline Irrtumswahrscheinlichkeit & $\alpha$ & $\%$ & $\begin{array}{l}\text { Wahrscheinlichkeit für einen Fehler 1. Art bzw. a-Fehler in einem Hypothesen- } \\
\text { test, z.B. in einem zweiseitigen t-Test. }\end{array}$ \\
\hline $\mathrm{Pi}$ & $\pi$ & - & $\begin{array}{l}\text { Kreiszahl bzw. Zahlenwert für das allgemeine Verhältnis zwischen Umfang und } \\
\text { Durchmesser eines Kreises }(3,14159) \text {. }\end{array}$ \\
\hline \multicolumn{4}{|c|}{ Chemische Elemente \& Verbindungen } \\
\hline Kohlenstoff & $\mathrm{C}$ & - & Symbol für das chemische Element Kohlenstoff. \\
\hline Kobalt & Co & - & Symbol für das chemische Element Cobalt. \\
\hline Chrom & $\mathrm{Cr}$ & - & Symbol für das chemische Element Chrom. \\
\hline Mangan & $\mathrm{Mn}$ & - & Symbol für das chemische Element Mangan. \\
\hline Nickel & $\mathrm{Ni}$ & - & Symbol für das chemische Element Nickel. \\
\hline Wolframcarbid & WC & - & $\begin{array}{l}\text { Chemische Verbindung aus den Elementen Wolfram und Kohlenstoff. Symbol } \\
\text { für Wolframcarbid bzw. Abkürzung für den Begriff Hartmetall. }\end{array}$ \\
\hline \multicolumn{4}{|l|}{ Tunnelbauwerk } \\
\hline Auftraggeber & $A G$ & - & Bauherr bzw. Auftraggeber eines Tunnelbauprojektes. \\
\hline Auftragnehmer & AN & - & Bauunternehmer bzw. Auftragnehmer eines Tunnelbauprojektes. \\
\hline TVM-Lieferant & TL & - & $\begin{array}{l}\text { Hersteller bzw. Lieferant der Tunnelvortriebsmaschine (TVM) für ein Tunnel- } \\
\text { bauprojekt. }\end{array}$ \\
\hline Referenzprojekt & $\mathrm{RP}$ & - & Abkürzung und Verweise für Referenzprojekt. \\
\hline Vortrieb & VT & - & Abkürzung und Verweise für Vortriebe. \\
\hline Tunnelvortriebsmaschine & TVM & - & Abkürzung und Verweise für Tunnelvortriebsmaschinen. \\
\hline Erddruckschild & EPB TVM & - & $\begin{array}{l}\text { Tunnelvortriebsmaschine mit vollflächigem Bodenabbau und erddruckgestütz- } \\
\text { ter Ortsbrust. }\end{array}$ \\
\hline Tunnelachse & TA & - & Zentrumslinie des auszubrechenden bzw. ausgebrochenen Tunnelhohlraums. \\
\hline Vortriebsrichtung & VTR & - & Fortbewegungsrichtung der TVM beim Bodenabbau parallel zur Tunnelachse. \\
\hline Geländeoberkante & GOK & - & Erdoberfläche. \\
\hline Tunnellänge & $\mathrm{L}_{\mathrm{TA}}$ & $\mathrm{m}$ & Gesamtlänge eines Vortriebs. \\
\hline Ausbruchvolumen & $\mathrm{V}_{\mathrm{TA}}$ & $\mathrm{m}^{3}$ & Summe des Bodenvolumens (fest) in einem Vortrieb. \\
\hline \multicolumn{4}{|c|}{ Auslegung der Hydroschild TVM } \\
\hline Schneidraddurchmesser & $\mathrm{D}_{\mathrm{TVM}}$ & $\mathrm{m}$ & $\begin{array}{l}\text { Durch die Werkzeuge auf dem Schneidrad der TVM abgebauter Tunneldurch- } \\
\text { messer. }\end{array}$ \\
\hline Schneidradradius & $\mathrm{R}_{\text {TVM }}$ & $\mathrm{m}$ & Radius des Schneidrades. \\
\hline Ortsbrustfläche & $A_{\text {TVM }}$ & $\mathrm{m}^{2}$ & Kreisfläche der Ortsbrust beim Bodenabbau (Gl. 8). \\
\hline Schneidradöffnungsverhältnis & Отим & $\%$ & Verhältnis der Öffnungsfläche des Schneidrades zur Ortsbrustfläche. \\
\hline $\begin{array}{l}\text { Fläche der Schneidrad- } \\
\text { befestigung }\end{array}$ & $A_{S B}$ & $\mathrm{~m}^{2}$ & $\begin{array}{l}\text { Fläche des Flanschrings bzw. der mitdrehenden Zentrumsplatte in der Frontflä- } \\
\text { che der Tauchwand (Gl. 4). }\end{array}$ \\
\hline Länge der Schildmaschine & $\mathrm{L}_{\mathrm{TVM}}$ & $\mathrm{m}$ & $\begin{array}{l}\text { Länge der Schildmaschine von der Frontfläche des Schneidrades bis zum } \\
\text { hinteren Ende des Schildschwanzes. }\end{array}$ \\
\hline Mittlere Breite Tübbingring & $\mathrm{l}_{(\mathrm{b})}$ & $\mathrm{m}$ & Mittlere Breite eines Tübbingrings in Vortriebsrichtung. \\
\hline
\end{tabular}




\begin{tabular}{|c|c|c|c|}
\hline Begriff & Symbol & Einheit & Bemerkung \\
\hline Masse der Nachläufer & $\mathrm{M}_{\mathrm{NL}}$ & $\mathrm{t}$ & Masse der an die Schildmaschine angehängten Nachläufer. \\
\hline \multirow[t]{2}{*}{ Förderleitung } & $\mathrm{FL}$ & - & Abkürzung für Förderleitung. \\
\hline & $\mathrm{FL}$ & - & Index für Formelzeichen mit Bezug auf die Förderleitung. \\
\hline Durchmesser der Förderleitung & $d_{F L}$ & $\mathrm{~mm}$ & Innendurchmesser der Förderleitung. \\
\hline \multirow[t]{2}{*}{ Speiseleitung } & SL & - & Abkürzung für Speiseleitung. \\
\hline & SL & - & Index für Formelzeichen mit Bezug auf die Speiseleitung. \\
\hline $\begin{array}{l}\text { Spurradius eines Abbauwerk- } \\
\text { zeuges }\end{array}$ & $r_{\mathrm{s}}$ & $\mathrm{mm}$ & $\begin{array}{l}\text { Radius der Schneidspur eines Abbauwerkzeuges in Bezug auf den Zentrums- } \\
\text { punkt des Schneidrades bis zur Mitte der Schneide des Abbauwerkzeuges. }\end{array}$ \\
\hline Außenradius & $r_{\mathrm{s} 2}$ & $\mathrm{~mm}$ & $\begin{array}{l}\text { Radius der Schneidspur eines Abbauwerkzeuges vom Zentrumspunkt des } \\
\text { Schneid-rades bis zur Außenkante der Schneide des Abbauwerkzeuges } \\
\text { (Abb. 63). }\end{array}$ \\
\hline Innenradius & $r_{\mathrm{s} 1}$ & $\mathrm{~mm}$ & $\begin{array}{l}\text { Radius der Schneidspur eines Abbauwerkzeuges vom Zentrumspunkt des } \\
\text { Schneid-rades bis zur Innenkante der Schneide des Abbauwerkzeuges } \\
\text { (Abb. 63). }\end{array}$ \\
\hline Spurbesatz & $\mathrm{k}_{\mathrm{s}}$ & - & $\begin{array}{l}\text { Anzahl der auf einer Schneidspur angebrachten identischen Abbauwerkzeuge } \\
\text { zum Bodenabbau in einer Schneidraddrehrichtung. }\end{array}$ \\
\hline Spacing & $\mathrm{S}$ & $\mathrm{mm}$ & Abstand in radialer Richtung zwischen benachbarten Schneidspuren. \\
\hline Spacing der Schneidrollen & $\mathrm{S}_{\mathrm{SR}}$ & $\mathrm{mm}$ & $\begin{array}{l}\text { Abstand in radialer Richtung zwischen benachbarten Schneidspuren der } \\
\text { Schneidrollen. }\end{array}$ \\
\hline Spacing der Schälmesser & $\mathrm{S}_{\mathrm{SM}}$ & $\mathrm{mm}$ & $\begin{array}{l}\text { Abstand in radialer Richtung zwischen benachbarten Schneidspuren der } \\
\text { Schälmesser. }\end{array}$ \\
\hline Spacing der Stichel & $\mathrm{S}_{\mathrm{ST}}$ & $\mathrm{mm}$ & $\begin{array}{l}\text { Abstand in radialer Richtung zwischen benachbarten Schneidspuren der } \\
\text { Stichel. }\end{array}$ \\
\hline $\begin{array}{l}\text { Winkelabstand in aktiver Rich- } \\
\text { tung }\end{array}$ & $\delta_{p}$ & $\circ$ & $\begin{array}{l}\text { Winkelabstand von einem Abbauwerkzeug zum nächstgelegenen identischen } \\
\text { Werkzeug auf der gleichen Schneidspur in Richtung des passiven Einsatzes. }\end{array}$ \\
\hline $\begin{array}{l}\text { Winkelabstand in passiver } \\
\text { Richtung }\end{array}$ & $\delta_{a}$ & $\circ$ & $\begin{array}{l}\text { Winkelabstand von einem Abbauwerkzeug zum nächstgelegenen identischen } \\
\text { Werkzeug auf der gleichen Spur in Richtung des aktiven Einsatzes. }\end{array}$ \\
\hline \multirow[t]{2}{*}{ Schneidrolle } & SR & - & Abkürzung für Schneidrollen. \\
\hline & SR & - & Index für Formelzeichen mit Bezug auf Schneidrollen. \\
\hline Vorlauf der Schneidrollen & $\mathrm{h}_{\mathrm{SR}}$ & $\mathrm{mm}$ & $\begin{array}{l}\text { Höhe der Schneide der Schneidrollen senkrecht über der Frontfläche des } \\
\text { Schneidradstahlbaus (Abb. 15). }\end{array}$ \\
\hline Schneidringbreite & $b_{S R}$ & $\mathrm{~mm}$ & Breite der Lauffläche eines Schneidrings (Abb. 10) \\
\hline $\begin{array}{l}\text { Maximaler radialer Abrieb am } \\
\text { Schneidring }\end{array}$ & $\mathrm{h}_{\mathrm{d}, \max (\mathrm{SR})}$ & $\mathrm{mm}$ & Verschleißgrenze für den radialen Abrieb eines Schneidringes (GI. 22). \\
\hline \multirow[t]{2}{*}{ Stichel } & ST & - & Abkürzung für Stichel. \\
\hline & ST & - & Index für Formelzeichen mit Bezug auf Stichel. \\
\hline Vorlauf der Stichel & $\mathrm{h}_{\mathrm{ST}}$ & $\mathrm{mm}$ & $\begin{array}{l}\text { Höhe der Schneide der Stichel senkrecht über der Frontfläche des Schneidrad- } \\
\text { stahlbaus (Abb. 15). }\end{array}$ \\
\hline Maximaler Abrieb Stichel & $\mathrm{h}_{\mathrm{d}, \max (\mathrm{ST})}$ & $\mathrm{mm}$ & $\begin{array}{l}\text { Verschleißgrenze der Stichel in Bezug auf den Abrieb in der Rückenfläche } \\
\text { senkrecht zur Ortsbrust (Abb. 52). }\end{array}$ \\
\hline Breite des Stichelkopfs & $b_{S T}$ & $\mathrm{~mm}$ & Breite der Schneide eines Stichelkopfs. \\
\hline \multirow[t]{2}{*}{ Schälmesser } & SM & - & Abkürzung für Schälmesser. \\
\hline & SM & - & Index für Formelzeichen mit Bezug auf Schälmesser \\
\hline Aktiv-Schälmesser & $\mathrm{SM}_{\mathrm{a}}$ & - & $\begin{array}{l}\text { Schälmesser, das zum Betrachtungszeitpunkt in aktiver bzw. bestimmungsge- } \\
\text { mäßer Richtung über die Ortsbrust geführt wird. }\end{array}$ \\
\hline Passiv-Schälmesser & $\mathrm{SM}_{\mathrm{p}}$ & - & $\begin{array}{l}\text { Schälmesser, das zum Betrachtungszeitpunkt in passiver bzw. entgegen der } \\
\text { bestimmungsgemäßen Richtung über die Ortsbrust geführt wird. }\end{array}$ \\
\hline Breite des Schälmessers & $b_{S M}$ & $\mathrm{~mm}$ & Breite der Schneide eines Schälmessers. \\
\hline Schneidwinkel & $\alpha_{c}$ & $\circ$ & $\begin{array}{l}\text { Schneidwinkel an der Schneide von Schälmessern (Abb. 12), Sticheln und } \\
\text { Räumern. }\end{array}$ \\
\hline Freiwinkel & $\beta_{c}$ & $\circ$ & $\begin{array}{l}\text { Freiwinkel im Rücken der Schneide von Schälmessern (Abb. 12), Sticheln und } \\
\text { Räumern. }\end{array}$ \\
\hline Vorlauf der Schälmesser & $\mathrm{h}_{\mathrm{SM}}$ & $\mathrm{mm}$ & $\begin{array}{l}\text { Höhe der Schneide der Schälmesser senkrecht über der Frontfläche des } \\
\text { Schneidradstahlbaus (Abb. 15). }\end{array}$ \\
\hline $\begin{array}{l}\text { Vorlauf des Schälmesserhal- } \\
\text { ters }\end{array}$ & $\mathrm{h}_{\mathrm{SMH}}$ & $\mathrm{mm}$ & $\begin{array}{l}\text { Höhe des Schälmesserhalters senkrecht über der Frontfläche des Schneidrad- } \\
\text { stahlbaus. (Abb. 59) }\end{array}$ \\
\hline Maximaler Abrieb Schälmesser & $\mathrm{h}_{\mathrm{d}, \max (\mathrm{SM})}$ & $\mathrm{mm}$ & $\begin{array}{l}\text { Verschleißgrenze der Schälmesser in Bezug auf den Abrieb in der Rückenflä- } \\
\text { che senkrecht zur Ortsbrust (Abb. 59). }\end{array}$ \\
\hline
\end{tabular}




\begin{tabular}{|c|c|c|c|}
\hline Begriff & Symbol & Einheit & Bemerkung \\
\hline \multirow[t]{2}{*}{ Räumer } & $\mathrm{RM}$ & - & Abkürzung für Räumer. \\
\hline & $\mathrm{RM}$ & - & Index für Formelzeichen mit Bezug auf Räumer. \\
\hline Vorlauf der Räumer & $\mathrm{h}_{\mathrm{RM}}$ & $\mathrm{mm}$ & $\begin{array}{l}\text { Höhe der Schneide der Räumer senkrecht über der Frontfläche des } \\
\text { Schneidradstahlbaus (Abb. 62). }\end{array}$ \\
\hline Vorlauf des Räumerhalters & $\mathrm{h}_{\mathrm{RMH}}$ & $\mathrm{mm}$ & $\begin{array}{l}\text { Höhe des Räumerhalters senkrecht über der Frontfläche des Schneidradstahl- } \\
\text { baus. (Abb. 62) }\end{array}$ \\
\hline Maximaler Abrieb Räumer & $\mathrm{h}_{\mathrm{d}, \max (\mathrm{RM})}$ & $\mathrm{mm}$ & $\begin{array}{l}\text { Verschleißgrenze der Räumer in Bezug auf den Abrieb in der Rückenfläche } \\
\text { senkrecht zur Ortsbrust (Abb. 62). }\end{array}$ \\
\hline \multicolumn{4}{|l|}{ Steuerung des Abbauprozesses } \\
\hline $\begin{array}{l}\text { Speicherprogrammierbare } \\
\text { Steuerung }\end{array}$ & SPS & - & Digitale Steuerung der TVM. \\
\hline Schneidraddrehzahl & $U$ & $\min ^{-1}$ & Drehzahl des Schneidrads beim Bodenabbau. \\
\hline Schneidraddrehgeschwindigkeit & $\omega_{\text {TVM }}$ & $\mathrm{rad} / \mathrm{s}$ & Drehgeschwindigkeit des Schneidrads beim Bodenabbau (GI. 1). \\
\hline Schneidgeschwindigkeit & $\mathrm{v}_{\mathrm{sc}}$ & $\mathrm{m} / \mathrm{s}$ & Bahngeschwindigkeit eines Abbauwerkzeuges auf der Schneidspur (Gl. 20). \\
\hline Aktiver Einsatz & a & - & Index für den aktiven Einsatz eines Werkzeuges (Kap. 3.2.1). \\
\hline Passiver Einsatz & $\mathrm{p}$ & - & Index für den passiven Einsatz eines Werkzeuges (Kap. 3.2.1). \\
\hline Vortriebsgeschwindigkeit & $\mathrm{V}_{\mathrm{TVM}}$ & $\mathrm{mm} / \mathrm{min}$ & Vortriebsfortschritt der TVM in Richtung Tunnelachse je Minute. \\
\hline Penetration & $\mathrm{p}$ & $\mathrm{mm} / \mathrm{U}$ & $\begin{array}{l}\text { Vortriebsfortschritt in Richtung Tunnelachse je Umdrehung des Schneidrades } \\
\text { (Gl. 2). }\end{array}$ \\
\hline Mittlere Penetration & $\mathrm{p}_{(\mathrm{b})}$ & $\mathrm{mm} / \mathrm{U}$ & Arithmetischer Mittelwert der Penetration $p$ über die Länge $\mathrm{I}_{(\mathrm{b})}$ eines Vortriebs b. \\
\hline Aktiv-Penetration & $\mathrm{p}_{\mathrm{a}}$ & $\mathrm{mm} / \mathrm{U}$ & $\begin{array}{l}\text { Anteil eines Abbauwerkzeuges an der Penetration } \mathrm{p} \text { bei aktivem Bodenabbau } \\
\text { (Gl. 40). }\end{array}$ \\
\hline Passiv-Penetration & $p_{p}$ & $\mathrm{~mm} / \mathrm{U}$ & $\begin{array}{l}\text { Anteil eines Abbauwerkzeuges an der Penetration } p \text { bei passivem Bodenabbau } \\
\text { (Gl. 41). }\end{array}$ \\
\hline Stützdruck & $\mathrm{P}_{\mathrm{SF}}$ & bar & $\begin{array}{l}\text { Druck der Stützflüssigkeit und des Druckluftkissens in der Abbaukammer und } \\
\text { Arbeitskammer der TVM (Abb. 17). }\end{array}$ \\
\hline \multirow[t]{2}{*}{ Stützflüssigkeit } & SF & - & Abkürzung für Stützflüssigkeit. \\
\hline & $\mathrm{SF}$ & - & Index für Formelzeichen mit Bezug auf die Stützflüssigkeit. \\
\hline Fördervolumen & $\mathrm{V}_{\mathrm{FL}}$ & $\mathrm{m}^{3} / \mathrm{h}$ & $\begin{array}{l}\text { Aus der Abbaukammer über die Förderleitung und Förderpumpen entnomme- } \\
\text { nes Volumen an Stützflüssigkeit je Minute. }\end{array}$ \\
\hline Speisevolumen & $\mathrm{V}_{\mathrm{SL}}$ & $\mathrm{m}^{3} / \mathrm{h}$ & $\begin{array}{l}\text { In die Abbaukammer über die Speiseleitung und Speisepumpen zugeführtes } \\
\text { Volumen an Stützflüssigkeit je Minute. }\end{array}$ \\
\hline $\begin{array}{l}\text { Fließgeschwindigkeit Förderlei- } \\
\text { tung }\end{array}$ & $\mathrm{v}_{\mathrm{SF}}$ & $\mathrm{m} / \mathrm{s}$ & Fließgeschwindigkeit der Stützflüssigkeit in der Förderleitung. \\
\hline \multirow[t]{3}{*}{ Dichte der Stützflüssigkeit } & $\rho_{\mathrm{SF}}$ & $\mathrm{g} / \mathrm{cm}^{3}$ & Dichte der Stützflüssigkeit in der Abbaukammer. \\
\hline & $\rho_{\mathrm{SL}}$ & $\mathrm{g} / \mathrm{cm}^{3}$ & Dichte der Stützflüssigkeit in der Speiseleitung. \\
\hline & $\rho_{\mathrm{FL}}$ & $\mathrm{g} / \mathrm{cm}^{3}$ & Dichte der Stützflüssigkeit in der Förderleitung. \\
\hline $\begin{array}{l}\text { Kinematische Viskosität der } \\
\text { Stützflüssigkeit }\end{array}$ & $v_{\mathrm{SF}}$ & $\mathrm{m}^{2} / \mathrm{s}$ & Kinematische Viskosität der Stützflüssigkeit in der Förderleitung (Gl. 21). \\
\hline REYNOLDS-Zahl & $\mathrm{R}_{\mathrm{e}}$ & - & Reynolds-Zahl der Stützflüssigkeit in der Förderleitung (Gl. 21). \\
\hline Schneidkraft & $\mathrm{F}_{\mathrm{C}}$ & $\mathrm{kN}$ & Resultierende Schneidkraft an einem Abbauwerkzeug beim Bodenabbau. \\
\hline Schneidwiderstand & $\mathrm{W}_{\mathrm{C}}$ & $\mathrm{kN}$ & Schneidwiderstand des Bodens beim Abbau mit einem Abbauwerkzeug. \\
\hline Eindringwiderstand & $\mathrm{W}_{\mathrm{TC}}$ & $\mathrm{kN}$ & $\begin{array}{l}\text { Widerstandskomponente von } \mathrm{W}_{\mathrm{C}} \text { parallel zu TA entgegen VTR beim Bodenab- } \\
\text { bau. }\end{array}$ \\
\hline Anpresskraft & $\mathrm{F}_{\mathrm{TC}}$ & $\mathrm{kN}$ & $\begin{array}{l}\text { Kraftkomponenten von } F_{C} \text { parallel zu TA in VTR an einem Abbauwerkzeug } \\
\text { beim Bodenabbau. }\end{array}$ \\
\hline Schneidwiderstand & $\mathrm{W}_{\mathrm{SC}}$ & $\mathrm{kN}$ & $\begin{array}{l}\text { Widerstandskomponente von } \mathrm{W}_{\mathrm{C}} \text { senkrecht zu TA bzw. entgegen der Schneid- } \\
\text { richtung eines Abbauwerkzeuges beim Bodenabbau. }\end{array}$ \\
\hline Schneidkraft & $\mathrm{F}_{\mathrm{SC}}$ & $\mathrm{kN}$ & $\begin{array}{l}\text { Kraftkomponenten von } F_{C} \text { senkrecht zu TA bzw. in Schneidrichtung eines } \\
\text { Abbauwerkzeuges beim Bodenabbau. }\end{array}$ \\
\hline $\begin{array}{l}\text { Vortriebswiderstand aus me- } \\
\text { chanischer Stützung }\end{array}$ & $\mathrm{W}_{\mathrm{ME}}$ & $\mathrm{kN}$ & $\begin{array}{l}\text { Widerstand parallel zu TA entgegen VTR durch mechanische Stützwirkung } \\
\text { zwischen Schneidradfrontfläche und Ortsbrust. }\end{array}$ \\
\hline $\begin{array}{l}\text { Vortriebskraft aus mechani- } \\
\text { scher Stützung }\end{array}$ & $\mathrm{F}_{\mathrm{ME}}$ & $\mathrm{kN}$ & $\begin{array}{l}\text { Kraft parallel zu TA in VTR durch mechanische Stützwirkung zwischen Schneid- } \\
\text { radfrontfläche und Ortsbrust. }\end{array}$ \\
\hline $\begin{array}{l}\text { Vortriebswiderstand aus der } \\
\text { Schneidradbefestigung }\end{array}$ & $\mathrm{W}_{\mathrm{SB}}$ & $\mathrm{kN}$ & $\begin{array}{l}\text { Vortriebswiderstand parallel zu TA entgegen VTR aus dem an der Fläche der } \\
\text { Schneidradbefestigung anliegenden Stützdruck } p_{S F} \text { (Gl. 4). }\end{array}$ \\
\hline
\end{tabular}




\begin{tabular}{|c|c|c|c|}
\hline Begriff & Symbol & Einheit & Bemerkung \\
\hline $\begin{array}{l}\text { Vortriebskraft aus der } \\
\text { Schneidradbefestigung }\end{array}$ & $\mathrm{F}_{\mathrm{SB}}$ & $\mathrm{kN}$ & 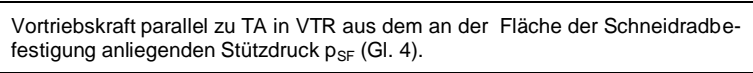 \\
\hline $\begin{array}{l}\text { Vortriebswiderstand Verschie- } \\
\text { bezylinder }\end{array}$ & $\mathrm{W}_{\mathrm{SV}}$ & $\mathrm{kN}$ & $\begin{array}{l}\text { Summe der an den Verschiebezylindern parallel zu TA entgegen VTR anlie- } \\
\text { genden Vortriebswiderstände (Gl. 5). }\end{array}$ \\
\hline $\begin{array}{l}\text { Vortriebskraft an den Verschie- } \\
\text { bezylindern }\end{array}$ & $\mathrm{F}_{\mathrm{SV}}$ & $\mathrm{kN}$ & $\begin{array}{l}\text { Summe der an den Verschiebezylindern parallel zu TA in VTR anliegenden } \\
\text { Vortriebskräfte (Gl. 6). }\end{array}$ \\
\hline Schneidradwiderstand & $\mathrm{W}_{\mathrm{SR}}$ & $\mathrm{kN}$ & $\begin{array}{l}\text { Vortriebswiderstand parallel zu TA entgegen VTR aus der Summe der An- } \\
\text { presskräfte } \mathrm{F}_{\mathrm{TC}} \text { der einzelnen Abbauwerkzeuge (Gl. 3). }\end{array}$ \\
\hline Schneidradanpresskraft & $\mathrm{F}_{\mathrm{SR}}$ & $\mathrm{kN}$ & $\begin{array}{l}\text { Vortriebskraft parallel zu TA in VTR aus der Summe der Anpresskräfte } F_{\text {TC }} \text { der } \\
\text { einzelnen Abbauwerkzeuge (Gl. 3). }\end{array}$ \\
\hline $\begin{array}{l}\text { Normierte Schneidradanpress- } \\
\text { kraft }\end{array}$ & $F_{\text {norm }}$ & $\mathrm{kN}$ & $\begin{array}{l}\text { Schneidradanpresskraft FSR normiert auf den Schneidraddurchmesser DTVM } \\
\text { und die Penetration } \mathrm{p} \text { (Gl. 66). }\end{array}$ \\
\hline $\begin{array}{l}\text { Vortriebswiderstand an den } \\
\text { Vortriebspressen }\end{array}$ & $\mathrm{W}_{\mathrm{VTP}}$ & $\mathrm{kN}$ & $\begin{array}{l}\text { Summe der an den Vortriebszylindern parallel zu TA entgegen VTR anliegen- } \\
\text { den Vortriebswiderstände (GI. 9). }\end{array}$ \\
\hline $\begin{array}{l}\text { Vortriebspressenkraft an den } \\
\text { Vortriebspressen }\end{array}$ & $\mathrm{F}_{\mathrm{VTP}}$ & $\mathrm{kN}$ & $\begin{array}{l}\text { Summe der an den Vortriebszylindern parallel zu TA in VTR anliegenden } \\
\text { Vortriebskräfte (Gl. 10). }\end{array}$ \\
\hline $\begin{array}{l}\text { Vortriebswiderstand aus dem } \\
\text { Stützdruck }\end{array}$ & $\mathrm{W}_{\mathrm{SF}}$ & $\mathrm{kN}$ & $\begin{array}{l}\text { Vortriebswiderstand parallel zu TA entgegen VTR aus dem an der Fläche der } \\
\text { Druckwand anliegenden Stützdruck pSF (Gl. 7). }\end{array}$ \\
\hline $\begin{array}{l}\text { Vortriebskraft aus dem Stütz- } \\
\text { druck }\end{array}$ & $\mathrm{F}_{\mathrm{SF}}$ & $\mathrm{kN}$ & $\begin{array}{l}\text { Vortriebskraft parallel zu TA in VTR aus dem an der Fläche der Druckwand } \\
\text { anliegenden Stützdruck p ps (Gl. 7). }\end{array}$ \\
\hline $\begin{array}{l}\text { Vortriebswiderstand aus der } \\
\text { Schildreibung }\end{array}$ & $\mathrm{W}_{\mathrm{RS}}$ & $\mathrm{kN}$ & $\begin{array}{l}\text { Reibungswiderstand parallel zu TA entgegen VTR durch den Kontakt zwischen } \\
\text { Schuldmantel und Boden. }\end{array}$ \\
\hline $\begin{array}{l}\text { Vortriebskraft aus der Schild- } \\
\text { reibung }\end{array}$ & $\mathrm{F}_{\mathrm{RS}}$ & $\mathrm{kN}$ & $\begin{array}{l}\text { Kraft parallel zu TA in VTR zur Überwindung der Schildreibung durch den } \\
\text { Kontakt zwischen Schuldmantel und Boden. }\end{array}$ \\
\hline $\begin{array}{l}\text { Vortriebswiderstd. a.d. Reibung } \\
\text { d. Schildschwanzdichtung }\end{array}$ & $\mathrm{W}_{\mathrm{SD}}$ & $\mathrm{kN}$ & $\begin{array}{l}\text { Reibungswiderstand parallel zu TA entgegen VTR durch den Kontakt zwischen } \\
\text { Schildschwanzdichtung und Tübbingen. }\end{array}$ \\
\hline $\begin{array}{l}\text { Vortriebskraft a. d. Reibung der } \\
\text { Schildschwanzdichtung }\end{array}$ & $\mathrm{F}_{\mathrm{SD}}$ & $\mathrm{kN}$ & $\begin{array}{l}\text { Kraft parallel zu TA in VTR zur Überwindung der Reibung durch den Kontakt } \\
\text { zwischen Schildschwanzdichtung und Tübbingen. }\end{array}$ \\
\hline Reibungskoeffizient Nachläufer & $\mu_{\mathrm{NL}}$ & - & $\begin{array}{l}\text { Reibungskoeffizient beim Kontakt zwischen den Laufwerken der Nachläufer und } \\
\text { dem Schienensystem bzw. Tübbingen. }\end{array}$ \\
\hline $\begin{array}{l}\text { Schleppwiderstand der Nach- } \\
\text { läufer }\end{array}$ & $\mathrm{W}_{\mathrm{NL}}$ & $\mathrm{kN}$ & $\begin{array}{l}\text { Reibungswiderstand parallel zu TA entgegen VTR beim Schleppen der Nach- } \\
\text { läufer. }\end{array}$ \\
\hline Schleppkraft der Nachläufer & $\mathrm{F}_{\mathrm{NL}}$ & $\mathrm{kN}$ & Kraft parallel zu TA in VTR zum Schleppen der Nachläufer. \\
\hline $\begin{array}{l}\text { Reibungswiderstand } \\
\text { Schneidradverschiebung }\end{array}$ & $\mathrm{W}_{\mathrm{RA}}$ & $\mathrm{kN}$ & $\begin{array}{l}\text { Summierter Reibungswiderstand in den Hydraulikzylindern der Schneidradver- } \\
\text { schiebung und dem Hauptlager des Schneidradantriebes parallel zu TA in VTR. }\end{array}$ \\
\hline $\begin{array}{l}\text { Reibungskraft Schneidradver- } \\
\text { schiebung }\end{array}$ & $\mathrm{F}_{\mathrm{RA}}$ & $\mathrm{kN}$ & $\begin{array}{l}\text { Summierter Reibungskraft in den Hydraulikzylindern der Schneidradverschie- } \\
\text { bung und dem Hauptlager des Schneidradantriebes parallel zu TA entgegen } \\
\text { VTR.. }\end{array}$ \\
\hline $\begin{array}{l}\text { Reibungswiderstand Vortriebs- } \\
\text { pressen }\end{array}$ & $\mathrm{W}_{\mathrm{RV}}$ & $\mathrm{kN}$ & $\begin{array}{l}\text { Summierter Reibungswiderstand in den Hydraulikzylindern der Vortriebspres- } \\
\text { sen parallel zu TA in VTR. }\end{array}$ \\
\hline Reibungskraft Vortriebspressen & $\mathrm{F}_{\mathrm{RV}}$ & $\mathrm{kN}$ & $\begin{array}{l}\text { Summierter Reibungskraft in den Hydraulikzylindern der Vortriebspressen } \\
\text { parallel zu TA entgegen VTR. }\end{array}$ \\
\hline Schneidraddrehmoment & $\mathrm{M}_{\text {TVM }}$ & $\mathrm{kNm}$ & Am Schneidrad beim Bodenabbau anliegendes Drehmoment (GI. 12). \\
\hline $\begin{array}{l}\text { Schneiddrehmoment Schneid- } \\
\text { rad }\end{array}$ & $\mathrm{M}_{\mathrm{SR}}$ & $\mathrm{kNm}$ & $\begin{array}{l}\text { Resultierendes Drehmoment aus der Summe der an den Abbauwerkzeugen } \\
\text { anliegenden Schneidmomente } M_{C S}(G l .11) .\end{array}$ \\
\hline $\begin{array}{l}\text { Normiertes Schneiddrehmo- } \\
\text { ment }\end{array}$ & $M_{\text {norm }}$ & $\mathrm{kNm}$ & $\begin{array}{l}\text { Schneiddrehmoment } M_{S R} \text { normiert auf den Schneidraddurchmesser } D_{\text {TVM }} \text { und } \\
\text { die Penetration } p \text { (Gl. 65). }\end{array}$ \\
\hline $\begin{array}{l}\text { Schneiddrehmoment Abbau- } \\
\text { werkzeug }\end{array}$ & $\mathrm{M}_{\mathrm{sc}}$ & $\mathrm{kNm}$ & $\begin{array}{l}\text { Resultierendes Drehmoment beim Bodenabbau eines einzelnen Abbauwerk- } \\
\text { zeuges aus der Schneidkraft } F_{C S} \text { und dem Spurradius } r_{s}(G l .11) \text {. }\end{array}$ \\
\hline Eigendrehmoment Schneidrad & $\mathrm{M}_{\mathrm{ES}}$ & $\mathrm{kNm}$ & Eigendrehmoment des Schneidrades. \\
\hline Rührmoment Schneidrad & $\mathrm{M}_{\mathrm{RS}}$ & $\mathrm{kNm}$ & $\begin{array}{l}\text { Resultierendes Drehmoment aus der Rotation des Schneidrades in der Stütz- } \\
\text { flüssigkeit. }\end{array}$ \\
\hline Reibungsmoment Schneidrad & $\mathrm{M}_{\mathrm{ME}}$ & $\mathrm{kNm}$ & $\begin{array}{l}\text { Resultierendes Drehmoment aus der Reibung zwischen Schneidrad und } \\
\text { Ortsbrust über bereits abgebauten Boden. }\end{array}$ \\
\hline Antriebsleistung & $\mathrm{P}_{\mathrm{TVM}}$ & kW & Die vom Schneidradantrieb beim Bodenabbau erzeugte Gesamtleistung (GI. 1). \\
\hline Spezifische Energie & $\mathrm{SE}_{\mathrm{TVM}}$ & $\mathrm{MJ} / \mathrm{m}^{3}$ & $\begin{array}{l}\text { Energie die mit der TVM zum Abbau eines Kubikmeters Boden (fest) eingesetzt } \\
\text { wird (Gl. 13). }\end{array}$ \\
\hline
\end{tabular}




\begin{tabular}{|c|c|c|c|}
\hline Begriff & Symbol & Einheit & Bemerkung \\
\hline \multicolumn{4}{|l|}{ Geotechnische Parameter } \\
\hline Überlagerung & $\mathrm{H}_{\mathrm{TA}}$ & $\mathrm{m}$ & Überlagerung der Tunnelachse, senkrecht zwischen GOK und TA. \\
\hline Index & i & - & $\begin{array}{l}\text { Index zur Nummerierung der Bodeneinheiten in der Überlagerung } \mathrm{H}_{\mathrm{TA}} \text { von TA } \\
\text { bis GOK.. }\end{array}$ \\
\hline Mächtigkeit & $\mathrm{H}_{\mathrm{i}}$ & $\mathrm{m}$ & $\begin{array}{l}\text { Mächtigkeit einer geotechnischen Einheit in der Überlagerung } \mathrm{H}_{\text {TA }} \text { oberhalb der } \\
\text { Tunnelachse TA. }\end{array}$ \\
\hline Wasserspiegel & $\mathrm{W}_{\mathrm{TA}}$ & $\mathrm{m}$ & Wasserspiegel senkrecht über der Tunnelachse TA. \\
\hline Erdbeschleunigung & $g$ & $\mathrm{kN} / \mathrm{t}$ & Zahlenwert der Erdbeschleunigung (9,81 kN/t) \\
\hline Feuchtwichte des Bodens & $\mathrm{Y}$ & $\mathrm{kN} / \mathrm{m}^{3}$ & DIN ISO TS 17892-2 (2005) \\
\hline $\begin{array}{l}\text { Wichte des Bodens unter Auf- } \\
\text { trieb }\end{array}$ & $\mathrm{Y}^{\prime}$ & $\mathrm{kN} / \mathrm{m}^{3}$ & DIN ISO TS $17892-2$ \\
\hline Korndurchmesser & $\mathrm{D}_{10}$ & $\mathrm{~mm}$ & $\begin{array}{l}\text { Korngröße einer Bodenprobe bei } 10 \% \text { Siebdurchgang nach } \\
\text { DIN ISO TS 17892-4 }\end{array}$ \\
\hline Korndurchmesser & $\mathrm{D}_{50}$ & $\mathrm{~mm}$ & $\begin{array}{l}\text { Korngröße einer Bodenprobe bei } 50 \% \text { Siebdurchgang nach } \\
\text { DIN ISO TS 17892-4 }\end{array}$ \\
\hline Korndurchmesser & $\mathrm{D}_{60}$ & $\mathrm{~mm}$ & $\begin{array}{l}\text { Korngröße einer Bodenprobe bei } 60 \% \text { Siebdurchgang nach } \\
\text { DIN ISO TS } 17892-4\end{array}$ \\
\hline Ungleichkörnigkeitszahl & $\mathrm{C}_{\mathrm{u}}$ & - & Ungleichkörnigkeitszahl einer Bodenprobe nach DIN 14688-2. \\
\hline Krümmungszahl & $\mathrm{C}_{\mathrm{c}}$ & - & Krümmungszahl einer Bodenprobe nach DIN 14688-2. \\
\hline Lagerungsdichte & $\mathrm{D}$ & $\%$ & DIN 18126 (1996), DIN 18127 (1997) \\
\hline Reibungswinkel & $\varphi^{\prime}$ & $\circ$ & DIN 18137, T1 (1990), DIN 18137, T2 (1990) \\
\hline Kohäsion & $c^{\prime}$ & $\mathrm{kN} / \mathrm{m}^{2}$ & DIN 18137, T1 (1990), DIN 18137, T2 (1990) \\
\hline Dynamische Kohäsion & $\mathrm{C}_{\mathrm{dyn}}$ & $\mathrm{kN} / \mathrm{m}^{2}$ & Dynamische Kohäsion des Bodens (nicht genormt). \\
\hline Dynamischer Reibungswinkel & $\varphi_{\text {dyn }}$ & $\circ$ & Dynamischer Reibungswinkel des Bodens (nicht genormt). \\
\hline Wassergehalt & w & $\%$ & DIN ISO TS $17892-12$ \\
\hline Fließgrenze & $\mathrm{W}_{\mathrm{L}}$ & $\%$ & DIN ISO TS 17892-12 \\
\hline Ausrollgrenze & $\mathrm{W}_{\mathrm{P}}$ & $\%$ & DIN ISO TS $17892-12$ \\
\hline Schrumpfgrenze & $\mathrm{w}_{\mathrm{S}}$ & $\%$ & DIN ISO TS $17892-12$ \\
\hline Plastizitätszahl & $\mathrm{I}_{\mathrm{P}}$ & $\%$ & DIN ISO TS $17892-12$ \\
\hline Konsistenzzahl & $\mathrm{I}_{\mathrm{C}}$ & - & DIN ISO TS $17892-12$ \\
\hline Porenanteil & $\mathrm{n}_{\text {por }}$ & $\%$ & DIN ISO TS 17892-2, DIN ISO TS 17892-3 \\
\hline Porenzahl & $e_{\text {por }}$ & - & DIN ISO TS 17892-2, DIN ISO TS 17892-3 \\
\hline Durchlässigkeitsbeiwert & $\mathrm{k}_{\mathrm{f}}$ & $\mathrm{m} / \mathrm{s}$ & Durchlässigkeitsbeiwert eines Bodens n. DIN 1830, T1 (1998) \\
\hline Mineralanteil & $a_{m}$ & $\%$ & Volumenanteil eines Minerals an einer Gesteinsprobe. \\
\hline Mineraldichte & $\rho_{\mathrm{m}}$ & $\mathrm{g} / \mathrm{cm}^{3}$ & Tab. 5 \\
\hline Schleifhärte nach RosIWAL & HRW & - & Tab. 5 \\
\hline Ritzhärte nach MOHS & $\mathrm{HMO}$ & - & Tab. 5 \\
\hline Härte nach VICKERS & HV & - & Tab. 5 \\
\hline Härte nach ROCKWELL & $\mathrm{HRC}$ & - & Tab. 5 \\
\hline Härte nach BRINELL & HBW & - & - \\
\hline Äquivalenter Quarzgehalt & ÄQu & $\%$ & $\begin{array}{l}\text { Ermittelt aus dem quantitativen Mineralbestand des Bodens und der Härte } \\
\text { HRW der Minerale (GI. 14) }\end{array}$ \\
\hline Konstante & $\mathrm{k}_{\mathrm{EPB}}$ & - & Dimensionslose Konstante zur Berechnung von $V_{\mathrm{EPB}}$ mit dem Wert 1000. \\
\hline Röntgendiffraktometeranalyse & RDA & - & Abkürzung Röntgendiffraktometeranalyse. \\
\hline Scherfestigkeit & $\mathrm{T}_{\mathrm{C}}$ & $\mathrm{kN} / \mathrm{m}^{2}$ & Scherfestigkeit des Bodens in der Ortsbrust (GI. 54 \& 73). \\
\hline Kontaktspannung & $\mathrm{T}_{\mathrm{act}}$ & $\mathrm{kN} / \mathrm{m}^{2}$ & Spannung zwischen Werkzeugoberfläche und Boden beim Bodenabbau. \\
\hline Normalspannung & $\sigma_{\mathrm{n}}$ & $\mathrm{kN} / \mathrm{m}^{2}$ & Effektive Normalspannung im Bruchkriterium nach MOHR-COULOMB (Gl. 58). \\
\hline Normalspannung & $\sigma_{\mathrm{n}(\mathrm{z})}$ & $\mathrm{kN} / \mathrm{m}^{2}$ & $\begin{array}{l}\text { Effektive Normalspannung im Bruchkriterium nach MOHR-COULOMB im geo- } \\
\text { technischen Homogenbereich } \mathrm{HGB}_{(\mathrm{z})}(\mathrm{Gl} .74) \text {. }\end{array}$ \\
\hline
\end{tabular}




\begin{tabular}{|c|c|c|c|}
\hline Begriff & Symbol & Einheit & Bemerkung \\
\hline $\begin{array}{l}\text { Vertikale Hauptnormal- } \\
\text { spannung }\end{array}$ & $\sigma_{\mathrm{zz}}$ & $\mathrm{kN} / \mathrm{m}^{2}$ & $\begin{array}{l}\text { Effektive vertikale Spannungskomponente parallel zur Ortsbrust und senkrecht } \\
\text { zu TA. (Gl. 55). }\end{array}$ \\
\hline $\begin{array}{l}\text { Horizontale Hauptnormal- } \\
\text { spannung }\end{array}$ & $\sigma_{\mathrm{yy}}$ & $\mathrm{kN} / \mathrm{m}^{2}$ & $\begin{array}{l}\text { Horizontale effektive Spannungskomponente parallel zu TA und senkrecht zur } \\
\text { Ortsbrust. }\end{array}$ \\
\hline $\begin{array}{l}\text { Horizontale Hauptnormal- } \\
\text { spannung }\end{array}$ & $\sigma_{\mathrm{xx}}$ & $\mathrm{kN} / \mathrm{m}^{2}$ & $\begin{array}{l}\text { Horizontale effektive Spannungskomponente parallel zur Ortsbrust und senk- } \\
\text { recht zu TA. }\end{array}$ \\
\hline Porenwasserdruck & u & $\mathrm{kN} / \mathrm{m}^{2}$ & Porenwasserdruck. \\
\hline Erdruhedruckbeiwert & $\mathrm{k}_{0}$ & - & - \\
\hline $\begin{array}{l}\text { Verschleißindex nach } \\
\text { WILMS (1997) }\end{array}$ & $V_{E P B}$ & - & Verschleißindex zur Beschreibung der Bodenabrasivität für EPB TVM (Gl. 18). \\
\hline F-Koeffizient & $\mathrm{F}$ & - & Verschleißkoeffizient n. SCHIMATZECK \& KNATZ (1976) (Gl. 17). \\
\hline Quarzanteil & $\mathrm{a}_{\mathrm{qz}}$ & $\%$ & Volumenanteil von Quarz im Gestein. \\
\hline Korngröße Quarz & $d_{\mathrm{qz}}$ & $\mathrm{mm}$ & Durchmesser der Quarzkörner im Gestein. \\
\hline Miller-Zahl & - & - & Kennwert zum Massenverlust eines Stahlblocks nach dem Miller-Test. \\
\hline Soil Abrasivity Test & SAT & - & Testbezeichnung \\
\hline SAT-Abrasivitätsindex & SAT & $\mathrm{mg}$ & Indexwert als Ergebnis aus dem SAT. \\
\hline CERCHAR-Abrasivitätsindex & CAI & - & AFNOR (2000) \\
\hline Spitzendurchmesser & $d_{v}$ & $\mathrm{~mm}$ & Durchmesser der Prüfspitze nach dem CIA-Test. \\
\hline LCPC-Abrasivitätsindex & LAK & $\mathrm{g} / \mathrm{t}$ & AFNOR (1990) \\
\hline LCPC-Brechbarkeitsindex & LBR & $\%$ & AFNOR (1990) \\
\hline Ausgangsmasse Stahlflügel & $m_{F A}$ & $g$ & Ausgangsmasse des Stahlflügels vor dem LCPC-Test. \\
\hline Endmasse Stahlflügel & $\mathrm{m}_{\mathrm{FV}}$ & g & Masse des Stahlflügels nach dem LCPC-Test. \\
\hline Probenmasse & $\mathrm{m}_{\mathrm{P}}$ & g & Eingesetzte Probenmasse im LCPC-Test. \\
\hline Soil Abrasivity Index & SAI & - & Indexwert zur Beschreibung der Bodenabrasivität (Gl. 67). \\
\hline Einaxiale Druckfestigkeit & UCS & $\mathrm{MPa}$ & DIN ISO TS 17892-7 \\
\hline Spaltzugfestigkeit & SPZ & $\mathrm{kN} / \mathrm{m}^{2}$ & ISRM (1978) \\
\hline \multicolumn{4}{|c|}{ Standgrößen und deren Berechnung } \\
\hline Vortrieb (Ring) & $b$ & - & Ringnummer bzw. Vortrieb eines Ringes. \\
\hline Einbaustationierung & $b_{0}$ & - & $\begin{array}{l}\text { Vortrieb (Ringnummer) bei Einbau eines Abbauwerkzeuges auf dem Schneid- } \\
\text { rad. }\end{array}$ \\
\hline Ausbaustationierung & $b_{v}$ & - & $\begin{array}{l}\text { Vortrieb (Ringummer) bei Ausbau eines Abbauwerkzeuges auf dem Schneid- } \\
\text { rad. }\end{array}$ \\
\hline Aktiv-Vortrieb (Ring) & $b_{a}$ & - & $\begin{array}{l}\text { Vortrieb bzw. Ring in dem ein Abbauwerkzeug durchgehend in Aktiv-Richtung } \\
\text { über die Ortsbrust geführt wurde. }\end{array}$ \\
\hline Passiv-Vortrieb (Ring) & $b_{p}$ & - & $\begin{array}{l}\text { Vortrieb bzw. Ring in dem ein Abbauwerkzeug durchgehend in Passiv-Richtung } \\
\text { über die Ortsbrust geführt wurde. }\end{array}$ \\
\hline Lösevolumen (Standvolumen) & $\mathrm{v}_{\mathrm{c}}$ & $\mathrm{m}^{3}$ & $\begin{array}{l}\text { Lösevolumen eines Abbauwerkzeuges zwischen Einbaustationierung } b_{0} \text { und } \\
\text { Ausbaustationierung } b_{v} \text { im Vortrieb (Gl. 39). }\end{array}$ \\
\hline Aktiv-Lösevolumen & $v_{c a}$ & $\mathrm{~m}^{3}$ & $\begin{array}{l}\text { Lösevolumen eines Abbauwerkzeuges beim Einsatz in aktiver bzw. bestim- } \\
\text { mungsgemäßer Richtung zwischen Einbau } \mathrm{b}_{0} \text { und Ausbaustationierung b b im } \\
\text { Vortrieb (Gl. 42). }\end{array}$ \\
\hline Passiv-Lösevolumen & $v_{c p}$ & $\mathrm{~m}^{3}$ & $\begin{array}{l}\text { Lösevolumen eines Abbauwerkzeuges beim passiven Einsatz bzw. entgegen } \\
\text { der bestimmungsgemäßen Richtung zwischen Einbau bo und Ausbaustationie- } \\
\text { rung b } b_{v} \text { im Vortrieb (Gl. 43). }\end{array}$ \\
\hline Vortriebsstrecke (Standstrecke) & $I_{c}$ & $\mathrm{~m}$ & $\begin{array}{l}\text { Vortriebsstrecke eines Abbauwerkzeuges, parallel zu TA zwischen Einbaustati- } \\
\text { onierung } b_{0} \text { und Ausbaustationierung bv (Gl. 30) }\end{array}$ \\
\hline Passiv-Vortriebsstrecke & $\mathrm{I}_{\mathrm{ca}}$ & & $\begin{array}{l}\text { Vortriebsstrecke eines Abbauwerkzeuges in Passiv-Richtung, parallel zu TA } \\
\text { zwischen Einbaustationierung } b_{0} \text { und Ausbaustationierung } b_{v} \text { (Gl. 31). }\end{array}$ \\
\hline Aktiv-Vortriebsstrecke & $I_{\mathrm{cp}}$ & & $\begin{array}{l}\text { Vortriebsstrecke eines Abbauwerkzeuges in Aktiv-Richtung, parallel zu TA } \\
\text { zwischen Einbaustationierung } b_{0} \text { und Ausbaustationierung } b_{v} \text { (Gl. 32). }\end{array}$ \\
\hline Laufweg (Standweg) & $\mathrm{s}_{\mathrm{c}}$ & $\mathrm{km}$ & $\begin{array}{l}\text { Der von einem Abbauwerkzeug entlang der Schneidspur zurückgelegt Weg } \\
\text { beim Bodenabbau zwischen Einbaustationierung } b_{0} \text { und Ausbaustationierung } \\
\text { bv (Gl. 35). }\end{array}$ \\
\hline Modalwert des Laufwegs & $\mathrm{S}_{\mathrm{c}, \bmod }$ & $\mathrm{km}$ & $\begin{array}{l}\text { Der Modalwert des Laufwegs } s_{c} \text { eines Werkzeugtyps innerhalb eines geotech- } \\
\text { nischen Homogenbereichs. }\end{array}$ \\
\hline Aktiv-Laufweg & $\mathrm{s}_{\mathrm{ca}}$ & $\mathrm{km}$ & $\begin{array}{l}\text { Der von einem Abbauwerkzeug entlang der Schneidspur zurückgelegt Weg in } \\
\text { Aktiv-Richtung beim Bodenabbau zwischen Einbaustationierung } b_{0} \text { und Aus- } \\
\text { baustationierung bv (Gl. 37). }\end{array}$ \\
\hline
\end{tabular}




\begin{tabular}{|c|c|c|c|}
\hline Begriff & Symbol & Einheit & Bemerkung \\
\hline Passiv-Laufweg & $\mathrm{S}_{\mathrm{cp}}$ & $\mathrm{km}$ & $\begin{array}{l}\text { Der von einem Abbauwerkzeug entlang der Schneidspur zurückgelegt Weg in } \\
\text { Passiv-Richtung beim Bodenabbau zwischen Einbaustationierung } b_{0} \text { und } \\
\text { Ausbaustationierung } b_{v}(G l .38) \text {. }\end{array}$ \\
\hline Betriebsstunden (Standzeit) & $t_{c}$ & $\mathrm{~h}$ & $\begin{array}{l}\text { Betriebsstunden eines Abbauwerkzeuges beim Bodenabbau zwischen Ein- } \\
\left.\text { baustationierung } b_{0} \text { und Ausbaustationierung } b_{\mathrm{v}} \text {. (Gl. } 49\right) \text {. }\end{array}$ \\
\hline Aktiv-Betriebsstunden & $\mathrm{t}_{\mathrm{ca}}$ & $\mathrm{h}$ & $\begin{array}{l}\text { Betriebsstunden eines Abbauwerkzeuges beim Bodenabbau in Aktiv-Richtung } \\
\text { zwischen Einbaustationierung } b_{0} \text { und Ausbaustationierung } b_{v}(G l .50) \text {. }\end{array}$ \\
\hline Passiv-Betriebsstunden & $\mathrm{t}_{\mathrm{cp}}$ & $\mathrm{h}$ & $\begin{array}{l}\text { Betriebsstunden eines Abbauwerkzeuges beim Bodenabbau in Aktiv-Richtung } \\
\text { zwischen Einbaustationierung } b_{0} \text { und Ausbaustationierung } b_{v}(G l .51) \text {. }\end{array}$ \\
\hline Verschleißrate & $\mathrm{C}_{\mathrm{v}}$ & - & $\begin{array}{l}\text { Allgemein durch Verschleiß verursachter weg-, volumen- oder zeitabhängiger } \\
\text { Massen-, Volumen- oder Längenverlust eines Abbauwerkzeuges (Gl. } 28 \text { \& 29). }\end{array}$ \\
\hline $\begin{array}{l}\text { Ausgangsmasse Abbau- } \\
\text { werkzeug }\end{array}$ & $m_{0}$ & g & Ausgangsmasse eines Abbauwerkzeuges beim Einbau auf dem Schneidrad. \\
\hline Endmasse Abbauwerkzeug & $\mathrm{m}_{\mathrm{v}}$ & g & $\begin{array}{l}\text { Endmasse eines Abbauwerkzeuges beim Ausbau auf dem Schneidrad nach } \\
\text { dem Einsatz zum Bodenabbau. }\end{array}$ \\
\hline Massenverlust Abbauwerkzeug & $m_{d}$ & g & $\begin{array}{l}\text { Massenverlust eines Abbauwerkzeuges durch Verschleiß beim Bodenabbau } \\
\text { zwischen Ein- und Ausbau auf dem Schneidrad (Gl. 25). }\end{array}$ \\
\hline $\begin{array}{l}\text { Ausgangsvolumen Abbau- } \\
\text { werkzeug }\end{array}$ & $\mathrm{v}_{0}$ & $\mathrm{~cm}^{3}$ & Ausgangsvolumen eines Abbauwerkzeuges beim Einbau auf dem Schneidrad. \\
\hline Endvolumen Abbauwerkzeug & $\mathrm{v}_{\mathrm{v}}$ & $\mathrm{cm}^{3}$ & $\begin{array}{l}\text { Endvolumen eines Abbauwerkzeuges beim Ausbau auf dem Schneidrad nach } \\
\text { dem Einsatz zum Bodenabbau. }\end{array}$ \\
\hline $\begin{array}{l}\text { Volumenverlust Abbau- } \\
\text { werkzeug }\end{array}$ & $v_{d}$ & $\mathrm{~cm}^{3}$ & $\begin{array}{l}\text { Volumenverlust eines Abbauwerkzeuges durch Verschleiß beim Bodenabbau } \\
\text { zwischen Ein- und Ausbau auf dem Schneidrad (Gl. 26) }\end{array}$ \\
\hline Ausgangshöhe Abbauwerkzeug & $\mathrm{h}_{0}$ & $\mathrm{~mm}$ & Ausgangshöhe eines Abbauwerkzeuges beim Einbau auf dem Schneidrad. \\
\hline Endhöhe Abbauwerkzeug & $\mathrm{h}_{\mathrm{v}}$ & $\mathrm{mm}$ & $\begin{array}{l}\text { Endhöhe eines Abbauwerkzeuges beim Ausbau auf dem Schneidrad nach dem } \\
\text { Einsatz zum Bodenabbau. }\end{array}$ \\
\hline Höhenverlust Abbauwerkzeug & $h_{d}$ & $\mathrm{~mm}$ & $\begin{array}{l}\text { Höhenverlust eines Abbauwerkzeuges durch Verschleiß beim Bodenabbau } \\
\text { zwischen Ein- und Ausbau auf dem Schneidrad (Gl. 27). }\end{array}$ \\
\hline Bohrzeit & $t_{(b)}$ & s & Zeit für den Vortrieb eines Ringes b. \\
\hline Aktiv-Bohrzeit & $t_{a(b)}$ & s & $\begin{array}{l}\text { Zeit für den Vortrieb eines Ringes } b_{a} \text {, in dem ein Abbauwerkzeug durchgehend } \\
\text { in Aktiv-Richtung über die Ortsbrust geführt wurde. }\end{array}$ \\
\hline Passiv-Bohrzeit & $t_{p(b)}$ & s & $\begin{array}{l}\text { Zeit für den Vortrieb eines Ringes } b_{p} \text { in dem ein Abbauwerkzeug durchgehend } \\
\text { in Passiv-Richtung über die Ortsbrust geführt wurde.. }\end{array}$ \\
\hline $\begin{array}{l}\text { Anzahl der Schneidrad- } \\
\text { umdrehungen }\end{array}$ & $\mathrm{n}_{\mathrm{u}}$ & - & Anzahl der Schneidradumdrehungen in einem definierten Vortriebsintervall. \\
\hline $\begin{array}{l}\text { Anzahl Schneidrad- } \\
\text { umdrehungen }\end{array}$ & $\mathrm{n}_{\mathrm{u}(\mathrm{b})}$ & - & Anzahl der Schneidradumdrehungen innerhalb eines Bohrhubes b (Gl. 36). \\
\hline Aktiv-Schneidradumdrehungen & $\mathrm{n}_{\mathrm{ua}(\mathrm{b})}$ & - & $\begin{array}{l}\text { Anzahl der Schneidradumdrehungen innerhalb eines Bohrhubes b, in dem ein } \\
\text { Abbauwerkzeug in Aktiv-Richtung über die Ortsbrust geführt wurde. }\end{array}$ \\
\hline $\begin{array}{l}\text { Passiv-Schneidrad- } \\
\text { umdrehungen }\end{array}$ & $\mathrm{n}_{\mathrm{up}(\mathrm{b})}$ & - & $\begin{array}{l}\text { Anzahl der Schneidradumdrehungen innerhalb eines Bohrhubes b, in dem ein } \\
\text { Abbauwerkzeug in Passiv-Richtung über die Ortsbrust geführt wurde. }\end{array}$ \\
\hline \multicolumn{4}{|l|}{ Prognosemodell } \\
\hline Index & z & - & $\begin{array}{l}\text { Ganzzahliger Index zur von Vortriebsbeginn an ansteigenden Nummerierung } \\
\text { der geotechnischen Homogenbereiche HGB. }\end{array}$ \\
\hline Index & $\mathrm{m}$ & - & $\begin{array}{l}\text { Ganzzahliger Index zur von Vortriebsbeginn an ansteigenden Nummerierung } \\
\text { der Vortriebsunterbrechungen } I_{v} \text { und } I_{e} \text {. }\end{array}$ \\
\hline Index & $\mathrm{n}$ & - & $\begin{array}{l}\text { Index für die Anzahl der Iterationen bzw. die Anzahl der Teilstrecken bei der } \\
\text { Schätzung der maximal erreichbaren Vortriebsstrecken } L_{\max (m+1 ; z+n)} \text {. }\end{array}$ \\
\hline Index & k & - & $\begin{array}{l}\text { Index für Anzahl der Teilstrecken bzw. angefahrenen Homogenbereiche in der } \\
\text { Vortriebsstrecke } L_{d, e(m+1 ; z+k)} \text {. }\end{array}$ \\
\hline \multirow[t]{5}{*}{$\begin{array}{l}\text { Geotechnische Homogen- } \\
\text { bereiche }\end{array}$} & $\mathrm{HGB}_{(\mathrm{z})}$ & - & Abkürzung für geotechnische Homogenbereiche mit dem Index (z). \\
\hline & $\mathrm{HGB}_{(\mathrm{z}+(\mathrm{n}-1))}$ & - & Abkürzung für geotechnische Homogenbereiche mit dem Index $(z+(n-1))$. \\
\hline & $\mathrm{HGB}_{(\mathrm{z}+\mathrm{n})}$ & & Abkürzung für geotechnische Homogenbereiche mit dem Index $(z+n)$. \\
\hline & $\mathrm{HGB}_{(\mathrm{z}+(\mathrm{k}-1))}$ & - & Abkürzung für geotechnische Homogenbereiche mit dem Index (z+(k-1)). \\
\hline & $\mathrm{HGB}_{(\mathrm{z}+\mathrm{k})}$ & - & Abkürzung für geotechnische Homogenbereiche mit dem Index $(z+k)$. \\
\hline
\end{tabular}




\begin{tabular}{|c|c|c|c|}
\hline Begriff & Symbol & Einheit & Bemerkung \\
\hline \multirow[t]{2}{*}{ Erwartungswert der Penetration } & $\mathrm{p}_{\mathrm{e}(\mathrm{z})}$ & $\mathrm{mm} / \mathrm{U}$ & Erwartungswert für der Penetration $\mathrm{p}_{\mathrm{e}(\mathrm{z})}[\mathrm{mm} / \mathrm{U}]$ im Homogenbereich $\mathrm{HGB}_{(\mathrm{z})}$. \\
\hline & $\mathrm{p}_{\mathrm{e}(z+n)}$ & $\mathrm{mm} / \mathrm{U}$ & Erwartungswert für der Penetration $\mathrm{p}_{\mathrm{e}(\mathrm{z})}[\mathrm{mm} / \mathrm{U}]$ im Homogenbereich $\mathrm{HGB}_{(\mathrm{z}+\mathrm{n})}$. \\
\hline $\begin{array}{l}\text { Erwartungswert der Aktiv- } \\
\text { Penetration }\end{array}$ & $\mathrm{p}_{\mathrm{a}, \mathrm{e}(\mathrm{z})}$ & $\mathrm{mm} / \mathrm{U}$ & $\begin{array}{l}\text { Erwartungswert der Aktiv-Penetration } \mathrm{p}_{\mathrm{a}, \mathrm{e}(\mathrm{z})}[\mathrm{mm} / \mathrm{U}] \text { im Homogenbereich } \mathrm{HGB}_{(\mathrm{z})} \\
(\mathrm{Gl} . \mathrm{80})\end{array}$ \\
\hline $\begin{array}{l}\text { Korrekturfaktor Schneidring- } \\
\text { breite }\end{array}$ & $\mathrm{f}_{\mathrm{b}}$ & - & $\begin{array}{l}\text { Faktor zur Korrektur des Laufweg } \mathrm{s}_{\mathrm{c}} \text { für Schneidrollen um die Schneidringbreite } \\
\mathrm{b}_{\mathrm{SR}}[\mathrm{mm}](\mathrm{Gl} .68) .\end{array}$ \\
\hline $\begin{array}{l}\text { Korrekturfaktor Schneidring- } \\
\text { breite }\end{array}$ & $f_{b(z)}$ & - & $\begin{array}{l}\text { Faktor zur Korrektur des Basiswertes } \mathrm{s}_{\mathrm{c}, \mathrm{b}(\mathrm{zz})} \text { für Schneidrollen um die Schneid- } \\
\text { ringbreite } \mathrm{b}_{\mathrm{SR}}[\mathrm{mm}] \text { im Homogenbereich } \mathrm{HGB}_{(\mathrm{z})}(\mathrm{Gl} .77) \text {. }\end{array}$ \\
\hline Korrekturfaktor Penetration & $f_{p}$ & - & $\begin{array}{l}\text { Faktor zur Korrektur des Laufweg } \mathrm{s}_{\mathrm{c}} \text { für Schälmesser, Stichel und Räumer um } \\
\text { die Penetration } \mathrm{p}[\mathrm{mm} / \mathrm{U}](\mathrm{Gl} .69) \text {. }\end{array}$ \\
\hline Korrekturfaktor Penetration & $f_{p(z)}$ & - & $\begin{array}{l}\text { Faktor zur Korrektur des Basiswertes } \mathrm{s}_{\mathrm{c}, \mathrm{b}(\mathrm{zz})} \text { für Schälmesser, Stichel und } \\
\text { Räumer um die Penetration } \mathrm{p}[\mathrm{mm} / \mathrm{U}] \text { im Homogenbereich HGB } \mathrm{HG}_{(\mathrm{z})}(\mathrm{Gl} .79) \text {. }\end{array}$ \\
\hline $\begin{array}{l}\text { Korrekturfaktor für vorlaufende } \\
\text { Stichel }\end{array}$ & $f_{h(z)}$ & - & $\begin{array}{l}\text { Faktor zur Korrektur des Basiswertes } \mathrm{s}_{\mathrm{c}, \mathrm{b}(\mathrm{z})} \text { für Schälmesser und Räumer um } \\
\text { die Auswirkungen vorlaufender Stichel im Homogenbereich } \mathrm{HGB}_{(\mathrm{z})}(\mathrm{Gl} .78) \text {. }\end{array}$ \\
\hline $\begin{array}{l}\text { Korrekturfaktor Verschleiß- } \\
\text { schutz }\end{array}$ & $f_{v(z)}$ & - & $\begin{array}{l}\text { Faktor zur Korrektur des Verschleißschutze im Homogenbereich } \mathrm{HGB}_{(\mathrm{z})} \\
\text { (Gl. 81). }\end{array}$ \\
\hline $\begin{array}{l}\text { Korrekturfaktor Schneidrad- } \\
\text { öffnungsverhältnis }\end{array}$ & $\mathrm{f}_{\mathrm{o}(\mathrm{z})}$ & - & $\begin{array}{l}\text { Faktor zur Korrektur des Verschleißschutze im Homogenbereich } \mathrm{HGB}_{(z)} \\
\text { (Gl. 81). }\end{array}$ \\
\hline Basiswert des Laufwegs & $\mathrm{S}_{\mathrm{c}, \mathrm{b}(\mathrm{z})}$ & $\mathrm{km}$ & $\begin{array}{l}\text { Basiswert des Laufwegs } \mathrm{S}_{\mathrm{c}, \mathrm{b}(z)} \text { in } \mathrm{HGB}_{(z)} \text { in der Verschleißprognose aus der } \\
\text { Korrelation mit dem SAI (Gl. } 75 \text { \& 76). }\end{array}$ \\
\hline \multirow[t]{2}{*}{ Erwartungswerte des Laufwegs } & $\mathrm{S}_{\mathrm{c}, \mathrm{e}(\mathrm{z})}$ & $\mathrm{km}$ & $\begin{array}{l}\text { Erwartungswert des Laufwegs } \mathrm{s}_{\mathrm{c}, \mathrm{e}(\mathrm{z})} \text { in } \mathrm{HGB}_{(\mathrm{z})} \text { in der Verschleißprognose } \\
\text { (Gl. 81 \& 82). }\end{array}$ \\
\hline & $\mathrm{S}_{\mathrm{c}, \mathrm{e}(\mathrm{z}+\mathrm{n})}$ & $\mathrm{km}$ & $\begin{array}{l}\text { Erwartungswert des Laufwegs } \mathrm{s}_{\mathrm{c}, \mathrm{e}(\mathrm{z}+\mathrm{n})} \text { in } \mathrm{HGB}_{(\mathrm{z}+\mathrm{n})} \text { in der Verschleißprognose } \\
\text { (Gl. } 81 \text { \&82). }\end{array}$ \\
\hline \multirow[t]{5}{*}{ Vortriebsunterbrechung } & $\mathrm{I}_{(\mathrm{m})}$ & - & Abkürzung für eine Vortriebsunterbrechung I mit dem Index (m). \\
\hline & $\mathrm{I}_{(\mathrm{m}+1)}$ & - & Abkürzung für eine Vortriebsunterbrechung I mit dem Index $(m+1)$. \\
\hline & $\mathrm{I}_{(\mathrm{m} ; \mathrm{z})}$ & - & $\begin{array}{l}\text { Abkürzung für eine Vortriebsunterbrechung I mit dem Index (m) im geotechni- } \\
\text { schen Homogenbereich mit dem Index }(\mathrm{z}) \text {. }\end{array}$ \\
\hline & $\mathrm{I}_{(\mathrm{m} ; \mathrm{z+1})}$ & - & $\begin{array}{l}\text { Abkürzung für eine Vortriebsunterbrechung I mit dem Index }(m) \text { im geotechni- } \\
\text { schen Homogenbereich mit dem Index }(z+1) \text {. }\end{array}$ \\
\hline & $\mathrm{I}_{(\mathrm{m}+1 ; z+\mathrm{k})}$ & - & $\begin{array}{l}\text { Abkürzung für eine Vortriebsunterbrechung I mit dem Index }(m) \text { im geotechni- } \\
\text { schen Homogenbereich mit dem Index }(z+k) \text {. }\end{array}$ \\
\hline \multirow[t]{3}{*}{$\begin{array}{l}\text { Maximal Vortriebsstrecken der } \\
\text { Abbauwerkzeuge }\end{array}$} & $\mathrm{L}_{\mathrm{c}(\mathrm{m})}$ & $m$ & $\begin{array}{l}\text { Maximal erreichbare Vortriebsstrecke eines einzelnen Abbauwerkzeuges c } \\
\text { ausgehend von der Vortriebsunterbrechung } \mathrm{I}_{(\mathrm{m}-1)} \text { bis zum Erreichen der Ver- } \\
\text { schleißgrenze (Gl. 83). }\end{array}$ \\
\hline & $\mathrm{L}_{c(\mathrm{~m}+1)}$ & $\mathrm{m}$ & $\begin{array}{l}\text { Maximal erreichbare Vortriebsstrecke eines einzelnen Abbauwerkzeuges } c \\
\text { ausgehend von der Vortriebsunterbrechung } I_{(m)} \text { bis zum Erreichen der Ver- } \\
\text { schleißgrenze (Gl. 83). }\end{array}$ \\
\hline & $\mathrm{L}_{\mathrm{c}(\mathrm{m}+1 ; z+n)}$ & $\mathrm{m}$ & $\begin{array}{l}\text { Maximal erreichbare Vortriebsstrecke eines einzelnen Abbauwerkzeuges } c \\
\text { ausgehend von der Vortriebsunterbrechung } I_{(m)} \text { bis zum Erreichen der Ver- } \\
\text { schleißgrenze in der Teilstrecke im Homogenbereich }(z+n) \text {. (Gl. 107). }\end{array}$ \\
\hline \multirow[t]{4}{*}{$\begin{array}{l}\text { Maximale Vortriebsstrecken der } \\
\text { TVM }\end{array}$} & $\mathrm{L}_{\max (\mathrm{m})}$ & $\mathrm{m}$ & $\begin{array}{l}\text { Maximal erreichbare Vortriebsstrecke der TVM ausgehend von der Vortriebsun- } \\
\text { terbrechung } I_{(\mathrm{m}-1)} \text { bis zum erreich der Verschleißgrenze des ersten Abbauwerk- } \\
\text { zeuges auf dem Schneidrad (GI. 84). }\end{array}$ \\
\hline & $\mathrm{L}_{\max (m+1)}$ & $\mathrm{m}$ & $\begin{array}{l}\text { Maximal erreichbare Vortriebsstrecke der TVM ausgehend von der Vortriebsun- } \\
\text { terbrechung } I_{(m)} \text { bis zum erreich der Verschleißgrenze des ersten Abbauwerk- } \\
\text { zeuges auf dem Schneidrad (Gl. 84). }\end{array}$ \\
\hline & $\mathrm{L}_{\max (\mathrm{m}+1 ; z)}$ & $\mathrm{m}$ & $\begin{array}{l}\text { Maximal erreichbare Vortriebsstrecke der TVM ausgehend von der Vortriebsun- } \\
\text { terbrechung } \mathrm{I}_{(\mathrm{m}-1)} \text { bis zum erreich der Verschleißgrenze des ersten Abbauwerk- } \\
\text { zeuges auf dem Schneidrad in der Teilstrecke in } \mathrm{HGB}_{(2)}(\mathrm{Gl} .102) .\end{array}$ \\
\hline & $\mathrm{L}_{\max (m+1 ; z+n)}$ & $\mathrm{m}$ & $\begin{array}{l}\text { Maximal erreichbare Vortriebsstrecke der TVM ausgehend von der Vortriebsun- } \\
\text { terbrechung } \mathrm{I}_{(\mathrm{m}-1)} \text { bis zum erreich der Verschleißgrenze des ersten Abbauwerk- } \\
\text { zeuges auf dem Schneidrad in der Teilstrecke in } \mathrm{HGB}_{(z+n)}(\mathrm{Gl} .108) \text {. }\end{array}$ \\
\hline \multirow[t]{6}{*}{$\begin{array}{l}\text { Vortriebsstrecke zwischen } \\
\text { Vortriebsunterbrechungen }\end{array}$} & $\mathrm{L}_{\mathrm{d}(\mathrm{m})}$ & $\mathrm{m}$ & Vortriebsstrecke zwischen den Vortriebsunterbrechungen $\mathrm{I}_{(\mathrm{m}-1)}$ und $\mathrm{I}_{(\mathrm{m})}$. \\
\hline & $L_{d(m+1)}$ & $\mathrm{m}$ & Vortriebsstrecke zwischen den Vortriebsunterbrechungen $I_{(m)}$ und $I_{(m+1)}$. \\
\hline & $L_{d, e(m)}$ & $\mathrm{m}$ & $\begin{array}{l}\text { Erwartungswert der Vortriebsstrecke zwischen den Vortriebsunterbrechungen } \\
\mathrm{I}_{(\mathrm{m}-1)} \text { und } \mathrm{I}_{(\mathrm{m})}(\mathrm{Gl} .85) \text {. }\end{array}$ \\
\hline & $L_{d, e(m+1)}$ & $\mathrm{m}$ & $\begin{array}{l}\text { Erwartungswert der Vortriebsstrecke zwischen den Vortriebsunterbrechungen } \\
\mathrm{I}_{(\mathrm{m})} \text { und } \mathrm{I}_{(\mathrm{m}+1)}(\mathrm{Gl} .93) \text {. }\end{array}$ \\
\hline & $\mathrm{L}_{\mathrm{d}, \mathrm{e}(\mathrm{m}+1 ; z)}$ & $\mathrm{m}$ & $\begin{array}{l}\text { Erwartungswert für den Streckenanteil der Vortriebsstrecke } L_{d, e(m+1)} \text { in } \mathrm{HGB}_{(z)} \\
\text { (Gl. 121) }\end{array}$ \\
\hline & $\mathrm{L}_{\mathrm{d}, \mathrm{e}(\mathrm{m}+1 ; \mathrm{z}+\mathrm{k})}$ & $\mathrm{m}$ & $\begin{array}{l}\text { Erwartungswert für den Streckenanteil der Vortriebsstrecke } L_{d, e(m+1)} \text { in } \mathrm{HGB}_{(z+k)} \\
\text { (Gl. 126). }\end{array}$ \\
\hline
\end{tabular}




\begin{tabular}{|c|c|c|c|}
\hline Begriff & Symbol & Einheit & Bemerkung \\
\hline \multirow[t]{7}{*}{ Partielle Laufwege } & $\mathrm{S}_{\mathrm{cd}(\mathrm{m})}$ & $\mathrm{km}$ & Partieller Laufweg des Abbauwerkzeuges c in der Vortriebsstrecke $L_{d(m)}$. \\
\hline & $\mathrm{S}_{\mathrm{cd}(\mathrm{m}+1)}$ & $\mathrm{km}$ & Partieller Laufweg des Abbauwerkzeuges $\mathrm{c}$ in der Vortriebsstrecke $\mathrm{L}_{\mathrm{d}(\mathrm{m}+1)}$. \\
\hline & $\mathrm{S}_{\mathrm{cd}, \mathrm{e}(\mathrm{m})}$ & $\mathrm{km}$ & $\begin{array}{l}\text { Erwartungswert des partiellen Laufwegs des Abbauwerkzeuges } c \text { in der } \\
\text { geplanten Vortriebsstrecke } L_{d, e(m)}(G l .86) \text {. }\end{array}$ \\
\hline & $\mathrm{S}_{\mathrm{cd}, \mathrm{e}(\mathrm{m}+1)}$ & $\mathrm{km}$ & $\begin{array}{l}\text { Erwartungswert des partiellen Laufwegs des Abbauwerkzeuges } c \text { in der } \\
\text { geplanten Vortriebsstrecke } L_{d, e(m+1)}(G l .95) \text {. }\end{array}$ \\
\hline & $\mathrm{S}_{\mathrm{cd}, \mathrm{e}(\mathrm{m}+1 ; \mathrm{z})}$ & $\mathrm{km}$ & $\begin{array}{l}\text { Erwartungswert des partiellen Laufwegs des Abbauwerkzeuges } c \text { in der } \\
\text { geplanten Vortriebsstrecke } L_{d, e(m+1 ; z)}(G l .103) \text {. }\end{array}$ \\
\hline & $\mathrm{S}_{\mathrm{cd}, \mathrm{e}(\mathrm{m}+1 ; \mathrm{z}+\mathrm{n})}$ & $\mathrm{km}$ & $\begin{array}{l}\text { Erwartungswert des partiellen Laufwegs des Abbauwerkzeuges } c \text { in der } \\
\left.\text { geplanten Vortriebsstrecke } L_{d, e(m+1 ; z+n)} \text { (Gl. } 105 \& 109\right) .\end{array}$ \\
\hline & $\mathrm{S}_{\mathrm{cd}, \mathrm{e}(\mathrm{m}+1 ; \mathrm{z}+\mathrm{k})}$ & $\mathrm{km}$ & $\begin{array}{l}\text { Erwartungswert des partiellen Laufwegs des Abbauwerkzeuges } c \text { in der } \\
\text { geplanten Vortriebsstrecke } L_{d, e(m+1 ; z+k)}(G l .124) \text {. }\end{array}$ \\
\hline \multirow[t]{7}{*}{ Partielle Ausnutzungsgrade } & $e_{c d(m)}$ & $\%$ & $\begin{array}{l}\text { Partieller Ausnutzungsgrad des Abbauwerkzeuges c in der Vortriebsstrecke } \\
L_{d(m)} .\end{array}$ \\
\hline & $e_{c d(m+1)}$ & $\%$ & $\begin{array}{l}\text { Partieller Ausnutzungsgrad des Abbauwerkzeuges } c \text { in der Vortriebsstrecke } \\
L_{d(m+1)} \text {. }\end{array}$ \\
\hline & $\mathrm{e}_{\mathrm{cd}, \mathrm{e}(\mathrm{m})}$ & $\%$ & $\begin{array}{l}\text { Erwartungswert des partiellen Ausnutzungsgrades des Abbauwerkzeuges c in } \\
\text { der geplanten Vortriebsstrecke } L_{d, e(m)}(\text { Gl. } 87) \text {. }\end{array}$ \\
\hline & $\mathrm{e}_{\mathrm{cd}, \mathrm{e}(\mathrm{m}+1)}$ & $\%$ & $\begin{array}{l}\text { Erwartungswert des partiellen Ausnutzungsgrades des Abbauwerkzeuges } c \text { in } \\
\text { der geplanten Vortriebsstrecke } L_{d, e(m+1)}(G l .96) \text {. }\end{array}$ \\
\hline & $\mathrm{e}_{\mathrm{cd}, \mathrm{e}(\mathrm{m}+1 ; z)}$ & $\%$ & $\begin{array}{l}\text { Erwartungswert des partiellen Ausnutzungsgrades des Abbauwerkzeuges } c \text { in } \\
\text { der geplanten Vortriebsstrecke } L_{d, e(m+1 ; z)}(G l .104) \text {. }\end{array}$ \\
\hline & $e_{c d, e(m+1 ; z+n)}$ & $\%$ & $\begin{array}{l}\text { Erwartungswert des partiellen Ausnutzungsgrades des Abbauwerkzeuges } \mathrm{c} \text { in } \\
\text { der geplanten Vortriebsstrecke } L_{d, e(m+1 ; z+n)}(G \mid .110) \text {. }\end{array}$ \\
\hline & $e_{c d, e(m+1 ; z+k)}$ & $\%$ & $\begin{array}{l}\text { Erwartungswert des partiellen Ausnutzungsgrades des Abbauwerkzeuges } c \text { in } \\
\text { der geplanten Vortriebsstrecke } L_{d, e(m+1 ; z+k)}(G l .125) \text {. }\end{array}$ \\
\hline \multirow[t]{4}{*}{$\begin{array}{l}\text { Metrische Stationierung der } \\
\text { Vortriebsunterbrechungen }\end{array}$} & $\mathrm{L}_{1(\mathrm{~m})}$ & $\mathrm{m}$ & $\begin{array}{l}\text { Kumulierte Vortriebsstrecke bis zur Vortriebsunterbrechung } I_{(m)} \text { bzw. metrische } \\
\text { Stationierung von } I_{(m)} \text {. }\end{array}$ \\
\hline & $L_{1(m+1)}$ & $\mathrm{m}$ & $\begin{array}{l}\text { Kumulierte Vortriebsstrecke bis zur Vortriebsunterbrechung } \mathrm{I}_{(\mathrm{m}+1)} \text { bzw. metri- } \\
\text { sche Stationierung von } \mathrm{I}_{(\mathrm{m}+1)} \text {. }\end{array}$ \\
\hline & $\mathrm{L}_{\mathrm{l}, \mathrm{e}(\mathrm{m})}$ & $\mathrm{m}$ & $\begin{array}{l}\text { Erwartungswert der kumulierten Vortriebsstrecke bis zur Vortriebsunterbre- } \\
\text { chung } I_{(m)} \text { bzw. metrische Stationierung von } I_{(m)}(G l .89) \text {. }\end{array}$ \\
\hline & $\mathrm{L}_{\mathrm{l}, \mathrm{e}(\mathrm{m}+1)}$ & $\mathrm{m}$ & $\begin{array}{l}\text { Erwartungswert der kumulierten Vortriebsstrecke bis zur Vortriebsunterbre- } \\
\text { chung } I_{(m+1)} \text { bzw. metrische Stationierung von } I_{(m+1)}(G l .94) \text {. }\end{array}$ \\
\hline \multirow[t]{4}{*}{$\begin{array}{l}\text { Ringnummern der Vortriebs- } \\
\text { unterbrechungen }\end{array}$} & $b_{1(m)}$ & - & Ringnummer der Vortriebsunterbrechung $I_{(m)}$. \\
\hline & $\mathrm{b}_{\mathrm{l}(\mathrm{m}+1)}$ & - & Ringnummer der Vortriebsunterbrechung $\mathrm{I}_{(\mathrm{m}+1)}$. \\
\hline & $\mathrm{b}_{\mathrm{I}, \mathrm{e}(\mathrm{m})}$ & - & Erwartungswert der Ringnummer der Vortriebsunterbrechung $\mathrm{I}_{(\mathrm{m})}(\mathrm{Gl} .90)$ \\
\hline & $\mathrm{b}_{\mathrm{I}, \mathrm{e}(\mathrm{m}+1)}$ & - & Erwartungswert der Ringnummer der Vortriebsunterbrechung $\mathrm{I}_{(\mathrm{m}+1)}(\mathrm{Gl} .90)$. \\
\hline \multirow[t]{4}{*}{ Kumulierte Laufwege } & $\mathrm{S}_{\mathrm{c}(\mathrm{m})}$ & $\mathrm{km}$ & $\begin{array}{l}\text { Kumulierter Laufweg des Abbauwerkzeuges c an der Vortriebsunterbrechung } \\
\mathrm{I}_{(\mathrm{m})} \text {. }\end{array}$ \\
\hline & $\mathrm{S}_{\mathrm{c}(\mathrm{m}+1)}$ & $\mathrm{km}$ & $\begin{array}{l}\text { Kumulierter Laufweg des Abbauwerkzeuges c an der Vortriebsunterbrechung } \\
I_{(m+1)} \text {. }\end{array}$ \\
\hline & $\mathrm{S}_{\mathrm{c}, \mathrm{e}(\mathrm{m})}$ & $\mathrm{km}$ & $\begin{array}{l}\text { Erwartungswert des kumulierten Laufwegs des Abbauwerkzeuges } \mathrm{c} \text { an der } \\
\text { Vortriebsunterbrechung } \mathrm{I}_{(\mathrm{m})} \text {. (Gl. 91). }\end{array}$ \\
\hline & $\mathrm{S}_{\mathrm{c}, \mathrm{e}(\mathrm{m}+1)}$ & $\mathrm{km}$ & $\begin{array}{l}\text { Erwartungswert des kumulierten Laufwegs des Abbauwerkzeuges } \mathrm{c} \text { an der } \\
\text { Vortriebsunterbrechung } \mathrm{I}_{(\mathrm{m}+1)}(\mathrm{Gl} .97) \text {. }\end{array}$ \\
\hline \multirow[t]{4}{*}{ Kumulierte Ausnutzungsgrade } & $e_{c(m)}$ & $\%$ & $\begin{array}{l}\text { Kumulierter Ausnutzungsgrad des Abbauwerkzeuges } \mathrm{c} \text { an der Vortriebsunter- } \\
\text { brechung } \mathrm{I}_{(\mathrm{m})} \text {. }\end{array}$ \\
\hline & $e_{c(m+1)}$ & $\%$ & $\begin{array}{l}\text { Kumulierter Ausnutzungsgrad des Abbauwerkzeuges c an der Vortriebsunter- } \\
\text { brechung } \mathrm{I}_{(\mathrm{m}+1)} \text {. }\end{array}$ \\
\hline & $e_{c, e(m)}$ & $\%$ & $\begin{array}{l}\text { Erwartungswert des kumulierten Ausnutzungsgrades des Abbauwerkzeuges } \mathrm{C} \\
\text { an der Vortriebsunterbrechung } \mathrm{I}_{(\mathrm{m})} \text {. (Gl. 92). }\end{array}$ \\
\hline & $e_{c, e(m+1)}$ & $\%$ & $\begin{array}{l}\text { Erwartungswert des kumulierten Ausnutzungsgrades des Abbauwerkzeuges c } \\
\text { an der Vortriebsunterbrechung } \mathrm{I}_{(\mathrm{m}+1)}(\mathrm{Gl} .98) \text {. }\end{array}$ \\
\hline \multirow[t]{5}{*}{$\begin{array}{l}\text { Länge geotechnischer Homo- } \\
\text { genbereiche }\end{array}$} & $L_{d(z)}$ & $\mathrm{m}$ & Länge des geotechnischen Homogenbereichs mit dem Index $(z)$. \\
\hline & $L_{d}(z+1)$ & $\mathrm{m}$ & Länge des geotechnischen Homogenbereichs mit dem Index $(z+1)$. \\
\hline & $\mathrm{L}_{d(z+n)}$ & $\mathrm{m}$ & Länge des geotechnischen Homogenbereichs mit dem Index $(z+n)$. \\
\hline & $\mathrm{L}_{\mathrm{d}(\mathrm{z}+(\mathrm{k}-1))}$ & $\mathrm{m}$ & Länge des geotechnischen Homogenbereichs mit dem Index $x_{(z+(k-1))}$. \\
\hline & $L_{d(z+k)}$ & $\mathrm{m}$ & Länge des geotechnischen Homogenbereichs mit dem Index $(z+k)$. \\
\hline
\end{tabular}




\begin{tabular}{|c|c|c|c|}
\hline Begriff & Symbol & Einheit & Bemerkung \\
\hline \multirow[t]{5}{*}{$\begin{array}{l}\text { Metrische Stationierung der } \\
\text { Grenzen zwischen HGB }\end{array}$} & $\mathrm{L}_{\mathrm{HGB}(\mathrm{z})}$ & $\mathrm{m}$ & $\begin{array}{l}\text { Kumulierte Länge der geotechnischen Homogenbereiche } \mathrm{L}_{d(z)} \text { bis } \mathrm{HGB}_{(z)} \text { bzw. } \\
\text { metrische Stationierung der Grenze zwischen } \mathrm{HGB}_{(z)} \text { und } \mathrm{HGB}_{(\mathrm{z}+1)}(\mathrm{Gl} .100) \text {. }\end{array}$ \\
\hline & $\mathrm{L}_{H G B}(z+1)$ & $\mathrm{m}$ & $\begin{array}{l}\text { Kumulierte Länge der geotechnischen Homogenbereiche } \mathrm{L}_{\mathrm{d}(\mathrm{z}+1)} \text { bis } \mathrm{HGB}_{(z+1)} \\
\text { bzw. metrische Stationierung der Grenze zwischen } \mathrm{HGB}_{(\mathrm{z}+1)} \text { und } \mathrm{HGB}_{(z+2)} \text {. }\end{array}$ \\
\hline & $\mathrm{L}_{H G B}(z+n)$ & m & $\begin{array}{l}\text { Kumulierte Länge der geotechnischen Homogenbereiche } L_{d(z+n)} \text { bis } \mathrm{HGB}_{(z+n)} \text { bzw. } \\
\text { metrische Stationierung der Grenze zwischen } \mathrm{HGB}_{(z+n)} \text { und } \mathrm{HGB}_{(z+(n+1))}(\mathrm{Gl} .115) \text {. }\end{array}$ \\
\hline & $\mathrm{L}_{\mathrm{HGB}(\mathrm{z}+(\mathrm{k}-1))}$ & $\mathrm{m}$ & $\begin{array}{l}\text { Kumulierte Länge der geotechnischen Homogenbereiche } \mathrm{L}_{\mathrm{d}(z+(k-1))} \text { bis } \mathrm{HGB}_{(z+(k-1))} \\
\text { bzw. metrische Stationierung der Grenze zwischen } \mathrm{HGB}_{(\mathrm{z}+(\mathrm{k}-1))} \text { und } \mathrm{HGB}_{(\mathrm{z}+\mathrm{k})} \\
\text { (Gl. 120). }\end{array}$ \\
\hline & $\mathrm{L}_{\mathrm{HGB}(z+k)}$ & $\mathrm{m}$ & $\begin{array}{l}\text { Kumulierte Länge der geotechnischen Homogenbereiche } L_{d(z+k)} \text { bis } \mathrm{HGB}_{(z+k)} \text { bzw. } \\
\text { metrische Stationierung der Grenze zwischen } \mathrm{HGB}_{(z+k)} \text { und } \mathrm{HGB}_{(z+(k+1))}(\mathrm{Gl} \text {. 120). }\end{array}$ \\
\hline $\begin{array}{l}\text { Ringnummern der Grenzen } \\
\text { zwischen HGB }\end{array}$ & $\mathrm{b}_{\mathrm{HGB}(\mathrm{z})}$ & - & Ringnummer an der Grenze zwischen $\mathrm{HGB}_{(\mathrm{z})}$ und $\mathrm{HGB}_{(\mathrm{z}+1)}(\mathrm{Gl} .101)$. \\
\hline \multirow[t]{2}{*}{$\begin{array}{l}\text { Ausnutzungsgrad eines Abbau- } \\
\text { werkzeuges zw. Ein- \& Ausbau }\end{array}$} & $\mathrm{e}_{\mathrm{cv}(\mathrm{m})}$ & $\%$ & $\begin{array}{l}\text { Ausnutzungsgrad des Abbauwerkzeuges } c \text { zwischen Einbau auf dem Schneidrad } \\
\text { bei } b_{0} \text { und Ausbau bei } b_{v} \text {. }\end{array}$ \\
\hline & $\mathrm{e}_{\mathrm{cv}, \mathrm{e}(\mathrm{m})}$ & $\%$ & $\begin{array}{l}\text { Erwartungswert des Ausnutzungsgrades des Abbauwerkzeuges c zwischen } \\
\text { Einbau auf dem Schneidrad bei } b_{0} \text { und Ausbau bei } b_{v} \text { bzw. } b_{l(m)} \text { in der Vortriebs- } \\
\text { unterbrechung } I_{(m)}(G l .136) \text {. }\end{array}$ \\
\hline \multirow[t]{2}{*}{$\begin{array}{l}\text { Laufweg eines Abbauwerkzeu- } \\
\text { ges zwischen Ein- und Ausbau }\end{array}$} & $\mathrm{S}_{\mathrm{cv}(\mathrm{m})}$ & $\mathrm{km}$ & $\begin{array}{l}\text { Laufweg des Abbauwerkzeuges } \mathrm{c} \text { zwischen Einbau auf dem Schneidrad bei } b_{0} \\
\text { und Ausbau bei } b_{v} \text {. }\end{array}$ \\
\hline & $\mathrm{S}_{\mathrm{cv}, \mathrm{e}(\mathrm{m})}$ & $\mathrm{km}$ & $\begin{array}{l}\text { Erwartungswert des Laufwegs des Abbauwerkzeuges c zwischen Einbau auf } \\
\text { dem Schneidrad bei } b_{0} \text { und Ausbau bei } b_{v} \text { bzw. } b_{l(m)} \text { in der Vortriebsunterbre- } \\
\text { chung } I_{(m)}(G l .135) \text {. }\end{array}$ \\
\hline \multirow[t]{2}{*}{$\begin{array}{l}\text { Anzahl Vortriebsunterbrech- } \\
\text { ungen über die Tunnelachse }\end{array}$} & $\mathrm{n}_{\mathrm{I}(\mathrm{TA})}$ & - & Anzahl der Vortriebsunterbrechungen $I_{(m)}$ über die Tunnelachse TA. \\
\hline & $\mathrm{n}_{\mathrm{I}, \mathrm{e}(\mathrm{TA})}$ & - & $\begin{array}{l}\text { Erwartungswert der Anzahl der Vortriebsunterbrechungen } \mathrm{I}_{(\mathrm{m})} \text { über die Tun- } \\
\text { nelachse TA. (Gl. 138). }\end{array}$ \\
\hline \multirow[t]{2}{*}{$\begin{array}{l}\text { Anzahl Werkzeugwechsel je } \\
\text { Vortriebsunterbrechung }\end{array}$} & $\mathrm{n}_{\mathrm{c}(\mathrm{m})}$ & - & $\begin{array}{l}\text { Anzahl der Abbauwerkzeuge } c \text {, die in der Vortriebsunterbrechung } I_{(m)} \text { gewech- } \\
\text { selt werden. }\end{array}$ \\
\hline & $\mathrm{n}_{\mathrm{c}, \mathrm{e}(\mathrm{m})}$ & - & $\begin{array}{l}\text { Erwartungswert der Anzahl der Abbauwerkzeuge c, die in der Vortriebsunter- } \\
\text { brechung } \mathrm{I}_{(\mathrm{m})} \text { gewechselt werden (Gl. 137) }\end{array}$ \\
\hline \multirow[t]{2}{*}{$\begin{array}{l}\text { Anzahl Werkzeugwechsel über } \\
\text { die Tunnelachse }\end{array}$} & $\mathrm{n}_{\mathrm{c}(\mathrm{TA})}$ & - & $\begin{array}{l}\text { Anzahl der Abbauwerkzeuge c, die in Summe über die Tunnelachse TA } \\
\text { gewechselt werden. }\end{array}$ \\
\hline & $\mathrm{n}_{\mathrm{c}, \mathrm{e}(\mathrm{TA})}$ & - & $\begin{array}{l}\text { Erwartungswert der Anzahl der Abbauwerkzeuge c, die in Summe über die } \\
\text { Tunnelachse TA gewechselt werden (Gl. 138). }\end{array}$ \\
\hline Index & s & - & Index für die Schneidspurnummer \\
\hline \multirow[t]{2}{*}{ Anzahl Werkzeugwechsel } & $\mathrm{n}_{\mathrm{c}(\mathrm{s})}$ & - & $\begin{array}{l}\text { Anzahl der Abbauwerkzeuge c, die je Schneidspur s in Summe über die } \\
\text { Tunnelachse TA gewechselt werden. }\end{array}$ \\
\hline & $\mathrm{n}_{\mathrm{c}, \mathrm{e}(\mathrm{s})}$ & - & $\begin{array}{l}\text { Erwartungswert der Anzahl der Abbauwerkzeuge c, die je Schneidspur in } \\
\text { Summe über die Tunnelachse TA gewechselt werden. }\end{array}$ \\
\hline Werkzeugkontrollzahl & $\mathrm{n}_{\mathbf{l}(\mathrm{Ic})}$ & - & $\begin{array}{l}\text { Durchschnittliche Anzahl der Vortriebsunterbrechungen zur Kontrolle und } \\
\text { Wechsel von Abbauwerkzeugen zwischen Einbau } b_{0} \text { und Ausbau b b eines } \\
\text { Abbauwerkzeuges c. }\end{array}$ \\
\hline Verschleißkosten & $\mathrm{K}_{\mathrm{v}}$ & $€$ & Tab. 23 \\
\hline $\begin{array}{l}\text { Erwartungswert Verschleiß- } \\
\text { kosten }\end{array}$ & $\mathrm{K}_{\mathrm{v}, \mathrm{e}}$ & $€$ & Tab. 23 \\
\hline $\begin{array}{l}\text { Abweichung Anzahl Werk- } \\
\text { zeugwechsel }\end{array}$ & $d_{n c}$ & $\%$ & $\begin{array}{l}\text { Abweichung zwischen dem Erwartungswert } n_{c, e(m)} \text { der Anzahl der Werkzeug- } \\
\text { wechsel und der tatsächlichen Anzahl der Werkzeugwechsel } n_{c(m)} \text {. }\end{array}$ \\
\hline Abweichung Laufweg & $d_{s c}$ & $\%$ & $\begin{array}{l}\text { Abweichung zwischen dem Erwartungswert } \mathrm{s}_{\mathrm{cv}, \mathrm{e}(\mathrm{m})} \text { des Laufwegs der Abbau- } \\
\text { werkzeuge aus dem Prognosemodell und dem tatsächlichen Laufweg } \mathrm{s}_{\mathrm{cv},(\mathrm{m})} \\
\text { (Gl. 139). }\end{array}$ \\
\hline Sicherheitsbeiwert & $Y_{n c}$ & - & $\begin{array}{l}\text { Sicherheitsbeiwert zur Korrektur des Erwartungswertes der Anzahl der Werk- } \\
\text { zeugwechsel } n_{c, e(m)} \text {. }\end{array}$ \\
\hline $\begin{array}{l}\text { Korrigierter Erwartungswert der } \\
\text { Anzahl Werkzeugwechsel }\end{array}$ & $\mathrm{n}_{\mathrm{c}, \gamma(\mathrm{m})}$ & - & $\begin{array}{l}\text { Mit } Y_{n c} \text { korrigierter Erwartungswert der Anzahl der Abbauwerkzeuge c, die in } \\
\text { der Vortriebsunterbrechung } I_{(m)} \text { gewechselt werden (Gl. 140). }\end{array}$ \\
\hline Sicherheitsbeiwert & $\mathrm{Y}_{\mathrm{sc}}$ & - & $\begin{array}{l}\text { Sicherheitsbeiwert zur Korrektur des Erwartungswertes für den Laufweg } \mathrm{s}_{\mathrm{c}, \mathrm{e}(\mathrm{z})} \\
\text { (Gl. 141). }\end{array}$ \\
\hline $\begin{array}{l}\text { Korrigierter Erwartungswert des } \\
\text { Laufweg }\end{array}$ & $\mathrm{S}_{\mathrm{cv}, \mathrm{\gamma}(\mathrm{m})}$ & - & $\begin{array}{l}\text { Mit } Y_{s c} \text { korrigierter Erwartungswert des Laufwegs des Abbauwerkzeuges } c \\
\text { zwischen Einbau auf dem Schneidrad bei } b_{0} \text { und Ausbau bei } b_{v} \text { bzw. } b_{(m)} \text { in der } \\
\text { Vortriebsunterbrechung } I_{(m)} \text {. }\end{array}$ \\
\hline Sicherheitsbeiwert & $\mathrm{YL}$ & - & $\begin{array}{l}\text { Sicherheitsbeiwert zur Korrektur des Erwartungswertes der Vortriebsstrecken } \\
L_{\max (m)} \text {. }\end{array}$ \\
\hline $\begin{array}{l}\text { Korrigierter Erwartungswert der } \\
\text { max. Vortriebsstrecken }\end{array}$ & $\mathrm{L}_{\max , Y(m)}$ & - & $\begin{array}{l}\text { Mit } y_{\llcorner} \text {korrigierte maximal erreichbare Vortriebsstrecke der TVM ausgehend von } \\
\text { der Vortriebsunterbrechung } I_{(m-1)} \text { bis zum erreich der Verschleißgrenze des } \\
\text { ersten Abbauwerkzeuges auf dem Schneidrad (Gl. 143). }\end{array}$ \\
\hline Kostentrendanalyse & KTA & - & Abkürzung Kostentrendanalyse. \\
\hline
\end{tabular}

